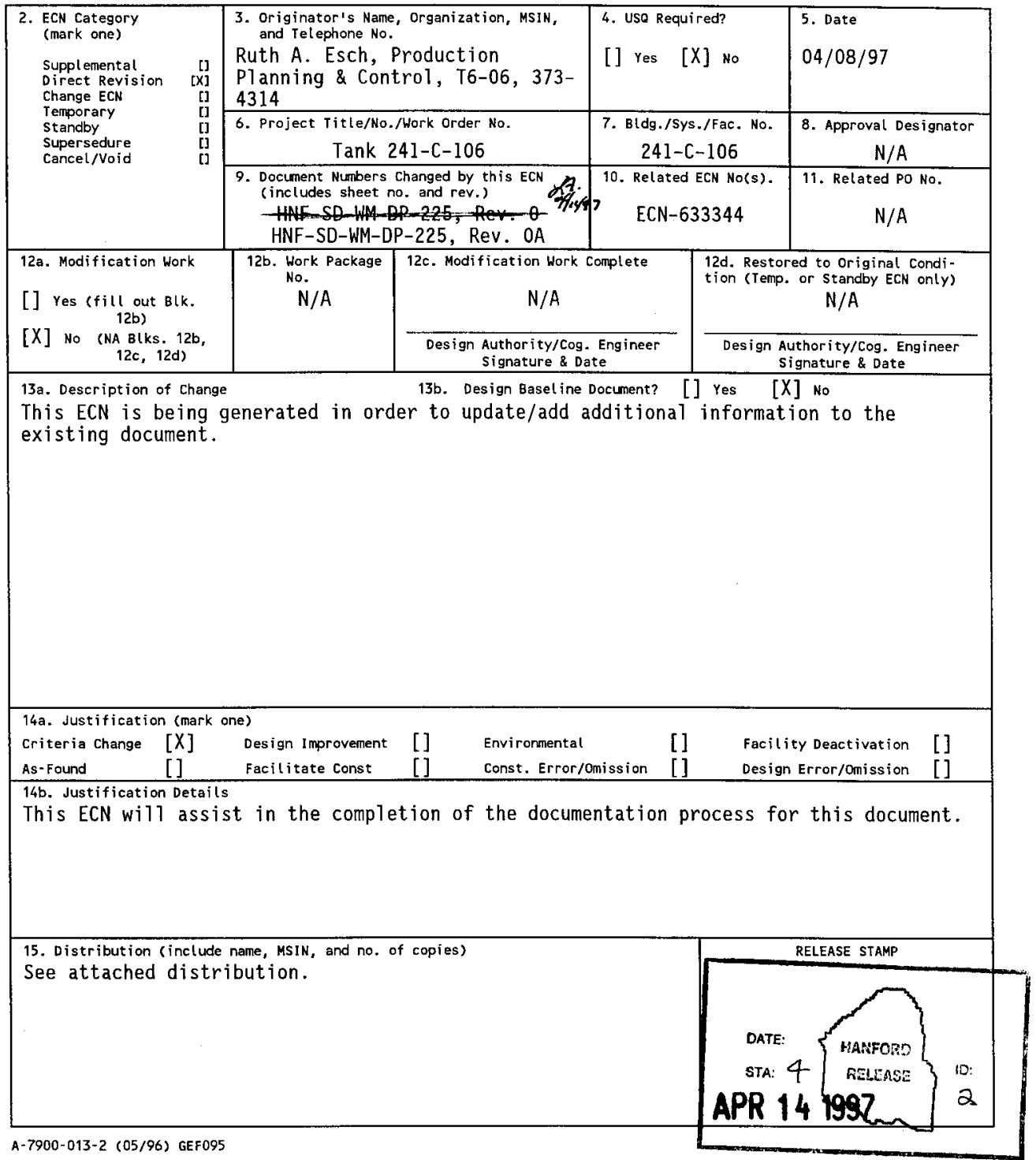




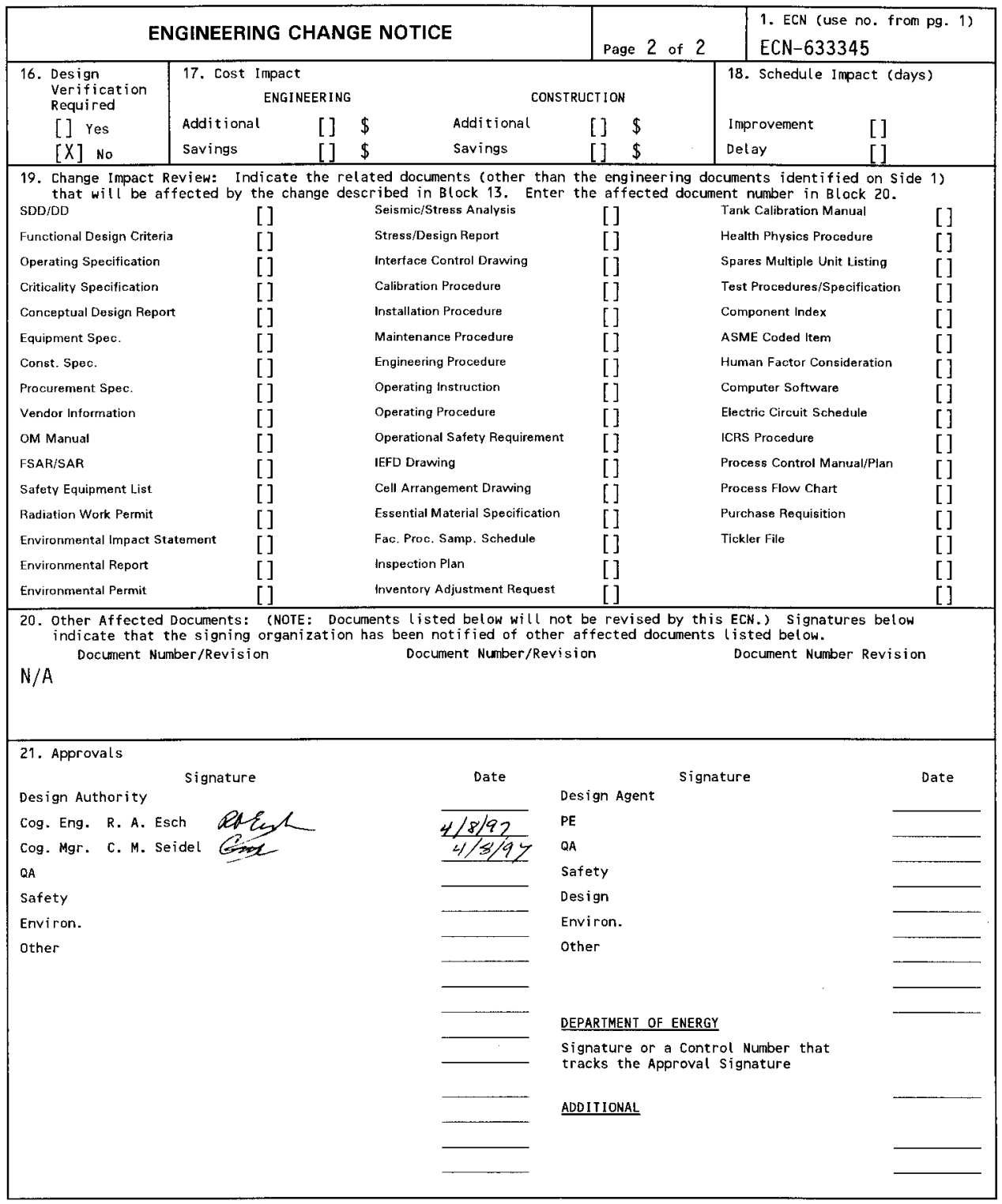




\title{
Tank Waste Remediation System (TWRS) Privatization Contractor Samples Waste Envelope D Material 241-C-106
}

\author{
Ruth A. Esch
}

Rust Federal Services of Hanford, Inc., Richland, WA 99352

U.S. Department of Energy Contract DE-AC06-96RL13200

$\begin{array}{lll}\text { EDT } / E C N: & \text { ECN-633345 } & \text { UC: } 2070 \\ \text { Org Code: } & 7576 B & \text { Charge Code: MD378 } \\ \text { B\&R Code: } & \text { EW } 3120074 & \text { Total Pages: } 232\end{array}$

Key Words: Tank, Waste, Remediation, System, TWRS, Privatization, Contractor Samples, Samples, Waste Envelope, Envelope D, D, Material, 241-C-106, C-106

Abstract: $\mathrm{N} / \mathrm{A}$

TRADEMARK DISCLAIMER. Reference herein to any specific comercial product, process, or service by trade name, trademark, manufacturer, or otherwise, does not necessarily constitute or imply its endorsement, recommendation, or favoring by the United States Government or any agency thereof or its contractors or subcontractors.

Printed in the United States of America. To obtain copies of this document, contact: Document Control Services, P.D. Box 950, Mailstop H6-08, Richland WA 99352, Phone (509) 372-2420; Fax (509) 376-4989.
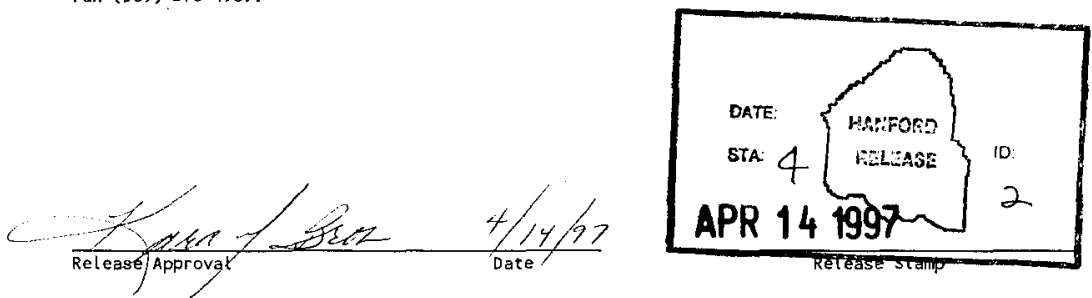

Approved for Public Release 


\section{RECORD OF REVISION}

(1) Document Number

HNF-SD-WM-DP-225

Page 1

(2) Jitle

Tank Waste Remediation System (TWRS) Privatization Contractor Samples Waste Envelope D

Material 24l-C-106

CHANGE CONTROL RECORD

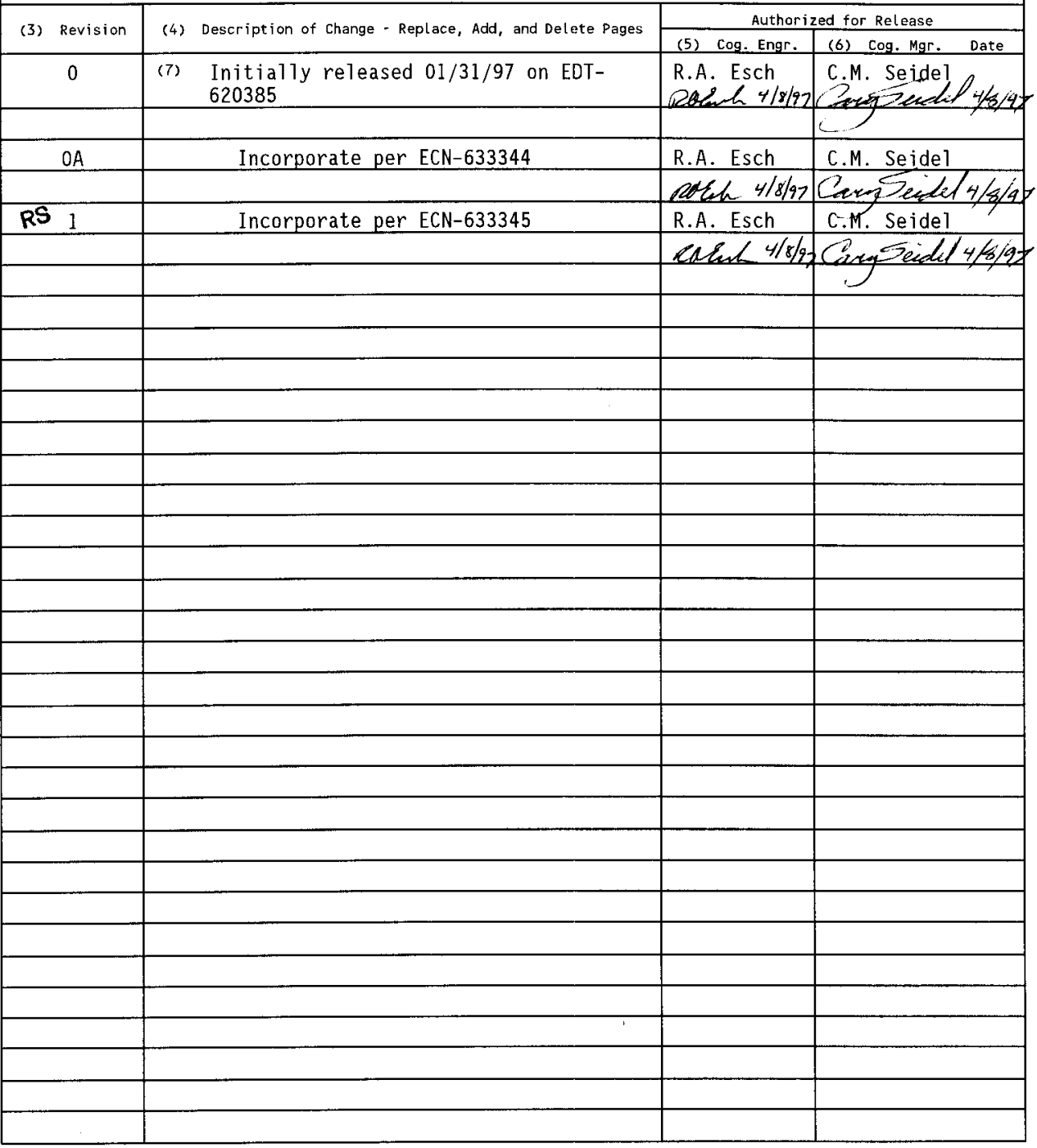


HNF-SD-WM-DP-225, REV. 1

TANK WASTE REMEDIATION SYSTEM (TWRS) PRIVATIZATION

CONTRACTOR SAMPLES

WASTE ENVELOPE D MATERIAL

241-C-106

\section{FINAL ANALYTICAL REPORT}

Prepared by: Michael W. Urie

Pacific Northwest National Laboratory (PNNL)

Analytical Chemistry Laboratory (ACL)

C-106 Project Manager

April 1, 1997 


\author{
Contributors \\ OP Bredt \\ KP Brooks \\ SK Fadeff \\ LR Greenwood \\ KJ Kuhl-Klinger \\ GJ Lumetta \\ EA Nelson \\ MM O'Neill \\ GK Patello \\ RT Steele \\ KJ Smith \\ JM Tingey \\ MW Urie \\ JJ Wagner \\ KD Wiemers \\ EJ Wyse
}


HNF-SD-WM-DP-225, REV. 1

Final Analytical Report - April 1, 1997 .

TANK WASTE REMEDIATION SYSTEM (TWRS) PRIVATIZATION CONTRACTOR SAMPLES

WASTE ENVELOPE D MATERIAL

C-106

FINAL ANALYTICAL REPORT 
Table of Contents

$1.0 \quad$ INTRODUCTION $\ldots \ldots \ldots \ldots \ldots \ldots \ldots \ldots \ldots \ldots \ldots \ldots \ldots \ldots \ldots \ldots$

1.1 Summary of Characterization Activities $\ldots \ldots \ldots \ldots \ldots \ldots \ldots \ldots \ldots \ldots$

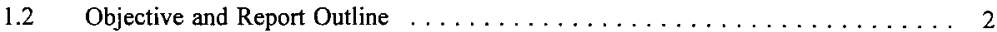

$2.0 \quad$ SAMPLE HANDLING $\ldots \ldots \ldots \ldots \ldots \ldots \ldots \ldots \ldots \ldots \ldots \ldots \ldots \ldots$

2.1 Initial Grab Sample Composite Preparation $\ldots \ldots \ldots \ldots \ldots \ldots \ldots \ldots \ldots$

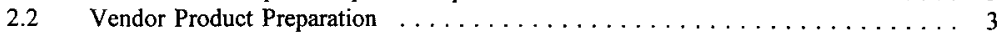

2.3 Analytical Sample Preparation $\ldots \ldots \ldots \ldots \ldots \ldots \ldots \ldots \ldots \ldots \ldots \ldots$

3.0 SUMMARY OF ANALYTICAL RESULTS AND ASSESSMENT OF DATA

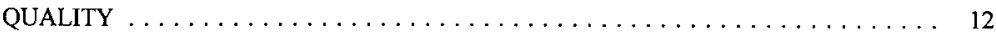

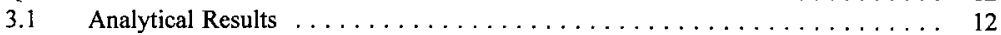

3.2 Data Quality - Comparison of Analytical Results to Detection Limits . . . . . . 17

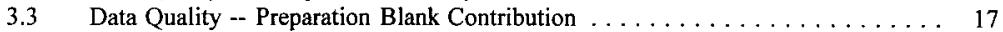

3.4 Data Quality -- Reproducibility $\ldots \ldots \ldots \ldots \ldots \ldots \ldots \ldots \ldots \ldots \ldots$

3.5 Data Quality -- Accuracy ... . . . . . . . . . . . . . . . 19

3.6 Preparation and Analysis Comparison for ICP-AES and ICP-MS Results . . . . 19

3.6.1 Evaluation of Differences Between ICP-AES and ICP-MS Results . . . . 24

3.6.2 Evaluation of ICP-AES and ICP-MS Differences Between Preparation

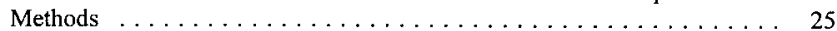

3.7 Analyte/Radionuclide Comparisons Based on Different Methods/Procedures . . . 26

4.0 VENDOR PRODUCT PROCESSING AND PHYSICAL MEASUREMENT $\ldots \ldots \ldots . .28$

4.1 Discrepancies Between Laboratory-Scale and Bench-Scale Results . . . . . . . . 28

4.2 Subsampling Adequacy of the Bench-Scale Processing Apparatus . . . . . . . 29

4.3 Problems Resulting in the Need for Revision 2 to LOI . . . . . . . . . . . 30

4.4 Discrepancies Between SAL and HLRF Weight Percent Solids Results . . . . . . 31

Evaluation of Mass Loss of Vendor Product $\ldots \ldots \ldots \ldots \ldots$

$5.0 \quad$ ORIGINAL MATERIAL $\ldots \ldots \ldots \ldots \ldots \ldots \ldots \ldots \ldots \ldots \ldots \ldots \ldots \ldots$

5.1 Comparison of Original Material (Dried Samples to Centrifuged Solid+Liquid) . . 35

$5.2 \quad$ Evaluation of Effect of Removal of Suspect Organic Layer . . . . . . . . . . 38

6.0 OBSERVATIONS, LIMITATIONS, and RECOMMENDATIONS . . . . . . . . 39

$6.1 \quad$ Data Acceptability . . . . . . . . . . . . . . . . . . . . 39

6.2 Laboratory-Scale and Bench-Scale Operations $\ldots \ldots \ldots \ldots \ldots \ldots \ldots \ldots \ldots \ldots$

6.3 Chemical and Physical Measurement Analysis . . . . . . . . . . . . . 40

$7.0 \quad$ REFERENCES $\ldots \ldots \ldots \ldots \ldots \ldots \ldots \ldots \ldots \ldots \ldots \ldots \ldots \ldots \ldots \ldots$ 


\section{HNF-SD-WM-DP-225, REV. 1

\section{List of Tables}

Table 2.1. Analyte Preparation and Analysis Methods for Reported Results . . . . . . . . . . 6

Table 2.2. Radionuclide Preparation and Analysis Methods for Reported Results . . . . . . . . . 9

Table 2.3. Physical Measurement Methods for Reported Results . . . . . . . . . . . . . 11

Table 3.1. Analyte Results -- Data Quality Summary . . . . . . . . . . . . . . . . 13

Table 3.2. Radionuclide Results -- Data Quality Summary . . . . . . . . . . . . . 15

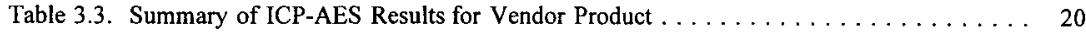

Table 3.4. Summary of ICP-MS Results for Vendor Product $\ldots \ldots \ldots \ldots \ldots \ldots \ldots \ldots \ldots$

Table 3.5. Analyte Comparison From Different Methods/Procedure for Vendor Product . . . . 27

Table 4.1. Summary of Physical Measurement for LOI Revisions 0,1 and $2 \ldots \ldots \ldots$

Table 5.1. Comparison of Original Material Slurry and Centrifuged Fractions . . . . . . . . 37

\section{List of Figures}

Figure 4.1. Material Losses During Sodium Adjustment Treatment (LOI Revision 1) . . . . . 34 


\section{List of Attachments}

Attachment 1: Tank Waste Remediation System Privatization Contractor Samples Analytical Summary Report, Revision 1. (Urie 1997)

Attachment 2: Tank Waste Remediation System Privatization Contractor Samples Analytical Summary Report, Addendum 1, Revision 1. (Kuhl-Klinger and Urie 1997)

Attachment 3: Letter Report "PNNL ACL Submittal of Organic and Radionuclide Results from Analysis of C-106 Original Material Supernate" (Urie 1996)

Attachment 4: Tank Waste Remediation System Privatization Contractor Samples Final Statistical Analysis Report. (Welsh 1997)

Attachment 5: Tank Waste Remediation System Privatization Contractor Samples -- Primary Analytical Data

5A - Analytical Service Requests and Test Instructions

$5 B$ - Chain of Custody

5C - Sample Preparation/Distribution Data Sheets

5D - Physical Measurements

$5 \mathrm{E}$ - Inorganic Analysis (Excluding ICP-AES and ICP-MS)

5F - ICP-AES

5G - ICP-MS

5H - Radiochemistry Analysis 


\section{HNF-SD-WM-DP-225, REV. 1}

Terms/Acronyms

$\begin{array}{ll}\text { ACL } & \text { Analytical Chemistry Laboratory } \\ \text { AEA } & \text { Alpha Energy Analysis } \\ \text { CVAA } & \text { Cold Vapor Atomic Absorption } \\ \text { DL } & \text { Detection Limit (Instrument Detection Limit) } \\ \text { DSC } & \text { Differential Scanning Calorimetry } \\ \text { EQL }{ }^{(1)} & \text { Estimated Quantitation Limit } \\ \text { GEA } & \text { Gamma Energy Analysis } \\ \text { HLRF } & \text { High-Level Radiation Facility } \\ \text { IC } & \text { Ion Chromatography } \\ \text { ICP-AES } & \text { Inductively Coupled Plasma Atomic Emission Spectrometry } \\ \text { ICP-MS } & \text { Inductively Coupled Plasma Mass Spectroscopy } \\ \text { ISE } & \text { Ion Specific Electrode } \\ \text { LCS } & \text { Laboratory Control Standard } \\ \text { LOI } & \text { Letter of Instruction } \\ \text { MDA } & \text { Minimum Detectable Activity } \\ \text { PNNL } & \text { Pacific Northwest National Laboratory } \\ \text { QC } & \text { Quality Control } \\ \text { RPD } & \text { Relative Percent Difference } \\ \text { RSD } & \text { Relative Standard Deviation } \\ \text { SAL } & \text { Shielded Analytical Laboratory } \\ \text { TWRS } & \text { Tank Waste Remediation System }\end{array}$

(1) For definition of these terms and a definition of QC terms see Analytical Summary Report (Urie 1997). 


\subsection{INTRODUCTION}

This report represents the Final Analytical Report on Tank Waste Remediation System (TWRS) Privatization Contractor Samples for Waste Envelope D. All work was conducted in accordance with "Addendum 1 of the Letter of Instruction (LOI) for TWRS Privatization Contractor Samples Addressing Waste Envelope D Materials - Revision 0, Revision 1, and Revision 2." (Jones 1996, Wiemers 1996a, Wiemers 1996b)

Tank 241-C-106 (C-106) was selected by TWRS Privatization for the Part 1A Envelope D high-level waste demonstration. Twenty bottles of Tank C-106 material were collected by Westinghouse Hanford Company using a grab sampling technique and transferred to the 325 building for processing by the Pacific Northwest National Laboratory (PNNL). At the 325 building, the contents of the twenty bottles were combined into a single Initial Composite Material. This composite was subsampled for the laboratory-scale screening test and characterization testing, and the remainder was transferred to the 324 building for bench-scale preparation of the Privatization Contractor Samples.

\subsection{Summary of Characterization Activities}

The C-106 material was characterized during five sequential stages of the Envelope D Vendor Product preparation. These stages are summarized as follows:

1) Laboratory-Scale Screening Test: Analyses were conducted during and after completing the laboratory-scale washing and leaching screening test. The laboratory-scale screening test was used to establish operational parameters for the bench-scale batch processing. Details of the screening test are provided by Lumetta (1996).

2) Original Material Characterization: Analyses were performed on the feed material used for the bench-scale processing. The feed material was a blend of the Initial Composite Material with distilled water (at a 3:2 Initial Composite Material to water ratio). This feed material (i.e., diluted Initial Composite Material) is referred to, in this and previous reports, as "Original Material". The analytical results establish the baseline concentration of the bench-scale processing feed material and are used to establish the effectiveness of the washing and leaching process. The analytical and radionuclide results of the Original Material reported by Urie (1997).

3) Vendor Product Characterization: Analyses were performed on the material resulting from the bench-scale batch processing per revision 0 of the LOI. This prepared Envelope D material is referred to, in this and previous reports, as "Vendor Product". The details of the bench-scale operation and the effectiveness of the washing and leaching process are discussed by Brooks (1997). The analytical and radionuclide results for the Vendor Product are reported by Urie (1997).

4) Sodium Adjustment Treatment: The Vendor Product did not meet the Envelope D specification for a few analytes/radionuclides, most importantly for sodium. Therefore, the Vendor Product was subjected to a decant/dilute sodium adjustment treatment, per revision 1 of the LOI, to reduce the sodium concentration. Following the decanted/diluted treatment, the Vendor Product is referred to, in this and previous reports, as "Final Vendor Product".

Estimates of the Final Vendor Product analyte and radionuclide concentrations were made 
based on the quantity of sodium removed and the percent solids of the Final Vendor Product. Kuhl-Klinger and Urie (1997) provide details of the sodium treatment process and the criteria used to estimate the Final Vendor Product concentration.

5) Final Vendor Product Characterization: The initial measurement of the weight percent solids and weight percent oxides of the Final Vendor Product were inconsistent with estimated values. Based on this and the variability of the settled solids in each of the Final Vendor Product bottles (ten bottles for each vendor), additional analyses (albeit, a limited set of analyses) were performed following reblending and rebottling per revision 2 of the LOI. These analyses were used to confirm the estimates reported for the Final Vendor Product following the sodium adjustment treatment. The result of the analyses of the Final Vendor Product are reported by Kuhl-Klinger and Urie (1997).

The analytical results for the Original Material and Vendor Product (including Final Vendor Product) have been presented and discussed in previous reports which are included as Attachment 1 (Urie 1997) and Attachment 2 (Kuhl-Klinger and Urie 1997). Also included are the letter report detailing organic and radiochemistry analyses performed on Original Material supernatant as Attachment 3 (Campbell 1996) and the Final Statistical Report (Welsh 1997) for the Vendor Product as Attachment 4. The details of the analytical performance and raw analytical data are presented in Attachment 5 (consisting of Sections $5 \mathrm{~A}$ through $5 \mathrm{H}$ ) for all stages of characterization, including the laboratory-scale screening test and bench-scale processing.

The final reports detailing the laboratory-scale sereening test and final bench-scale preparation of the Vendor Product are provided by Lumetta (1996) and Brooks (1997), respectively. Although these reports are not included as Attachments, laboratory-scale test and bench-scale processing operations which potentially affected Vendor Product analyte concentrations are included in this report.

\subsection{Objective and Report Outline}

The overall objective of this report is to evaluate the quality of the analytical data reported, to identify any limitations or discrepancies in the material processing, sampling, or preparation/analysis methods which may impact future characterization efforts, and to provide a basis for the development of the High-Level Waste feed characterization/validation methods. Results from analyses of the Vendor Product and Final Vendor Product are emphasized in this report; however, most of this evaluation also is applicable to the analysis of the Original Material. Each Section in this report is summarized below:

Section 2 provides a summary of the sampling, preparations, and analysis methods used. This includes initial grab sampling, compositing of the grab samples, subsampling for the laboratory-scale screening test, subsampling of the Original Material and Vendor Product during the bench-scale batch processing, and the analytical sampling and analysis methods.

Section 3 evaluates the data quality for the inorganic and radiochemistry results obtained on the Envelope D Privatization Samples. This includes evaluation of the precision and accuracy of the analytical techniques used to characterize the Vendor Product and Final Vendor Product, as well as an evaluation of the analytical sampling methods and the dissolution techniques used to prepare the sample for analysis. A limited set of analyses were performed on the Final Vendor Product following the reblending/rebottling operations, with these analyses providing confirmation of the estimated 
(calculated) concentration of the Final Vendor Product following the sodium adjustment treatment. If analyses were performed on the Final Vendor Product and reported by Kuhl-Klinger and Urie (1997), then these results are evaluated for data quality. Otherwise, the Vendor Product data is used for evaluation.

Section 4 evaluates the data quality for the physical measurements, and identifies problems or concerns related to the laboratory-scale screening test and bench-scale batch processing. Although the reports by Lumetta (1996) and Brooks (1997) address most of the issues related to these processes, the key issues are summarized and evaluated for impact on the reported results.

Section 5 provides an evaluation of the analytical processes by comparing the analysis of the Original Material to the summation of results for the centrifuged solids and centrifuged liquid of the Original Material. The objective is to identify discrepancies which might impact approaches to future characterization efforts or that may impact the interpretation of the analytical results. This section also addresses the impact of removing a suspected organic layer from the Original Material prior to characterization.

Section 6 provides a summary of observations, limitations, and recommendations.

\subsection{SAMPLE HANDLING}

\subsection{Initial Grab Sample Composite Preparation}

Twenty slurry grab samples from tank 241-C-106 were taken from June 21, 1996 through June 24, 1996 at the East Tank Farms (Conners 1996). All the samples were taken from riser 7 at a sample elevation from 406 to 414 inches. These grab samples were received at the 325 building from June 22, 1996 through June 26, 1996. . Due to the dose rates of these samples, the samples were transferred into the High-Level Radiation Facility (HLRF) hot cells where they were homogenized and composited in a series of steps. Each sample was individually homogenized with a high speed mixer, in an ice bath used to control the temperature of the samples during homogenization. The individually homogenized samples were blended together to form a single homogenized C-106 Initial Composite Material. A small aliquot of the Initial Composite Material was transferred to the Shielded Analytical Laboratory (SAL) for the laboratory-scale screening test, while the remainder of the Initial Composite Material was transferred to the 324 building for the bench-scale batch preparation of the Vendor Product.

\subsection{Vendor Product Preparation}

The laboratory-scale washing and caustic leaching screening test performed on C-106 Initial Composite Material is described by Lumetta (1996). The results from this testing were used (as available) to establish the sludge washing treatment parameters for the bench-scale batch preparation of the Vendor Product. Prior to initiating the bench-scale batch processing, approximately 3000 grams of C-106 Initial Composite Material was blended with approximately 2000 grams of deionized water. At this stage, four $125-\mathrm{ml}$ subsamples identified as Original Material were extracted from the bench-scale processing apparatus and sent to the 325 building for physical and chemical characterization. A fifth sample was removed for archive. 


\section{HNF-SD-WM-DP-225, REV. 1}

Final Analytical Report - April 1, 1997

As with the laboratory-scale screening test, two caustic leaches and three inhibited water washes were performed during the bench-scale batch processing. During this processing, each leach or wash step was followed by gravity settling and supernatant removal at $85^{\circ} \mathrm{C}$ for leach and ambient temperature for wash to separate the liquid and solid phases. To evaluate the bench-scale processing, the supernatants removed following each leaching and washing operation were transferred to the 325 building SAL for chemical characterization. The analytical results for these supernatants are included in Attachment 5 for completeness; however, no discussion of the analytical results for these supernatants is included in this report.

At the completion of the bench-scale sludge washing/leaching processing, the resulting material (i.e., Vendor Product) was thoroughly mixed and four subsamples were collected and sent to the 325 building for subsequent physical and chemical characterization. A fifth sample was collected for archive. Also, two 1-L containers of Vendor Product (one containing $1624 \mathrm{~g}$ and the other $1523 \mathrm{~g}$ ) were transferred to the 325 building for subsequent bottling and shipping to the two privatization contractors. The details of the bench-scale processing tasks, procedures, and data evaluation are described by Brooks (1997).

\subsection{Analytical Sample Preparation}

Three of the four subsamples of Original Material and three of the four subsamples of Vendor Product were distributed to the SAL for drying and subsequent analytical sample preparation and/or direct distribution for chemical analysis. The remaining subsamples of the Original Material and Vendor Product were distributed to the HLRF for density, weight loss on drying and firing measurements, and additional physical testing. The preparation methods and analysis procedures used for reporting the results for the Vendor Product and Final Vendor Product are presented in Tables 2.1 - 2.3.

A full explanation of the drying and blending operations performed on the Original Material and Vendor Product chemical and radionuclide characterization samples is detailed in the Analytical Summary Report (Urie 1997). In summary, three subsamples each of Original Material and Vendor Product were dried individually. The dried solids were medium brown in color and the evaporated supernatant left a white coating on top of the dried brown solid layer. No indication of spattering, foaming, etc. during drying was noted. Each dried subsample was manually blended, and then divided into two portions (i.e., a sample and a duplicate) for further sample preparation (e.g., acid digestion) or direct chemical analysis.

For the Original Material subsamples, a clear liquid layer was observed forming on top of each subsample within minutes after transferring the slurries to beakers for drying. The layer was suspected to be organic in nature, because the layer separated rapidly, was distinct from the slurry, and coated the beaker walls when swirled. Due to the concern with drying any material having an organic top layer, the suspected layer was removed. The results of the organic and radiochemical screening of this suspect organic layer is provided in a separate letter report (Campbell 1996), and the effect of the removal of this liquid layer on the Original Material analytical results is discussed in Section 5.2 of this report.

Additional preparations and analyses were performed on the Final Vendor Product. In this case, four bottles of Final Vendor Product were subjected to the same processing as those performed on the three subsamples of the Vendor Product. However, only one of the four Final Vendor Product samples were prepared in duplicate. The average results reported for the Vendor Product and Final Vendor 
Product were calculated from six results for the Vendor Product (i.e., sample and duplicate on each subsample) and five results for the Final Vendor Product (i.e., four samples and one duplicate).

Sample preparations conducted in the SAL included potassium hydroxide-potassium nitrate fusion utilizing nickel crucibles (PNL-ALO-115), sodium peroxide-sodium hydroxide-sodium chloride fusion utilizing zirconium crucibles (PNL-ALO-116), nitric acid-hydrochloric acid digestion (PNL-ALO-129), and water leaching (PNL-ALO-103). Throughout this report these preparation procedures are referred to as $\mathrm{KOH}$ fusion, $\mathrm{Na}_{2} \mathrm{O}_{2}$ fusion, acid digestion, and water leaching, respectively.

All samples were prepared for analysis from the dried slurries. Fusions, digestions, leaches, and direct analyses (except the SAL bulk weight percent solids) were performed on the sample and duplicate portions of each Original Material and Vendor Product dried subsample. Quality control (QC) was applied to the analytical preparation batch in accordance with the governing quality assurance plan (Kuhl-Klinger 1996). The batch QC incorporated the use of preparation blanks, spiked blanks or Laboratory Control Standards (LCS), duplicate samples, matrix spiked samples, and radiochemical tracers, where available and appropriate for use. Sections 3.1.1 to 3.1.3 of the Analytical Summary Report (Urie) provide further details on the fusion, digestion, and leaching procedures used.

Limited analytical and radionuclide characterization was performed on centrifuged solids and centrifuged supernatants which were generated during physical characterization operations conducted in the HLRF. The Original Material centrifuged solids were prepared for chemical characterization using the same preparation methods as used for the dried slurry subsamples. Depending on the analysis method, the Original Material and Vendor Product supernatants were either analyzed directly or subjected to a nitric acid-hydrochloric acid digestion (PNL-ALO-128) prior to analysis. The details of the centrifuged solids and supernatant preparation are discussed in Section 4.5 of the Analytical Summary Report (Urie 1997). A comparison of the summation of the concentration of Original Material centrifuged solids and centrifuged supernatant to the dried Original Material subsamples is discussed in Section 5.1 of this report. A similar comparison of the Vendor Product centrifuged solids/supernatant is not available, since the Vendor Product centrifuged solids were not analyzed. 
HNF-SD-WM-DP-225, REV. 1

Table 2.1. Analyte Preparation and Analysis Methods for Reported Results

\begin{tabular}{|c|c|c|c|c|}
\hline Material & Analyte & $\begin{array}{l}\text { Preparation } \\
\text { Method }\end{array}$ & $\begin{array}{l}\text { Analysis } \\
\text { Method }\end{array}$ & Procedure \\
\hline VP & $\mathrm{Ag}$ & PNL-ALO-116 & ICP-AES & PNL-ALO-211 \\
\hline FVP & $\mathrm{Ag}$ & PNL-ALO-115 & ICP-AES & PNL-ALO-211 \\
\hline VP/FVP & $\mathrm{Al}$ & PNL-ALO-115 & ICP-AES & PNL-ALO-211 \\
\hline VP & As & PNL-ALO-115 & ICP-MS & PNL-ALO-280 \\
\hline VP & B & PNL-ALO-115 & ICP-MS & PNL-ALO-280 \\
\hline VP/FVP & $\mathrm{Ba}$ & PNL-ALO-116 & ICP-AES & PNL-ALO-211 \\
\hline VP & $\mathrm{Be}$ & PNL-ALO-129 & ICP-AES & PNL-ALO-211 \\
\hline VP & $\mathrm{Bi}$ & PNL-ALO-129 & ICP-AES & PNL-ALO-211 \\
\hline FVP & $\mathrm{Bi}$ & PNL-ALO-116 & ICP-AES & PNL-ALO-211 \\
\hline VP/FVP & $\mathrm{Ca}$ & PNL-ALO-129 & ICP-AES & PNL-ALO-211 \\
\hline VP & $\mathrm{Cd}$ & PNL-ALO-115 & ICP-MS & PNL-ALO-280 \\
\hline FVP & $\mathrm{Cd}$ & PNL-ALO-129 & ICP-AES & PNL-ALO-211 \\
\hline VP & $\mathrm{Ce}$ & PNL-ALO-116 & ICP-MS & PNL-ALO-280 \\
\hline VP & $\mathrm{Co}$ & PNL-ALO-115 & ICP-MS & PNL-ALO-280 \\
\hline VP & $\mathrm{Cr}$ & PNL-ALO-115 & ICP-MS & PNL-ALO-280 \\
\hline FVP & $\mathrm{Cr}$ & PNL-ALO-115 & ICP-AES & PNL-ALO-211 \\
\hline VP & $\mathrm{Cs}$ & PNL-ALO-115 & ICP-MS & PNL-ALO-280 \\
\hline VP & $\mathrm{Cu}$ & PNL-ALO-115 & ICP-MS & PNL-ALO-280 \\
\hline FVP & $\mathrm{Cu}$ & PNL-ALO-129 & ICP-AES & PNL-ALO-211 \\
\hline VP & Dy & PNL-ALO-115 & ICP-MS & PNL-ALO-280 \\
\hline VP & Er & PNL-ALO-115 & ICP-MS & PNL-ALO-280 \\
\hline VP & $\mathrm{Eu}$ & PNL-ALO-115 & ICP-MS & PNL-ALO-280 \\
\hline VP/FVP & $\mathrm{Fe}$ & PNL-ALO-115 & ICP-AES & PNL-ALO-211 \\
\hline VP & Gd & PNL-ALO-115 & ICP-MS & PNL-ALO-280 \\
\hline VP & $\mathrm{Hg}$ & Direct & CVAA & PNL-ALO-131/201 \\
\hline VP & Ho & PNL-ALO-115 & ICP-MS & PNL-ALO-280 \\
\hline VP & $\mathrm{K}$ & PNL-ALO-129 & ICP-AES & PNL-ALO-211 \\
\hline VP & $\mathrm{La}$ & PNL-ALO-116 & ICP-MS & PNL-ALO-280 \\
\hline FVP & $\mathrm{La}$ & PNL-ALO-129 & ICP-AES & PNL-ALO-211 \\
\hline VP & $\mathrm{Li}$ & PNL-ALO-129 & ICP-AES & PNL-ALO-211 \\
\hline VP & $\mathrm{Mg}$ & PNL-ALO-116 & ICP-MS & PNL-ALO-280 \\
\hline
\end{tabular}


HNF-SD-WM-DP-225, REV. 1

Table 2.1. Analyte Preparation and Analysis Methods for Reported Results

\begin{tabular}{|c|c|c|c|c|}
\hline Material & Analyte & $\begin{array}{c}\text { Preparation } \\
\text { Method }\end{array}$ & $\begin{array}{l}\text { Analysis } \\
\text { Method }\end{array}$ & Procedure \\
\hline FVP & $\mathrm{Mg}$ & PNL-ALO-115 & ICP-AES & PNL-ALO-211 \\
\hline VP & Mn & PNL-ALO-115 & ICP-MS & PNL-ALO-280 \\
\hline VP & Mo & PNL-ALO-115 & ICP-MS & PNL-ALO-280 \\
\hline VP/FVP & $\mathrm{Na}$ & PNL-ALO-129 & ICP-AES & PNL-ALO-211 \\
\hline VP & $\mathrm{Nb}$ & PNL-ALO-115 & ICP-MS & PNL-ALO-280 \\
\hline VP/FVP & $\mathrm{Nd}$ & PNL-ALO-129 & ICP-AES & PNL-ALO-211 \\
\hline VP & $\mathrm{Ni}$ & PNL-ALO-116 & ICP-MS & PNL-ALO-280 \\
\hline FVP & $\mathrm{Ni}$ & PNL-ALO-116 & ICP-AES & PNL-ALO-211 \\
\hline VP & $\mathrm{p}$ & PNL-ALO-115 & ICP-AES & PNL-ALO-211 \\
\hline FVP & $\mathrm{P}$ & PNL-ALO-116 & ICP-AES & PNL-ALO-21 I \\
\hline VP & $\mathrm{Pb}$ & PNL-ALO-129 & ICP-AES & PNL-ALO-211 \\
\hline FVP & $\mathrm{Pb}$ & PNL-ALO-116 & ICP-AES & PNL-ALO-211 \\
\hline VP & $\mathrm{Pd}$ & PNL-ALO-116 & ICP-MS & PNL-ALO-280 \\
\hline VP & $\mathrm{Pr}$ & PNL-ALO-116 & ICP-MS & PNL-ALO-280 \\
\hline VP & $\mathrm{Pt}$ & PNL-ALO-115 & ICP-MS & PNL-ALO-280 \\
\hline VP & $\mathrm{Rb}$ & PNL-ALO-116 & ICP-MS & PNL-ALO-280 \\
\hline VP & $\mathrm{Re}$ & PNL-ALO-115 & ICP-MS & PNL-ALO-280 \\
\hline VP & $\mathrm{Rh}$ & PNL-ALO-116 & ICP-MS & PNL-ALO-280 \\
\hline VP & Ru & PNL-ALO-115 & ICP-MS & PNL-ALO-280 \\
\hline VP & $\mathrm{Sb}$ & PNL-ALO-129 & ICP-MS & PNL-ALO-280 \\
\hline VP & $\mathrm{Se}$ & PNL-ALO-129 & ICP-AES & PNL-ALO-211 \\
\hline VP/FVP & $\mathrm{Si}$ & PNL-ALO-115 & ICP/AES & PNL-ALO-211 \\
\hline VP & Sn & PNL-ALO-116 & ICP-MS & PNL-ALO-280 \\
\hline VP & Sr & PNL-ALO-116 & ICP-MS & PNL-ALO-280 \\
\hline FVP & $\mathrm{Sr}$ & PNL-ALO-115 & ICP/AES & PNL-ALO-211 \\
\hline VP & $\mathrm{Ta}$ & PNL-ALO-116 & ICP-MS & PNL-ALO-280 \\
\hline VP & $\mathrm{Tb}$ & PNL-ALO-115 & ICP-MS & PNL-ALO-280 \\
\hline VP & $\mathrm{Te}$ & PNL-ALO-115 & ICP-MS & PNL-ALO-280 \\
\hline FVP & $\mathrm{Te}$ & PNL-ALO-129 & ICP-AES & PNL-ALO-211 \\
\hline VP/FVP & Th & PNL-ALO-129 & ICP-AES & PNL-ALO-211 \\
\hline $\mathrm{VP}$ & $\mathrm{Ti}$ & PNL-ALO-116 & ICP-MS & PNL-ALO-280 \\
\hline
\end{tabular}


HNF-SD-WM-DP-225, REV. 1

Table 2.1. Analyte Preparation and Analysis Methods for Reported Results

\begin{tabular}{|c|c|c|c|c|}
\hline Material & Analyte & $\begin{array}{l}\text { Preparation } \\
\text { Method }\end{array}$ & $\begin{array}{l}\text { Analysis } \\
\text { Method }\end{array}$ & Procedure \\
\hline VP & $\mathrm{Tl}$ & PNL-ALO-129 & ICP-AES & PNL-ALO-211 \\
\hline FVP & $\mathrm{Tl}$ & PNL-ALO-116 & ICP-AES & PNL-ALO-211 \\
\hline VP & $\mathrm{Tm}$ & PNL-ALO-115 & ICP-MS & PNL-ALO-280 \\
\hline VP & $\mathrm{U}$ & PNL-ALO-115 & ICP-MS & PNL-ALO-280 \\
\hline FVP & $\mathbf{U}$ & PNL-ALO-115 & Fluorimetry & PNL-ALO-445 \\
\hline VP & $\mathrm{V}$ & PNL-ALO-129 & ICP-MS & PNL-ALO-280 \\
\hline VP & W & PNL-ALO-115 & ICP-MS & PNL-ALO-280 \\
\hline VP & $\mathrm{Y}$ & PNL-ALO-129 & ICP-AES & PNL-ALO-211 \\
\hline VP & $\mathrm{Yb}$ & PNL-ALO-115 & ICP-MS & PNL-ALO-280 \\
\hline VP & $\mathrm{Zn}$ & PNL-ALO-115 & ICP-MS & PNL-ALO-280 \\
\hline VP/FVP & $\mathrm{Zr}$ & PNL-ALO-115 & ICP-AES & PNL-ALO-211 \\
\hline VP & $\mathrm{NH}_{3}$ & PNL-ALO-103 & ISE & PNL-ALO-226 \\
\hline VP/FVP & $\mathrm{C}_{2} \mathrm{O}_{4}$ & PNL-ALO-103 & $\mathrm{IC}$ & PNL-ALO-212 \\
\hline VP/FVP & $\mathrm{Cl}$ & PNL-ALO-103 & IC & PNL-ALO-212 \\
\hline VP & $\mathrm{CN}$ & Direct & Colorimetic & PNL-ALO-285/289 \\
\hline VP/FVP & $\mathrm{F}$ & PNL-ALO-103 & $1 \mathrm{C}$ & PNL-ALO-212 \\
\hline VP/FVP & $\mathrm{NO}_{2}$ & PNL-ALO-103 & IC & PNL-ALO-212 \\
\hline VP/FVP & $\mathrm{NO}_{3}$ & PNL-ALO-103 & IC & PNL-ALO-212 \\
\hline VP/FVP & $\mathrm{PO}_{4}$ & PNL-ALO-103 & IC & PNL-ALO-212 \\
\hline VP/FVP & $\mathrm{SO}_{4}$ & PNL-ALO-103 & IC & PNL-ALO-212 \\
\hline VP & TIC/TOC & Direct & Hot Persulfate & PNL-ALO-381 \\
\hline FVP & $\mathrm{pH}$ & Direct & Potentiometric & PNL-ALO-225 \\
\hline FVP & $\mathrm{OH}$ & Direct & Titration & PNL-ALO-228 \\
\hline
\end{tabular}

CVAA = Cold Vapor Atomic Absorption

FVP $=$ Final Vendor Product

IC = Ion Chromatography

ICP-AES = Inductively Coupled Plasma Atomic Emission Spectrometry

ICP-MS = Inductively Coupled Plasma - Mass Spectroscopy

ISE = Ion Specific Electrode

$\mathrm{VP}=$ Vendor Product 
HNF-SD-WM-DP-225, REV. 1

Table 2.2. Radionuclide Preparation and Analysis Methods for Reported Results ${ }^{(1)}$

\begin{tabular}{|c|c|c|c|}
\hline Radionuclide & Prep Method & Analysis Method & Procedure \\
\hline Total Alpha & PNL-ALO-115 & alpha counting & PNL-ALO-420/421 \\
\hline Total Beta & PNL-ALO-115 & beta counting & PNL-ALO-430/431 \\
\hline${ }^{110 \mathrm{~m}} \mathrm{Ag}$ & PNL-ALO-115 & GEA & PNL-ALO-450 \\
\hline${ }^{241} \mathrm{Am}$ & PNL-ALO-115 & separation, AEA & PNL-ALO-417/422 \\
\hline${ }^{243} \mathrm{Am}$ & PNL-ALO-115 & separation, AEA & PNL-ALO-417/422 \\
\hline${ }^{14} \mathrm{C}$ & Direct & oxidize, LSC & PNL-ALO-428/474 \\
\hline${ }^{113 \times} \mathrm{Cd}^{(2)}$ & N/A & Not Measured & $\mathrm{N} / \mathrm{A}$ \\
\hline${ }^{144} \mathrm{Ce}\left({ }^{144} \mathrm{Pr},{ }^{144 \mathrm{~m}} \mathrm{Pr}\right)$ & PNL-ALO-115 & GEA & PNL-ALO-450 \\
\hline${ }^{242} \mathrm{Cm}\left({ }^{242} \mathrm{Am},{ }^{242 \mathrm{~m}} \mathrm{Am}\right)$ & PNL-ALO- 115 & separation, AEA & PNL-ALO-417/422 \\
\hline${ }^{243+244} \mathrm{Cm}$ & PNL-ALO-115 & separation, AEA & PNL-ALO-417/422 \\
\hline${ }^{60} \mathrm{Co}$ & PNL-ALO-115 & GEA & PNL-ALO-450 \\
\hline${ }^{134} \mathrm{Cs}$ & PNL-ALO-115 & GEA & PNL-ALO-450 \\
\hline${ }^{135} \mathrm{Cs}$ & PNL-ALO-115 & TIMS & PNL-ALO-452 \\
\hline${ }^{137} \mathrm{Cs}\left({ }^{137} \mathrm{Ba}\right)$ & PNL-ALO-115 & GEA & PNL-ALO-450 \\
\hline${ }^{152} \mathrm{Eu}$ & PNL-ALO-115 & GEA & PNL-ALO-450 \\
\hline${ }^{154} \mathrm{Eu}$ & PNL-ALO-115 & GEA & PNL-ALO-450 \\
\hline${ }^{135} \mathrm{Eu}$ & PNL-ALO-115 & GEA & PNL-ALO-450 \\
\hline${ }^{55} \mathrm{Fe}$ & PNL-ALO-115 & $\begin{array}{c}\text { separation, X-ray } \\
\text { count }\end{array}$ & PNL-ALO-435/450 \\
\hline${ }^{3} \mathrm{H}^{(3)}$ & N/A & Not Measured & N/A \\
\hline${ }^{129} \mathrm{I}$ & PNL-ALO-129 & ICP-MS & PNL-ALO-280 \\
\hline${ }^{93 m} \mathrm{Nb}$ & PNL-ALO-115 & separation, beta count & PNL-ALO-4003 \\
\hline${ }^{59} \mathrm{Ni}$ & PNL-ALO-116 & $\begin{array}{c}\text { separation, X-ray } \\
\text { count }\end{array}$ & PNL-ALO-495/450 \\
\hline
\end{tabular}


HNF-SD-WM-DP-225, REV. 1

Table 2.2. Radionuclide Preparation and Analysis Methods for Reported Results ${ }^{(1)}$

\begin{tabular}{|c|c|c|c|}
\hline Radionuclide & Prep Method & Analysis Method & Procedure \\
\hline${ }^{63} \mathrm{Ni}$ & PNL-ALO-116 & separation, LSC & PNL-ALO-495/450 \\
\hline${ }^{237} \mathrm{~Np}$ & PNL-ALO-116 & ICP-MS & PNL-ALO-280 \\
\hline${ }^{107} \mathrm{Pd}$ & PNL-ALO-129 & ICP-MS & PNL-ALO-280 \\
\hline${ }^{238} \mathrm{Pu}$ & PNL-ALO-115 & separation, AEA & PNL-ALO-417/422 \\
\hline${ }^{239} \mathrm{Pu}$ & PNL-ALO-116 & ICP-MS & PNL-ALO-280 \\
\hline${ }^{240} \mathrm{Pu}$ & PNL-ALO-129 & ICP-MS & PNL-ALO-280 \\
\hline${ }^{239+240} \mathrm{Pu}$ & PNL-ALO-115 & separation, AEA & PNL-ALO-417/422 \\
\hline${ }^{241} \mathrm{Pu}$ & PNL-ALO-115 & separation, LSC & PNL-ALO-417/474 \\
\hline${ }^{242} \mathrm{Pu}$. & PNL-ALO-115 & separation, AEA & PNL-ALO-417/422 \\
\hline${ }^{106} \mathrm{Ru}\left({ }^{106} \mathrm{Rh}\right)$ & PNL-ALO-115 & GEA & PNL-ALO-450 \\
\hline${ }^{125} \mathrm{Sb}\left({ }^{125 \mathrm{~m}} \mathrm{Te}\right)$ & PNL-ALO-115 & GEA & PNL-ALO-450 \\
\hline${ }^{79} \mathrm{Se}$ & PNL-ALO-115 & distillation, LSC & PNL-ALO-440/474 \\
\hline${ }^{151} \mathrm{Sm}+{ }^{147} \mathrm{Pm}$ & PNL-ALO-115 & separation, LSC & PNL-ALO-417/474 \\
\hline${ }^{113} \mathrm{Sn}\left({ }^{113 \mathrm{~m}} \mathrm{In}\right)$ & PNL-ALO-115 & $\begin{array}{l}\text { separation, } X \text {-ray and } \\
\text { gamma count }\end{array}$ & PNL-ALO-453/450 \\
\hline${ }^{119 m} \mathrm{Sn}$ & PNL-ALO-115 & $\begin{array}{l}\text { separation, } X \text {-ray and } \\
\text { gamma count }\end{array}$ & PNL-ALO-453/450 \\
\hline${ }^{121 \mathrm{~m}} \mathrm{Sn}$ & PNL-ALO-115 & $\begin{array}{l}\text { separation, X-ray and } \\
\text { gamma count }\end{array}$ & PNL-ALO-453/450 \\
\hline${ }^{126} \mathrm{Sn}\left({ }^{126} \mathrm{Sb},{ }^{126 m} \mathrm{Sb}\right)$ & PNL-ALO-115 & $\begin{array}{l}\text { separation, X-ray and } \\
\text { gamma count }\end{array}$ & PNL-ALO-453/450 \\
\hline${ }^{90} \mathrm{Sr}\left({ }^{90} \mathrm{Y}\right)$ & PNL-ALO-115 & separation, beta count & PNL-ALO-476/431 \\
\hline${ }^{99} \mathrm{Tc}$ & PNL-ALO-129 & ICP-MS & PNL-ALO-280/281 \\
\hline${ }^{232} \mathrm{Th}$ & PNL-ALO-116 & ICP-MS & PNL-ALO-280 \\
\hline${ }^{233} \mathrm{U}$ & PNL-ALO-129 & ICP-MS & PNL-ALO-280/282 \\
\hline${ }^{234} \mathrm{U}$ & PNL-ALO-129 & ICP-MS & PNL-ALO-280/282 \\
\hline
\end{tabular}


Table 2.2. Radionuclide Preparation and Analysis Methods for Reported Results ${ }^{(1)}$

\begin{tabular}{|c|c|c|c|}
\hline Radionuclide & Prep Method & Analysis Method & Procedure \\
\hline${ }^{235} \mathrm{U}$ & PNL-ALO-129 & ICP-MS & PNL-ALO-280/282 \\
\hline${ }^{236} \mathrm{U}$ & PNL-ALO-129 & ICP-MS & PNL-ALO-280/282 \\
\hline${ }^{238} \mathrm{U}$ & PNL-ALO-116 & ICP-MS & PNL-ALO-280/282 \\
\hline${ }^{93} \mathrm{Zr}$ & PNL-ALO-115 & separation, LSC & PNL-ALO-4007/474 \\
\hline
\end{tabular}

$\mathrm{AEA}=$ Alpha Energy Analysis

$\mathrm{GEA}=$ Gamma Energy Analysis

$\mathrm{VP}=$ Vendor Product

FVP $=$ Final Vendor Product

LSC $=$ Liquid Scintillation Count

ICP-MS = Inductively Coupled Plasma Mass Spectroscopy

TIMS = Thermal Ionization Mass Spectroscopy

(1) Radiochemistry performed on Vendor Product only, not on Final Vendor Product.

(2) No method available for determining ${ }^{113 \mathrm{~m}} \mathrm{Cd}$.

(3) Tritium analysis was not performed because all samples were dried, and tritium loss was assumed.

Table 2.3. Physical Measurement Methods for Reported Results

\begin{tabular}{|c|c|c|c||}
\hline Material & Physical Test & Analysis Method & Procedure \\
\hline \hline VP & Vol\% Solids & $\begin{array}{c}\text { Volume/Height } \\
\text { Measurement }\end{array}$ & PNL-ALO-501 \\
\hline VP/FVP & Density & $\begin{array}{c}\text { Volume/Mass } \\
\text { Measurement }\end{array}$ & PNL-ALO-501 \\
\hline VP/FVP & Wt\% Solids & $\begin{array}{c}\text { Gravimetric } \\
\left.\text { (Dried at } 105^{\circ} \mathrm{C}\right)\end{array}$ & PNL-ALO-501/504 \\
\hline VP/FVP & Wt\% Oxides & $\begin{array}{c}\text { Gravimetric (Fired } \\
\left.\text { at } 1050^{\circ} \mathrm{C}\right)\end{array}$ & PNL-ALO-501 \\
\hline VP & $\begin{array}{l}\text { Shear Strength/Apparent } \\
\text { Viscosity/Yield Strength }\end{array}$ & Viscometer & PNL-ALO-501/502 \\
\hline VP & Heat Capacity & DSC & PNL-ALO-508 \\
\hline
\end{tabular}

$\mathrm{DSC}=$ Differential Scanning Calorimetry

FVP $=$ Final Vendor Product

$\mathrm{VP}=$ Vendor Product 


\subsection{Analytical Results}

All analytical results for the Vendor Product and Final Vendor product have been reported previously (Urie 1997, Kuhl-Klinger and Urie 1997). A summary of the best available analytical results and data quality assessment for the inorganic analyses and radionuclide analyses performed on Vendor Product and Final Vendor Product are presented in Table 3.1 and Table 3.2, respectively. The "Method" identifies both the analysis methods and preparation method used to generate the reported analytical results, and "VP or FVP" identifies the material analyzed (i.e., either Vendor Product or Final Vendor Product"). Where available, the analytical results for the Final Vendor Product are used for data quality evaluation. Where not available, the Vendor Product results are used for data quality evaluation and comparison to the Envelope D specification. The data quality evaluation is expected to be applicable to the Original Material analysis as well.

The "Average" is the mean analyte or radionuclide concentration for the six analyses on the three subsamples of the Vendor Product, and the five analyses on the four samples of Final Vendor Product. The reported "Average" result uses only results which are above the detection limit; i.e., any results less than the detection limit are not included in the average. This approach has been taken to ensure that averages are not biased low by the less than results, and that the highest (i.e., most conservative) average is used for comparison to the Envelope D specification. If all measurements for an analyte or radionuclide are below the detection limit, the "Average" result is reported as the highest less than value, and for these values the relative standard deviation (RSD) is not applicable ("NA").

Although there may be six sets of analytical data for some analytes resulting from all possible combinations of two analytical techniques (Inductively coupled plasma atomic emission spectrometry [ICP-AES] and inductively coupled plasma mass spectroscopy [ICP-MS]) and three sample preparation techniques ( $\mathrm{KOH}$ fusion, $\mathrm{Na}_{2} \mathrm{O}_{2}$ fusion, and acid digestion), only one set of data is selected for reporting and data quality evaluation (e.g., ICP-AES acid digestion). In selecting this set of data, an effort has been made to evaluate the characteristics of the analytical technique and the limitation of the preparation method. In general, where the preparation methods are considered equivalent and the measured result for the analyte (by either ICP-AES or ICP-MS) is above the estimated quantitation limit (EQL), the highest reported average is selected. The exceptions to this are where: a) the relative standard deviation for the analysis set is greater than $10 \%$; b) processing blanks are greater than $5 \%$ of the reported results; c) the ICP-AES is near the EQL; and/or d) quality control failures are considered significant (e.g., matrix spike recovery at $30 \%$ or LCS recovery at $200 \%$ ). Where the only reported results for ICP-AES and ICP-MS are less than values (i.e., beryllium, potassium, selenium, thallium, and yttrium), the ICP-AES results are used for evaluation since the ICP-AES results are considered the more reliable and provide the most conservative less than value. 


\begin{tabular}{|c|c|c|c|c|c|c|c|c|c|c|c|c|}
\hline 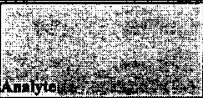 & 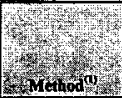 & $\begin{array}{l}\text { ver } \\
\text { ror } \\
\text { rup }\end{array}$ & minge & 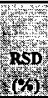 & 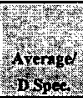 & 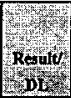 & 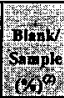 & 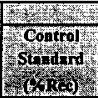 & 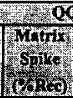 & 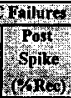 & 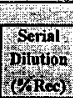 & $\begin{array}{l}\text { Cont } \\
\text { Not } \\
\text { Spiked }\end{array}$ \\
\hline $\mathrm{Ag}$ & ICP NiF & FVP & $1.62 \mathrm{E}+03$ & 38 & 0.726 & 36 & nbd & & & & & \\
\hline A) & ICP NiF & FVP & $6.68 \mathrm{E}+04$ & 7 & 0.462 & 614 & 0.5 & & & & & \\
\hline As & ICP-MS NiF & $\mathrm{VP}$ & $2.86 \mathrm{E}+02$ & 15 & 0.222 & 286 & 4 & 127 & & 156 & & \\
\hline B & ICP-MS NiF & VP & $2.29 \mathrm{E}+02$ & 36 & 0.022 & 229 & 4 & & & & & $\mathrm{x}$ \\
\hline $\mathrm{Ba}$ & ICP $\mathrm{ZrF}$ & FVP & $5.44 \mathrm{E}+02$ & 3 & 0.012 & 30 & nbd & & & & & \\
\hline $\mathrm{Be}$ & ICP Acid & $\overline{V P}$ & $<1.00 \mathrm{E}+00$ & $\mathrm{~N} / \mathrm{A}$ & 60.002 & 1 & nbd & & & & & \\
\hline$B i$ & ICP ZrF & FVP & $6.60 \mathrm{E}+02$ & 5 & 0.019 & 4 & nbd & & & & & \\
\hline $\mathrm{Ca}$ & ICP Acid & FVP & 4. $83 \mathrm{E}+03$ & 7 & 0.068 & 78 & 1 & & & & 12.3 & \\
\hline Cd & ICP Acid & FVP & $8.40 \mathrm{E}+01$ & 4 & 0.003 & 23 & nbd & & & & & \\
\hline Ce & ICP-MS $Z_{r} F$ & $\mathrm{VP}$ & $2.02 \mathrm{E}+02$ & 11 & 0.031 & 202 & nbd & 49 & & & & \\
\hline Co & ICP-MS NiF & $\mathrm{VP}$ & $2.60 \mathrm{E}+01$ & 24 & 0.007 & 26 & nbd & & & & & \\
\hline $\mathrm{Cr}$ & ICP NiF & FVP & $8.06 \mathrm{E}+02$ & 5 & 0.143 & 22 & nbd & & & & & \\
\hline Cs & ICP-MS NiF & VP & $1.80 \mathrm{E}+01$ & 22 & 0.004 & 18 & 4 & & & & & \\
\hline $\mathrm{Cu}$ & ICP Acid & FVP & $1.30 \mathrm{E}+02$ & 6 & 0.040 & 21 & 2 & & & & & \\
\hline Dy & ICP-MS NiF & VP & $2.02 \mathrm{E}+01$ & 37 & 0,098 & 20 & 13 & 193 & & & & \\
\hline $\mathrm{Er}$ & ICP-MS NiF & $\mathrm{VP}$ & $1.31 \mathrm{E}+0 \mid$ & 36 & 0.509 & 13 & 13 & & & & & $\overline{\mathbf{x}}$ \\
\hline Eu & ICP-MS NiF & $\mathrm{VP}$ & $7.86 \mathrm{E}+00$ & 75 & 0.061 & 8 & nbd & ND@1 $1 \mu \mathrm{g} / \mathrm{g}$ & & & & \\
\hline $\mathbf{F e}$ & ICP NiF & FVP & $1.58 \mathrm{E}+05$ & 4 & 0.614 & 3493 & 0.1 & & & & & \\
\hline Gd & ICP-MS NiF & $\mathrm{VP}$ & $2.50 \mathrm{E}+01$ & 28 & 0.323 & 25 & nbd & & & & & $\mathrm{x}$ \\
\hline Ho & ICP-MS NiF & VP & $6.07 \mathrm{E}+00$ & 25 & 0.236 & 6 & nbd & 395 & & & & \\
\hline $\mathbf{K}$ & ICP Acid & $\mathrm{VP}$ & $<4.92 \mathrm{E}+02$ & $\mathrm{~N} / \mathrm{A}$ & $<0.047$ & $T$ & nbd & & & & & \\
\hline $\mathrm{La}$ & ICP Acid & FVP & $1.12 \mathrm{E}+02$ & 3 & 0.004 & 9 & nbd & & & & & \\
\hline $\mathrm{Li}$ & ICP Acid & VP & $4.60 \mathrm{E}+01$ & 65 & 0.042 & 6 & nibd & & & & & \\
\hline $\mathrm{Mg}$ & ICP NiF & FVP & $1.48 \mathrm{E}+03$ & 5 & 0.075 & 8 & nbd & & & & & \\
\hline $\mathrm{Mn}$ & ICP $Z_{r F}$ & FVP & $3.20 \mathrm{E}+03$ & 3 & 0.055 & 35 & nbd & & & & & \\
\hline Mo & ICP-MS NiF & VP & $5.70 \mathrm{E}+01$ & 24 & 0.011 & 57 & 9 & 258 & & & & \\
\hline $\mathrm{Na}$ & ICP Acid & FVP & $1.20 \mathrm{E}+05$ & 6 & 1.048 & 3224 & 0.1 & & & & 10.9 & \\
\hline Nb & ICP-MS NiF & VP & $3.38 \mathrm{E}+01$ & 77 & 0.437 & 34 & nbd & & & & & $\mathrm{x}$ \\
\hline Nd & ICP Acid & FVP & $3.32 \mathrm{E}+02$ & 4 & 0.021 & 13 & nbd & & & & & \\
\hline $\mathrm{Ni}$ & ICP $\mathrm{ZrF}$ & FVP & $1.91 E+03$ & 6 & 0.086 & 35 & 5 & & & & & \\
\hline $\bar{P}$ & $\mathrm{ICP} \mathrm{ZrF}$ & FVP & $4.81 \mathrm{E}+03$ & 4 & 0.239 & 27 & nbd & & & & & \\
\hline Pb & ${ }_{1 C P} \mathrm{ZFF}$ & FVP & 4.12E+03 & 3 & 0.392 & 23 & nbd & & & & & \\
\hline $\mathrm{Pd}$ & ICP-MS ZrF & VP & $2.74 E+01$ & 19 & 0.027 & 27 & nbd & & & & & \\
\hline Pr & ICP-MS Z $\mathrm{rF}$ & $\mathrm{vp}$ & $8.41 E+01$ & 6 & 0.030 & 84 & nbd & & & & & $\mathrm{x}$ \\
\hline Pt & ICP-MS NiF & VP & $3.07 \mathrm{E}+01$ & 25 & 1.192 & 31 & 14 & & & & & \\
\hline$\overline{\mathrm{Rb}}$ & ICP-MS ZrF & $\overline{\mathrm{VP}}$ & $7.68 \mathrm{E}+00$ & 38 & 0.005 & 8 & nbd & & & & & \\
\hline Re & ICP-MS NiF & VP & $6.61 \mathrm{E}+00$ & 31 & 0.009 & 7 & 18 & & & & & $\mathrm{x}$ \\
\hline $\mathrm{Rh}$ & ICP-MS ZrF & VP & $1.44 E+02$ & 11 & 0.140 & 144 & nbd & & & & & \\
\hline Ru & ICP-MS NiF & VP & $9.57 \mathbf{E}+01$ & 7 & 0.034 & 96 & nbd & & & & . & \\
\hline S (Not Analyzed) & -- & $\ldots$ & - & --. & - & $\ldots$ & nbd & & & & & \\
\hline
\end{tabular}




\begin{tabular}{|c|c|c|c|c|c|c|c|c|c|c|c|c|}
\hline 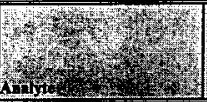 & 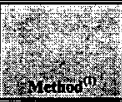 & 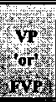 & 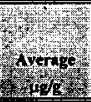 & Rsp & Pros & Rerittix & Bing & Sontrol & 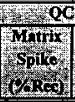 & 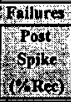 & Kring & Not \\
\hline Sb & ICP-MS Acid & VP & $7,00 \mathrm{E}-01$ & 41 & 0,0001 & 35 & nbd & 32 & & & & \\
\hline $\mathrm{Se}$ & ICP Acid & VP & $<3.69 E+02$ & $\mathrm{~N} / \mathrm{A}$ & $<0.090$ & 1 & nbd & & & & & \\
\hline $\mathrm{Si}$ & ICP NiF & FVP & $8.68 \mathrm{E}+04$ & 6 & 0.461 & 96 & nbd & & & & & \\
\hline $\mathrm{Sn}$ & ICP-MS ZrF & $\mathrm{VP}$ & $1.02 \mathrm{E}+02$ & 28 & 0.360 & 102 & 2 & & & & & \\
\hline Sr & ICP NiF & FVP & $1,00 E+02$ & 4 & 0,036 & 4 & nbd & 129 & & & & \\
\hline $\mathrm{Ta}$ & ICP-MS ZrF & VP & $2.86 \mathrm{E}+01$ & 36 & 0.139 & 29 & nbd & & & & & $\mathrm{X}$ \\
\hline To & ICP-MS NiF & VP & $8.20 \mathrm{E}+00$ & 19 & 0.318 & 8 & nbd & & & & & \\
\hline $\mathrm{Te}$ & ICP-MS NiF & VP & $4.24 \mathrm{E}+02$ & 20 & 0.344 & 424 & 5 & & & & & \\
\hline $\mathrm{Th}$ & ICP Acid & FVP & $4.28 \mathrm{E}+02$ & 6 & 0.847 & 2 & nbd & & & & & \\
\hline $\mathrm{Ti}$ & ICP $\mathrm{ZrF}$ & FVP & $1.24 \mathrm{E}+03$ & 3 & 0.127 & 27 & nbd & & & & & \\
\hline$\pi$ & ICP $\mathrm{ZrF}$ & VP & $<1,23 \mathrm{E}+02$ & $\mathrm{~N} / \mathrm{A}$ & $<0.034$ & 1 & nbd & & & & & \\
\hline $\mathrm{Tm}$ & ICP-MS NiF & VP & $2.64 \mathrm{E}+00$ & 52 & 0.102 & 3 & nbd & & & & & $\mathrm{x}$ \\
\hline $\mathrm{U}$ & U-Laser & FVP & $2.65 \mathrm{E}+03$ & 0.01 & 0,018 & 16 & 1 & & & & & \\
\hline $\mathrm{v}$ & ICP-MS Acid & VP & $3.72 \mathrm{E}+01$ & 18 & 0.144 & 1860 & 11 & & & & & \\
\hline W & ICP-MS NiF & VP & $4.98 \mathrm{E}+02$ & 15 & 0.261 & 498 & 2 & 244 & & & & \\
\hline$Y$ & ICP Acid & VP & $<8.60 \mathrm{E}+01$ & $\mathrm{~N} / \mathrm{A}$ & $<0.067$ & 1 & nbd & & & & & \\
\hline $\mathrm{Yb}$ & ICP-MS NiF & $\mathrm{VP}$ & $1.26 \mathrm{E}+01$ & 49 & 0.489 & 13 & 11 & 1208 & & & & \\
\hline $\mathrm{Zn}$ & ICP-MS NiF & VP & $3.06 \mathrm{E}+02$ & 23 & 0.091 & 306 & 2 & 50 & & & & \\
\hline $\mathrm{zr}$ & $\mathrm{ICP} \mathrm{NiF}$ & FVP & $1.25 \mathrm{E}+03$ & 38 & 0.009 & 14 & nbd & & & & & \\
\hline $\mathrm{Br}$ & IC water leach & VP & $<1.16 \mathrm{E}+02$ & $\mathrm{~N} / \mathrm{A}$ & $N / A$ & 1 & nbd & & & & & \\
\hline$F$ & IC water leach & VP & $<2.91 \mathrm{E}+03$ & $\mathrm{~N} / \mathrm{A}$ & $<0 . \overline{103}$ & $i$ & nbd & & 144 & & & \\
\hline $\mathrm{Cl}$ & IC water leach & VP & $1.26 \mathrm{E}+02$ & 21 & 0.049 & 1 & 27 & & & & & \\
\hline $\mathrm{CN}$ & Direct & $\mathrm{VP}$ & $9.50 \mathrm{E}+00$ & 9 & 0.001 & 27 & 4 & & & & & \\
\hline $\mathrm{Hg}$ & Direct & VP & $3.43 \mathrm{E}+02$ & 5 & 0.444 & 7 & nbd & & & & & \\
\hline $\mathrm{NO}_{2}$ & IC water leach & FVP & $4.00 \mathrm{E}+02$ & 0 & $\mathrm{~N} / \mathrm{A}$ & 2 & nbd & & & & & \\
\hline $\mathrm{NO}_{3}$ & IC water leach & FVP & $5.25 \mathrm{E}+02$ & 10 & N/A & 2 & 25 & & & & & \\
\hline $\mathrm{NO}_{2} \& \mathrm{NO}_{3}$ & IC water leach & FVP & $9.25 \mathrm{E}+02$ & N/A & 0.010 & N/A & $\mathrm{N} / \mathrm{A}$ & & & & & \\
\hline $\mathrm{PO}_{4}$ & IC water leach & FVP & $2.75 E+02$ & 16 & N/A & 1 & nbd & & & & & \\
\hline $\mathrm{SO}_{4}$ & IC water leach & FVP & $3.25 \mathrm{E}+02$ & 14 & $\mathrm{~N} / \mathrm{A}$ & 2 & 28 & & & & & \\
\hline $\mathrm{C}_{2} \mathrm{O}_{4}$ & IC water leach & FVP & $8.33 E+04$ & 0.4 & N/A & 31 & nbd & & & & & \\
\hline $\mathrm{NH}_{3}$ & ISE water leach & $\overline{V P}$ & $2.13 \mathrm{E}+01$ & 43 & 0.002 & 3 & 38 & & & & & \\
\hline $\mathrm{TIC}\left(\mathrm{CO}_{3}\right)$ & Direct & VP & $5.30 \mathrm{E}+03$ & [1 & 0.111 & 589 & N/A & & & & & \\
\hline TOC & Direct & VP & $4.79 E+04$ & 7 & 0.547 & 2994 & N/A & & & & & \\
\hline $\mathrm{TC}$ & Direct & VP & $5.22 \mathrm{E}+04$ & 6 & $\mathrm{~N} / \mathrm{A}$ & $\mathrm{N} / \mathrm{A}$ & $\mathrm{N} / \mathrm{A}$ & & & & & \\
\hline $\begin{array}{l}\mathrm{pH} \\
\mathrm{OH}(\mathrm{N})\end{array}$ & $\begin{array}{l}\text { Direct } \\
\text { Direct }\end{array}$ & $\begin{array}{l}\text { FVP } \\
\text { FVP }\end{array}$ & $\begin{array}{l}11.7 \\
0.021\end{array}$ & $\begin{array}{l}1 \\
6\end{array}$ & $\begin{array}{l}\text { N/A } \\
\text { N/A }\end{array}$ & $\begin{array}{l}\text { N/A } \\
\text { N/A }\end{array}$ & $\begin{array}{l}\mathrm{N} / \mathrm{A} \\
\mathrm{N} / \mathrm{A}\end{array}$ & & & & & \\
\hline $\begin{aligned} \text { (I) Method: } \mathrm{NiF}= & \mathrm{KOH} F \\
\text { Acid }= & \mathrm{HNO} / \mathrm{H} \\
\text { (2) "nbd" }= & \text { No blank concer }\end{aligned}$ & $\begin{array}{l}\text { on (PNL-ALO-1 } \\
\text { Digestion (PNL } \\
\text { tion detected in }\end{array}$ & $\begin{array}{l}\mathrm{ZrF} \\
\mathrm{O}-129 \\
\text { eess bl }\end{array}$ & $\begin{array}{l}{ }_{2} \mathrm{O}_{2} \text { Fusio } \\
\text { /ater Lea } \\
\text { (i.c., blan }\end{array}$ & 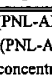 & $\begin{array}{l}116) ; \\
\text { (103). } \\
\text { in }<\text { DL). }\end{array}$ & & & $\begin{array}{l}N / A=\text { Not App } \\
V P=\text { Vendor } \\
\text { FVP = Final } V\end{array}$ & $\begin{array}{l}\text { Plicable; } \\
\text { Product } \\
\text { endor Pr }\end{array}$ & $=1$ & ted & \\
\hline
\end{tabular}




\begin{tabular}{|c|c|c|c|c|c|c|c|c|c|c|c|c|}
\hline 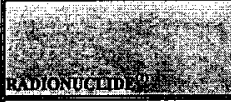 & 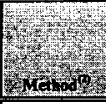 & 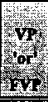 & $\lim _{\operatorname{lin}}$ & Kst & 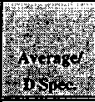 & $2 \ln 2$ & (3ing & Kcontrol & 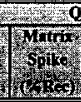 & 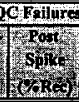 & 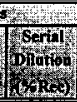 & Not \\
\hline Ag- $110 \mathrm{~m}$ & RNiF & $\overline{V P}$ & $<9 \mathrm{E}-02$ & $\mathrm{~N} / \mathrm{A}$ & $<349.4$ & 1 & $n \mathrm{bd}$ & & & & & \\
\hline$A m-241$ & RNiF & VP & $3.15 \mathrm{E}+00$ & 23 & 0.003 & 7875 & nbd & & & & & \\
\hline Am-243 & RNiF & VP & $<2 \mathrm{E}-02$ & $\mathrm{~N} / \mathrm{A}$ & $<0.155$ & 1 & nbd & & & & & \\
\hline |C-14 & Direct & $\mathrm{VP}$ & $2.87 \mathrm{E}-03$ & 191 & 0.056 & 4 & $\mathrm{~N} / \mathrm{A}$ & & & & & \\
\hline Cd- 113 (not measured) & - & - & - & - & 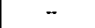 & - & - & & & & & \\
\hline Ce-144(Pr-144+Pr-144m) & $\mathrm{RNiF}$ & $\mathrm{VP}$ & $<2 E+00$ & $\mathrm{~N} / \mathrm{A}$ & $<0.776$ & 1 & nbd & & & & & \\
\hline $\mathrm{Ce}-144(\mathrm{Pr}-144+\mathrm{Pr}-144 \mathrm{~m})$ & RNiF & VP & $<2 \mathrm{E}+00$ & $\mathrm{~N} / \mathrm{A}$ & $<0.776$ & $t$ & nbd & & & & & \\
\hline Ce-144(Pr-144+Pr-144m) & RNiF & VP & $<6 \mathrm{E}-03$ & $\mathrm{~N} / \mathrm{A}$ & $<2.328$ & 1 & nbd & & & & & \\
\hline Cm-242(Am-242+Am-242m) & RNiF & VP & $8.07 E-03$ & 21 & 0.008 & 404 & nbd & & & & & \\
\hline Cm-242(Am-242+Am-242m) & $\mathrm{RNiF}$ & $\mathrm{VP}$ & $9.76 \mathrm{E}-03$ & 21 & 0.012 & 404 & nbd & & & & & \\
\hline Cm-242(Am-242+Am-242m) & $\mathrm{RNiF}$ & VP & $9.76 \mathrm{E}-03$ & 21 & 0.012 & 404 & nbd & & & & & \\
\hline $\mathrm{Cm}-243+\mathrm{Cm} \cdot 244$ & $\mathrm{RNiF}$ & $\mathrm{VP}$ & $9.86 \mathrm{E}-02$ & 22 & 3827 & 4930 & 1 & & & & & \\
\hline $\mathrm{C} m-243+\mathrm{Cm}-244$ & $\mathrm{RNiF}$ & $\mathrm{VP}$ & $9.86 \mathrm{E}-02$ & 22 & 0.004 & 4930 & 1 & & & & & \\
\hline Co-60 & RNiF & VP & $4.91 \mathrm{E}-01$ & 8 & 0.006 & 123 & nbd & & & & & \\
\hline Cs-134 & RNiF & $\mathrm{VP}$ & $<3 \mathrm{E}-01$ & N/A & $<0.002$ & 1 & nbd & & & & & \\
\hline Cs-135 & $\mathrm{RNiF}$ & VP & $2.86 \mathrm{E}-03$ & 5 & 0.004 & N/A & N/A & & & & & \\
\hline $\mathrm{Cs-137(Ba-137)}$ & RNiF & $\mathrm{vp}$ & $4.96 \mathrm{E}+02$ & 5 & 0.006 & 24800 & 0.03 & & & & & \\
\hline $\mathrm{Cs}=137(\mathrm{Ba}-137)$ & RNiF & VP & $4.69 \mathrm{E}+02$ & 5 & 0.006 & 24800 & 0.03 & & & & & \\
\hline Eu-152 & RNiF & VP & $<2 \mathrm{E}+00$ & $\mathrm{~N} / \mathrm{A}$ & $<0.518$ & 1 & nbd & & & & & \\
\hline Eu-154 & $\mathrm{RNiF}$ & $\mathrm{VP}$ & $3.79 \mathrm{E}+00$ & 4 & 0.009 & 190 & nbd & & & & & \\
\hline Eu-155 & RNiF & VP & $3.13 \mathrm{E}+00$ & 10 & 0.013 & 157 & nbd & & & & & \\
\hline Fe-55 & RNiF & VP & $1.53 \mathrm{E}-0 \mathrm{I}$ & 5 & 0.006 & 17 & nbd & 115 & & & & \\
\hline H-3 (not Measured) & -- & -- & - & .. & - & - & -- & & & & - & \\
\hline $1-129$ & ICP-MS Acid & VP & $2.20 \mathrm{E}-04$ & 14 & 0.099 & 1 & nbd & & & & & $\mathrm{x}$ \\
\hline $\mathrm{Nb}-93 \mathrm{~m}$ & RNiF & VP & $2.34 \mathrm{E}-01$ & 56 & 0.104 & 1 & nbd & 71 & & & & \\
\hline $\mathrm{Ni}-59$ & $\mathrm{RNiF}$ & VP & $2.05 \mathrm{E}-02$ & 24 & 0.057 & 1 & nbd & & & & & \\
\hline $\mathrm{Ni}-63$ & RNiF & VP & $2.40 \mathrm{E}+00$ & 6 & 0.058 & 1200 & nbd & & & & & \\
\hline $\mathrm{Np}-237$ & ICP-MS ZrF & VP & $3.92 \mathrm{E}-03$ & 20 & 0.007 & 6 & 4 & & & & & $\bar{x}$ \\
\hline $\mathrm{Pd}-107$ & ICP-MS Acid & VP & $1.10 \mathrm{E}-03$ & 118 & 0.011 & 4 & nbd & & & & & $\mathrm{x}$ \\
\hline$P u=238$ & RNiF & VP & $6.31 \mathrm{E}-01$ & 5 & 0.223 & 31550 & 0.2 & & & & & \\
\hline Pu-239 & ICP-MS ZrF & VP & $1.52 \mathrm{E}+00$ & 7 & 0.063 & 25 & 2 & & & & & $\mathrm{x}$ \\
\hline$P u-240$ & ICP-MS Acid & VP & $1.30 \mathrm{E}-01$ & 8 & 0.019 & 26 & nbd & & & & & $\mathbf{x}$ \\
\hline Pu-239+Pu-240 & RNiF & $\mathrm{VP}$ & $2.99 \mathrm{E}+00$ & 5 & N/A & 149500 & nbd & & & & & \\
\hline $\mathrm{Pu}-241$ & RNiF & VP & $6.96 \mathrm{E}+00$ & 6 & 0.039 & 17 & 0.4 & & & & & \\
\hline Pu-242 & RNiF & VP & $<2 \mathrm{E}-02$ & $\mathrm{~N} / \mathrm{A}$ & $<10.93$ & 1 & nbd & & & & & \\
\hline$R u-106(\mathrm{Rh}-106)$ & RNiF & $\mathrm{VP}$ & $<3 \mathrm{E}+00$ & $\mathrm{~N} / \mathrm{A}$ & $<0.582$ & 1 & nbd & & & & & \\
\hline Ru-106(Rh-106) & RNiF & $\mathrm{VP}$ & $<3 \mathrm{E}+00$ & $\mathrm{~N} / \mathrm{A}$ & $<0.582$ & 1 & nbd & & & & & \\
\hline
\end{tabular}




\begin{tabular}{|c|c|c|c|c|c|c|c|c|c|c|c|c|}
\hline 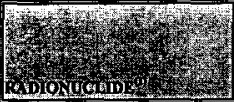 & W & Whe & 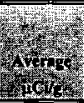 & 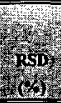 & $\log (x)$ & Sid & (ming & (6) & Minting & 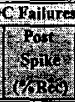 & hor & 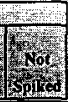 \\
\hline $5 \mathrm{~b}-125(\mathrm{Te}-125 \mathrm{~m})$ & RNiF & $\sqrt{P}$ & $<2 E+\infty$ & N/A & $<0.008$ & 1 & sbd & & & & & \\
\hline$S b-125(T e-125 m)$ & $\mathrm{RNiF}$ & VP & $<4 \mathrm{E}-01$ & N/A & $<0.006$ & 1 & nbd & & & & & \\
\hline Se-79 & $\mathrm{RNiF}$ & VP & $<2 \mathrm{E}-04$ & $\mathrm{~N} / \mathrm{A}$ & $<0.019$ & 1 & nbd & & & & & \\
\hline $\operatorname{Sm-151(Pm-147)}$ & RNiF & VP & $9.23 \mathrm{E}+01$ & 5 & 0.039 & 115 & 0.02 & & & & & \\
\hline $\mathrm{Sm}-151(\mathrm{Pm}-147)$ & RNiF & $\mathrm{VP}$ & $9.23 \mathrm{E}+01$ & 5 & 0.022 & 115 & 0.02 & & & & & \\
\hline Sn-113(In-113) & $\mathrm{RNiF}$ & VP & $<\mathrm{LE}-02$ & $\mathrm{~N} / \mathrm{A}$ & $<0.206$ & $\overline{1}$ & nbd & & & & & \\
\hline $\operatorname{Sn}-113(\mathrm{In}-113)$ & RNiF & VP & $<1 \mathrm{E}-02$ & N/A & $<0.206$ & 1 & nbd & & & & & \\
\hline Sn-119m & RNiF & $\mathrm{VP}$ & $<4 \mathrm{E}-02$ & $\mathrm{~N} / \mathrm{A}$ & $<155.3$ & 1 & nbd & & & & & \\
\hline $\mathrm{S}_{\mathrm{n}-121 \mathrm{~m}}$ & RNiF & VP & $1.23 \mathrm{E}+00$ & 43 & 5.305 & 14 & nbd & & & & & \\
\hline $\mathrm{Sn}-126(\mathrm{Sb}-126 \mathrm{~m}, \mathrm{Sb}-126)$ & RNiF & $\mathrm{VP}$ & $1.15 \mathrm{E}-2$ & 28 & 0.009 & 2 & nbd & & & & & \\
\hline $\mathrm{Sn}-126(\mathrm{Sb}-126 \mathrm{~m}, \mathrm{Sb}-126)$ & RNiF & $\overline{\mathrm{VP}}$ & $1.15 \mathrm{E}-2$ & 28 & 0.013 & 2 & nbd & & & & & \\
\hline $\mathrm{Sn}-126(\mathrm{Sb}-126 \mathrm{~m}, \mathrm{Sb}-126)$ & RNiF & VP & 1.61E-3 & 28 & 0.013 & 2 & nbd & & & & & \\
\hline Sr-90(Y-90) & RNiF & VP & $7.30 \mathrm{E}+02$ & 6 & 0.009 & 104 & 0,01 & & & & & \\
\hline $\mathrm{St}-90(Y-90)$ & RNiF & VP & $7.30 \mathrm{E}+02$ & 6 & 0.009 & 104 & 0.01 & & & & & \\
\hline Te-99 & ICP-MS Acid & $\mathrm{VP}$ & $5.60 \mathrm{E}-03$ & 17 & 0.00005 & 16 & nbd & & & & & $\mathrm{x}$ \\
\hline Th-232 & ICP-MS ZrF & VP & $5.48 E-05$ & 33 & $\mathrm{~N} / \mathrm{A}$ & 548 & $n b d$ & & & & & $\mathrm{x}$ \\
\hline U.233 & ICP-MS Acid & VP & $-1.7 \mathrm{E}-03$ & 20 & 65.99 & 9 & nbd & & & & & $\mathrm{x}$ \\
\hline U.234 & ICP-MS Acid & VP & $7.70 \mathrm{E}-04$ & 18 & 0.039 & 8 & nbd & & & & & $\mathrm{x}$ \\
\hline U.235 & ICP-MS Acid & VP & $2.80 \mathrm{E}-05$ & 1 & 0.034 & 700 & nbd & & & & & $\mathbf{x}$ \\
\hline U.236 & ICP-MS Acid & VP & $1.80 \mathrm{E}-05$ & 14 & 0.009 & 18 & nbd & & & & & $x$ \\
\hline$\overline{\mathrm{U}-\overline{238}}$ & ICP-MS ZrF & VP & $6.71 \mathrm{E}-04$ & 3 & 0.045 & 1997 & 0.01 & & & & & $\bar{x}$ \\
\hline $\mathrm{Zr}-93$ & $\mathbf{R N i F}$ & VP & $<6 \mathrm{E}-01$ & N/A & $<0.166$ & 1 & nbd & & & & & \\
\hline Total Alpha & RNiF & VP & $6.78 \mathrm{E}+00$ & 3 & $N / A$ & 6780 & 0.04 & & & & & \\
\hline Total Beta & RNiF & $V_{P}$ & $2.05 \mathrm{E}+03$ & 6 & N/A & 410000 & 0.01 & & & & & \\
\hline
\end{tabular}

(I) The "bolded" radionuclides of the parent/daughter pairs are used to compare to the Envelope $\mathrm{D}$ specification; the daughter "averages" have been corrected for the decay fraction.

(2) Method: $\mathrm{NiF}=\mathrm{KOH}$ Fusion (PNL-ALO-115); $\mathrm{ZrF}=\mathrm{Na}_{2} \mathrm{O}_{2}$ Fusion (PNL-ALO-116); Atid = $\mathrm{HNO}_{3} / \mathrm{HCl}$ Digestion (PNL-ALO-129); Water Leach (PNL-ALO-103)

FVP $=$ Final Vendor Product

$\mathrm{N} / \mathrm{A}=$ Not Applicable

(3) For ICPMS the ratio is "Results/DL".

(4) "nbd" = No blank concentration detected in process blank (i.e., blank concentration $<D L$ or $<M D A$ ).

严 
The primary objective for the characterization analysis was to demonstrate that the Final Vendor Product complied with the Envelope D specifications (Jones 1996). The specifications are in grams per liter $(\mathrm{g} / \mathrm{L})$ at 31 grams of oxide for analytes of interest, and curies per liter $(\mathrm{Ci} / \mathrm{L})$ at 31 grams of oxide for radionuclides of interest. The "Average/D Spec." uses the average analyte (or radionuclide) result in $\mu \mathrm{g} / \mathrm{g}$ (or $\mu \mathrm{Ci} / \mathrm{g}$ ) converted to $\mathrm{g} / \mathrm{L}$ (or $\mathrm{Ci} / \mathrm{L}$ ) using the weight percent solids and weight percent oxides. Sections 3.3.1 and 3.3.2 of the Analytical Summary Report Addendum 1 (Kuhl-Klinger and Urie 1997) provide an explanation of the equations used to calculate the $\mathrm{g} / \mathrm{L}$ (or $\mathrm{Ci} / \mathrm{L}$ ) average results. Values for "Average/D Spec." less than 1.00 indicate that the average measured concentration of the material (either Vendor Product or Final Vendor Product) is within the Envelope D specification.

\subsection{Data Quality - Comparison of Analytical Results to Detection Limits}

The "Result/DL" or "Result/MDA" is the ratio of the average result to the reported detection limit (DL) or minimum detected activity (MDA). This ratio gives an indication of the reliability of the reported analytical measurement. The magnitude of this ratio has different significance for the various methods used for sample analysis. Typically, the EQL for a method is ten times DL, or equal to the lowest calibration standard used to calibrate the method. Therefore, for analyte concentration less than five to ten times the DL (i.e., Result/DL <5), the variabi]ity of the analytical measurement is anticipated to be quite high. However, because of the high sensitivity (i.e., very low DL) of ICP-MS, a ratio less than 10 to 20 times DL is more reasonable for complex matrices such as C-106 waste. For radiochemistry measurements, the MDA provides an indication of the practical minimum activity which can be measured. Besides the MDA, propagated uncertainties and mean differences are used to used to evaluate radiochemical results near the MDA; see Section 4.4 of the Analytical Summary Report (Urie 1997) for explanation of uncertainty and mean difference evaluations. The variability in analytical results caused by measurements near the DL or MDA is most often reflected in a large RSD and high uncertainties for radiochemistry measurement. As can be seen in Table 3.1 and Table 3.2, over half of the reported ratios are low (i.e., <20), indicating the reported average results should be used with discretion. Where detection limits are not available for a method, the "Results/DL" or "Result/MDA" is designated as "N/A".

\subsection{Data Quality -- Preparation Blank Contribution}

The presence of detectable levels of an analyte in the preparation blank is an indication of potential contamination during sample preparation or analysis. The "Blank/Sample" summarizes the contribution from the preparation blank, and is expressed as a percentage of the sample concentration for each analyte or radionuclide. Blank contributions greater than $5 \%$ of the sample concentration are considered significant and exceed the limits establish by the governing QA Plan, MCS-033 (Kuhl-Klinger 1996). Dysprosium, erbium, molybdenum, nickel, platinum, rhenium, vanadium, ytterbium, chloride, nitrate, sulfate, and ammonia have blank contributions exceeding $5 \%$. With the exception of molybdenum, nickel, platinum, and vanadium, the analytes and their respective blank concentrations are at or near the DL, which may account for the high (i.e., $>5 \%$ ) contribution from the blank. With molybdenum, nickel, platinum, and vanadium at concentrations at least 31 times the DL, these blank contaminants contribute significantly to the uncertainty (both precision and accuracy) of the reported results. Nickel is a common, low-level contaminant in the $\mathrm{Na}_{2} \mathrm{O}_{2}$ fusion preparations; the source of the molybdenum, platinum, and vanadium blank contamination is unknown. 
A few analytes (e.g., boron, calcium, copper, iron, sodium, uranium, and zinc) are common contaminants from the fusion and acid digestion preparations performed in the SAL hot cells. The blank concentration for these analytes contribute less that $5 \%$ to the sample concentration; therefore, the impact of these blank concentrations is considered to be insignificant.

\subsection{Data Quality - Reproducibility}

The "RSD" in Table 3.1 and Table 3.2 is the RSD of the mean for the data set reported. For this report, the RSD is used as the measure of reproducibility for the Vendor Product and Final Vendor Product results. With six measurements for the Vendor Product (i.e., three subsamples analyzed in duplicate) and five measurements for the Final Vendor Product (i.e., four samples with one being analyzed in duplicate), RSDs less than $10 \%$ are used to indicate high quality results. Subsampling variability (i.e., either bench-scale batch processing subsampling, SAL sample/duplicate splitting, or analysis preparation subsampling) is suggested when the RSD results are high (i.e., $>10 \%$ ) and the "Result/DL" results indicate the measured analyte (or radionuclide) is 10-20 times above the DL (or MDA).

For the reported analyte and radionuclide averages which are above the DL, approximately half of the results have RSD values above $10 \%$. Based on the "Result/DL" and "Result/MDA" values, most of the large RSDs are the result of the analytes being at low concentration (i.e., within 5 to 20 times the DL or MDA). At these concentrations, reproducible results are difficult to obtain due to sample inhomogeneity. There are a few analytes where the results variability can be explained by potential contamination during sample preparation (i.e., vanadium) or from high standard carryover resulting in non-reproducible, biased high results (i.e., ${ }^{14} \mathrm{C}$ ).

When the average results with RSD values exceeding $10 \%$ are evaluated with the "Results/DL (or MDA)" and "Average/D Spec." values, there are only a few analytes and radionuclides which are impacted by the high RSD values. For those radionuclides which exceed the Envelope D specification by over 5 times (i.e., ${ }^{243} \mathrm{Cm},{ }^{121 m} \mathrm{Sn}$ and ${ }^{233} \mathrm{U}$ ), the effect of large RSD values (e.g., $20 \%$ to $40 \%$ ) is negligible. Regardless of the variability of the results, the results remain significantly over the specification. For analytes with results within $25 \%$ to $120 \%$ of the Envelope D specification, high RSD values (e.g., $20 \%$ to $80 \%$ ) can have a significant effect if the a $95 \%$ confidence limit is used to evaluate the acceptability of the material. Silver, erbium, gadolinium, niobium, platinum (an analyte exceeding the Envelope D specification), tin, and ytterbium fall in this category.

For the Vendor Product (only), Welsh (1997) provides a thorough evaluation of the variability associated with both the sampling and analysis. Welsh addresses all analyses performed on the Vendor Product, and uses the three subsamples of Vendor Product from the bench-scale batch processing, analyzed in duplicate, to evaluate both the subsampling and analytical components of the variability. The differences between the three subsample results are used in determining the sampling variability; whereas, the differences between the duplicate measurements for each subsample are used in determining the analytical variability. The statistical results and evaluation are not reproduced in this report. Since analytical variabilities (and associated analysis problems) were similar for the analysis of the Original Material, Vendor Product, and Final Vendor Product, the quality of the data generated for all materials should be similar and can be used as a base guidance for future analyses of washed sludges. 


\subsection{Data Quality - Accuracy}

The accuracy of the reported results is evaluated from laboratory standards or matrix spikes processed and analyzed with the samples, or from post-digestion spiking or serial dilution during the analysis of the samples. In most cases the batch quality control and analytical quality control sample results are within the requirements defined by the governing QA plan, MCS-033 (Kuhl-Klinger 1996). Table 3.1 and Table 3.2 present only those few cases where the LCS, Matrix Spike, Post-Digestion Spike, or Serial Dilution analysis failed to meet the QA Plan requirements. Where the QC sample failures occurred, the measured recovery values or percent difference values (for serial dilution) are reported.

Where no LCS was available and Matrix Spikes (or Post-digestion spikes) were not performed, the accuracy of the reported result can not be determined per the governing QA plan. The analytes which have no preparation QC to support an evaluation of the accuracy are indicated by an "X" under "Not Spiked". For these analytes, the analytical QC samples (e.g., instrument blanks and continuing calibration verifications, interference checks, etc.) were acceptable and met the QA plan requirements. Analytes and radionuclides with only analytical QC to support the reported result are praseodymium, rhenium, thulium, iodine-129, neptunium-237, technetium-99, palladium-107, and plutonium and uranium isotopes determined by ICP-MS.

Most of the LCS, Matrix Spike, or Post-digestion Spike failures resulted from recoveries exceeding the upper control limits for the standards. If the average reported results are corrected for these standard recoveries, the actual average concentration for these analytes is lower. Thus, there is a high probability that the measured average results are biased high. The analytes and radionuclides which may be biased high, based on high standard recoveries, are arsenic, dysprosium, holmium, molybdenum, strontium, tungsten, ytterbium, fluoride, and iron- 55 . There were some interferences noted for the fluoride measurement, possibly caused by the excess oxalate or co-eluting organic anions. Of more concern are the analytes and radionuclides which demonstrated low standard recoveries, since the measured average value may be significantly lower than the "true" concentration. Those analytes and radionuclides that may be biased low are cerium, antimony, zinc, and niobium-93m. However, the concentrations of these analytes and radionuclides do not exceed $10 \%$ of the Envelope D specification. Even considering the potential that the reported average result is biased low, there is little probability of exceeding the specification.

The $\mathrm{pH}$ and $\mathrm{OH}$ measurements were performed on the Final Vendor Product per procedures PNL-ALO-225 and PNL-ALO-228, respectively. Although both measurements demonstrated good precision, the $\mathrm{pH}$ results are a better estimate of the alkaline properties of the Final Vendor Product because the free hydroxide in the samples is too low to measure accurately by titration.

\subsection{Preparation and Analysis Comparison for ICP-AES and ICP-MS Results}

For a number of analytes, it is possible to compare ICP-AES results to the ICP-MS results; thus providing additional confidence in the results and gaining additional information on the chemistry of the Vendor Product waste material. Table 3.3 summarizes the ICP-AES results for all the preparation methods (i.e., $\mathrm{KOH}$ fusion, $\mathrm{Na}_{2} \mathrm{O}_{2}$ fusion, and acid digestion) and Table 3.4 summaries the ICP-MS results. Each of the tables identifies those analytes for which there may be significant preparation method differences reported for the specific analysis method. Each table also identifies those analytes for which there may be significant differences between the analysis methods. 
Table 3.3. Summary of ICP-AES Results for Vendor Product

\begin{tabular}{|c|c|c|c|c|c|c|c|c|c|c|c|}
\hline 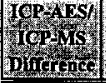 & 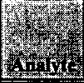 & 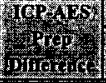 & Mrat & 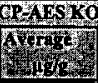 & 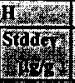 & 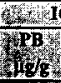 & 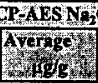 & 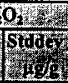 & W" & 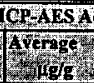 & $\frac{\mathrm{de}}{\mathrm{spd}}$ \\
\hline \multirow{5}{*}{$x$} & $\mathbf{A g}$ & $x$ & - & 1,030 & 240 & [50] & 3,180 & 680 & - & 76 & 5 \\
\hline & Al & & [248] & 51,200 & 2300 & [218] & 49,500 & 2400 & [16] & 47,900 & 1300 \\
\hline & As & & - & $<479$ & $n / a$ & - & $<510$ & n/a & - & $<123$ & $n / a$ \\
\hline & B & & [90] & [303] & 98 & - & [154] & 35 & [119] & 225 & 62 \\
\hline & Ba & & - & 407 & 19 & [25] & 437 & 22 & 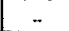 & 415 & 10 \\
\hline \multirow{10}{*}{$\mathrm{x}$} & $\mathrm{Be}$ & & - & $<9$ & $n / a$ & - & $<9$ & $\mathrm{n} / \mathbf{a}$ & - & $<1$ & $n / \mathbf{s}$ \\
\hline & $\mathrm{Bi}$ & & - & [538] & 14 & - & [582] & 58 & -. & 430 & 11 \\
\hline & $\mathrm{Ca}$ & $x$ & {$[1,120]$} & 6,690 & 720 & {$[4,180]$} & 9,850 & 1000 & [71] & 3,840 & 93 \\
\hline & Cd & & - & [81] & 2 & - & [81] & 5 & - & 73 & 13 \\
\hline & Ce & & - & $<523$ & $n / u$ & - & $<\mathbf{1 , 5 8 0}$ & $n / \mathbf{a}$ & - & [299] & 11 \\
\hline & $\mathrm{Co}$ & & - & $<87$ & $n / a$ & - & $<93$ & $\mathrm{~N} / \mathrm{B}$ & - & [28] & 1 \\
\hline & $\mathrm{Cr}$ & $x$ & [63] & 802 & 38 & [45] & 700 & 46 & - & 533 & 12 \\
\hline & $\mathrm{Cs}-133$ & & חים & $\mathbf{n}^{\prime} \mathbf{m}$ & w'm & $\mathrm{n} / \mathrm{m}$ & $n / m$ & $n^{\prime m}$ & $\mathrm{n} / \mathrm{m}$ & $n / m$ & $\mathrm{wm}$ \\
\hline & Cs-135 & & $n / m$ & $\mathbf{n} / \mathbf{m}$ & $w / m$ & $\mathrm{~nm}$ & $\pi / m$ & $n / m$ & $\mathrm{n} / \mathrm{m}$ & $\mathbf{n} / \mathbf{m}$ & $\mathrm{n} / \mathrm{m}$ \\
\hline & $\mathrm{Cu}$ & & [49] & [109] & 5 & [49] & [109] & 15 & - & 78 & 2 \\
\hline \multirow{10}{*}{$\mathrm{x}$} & Dy & & - & $<87$ & $\alpha / a$ & - & $<93$ & $\pi / \mathrm{s}$ & -- & $<12$ & $n / a$ \\
\hline & Er & & $n / m$ & $\mathbf{n} / \mathbf{m}$ & $\mathbf{n} / \mathbf{m}$ & $\mathrm{nm}$ & $\pi / m$ & $n^{\prime} / \mathbf{m}$ & $\mathrm{n} / \mathrm{m}$ & $n / m$ & $n / m$ \\
\hline & Eu & & - & $<174$ & $n / a$ & - & $<185$ & $\pi / \mathbf{a}$ & .. & $<25$ & $\sqrt{ } / a$ \\
\hline & $\mathrm{Fe}$ & & [268] & 141,000 & 7600 & [270] & 131,000 & 7800 & [9] & 137,000 & 3200 \\
\hline & Gd & & $n / m$ & $n / \boldsymbol{m}$ & $\mathrm{n} / \mathrm{m}$ & $\mathrm{n} / \mathrm{m}$ & $\mathbf{n} / \mathbf{m}$ & $n / m$ & $n / m$ & $n / m$ & $\mathrm{n} / \mathrm{m}$ \\
\hline & $\mathrm{Hg}$ & & $n / m$ & $\mathbf{n} / \mathbf{m}$ & $\mathrm{n} / \mathrm{m}$ & $\mathrm{n} / \mathrm{m}$ & $n / m$ & $\pi^{\prime} \mathbf{m}$ & $\mathrm{n} / \mathrm{m}$ & $n ' m$ & $\mathrm{n} / \mathrm{m}$ \\
\hline & Ho & & $n / m$ & $\mathbf{a} / \mathbf{m}$ & $\mathrm{a} / \mathrm{m}$ & n'm & $n / m$ & $n^{\prime} / \mathbf{m}$ & $n / m$ & $n / m$ & $n / m$ \\
\hline & I-129 & & $n / m$ & $\mathbf{n} / \mathbf{m}$ & $n^{\prime} / \mathbf{n}$ & $n / m$ & $n / m$ & $n / m$ & $\mathrm{~N} / \mathrm{m}$ & $n / m$ & $\mathrm{n} / \mathrm{m}$ \\
\hline & $\mathbf{K}$ & & nir & $n / u$ & $n / a$ & {$[6,930]$} & {$[7,590]$} & 1700 & - & $<492$ & $\sqrt{ } / \mathbf{a}$ \\
\hline & La & & - & [119] & 9 & - & {$[164]$} & 26 & .. & [96] & 3 \\
\hline \multirow{15}{*}{$\mathbf{x}$} & $\mathbf{L i}$ & & - & {$[90]$} & 6 & [59] & [94] & 25 & - & 46 & 30 \\
\hline & Mg & $x$ & -. & {$[1,360 \mid$} & 130 & - & {$[1,310]$} & 99 & - & 754 & 23 \\
\hline & Mn & & [191] & 3,140 & 140 & - & 2,590 & 160 & - & 2,600 & 60 \\
\hline & Mo & & - & $<87$ & $n / a$ & - & $<93$ & nia & - & {$[27]$} & 1 \\
\hline & $\mathrm{Na}$ & $x$ & 2,990 & 142,000 & 9000 & $\pi / u$ & niu & $n / 4$ & [161] & 162,000 & 8000 \\
\hline & Nd & & - & [379] & 31 & [254] & [517] & 59 & - & 282 & 8 \\
\hline & $\mathbf{N i}$ & $\mathbf{x}$ & a'u & $n / u$ & $n / a$ & 597 & 1,660 & 120 & - & 1,130 & 31 \\
\hline & $N_{p}-237$ & & $n / m$ & $\mathbf{m} / \mathrm{m}$ & $\pi / n$ & $n / m$ & $\mathbf{n} / \mathbf{m}$ & $N m$ & $\pi n$ & $\mathbf{w} / \mathbf{m}$ & $n / m$ \\
\hline & $\mathbf{P}$ & $x$ & - & 3,330 & 370 & [239] & 3,840 & 670 & - & 1,340 & 66 \\
\hline & $\mathrm{Pb}$ & & - & 2,950 & 510 & [203] & 3,530 & 210 & - & 3,430 & 78 \\
\hline & $\mathbf{P d}$ & & - & $<1,310$ & $n / a$ & - & $<1,390$ & $n / \mathbf{a}$ & - & $<369$ & $n / \mathbf{a}$ \\
\hline & Pd-107 & & $n / m$ & $n / m$ & $\mathbf{n} / \mathbf{m}$ & $\mathbf{n}^{\prime} \mathbf{m}$ & $\mathbf{a} / \mathbf{m}$ & $\mathrm{Nm}$ & $n / m$ & $\mathbf{m} / \mathbf{m}$ & $\mathrm{N} / \mathrm{m}$ \\
\hline & Pr & & $n / m$ & $n / m$ & $\mathbf{n} / \mathbf{m}$ & $\mathrm{n} / \mathrm{m}$ & $\mathrm{n} / \mathrm{m}$ & $n / m$ & $n / m$ & $\mathbf{n} / \mathbf{m}$ & $n / m$ \\
\hline & & & $\mathrm{n} / \mathrm{m}$ & $n / m$ & $\mathrm{n} / \mathrm{m}$ & $\mathbf{n} / \mathbf{m}$ & $n / m$ & $\pi / m$ & $n / m$ & $\mathbf{w} / \mathbf{m}$ & $n^{\prime} \mathbf{m}$ \\
\hline & Pu-239 & & $\mathrm{n} / \mathrm{m}$ & $\mathbf{n} / \mathbf{m}$ & $n / m$ & $n / m$ & $n / m$ & $\mathrm{~N} / \mathrm{m}$ & $\pi / m$ & $\mathbf{m} \mathbf{m}$ & $\mathrm{n} / \mathrm{m}$ \\
\hline
\end{tabular}


Table 3.3. Summary of ICP-AES Results for Vendor Product (Cont.)

\begin{tabular}{|c|c|c|c|c|c|c|c|c|c|c|c|}
\hline 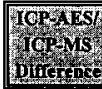 & 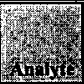 & 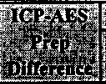 & the & 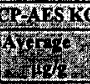 & Foder & \multicolumn{3}{|c|}{ 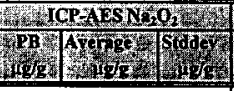 } & $\frac{1}{3 x}$ & 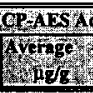 & 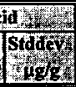 \\
\hline & $\mathrm{Pu}-240$ & & $\mathrm{n} / \mathrm{m}$ & $\mathrm{n} / \mathbf{m}$ & $n^{\prime} / m$ & $\mathrm{n} / \mathrm{m}$ & $w^{\prime / m}$ & $\mathbf{n} / \mathbf{m}$ & $\mathrm{a} / \mathrm{m}$ & $\mathbf{w} / \mathrm{m}$ & $\mathrm{N} / \mathrm{m}$ \\
\hline . & Pu-242 & & $\mathrm{n} / \mathrm{m}$ & $\mathbf{n} / \mathbf{m}$ & $n^{\prime} m$ & $\pi / m$ & $\mathbf{m} / \mathbf{m}$ & $\mathbf{n}^{\prime} \mathbf{m}$ & $\mathbf{n} / \mathbf{m}$ & $n / m$ & $\mathrm{n} / \mathrm{m}$ \\
\hline & $\mathbf{R b}$ & & $n / m$ & $\mathbf{a} / \mathbf{m}$ & 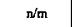 & $n / m$ & $n / m$ & $n$ & $n / m$ & $n / m$ & $n / m$ \\
\hline & $\mathrm{Re}$ & & $\mathrm{n} / \mathrm{m}$ & $\mathbf{a} / \mathbf{m}$ & $n / m$ & $\omega / m$ & $\mathbf{n} / \mathbf{m}$ & $n / m$ & $\mathrm{w} / \mathrm{m}$ & $n / m$ & $\mathrm{~N} / \mathrm{m}$ \\
\hline & $\mathbf{R h}$ & & - & $<523$ & $\mathrm{n} / \mathrm{a}$ & -. & $<556$ & nia & - & $<74$ & $\mathrm{~N} / \mathrm{a}$ \\
\hline & $\mathbf{R u}$ & & - & $<1,740$ & $n / a$ & 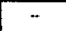 & $<1,850$ & $n / \mathbf{a}$ & 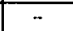 & $<738$ & $\bar{a} / \mathrm{a}$ \\
\hline & Sb & & - & $<871$ & $\mathrm{n} / \mathrm{a}$ & - & $<927$ & $n^{\prime 2}$ & - & $<74$ & $n / a$ \\
\hline & Se & & - & $<610$ & $w / a$ & - & $<649$ & $\mathrm{n} / \mathrm{s}$ & - & $<369$ & $n / a$ \\
\hline & $\mathbf{S i}$ & $\mathbf{x}$ & - & 68,900 & 4300 & -- & 64,600 & 3900 & [165] & 2,800 & 430 \\
\hline & Sn & & - & $<3,920$ & $n / a$ & - & $<4,170$ & $\pi / a$ & - & $<4,060$ & $n / a$ \\
\hline & $\mathbf{S r}$ & $x$ & - & [79] & 5 & [71] & [145] & 12 & - & 54 & 1 \\
\hline & $\mathbf{T a}$ & & $\mathrm{n} / \mathrm{m}$ & $\mathbf{n} / \mathbf{m}$ & $\mathrm{n} / \mathrm{m}$ & $n / m$ & a/m & $\mathrm{n} / \mathrm{m}$ & $n / m$ & $m / m$ & $n / m$ \\
\hline & $\mathrm{Tb}$ & & nim & $n / m$ & $n^{\prime} / \mathbf{m}$ & $n^{\prime} m$ & $m / m$ & $n / m$ & $n / m$ & $\mathbf{n} / \mathbf{m}$ & $n / m$ \\
\hline & Тe-99 & & $\mathrm{w} / \mathrm{m}$ & $n / m$ & $n^{\prime} m$ & $\mathbf{n}^{\prime} \mathbf{m}$ & $n / m$ & $n / m$ & $n / m$ & $\mathbf{n}^{\prime} \mathbf{m}$ & $n^{\prime} m$ \\
\hline$x$ & $\mathrm{Te}$ & & - & $<2,610$ & $n / 2$ & - & $<2,780$ & $\mathrm{n} / \mathrm{a}$ & - & 523 & 12 \\
\hline & Th & & 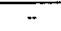 & $<1,740$ & $n / \mathbf{a}$ & - & $<1,850$ & $n / \mathbf{a}$ & - & [349] & 5 \\
\hline & $\mathrm{Ti}$ & $\mathrm{x}$ & - & 1,030 & 79 & - & 1,020 & 100 & - & 350 & 11 \\
\hline & TI & & - & $<871$ & nis & - & $<927$ & $\pi / \mathbf{a}$ & - & $<123$ & $n / a$ \\
\hline & $\mathrm{Tm}$ & & $\mathbf{n}^{\prime} \mathbf{m}$ & $n / m$ & $\mathrm{~N}^{\prime} \mathrm{m}$ & $n^{\prime} m$ & $n / m$ & n'm & $n / m$ & $\mathbf{n} / \mathbf{m}$ & $n^{\prime} m$ \\
\hline & U (total) & & - & $<4,360$ & nja & - & $<6,950$ & $n / 2$ & - & {$[1,950]$} & 37 \\
\hline & U-233 & & $\mathrm{n} / \mathrm{m}$ & $\mathrm{n} / \mathrm{m}$ & $\mathrm{n} / \mathrm{m}$ & $n / m$ & $n / m$ & $\mathrm{n} / \mathrm{m}$ & $\mathrm{r} / \mathrm{m}$ & $\mathbf{n} / \mathbf{m}$ & $\mathrm{n} / \mathrm{m}$ \\
\hline & $\mathrm{U}-\mathbf{2 3 4}$ & & $n / m$ & $n / m$ & $\pi / m$ & $n / m$ & $\mathrm{n} / \mathrm{m}$ & $n / m$ & $n j \mathrm{~m}$ & $n / m$ & $n^{\prime} \mathbf{m}$ \\
\hline & U-235 & & $n^{\prime} \mathbf{m}$ & $\mathrm{n} / \mathrm{m}$ & $n^{\prime} m$ & $\mathbf{n} / \mathbf{n}$ & $n / m$ & $n / m$ & $n m$ & $n / m$ & $\pi / m$ \\
\hline & U-236 & & $n / m$ & $n / m$ & $n / m$ & $n / m$ & $n / m$ & $\pi / m$ & $\mathrm{n} / \mathrm{m}$ & W/a & $\mathrm{n} / \mathrm{m}$ \\
\hline & U-238 & & $\mathrm{n} / \mathrm{m}$ & $n / m$ & $n / m$ & $\mathrm{n} / \mathrm{m}$ & $\mathbf{m} / \mathbf{m}$ & $\pi / m$ & $\mathrm{nim}$ & $n / m$ & $n / m$ \\
\hline & $\mathrm{v}$ & & - & $<87$ & $n^{\prime} \mathbf{a}$ & - & $<93$ & $n / 3$ & . & $<37$ & $\pi / n$ \\
\hline & w & & - & $<3,480$ & $n^{\prime} \mathbf{a}$ & - & $<3,710$ & $\mathbf{n} / \mathbf{a}$ & - & $<492$ & $n / \mathbf{z}$ \\
\hline & $y$ & & - & $<131$ & $n^{\prime} \mathbf{a}$ & - & $<139$ & $n / \mathbf{a}$ & - & $<86$ & $\pi / \mathbf{s}$ \\
\hline & $\mathbf{Y b}$ & & $\sqrt{ } / \mathrm{m}$ & $n / m$ & $\mathrm{n} / \mathrm{m}$ & $\sqrt[n]{m}$ & $n / m$ & $\mathrm{~N} / \mathrm{m}$ & $n / m$ & $a / m$ & $\mathrm{n} / \mathrm{m}$ \\
\hline & $\mathrm{Zn}$ & & - & [183] & 25 & - & [161] & 14 & [16] & [116] & 3 \\
\hline$x$ & $\mathbf{Z r}$ & $x$ & - & 1,110 & 530 & $n / u$ & $\mathrm{n} / \mathrm{u}$ & $\mathrm{d} / \mathrm{a}$ & - & 661 & 200 \\
\hline
\end{tabular}

ICP-AES/ICP-MS Difference: " $x$ " = Differences between ICP-AES and ICP-MS results should be evaluated. ICP-AES Prep Difference: " $\mathrm{x}$ " = Differences between $\mathrm{KOH} / \mathrm{Na}_{2} \mathrm{O}_{2} / \mathrm{Acid}$ prep for ICP-AES should be evaluated. " $\mathrm{n} / \mathrm{m}$ " = not measured; " $\mathrm{n} / \mathrm{a}^{\text {" }}=$ not applicable; " $\mathrm{n} / \mathrm{u}^{\mathrm{l}}=$ not used (matrix issue) "<" = result <IDL; "[ ]" = results > IDL but $<$ EQL 
Table 3.4. Summary of ICP-MS Results for Vendor Product

\begin{tabular}{|c|c|c|c|c|c|c|c|c|c|c|c|}
\hline 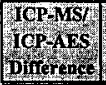 & 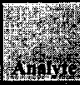 & 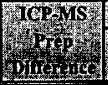 & $\frac{1}{4}$ & 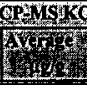 & 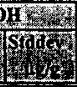 & 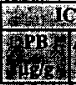 & 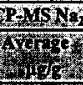 & 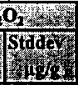 & 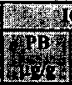 & 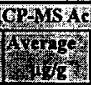 & 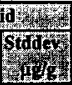 \\
\hline \multirow{5}{*}{$\mathbf{x}$} & Ag & & and & $\mathrm{n} / \mathrm{u}$ & $\pi / 4$ & $n / n$ & $a / m$ & $n / \imath$ & $n / u$ & $n / u$ & $\mathbf{w} / \mathbf{a}$ \\
\hline & Al & & nú & $n / u$ & $n / \mathbf{a}$ & $\pi / 4$ & $a / u$ & $n / a$ & 8.22 & 33,433 & 2425 \\
\hline & As & & 12.3 & 286 & 44 & 6.6 & 256 & 55 & 0.97 & 155 & 12 \\
\hline & B & & 8.2 & 229 & 83 & 2.2 & 101 & 03 & 50.3 & 186 & 62 \\
\hline & $\mathbf{B a}$ & & 3.35 & 412 & 19 & $<\mathbf{I}$ & 427 & 14 & 0.10 & 362 & 10 \\
\hline \multirow{10}{*}{$\mathbf{x}$} & $\mathbf{B e}$ & & n'm & $n / m$ & $\mathrm{~N} / \mathrm{m}$ & $a_{\mathrm{m}}$ & $m^{\prime \prime m}$ & $n / m$ & $n^{\prime} \mathbf{m}$ & $\mathrm{a} / \mathbf{m}$ & $\pi / m$ \\
\hline & & & $\mathrm{n} / \mathrm{m}$ & $\mathbf{n} / \mathbf{m}$ & $\mathbf{n} / \mathbf{m}$ & $\mathbf{n} / \mathbf{m}$. & $\mathbf{a} / \mathbf{m}$ & $\mathbf{n} / \mathbf{m}$ & $\mathrm{N} / \mathrm{m}$ & $\mathbf{a} / \mathbf{m}$ & $\mathrm{n} / \mathrm{m}$ \\
\hline & $\mathrm{Ca}$ & $\mathrm{x}$ & 65.3 & 1,723 & 218 & 133 & 3,767 & 336 & 84.0 & 9,573 & 1124 \\
\hline & Cd & & 1.54 & 90.6 & 11.4 & $<1$ & 74.1 & 8.6 & 0.05 & 58.1 & 1.7 \\
\hline & $\mathrm{Ce}$ & & 0.15 & 160 & 28 & $<1$ & 202 & 23 & 0.03 & 160 & 6 \\
\hline & $\mathrm{Co}$ & & 4 & 26 & 6 & 4 & 21.8 & 2.3 & 0.03 & 15.7 & 0.5 \\
\hline & $\mathbf{C r}$ & $\mathbf{x}$ & 11.9 & 774 & 84 & 5.99 & 739 & 47 & 0.67 & 426 & 11 \\
\hline & Cs-133 & & 0.70 & 18.0 & 4.0 & 4 & 7.18 & 2.17 & $<0.02$ & 10.4 & 0.7 \\
\hline & Cs-135 & & $\mathbf{n} / \mathbf{a}$ & $\pi / 2$ & $n / \mathbf{a}$ & $n / a$ & $\alpha / n$ & $\mathrm{n} / \mathrm{a}$ & $\pi / s$ & $n / n$ & $\pi / 2$ \\
\hline & $\mathrm{Cu}$ & $\mathrm{x}$ & $<1$ & 21.7 & 96 & 1.2 & 156.3 & 12.7 & 0.4 & 110.0 & 20 \\
\hline \multirow{10}{*}{$\mathrm{x}$} & Dy & $x$ & 2.68 & 20.2 & 75 & $<1$ & 3.60 & 1.84 & 0.04 & 1.47 & 0.09 \\
\hline & $\mathbf{E r}$ & $x$ & 1.73 & 13.1 & 4.7 & $<1$ & $<1$ & $n / a$ & $\infty .02$ & 0.8 & 0.0 \\
\hline & Eu & & $<1$ & 7.86 & 5.91 & $<1$ & 7.84 & 1.26 & 0.02 & 6.46 & 0.24 \\
\hline & $\mathrm{Fe}$ & & $n^{\prime \prime u}$ & $n / u$ & $n / a$ & ه & nit & n/a & 20.2 & 109,700 & 8717 \\
\hline & Gd & $\mathrm{x}$ & $<$ & 25.0 & 6.9 & $<1$ & 12.1 & 3.4 & $<0.02$ & 13.6 & 1.0 \\
\hline & $\mathbf{H g}$ & & $n / a$ & $n / n$ & $n / a$ & $n / \mathbf{a}$ & $\mathbf{a} / \mathbf{2}$ & $n / \mathbf{a}$ & $\pi / \mathrm{s}$ & $\mathbf{a} / \mathbf{m}$ & $\mathbf{n} / \mathbf{a}$ \\
\hline & Ho & $\mathbf{x}$ & $<1$ & 6.07 & 1.53 & $<1$ & 1.11 & $\pi / 2$ & $<0.02$ & 0.14 & 0.04 \\
\hline & $1-129$ & & $n / u$ & $n / u$ & $n / a$ & $<20$ & $<20$ & $n / 2$ & $<1$ & 1.27 & 0.25 \\
\hline & $\mathbf{K}$ & & $n / m$ & $\mathbf{n} / \mathbf{m}$ & $w m$ & $\mathrm{n} / \mathrm{m}$ & D/m & $n / m$ & $\mathrm{Nm}$ & $n^{\prime} \mathbf{m}$ & $\mathrm{n} / \mathrm{m}$ \\
\hline & $\mathrm{La}$ & & 0.63 & 80.6 & 12.1 & $<1$ & 92.1 & 14.5 & $<0.02$ & 84.9 & 3.4 \\
\hline \multirow{15}{*}{$x$} & $\hat{L} \mathbf{i}$ & & $\mathrm{n} / \mathrm{m}$ & $\mathbf{n} / \mathbf{m}$ & n'm & $n / m$ & $\mathrm{n} / \mathrm{m}$ & $\pi / m$ & $\mathrm{w} / \mathrm{m}$ & $\mathrm{m}^{\prime} \mathrm{mt}$ & $n / m$ \\
\hline & $\mathbf{M g}$ & $x$ & 2.6 & 1,220 & 156 & 7.05 & 1,257 & 81 & 2.87 & 446 & 17 \\
\hline & $\mathbf{M n}$ & & 19,3 & 2,807 & 206 & 3.63 & 2,558 & 126 & 4.00 & 2,043 & 115 \\
\hline & Mo & $\mathrm{x}$ & 5.14 & 57.0 & 13.7 & 1.30 & 27.9 & 4.5 & 0.07 & 9.60 & 0.60 \\
\hline & $\mathbf{N a}$ & $x$ & nit & $\mathrm{a} / \mathrm{u}$ & $n / a$ & $n \mu$ & $n / u$ & $n / \mathbf{x}$ & 27.9 & 181,667 & 27171 \\
\hline & Nd & & 5.49 & 233 & 34 & $<$ & 224 & 22 & 0.07 & 193 & 4 \\
\hline & $\mathbf{N i}$ & $x$ & $n / 4$ & $n / u$ & $n / 2$ & 30.5 & 1,605 & 62 & 7.40 & 948 & 56 \\
\hline & $N_{p}-237$ & & $n / u$ & $\mathrm{a} / \mathrm{u}$ & $\mathrm{n} / \mathrm{a}$ & $<1$ & 5.57 & 1.12 & $<0.02$ & 4.48 & 0.19 \\
\hline & $P$ & & $n / m$ & $a / m$ & $\mathrm{n} / \mathrm{m}$ & $n^{\prime} m$ & $\mathbf{m} / \mathbf{m}$ & $n / m$ & $\mathbf{n} / \mathbf{m}$ & $\mathrm{n} / \mathrm{m}$ & $\mathrm{n} / \mathrm{m}$ \\
\hline & $\mathrm{Pb}$ & & n'o & $\pi / \boldsymbol{u}$ & $\pi / 2$ & 2.76 & 2,553 & 95 & 8.31 & 2,853 & 192 \\
\hline & Pd & & $<1$ & 23.5 & 13.1 & $<1$ & 27.4 & 5.1 & 0.1 & 10.0 & 0.7 \\
\hline & Pd-107 & & $n^{\prime} u$ & $n / u$ & $n / \mathbf{a}$ & n'u & $a / n$ & $n / \mathbf{a}$ & $<0.5$ & 2.20 & 2.43 \\
\hline & $\mathrm{Pr}$ & & $<1$ & 77.1 & 10.6 & $<1$ & 84.1 & 5.3 & 0.02 & 70.5 & 2.0 \\
\hline & $\mathrm{Pt}$ & $\mathbf{x}$ & 4.38 & 30.7 & 7.7 & $<1$ & 5.19 & 1.66 & 0.10 & 0.2 & 0.1 \\
\hline & Pu-239 & $x$ & $\pi / u$ & $\mathbf{n} / \mathbf{u}$ & $\mathbf{n} / \mathbf{s}$ & $<1$ & 24.6 & 1.7 & 40.02 & 7.34 & 0.37 \\
\hline
\end{tabular}


Table 3.4. Summary of ICP-MS Results for Vendor Product (Cont.)

\begin{tabular}{|c|c|c|c|c|c|c|c|c|c|c|c|}
\hline \multirow[t]{6}{*}{ 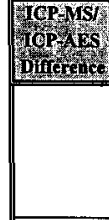 } & $x^{2}$ & 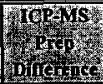 & $\frac{1}{4 x}$ & 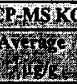 & 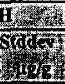 & mas & 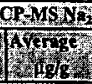 & 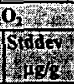 & 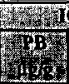 & 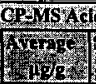 & (d ${ }^{2}$ \\
\hline & Pu-240 & & a'u & $n^{\prime} / u$ & $n / 2$ & $<1$ & $<1$ & $\pi / 2$ & $\$ .02$ & 0.56 & 0.04 \\
\hline & Pu-242 & & a's & $\mathbf{n} / \mathbf{u}$ & $n / \mathbf{n}$ & $<1$ & $<1$ & $n / 2$ & $<0.02$ & 0.02 & 0.00 \\
\hline & Rb & $\mathrm{x}$ & n's & $w^{\prime \prime u}$ & $\pi / \mathbf{a}$ & $<1$ & 7.68 & 2.92 & 0.2 & 1.10 & 0.11 \\
\hline & $\operatorname{Re}$ & $\mathbf{x}$ & 1.16 & 6.61 & 2.06 & $<1$ & 1.00 & $n / 2$ & 0.02 & 0.08 & 0.03 \\
\hline & Rh & $x$ & $<1$ & 36.9 & 41.0 & $<1$ & 144 & 16 & $<0.02$ & 104.7 & 3.7 \\
\hline \multirow[b]{10}{*}{$\mathbf{x}$} & Ru & $x$ & $<1$ & 95.7 & 6.5 & n'u & n'v & $\pi / 2$ & 0.1 & 54.2 & 1.5 \\
\hline & $\mathbf{S b}$ & & $<1$ & $<1$ & $n / a$ & $<1$ & $<1$ & $n / a$ & $<0.02$ & 0.73 & 0.25 \\
\hline & Se & & n & $\mathbf{n} / \mathbf{u}$ & $\pi / \mathbf{n}$ & $n / u$ & $n / n$ & $\mathbf{n}^{\prime} \mathbf{a}$ & n'u & w/u & $n / \mathbf{a}$ \\
\hline & $\mathbf{S i}$ & & $n^{\prime} m$ & $\mathrm{~m} / \mathrm{m}$ & $\mathrm{n} / \mathrm{m}$ & Nim & $\mathrm{n} / \mathrm{m}$ & $\mathrm{n} / \mathrm{m}$ & $n / m$ & $\mathbf{n} / \mathbf{m}$ & $n / m$ \\
\hline & Sn & $x$ & 4 & 29.3 & 28.5 & 1.73 & 102 & 29 & $<0.02$ & 34.8 & 1.5 \\
\hline & $\mathrm{Sr}$ & $x$ & $<1$ & 59 & 15 & 6.87 & 150 & 13 & 0.07 & 45.1 & 0.9 \\
\hline & $\mathbf{T a}$ & & 1.84 & 21.3 & 9.9 & $<1$ & 28.6 & 10.2 & 0.09 & 10.4 & 2.9 \\
\hline & Tb & $x$ & $<1$ & 8.20 & 1.55 & $<1$ & 2.34 & 0.78 & 0.02 & 1.46 & 0.09 \\
\hline & Te-99 & & $<1$ & $<1$ & $n / a$ & $n / u$ & $\mathbf{n} / \mathbf{u}$ & $n / a$ & 0.048 & 0.330 & 0.058 \\
\hline & $\mathrm{Te}$ & $x$ & 22.0 & 424 & 85 & 4.4 & 288 & 99 & 1.74 & 203 & 8 \\
\hline \multirow{10}{*}{ A } & Th & $\mathrm{x}$ & $\mathrm{m} / \mathrm{u}$ & $n / w$ & $n / a$ & 4 & 499 & 163 & $<0.2$ & 185 & 6 \\
\hline & $\mathrm{Ti}$ & $x$ & 4.4 & 1,124 & 121 & 3.20 & 1,313 & 147 & 0.4 & 358 & 12 \\
\hline & $\mathrm{Tl}$ & & $n / m$ & $\mathrm{n} / \mathrm{m}$ & $\pi / m$ & $n^{\prime} / m$ & $\mathbf{n} / \mathbf{m}$ & $\mathrm{n} / \mathrm{m}$ & $n / m$ & $n / m$ & $n / m$ \\
\hline & $\mathrm{Tm}$ & $x$ & $<1$ & 2.64 & 1.36 & $<1$ & 1.17 & 0.10 & $<0.02$ & 0.06 & 0.02 \\
\hline & U (total) & & $<1$ & 1,897 & 132 & $<1$ & 2,010 & 66 & 2.12 & 1,905 & 72 \\
\hline & $\mathrm{U}-233$ & & wis & $\mathbf{w} / \mathrm{u}$ & $\mathrm{n} / \mathrm{a}$ & nos & $n^{\prime \prime u}$ & wa & 0,02 & 0.17 & 0.03 \\
\hline & U-234 & & $\mathbf{n}^{\prime} \mathbf{u}$ & n/u & $n / a$ & n/u & $\mathbf{a} / \mathbf{u}$ & n/a & $<0.02$ & 0.12 & 0.02 \\
\hline & $\mathrm{U}-235$ & & n'u & n/u & $n / a$ & nu & $n / w$ & $n / a$ & $<0.02$ & 13.0 & 0.8 \\
\hline & U-236 & & n'u & $\mathbf{n} / \mathbf{u}$ & $\pi / \mathbf{a}$ & $\pi / 4$ & $n / u$ & $n / a$ & $<0.02$ & 0.29 & 0.04 \\
\hline & U-238 & & $<1$ & 1,887 & 132 & 4 & 1,998 & 63 & 2.11 & 1,895 & 72 \\
\hline \multirow[b]{6}{*}{$\lambda$} & V & & $\alpha / 2$ & $\mathbf{m}^{\prime} \mathbf{u}$ & $n / \mathbf{a}$ & nu & $a / u$ & $n / a$ & 4 & 37.2 & 6.8 \\
\hline & w & & 11.6 & 498 & 74 & 6.6 & 357 & 112 & 0.9 & 222 & 15 \\
\hline & $\mathbf{Y}$ & & $n / m$ & $n / m$ & $n / m$ & $n / m$ & $n / m$ & $\mathbf{n} / \mathbf{m}$ & $n^{\prime} / m$ & n'm & $n / m$ \\
\hline & $\mathbf{Y b}$ & $x$ & 1.39 & 12.6 & 6.2 & $<1$ & 3.24 & 1.48 & 0.02 & 0.2 & 0.1 \\
\hline & $2 n$ & $\mathrm{x}$ & 5.21 & 306 & 71 & 1.50 & 234 & 44 & 5.95 & 99.3 & 6.7 \\
\hline & $\mathbf{Z r}$ & $x$ & 199 & 433 & 230 & $\mathbf{n}^{\prime} \mathbf{u}$ & $n / \mathbf{u}$ & $n / \mathbf{z}$ & 0.25 & 256 & 67 \\
\hline
\end{tabular}

ICP-MS/LCP-AES Difference: " $x$ " $=$ Differences between ICP-AES and ICP-MS results should be evaluated ICP-MS Prep Difference; " $\mathrm{x}$ " = Differences between $\mathrm{KOH} / \mathrm{Na}_{2} \mathrm{O}_{2} / \mathrm{Acid}$ prep for ICP-AES should be evaluated $" \mathrm{n} / \mathrm{m}$ " = not measured; " $\mathrm{n} / \mathrm{a} \mathrm{n}$ = not applicable; " $\mathrm{n} / \mathrm{u}$ = nat used (matrix issue or data unusable)

$"<"=$ results less than EQL 
Comparison of the results generated by ICP-AES and ICP-MS (i.e., analysis methods) is presented only for the Vendor Product. A comparison is not possible for the Final Vendor Product, since only ICP-AES analyses were performed. Also, since the ICP-AES and ICP-MS comparative results for the Original Material are similar to the Vendor Product, no comparison of the Original Material ICP-AES and ICP-MS results is presented in this report.

For analytes with concentrations exceeding the ICP-AES EQL, ICP-AES and ICP-MS differences exceeding $20 \%$ are considered significant. For analytes with concentrations lower than the ICP-AES EQL, comparison between ICP-AES and ICP-MS are difficult due to the high uncertainty of the ICP-AES result. For analytes exceeding the ICP-AES EQL, the ICP-AES and ICP-MS results from the same preparation method agree quite well, except for calcium and zirconium (and possibly aluminum, iron, and tellurium). Although the difference in the sodium results between ICP-AES and ICP-MS is acceptable per the criteria set forth in this assessment (i.e., ICP-MS is about $12 \%$ higher than ICP-AES), the difference is of concern since a major component of the Vendor Product is sodium, and sodium will be used as a metric for privatization contractor service payment. As is expected, for each analysis method, there are significant differences between the results for each of the preparation methods.

\subsubsection{Evaluation of Differences Between ICP-AES and ICP-MS Results}

As stated above, the differences between the analysis methods (for those analytes detectable by ICP-AES) were for calcium, zirconium, and possibly aluminum, iron, sodium, and tellurium. It is important to note that ICP-AES and ICP-MS Vendor Product analyses were performed on the same dissolution (only different aliquots), as were all batch quality control samples. Table 3.3 (ICP-AES) and Table 3.4 (ICP-MS) identify analytes which show significant difference between the analysis methods. The "ICP-AES/ICP-MS Difference" column contains an "X" if, based on the standard deviation and variability of the results between analysis methods (except for sodium), the difference between average results is considered significant.

Calcium: The first issue is the high (and differing) blanks observed in the ICP-AES analysis and not in the ICP-MS analysis. This has a significant impact on the gross ICP-AES result; however, correcting for the blank (or more probably spectral background) the results should (but do not) compare favorably to the ICP-MS for each specific preparation. The second issue is that the LCS and post-digestion spikes provided excellent recoveries for the ICP-AES analysis; however, the LCS recovered very poorly for the ICP-MS. This is the primary reason for selecting the ICP-AES acid digestion results. Also, no apparent interferences were noted in wavescans for the ICP-AES.

Zirconium: The difference between the ICP-AES and ICP-MS is about a factor of 2.5, with ICP-AES being higher. This ratio is consistent between the preparations (i.e., $\mathrm{KOH}$ fusion and acid digestion), suggesting either a high bias for the ICP-AES or a low bias for the ICP-MS. The quality control results for the ICP-MS were unacceptable and the recoveries were all high which would indicate that the reported results for ICP-MS are high. This is inconsistent with the difference between the methods. ICP-AES results were chosen primarily because of the ICP-MS QC sample failures.

Iron: The ICP-MS QC was unacceptable and biased high. However, the single average ICP-MS result (from acid digestion) is significantly lower than the ICP-AES result from the 
acid digestion, as well as the ICP-AES iron results from the two fusion preparations. The ICP-AES results are considered more reliable than the ICP-MS results primarily due to the ICP-MS QC failures and the consistency of the ICP-AES iron results between preparation methods (i.e., 131,000 to $141,000 \mu \mathrm{g} / \mathrm{g}$ ).

Aluminum: The ICP-AES aluminum analyses are consistent between the three different preparations (i.e., 47,900 to $51,200 \mu \mathrm{g} / \mathrm{g}$ ), and all the QC associated with the ICP-AES aluminum analysis were within acceptable limits. The ICP-MS reported only results for the acid digestion and the $\mathrm{QC}$ related to the aluminum analysis had poorer recoveries than the ICP-AES. Based on this, the ICP-AES analysis results were selected for evaluation and reporting. In general, the ICP-AES provides better aluminum results for samples containing very high aluminum concentrations (e.g., $>3 \%$ ) than the ICP-MS.

Tellurium: The ICP-AES reported result from the acid digestion is only slightly higher than the EQL, and based on this and peak wavescans, there is a high probability that the tellurium result by the ICP-AES is biased high by as much as a factor of two. Although post spike recoveries were good for the ICP-AES, these recoveries are based on concentration differences and do not reflect potential background or interelement interferences which may not be adequately corrected for in the reported results. Based on this and the acceptable ICP-MS control standard recoveries, the ICP-MS result from the $\mathrm{KOH}$ fusion was selected for evaluation and reporting.

Sodium: Based on the relatively high RSD for the ICP-MS results, there appears to be no statistical difference between the ICP-MS and ICP-AES results. The ICP-AES recovery on the LCS was within acceptable limits, although the recovery was slightly low at $90 \%$. Therefore, there remains an uncertainty as to the "true" concentration of the sodium in the Vendor Product; however, the average sodium concentration by both ICP-AES and ICP-MS exceeded the Envelope D specification.

\subsubsection{Evaluation of ICP-AES and ICP-MS Differences Between Preparation Methods}

Table 3.3 (ICP-AES) and Table 3.4 (ICP-MS) identify analytes which show significant differences between the preparation methods (within each analysis method). The "Prep Difference" column contains an "X" if, based on the standard deviation and the average concentration (i.e., if above or below the analysis method EQL), the average results between preparation methods differs by $20 \%$. The differences reported for the greatest majority of these analytes can be attributed either to the varying contributions from blanks (e.g., calcium or nickel), the ruggedness of the various preparation methods for specific analytes (e.g., acid digestion may not fully solubilize certain analytes, such as chromium, bismuth, silver, titanium, phosphorus, silicon, zirconium, platinum group metal, etc.) or to the measured analyte concentration (e.g., fusion preparations may dilute an analyte too near to the detection limit). The exceptions to this appear to be differences measured between the two fusion methods for copper, molybdenum, platinum, rhenium, tin, and strontium ICP-MS results. Copper, molybdenum, tin, and strontium ICP-AES results were more consistent than ICP-MS, and an error in the ICP-MS results for one or both of the fusions is suspected.

Although the acid digestion showed fewer interferences than the fusion preparations (for both the ICP-MS and ICP-AES), the acid digestion results were consistently lower than those from the fusion preparations. Based on the preparation observations (i.e., residue remained following the acid 


\section{HNF-SD-WM-DP-225, REV. 1}

digestion preparation) and the consistently lower results, the acid digestion results are considered less reliable than those from the fusion preparations. Also, the variability in the calcium results can not be explained. The fusion dissolution method should provide reasonable results for calcium, with the acid digestion less likely to provide full dissolution. The difference between the $\mathrm{KOH}$ fusion and acid digestion of over five times, with the acid digestion showing the highest concentration, is inconsistent with other ICP-MS results (i.e., Original Material results) and all ICP-AES results.

For the acid digestion ICP-AES silver analysis, the matrix spike recovery for the acid digestion was only $12 \%$, indicating significant matrix/procedure problems. The additional chloride in the $\mathrm{Na}_{2} \mathrm{O}_{2}$ fusion procedure may enhance the solubilization of the silver; however, this has not been confirmed in presence of high sodium and aluminum concentrations. The silver results from the Original Material, Vendor Product, and Final Vendor Product suggest that the higher chloride in the $\mathrm{Na}_{2} \mathrm{O}_{2}$ fusion assisted solubilization, but was still inadequate as evidenced by the high RSD values.

\subsection{Analyte/Radionuclide Comparisons Based on Different Methods/Procedures}

Besides the analytes measured by ICP-AES and ICP-MS, there are a few additional analytes which have been analyzed (either directly or indirectly) by more than one method. For these cases, it is possible to compare the results from the different methods. Comparing the results from different methods can provide additional confidence in the reported results. A comparison of these methods is summarized in Table 3.5 .

The comparison between the methods is very good, except for phosphorus by ICP-AES and IC and the summation of ${ }^{239} \mathrm{Pu}$ and ${ }^{240} \mathrm{Pu}$ by ICP-MS and radiochemistry. Of particular interest is the comparison of the carbon measured by the TOC hot persulfate method and carbon measured as oxalate by IC. The results suggest that nearly all, if not all, of the organic carbon is in the form of oxalate.

The ICP-AES analysis measures the total phosphorus and IC measures only the soluble phosphate present in the water leach of the Vendor Product. These methods will only produce comparable results if all the phosphorus is present as water soluble phosphates. Because the ICP-AES result for phosphorus is significantly greater than the IC result, there is an indication that majority of the phosphorus is present as a non-water soluble species.

The radiochemical ${ }^{239+240} \mathrm{Pu}$ determinations were performed on the samples prepared from the KOH fusion only. The fusion preparation technique is an excellent method for dissolution of refractories (such as plutonium oxides). The subsequent radiochemical separations were performed on an aliquot of the acidified $\mathrm{KOH}$ melt using well-established separation procedures. The ${ }^{239} \mathrm{Pu}$ and ${ }^{240} \mathrm{Pu}$ were measured together (along with ${ }^{238} \mathrm{Pu}$ ) by alpha energy spectrometry. Inductively coupled plasma mass spectroscopy measured the ${ }^{239} \mathrm{Pu}$ and ${ }^{240} \mathrm{Pu}$ separately on the $\mathrm{Na}_{2} \mathrm{O}_{2}$ fusion and acid digestion preparation. As expected, due to the uncertainty of the solubilization of plutonium by the acid digestion, the ICP-MS ${ }^{239} \mathrm{Pu}$ and ${ }^{240} \mathrm{Pu}$ concentrations for the acid digested samples were considerably lower than the concentrations measured for the fusion samples. When adjusted for the specific activity, the ICP-MS ${ }^{239} \mathrm{Pu}$ and ${ }^{240} \mathrm{Pu}$ results from the $\mathrm{Na}_{2} \mathrm{O}_{2}$ fusion preparation should provide similar results to radiochemistry results from the $\mathrm{KOH}$ fusion preparation; however, the radiochemistry results are nearly twice those of the ICP-MS. Although the ${ }^{239} \mathrm{Pu}$ and ${ }^{240} \mathrm{Pu}$ results from the radiochemistry analysis are a sum of the two isotopes (i.e., separate isotopes are not available for comparison to the Envelope D specification), the radiochemistry result is considered most accurate. 
To confirm this conclusion, the ICP-MS results and radiochemical results for the Original Material were compared to the Vendor Product results. In general, the concentrations for refractory and/or rare earth analytes analyzed by ICP-MS on the $\mathrm{Na}_{2} \mathrm{O}_{2}$ fusion preparations (e.g., cadmium, chromium, copper, manganese, cerium, lanthanum, and neodymium) were approximately two times higher in the Vendor Product than in the Original Material. Plutonium should demonstrate a similar increase in concentration. However, the average activity concentration for the ICP-MS ${ }^{239 / 240} \mathrm{Pu}$ on the Vendor Product is only half the average activity concentration measured on the Original Material (i.e., 1.53 $\mu \mathrm{Ci} / \mathrm{g}$ versus $2.37 \mu \mathrm{Ci} / \mathrm{g}$ ). The radiochemistry average ${ }^{239+240} \mathrm{Pu}$ result for the Vendor Material was twice the result measured on the Original Material (i.e., $2.99 \mu \mathrm{Ci} / \mathrm{g}$ versus $1.55 \mu \mathrm{Ci} / \mathrm{g}$ ), consistent with expectations.

Table 3.5. Analyte Comparison From Different Methods/Procedure for Vendor Product ${ }^{(1)}$

\begin{tabular}{|c|c|c|c|c|c|c|}
\hline Analyte & Units & Method/Procedure & $\begin{array}{l}\text { Average } \\
\text { Result }\end{array}$ & $\mathbf{R S O}^{(2)}$ & $\begin{array}{l}\text { Compared } \\
\text { Results }^{(3)}\end{array}$ & RPD \\
\hline \multirow{2}{*}{ Carbon } & \multirow{2}{*}{$\mu g / g$} & TOC by Hot Persulfate & 47,900 & 7 & 47,900 & \multirow{2}{*}{3.2} \\
\hline & & Oxalate by IC / 3.66 & 170,00 & 8 & 46,400 & \\
\hline \multirow{2}{*}{ Phosphorus } & \multirow{2}{*}{$\mu \mathrm{g} / \mathrm{g}$} & Phosphorus by ICP & 3,330 & 11 & 3,330 & \multirow{2}{*}{194} \\
\hline & & Phosphate by IC / 3.07 & 160 & 23 & 52 & \\
\hline \multirow{2}{*}{$\begin{array}{l}\text { Total } \\
\text { Alpha }^{(4,5)}\end{array}$} & \multirow{2}{*}{$\mu \mathrm{Ci} / \mathrm{g}$} & Alpha Counting & 6.78 & 3 & 6.78 & \multirow[b]{2}{*}{0.4} \\
\hline & & $\Sigma^{238,239+240} \mathrm{Pu}+{ }^{241} \mathrm{Am}$ & $\begin{array}{c}0.61+2.99+ \\
3.15\end{array}$ & $<20$ & 6.75 & \\
\hline \multirow{2}{*}{ Total Beta ${ }^{(5)}$} & \multirow{2}{*}{$\mu \mathrm{Ci} / \mathrm{g}$} & Beta Counting & 2050 & 6 & 2050 & \multirow{2}{*}{4.7} \\
\hline & & $\sum{ }^{137} \mathrm{Cs}+{ }^{90} \mathrm{Sr}\left({ }^{90} \mathrm{Y}\right)$ & $\begin{array}{c}496+730+ \\
730\end{array}$ & $<6$ & 1956 & \\
\hline \multirow{2}{*}{${ }^{239+240} \mathrm{Pu}$} & \multirow{2}{*}{$\mu \mathrm{Ci} / \mathrm{g}$} & ICP-MS ${ }^{239} \mathrm{Pu}+{ }^{240} \mathrm{Pu}$ & $1.52+0.13$ & $<8$ & 1.65 & \multirow{2}{*}{58} \\
\hline & & Separations/AEA, ${ }^{239+240} \mathrm{Pu}$ & 2.99 & 5 & 2.99 & \\
\hline
\end{tabular}

(1) Average reported results for the Vendor Product are summarized in Analytical Summary Report (Urie).

(2) RSD based on average reported result. Where multiple results are summed, " $<$ " indicates that each RSD value is less than highest RSD, which is the one reported.

(3) For comparison, the reported results are converted to the analyte/radionuclide of interest by either adjusting for stoichiometry or summing the appropriate radionuclides.

(4) Only the radiochemistry results for the Vendor Product are compared (i.e, the ${ }^{239+240} \mathrm{Pu}$ is from separations/AEA not from ICP-MS).

(5) Only alpha-emitters and beta-emitters with significant activity are summed for comparison. 


\subsection{VENDOR PRODUCT PROCESSING AND PHYSICAL MEASUREMENT}

From the Vendor Product processing and physical measurement activities, numerous issues have been identified which require further evaluation and clarification. Issues addressed in this report are the difference in the sodium concentration of the laboratory-scale product and the bench-scale processing Vendor Product, the adequacy of the subsampling of the Vendor Product (and Original Material) from the bench-scale processing apparatus, the transferring/bottling procedure used to prepare the initial Vendor Product bottles for shipping, the weight percent solids discrepancies between the HLRF, and SAL, and the apparent loss of about 230 grams of Vendor Product.

\subsection{Discrepancies Between Laboratory-Scale and Bench-Scale Results}

The laboratory-scale screening test was conducted to provide operational parameters for the bench-scale batch preparation of the Vendor Product. Since the bench-scale processing was initiated prior to completion of the laboratory-scale screening test, these parameters were used by the benchscale processing, but only as available. The laboratory-scale screening test prepared a product which met the Envelope D Specifications for selected analytes measured, including sodium. However, following the bench-scale processing, the sodium concentration in the Vendor Product was determined to exceed the Envelope D Specification (and was about 50\% higher than the laboratory-scale product). The reason for the discrepancy is hypothesized as follows:

Lower Weight Percent Solids: The water wash steps during the laboratory screening test were performed at a lower weight percent solids than those used during the bench-scale processing. Therefore, more water was used in the laboratory-scale screening test to wash out the sodium from a diluted Initial Composite Material than in the bench-scale processing. The goal of the "baseline" enhanced sludge washing is to obtain five weight percent solids during the washes. For the laboratory-scale screening test, this requires estimating the fraction of solids that will dissolve (usually assumed to be $30 \%$ of the water insoluble sludge). The bench-scale processing then uses the actual dissolution results of the laboratory-scale screening test to determine the quantity of inhibited water to use for the water washes.

The bench-scale processing used the calculated laboratory-scale screening test initial solids concentrations and final solids concentration to determine the required inhibited water addition to obtain five weight percent solids. However, results of measurements, following the bench-scale processing, determined the weight percent solids of the bench-scale processing feed material (Original Material) to be about $20 \%$ higher than expected. Thus, instead of obtaining five weight percent solids during washing, the slurry was estimated to be between six to seven weight percent solids. This is about two times the solids concentration used during the laboratory-scale testing, and it is anticipated that the washing efficiency was reduced.

On evaluation of the laboratory-scale data following in bench-scale processing, it was determined that the laboratory-scale screening test underestimated the solids dissolution and obtained a three weight percent (instead of five weight percent) slurry during water washes. Using this data resulted in the higher bench-scale processing weight percent solids and reduced washing efficiency. 
High Sodium Oxalate Concentration: The Original Material (feed material to the bench-scale processing) appears to contain a significant quantity of sodium oxalate. Oxalate ions represented approximately $20 \%$ (by weight) of the Original Material (on a dry weight basis), and a significant fraction of this oxalate appears to be sparingly soluble sodium oxalate. During the caustic leach, the high sodium concentration from the $\mathrm{NaOH}$ results in the equilibrium shifting toward the solid phase, thus preventing the sodium from being washed from the sludge during these steps. Once the sodium concentration is decreased during the water washes, the sodium oxalate began to dissolve, but only as its solubility equilibrium allows. The amount of sodium oxalate removed is a function of the total volume of water used in washing.

In summary, it appears that a proportionally smaller quantity of water was used in the bench-scale processing than in the laboratory-scale screening test. Therefore, a smaller fraction of the sparingly soluble sodium oxalate was removed in the washing process, resulting in a higher concentration of sodium in the Vendor Product.

\subsection{Subsampling Adequacy of the Bench-Scale Processing Apparatus}

The bench-scale batch processing apparatus was not validated to insure that the sampling method used to obtain the Original Material and Vendor Product subsamples for characterization and physical measurement provided subsamples representative of the bulk material being processed. The corrective actions related to changes in the bench-scale apparatus or operating parameters is beyond the scope of this report. However, the data from the Original Material, the Vendor Product, and the Final Vendor Product can be used to determine whether the subsampling method provides consistent and representative subsamples. Based on the data available, the subsampling appears very adequate for producing representative samples for the C-106 material.

Three subsamples each of the Original Material and Vendor Product were characterized in duplicate, thus providing analytical data to evaluate the bench-scale processing subsampling uncertainties (i.e., comparison of the analytical results for the three subsamples) and to evaluate the analytical uncertainties associated with the sample preparation (comparison of duplicates). In general, there is very good agreement between the analytes and radionuclides when comparing the results for the three subsamples for both the Original Material and Vendor Product. This is evident by the RSDs (Table 3.1 and Table 3.2) for concentrations significantly above the DL or MDA. Also, for the analytes and radionuclides considered to be insoluble during the washing and leaching processing, the Vendor Product results are consistently about twice the Original Material results (which is expected due to removal of soluble components from the solids). Additional supporting data detailing the sampling variability is provided by the Final Statistical Analysis Report (Welsh 1997). However, this only provides an indication that the bench-scale processing apparatus can provide "reproducible" subsamples, but not necessarily "representative" subsamples.

The analysis of the Final Vendor Product provides a further indication that the subsamples from the bench-scale processing apparatus are representative. In order to perform the decant/dilute sodium treatment operations, all Vendor Product settled solids were transferred to a single container, diluted with water, and blended to obtain a homogeneous composite. After the recompositing and rebottling operation performed per LOI revision 2, additional characterization was performed on the Final Vendor Product to confirm the estimated Final Vendor Product concentrations calculated from the Vendor Product results and decant/dilute parameters. The analysis of the Final Vendor Product 
produced results consistent with expectations (i.e., the results agreed with the Vendor Product adjusted for decanting the Vendor Product supernatant and diluting with water) as described by Kuhl-Klinger and Urie (1997). The Final Vendor Product analyses were performed on subsamples initially pipetted in HLRF from the homogenized recomposite. Since this HLRF "stir-pipet" subsampling operation is independent of the bench-scale "pumping" subsampling operation, and since the result from both subsampling operations are analytically consistent, both subsampling methods are considered to provide representative subsamples.

\subsection{Problems Resulting in the Need for Revision 2 to LOI}

The initial bottling of the Final Vendor Product was accomplished by pouring the composited Final Vendor Product into twenty-two $125-\mathrm{mL}$ sample bottles (twenty bottles for the two privatization contractors and two bottles for physical testing). The composited Final Vendor Product was stirred between pouring into each bottle. After the material in these twenty-two bottles had settled (approximately 3 days was required for the material to settle sufficiently such that no further settling was observed), the amount of solids in each bottle varied by as much as $30 \%$ (by volume). Also, the weight percent solids determined on the material collected for physical testing indicated a significantly higher solids content than anticipated (i.e., $21.5 \%$ versus an estimated $11.5 \%$ based on the decant/dilute sodium adjustment treatment data). Based on the discrepancy of the weight percent solids and the variability of the solids content in each bottle, the Final Vendor Product was recomposited and rebottled per revision 2 of the LOI. Section 4.4 provides additional details on the weight percent solids discrepancy.

In order to ensure that each vendor bottle contained approximately equal quantities of solids and to obtain more representative weight percent solids data than previously measured, the twenty-two bottles of Final Vendor Product and all additional Final Vendor Product were recomposited (i.e., transferred to a single container). Each of the bottles was rinsed with the settled supernatant to obtain essentially all of the solids. The recomposited Final Vendor Product was stirred with a magnetic stirrer and a stir bar to obtain a homogeneous composite. The Final Vendor Product was transferted back into the twenty-two $125-\mathrm{mL}$ bottles by pipetting the samples while stirring the composite. The Final Vendor Product remaining after filling the vendor bottles was transferred to a $1-\mathrm{L}$ bottle for archiving. After settling, each of the twenty vendor bottles contained approximately the same volume of settled solids (i.e., no visual difference in the volume of settled solids in these bottles was observed after three days). The ratio of the volume of settled solids to total volume for two bottles (C106-FP-V1-3 and C106-FP-V 1-4) were measured. This ratio was 0.26 for both bottles. The qualitative visual inspection of the settled solids volume provided an indication that the contents (i.e., Final Vendor Product) of each privatization contractor bottle were considered representative of the bulk composite material.

The stir-pour, stir-pour, etc. transferring method appeared to be inadequate for ensuring that representative samples are transferred, particularly for materials with a similar ratio of settled solids to total volume. The inadequacy of this transfer method was primarily due to the settling behavior of the Final Vendor Product. It appeared that the heavier solids settled quickly; however, due to the opaqueness of the material, visual observation did not indicate that any settling had occurred. Visually, it appeared that the composite remained homogenous during the pouring process, whereas significant quantities of solids had settled. The continuous stirring and pipetting transfer method appeared to maintain a more consistent composite throughout the transferring operations. 


\subsection{Discrepancies Between SAL and HLRF Weight Percent Solids Results}

Table 4.1 provides a summary of the physical measurements (excluding rheology and heat capacity) performed on the Vendor Product and Final Vendor Product. Details and evaluation of the physical measurement results (including rheology and heat capacity) are described by Urie (1997). Based on the RSD and RPD values in Table 4.1, the reproducibility of the physical measurements is very good; however, due to the unavailability of a control standard, the accuracy of these results is unknown. All physical measurements met the quality control requirements of the governing QA plan, MCS-033 (Kuhl-Klinger 1996).

Per revision 0 of the LOI, 100 grams of Envelope D material (dry weight basis) was to be available for each privatization contractor. Since the material was being shipped/transferred to each vendor as a slurry, accurate weight percent solids results were required to ensure that each vendor received at least 100 grams. Based on limited weight percent solids results, there appeared to be some inconsistencies between the results measured by the HLRF and those measured by the SAL. In general, the physical measurements performed in the HLRF use relatively small aliquots (1-3 grams) of the product slurries. Although the results are consistent, as evidenced by the good RPDs or RSDs, the use of these small sample aliquots may bias the results because of the difficulty in obtaining representative subsamples from small subaliquots of slurry. Based on the measured weight percent solids of the Vendor Product (14.4\% HLRF and $14.8 \%$ SAL) and the estimated weight percent solids of Final Vendor Product $(11.5 \%)$ following the decant/dilute sodium adjustment treatment (LOI Revision 1) and recompositing/rebottling operation (LOI Revision 2), the HLRF weight percent solids of $15.2 \%$ on the Final Vendor Product is inconsistent with expectations. However, the SAL weight percent solids for the Vendor Product and Final Vendor Product appear to be consistent with the expected results based on the decant supernatant analysis and decant/dilution data available. The SAL weight percent solids were obtained from drying the entire contents of the subsample (i.e., 30 to 100 grams of slurry). Although the drying times are considerably longer, and there is more opportunity for loss due to spattering, etc., the weight percent solids results obtained on the "bulk" 30 to 100 gram subsamples were considered the most reliable and were more conservative, and were used to determine the quantity of dry-equivalent Final Vendor Product supplied to each privatization contractor. 


\section{HNF-SD-WM-DP-225, REV. 1}

Table 4.1. Summary of Physical Measurement for LOI Revisions 0, 1 and 2

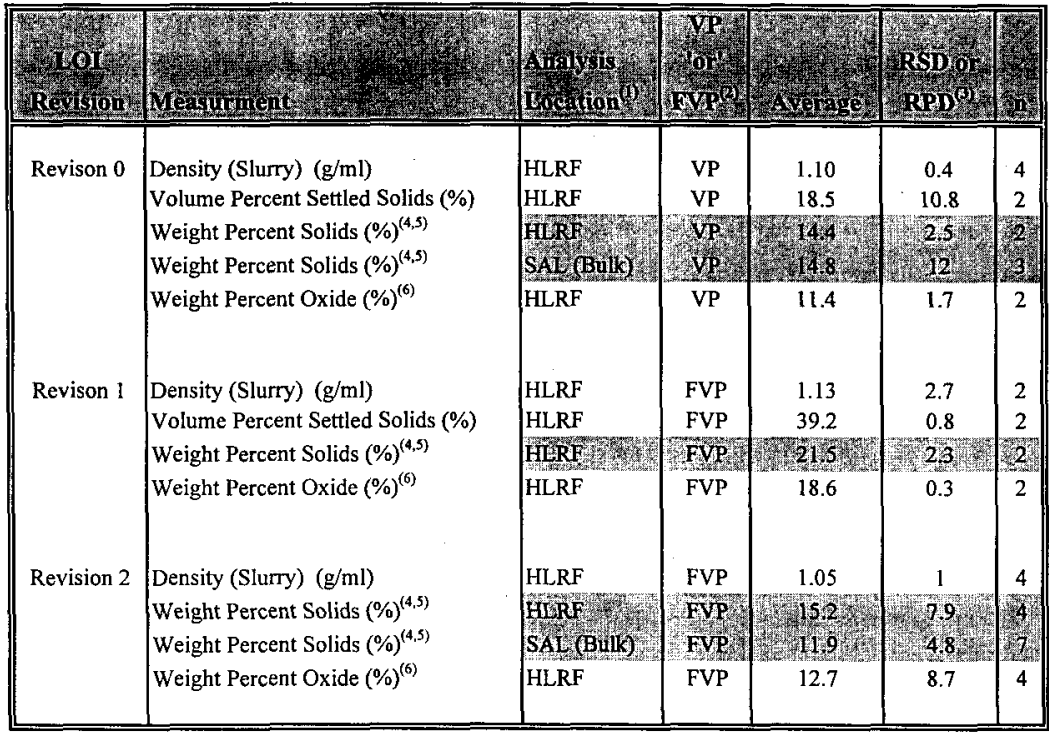

(1) $\mathrm{HLRF}=$ High-Level Radation Facility; SAL = Shielded Analytical Laboratory

(2) $\mathrm{VP}=$ Vendor Product; FVP $=$ Final Vendor Product

(3) RPD if $n=2$; RSD if $n>2$

(4) Sample size 1-3 $\mathrm{g}$ for HLRF measurements, $30-100 \mathrm{~g}$ for bulk SAL measurements

(5) Weight Percent Solids at $105^{\circ} \mathrm{C}$

(6) Weight Percent Oxides as $1050^{\circ} \mathrm{C}$ 


\section{HNF-SD-WM-DP-225, REV. 1}

\subsection{Evaluation of Mass Loss of Vendor Product}

Following the bench-scale batch processing, the Vendor Product was transferred from the 324 building to the HLRF. The contents of the two of the Vendor Product containers (SPD1-C106-051 and SPD1-C106-052) were to provide the material for shipment/transfer to the privatization contractors. There was 1624 grams of Vendor Product in container SPD1-C106-051 and 1523 grams in container SPD1-C106-52 (a total of $3147 \mathrm{~g}$ ) when the containers were transferred from the 324 building. However, following the decant/dilute sodium adjustment treatment, only 2914 grams of Vendor Product was accounted for (a discrepancy of $233 \mathrm{~g}, 30 \mathrm{~g}$ of which can be attributed to viscosity sample). The Vendor Product accounted for following the decant/dilute sodium treatment is the sum of the SPD1-C106-051 Supernatant (1044.7 g), SPD1-C106-052 Supernatant (957.6 g) and the SPD1-C106-051+052 Settled Solids $(911.6 \mathrm{~g})$. Figure 4.1 provides the details of the handling of the contents of the SPD1-C106-051 and SPD1-C106-052 containers, and provides an indication as to where potential material losses may occur. All weight presented in Figure 4.1 are measured, except for the viscosity and shear strength samples which are estimates based on the quantity of material needed for analysis. Kuhl-Klinger and Urie (1997) provide the details of the decant/dilute sodium adjustment treatment.

In investigating the possible losses (or subsampling) of the Vendor Product, a detailed evaluation of the handling, testing, and sodium adjustment operations was performed. The losses fall into three categories as follows:

Subsampling Losses: Of the 3147 grams of Vendor Product, approximately 30 grams of slurry from SPDI-C106-051 was used for viscosity analysis and not returned to the container. The sample for shear strength analysis (approximately 150 grams of settled solids) was also removed from this container; however, this sample material was recombined following the analysis. There are small losses (2-3 grams) associated with the shear strength analysis (e.g., some material remains on the shear vane).

Holdup/Transfer Losses: For the decant/dilute sodium adjustment treatment, the supernatant from the settled Vendor Product remaining in SPD1-C106-051 and SPD1-C106-052, after removal of the viscosity and shear strength subsamples, was decanted and combined with the supernatant removed from the shear strength subsample. The losses associated with residue holdup in the two initial containers (SPD1-C106-051 and SPD1-C106-052) and other container surfaces (e.g. pipets) are considered to be about $0.5 \%$ to $1 \%$ of the quantity of material handled, or potentially about 30 grams. Previous experience also indicates that significant quantities of material are lost during material transfer operations. Since the Vendor Product is a fluid slurry, the loss is minimized. For this type of slurry, the estimated mass lost during transfers is about $0.5 \%$ to $1 \%$ of the material mass transferred, or potentially another 30 grams. 


\section{HNF-SD-WM-DP-225, REV. 1}

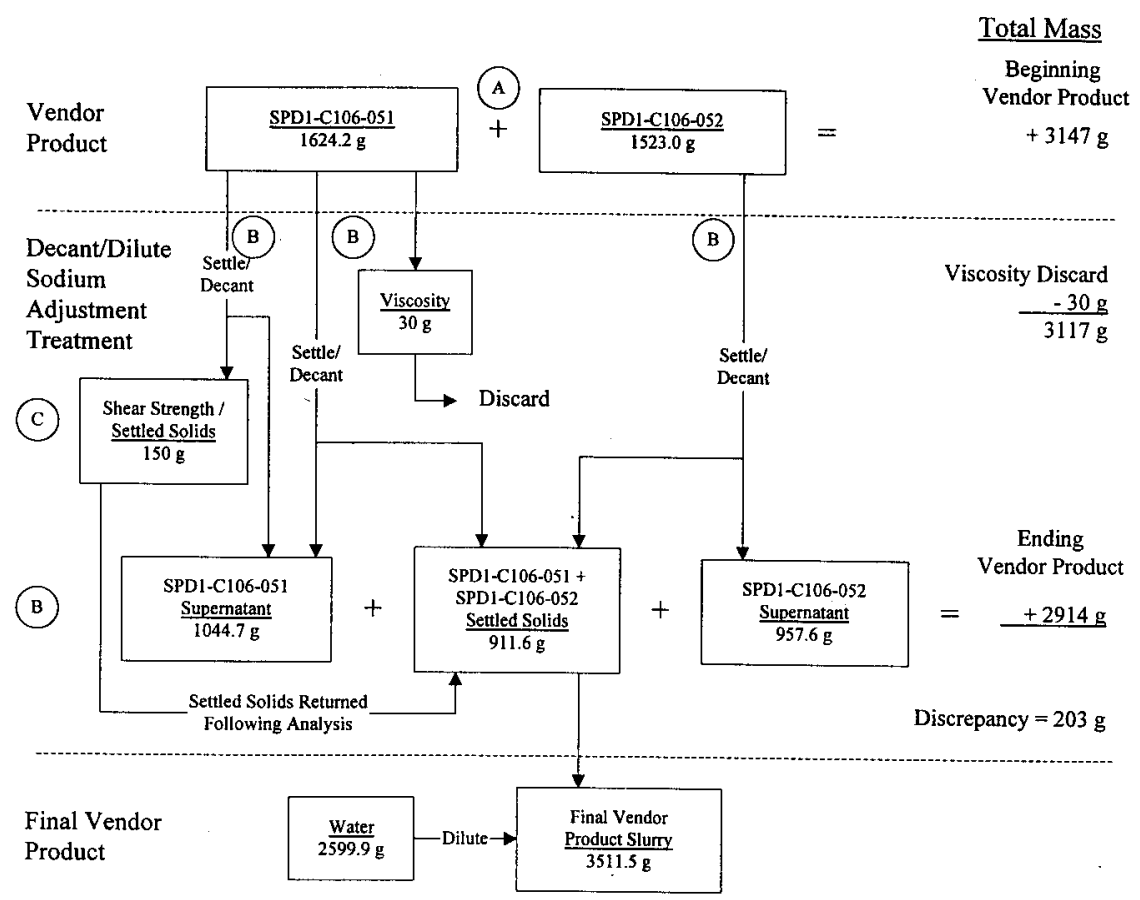

Losses

A) 1) Evaporation; 2) Residue Left on Bottles SPDI-C106-051 and SPD1-C106-052

B) 1) Transfers; holdup on pipets, minor spill, etc.; 2) Evaporation Losses

C) 1) Evaporation; 2) Residue Left on Shear Strength Container;

3) Residue Left on Shear Vane

Figure 4.1. Material Losses During Sodium Adjustment Treatment (LOI Revision 1) 
Evaporation Losses: The majority of the loss is most likely due to evaporation occurring primarily during handling of the material (e.g., open containers during decanting and testing expose high surface areas for evaporation). Due to the hot cell temperatures, it is estimated that as much as $2-3 \%$ of the material evaporated (as water) during open container handling. Also, considerable evaporative losses from closed containers have been observed during storage. A mass loss of approximately $2 \%$ was observed over a month period while the Final Vendor Product bottles (capped 125-ml bottles) were stored in the HLRF hot cell while awaiting shipping/transferring to the privatization contractors (See data in Appendix 5D, Final Vendor Product Masses). The Vendor Product container, SPD1-C106-051 and SPD1-C106052, were stored in the HLRF hot cells for approximately two months prior to opening and beginning the decant/dilute sodium treatment. Therefore, similar evaporation losses are anticipated (i.e., from 120 to 180 gram of water).

On further evaluation, the impact of this loss is considered insignificant on the data reported for the Final Vendor Product. The residual Vendor Product slurry which was left on pipettes, bottles, and working surfaces during transfers and handling only impacts the amount of Final Vendor Product available, and sufficient Final Vendor Product was available to meet the needs of the privatization contractors. The evaporation of water from the Vendor Product does increase the weight percent solids (and weight percent oxides) in the Vendor Product and the analyte concentration of the slurry. However, this evaporation does not impact the concentration of the analytes based on a dry weight basis. If all 203 grams of the unaccounted Vendor Product (233 grams - 30 gram Viscosity subsample sample) was due to the evaporation of the water, the weight percent solids would be $6.5 \%$ higher ( 15.3 weight percent solids instead of 14.4 weight percent solids [HLRF data]). The weight percent solids (and weight percent oxides) were measured on the Final Vendor Product following the recomposting and rebottling per revision 2 of the LOI; therefore, the weight percent solids measurements on the Vendor Product do not impact the quantity of solids provided to the privatization contractors.

\subsection{ORIGINAL MATERIAL}

\subsection{Comparison of Original Material (Dried Samples to Centrifuged Solid+Liquid)}

To provide additional information on the reliability of the analytical measurements, the Original Material slurry was analyzed as a slurry (after drying) and as solids and liquid fractions (after centrifuging). The dried slurry samples consist of all the solids plus the dissolved salts in the slurry liquid. The solids and liquid fraction samples were obtained by centrifuging the Original Material slurry and separating the solids and supernatant liquid fractions by decanting/pipetting the supernatant liquid.

The results for the dried Original Material have been presented by Urie (1997), and consist of duplicate analyses on three subsamples (SPDL1-C106-003, -004, and -005) extracted from the benchscale processing apparatus just prior to beginning the sludge washing operations. A fourth subsample (SPDL1-C106-002) was collected for physical measurements testing, and was the source of the solid and liquid fractions which were analyzed in duplicate for a limited set of analyses. An aliquot of the fourth subsample was centrifuged and the solids and liquid fractions separated. The solid fraction was analyzed in duplicate by ICP-AES following $\mathrm{KOH}$ (PNL-ALO-115) and $\mathrm{Na}_{2} \mathrm{O}_{2}$ (PNL-ALO-116) fusions and by IC following water leaching (PNL-ALO-103). The liquid fraction was analyzed in duplicate by ICP-AES following an acid digestion (PNL-ALO-128) and directly by IC following filtering and dilution. To provide the information necessary to recombine the analyzed fractions, 
weight percent solids (wet) of the centrifuged aliquot $\left(43.4 \%\right.$ ), weight percent solids (dried at $105^{\circ} \mathrm{C}$ ) of the centrifuged solids fraction $(51.7 \%)$, and density of the centrifuged liquid fraction $(1.07 \mathrm{~g} / \mathrm{ml})$ were measured. To allow all results to be normalized to slurry concentration, the weight percent solids (dried at $105^{\circ} \mathrm{C}$ ) of the Original Material slurry (29.2\%) was determined.

The averaged ICP-AES fusion results ( $\mathrm{KOH}$ and $\mathrm{Na}_{2} \mathrm{O}_{2}$ ) and the IC analyses results from the dried Original Material slurry were compared to the summation of concentrations of the Original Material centrifuged solids and centrifuged liquid. Table 5.1 presents the comparison of the Original Material slurry to the summation of the centrifuged solids/liquid fractions. Although not shown on the table, the uncertainty of the analytical results between the duplicates (and particularly between the two fusion results) is approximately $20 \%$ for many analytes, and the uncertainties in the physical measurements used to combine and normalize the results are about $3 \%$ for each measurement. Also, some measurements used very small sub-aliquots (e.g. centrifuged solids weight percent solids dried at 105 ${ }^{\circ} \mathrm{C}$ used only 1 gram); this adds additional subsampling uncertainty to the results.

Except for a few analytes, the average concentrations for the slurry and centrifuged solid/liquid fractions are within $\pm 30 \%$. Silver, barium, chromium, phosphorus, lead, uranium, nitrate, and phosphate are near or below the EQL and are presented for information only due to high uncertainties. Considering that both the phosphonus and phosphate in the centrifuged liquid were near the EQL, the ICP-AES and IC results compared reasonably well (i.e., $0.020 \% \mathrm{PO}_{4}$ as $\mathrm{P}$ from ICP and $0.013 \% \mathrm{PO}_{4}$ from IC). For the other analytes, the comparison may be considered bounding, considering the uncertainties associated with each analysis/measurement, the centrifuged fractions were extracted from a different subsample than the subsamples used for determining the slurry concentration, and the analytical work on the slurry and centrifuged fractions were performed two months apart.

Of particular interest is the sodium and oxalate of the centrifuged liquid fraction, since the analysis is very high in sodium $(2.38 \mathrm{wt} \%)$ and low in oxalate and other measured anions (total anion $=1.17 \mathrm{wt} \%$ ). Assuming all the anions are from sodium compounds, only about $30 \%$ of the total sodium (i.e., $0.7 \mathrm{wt} \%$ ) can be attributed to these compounds; the remainder most probably being from sodium hydroxide. The oxalate is considerably lower than would be expected from the quantity of oxalate soluble in the water leaches of both the centrifuged solids fraction and the dried Original Material slurry. However, when compared to the oxalate concentration in the Original Material (5.9 $w t \%)$, the oxalate concentration in the centrifuged liquid fraction is similar to the concentration of oxalate determined in the suspected organic layer (Campbell 1996); i.e, $0.34 \mathrm{wt} \%$ versus about 0.6 wt \%. Based on other work (Russell, et. al 1996; Smith, et.al 1996) the solubility of various oxalates appears to decrease as the $\mathrm{pH}$ increases. At a lower $\mathrm{pH}$, the solubility of the various oxalates (e.g. sodium, iron, aluminum) increase, resulting in the higher oxalate concentration.

Although the $\mathrm{pH}$ of the centrifuged solids water leach samples was not measured, the $\mathrm{pH}$ is expected to be lower than the $\mathrm{pH}$ of the centrifuged liquid because the majority of the centrifuged liquid fraction (with a pH near 12) was removed prior to water leaching of the centrifuged solids. At this lower $\mathrm{pH}$, the concentration of oxalates should increase, as is observed in the centrifuged solids leachates. An ICP-AES analysis on the water leach samples would identify the associated cations; however, no ICP-AES analysis was performed on the water leaches. Also, based on the data available, it is not possible to determine if all of the oxalate present in the Original Material slurry or the centrifuged solids was extracted by the water leaching (i.e., the oxalate may be in solubility equilibrium). 
Table 5.1. Comparison of Original Material Slurry and Centrifuged Fractions

\begin{tabular}{|c|c|c|c|c|c|c|}
\hline \multirow{4}{*}{ Analytents } & \multicolumn{3}{|c|}{ Centrifuged Orig Mat'1 SPDL1-C106-002 } & \multirow{2}{*}{\multicolumn{2}{|c|}{$\begin{array}{l}\text { Original Material } \\
\text { SPDL1-9106-003/005 }\end{array}$}} & \multirow{4}{*}{ 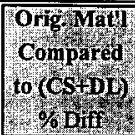 } \\
\hline & Centrifuged & Decant & Total & & & \\
\hline & Solidis & Liquid & $(\mathrm{CS}+\mathrm{DH})^{(2)}$ & Uncortd & Corrd & \\
\hline & Whosliring & Wo\% Slumy & Wio slurry & Wto $\%$ & $\mathrm{~W} \%$ & \\
\hline Ag & 0.011 & $<0.001$ & 0.011 & 0.046 & 0.046 & 76 \\
\hline Al & 1.197 & $<0.001$ & 1.197 & 1.079 & 1.079 & -11 \\
\hline $\mathbf{B a}$ & 0.007 & $<0.001$ & 0.007 & 0.006 & 0.006 & -20 \\
\hline $\mathrm{Ca}^{\mathrm{KOH}}$ & 0.037 & $<0.001$ & 0.037 & 0.074 & 0.074 & 50 \\
\hline $\mathbf{C r}$ & 0.019 & $<0.001$ & 0.019 & 0.017 & 0.017 & -15 \\
\hline $\mathrm{Fe}$ & 2.429 & $<0.001$ & 2.429 & 1.942 & 1.942 & -25 \\
\hline Mn & 0.053 & $<0.001$ & 0.053 & 0.044 & 0.044 & -20 \\
\hline $\mathrm{Na}$ & 3.797 & 2.376 & 6.173 & 6.472 & 6.786 & 9 \\
\hline $\mathrm{Ni}$ & 0.018 & $\mathrm{n} / \mathrm{d}$ & 0.018 & 0.016 & 0.016 & -13 \\
\hline $\mathbf{P}$ & 0.100 & 0.007 & 0.107 & 0.101 & 0.102 & -4 \\
\hline $\mathbf{P b}$ & 0.062 & $<0.001$ & 0.062 & 0.052 & 0.052 & -20 \\
\hline Si & 0.664 & $<0.001$ & 0.664 & 0.988 & 0.988 & 33 \\
\hline $\mathrm{Ti}$ & 0.009 & $<0.001$ & 0.009 & 0.013 & 0.013 & 29 \\
\hline $\mathbf{U}$ & 0.013 & 0.032 & 0.045 & 0.057 & 0.062 & 29 \\
\hline $\mathbf{Z r}$ & 0.013 & 0.005 & 0.018 & 0.017 & 0.017 & -2 \\
\hline Wryty & $\begin{array}{l}\text { 10pyass } \\
\text { Total (Cs) }\end{array}$ & IOPAES & 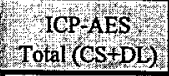 & Wrater & 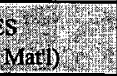 & 2. \\
\hline Writy & 8.43 & 2.42 & 10.85 & 10.93 & 11.25 & 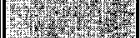 \\
\hline & $<0$ & & & 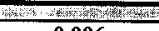 & 然 & Mth \\
\hline $\mathrm{Cl}$ & $<0.001$ & $<0.001$ & $\mathrm{n} / \mathrm{d}$ & 0.006 & 0.006 & $n / d$ \\
\hline $\mathrm{NO}_{2}$ & 0.247 & 0.627 & 0.874 & 0.691 & 0.756 & -16 \\
\hline $\mathrm{NO}_{3}$ & $<0.001$ & 0.024 & 0.024 & 0.031 & 0.031 & 23 \\
\hline $\mathrm{PO}_{4}$ & 0.045 & 0.013 & 0.058 & 0.033 & 0.034 & -70 \\
\hline $\mathrm{SO}_{4}$ & 0.087 & 0.169 & 0.256 & 0.202 & 0.219 & -17 \\
\hline $\mathrm{C}_{2} \mathrm{O}_{4}$ & 4.071 & 0.336 & 4.407 & 5.849 & 5.903 & 25 \\
\hline r. & 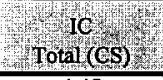 & Total $(\mathrm{Db})$ & Total (CS DLY & Total $(\mathrm{Orig}$ & 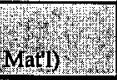 & Wy \\
\hline $4 x^{4}+x^{2}$ & 4.45 & 1.17 & 5.62 & 6.81 & 6.95 & (x) \\
\hline
\end{tabular}

(1) For all CS, DL and OM anlaytical/physical results see Analytical Summary Report . Averaged ICP results from $\mathrm{KOH}+\mathrm{Na2O} 2$ fusions used for comparison purposes.

All result have been blank corrected for comparison; $U$ on $O M$ is from acid digestion.

(2) $\mathrm{CS}=$ Centrifuged Solids; $\mathrm{DL}=$ Decant Liquid; $\mathrm{OM}=$ Original Material

(3) $\mathrm{CS} \mathrm{wt} \%=\mu \mathrm{g} / \mathrm{g}$ result * $(\mathrm{g} / \mu \mathrm{g} * 100)[0.0001]^{*}(\mathrm{wt} \% \mathrm{CS}$ wet $/ 100)[0.434] *(\mathrm{wt} \% \mathrm{CS} \mathrm{dry} / 100)$ [0.517]

(4) $\mathrm{DL} \mathrm{wt} \%=\mu \mathrm{g} / \mathrm{ml}$ result * $(\mathrm{g} / \mu \mathrm{g} * 100)[0.0001] *(1-\mathrm{wt} \% \mathrm{CS}$ wet $)$ [0.566] / DL density [1.07]

(5) $\mathrm{OM}$ wt $\%=\mu \mathrm{g} / \mathrm{g}$ result * $(\mathrm{g} / \mu \mathrm{g} * 100)[0.0001] *$ (OM wt\% solids on drying $/ 100)$ [0.292]

(6) See Section 5.2 for Corr'd Wt\% calculation

(7) $\mathrm{n} / \mathrm{d}=$ measured analyted not detected above $0.0005 \%$ 


\subsection{Evaluation of Effect of Removal of Suspect Organic Layer}

The three Original Material slurry subsamples (SPDL1-C106-003, -004, and -005) were processed for chemical analysis in the SAL. Each of these subsamples, when transferred to beakers for drying, developed a clear layer on top of the slurry material. This clear layer was suspected to be an organic layer, and comprised about $10 \%$ of the total volume of each sample. The details of the removal of the layer and subsequent organic and radiochemistry screening are reported by Urie (1997), and the results of the organic screening are reported by Campbell (1996). Since this layer was removed from the subsample prior to drying, the effect of the removal of $10 \%$ of the Original Material slurry supernatant on the reported Original Material results has been evaluated.

Based on an initial volume of the Original Material slurry samples $(100 \mathrm{ml}$ each $)$, the density of the slurry $(1.25 \mathrm{~g} / \mathrm{ml})$, the volume of layer removed (10\% of the total volume), and the density of the layer removed $(1.07 \mathrm{~g} / \mathrm{ml})$, the reduction in the quantity of supernatant is estimated to be about $12 \%$. Based on the analytical results from the centrifuged liquid (See Table 5.1), the only analyte with any significant concentration in the supernatant is sodium (and possibly nitrite and oxalate).

Since $12 \%$ of the supernatant was removed from the Original Material samples prior to drying, the Original Material results are biased low for those analytes measured in the supernatant. This bias can estimated by reducing (by $12 \%$ ) the concentration determined in the centrifuged liquid and calculating a new total weight percent slurry [e.g., (CS Na 3.797)+(DL Na 2.376)-((DL Na 2.376)*0.12) = 5.89], and then ratioing this total to the original total (e.g., $6.17 / 5.89=1.05$ ). Based on this rough estimate, the reported sodium value for the Original Material is low by $5 \%$. Following the same logic, the reported nitrite result is about $10 \%$ low and the reported oxalate is about $1-2 \%$ low. Although an estimate, the "corrected" Original Material slurry results have been used for the direct comparison in Table 5.1. Only the chemical and radiochemical results are affected by the removal of the suspected organic layer, since the physical measurements (including weight percent solids) were performed on an independent subsample.

At the same time the organic screening was performed on the layer removed from the Original Material, total alpha activity and total beta activity were also performed. The total alpha activity for the supernatant layer was about $0.2 \mu \mathrm{Ci} / \mathrm{g}$, and the total beta activity was $33 \mu \mathrm{Ci} / \mathrm{g}$. Again, the removal of about $12 \%$ of the supernatant has little effect on the final reported alpha and beta activities for the Original Material $(3.2 \mu \mathrm{Ci} / \mathrm{g}$ and $1220 \mu \mathrm{Ci} / \mathrm{g}$, respectively), since the majority of the activity appears to be in the solids fraction. 


\subsection{OBSERVATIONS, LIMITATIONS, and RECOMMENDATIONS}

\subsection{Data Acceptability}

In general, the analytical, radiochemical, and physical measurement results are fundamentally sound and provide a solid basis for full characterization of the Privatization Contractor Samples. As such, the analytical results should be accepted as representing the nominal concentration of the Final Vendor Product.

\subsection{Laboratory-Scale and Bench-Scale Operations}

Observation: Due to programmatic schedule requirements, the bench-scale processing was initiated prior to completion of the laboratory-scale screening test. Every effort was made to evaluate the laboratory-scale results for each washing/leaching step prior to beginning these steps in the bench-scale processing. However, the failure to recognize that the laboratory-scale test washing/leaching steps were conducted at a weight percent solids lower than target (i.e., $3 \mathrm{wt} \%$ versus a target of $5 \mathrm{wt} \%$ ) resulted in selecting bench-scale processing operating parameters that reduced the washing/leaching efficiency. Because of the decrease in washing efficiency for the bench-scale processing operation, more insoluble sodium oxalate than anticipated remained in the solids and contributed to the sodium concentration which exceeded the Envelope D specification.

Recommendation: Fully evaluate laboratory-scale screening test parameters for acceptability and assess the results against the material specification prior to initiating bench-scale processing activities.

Observation: There were only a selected set of analyses performed on the laboratory-scale screening test product. These analyses were primarily selected based on previous history of similar sludge washing tests, and did not include analytical screening for major components other than those measured by a simultaneous ICP-AES procedure and a specific IC procedure. For the C-106 material, a measurement of the oxalate concentration may have increase awareness of solubility issues and allowed adjustments to the laboratory-scale screening test and bench-scale processing operating parameters to mitigate the high sodium problem.

Recommendation: To better understand potential washing/leaching limitations, characterization of the initial feed material should include measurement of oxalate and complexing metal ions.

Observation: The bench-scale processing apparatus was not evaluated for the ability to provide representative subsamples for chemical/radiochemical analysis and physical measurements. Although the results from the Vendor Product and Final Vendor Product can be used to verify the apparatus provided representative samples for the $\mathrm{C}-106$ material, other material types (e.g., materials with significantly different settling rates) may not behave the same in the apparatus.

Recommendation: It is important to establish a bench-scale apparatus test which can evaluate or demonstrate subsampling reproducibility prior to beginning new (and different) material processing activities. 


\subsection{Chemical and Physical Measurement Analysis}

\section{General Observations:}

- The spike recoveries for arsenic, dysprosium, holmium, molybdenum, strontium, tungsten, and yttrium were high; therefore, the reported results may be biased high. Also, the spike recoveries for cerium, antimony, zinc, and niobium-93m were low, indicating a potential that the reported results may be biased low. All of these analytes are present at less than $30 \%$ of the Envelope D specification. Therefore, for the Final Vendor Product (C-106), the accuracy resulting from evaluation of these spike recoveries is not of concern.

- Beryllium, potassium, selenium, thorium and yttrium concentrations were below the detection limit for all analyses conducted.

- Total alpha activity and total beta activity measurement provide greater than $95 \%$ accountability for the major alpha and beta emitting radionuclides.

- Preparation blank concentrations exceeding $5 \%$ of the measured sample concentration were observed for nickel, molybdenum, platinum, and vanadium. The source of the molybdenum, platinum, and vanadium contamination is unknown at this time. The platinum concentration exceeded the Envelope D specification by $20 \%$, and the contribution from the blank adds to the uncertainty of the reported result.

- Major differences between the ICP-MS and ICP-AES results were reported for calcium, zirconium, iron, aluminum, tellurium, and sodium. Inductively coupled plasma mass spectroscopy differences were reported between the two fusion preparations ( $\mathrm{KOH}$ and $\mathrm{Na}_{2} \mathrm{O}_{2}$ ) for copper, molybdenum, platinum, rhenium, tin, and strontium. These differences can not be explained at this time. The unexplained difference in sodium concentration is of particular concern, as it is the metric for service payment.

- Comparison of analysis of the Original Material slurry with "centrifuged solids plus centrifuged supernatant" resulted in agreement of $\pm 30 \%$, except for a few trace and minor analytes near the EQL: silver, barium, chromium, phosphorus, lead, uranium, nitrate, and phosphate.

- Hot cell sample handling (e.g., shear strength analysis, compositing, blending, etc.) may result in evaporative losses as high as $10 \%$. Evaporation losses of $2 \%$ were observed for closed Final Vendor Product bottles following storage of the bottles for one month in the HLRF hot cells.

Observation/Limitation: Based on the results from the Original Material and Vendor Product, the ICP-MS experienced significant interferences when analyzing dissolutions from either fusion procedure. This was most dramatic on the Vendor Product for the very low concentration analytes, but also apparent in some major constituents. When the interference was severe, the data had to be flagged as "not used". When the ICP-MS results are within 20 times the DL, the results need to be considered for information only. Also, when very large differences exist between the $\mathrm{KOH}$ and $\mathrm{Na}_{2} \mathrm{O}_{2}$ fusion, the results are suspect. 
Observation/Limitation: The ICP-MS results for the Original Material are considered more reliable than the results for the Vendor Product. The Vendor Product analyses were the more important (i.e., comparison to the Envelope D specification), and the ICP-MS analyses were performed first on the Vendor Product due to schedule milestones. The ICP-MS analysis and data handling protocols were established on the Vendor Product, and then used for analyzing the Original Material. A number of analysis problems were encountered in the "initial" (i.e., Vendor Product) ICP-MS analysis (Urie 1997, Appendix A) which were related to the C-106 matrix and the large number of analytes and radionuclides measured. Many of analytes and radionuclides measured in the Vendor Product had to be flagged as unusable or not used (i.e., $n / u$ in data tables) because of these analysis problems. The analysis of the Original Material took considerably less time and the QC sample results were significantly better than for the Vendor Product.

Observation/Limitation: Silver, erbium, gadolinium, niobium, platinum, tin, and ytterbium reported results are at $40 \%$ to $120 \%$ of the Envelope D specification. Validation of these analytes may be subjected to higher uncertainty since their measurement had high relative standard deviations.

Observation/Limitation: The phosphorus analysis by IC measures only soluble phosphates. The total phosphorus concentration is obtained by ICP-AES. Like phosphorus, sulfur analysis by IC measures only soluble sulfates. The PNNL Analytical Chemistry Laboratory currently has no ICP-AES capability for measuring total sulfur.

Observation/Limitation: Although not discussed in this report, the shear strength analysis and heat capacity analysis did not meet analysis expectations. As reported by Urie (1997), the shear strength instrumentation/procedure used by PNNL was unable to measure the low shear strengths required, and the heat capacity was estimated but could not be measured.

Observation: The acid digestion procedure used for the $\mathrm{C}-106$ analyses was a well-established nitric acid-hydrochloric acid dissolution procedure. When the results from this acid digestion are compared to the results from the two fusion procedures used, the acid digestion results are consistently lower. Typically, acid digestion methods provide solutions with less blank contamination, less analyte dilution (i.e., higher sensitivity), and fewer matrix interferences than the fusion methods. Unfortunately, the acid digestion procedure used apparently provides incomplete dissolution for the complex C-106 material.

Recommendation: To gain the benefits of acid digestion dissolution (i.e., less contamination, higher sensitivity, and fewer interferences), a more rugged acid digestion procedure needs to be developed which will provide better dissolution for complex waste material.

Observation: Spike recovery information was not available for ICP-MS measurements of praseodymium, thulium, iodine-129, neptunium-237, technetium-99, palladium-107, and plutonium and uranium isotopes. Also, there was no other appropriate standard information which could be used to assess the accuracy of the reported results.

Recommendation: In the absence of spiking information which is used to evaluate the accuracy of the measured result, other acceptable methods for evaluating accuracy should be implemented and reported. 
Observation: The accuracy of the measured analytical results is obtained from evaluating the recovery of standards: LCSs, Blank Spikes, Matrix Spikes, or Post-Digestion Spikes. Each of these standards provides information to estimate the accuracy of the analytical measurement. However, there are no LCSs available which match the matrix of the C-106 material (or any other tank waste). The Blank Spike, Matrix Spike, and Post-Digestion Spike standards are merely solution standards added to the sample matrix (or blank) at different points in the preparation and may not reflect the dissolution characteristics of the sample matrix material.

Recommendation: To better evaluate the accuracy of the measured concentrations in tank sludge waste material, control standards more representative of tank waste material need to be "certified" and used as the LCS by laboratories performing analyses on tank sludge waste.

Observation: The average ICP-MS result for sodium was $12 \%$ higher than the ICP-AES result for sodium. Based on an evaluation of all ICP-AES and ICP-MS data, it is difficult to determine the "true" sodium concentration.

Recommendation: In order to ensure accurate sodium concentrations, improvements should be made in ICP-AES and/or ICP-MS sodium analysis or an alternate method identified.

Observation: From the results for the anion measurements performed on water leaches of the dried Original Material, Vendor Product, and Final Vendor Product, it is apparent that the reported results represent only the anions soluble at a 1:100 leaching ratio. There is data to suggest that the oxalate did not completely dissolve at this $1: 100$ leaching ratio.

Recommendation: To better establish the concentration of anions which may be in excess at these leaching ratios, multiple leaching ratios (e.g., 1:100, 1:200, 1:500) should be used to establish the "true" concentration of these soluble anions. Also, ICP-AES analyses may be performed on all water leached sample to assist in establishing the soluble compound.

Observation: The difference observed between the weight percent solids determinations made in the SAL and in the HLRF can be attributed to sample size differences (i.e., 1-3 $\mathrm{g}$ in HLRF and 30-100 $\mathrm{g}$ in SAL) and the ability to obtain representative samples when subsampling (particularly, for remote subsampling in hot cells). Although many times it is necessary to use small subsample to conserve material, these small subsamples can inject biases into the final result. This is particularly true when subsampling slurries containing reasonably high levels of solids (e.g., 10-20 wt\%). The typical subsampling method used is to vigorously stir the slurry while extracting a subsample by pipet. If the solids are not uniformly distributed throughout the bulk sample by this technique, the location of the pipet tip potentially can alter the measured results. In such cases, the samples withdrawn from the bulk may be very reproducible, but not representative of the bulk material. Worse, different waste materials may behave differently using the same stirring/extracting procedure.

Recommendation: Subsampling and subaliquotting techniques should be evaluated for appropriateness prior to use, and whenever possible, large subsamples should be used for more accurate weight percent solids measurements. 


\subsection{REFERENCES}

Brooks, KP, et. al. 1997: Bench-Scale Enhanced Sludge Washing and Gravity Settling of Hanford Tank C-106 Sludge. PNNL-11432, Pacific Northwest National Laboratory, Richland, Washington.

Conners, JM. 1996. Grab Sampling and Analysis Plan for Tank 241-C-106 (In Support of the Privatization Request for Proposal). WHC-SD-WM-TSAP-104. Westinghouse Hanford Company. Richland, Washington.

Jones, TE. 1996. Addendum 1 of the Letter of Instruction (LOI) for TWRS Privatization Contractor Samples Addressing Waste Envelope D Materials, Revision 0. Pacific Northwest National Laboratory, Richland, Washington.

Kuhl-Klinger, KJ. 1996. Quality Assurance Plan for Activities Conducted by the Analytical Chemistry Laboratory $(A C L)$. MCS-033, Pacific Northwest National Laboratory, Richland, Washington.

Kuhl-Klinger KJ and MW Urie. 1997. Tank Waste Remediation System Privatization Contractor Samples Analytical Summary Report, Addendum 1, Revision 2. Pacific Northwest National Laboratory, Richland, Washington.

Lumetta, GJ, et. al. 1996. Washing and Caustic Leaching of Hanford Tank C-106 Sludge. PNNL-1 1381, Pacific Northwest National Laboratory, Richland, Washington.

Russell, RL, et. al. 1996. Fiscal Year 1996 Laboratory Scale Studies of Cesium Elution in Tank 8D-1. PNNL-11274, Pacific Northwest National Laboratory, Richland, Washington.

Smith, HD, et. al. 1996. Fiscal Year 1996 Laboratory Scale Studies of the WVNS Flowsheet for Remediation of Tank $8 D-1$ and Tank 8D-2. PNNL-11409, Pacific Northwest National Laboratory, Richland, Washington.

Campbell, JA. 1996. Letter Report submitted by MW Urie to CM Seidel, "PNNL ACL Submittal of Organic and Radionuclide Results from Analysis of C-106 Original Material Supernate". October 31, 1996.

Urie, MW. 1997. Tank Waste Remediation System Privatization Contractor Samples Analytical Summary Report. Pacific Northwest National Laboratory, Richland, Washington.

Welsh, TL. 1997. Tank Waste Remediation System Privatization Contractor Samples Final Statistical Analysis Report. Babcock \& Wilcox Protec, Inc., Richland, Washington.

Wiemers, KD. 1996a. Addendum 1 of the Letter of Instruction (LOI) for TWRS Privatization Contractor Samples Addressing Waste Envelope D Materials, Revision 1. Pacific Northwest National Laboratory, Richland, Washington.

Wiemers, KD. 1996b. Addendum I of the Letter of Instruction (LOI) for TWRS Privatization Contractor Samples Addressing Waste Envelope D Materials, Revision 2. Pacific Northwest National Laboratory, Richland, Washington. 
HNF-SD-WM-DP-225, REV. 1

\section{TANK WASTE REMEDIATION SYSTEM (TWRS) PRIVATIZATION CONTRACTOR

\author{
ENVELOPE D SAMPLES \\ (C-106)
}

ATTACHMENT 1

ANALYTICAL SUMMARY REPORT

\section{March 1997}

Pacific Northwest National Laboratory 
HNF-SD-WM-DP-225, REV. 1

Analytical Summary Report - January 28, 1997

TANK WASTE REMEDIATION SYSTEM (TWRS) PRIVATIZATION CONTRACTOR SAMPLES

\section{WASTE ENVELOPE D MATERLAL}

241-C-106

ANALYTICAL SUMMARY REPORT

Prepared by: Michael W. Urie

Pacific Northwest National Laboratory (PNNL)

Analytical Chemistry Laboratory (ACL)

C-106 Project Manager

January 28, 1997 
HNF-SD-WM-DP-225, REV. 1

Analytical Summary Report - January 28, 1997

\author{
Contributors \\ PD Anderson \\ DL Baldwin \\ DL Bellofatto \\ PK Berry \\ JP Bramson \\ OP Bredt \\ RE Brinson Jr \\ KP Brooks \\ SK Fadeff \\ MW Goheen \\ LR Greenwood \\ IC Henry \\ FV Hoopes \\ GS Klinger \\ KJ Kuhl-Klinger \\ GJ Lumetta \\ MP Marrott \\ RL Myers \\ EA Nelson \\ MM O'Neill \\ D Ortiz \\ GK Patello \\ KA Poeppel \\ KG Rappe \\ RT Ratner \\ MR Reid \\ GM Richardson \\ DE Rinehart \\ DR Sanders \\ RT Steele \\ KJ Smith \\ $\mathrm{CZ}$ Soderquist \\ MJ Steele \\ RG Swoboda \\ KK Thomas \\ JM Tingey \\ SM Tingey \\ TL Trang-Le \\ MW Urie \\ JJ Wagner \\ EJ Wyse
}


HNF-SD-WM-DP-225, REV. 1

Analytical Summary Report - January 28, 1997

TANK WASTE REMEDIATION SYSTEM (TWRS) PRIVATYZATION CONTRACTOR SAMPLES

WASTE ENVELOPE D MATERIAL

C-106 


\section{EXECUTIVE SUMMARY}

This report represents the Analytical Summary Report on TWRS Privatization Contractor Samples conducted in support of the Tank Waste Remediation System (TWRS) Privatization Contractor Samples, Waste Envelope D, C-106 characterization testing. All work performed was conducted in accordance with "Addendum 1 of the Letter of Instruction (LOI) for TWRS Privatization Contractor Samples Addressing Waste Envelope D Materials - Revision 0," (Jones, 1996).

Tank 241-C-106 (C-106) was selected by TWRS Privatization for the Part 1A Envelope D high-level waste demonstration. Twenty grab samples of $\mathrm{C}-106$ were composited and characterized during three sequential stages of Envelope D sample preparation: 1) after dilution with water prior to bench-scale processing ("Original Material"); 2) after the bench-scale treatment per revision 0 of the LOI ("Vendor Product"); and 3) after decant/dilute treatment per revision 1 of the LOI ("Final Vendor Product"). This report includes only analytical results for the Vendor Product (Envelope D, Privatization Contractor Samples) and the Original Material.

The primary purpose of this report is to provide the chemical and radiochemical results for the Vendor Product for comparison to the Envelope D specification. A summary of the "most reasonable" averaged results compared to the Envelope D specification is presented in Table ES-1. The results are presented in units of $\mathrm{g} / \mathrm{L}$ and $\mathrm{Ci} / \mathrm{L}$ (at 31 gram waste oxide) for comparison with the envelope specification and in $\mu \mathrm{g} / \mathrm{g}$ and $\mu \mathrm{Ci} / \mathrm{g}$ dried solids for direct comparison to the analytical results.

The Vendor Product resulting from the treatment defined in the LOI Revision 0 exceeds the Envelope $\mathrm{D}$ specifications for $\mathrm{Na}$ and ${ }^{121 \mathrm{~m}} \mathrm{Sn}$. Additionally, a trace component (Pt) and a trace radionuclide $\left({ }^{233} \mathrm{U}\right)$ were found to exceed the envelope specification, and three radionuclides $\left({ }^{10 \mathrm{~m}} \mathrm{Ag},{ }^{242} \mathrm{Pu}\right.$, and ${ }^{119 m} \mathrm{Sn}$ ) could not be verified due to analytical detection limitations. Also, the results for ${ }^{243} \mathrm{Cm} /{ }^{44} \mathrm{Cm}$ and ${ }^{144} \mathrm{Ce} /{ }^{144} \mathrm{Pr} /{ }^{144 m} \mathrm{Pr}$ exceeded the specification; but only if the results are compared to the Envelope D specifications for ${ }^{243} \mathrm{Cm}$ (as a trace radionuclide) and for ${ }^{144 \mathrm{~m}} \mathrm{Pr}$, respectively. Based on the total activity measured, the specifications for ${ }^{244} \mathrm{Cm},{ }^{144} \mathrm{Ce}$, and ${ }^{144} \mathrm{Pr}$ were not exceeded.

Viscosity and shear strength did not meet the LOI Revision 0 specifications. The viscosity was lower than the specification, and the shear strength measured detection limit was higher than the specification 
limit. Compliance with these physical properties specifications are not required for shipping the Vendor Product to the vendors.

Inductively coupled plasma-atomic emission spectroscopy (ICP-AES) and inductively coupled plasma-mass spectroscopy (ICP-MS) were performed on multiple sample preparations for many of the analytes of interest. Generally, the highest reliable averaged result (considering the average, the standard deviation, and preparation blank performance) was reported. Table ES-1 indicates which analytical method and preparative technique were selected for comparison. When results fell below the estimated quantitation limit (EQL) or the instrument detection limit (IDL), the lowest reported "less than" value was used. Results preceded by a less than ("<") indicate that the analyte was not detected. In general, when one average was above the EQL and other averages were below the EQL, the result above the EQL was reported.

Additional processing was performed per "Addendum 1 of the Letter of Instruction (LOI) for TWRS Privatization Contractor Samples Addressing Waste Envelope D Materials - Revision 1," (Wiemers 1996). This additional processing and the adjustments made to establish the concentration of the Final Vendor Product are detailed in Addendum 1 to this report (Kuhl-Klinger 1997).

A summary of Original Material "most reasonable" average results is presented int Table ES-2. The average results are compared to the Envelope D specification for direct comparison to the Vendor Product. The results are presented in units of $g / L$ and $C i / L$ (at 31 gram waste oxide) for comparison with the envelope specification and in $\mu \mathrm{g} / \mathrm{g}$ and $\mu \mathrm{Ci} / \mathrm{g}$ for direct comparison to the analytical results. 
Analytical Summary Report - January 28, 1997

Table ES.1. Comparision of Vendor Product Results with Envelope D Specification

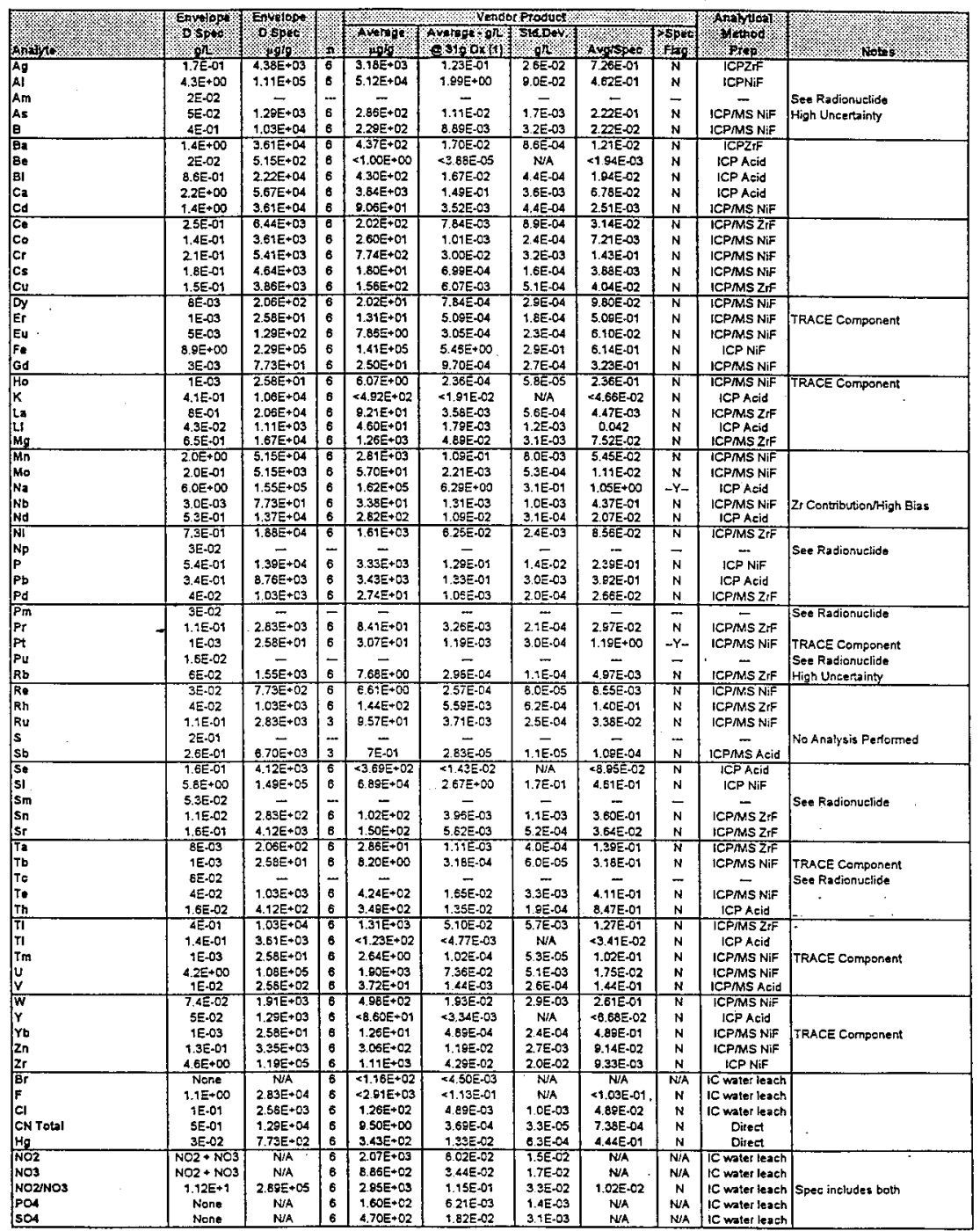


Analytical Summary Report - January 28, 1997

Table ES-1. Comparision of Vendor Product Results with Envelope D Specification

\begin{tabular}{|c|c|c|c|c|c|c|c|c|c|c|}
\hline Annth & Espolope & popos & $\pi$ & Aversot & 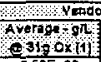 & Product & Avospect & Sopect & 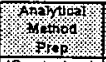 & Nots $s$ \\
\hline $\mathrm{C2O}$ & None & N/A & 6 & $170 E+05$ & $6.59 E+00$ & 5.E.01 & N/A & $\mathrm{N} / \mathrm{A}$ & IC water leach & \\
\hline NH3 & 5E-01 & $1.29 E+04$ & 6 & $2.13 E+01$ & 5.25E-04 & 3.5E-04 & $1.65 E-03$ & $\mathbf{N}$ & ISE water leach & $N^{*}(17 / 14)-$ High Blank \\
\hline TIC (COs) & $1.86 E+D$ & $4,79 E+04$ & 6 & $5.30 \mathrm{E}+03$ & 2.08E-01 & 2. 2E-O2 & $1,11 E-01$ & $\mathbf{N}$ & Direct & \\
\hline TOC & $3.4 E+00$ & $8.76 E+04$ & 8 & $4.79 E+04$ & $1.86 E+D 0$ & $1.3 E=01$ & $5.47 \mathrm{E}-01$ & N & Direct & \\
\hline TC & None & N/A & 6 & $5.22 \mathrm{E}+04$ & $203 E+\infty$ & 1.2E-01 & NA & $N / A$ & Direct & Sum of TOC \& TIC \\
\hline Aadolitudese & chis & acts & & Av + ocilos & AV ck-1 & socil & & & & \\
\hline$A g-110 \mathrm{~m}$ & $1.00 E-08$ & $2.58 \mathrm{E}-04$ & 6 & $\alpha 9 E-02$ & $<3.495-06$ & N/A & $<3.48 E+02$ & $-Y-$ & RNiF & \\
\hline Am-241 & 4.30E-02 & $1.11 E+03$ & 8 & 3.15E+00 & $1.22 E-04$ & 2. BE.OS & $2.84 E-03$ & $\mathbf{N}$ & RNiF & \\
\hline$A m-243$ & $5.00 \mathrm{E}-06$ & $1.29 E=01$ & 6 & $\angle 2 E-02$ & $<7.76 E-07$ & NA & $<1.5 \$ E-01$ & $N$ & RNiF & \\
\hline$c-14$ & $2.00 E-06$ & $5.15 E-02$ & 6 & 287E-03 & 1.11E-O & $2.1 E .07$ & $5.57 E-02$ & $\mathbf{N}$ & Direct & Poor Analys is Precision \\
\hline $\begin{array}{l}\text { Ce-1 } 144(\text { Pr-144+144m) } \\
\text { Ce-144(Pr-144+144m) }\end{array}$ & $1.00 E-04$ & $2.58 E+00$ & $\begin{array}{l}6 \\
6\end{array}$ & $<2 E+00$ & $\begin{array}{l}<7.78 E-05 \\
<7.785-05\end{array}$ & $N / A$ & $\begin{array}{l}<7.76 \mathrm{E}+01 \\
<7.76 \mathrm{E}-01\end{array}$ & $\stackrel{N}{N}$ & $\begin{array}{l}\text { RNiF } \\
\text { RNiF }\end{array}$ & Ce-144 Specification \\
\hline & $1.00 \mathrm{E}+04$ & $2.58 E+00$ & 6 & $<2=+\infty$ & $<7.70 E-05$ & $N / A$ & $<7,76 E-01$ & $\mathrm{~N}$ & RNiF & Pr.144 Specification \\
\hline $\begin{array}{l}\text { Co-144(Pr-144+144m) } \\
\text { Cm-242(Am-242+242m) }\end{array}$ & $\begin{array}{l}1.00 \mathrm{E}-07 \\
3.70 \mathrm{E}-05\end{array}$ & $\begin{array}{l}2.58 E-03 \\
9.53 E-01\end{array}$ & $\begin{array}{l}6 \\
6\end{array}$ & $\begin{array}{l}<2 E+00 \\
8.07 E-03\end{array}$ & $\begin{array}{l}-7.76 E-05 \\
3.19 E-07\end{array}$ & $\begin{array}{c}\text { N/A } \\
6.55-0 \mathrm{~B}\end{array}$ & $\begin{array}{l}<7.76 E+02 \\
8.47 E-03\end{array}$ & $\begin{array}{c}-\lambda- \\
-\end{array}$ & $\begin{array}{l}\text { RNiF } \\
\text { RNiF }\end{array}$ & $\begin{array}{l}\text { Pt.144m Specification } \\
\text { cm-242 Specfication }\end{array}$ \\
\hline$C m-242(A m-242+242 m)$ & $3.905-05$ & 7.95E-01 & 6 & 8.07E-03 & $3.13 E=07$ & 6.5E-08 & $1.01 \mathrm{E}-02$ & $\mathbf{N}$ & RNIF & Amr242 Spectication \\
\hline Cm-242(Am-242+242m) & $3.20 E-05$ & $0.24 E-01$ & 6 & 8.07E-03 & $3.13 E-07$ & $6.5 E-08$ & $9.79 \mathrm{E}-03$ & N & RNiF & Am-242m Speeification \\
\hline$C m-243+244$ & $1.00 E-09$ & $2.58 E-05$ & 6 & 9.86E-02 & $3.83 E-06$ & 8.5E-07 & $3.83 E+03$ & $-Y_{-}-$ & RNiF & $C_{r+243}$ Specification \\
\hline $\begin{array}{l}C_{m-243+244} \\
C_{0-60}\end{array}$ & $\begin{array}{l}9.30 E-04 \\
3.00 E-03\end{array}$ & $\begin{array}{l}2.40 E+01 \\
7.73 E+01\end{array}$ & $\begin{array}{l}6 \\
6\end{array}$ & $\begin{array}{l}9.86 E-02 \\
4.61 E-01\end{array}$ & $\begin{array}{l}3.83 \mathrm{E}-06 \\
1.09 \mathrm{E}-05\end{array}$ & $\begin{array}{l}\text { 8.5E-07 } \\
1.5 \mathrm{E}+08\end{array}$ & $\begin{array}{l}4.12 \mathrm{E}-03 \\
6.35 \mathrm{E}-03 \\
\end{array}$ & $\stackrel{N}{N}$ & $\begin{array}{l}\text { RNiF } \\
\text { RNiF }\end{array}$ & Cm-244 Specification \\
\hline Cs-134 & $6.80 E-0.3$ & $1.75 E+02$ & 6 & $<3 E-01$ & $<1,16 E-05$ & $N / A$ & $<1.71 \mathrm{E}-03$ & $\mathrm{~N}$ & RNiF & \\
\hline Cs.135 & $3.00 E-05$ & 7.73E-01 & 6 & 2.85E-C3 & 1.99E-07 & $5.8 E .09$ & 3.70E-03 & $\mathrm{N}$ & RNiF & \\
\hline Cs-137(Ba-137) & $3.00 E+\infty$ & $7.73 E * 04$ & 6 & $4.96 E+02$ & $1.925-02$ & $1.0 E-03$ & $6.42 E-03$ & $\mathrm{~N}$ & RNiF & Cs-197 Specification \\
\hline Cs-137(Ba-137) & $3.00 E+\infty 0$ & $7.73 E+04$ & 6 & $4.96 E+02$ & $1.93 E-02$ & $1.0 E-03$ & $6.42 \mathrm{E} .03$ & $N$ & RNiF & 5a.137 Specification \\
\hline EU-152 & $1.50 \mathrm{E}-04$ & $3.86 E+\infty$ & 6 & $<2 E+\infty$ & $+7.765-05$ & NA. & $<5.18 \mathrm{E}-01$ & $\mathrm{~N}$ & RNiF & \\
\hline EL+154 & $1.60 E-02$ & $4.12 \mathrm{E}+02$ & 6 & $3.79 E+\infty$ & $1.47 \mathrm{E}-04$ & $5.2 \mathrm{E}-06$ & 9.19E-C3 & $\mathrm{N}$ & RN:F & \\
\hline EU-15S & Q.00E-03 & $2.32 \mathrm{E}+02$ & 6 & $3.13 E+\infty 0$ & 1.29E-04 & $1.2 \mathrm{E}-05$ & $1.35 \mathrm{E}-02$ & $\mathbf{N}$ & RNiF & \\
\hline$F=-55$ & $1.00 E .03$ & $2.58 E+01$ & 6 & $1.53 E-01$ & $5.945-05$ & $3.1 E \cdot 07$ & $5.94 E-03$ & $N$ & RNiF & \\
\hline $1-129$ & Q.00E-08 & $2.32 E-03$ & 6 & 2.20E-04 & $8.54 E .09$ & 2.3E-0S & 9.49E-02 & $N$ & ICPMMS Acid & Potential Prep Losses \\
\hline $\mathrm{Nb}-93 \mathrm{~m}$ & 8.70E-05 & $2.24 \mathrm{E} \bullet 00$ & 6 & 2.34E-01 & $9.05 \mathrm{E}-08$ & $8.1 \mathrm{E}-08$ & $1.04 \mathrm{E}-01$ & $\mathrm{~N}$ & RNiF & \\
\hline NII-59 & 1.40E-05 & 3.61E-01 & 5 & $2.05 E-02$ & $7.96 \mathrm{E}-07$ & $8.0 E .07$ & $5.68 \mathrm{E} \cdot 02$ & $N$ & RNiF & \\
\hline $\mathrm{N} \mid-63$ & $1.60 \mathrm{E}-03$ & 4.12E +01 & 6 & $2.40 E+D O$ & $9.32 E-05$ & 9.3E-05 & $5.82 E-02$ & $\mathrm{~N}$ & RNiF & \\
\hline Np-237 & 2.30E-05 & 5.93E-01 & 6 & 3.S2E-03 & $1.53 \Xi-07$ & $3.0 E .08$ & $6.65 \mathrm{E}-03$ & N & ICPMS ZRF & \\
\hline$P d-107$ & 4.00E.06 & 1.05E-0 & 6 & $1,10 E-03$ & $4.27 \mathrm{E}-08$ & $5.0 E-08$ & $1.07 \mathrm{E}-02$ & $N$ & ICPIMS Acid & \\
\hline PU-238 & $1.90 E-04$ & $2.85 E+\infty 0$ & 6 & 6.31E-01 & $2.45 \mathrm{E}-05$ & $1.4 \mathrm{E}-06$ & $2.23 \mathrm{E} \cdot 01$ & $\mathrm{~N}$ & RNiF & \\
\hline Pu-239 & $9.50 E-04$ & $2.45 E+01$ & 6 & $1.53 E+00$ & 5. $54 E-05$ & 4.1E-05 & $6.25 E+02$ & $\mathrm{~N}$ & ICPIMS ZZFF & \\
\hline PU.240 & $2.60 \mathrm{E}-0.4$ & $6.70 E+00$ & 6 & $1.27 E .01$ & 4.935.05 & $3.8 \mathrm{E}-07$ & $1.90 \mathrm{E} \cdot 02$ & $\mathrm{~N}$ & ICРMS ZrF. & \\
\hline $\mathrm{Pu}+241$ & $6.90 E-03$ & $1.78 E+02$ & 6 & $6.95 E+\infty 0$ & 270E-04 & $1.6 \mathrm{E}-0.5$ & $3.92 \mathrm{E}-\mathrm{C2}$ & $N$ & $\mathrm{RNiF}$ & \\
\hline Pu-242 & $7.10 E-08$ & $1.82 E-03$ & 6 & $<2 E-02$ & $47.765-07$ & N/A & $<1.09 \mathrm{E}+01$ & $-Y-$ & RNiF & \\
\hline Ru-106(Rh-105) & $200 E-04$ & $5.15 E+00$ & 6 & $<3 E+00$ & $<9.165-04$ & N/A. & -5.82E-01 & $\mathrm{N}$ & RNiF & Ru+106 Specification \\
\hline Ru-106(Rh-106) & $2.00 E-04$ & $5.15 E+\infty 0$ & 6 & $\angle 3 E+D 0$ & $41.16 \mathrm{E}-04$ & N/A & $\angle 582 E-01$ & $N$ & RNiF & Rh-106 Specification \\
\hline Sb-12S(Te-125m) & $1.00 E-02$ & $2.58 E+02$ & 6 & $<2 E+\infty 0$ & $<7.75 E .05$ & N/A & $<7.76 E-03$ & $N$ & RNiF & St. 125 Specification \\
\hline$S b-\{25(T e-125 m)$ & $3.005-03$ & $7.735+01$ & 6 & $<2 E+\infty$ & $c 7.765-05$ & N/A & $<2.59 E-02$ & N & RNiF & $T=-125 \mathrm{~m}$ 5pecification \\
\hline Se-79 & $4.20 \mathrm{E}-07$ & $1.08 \mathrm{E} .02$ & 6 & $<2 E .04$ & $<8.15 E .09$ & N/A & $<1.945 .02$ & N & RNiF & \\
\hline$(5 m-151(P, n-147)$ & $9.30 \mathrm{E}-02$ & $2.40 E+03$ & 8 & $9.23 E+04$ & $3.55 E-03$ & $1.9 E-04$ & $3.25 \mathrm{E} \cdot 02$ & $\mathrm{~N}$ & RNiF & Sm-151 Specification \\
\hline Sm-151(Prn-147) & $1.6 \overline{0 E-01}$ & $4.12 E \div 03$ & 6 & $9.23 E+01$ & $3.55=03$ & $1.9 E-04$ & 2.24E-02 & $\mathrm{N}$ & RtViF & Pm-147 Specification \\
\hline $5 n-113(1 n-113)$ & $188 \mathrm{E}-05$ & $464 E-02$ & 6 & $<9.02$ & $<3.88 E-07$ & N/A & $<2.05 E .01$ & $\mathbf{N}$ & RNiF & Sn-113 Specification \\
\hline $5 n-113(\ln -113)$ & $1.88 E-06$ & 4.84E-02 & 6 & $<1 E .02$ & $<389 E-07$ & N/A & $<2.06 \mathrm{E}-01$ & $N$ & RNiF & In-113 Specification \\
\hline Sn-119m & $1.00 E-08$ & $2.59 E-04$ & 6 & $<4 E-02$ & $41.55 E .05$ & N/A & $<1.55 \mathrm{E}+02$ & $-Y_{-}$ & RNiF & \\
\hline $5 n-121 m$ & 9.00E-06 & $2.32 E-01$ & 6 & $1.23 E+00$ & $4.77 E-05$ & 2.1E-05 & $5.31 E+00$ & $-Y .-$ & RNIF & \\
\hline (Sn-126(Sb-126m,Sb-126) & $480 E-05$ & $1.24 E=000$ & 6 & T.15E.2 & 4.465 .07 & 1.220 .07 & 9.30E-03 & $N$ & RNiF & Sn-126 Specification \\
\hline Sn-125(Sb-125m,Sb-126) & $3.43 \mathrm{E} \cdot 05$ & 8.84E-01 & 5 & $1.15 E-2$ & 4,46E-07 & $1.2 E-07$ & $1.30 E-02$ & N & RNEF & Sb-126m Specification \\
\hline Sn-126(Sb-126m,Sb-126) & $483 \mathrm{E} \cdot 05$ & $1.24 E .01$ & 8 & $1.15 E-2$ & 4.46E.07 & $1.2 \mathrm{E} \cdot 07$ & $9.24 E-02$ & $\mathbf{N}$ & RNiF & Sb-126 Specification \\
\hline $\begin{array}{l}\text { Sr-90(Y-90) } \\
\text { Sr-90(Y+90) }\end{array}$ & $\begin{array}{l}3.70 E+00 \\
3.10 E+00 \\
\end{array}$ & $\begin{array}{l}7.99 E+04 \\
7.89 E+04\end{array}$ & $\begin{array}{l}6 \\
6 \\
\end{array}$ & $\begin{array}{l}7.30 \mathrm{E}+02 \\
7.30 \mathrm{E}+02 \\
\end{array}$ & $\begin{array}{l}2 . E 3 E-C 2 \\
2.83 E \cdot C 2\end{array}$ & $\begin{array}{l}1.7 \mathrm{E}-03 \\
1.7 \mathrm{E}-03 \\
\end{array}$ & $\begin{array}{l}9.14 E-03 \\
9.14 E \cdot 03 \\
\end{array}$ & $\begin{array}{l}N \\
N\end{array}$ & $\begin{array}{l}\text { RNiF } \\
\text { RNiF }\end{array}$ & $\begin{array}{l}\text { Sr-90 Specificstion } \\
\text { Y-90 Specification }\end{array}$ \\
\hline Te-99 & $4.50 E-03$ & $9.16 \mathrm{E}+02$ & 6 & $1.32 \bar{E}-02$ & $5.12 E-07$ & $3.0 E-08$ & $1.14 E-04$ & $\mathrm{~N}$ & ICPIMS NiF & - \\
\hline Th+232 & See Th" & See "Th" & 6 & $5.48 E-05$ & 2.13E-09 & $7.0 \mathrm{E} \cdot 10$ & N/A & $N / A$ & ICPMS Z $t F$ & \\
\hline U-233 & $1.00 E-09$ & $2.58 E-05$ & 6 & 1.7E-03 & $6.55-08$ & 1.3E.08 & $6.60 E+01$ & $-Y_{-}$ & ICPMMS Acid & TRACE Comp - Th Interferes \\
\hline U-234 & $7,705-07$ & 1.98E-02 & 6 & $7.70 E-04$ & 2.69E-08 & 4.95 .09 & $3.88 \mathrm{E}-02$ & $N$ & ICPMS AEId & \\
\hline U-235 & $320 E .08$ & 8.24E-04 & 6 & $280 E \cdot 05$ & $1.09 E-09$ & 8.7E.12 & $3.40 E-02$ & $N$ & ICPMS Acid & \\
\hline U-236 & 8.205 .08 & 2.11E.03 & 6 & $1,805-05$ & $6.995-10$ & $7.6 \bar{E}-11$ & $8.525-03$ & $\mathrm{~N}$ & ICPMS AEId & \\
\hline$U-238$ & 5.805 .07 & 1.45E-02 & 6 & 6.37E-OA & 2.47E-0B & $1.7 E=08$ & 4.26E-02 & $N$ & ICPMS Z.JF & \\
\hline$Z r-93$ & $1.40 E-04$ & $3.61 E+\infty$ & 6 & $<\$ E-01$ & -2.33E-05 & N/A & $<1.66 E-04$ & $\mathbf{N}$ & RNiF & \\
\hline Total Alpha & none & N/A & 6 & $6.78 \mathrm{E}+00$ & 2.ESE.04 & B.7E-06 & NS/A & N/A & RNiF & \\
\hline Total Beta. & mone & NiA & 8 & $2.05 E+03$ & $7.05 E+02$ & 4.6E-03 & N/A & N/A & RNiF & \\
\hline Phorspopest & & & 皮 & W. & Vendg froduct & & & & & \\
\hline & 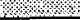 & 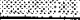 & & HALRT & \$स & & & & 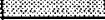 & \\
\hline Wti solds Q $105 c-(\%)$ & $2.5 \cdot 13$ & & & 144 & 148 & & & & & See Note 2 \\
\hline$w t \%$ oxld $\Phi^{1050 c}-(\%)$ & & & & 11.5 & - & & & & & \\
\hline Total OxId $-(g \Omega)$ & $25 \cdot 100$ & & & 126.5 & - & & & & & \\
\hline Slurry Density - (g/m) & $1.02 \cdot 1.10$ & & & 1.10 & 一 & & & & & \\
\hline $\begin{array}{l}\text { Settled Sollds - Nol\%) } \\
\text { pH }\end{array}$ & $\begin{array}{l}7.95 \\
>10\end{array}$ & & & $\begin{array}{l}18.50 \\
-\end{array}$ & $\overline{-}$ & & & & & \\
\hline
\end{tabular}

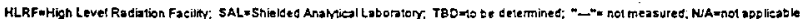

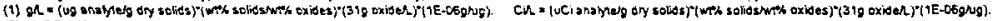

(2) HiRF with solids performed in duplicate on 3 g sample abquois, SAL wik solids pertomed on single, $>100$ g butk samples 
Analytical Summary Report - January 28, 1997

Table ES-2. Original Material Result Summary

\begin{tabular}{|c|c|c|c|c|c|c|c|c|c|c|}
\hline Whato & $\begin{array}{l}\text { Envelope } \\
\text { Dsposp }\end{array}$ & Epropes & 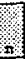 & Averots & 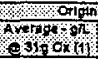 & Matorlal & Axos 5 & Spot & Anthods & \\
\hline$A D$ & 1.7E-01 & $4.71 E+03$ & 6 & $209 E+03$ & 8.64E-02 & $8.3 E+01$ & $5,0.0 \mathrm{E}-01$ & $\mathrm{~N}$ & ICPZA & \\
\hline AI & $4.3 E+\infty 0$ & $9.04 E+05$ & 6 & $3.73 E+04$ & $1.54 \mathrm{E}+00$ & $1.9 E+03$ & $3.59 \mathrm{E}-01$ & $N$ & ICPNiF & \\
\hline An & $2 E-02$ & - & - & - & - & $\bar{z}$ & - & - & - & See Radionuclide \\
\hline As & 5E-02 & $1.21 E+03$ & 6 & $3.49 E+01$ & 1.44E.03 & $1.3 E+01$ & $2.89 E-02$ & $\mathbf{N}$ & ICPMS ZrF & High Uncerainty \\
\hline$B$ & $4 E-01$ & 8.68E+03 & 6 & $1.08 E+02$ & $4.46 \mathrm{E} \cdot \mathrm{C3}$ & $2.6 E+01$ & $1.125-02$ & $N$ & ICPMMS ZfF & \\
\hline$\overline{B a}$ & $1.4 E+00$ & $339 E+04$ & 6 & $2,17 E+02$ & $8.97 E .03$ & $9.0 E+00$ & $8.41 E+03$ & $\bar{N}$ & ICPZ̈F & \\
\hline g. & $2 E-02$ & $4.24 E+02$ & G & $<2.00 E-01$ & $\times 8.27 E-06$ & NA & $<4.13 E-04$ & $\mathbf{N}$ & ICP Acid & \\
\hline BI & $8.6 E-01$ & $208 E+04$ & 6 & $1.025+02$ & 6.705 .03 & $2.0 E+01$ & $7.79 \mathrm{E}-03$ & $N$ & ICP Acid & High Uncertainty - Below EOL, \\
\hline $\mathrm{Ca}$ & $2.2 E+\infty$ & $5.32 \mathrm{E}+0.4$ & 6 & $1.705+03$ & 7.03E.02 & $1.2 \mathrm{E}+02$ & $3.19 E-02$ & $\mathbf{N}$ & ICP Acid & \\
\hline cd & $1.4 E+00$ & $3.30 \mathrm{E}+04$ & 6 & $2.91 E+01$ & $1.20 \mathrm{E}-03$ & $3.0 E+00$ & 8.59E-04 & N & ICPMSS Acid & . \\
\hline CE & $2.5 E-01$ & $6.05 E+03$ & 6 & $9.46 E+01$ & 391E.03 & $6.9 E+\infty 0$ & $1.55 E=02$ & $N$ & ICPMMS AcId & \\
\hline Co & 1.4E-01 & $3.38 E+03$ & 6 & $4.10 \mathrm{E}+01$ & $1.69 \mathrm{E}-03$ & $3.0 E+00$ & 1.21E-02 & N & ICPMSS NiF & \\
\hline cr & 2.1E-01 & $5.08 E+03$ & 6 & $6.37 \mathrm{E}+02$ & $2.63 E-02$ & $2.9 E+01$ & $1.25 \mathrm{E}-01$ & N & ICPMS NIF & \\
\hline Cs & 1.8E.01 & 4,35E+03 & 6 & 8.10E+00 & 3.35E-04 & 8. OE-01 & $1.86 E-03$ & N & ICPMS Acid & \\
\hline $\mathrm{Cu}$ & $1.5 \mathrm{E}-01$ & $3.63 E \div 03$ & 6 & $7.76 \mathrm{E}+0 \mathrm{t}$ & $3.21 E-03$ & $4.1 E \cdot 00$ & $2.14 \mathrm{E}-02$ & $\mathrm{~N}$ & ICPMS ZrF & \\
\hline Dy & $8 E-03$ & $1.94 E+02$ & 6 & $<5.00 \mathrm{E}+00$ & $* 2.07 E-04$ & NTA & $<2.58 E-02$ & $\bar{N}$ & ICP/MS Acid & \\
\hline Er & TE-03 & $2.42 E+01$ & 6 & $<5.00 \mathrm{E}+00$ & $<207 E-04$ & N/A & $<2.07 E-01$ & $\mathbf{N}$ & ICPMS Acid & TRACE Component \\
\hline Eu & SE.03 & $1.21 E+02$ & 6 & $<5.00 E+00$ & $<2.07 E \cdot 04$ & N/A & $<4.13 E-02$ & N & ICPMMS Acid & \\
\hline Fe & $8.9 E+00$ & $2.15 E+0 S$ & 6 & $6.78 \mathrm{E}+04$ & $2.80 E+00$ & $2.7 E+03$ & $3.15 E-01$ & N & ICP NiF & \\
\hline Gd & 3E. 03 & $7.265+01$ & 6 & 1.31:E+01 & 5.41E-04 & $3.8 E+\infty$ & 1.BOE-01 & $N$ & ICPMMS ZFF & \\
\hline Ho & $\mathrm{TE} \cdot \mathrm{CB}$ & $2.42 \mathrm{E}+09$ & $\overline{8}$ & $25.00 E+\infty$ & $\times 2.07 E-04$ & N/A & $\times 2.07 E-01$ & $\bar{N}$ & ICPMMS Acid & TRACE Component \\
\hline$k$ & 4.1E-01 & $9.925+03$ & 6 & 8.11E+02 & $3.35 E-02$ & N/A & $8.18 \mathrm{E}-02$ & $\mathrm{~N}$ & ICP Acid & High Uncertainty - Below EQL \\
\hline $\operatorname{lag}_{1}$ & 8E-01 & $1.94 E+04$ & 6 & 5. $12 E+01$ & 2.12E= 03 & $1.9 E+\infty$ & 2.65E-03 & N & ICPMSS Acid & Hith lnomeints Relou EO \\
\hline LI & $\begin{array}{l}4.3 E-02 \\
6.5 E-01\end{array}$ & $\begin{array}{l}1.02 \mathrm{E}+03 \\
1.57 \mathrm{E}+04\end{array}$ & $\begin{array}{l}6 \\
6 \\
\end{array}$ & $\begin{array}{l}8.00 \mathrm{E}+00 \\
3.31 \mathrm{E}+02\end{array}$ & $\begin{array}{l}3.31 E-04 \\
1.37 E-02\end{array}$ & $\begin{array}{l}1.0 \mathrm{E}+00 \\
1.1 E+01\end{array}$ & $\begin{array}{c}0.000 \\
2.10 \mathrm{E}-02\end{array}$ & $\begin{array}{l}\mathrm{N} \\
\mathrm{N}\end{array}$ & $\begin{array}{l}\text { ICP Acid } \\
\text { ICP Acid }\end{array}$ & High Uncertainty - Below EOL \\
\hline Mn & $20 E+00$ & $484 \mathrm{E}+04$ & 6 & $9.27 \mathrm{E}+03$ & $5.25 E-02$ & $5.5 E+01$ & $2.62 E-02$ & $\mathrm{~N}$ & ICP $2 \mathrm{~F}$ & \\
\hline mo & $20 \mathrm{E}-01$ & $4.84 E+03$ & 6 & $6.50 E+01$ & $2.62 E-03$ & $1.3 E+01$ & $1.34 \mathrm{E}-02$ & $\mathbf{N}$ & ICPMS NiF & \\
\hline $\mathrm{Na}$ & $6.0 E+00$ & $1.45 E+05$ & 6 & $2.25 E+05$ & $9.30 \mathrm{E}+00$ & $6.3 E+03$ & $1.55 E+\infty 0$ & $-Y-$ & ICP Acid & \\
\hline $\begin{array}{l}\text { Nb } \\
\text { Nd }\end{array}$ & $\begin{array}{l}\text { 3. DE-03 } \\
\text { 5.3E-01 }\end{array}$ & $\begin{array}{l}7.26 \mathrm{E}+01 \\
1.28 \mathrm{E}+04\end{array}$ & $\begin{array}{l}6 \\
6 \\
\end{array}$ & $\begin{array}{l}3.22 \mathrm{E}+01 \\
1.24 E+02\end{array}$ & $\begin{array}{l}1.23 \mathrm{E}-03 \\
5.73 \mathrm{E}-03\end{array}$ & $\begin{array}{l}1.3 E+01 \\
1.7 E+01\end{array}$ & $\begin{array}{l}4.44 E-01 \\
9.67 E-03\end{array}$ & $N$ & $\begin{array}{l}\text { ICPMMS Acid } \\
\text { ICPMS Acid }\end{array}$ & 2. Contribution/ligh Bias \\
\hline NI & $7.3 E-01$ & $1.77 E+04$ & 6 & $5.15 E+02$ & $2.13 E-02$ & $2.4 E+01$ & $2.92 E-02$ & $N$ & ICP Ació & \\
\hline Np & $3 E \cdot C 2$ & - & - & - & - & - & - & - & $\ldots$ & See Radionuclide \\
\hline$P$ & 5. 4E-01 & $1.37 E+04$ & 6 & $3.71 E+03$ & $1.53 E-01$ & $1.3 E+02$ & $2.84 E-01$ & $N$ & ICP NiF & \\
\hline$P D$ & $3.4 E-01$ & $8.23 E+03$ & 6 & $1.81 E+03$ & $7.48 E-02$ & $8.3 E+01$ & $220=-01$ & $\mathrm{~N}$ & ICP Acid & \\
\hline Pd & $-4 E-02$ & $9.68 E+02$ & 6 & $3.37 E+01$ & $1,395-03$ & $5.2 E+\infty$ & $3.48 E .02$ & $\mathrm{~N}$ & ICPMS ZrF & \\
\hline Pm & 3E-02 & - & 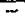 & - & - & $\cdots$ & - & 一 & - & See Ra dienvelide \\
\hline Pr & 1.1E-01 & $2.66 E+03$ & $\mathbf{E}$ & $3.66 E+C 7$ & $1,51 \mathrm{E}-03$ & $3.5 E+00$ & $1.38 E \cdot 02$ & $\mathbf{N}$ & ICPMS ZrF & \\
\hline Pt & $1 E \cdot 03$ & $2.42 E+01$ & 6 & $<500 E+00$ & $<2.07 E-04$ & $N / A$ & $<2.07 E-01$ & $N$ & ICPMS ZrF & TRACE Component \\
\hline Pu & 1.SE-02 & $-\cdot$ & - & - & - & - & - & $\ldots$ & $\rightarrow$ & See Radionuclide \\
\hline RE & 6E-02 & $1.45 E+03$ & 5 & $4.60 E+00$ & 1.905 .04 & $1.4 ㄷ ㅏ ㅇ=00$ & 3.17E-03. & $\mathrm{N}$ & ICPMS ZFF & High Uncertointy \\
\hline Re & 2E.02 & $7.26 E+02$ & 6 & - & - & $=$ & - & $\cdots$ & - & Not Anlalyzed \\
\hline Rh & $4 E-02$ & $9.68 \mathrm{E}+02$ & 6 & $1.59 \mathrm{E} * 01$ & 6.57E-04 & $1,2 E+\infty$ & $1.64 E-02$ & $N$ & ICPMS Reid & \\
\hline Ru & $1.1 E=01$ & $2.66 E+03$ & 6 & $1.05 E+02$ & $434 \mathrm{E}-03$ & $6.0 E+00$ & $395 \mathrm{E}-02$ & $N$ & ICPMS NiF & \\
\hline 5 & $2 \mathrm{E}-01$ & - & 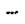 & - & - & - & - & - & $\cdots$ & No Analysis Pertormed \\
\hline SD & $2.6 \mathrm{E}-01$ & $6.29 \mathrm{E}+03$ & 6 & $6.7 \mathrm{E}+00$ & 2.77E. 04 & $4.0 E .01$ & $1,07 \mathrm{E}-03$ & $\mathrm{~N}$ & ICPIMS ZrF & \\
\hline si & $1,6 \mathrm{E}-09$ & $3.87 \mathrm{E}+03$ & 6 & $2.90 E+02$ & $8.68 \mathrm{E} \cdot 03$ & $8.0 \mathrm{E}+20$ & $5.43 \mathrm{E} \cdot 02$ & $\bar{N}$ & ICPAcid & High Uncertainty - Below EOL \\
\hline$\$ 1$ & $5.8 E+00$ & $1.40 E+05$ & 6 & $3.32 E+04$ & $1.355+\infty$ & $1.2 E+03$ & 2.57E-01 & $N$ & ICP ZrF & \\
\hline $\sin$ & $5.3 E-02$ & - & - & - & - & - & - & - & - & See Radionuclide \\
\hline$\$ n$ & $1.1 \mathrm{E}-02$ & $2.66 \mathrm{E} \bullet 02$ & 6 & $2.60 \mathrm{E}+01$ & $1.07 E-03$ & $2.3 E+\infty$ & $0.77 \mathrm{E}-02$ & $\mathrm{~N}$ & ICPIMS Aeid & \\
\hline Sr & 1.6E-01 & $3.87 E+03$ & 6 & $1.24 E+01$ & $5.13 E .04$ & $1.1 E+00$ & $3.20 E .03$ & $N$ & ICPMSSAcid & \\
\hline $\mathbf{T a}$ & $8 E-03$ & $1.94 E+02$ & 6 & $1.27 \mathrm{E} \div 01$ & $5.25 E-04$ & $3.0 E+00$ & $6.56 E-02$ & $\mathrm{~N}$ & ICPMS AEId & \\
\hline To & $1 E-03$ & $2.42 E+01$ & 6 & $<5.00 E+\infty$ & $<2$ 07E.04 & $\mathrm{N} / \mathrm{A}$ & $<2.07 E .01$ & N & ICPMMSAcid & TRACE Component \\
\hline TC & $8 E-02$ & - & - & - & $\ldots$ & - & - & - & - & See Radionuclide \\
\hline |Te & $4 \mathrm{E}-02$ & $9.66 \mathrm{E}+02$ & 6 & $2.64 E+01$ & 1. $09 E-03$ & $6.1 E+00$ & $2.73 E-02$ & $\mathbf{N}$ & ICPMS Acid & \\
\hline th & $1.6 \mathrm{E}-02$ & $3.87 E+02$ & 6 & $24.69 \mathrm{E}+01$ & 29.94E-03 & $\mathrm{N} / \mathrm{A}$ & $\leq 1,21 E-01$ & $N$ & ICP Acid & -. \\
\hline Ti & $4 \mathrm{E}-01$ & $9.68 E+03$ & 6 & $5.64 E+02$ & $2.33 E .02$ & $5.3 E+01$ & $5.83 E-02$ & $\mathrm{~N}$ & ICPMS NIF & \\
\hline II & $1.4 E-01$ & $3.39 E+03$ & 6 & $<2.35 E+01$ & $<9.71 E-04$ & NiA & $<6.84 E .03$ & N & ICP Acid & \\
\hline Im & IE-03 & $2.42 E+01$ & 6 & $<5,00 E+00$ & $<2.07 E-04$ & NiA & $<2.07 E-01$ & N & ICPMS ACid & TRACE Component \\
\hline $\mathbf{u}$ & $\begin{array}{c}4.2 E+00 \\
1 E-02\end{array}$ & $\begin{array}{l}1.02 E+05 \\
2.42 E+02\end{array}$ & 6 & $\begin{array}{l}1.13 E+03 \\
8.80 E+01 \\
\end{array}$ & $\begin{array}{l}\text { 4. } 58 E-02 \\
\text { 4.09E-.03 }\end{array}$ & $\begin{array}{l}1.4 E+02 \\
3.0 E+00\end{array}$ & $\begin{array}{l}1.11 E-02 \\
\text { A.09E.01 }\end{array}$ & $\begin{array}{l}\mathbf{N} \\
\mathbf{N}\end{array}$ & $\begin{array}{l}\text { ICPMS ZnF } \\
\text { ICPMS Acid }\end{array}$ & High Elank \\
\hline$w$ & $7.4 E .02$ & $1.79 \mathrm{E}+03$ & 6 & $3.96 E+02$ & 1.65E-02 & $1.1 E+01$ & $2.23 E-01$ & $\mathbf{N}$ & ICPIMS Aeid & \\
\hline |Y & 5E-02 & $1.21 E+03$ & 6 & $3.00 E+01$ & 1.61E.03 & $2.0 E+\infty$ & $3.22 E-02$ & $\mathbf{N}$ & ICP Acid & \\
\hline ro & $1 E-03$ & $2.42 E+0 t$ & B & $<5.00 E+00$ & $<2.07 E .04$ & N/A & <2.07E-01 & $\mathrm{N}$ & ICPMS Acid & TFACE Component \\
\hline $2 n$ & 1.3E+01 & $3.15 E+03$ & 6 & $1.87 E+02$ & 7.73E.C3 & $1.8 E+01$ & $5.85 E-02$ & N & ICPMMS NiF & High Elank \\
\hline zr & 4. $5 E+00$ & $1.11 E+0 S$ & 6 & $3.68 \mathrm{E}+02$ & $1.51 \mathrm{E} \cdot 02$ & 7. $g E+01$ & $3.29 E-03$ & $\mathrm{~N}$ & ICP Acid & \\
\hline$\overline{B r}$ & None & N/A & 6 & $<131 E+02$ & <5.41E.03 & NIA & NA & $\mathrm{N} / \mathrm{A}$ & IC water leseh & \\
\hline$F$ & $1.1 E+50$ & $2.66 E-04$ & 6 & $<3.27 E-03$ & $<1.35 E-01$ & N/A & $<1.23 E .01$ & $\mathrm{~N}$ & IC water beach & \\
\hline cl & $1 E-01$ & $2.42 \mathrm{E}+03$ & 6 & $2.26 \mathrm{E}+02$ & 9.34E-03 & $1.7 E+01$ & 0.34E-02 & $\mathrm{N}$ & IC water leseh & \\
\hline CN Total & SE-01 & $1.21 E+0.8$ & 6 & $6.20 E+01$ & 2.58E-03 & $5.3 E+\infty 0$ & $5.15 E-03$ & $\mathbf{N}$ & Direct & \\
\hline Ho & 3E-02 & $7.26 E+02$ & 6 & $1.60 E+02$ & $6.61 E+03$ & $1.1 E+01$ & $2.20 E-01$ & $\mathbf{N}$ & Direct & \\
\hline NO2 & $\mathrm{NO} 2+\mathrm{NO} 3$ & $\bar{N} / A$ & 6 & $2.37 E+04$ & S.60E-0i & $2.8 E+03$ & NAA & $N / A$ & IC water leach & \\
\hline NO3 & $\mathrm{NO} 2 \cdot \mathrm{NO} 3$ & NUA & 6 & $1,17 E+03$ & 4. E-AE-02 & $0.3 E * 01$ & N/R & N/A & IC water leach & \\
\hline NO2NOS & $1.12 E+1$ & $2.73 E+O S$ & 6 & $2.49 E+04$ & $1.02 E+\infty 0$ & 3.9E-02 & Q.13E-O2 & $N$ & ic watet teach & Spec includes both \\
\hline pod & None & N/A & 6 & $1.13 E+03$ & $4.67 E-02$ & $0.9 E+01$ & $N / A$ & N/A & IC water leach & \\
\hline so4 & None & NA & 6 & $5.99 E+03$ & $289 \mathrm{E}-01$ & $1.3 \mathrm{E}+03$ & N/A & $\mathrm{N} / \mathrm{A}$ & ic water beach & \\
\hline
\end{tabular}


HNF-SD-WM-DP-225, REV. 1

Analytical Summary Report - January 28, 1997

Table ES-2. Original Material Result Summary

\begin{tabular}{|c|c|c|c|c|c|c|c|c|c|c|}
\hline anstion & Enveppo & $\begin{array}{l}\text { Fnveppe } \\
\text { ospop } \\
\text { nolo }\end{array}$ & \# & 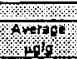 & 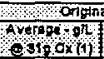 & Malertat & Whop & 35 & 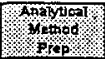 & $16 \%$ nols \\
\hline C204 & None & $N / A$ & 6 & $2.00 E+05$ & $8.2 \mathrm{tE}+\infty$ & $9.0 E+04$ & $\mathrm{~N} / \mathrm{A}$ & N/A & fC water leach & \\
\hline $\mathrm{NH3}$ & 5E-04 & $1.21 E+04$ & 6 & $212 E+01$ & $8.765-04$ & $6.5 E+\infty 0$ & $1.75 \mathrm{E}-03$ & $\mathbf{N}$ & iSE woter lesch & $N=(17 / 14)$ - High Blank \\
\hline (Tic (cO3) & $1.86 E=0$ & $4.50 E+04$ & 6 & $277 \mathrm{E}+04$ & $1.14 \mathrm{E}+00$ & $1.8 E+03$ & 6. 16E-01 & N & Direct & \\
\hline $\begin{array}{l}\text { Tos } \\
\text { TC }\end{array}$ & $\begin{array}{c}\text { 3.4E+00 } \\
\text { Nene }\end{array}$ & $\begin{array}{l}\text { 8. } 23 E+04 \\
N / A\end{array}$ & 6 & $\begin{array}{l}5.54 E+04 \\
8.34 E+04\end{array}$ & $\begin{array}{l}2.29 E+\infty 0 \\
3.43 E+\infty\end{array}$ & $\begin{array}{l}2.2 E+03 \\
2.6 E+03\end{array}$ & $\begin{array}{c}6.73 E \cdot 01 \\
\text { NAA }\end{array}$ & $\underset{N}{N}$ & $\begin{array}{l}\text { Direct } \\
\text { Direct }\end{array}$ & Sum of TOC \& ThC \\
\hline Retolwotos & on: & $\mathrm{HCOO}$ & & Ang tolfo & $\mathrm{AVGCUL \textrm {J }}$ & $50 \mathrm{cth}$ & & & & \\
\hline$\overline{A g-110 m}$ & $1.00 \mathrm{E}-08$ & $2.42 E-04$ & 6 & $\angle 7 E-02$ & $\angle 2.89 E .05$ & N/A & $<2.89 E+02$ & $-Y-$ & RNiF & \\
\hline Am-241 & 4.30E.02 & $1.04 E+03$ & 6 & $1.55 E+00$ & 6.A15.05 & $3.0 E-01$ & $1.49 E-03$ & $\mathrm{~N}$ & RNiF & GEA \\
\hline Am-243 & 5.00E-08 & $1.21 \mathrm{E}-01$ & 6 & $<2 E-02$ & $<8.27 \mathrm{E}-07$ & NA & C1.65E-01 & $\mathbf{N}$ & RNiF & \\
\hline$c-14$ & $2.00 E-66$ & $4,84 E-02$ & 6 & $6.85 E-03$ & $2.81 E .07$ & $9.1 E .03$ & 1.41E.01 & $N$ & Direct & Poot Precision/Cartyover \\
\hline$C=144(\operatorname{Pr}-144+944 m)$ & $1.00 E-04$ & $2.42 E+\infty 0$ & 6 & $<2 E+00$ & $48.27 E+05$ & N/A & $-8.27 \mathrm{E}-01$ & $N$ & RNiF & Ce-144 Specification \\
\hline Ct-144(Pr-144+144m) & $1.00 E-04$ & $2,42 E+00$ & $\dot{6}$ & $<2 E+00$ & $<8.27 \mathrm{E} .05$ & N/A & $<8.27 E-01$ & $\mathbf{N}$ & RNiF & PI-144 Specification \\
\hline CE-144(Pr-144+144m) & 1. DOE-D7 & 2.42E-03 & 6 & $<2 E+00$ & $48.27 \mathrm{E}-05$ & NA & $<8.27 \mathrm{E}+02$ & $-Y-$ & RNiF & Pr-144m Spocification \\
\hline$(\mathrm{Cm}-242(A \mathrm{~m}-242+242 \mathrm{~m})$ & $3.70 E-05$ & $8.85 \mathrm{E}-01$ & 6 & $3.27 E-03$ & $1.355-07$ & $1.1 E-03$ & $3.65 E-03$ & $\mathrm{~N}$ & RNiF & $C_{m-242}$ Specricatien \\
\hline $\bar{C} m-242(A m-242+242 m)$ & 3.10E-05 & $7.50 E-01$ & 6 & $3.27=-03$ & $9.35 E .07$ & $1.1 \mathrm{E} \cdot 03$ & $4.36=-03$ & $\bar{N}$ & RNiF & Am-242 Specification \\
\hline$C m-242(A m-242+242 m)$ & $3.20 E-05$ & $7,74 E-01$ & 6 & $3.27 E-03$ & $1.25=07$ & $1.1 E-03$ & 4.22E-03 & $\mathrm{N}$ & RNiF & Am-242m Specirication \\
\hline $\mathrm{Cn}-243+244$ & $1.00 E .09$ & $2.42 \mathrm{E}-05$ & 6 & $2.84 E-02$ & $1.57 \mathrm{E}-06$ & $8.6 \mathrm{E} \cdot 03$ & $1.17 \mathrm{E}+03$ & $-Y$ & RNiF & Cm-243 Specification \\
\hline $\begin{array}{l}\mathrm{C}_{\mathrm{m}-243+244} \\
\mathrm{Co}-50\end{array}$ & $\begin{array}{l}9.30 E-04 \\
3.00 E-03\end{array}$ & $\begin{array}{l}2.25 \mathrm{E}+01 \\
7.26 \mathrm{E}+01\end{array}$ & 6 & $\begin{array}{l}2.84 \mathrm{E}-02 \\
2.36 \mathrm{E}-01\end{array}$ & $\begin{array}{l}1.17 E .05 \\
9.75 E .08\end{array}$ & $\begin{array}{l}8.6 E-03 \\
1.7 E-02\end{array}$ & $\begin{array}{l}1.26 E-03 \\
3.25 E-03\end{array}$ & $\begin{array}{l}\mathbf{N} \\
\mathbf{N}\end{array}$ & $\begin{array}{l}\text { RNIF } \\
\text { RNiF }\end{array}$ & Cm-244 Specifieation \\
\hline $\mathrm{Cs-134}$ & $6.802-03$ & $1.65 E+02$ & 6 & $\triangle 3 E .01$ & <124E-05 & N/A & $<1.82 E-03$ & $\mathbf{N}$ & RNiF & \\
\hline Cs-135 & $3.00 E-05$ & $7.2 \mathrm{EE}-01$ & 6 & 2.96E-03 & $1.22 E .07$ & 7.5E-05 & 4.08E.03 & $\mathbf{N}$ & RNiF & \\
\hline Cs.137(8a-137) & $3.00 E+00$ & $7.26 E+04$ & 6 & $5.15 E+02$ & 2. $13 E-02$ & $1,4 E+01$ & $7.10 \mathrm{E}-\mathrm{CS}$ & $N$ & RNiF & C8-137 Specifieation \\
\hline Cs-137(Ba.137) & $3.00 E+00$ & $7.26 E+04$ & 6 & $5.15 E+02$ & $2.13 ㄷ .02$ & $\$ .4 E+01$ & 7.9OE.C3 & $\mathbf{N}$ & RNiF & Ea.137 Specification \\
\hline EU-152 & 1.50E-04 & $3.63 E+00$ & 6 & $<2 E+\infty$ & $<8.27 E .05$ & $\mathrm{~N} / \mathrm{A}$ & $<5.51 E .01$ & $\mathbf{N}$ & RNiF & \\
\hline Eu-154 & $1.60 E-02$ & $3.87 E+02$ & B & $1.56 \mathrm{E}+\infty 0$ & $6.45 E-05$ & $1.1 E-01$ & $4.03 E-03$ & $N$ & RNiF & \\
\hline EU-155 & $9.00 E-03$ & $2.18 \mathrm{E}+02$ & 6 & $1.23 E+\infty 0$ & $508 E-05$ & $3.0 \mathrm{E}-01$ & $5.65 E-03$ & N & RNiF & \\
\hline $\mathrm{Fe}-\mathbf{5 5}$ & $1,00 E=03$ & $2.42 \mathrm{E}+01$ & a & 6. $25 E .02$ & 2.58E-06 & $1.7 \mathrm{E} .02$ & $2.58 E-03$ & $\mathbf{N}$ & RNIF & \\
\hline $1-129$ & 9.00E-08 & $2.18 E-03$ & 6 & 1.90E-03 & $7.85 E .08$ & 4.2E-C4 & $8.73 E-01$ & $\mathbf{N}$ & ICPMS Acid & Potential Prep Losses \\
\hline Wb-93m & $8.70 E-05$ & $2.10 E+\infty 0$ & 6 & $\ldots$ & - & - & $\ldots$ & - & - & Not $/$ \\
\hline Ni159 & $1.40 E .05$ & $3.39 E-01$ & 6 & $<300 \mathrm{E}-02$ & $<1.96 \mathrm{E}-06$ & $\overline{N / A}$ & $<8 \overline{32 E-02}$ & $\mathbf{N}$ & RNiF & \\
\hline $\mathrm{NI}-63$ & $1.60 E-03$ & $3.87 E+01$ & 6 & $1.17 E+\infty 0$ & 4.84E.05 & 8.2E.02 & $3.02 E-02$ & $\mathbf{N}$ & $\mathrm{FNiF}$ & \\
\hline$N p-237$ & $2.30 E-05$ & $5.58 E-01$ & 6 & $1.50 \mathrm{E} .03$ & 6.20 E.05 & $2.15-04$ & $2.70 \mathrm{E}-03$ & $N$ & ICPMMS Acid & \\
\hline$P d-107$ & A. ODE-06 & $9.68 \mathrm{~B}-02$ & 6 & 5.80E-03 & $2.40 E-07$ & $3.8 E .03$ & 5.99E-02 & $N$ & ICPMSS Acid & \\
\hline Pu-238 & $1.10 E-04$ & $2.66 \Xi+00$ & 6 & 3.47E-01 & $1.43 \equiv .05$ & 4.7E.02 & $1.30 \mathrm{E}-01$ & $\mathrm{~N}$ & RNIF & \\
\hline$P U-239$ & $9.50 \mathrm{E}-04$ & $2.30 E+09$ & 5 & $1.30 E+D 0$ & $5.37 \mathrm{E} .05$ & $2.8 \mathrm{E} \cdot 01$ & $5.66 \mathrm{E} \cdot 02$ & $\mathrm{~N}$ & ICPIMS NIF & \\
\hline Pu-240 & $2.60 E-04$ & $6.29 E+00$ & 6 & $1.70 E-01$ & $7.03 E-06$ & $3.7 E-02$ & $2.70 E-02$ & $N$ & ICPMASACId & \\
\hline Pu-241 & 6.90E-03 & $1.67 E+02$ & $B$ & $3.68 E+\infty$ & $1.52 E .04$ & $1.1 E+00$ & $2.20 E \cdot 02$ & $N$ & RNiF & \\
\hline Pu-242 & $7.90 E-08$ & $1.72 \mathrm{E}-03$ & 6 & $<2 E-02$ & $<8.27 E .07$ & N/A & $<1.18 E \div 01$ & $-Y-$ & RNiF & \\
\hline RU-10s $(R h-105]$ & $2.00 E-04$ & 4.84E $+\infty 0$ & 5 & $<3 E+00$ & $\$ 1.24 E-04$ & N/A & $\angle 6.20 E-01$ & $\mathbf{N}$ & RNiF & Ru-106 Specification \\
\hline RU-105\{Rh-105) & $2.00 \mathrm{E}-04$ & $4.84 E+00$ & 5 & $<3 E+00$ & $<1.24 \mathrm{E}-04$ & N/A & $66.20 E-01$ & $\mathbf{N}$ & RNiF & Rh-106 Spesifieation \\
\hline (Sb-125[Te-125m] & $1.00 E-02$ & $2.42 E+02$ & 6 & $<2 E+\infty 0$ & $<827 E .05$ & $\mathbf{N} / \mathbf{A}$ & $<8.27 E-03$ & $\mathbf{N}$ & RNiF & Sb-125 Specification \\
\hline (Sb-125(Te-125m) & $3.006-03$ & $7.26 \mathrm{E}+01$ & 6 & $<2 E+\infty O$ & $\angle 8.27 E .05$ & NiA & $<2.76 \mathrm{E}-02$ & $\mathbf{M}$ & RNiF & Te-125m Spesification \\
\hline Se.79 & $4.20 E .07$ & $1.02 E-02$ & 6 & $<2 E .03$ & $<9.92 E .08$ & N/A & $<2.36 E-01$ & N & RNiF & \\
\hline Sm-151(Pn-147) & $9.30 E-02$ & $2.25 E+03$ & 6 & $4.52 E+01$ & $1.87 E \cdot 03$ & $1.0 E+01$ & 2.01E.02 & $\mathbf{N}$ & RNiF & Sm-151 Specification \\
\hline $5 m \cdot 151\left(P_{m+147}\right)$ & $1.60 E-01$ & $3.67 E \cdot 03$ & 6 & $4.52 E+01$ & $1.87 E .03$ & $1.0 E+01$ & $1.17 E-02$ & $\mathrm{~N}$ & RNiF & Pm-147 Specification \\
\hline $\operatorname{sn}-113(\ln -113)$ & $1.8 \mathrm{BE}-06$ & $4.55 \mathrm{E} \cdot 02$ & 8 & $<3 E .02$ & $<1.24 E .05$ & N/A & $\$ 6.605 .01$ & $\mathbf{N}$ & RNIF & Sn-113 Specification \\
\hline $\operatorname{sn}+113(\mid n+113)$ & $1.88 E-06$ & $4.55 \mathrm{E}-02$ & 6 & CEE.02 & $<1.24 E-06$ & N/A & $\angle 6.60 E-01$ & $\mathbf{N}$ & RNiF & in-113 Specification \\
\hline Sn-119m & $1.00 E-08$ & $2.42 E-04$ & 6 & <4E-01 & <1.ESE-05 & N/A & $<1,65 E+03$ & $-Y_{-}$ & RINIF & \\
\hline $5 n+121 m$ & Q.00E-06 & $2.18 E-01$ & 6 & $<3 E+\infty$ & $\times 1,24=04$ & NIA & $\angle 38 E+01$ & $-Y=$ & RNiF & \\
\hline Sn-126(Sb-126m, Sb-126) & 4.800 .05 & $1.16 E+00$ & B & $<5.02$ & $<1.24 E .06$ & NIA & $<2.58 \mathrm{E}+02$ & $N$ & RNiF & Sn-126 Spectrication \\
\hline Sn-125isb-126m,st-125) & $3.43 \varepsilon-05$ & 8.30E-01 & 6 & $<3 E .02$ & $<1.24 E-06$ & N/A & $<3.82 E-02$ & $\mathrm{~N}$ & RNiF & Sb-126m Specification \\
\hline$(s n-125(5 b-126 m, 5 b-126)$ & $4.83 E-05$ & $1.17 E-01$ & 6 & $43 E-02$ & $\leqslant 1.24 \mathrm{E} .08$ & NIA & $\times 2.57 E-01$ & $N$ & RNiF & Sb-126 Specification \\
\hline $5 r-90(Y-90)$ & $3.10 E+\infty 00$ & $7.50 E+04$ & 6 & $3.54 \mathrm{E}+02$ & $1.46 E-02$ & $1.1 E+09$ & $4.72 E-03$ & $\mathrm{~N}$ & RNiF & St.9o.Specifieation \\
\hline Sr-9o(Y-90) & $3.10 E+\infty 0$ & $7.50 E+04$ & 6 & $3.54 \mathrm{E}+02$ & $1.46 E-02$ & $1.1 E+01$ & $4.72 \mathrm{E}-03$ & $\mathrm{~N}$ & RiNiF & Y.90 Specification \\
\hline $\begin{array}{l}\text { Te-99 } \\
\text { Th-232 }\end{array}$ & $\begin{array}{l}4.50 E-03 \\
\text { See } 7 \mathrm{Th}^{-1}\end{array}$ & $\begin{array}{l}\text { 1.09E+D2 } \\
\text { Sev " Th" }\end{array}$ & $\begin{array}{l}6 \\
6\end{array}$ & $\begin{array}{l}3.00 E-02 \\
3.90 E-06\end{array}$ & $\begin{array}{l}1.24 E-06 \\
1.61 E-10\end{array}$ & $\begin{array}{l}3.2 E-03 \\
9.7 E-07\end{array}$ & $\begin{array}{c}2.76 E-04 \\
N / R\end{array}$ & $\underset{N / A}{N}$ & $\begin{array}{l}\text { ICPIMS Acid } \\
\text { ICPMS NBF }\end{array}$ & - \\
\hline U-233 & $1.00 E-09$ & $2.42 \mathrm{E}-05$ & 8 & L4E-03 & $\times 1.55 E-07$ & N/A & $49.65 E+02$ & $-Y=$ & ICPMS Acid & TRACE COITP - Th Interferes \\
\hline U.234 & $7.70 E-07$ & 1. $08 E-02$ & 6 & $<1 E-03$ & $<4.13 E-08$ & N/A & *5.37E.02 & $N$ & ICPMSS Acid & \\
\hline U-235 & $3.20 E-0 B$ & $7.74 E .04$ & 6 & 1.6OE.05 & 5.61E-10 & 1.5E-0B & 2.07E-02 & $\mathrm{N}$ & ICPMS Acid & \\
\hline 0.236 & $6.20 E-08$ & 1.985 .03 & 1 & 2.60E-C5 & $1.07 E-09$ & $\mathrm{~N} / \mathrm{A}$ & $1.31 \mathrm{E}-02$ & $N$ & ICPIMSAEIS & Five of Six Results <EOL \\
\hline U-238 & $5.80 E \cdot 07$ & 1. $40 E-02$ & 6 & $3.86 E .04$ & $1,59 E-0.8$ & 2.9E-05 & 2.61E.02 & N & ICPIMS A cid & \\
\hline $2 r-93$ & $1.40 E-04$ & $3.39 E+\infty$ & 6 & $4.72 \mathrm{E}-02$ & $1.95=06$ & $1.8 E-02$ & $1.38 \mathrm{E}-02$ & $N$ & RNiF & \\
\hline Total Alpha & none & NA & 6 & $329 E+00$ & 1.33E-04 & 4.5E.01 & N/A & N/A & RNiF & \\
\hline Total Beta & none & N/A & 6 & $1,22 E+03$ & $5.04 E-02$ & $4.4 E+01$ & N/A & N/A & RWiF & \\
\hline 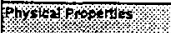 & 14 & & & \%ै। & olnatiatiter & . & & 6 & & \\
\hline WE sellds entosc $-[\%]$ & $\frac{1.43}{2.5 \cdot 13}$ & & & $\frac{\pi L E F}{28.2}$ & $\frac{5 R}{N / A}$ & & & & & See Note 2 \\
\hline$w r \%$ oxide e $1050 c-\{\%\}$ & & & & 21.8 & - & & & & & \\
\hline Total Oxlou - (gh) & 25.100 & & & & - & & & & & \\
\hline Slurry Density - $(g / m \mid\}$ & $1.02 \cdot 1,10$ & & & 1.25 & - & & & & & \\
\hline $\begin{array}{l}\text { Settled Solids - (Nor\%) } \\
\text { pit }\end{array}$ & $\begin{array}{l}7.95 \\
>10\end{array}$ & & & $\begin{array}{l}\text { C. } 80 \\
-\end{array}$ & - & & & & & \\
\hline
\end{tabular}

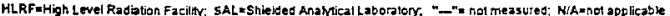

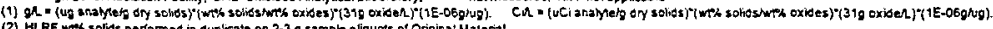

(2) HLRF wroh solists periomed in ouplicate on $2.3 \mathrm{~g}$ sample afiquots of Originat Materiat

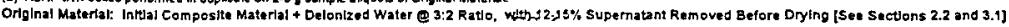




\title{
Definition of Terms used in Table ES-1
}

\author{
Avg/Spec \\ Flag $>$ Spec \\ n \\ N/A \\ $<$ \\ $\mathrm{ICPZrF}$ \\ ICP/MS ZrF \\ $\mathrm{RZrF}$ \\ ICPNiF \\ ICP/MS NiF \\ $\mathrm{RNiF}$ \\ ICP Acid \\ ICP/MS Acid \\ IC Water Leach \\ ISE Water Leach \\ Direct \\ Average result divided by Envelope D specification. \\ $\mathrm{N}$ means result does not exceed Envelope $\mathrm{D}$ specification, $\mathrm{Y}$ indicates result \\ exceeds specification. \\ Number of subsamples (including duplicates) used to generate the average and \\ standard deviation. \\ Not Applicable \\ Analyte/Radionuclide of interest was not detected above the instrument \\ detection limit \\ $\mathrm{Na}_{2} \mathrm{O}_{2}$ fusions ( $\mathrm{Zr}$ crucible) analyzed by ICP-AES \\ $\mathrm{Na}_{2} \mathrm{O}_{2}$ fusions ( $\mathrm{Zr}$ crucible) analyzed by ICP-MS \\ $\mathrm{Na}_{2} \mathrm{O}_{2}$ fusions ( $\mathrm{Zr}$ crucible) analyzed for radionuclide \\ $\mathrm{KOH}$ fusions (Ni crucible) analyzed by ICP-AES \\ $\mathrm{KOH}$ fusions (Ni crucible) analyzed by ICP-MS \\ $\mathrm{KOH}$ fusions (Ni crucible) analyzed for radionuclide \\ Acid digestion analyzed by ICP-AES \\ Acid digestion analyzed by ICP-MS \\ Water leach preparation analyzed by IC \\ Water leach preparation analyzed by ISE \\ Analysis performed on direct aliquots of the material
}




\section{TERMS/ACRONYMS/DEFINITIONS}

$\begin{array}{ll}\text { ACL } & \text { Analytical Chemistry Laboratory } \\ \text { AEA } & \text { Alpha Energy Analysis } \\ \text { CVAA } & \text { Cold Vapor Atomic Absorption } \\ \text { GEA } & \text { Gamma Energy Analysis } \\ \text { HLRF } & \text { High Level Radiochemistry Facility } \\ \text { IC } & \text { Ion Chromatography } \\ \text { ICP-AES } & \text { Inductively Coupled Plasma - Atomic Emission Spectrometry } \\ \text { ICP-MS } & \text { Inductively Coupled Plasma - Mass Spectroscopy. } \\ \text { ISE } & \text { Ion Specific Electrode } \\ \text { LEP } & \text { Low-Energy Photon (counting) } \\ \text { LOI } & \text { Letter of Instruction } \\ \text { LSC } & \text { Liquid Scintillation counting } \\ \text { m/z } & \text { mass-to-charge ratio (ICP-MS) } \\ \text { PNNL } & \text { Pacific Northwest National Laboratory } \\ \text { QC } & \text { Quality Control } \\ \text { SAL } & \text { Shielded Aralytical Laboratory } \\ \text { TIMS } & \text { Thermal Ionization Mass Spectroscopy } \\ \text { TWRS } & \text { Tank Waste Remediation System }\end{array}$

Quality Control Definitions:

Estimated Quantitation Level (EQL): The EQL is the lowest measurement that can be reliably achieved within specific limits of precision and accuracy during routine operating conditions. Typically, the EQL is either ten times the IDL or is the lowest calibration standard, whichever is appropriate for a specific method. Results reported to the EQL have been adjusted for sample preparation and analytical dilution factors.

Instrument Detection Limit (IDL): The IDL is the minimum concentration that can be measured and reported within $99 \%$ confidence that the value is above zero. Generally, the IDL is determined by the repeated analysis of standards with concentrations at or near the estimated IDL. Results reported to the IDL have been adjusted for sample preparation and analytical dilution factors. 


\section{HNF-SD-WM-DP-225, REV. 1}

\section{Analytical Summary Report - January 28, 1997}

Mean Difference (MD): The MD determines if two results are statistically different at the $95 \%$ confidence level. MD results greater or equal to 1.96 indicate there is a $95 \%$ confidence that the two results are not equal. The MD is typically used to evaluate radionuclide results.

Minimum Detectable Activity (MDA): The MDA is applied to nuclear counting and is defined as the level of activity that is practically achievable by a measurement system using a specified counting time for a specific sample quantity and sample dilution factor.

Relative Percent Difference (RPD): The RPD expresses the precision of duplicates. Generally, RPD values less than $20 \%$ indicate acceptable precision.

Quality Control Sample Type Definitions:

Duplicate (D): A second subsample (or aliquot) of the "sample to be analyzed" that is treated/processed the same as the initial subsample in order to determine the precision of the method.

Laboratory Blank: An analytical control used by the laboratory to monitor specific processes for potential contamination.

Laboratory Control Sample (LCS): A control sample of known composition. The laboratory control sample is analyzed using the same sample preparation, reagents, and analytical methods that are used for samples.

Matrix Spike/Matrix Spike Duplicate (MS/MSD): A subsample (or aliquot) of a sample spiked with a known quantity of a specific analyte of interest and subjected to the entire analytical procedure in order to indicate the appropriateness of the method to the sample matrix by measuring recovery.

Preparation Blank (PB): An analytical control that contains processing reagents only, which is carried through the entire analytical procedure and used to identify potential processing contamination.

Replicate: A replicate is a second analysis of a single sample by the same method, and usually applies to solutions. For solids, a single subsample analyzed twice may be defined as a replicate analysis; in contrast to a duplicate analysis, where two subsamples are analyzed. 
TABLE OF CONTENTS

2.0 PRELIMINARY SAMPLE HANDLING $\ldots \ldots \ldots \ldots \ldots \ldots \ldots \ldots \ldots$

$2.1 \quad$ Laboratory-Scale Screening Test $\ldots \ldots \ldots \ldots \ldots \ldots \ldots \ldots \ldots 6$

2.2 Bench-Scale Vendor Product Batch Preparation $\ldots \ldots \ldots \ldots \ldots \ldots 8$

2.3 Chemical, Radiochemical, and Physical Characterization $\ldots \ldots \ldots \ldots 10$

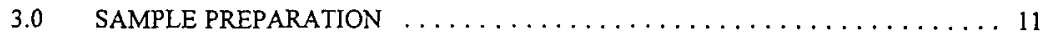

3.1 Preparations for Chemical Analyses $\ldots \ldots \ldots \ldots \ldots \ldots \ldots \ldots$

3.1 .1 Acid Digestion Preparation $\ldots \ldots \ldots \ldots \ldots \ldots \ldots \ldots \ldots \ldots$

3.1.2 Potassium Hydroxide and Sodium Peroxide Fusion Preparations . . 14

3.1 .3 Water leaching Preparation $\ldots \ldots \ldots \ldots \ldots \ldots \ldots \ldots \ldots$

4.0 INORGANIC, RADIOCHEMICAL, AND PHYSICAL ANALYSIS $\ldots \ldots \ldots \ldots 19$

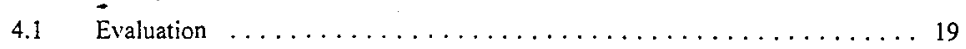

4.1.1 Analytes/Radionuclides/Physical Properties Exceeding Specification . 19

4.1.2 Radionuclides/Analytes Unable to be Evaluated Against Specification 20

4.1.3 Radionuclides/Analytes within 50\% of the Specification .......21

4.2 Physical Measurements -- Vendor Product and Original Material ...... 21

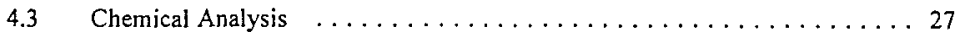

4.3.1 Inductively Coupled Plasma Atomic Emission Spectrometry

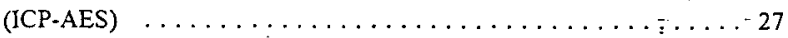

4.3.1.1 ICP-AES - Vendor Product (Table 4.3 to Table 4.5) ... 27

4.3.1.2 ICP-AES - Original Material (Table 4.6 to Table 4.8) $\ldots 28$

4.3.2 Inductively Coupled Plasma Mass Spectrometry (ICP-MS) $\ldots \ldots 28$

4.3.2.1 ICP-MS - Vendor Product (Table 4.9 to Table 4.14.) . .. 28

4.3.2.2 ICP-MS - Original Material (Table 4.15 to Table 4.20) . . . 29 
TABLE OF CONTENTS (Cont.)

4.3.3 Ion Chromatography (IC) for Anions (Table 4.21 and Table 4.22) . 29

4.3.4 Ion Specific Electrode for Ammonia (Table 4.21 and Table 4.22) .. 30

4.3.5 Cold Vapor Atomic Absorption for Mercury

(Table 4.21 and Table 4.22 ) ............... 30

4.3.6 Cyanide (Table 4.21 and Table 4.22) $\ldots \ldots \ldots \ldots \ldots \ldots$

4.3.7 Total Carbon - Inorganic and Organic Carbon

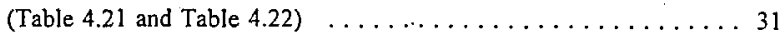

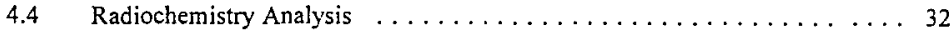

4.4.1 Radiochemistry - Vendor Product (Table 4.23) . . . . . . . 32

4.4.2 Radiochemistry - Origina! Material (Table 4.24) . . . . . . . . 37

4.5 Additional Sample Analyses -- Centrifuged Solids and Decanted Liquids ... 42

4.5.1 Preparation of Vendor Product (Decant Liquid) . . . . . . . . 43

4.5.2 Preparation of Original Material

- (Centrifuged Solids \& Decanted Liquid) . . . . . . . . . . . 43

4.5 .3 Chemical Analysis . . . . . . . . . . . . . . . 43

4.5.4 Radiochemical Analysis $\ldots \ldots \ldots \ldots \ldots \ldots \ldots \ldots \ldots \ldots$

$5.0 \quad$ REFERENCES $\ldots \ldots \ldots \ldots \ldots \ldots \ldots \ldots \ldots \ldots \ldots \ldots$

APPENDIX $A \ldots \ldots \ldots \ldots \ldots \ldots \ldots \ldots \ldots \ldots \ldots \ldots \ldots \ldots \ldots$ 


\section{HNF-SD-WM-DP-225, REV. 1}

Analytical Summary Report - January 28, 1997

\section{LIST OF TABLES}

1.1 Analytical Requirements for Envelope D Analytes/Radionuclides

$2.1 \quad 241-\mathrm{C}-106$ Sample Receipt Information $\ldots \ldots \ldots \ldots \ldots \ldots \ldots \ldots \ldots$

$2.2 \quad$ C-106 Material Transfer from Building 324 to $325 \ldots \ldots \ldots \ldots \ldots \ldots$

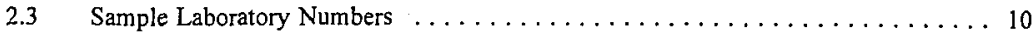

4.1 Vendor Product Bulk Weight Percent Solids Measurements $\ldots \ldots \ldots \ldots \ldots \ldots 21$

4.2 Physical Measurements -- Vendor Product and Original Material . . . . . . . . 22

4.3 ICP-AES KOH Fusion -- Vendor Product $\ldots \ldots \ldots \ldots \ldots \ldots \ldots \ldots \ldots \ldots \ldots \ldots$

4.4 ICP-AES $\mathrm{Na}_{2} \mathrm{O}_{2}$ Fusion -- Vendor Product $\ldots \ldots \ldots \ldots \ldots \ldots \ldots \ldots \ldots \ldots$

4.5 ICP-AES Acid Digestion -- Vendor Product $\ldots \ldots \ldots \ldots \ldots \ldots \ldots \ldots$

$4.6 \quad$ ICP-AES KOH Fusion -- Original Material $\ldots \ldots \ldots \ldots \ldots \ldots \ldots$

4.7 ICP-AES $\mathrm{Na}_{2} \mathrm{O}_{2}$ Fusion -- Original Material $\ldots \ldots \ldots \ldots \ldots \ldots \ldots$

$4.8 \quad$ ICP-AES Acid Digestion .. Original Material $\ldots \ldots \ldots \ldots \ldots \ldots \ldots \ldots \ldots$

4.9 ICP/MS KOH Fusion -- Vendor Product $\ldots \ldots \ldots \ldots \ldots \ldots \ldots \ldots \ldots$

4.10 ICPMS Isotopes KOH Fusion -- Vendor Product $\ldots \ldots \ldots \ldots \ldots \ldots \ldots \ldots$

4.11 ICP/MS $\mathrm{Na}_{2} \mathrm{O}_{2}$ Fusion -- Vendor Product $\ldots \ldots \ldots \ldots \ldots \ldots \ldots \ldots \ldots$

4.12 ICPMS Isotopes $\mathrm{Na}_{2} \mathrm{O}_{2}$ Fusion.- Vendor Product $\ldots \ldots \ldots \ldots \ldots \ldots$

4.13 ICP/MS Acid Digestion .. Vendor Product $\ldots \ldots \ldots \ldots \ldots \ldots \ldots \ldots$

4.14 ICP/MS Isotopes Acid Digestion -. Vendor Product $\ldots \ldots \ldots \ldots \ldots \ldots \ldots$

4.15 ICPAS KOH Fusion -- Original Material $\ldots \ldots \ldots \ldots \ldots \ldots \ldots \ldots \ldots \ldots \ldots \ldots$

4.16 ICP/MS Isotopes KOH Fusion .- Original Material $\ldots \ldots \ldots \ldots \ldots \ldots \ldots \ldots$

4.17 ICPMS $\mathrm{Na}_{2} \mathrm{O}_{2}$ Fusion -- Original Material $\ldots \ldots \ldots \ldots \ldots \ldots \ldots \ldots$

$4.18 \mathrm{ICP} / \mathrm{MS}$ Isotopes $\mathrm{Na}_{2} \mathrm{O}_{2}$ Fusion - Original Material $\ldots \ldots \ldots \ldots \ldots \ldots$

4.19 ICP/MS Acid Digestion -- Original Material $\ldots \ldots \ldots \ldots \ldots \ldots \ldots \ldots \ldots \ldots$

4.20 ICP/MS Isotopes Acid Digestion -. Original Material $\ldots \ldots \ldots \ldots \ldots$ 
HNF-SD-WM-DP-225, REV. 1

Analytical Summary Report - January 28, 1997

\section{LIST OF TABLES (Cont.)}

4.21 Wet Chemistry Analysis -- Ventor Product $\ldots \ldots \ldots \ldots \ldots \ldots \ldots \ldots \ldots \ldots$

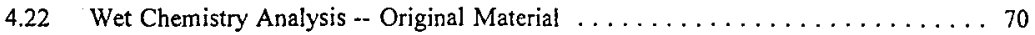

4.23 Radiochemical Analysis -- Vendor Product $\ldots \ldots \ldots \ldots \ldots \ldots \ldots \ldots$

4.24 Radiochemical Analysis -. Original Material $\ldots \ldots \ldots \ldots \ldots \ldots \ldots$

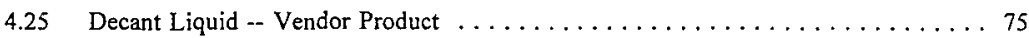

4.26 Centrifuged Solids/Decant Liquid -- Original Material $\ldots \ldots \ldots \ldots \ldots \ldots$

\section{LIST OF FIGURES}

3.1 Vendor Product Sample Preparation $\ldots \ldots \ldots \ldots \ldots \ldots \ldots \ldots \ldots \ldots$

3.2 Original Material Sample Preparation $\ldots \ldots \ldots \ldots \ldots \ldots \ldots \ldots \ldots$

3.3 Vendor Product Preparations and Analyses $\ldots \ldots \ldots \ldots \ldots \ldots \ldots \ldots$

3.4 Original Material Preparations and Analyses $\ldots \ldots \ldots \ldots \ldots \ldots \ldots \ldots$ 


\subsection{INTRODUCTION}

This report represents the Analytical Summary Report on Tank Waste Remediation System (TWRS) Privatization Contractor Samples conducted in support of the TWRS Privatization Contractor Samples, Waste Envelope D, C-106 characterization testing. All work performed was conducted in accordance with "Addendum 1 of the Letter of Instruction (LOI) for TWRS Privatization Contractor Samples Addressing Waste Envelope D Materials - Revision 0," (Jones, 1996). All work was conducted under the direction of the assigned Pacific Northwest National Laboratory (PNNL) Analytical Chemistry Laboratory (ACL) Project Manager.

Tank 241-C-106 (C-106) was selected by TWRS Privatization for the Part IA Envelope D high-level waste demonstration. Twenty bottles of Tank C-106 material were collected using the grab sampling teclinique and transferred to the 325 building. The contents of the twenty bottles were composited and subsampled for the laboratory-scale screening test and characterization testing. Envelope D material bench-scale batch preparation was performed in the 324 building, followed by characterization testing in the 325 building.

The C-106 composite batch material was characterized during three sequential stages of Envelope D sample preparation: 1) after initial dilution with water prior to beginning the bench-scale processing ("Original Material"); 2) after bench-scale caustic leach and inhibited water wash treatment per revision 0 of the LOI ("Vendor Product"); and 3) after decant and dilute treatment per revision 1 of the LOI ("Final Vendor Product").

This report is one of a series of reports addressing the C-106 vendor sample preparation and characterization. This summary report includes analytical results on the Envelope $D$ Privatization Vendor Product and the Original Material. The final vendor product results are provided in Addendum 1 to this report (Urie 1997). A subsequent Analytical Final Report (in progress) will include an assessment of the preparation and analytical methods, all raw data, and an evaluation of lessons learned. The final reports detailing the laboratory-scale screening and final bench-scale preparation are provided by Lumetta (1996) and Brooks (1997), respectively. Statistical reports for the C-106 analytical data will be prepared by TL Welsh (in progress).

The Envelope D analytes of interest (non-radionuclide and radionuclide), recommended methods, and estimated quantitation levels are defined in revision 0 of the LOI. Table 1.1 summarizes the LOI's analytical requirements for the Vendor Product. In many cases the recommended method for nonradionuclides is ICP-AES. Due to the low concentrations of many of the analytes of interest, ICP-MS results have been reported.

The sample handling, preparation, and analyses are described in Sections 2, 3, and 4, respectively. 
HNF-SD-WM-DP-225, REV. 1

Analytical Summary Report - January 28, 1997

Table 1.1 Analytical Requirements for Envelope D Analytes/Radionuclides

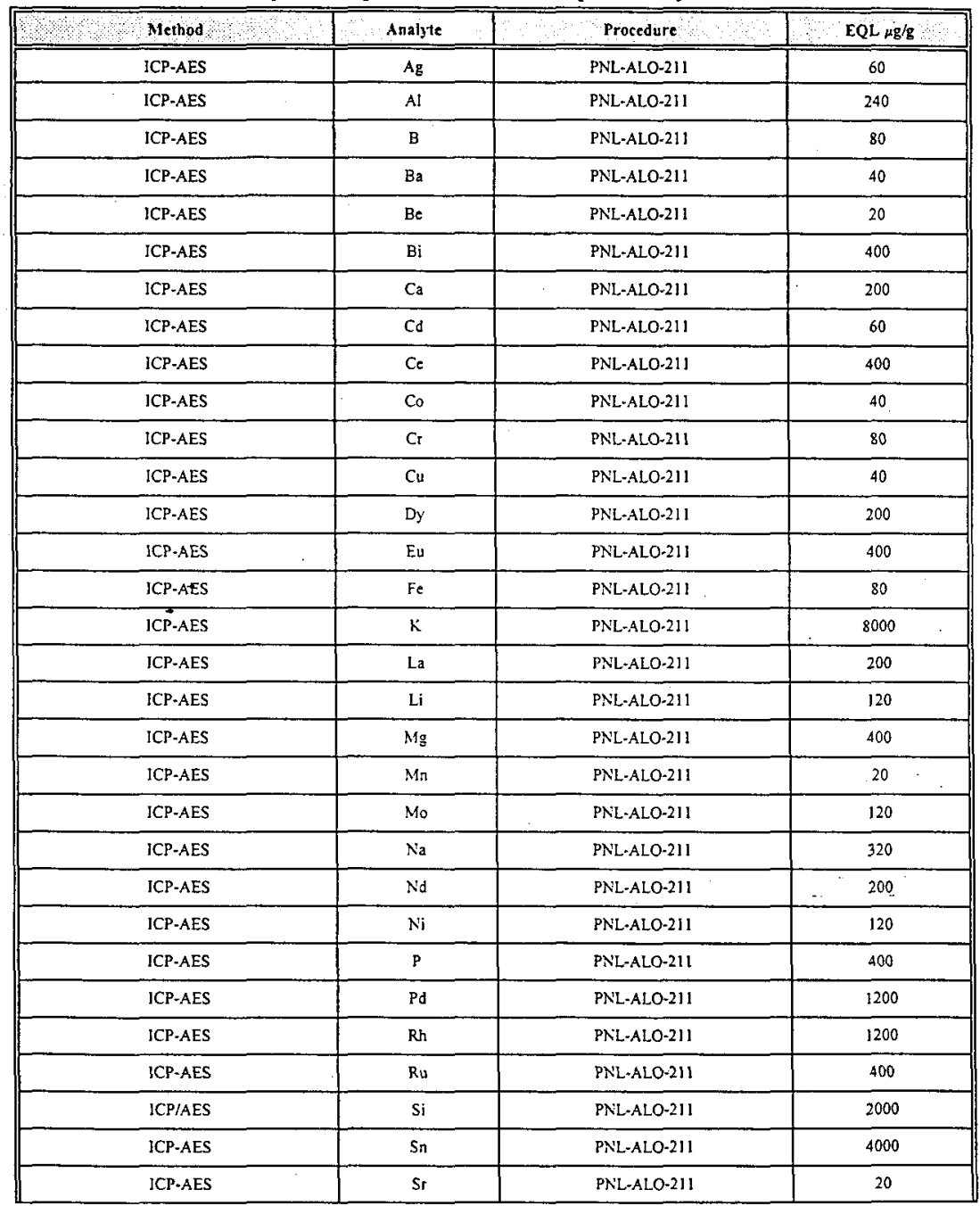


HNF-SD-WM-DP-225, REV. 1

Analytical Summary Report - January 28, 1997

\begin{tabular}{|c|c|c|c|}
\hline Method & Analyte & Procedure & $\mathrm{EQL} \mu \mathrm{g} / \mathrm{g}$ \\
\hline ICP-AES & Te & PNL-ALO-211 & 2000 \\
\hline ICP-AES & Th & PNL-ALO-211 & 3200 \\
\hline ICP-AES & $\mathrm{Ti}$ & PNL-ALO-211 & 20 \\
\hline ICP-AES & $\mathrm{U}$ & PNL-ALO-211 & 8000 \\
\hline ICP-AES & $\mathrm{v}$ & PNL-ALO-211 & 40 \\
\hline ICP-AES & W & PNL-ALO-211 & 2000 \\
\hline ICP-AES & $\mathrm{Y}$ & PNL-ALO-21] & 40 \\
\hline ICP-AES & $\mathrm{Zn}$ & PNL-ALO-211 & 80 \\
\hline ICP-AES & $\mathrm{Zr}$ & PNL-ALO-211 & 40 \\
\hline ICP-MS & Cs & PNL-ALO-280 & 0.2 \\
\hline ICP-MS & Gd & PNL-ALO-280 & 0.2 \\
\hline ICP-MS & $\mathrm{Nb}$ & PNL-ALO-280 & 0.2 \\
\hline ICP-MS & $\operatorname{Pr}$ & PNL-ALO-280 & 0.2 \\
\hline ICP-MS & $\mathrm{Rb}$ & PNL-ALO-280 & 0.2 \\
\hline ICP-MS & $\operatorname{Re}$ & PNL-ALO-280 & 0.2 \\
\hline ICP-MS & $\mathrm{ra}$ & PNL-ALO-280 & 0.2 \\
\hline ICP-AES / ICP-MS & As & PNL-ALO-211/280 & $4800 / 20$ \\
\hline ICP-AES / ICP-MS & $\mathrm{Cd}$ & PNL-ALO-211/280 & $300 / 6$ \\
\hline ICP-AES / ICP-MS : & $\mathrm{Pb}$ & PNL-ALO-211 / 280 & $1200 / 2$ \\
\hline ICP-AES / ICP-MS & $\mathrm{Sb}$ & PNL-ALO-211 / 280 & $9000 / 4$ \\
\hline ICP-AES / ICP-MS & Se & PNL-ALO-211 / 280 & $6000 / 100$ \\
\hline ICP-AES / ICP-MS & $\mathrm{T}$ & PNL-ALO-211 / 280 & $9000 /(\mathrm{n} / \mathrm{a})$ \\
\hline $\mathrm{CN}$ & $\mathrm{ON}$ & PNL-ALO-287/289/285 & 0.1 \\
\hline CVAA & $\mathrm{Hg}$ & PNL-ALO-13]/201 & 0.05 \\
\hline IC & $\mathrm{Cl}$ & PNL-ALO-212 & 50 \\
\hline IC & $\mathrm{F}$ & PNL-ALO-212 & 50 \\
\hline IC & $\mathrm{NO}_{2}$ & PNL-ALO-212 & 100 \\
\hline IC & $\mathrm{NO}$ & PNL-ALO-212 & 100 \\
\hline IC & So, & PNL-ALO-212 & 100 \\
\hline $1 \mathrm{C}$ & $\mathrm{PO}_{4}$ & PNL-ALO-212 & 100 \\
\hline Hot Persulfate & TICTOC & PNL-ALO-381/382/383 & 1 \\
\hline Ion Specific Electrode & $\mathrm{NH}_{4}-\mathrm{N}$ & PNL-ALO-226 & 0.1 \\
\hline
\end{tabular}


HNF-SD-WM-DP-225, REV. 1

Analytical Summary Report - January 28, 1997

\begin{tabular}{|c|c|c|c|}
\hline और Method & Radionuclide & Procedure & $\mathrm{EQL}_{\mu \mathrm{CV} / \mathrm{g}}$ \\
\hline GEA & ${ }^{\mathrm{N}} \mathrm{CO}$ & PNL-ALO-450 & 200 \\
\hline GEA & ${ }^{10} x_{R u(i k i k h)}$ & PNL-ALO-450 & 2000 \\
\hline GEA & ${ }^{111 m} \mathrm{Ag}$ & PNL-ALO-450 & 200 \\
\hline GEA & ${ }^{123} \mathrm{Sb}\left({ }^{133^{2 n} \mathrm{~T}} \mathrm{Te}\right)$ & PNL-ALO-450 & 500 \\
\hline GEA & ${ }^{1,4} \mathrm{Cs}$ & PNL-ALO-450 & 200 \\
\hline GEA & ${ }^{157} \mathrm{Cs}\left({ }^{112} \mathrm{Ba}\right)$ & PNL-ALO-450 & 200 \\
\hline GEA & ${ }^{34} \mathrm{Ce}\left({ }^{144} \mathrm{Pr},{ }^{14 \pi m} \mathrm{Pr}\right)$ & PNL-ALO-450 & 200 \\
\hline GEA & ${ }^{192} \mathrm{Eu}$ & PNL-ALO-450 & 1000 \\
\hline GEA & ${ }^{134} \mathrm{Eu}$ & PNL-ALO-450 & 1000 \\
\hline GEA & ${ }^{135} \mathrm{Eu}$ & PNL-ALO-450 & 1000 \\
\hline separation-->beta count & ${ }^{x} \mathrm{Sr}(\mathrm{Y})$ & PNL-ALO-476/431 & 100 \\
\hline separation --> beta count & ${ }^{n} \mathrm{Zr}\left({ }^{n} \mathrm{n} \mathrm{N} \mathrm{b}\right)$ & PNL-ALO-478 (modified) & 10 \\
\hline separation-->beta count & ${ }^{12} \mathrm{Pm}+1{ }^{1 / 1} \mathrm{Sm}$ & PNL-ALO-474 & TBD \\
\hline separation $\rightarrow$ AEA & ${ }^{2} \mathrm{Pu}$ & PNL-ALO-417/422 & 100 \\
\hline separation-->AEA & $2 \operatorname{sensenpu}$ & PNL-ALO-417/422 & 100 \\
\hline separation $\rightarrow>A E A$ & $\because \mathrm{Pu}$ & PNL-ALO-417/422 & 100 \\
\hline separation-->AEA & $\because A m$ & PNL-ALO-4 1 7/422 & 100 \\
\hline separation $->A E A$ & $\because \mathrm{Am}$ & PNL-ALO-417/422 & 100 \\
\hline separation-->AEA & $" * \mathrm{Cm}\left({ }^{*} \mathrm{Am},{ }^{* m_{\mathrm{m}}} \mathrm{Am}\right)$ & PNL-ALO-417/422 & 100 \\
\hline separation-->AEA & ${ }^{x y-2 x} \mathrm{Cm}$ & PNL-ALO-417/422 & 100 \\
\hline separation - > X-ray and gamma count & ${ }^{11} \operatorname{Sn}(1+3 m ! n)$ & PNL-ALO-453 & 200 \\
\hline separation $->X$-ray and gamma count & 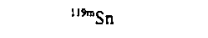 & PNL-ALO-453 & 500 \\
\hline separation-->X-ray and gamma count & $\operatorname{lntm} S n$ & PNL-ALO- 453 & 800 \\
\hline separation-->X-ray and gamma count & ${ }^{126} \mathrm{Sn}\left({ }^{1-6} \mathrm{Sb},{ }^{1 \times \tan } \mathrm{Sb}\right)$ & PNL-ALO-453 & 200 \\
\hline separation $\cdots>$ X-ray count & ${ }^{\text {ss }} \mathrm{Fe}$ & PNL-ALO-435 & 100 \\
\hline separation $\rightarrow>X$-ray count & shi & PNIL-ALO-495/450 & 100 \\
\hline separation-->liquid scintillation count & ${ }^{6} \mathrm{Ni}$ & PNL-ALO-495/450 & 10 \\
\hline separation -->liquid scintillation count & ${ }^{21} \mathrm{Pu}$ & PNL-ALO-417/474! & 100 \\
\hline oxidize-->liquid scintillation & ${ }^{14} \mathrm{C}$ & PNL-ALO-428/474 & 10 \\
\hline
\end{tabular}


HNF-SD-WM-DP-225, REV. 1

Analytical Summary Report - January 28, 1997

\begin{tabular}{|c|c|c|c|}
\hline \& Method & Radionuclide & Procedure & $\mathrm{EQL} \mu \mathrm{CL} / \mathrm{g}$ \\
\hline distillation-->liquid scintillation & ${ }^{7 \mathrm{Se}}$ & PNL-ALO-440/474 & 10 \\
\hline thermal jonization mass spectrometry & ${ }^{i n s} \mathrm{Cs}$ & PNL-ALO-452 & TBD \\
\hline ICP-MS & "Tc & PNL-ALO-280/281 & 0.2 \\
\hline ICP-MS & ${ }^{163} \mathrm{Pd}$ & PNL-ALO-280 & 0.2 \\
\hline ICP-MS & 191 & PNL-ALO-280 & 4 \\
\hline ICP-MS & No & PNL-ALO-280 & 0.2 \\
\hline ICP-MS & ${ }^{279} \mathrm{Pu}$ & PNL-ALO-280 & 0.2 \\
\hline $\mathrm{ICP}-\mathrm{MS}$ & ${ }^{241} \mathrm{Pu}$ & PNL-ALO-280 & 0.2 \\
\hline ICP-MS & ${ }^{241} \mathrm{Pu}+{ }^{241} \mathrm{Am}$ & PNL-ALO-280 & 0.2 \\
\hline ICP-MS & ${ }^{24} \mathrm{Pu}$ & PNL-ALO-280 & 0.2 \\
\hline ICP-MS & ${ }^{i n} \mathrm{Th}$ & PNL-ALO-280 & 0.2 \\
\hline $\mathrm{ICP} \cdot \mathrm{MS}$ & $\because " \mathrm{U}$ & PNL-ALO-280/282 & 0.2 \\
\hline ICP-MS & $: 4 \mathrm{U}$ & PNL-ALO-280/282 & 0.2 \\
\hline ICP-MS & $\because \mathrm{U}$ & PNL-ALO-280/282 & 0.2 \\
\hline ICP-MS & $=\mathrm{v}_{\mathrm{n}} \mathrm{U}$ & PNL-ALO-280/282 & 0.2 \\
\hline ICP-MS & ${ }^{2 n} \mathrm{U}$ & PNL-ALO-280/282 & 0.2 \\
\hline & ${ }^{163 \pi} \mathrm{Cd} *$ & Test Plan & $T B D$ \\
\hline
\end{tabular}

- Curently there is no method for $113 \mathrm{mCd}$, and ORJGEN must be used to estimate the concentration. However, a method has recently been proposed, and would be ajalyzed under a test plan, if approved. 
Tank C-106 material was sampled 6/21/96 through 6/24/96 and transferred to the 325 building High Level Radiochemistry Facility (HLRF) 6/22/96 through 6/26/96. Sampling information from chain of custody data is presented in Table 2.1.

Twenty grab samples from C-106 were received from the East Tank Farms. Each grab sample was transported to the 325 building in a $125 \mathrm{~mL}$ jar shielded by a B Plant shipping container. Due to the dose rates of these samples, the samples were transferred into a hot cell where they were homogenized and composited in a series of steps. Each sample was individually homogenized with a high speed mixer (OMNI Mixer) according to PNNL technical procedure PNL-ALO-135, "Laboratory Procedure for Homogenization of Solutions, Slurries and Sludges", section 3.0. An ice bath was used to control the temperature of the samples during homogenization.

The homogenized samples were transferred to a 2 liter carboy until the carboy was approximately $3 / 4$ full $(\approx 1.5$ Liters or 12 samples). The homogenized samples were composited by stirring for approximately 15 minutes with a mechanical stirring motor and a Teflon stirring rod. Approximately $750 \mathrm{~mL}$ of this sample was transferred to a 1 liter jar, and the remaining homogenized samples were transferred to the carboy where they were composited by the same procedure as the previous samples. In order to prepare a homogeneous composite, a series of cuts were made between the 1 liter jar and the 2 liter carboy with stirring between each cut. The homogenized composite was then split between the 1 liter jar and 2 liter carboy. The homogenized composite represents the Initial Composite Material. A 15 gram aliquot of the Initial Composite Material was taken and transferred to the Shielded Analytical Laboratory (SAL) for the laboratory-scale screening test.

\subsection{Laboratory-Scale Screening Test}

The screening test was conducted in accordance with PNNL technical procedure PCS-TP-511-1, Rev. 3. This screening test was designed to test whether washing with dilute hydroxide solution or a combination of caustic leaching and dilute hydroxide washing would yield a solids product (i.e., Vendor Product) which conforms to Envelope D specifications. In the screening test, approximately 15 grams of Initial Composite Material was mixed with approximately 29 grams of water. This test material was divided into five portions; for "simple" sludge washing, "enhanced" sludge washing, clemical analysis, particle size, and microscopy studies.

To determine the chemical composition of the untreated, Initial Composite Material, one 2.2 gram slurry portion was dried for chemical analysis, while two small portions (i.e., 0.7 grams and 0.5 grams slumy) were set aside for particle-size analysis and microscopy studies. A 4.5 gram slurry portion of the test material was washed three successive times with $0.01 \mathrm{M} \mathrm{NaOH}$ to determine the effects of "simple" sludge washing; for each wash, approximately 17 grams of wash solution was used per gram of dry sludge solids. Each wash consisted of mixing the sludge/ $0.01 \mathrm{M} \mathrm{NaOH}$ mixture for 1 hour at $100^{\circ} \mathrm{C}$, allowing to cool to ambient temperature, centrifuging, then decanting the wash liquor. The washed solids were dried to constant weight at $105^{\circ} \mathrm{C}$ and analyzed. 
HNF-SD-WM-DP-225, REV. 1

Analytical Summary Report - January 28, 1997

Table 2.1 241-C-106 Sample Receipt Information'

\begin{tabular}{|c|c|c|c|c|c|c|c|c|}
\hline Sample & $\begin{array}{l}\text { Tank } \\
\text { Riser }\end{array}$ & $\begin{array}{l}\text { Date } \\
\text { Sampled }\end{array}$ & $\begin{array}{c}\text { Sampling } \\
\text { Depth } \\
\text { (inches) }\end{array}$ & $\begin{array}{l}\text { Dose@ } \\
\text { Contact } \\
\text { (Rhr) }\end{array}$ & $\begin{array}{l}\text { Expected } \\
\text { Liquid } \\
\text { Content }\end{array}$ & $\begin{array}{l}\text { Expected } \\
\text { Solid } \\
\text { Content }\end{array}$ & $\begin{array}{l}\text { Date } \\
\text { Received } \\
\text { In } 325\end{array}$ & $\begin{array}{c}\text { Shipment } \\
\#\end{array}$ \\
\hline $6 C-96-18$ & $\mathrm{Cl} 106 / 7$ & $6 / 21 / 96$ & 406 & 1.5 & $30 \%$ & $70 \%$ & $6 / 22 / 96$ & 37406 \\
\hline $6 C-96-19$ & $\mathrm{C} 106 / 7$ & $6 / 21 / 96$ & 406 & 1.0 & $20 \%$ & $80 \%$ & $6 / 22 / 96$ & 37406 \\
\hline $6 \mathrm{C}-96-20$ & $\mathrm{C} 106 / 7$ & $6 / 21 / 96$ & 407 & 2.4 & $20 \%$ & $80 \%$ & $6 / 22 / 96$ & 37405 \\
\hline $6 C-96-21$ & $\mathrm{C} 106 / 7$ & $6 / 21 / 96$ & 408 & 1.5 & $20 \%$ & $80 \%$ & $6 / 22 / 96$ & 37408 \\
\hline $6 C-96-22$ & $\mathrm{C} 106 / 7$ & $6 / 21 / 96$ & 408 & 2.0 & $20 \%$ & $80 \%$ & $6 / 22 / 96$ & 37408 \\
\hline $6 C-96-23$ & C106/7 & $6 / 21 / 96$ & 409 & 1.1 & $20 \%$ & $80 \%$ & $6 / 22 / 96$ & 37407 \\
\hline $6 C-96-24$ & $\mathrm{Cl} 106 / 7$ & $6 / 21 / 96$ & 409 & 2.0 & $30 \%$ & $70 \%$ & $6 / 22 / 96$ & 37410 \\
\hline $6 C-96-25$ & $C_{106 / 7}$ & $6 / 21 / 96$ & 411 & 1.0 & $20 \%$ & $80 \%$ & $6 / 22 / 96$ & 37410 \\
\hline $6 C \cdot 96-26$ & $\mathrm{C} 106 / 7$ & $6 / 21 / 96$ & 411 & 1.0 & $20 \%$ & $80 \%$ & $6 / 22 / 96$ & 37409 \\
\hline $6 C-96-27$ & $\mathrm{C} 106 / 7$ & $6 / 21 / 96$ & 411 & 2.0 & $20 \%$ & $80 \%$ & $6 / 22 / 96$ & 37411 \\
\hline $6 C-96-28$ & $\mathrm{C} 106 / 7$ & $6 / 21 / 96$ & 413 & 2.3 & $20 \%$ & $.80 \%$ & $6 / 22 / 96$ & 37411 \\
\hline $6 C-96-29$ & Ci06/7 & $6 / 21 / 96$ & 413 & 1.8 & $20 \%$ & $80 \%$ & $6 / 22 / 96$ & 37412 \\
\hline $6 C-96-30$ & C106/7 & $6 / 23 / 96$ & 413 & 2.5 & $50 \%$ & $50 \%$ & $6 / 24 / 96$ & 37414 \\
\hline $6 C-96-31$ & $\mathrm{C} 106 / 7$ & $6 / 23 / 96$ & 413 & 2.3 & $50 \%$ & $50 \%$ & $6 / 24 / 96$ & 37413 \\
\hline $6 C-96-32$ & $\mathrm{Cl} 106 / 7$ & $6 / 23 / 96$ & 414 & 1.9 & $50 \%$ & $50 \%$ & $6 / 24 / 96$ & 37413 \\
\hline $6 C-96-33$ & $\mathrm{Cl06} / 7$ & $6 / 23 / 96$ & 414 & 1.5 & $50 \%$ & $50 \%$ & $6 / 25 / 96$ & 37415 \\
\hline $6 C-96-34$ & $\mathrm{Cl} 106 / 7$ & $6 / 24 / 96$ & 414 & 1.4 & $50 \%$ & $50 \%$ & $6 / 25 / 96$ & 37415 \\
\hline $6 C-96-35$ & $\mathrm{Cl} 106 / 7$ & $6 / 24 / 96$ & 417 & 1.5 & $50 \%$ & $50 \%$ & $6 / 25 / 96$ & 37416 \\
\hline $6 C-96-36$ & $\mathrm{Cl} 06 / 7$ & $6 / 24 / 96$ & 414 & 1.7 & $50 \%$ & $50 \%$ & $6 / 26 / 96$ & 37417 \\
\hline $6 C \cdot 96-37$ & $C 106 / 7$ & $6 / 24 / 96$ & 414 & 2.0 & $50 \%$ & $50 \%$ & $6 / 26 / 96$ & 37417 \\
\hline
\end{tabular}

'Reference: Process Memo, RG Brown to KD Wiemers, Request for Sludge Sample From Tank 241-C-106. June 17, 1996.

The remainder of the test material was subjected to an "enhanced" sludge washing test; two caustic and three inhibited water washes. The first caustic leach step consisted of mixing the sludge $/ \mathrm{NaOH}$ mixture (slumy contained 3.3 weight percent solids and the final free hydroxide concentration was $2.6 \mathrm{M}$ ) for 5 hours at $100^{\circ} \mathrm{C}$, allowing the mixture to cool to ambient temperature, allowing the sludge 
solids to settle by gravity, then decanting the liquid. The second caustic leach was done in a similar manner, but the slurry contained 3.9 weight percent solids and the final free hydroxide concentration was $3.0 \mathrm{M}$. The leached solids were washed three successive times with inhibited water (i.e., $0.01 \mathrm{M} \mathrm{NaOH} / 0.01 \mathrm{M} \mathrm{NaNO}_{2}$ ); these washes consisted of mixing the sludge/wash mixture for 0.5 hours at ambient temperature, allowing the solids to settle by gravity, then removing the wash liquor. The leached solids were dried to constant weight at $105^{\circ} \mathrm{C}$ and analyzed.

The laboratory-scale washing and caustic leaching tests performed on C-106 sludge material are detailed in report PNNL-11381 (Lumetta 1996). Although no analytical results associated with the laboratory-scale testing are presented in this report, the results from this testing were used (as available) to establish the sludge washing treatment for the bench-scale batch preparation.

\subsection{Bench-Scale Vendor Product Batch Preparation}

After removing the material for the laboratory-scale testing, all of the remaining composited C-106 material was transferred to the 324 building for Envelope D final batch processing (Enhanced Sludge Washing). Batch processing followed Test Plan Number TWRS-95-3.1A which was modified for C-106 batch preparation based upon performance of the laboratory-scale screening test. Approximately 3000 grams of C-106 Initial Composite Material was blended with approximately 2000 grams of deionized water prior to initiating the enhanced sludge washing final batch processing. At this stage, four subsamples identified as Original Batch Material (hereafter referred to as Original Material unless otherwise noted; see Table 2.2) were extracted from the process and sent to the 325 building for physical and chemical characterization. A fifth subsample was collected for archive.

As with the laboratory-scale testing, two caustic leaches and 3 inhibited water washes were performed. Each leach or wash step was followed by gravity settling and supernatant removal to separate the liquid and solid phases. The first caustic leach added $10 \mathrm{M} \mathrm{NaOH}$ and deionized water to the sludge and obtained a $2.74 \mathrm{M}$ free hydroxide solution with 5.7 weight percent caustic-insoluble solids. The slurry was mixed at $100^{\circ} \mathrm{C}$ for 5 hours and allowed to settle at $85^{\circ} \mathrm{C}$. The supernatant was then decanted in preparation for the second caustic leach. The second caustic leach utilized $3 \mathrm{M} \mathrm{NaOH}$ to obtain 5.8 weight percent caustic-insoluble solids with $3.22 \mathrm{M}$ free hydroxide; the same mixing and settling temperatures were used as in the first caustic leach. Each of the subsequent three water washes utilized inhibited water (i.e., $0.01 \mathrm{M} \mathrm{NaOH} 0.01 \mathrm{M} \mathrm{NaNO}$ ) added to obtain approximately 6 weight percent solids concentration. For each wash, the sludge was mixed, settled at ambient temperature, and the supernatant decanted. The bench-scale processing tasks, procedures, and data evaluation are presented in report PNNL-1 1432 (Brooks 1997).

Following final batch processing, the resulting material (Vendor Product) was thoroughly mixed and five subsamples were collected for subsequent physical and chemical characterization and archive. The four Vendor Product subsamples and all additional pumpable Vendor Product material (two $1 \mathrm{~L}$ containers) were collected and delivered to the 325 building (See Table 2.2); the remaining subsample was archived in the 324 building. Besides the Vendor Product material, the remaining Initial Composite Material (approximately $800 \mathrm{~g}$ for archiving) and samples of the supernatants from each caustic leach and water wash (for analysis) were transferred to the 325 building. 
HNF-SD-WM-DP-225, REV. 1

Analytical Summary Report - January 28, 1997

Table 2.2 C-106 Material Transfer from Building 324 to 325

\begin{tabular}{|c|c|c|}
\hline 324 Sa mple ID & Net Weight (g) & Description \\
\hline $\begin{array}{l}\text { SPDL1-C106-003/005 } \\
\text { and SPDL1-C106-002 }\end{array}$ & 507 & Original "Batch" Material (2) \\
\hline SPD1-C106-010 & 16.4 & Supematant from First Caustic Leach \\
\hline SPD 1-C106-015 & 22.8 & Supematant from Second Caustic Leach \\
\hline SPD I-C106-020 & 22.4 & Supernatant from First Water Wash \\
\hline SPD1-C106-024 & 13.0 & Supernatant from Second Water Wash \\
\hline SPD1-C106-029 & 23.2 & Supematant from Third Water Wash \\
\hline SPD1-C106-046 & 90.7 & $\begin{array}{l}\text { Vendor Product } \\
\text { (Leached \& Washed Sludge) }\end{array}$ \\
\hline SPD 1-C106.047 & 70.3 & $\begin{array}{l}\text { Vendor Product } \\
\text { (Leached \& Washed Sludge) }\end{array}$ \\
\hline SPD1-C106-048 & 84.7 & $\begin{array}{l}\text { Vendor Product } \\
\text { (Leached \& Washed Sludge) }\end{array}$ \\
\hline SPD1-C106-049 & 62.8 & $\begin{array}{l}\text { Vendor Product } \\
\text { (Leached \& Washed Sludge) }\end{array}$ \\
\hline C-106 & 800 & Initial Composite Material ${ }^{(b)}$ \\
\hline SPDI-C106-05! & 1624 & $\begin{array}{l}\text { Vendor Product } \\
\text { (Leached \& Washed Sludge) }\end{array}$ \\
\hline SPD 1-C106-052 & 1523 & $\begin{array}{l}\text { Vendor Product } \\
\text { (Leached \& Washed Sludge) }\end{array}$ \\
\hline
\end{tabular}

(a) Original Batch Material: Initial Composite Material and Water mixed at 3:2 ratio to provide initial pumpable material for bench-scale testing.

(b) Initial Composite Material: Composited material from twenty C-106 grab samiples; nö additional water added. 


\subsection{Chemical, Radiochemical, and Physical Characterization}

Table 2.3 provides a summary list of all the samples processed and analyzed per revision 0 of the LOI. Additional details of the characterization schemes are provided in Section 3.

Table 2.3 Sample Laboratory Numbers

\begin{tabular}{|c|c|c|}
\hline $\begin{array}{l}\mathrm{ACL} \text { Lab } \\
\text { Number }\end{array}$ & Sample ID & Material Description and Purpose \\
\hline $96-5892$ & SPDL1-C106-003 & Original Batch Material - Chemical Analysis \\
\hline $96-5893$ & SPDL1-C106-004 & Original Batch Material - Chemical Analysis \\
\hline $96-5894$ & SPDL1-C106-005 & Original Batch Material - Chemical Analysis \\
\hline $96-5945$ & SPDL1-C106-002 & Original Batch Material - Physical Testing \\
\hline $96-5946$ & $\mathrm{C} 106-002-\mathrm{CS}$ & Centrifuged Solids from SPDL1-C106-002 - Chemical Analysis \\
\hline $96-5947$ & C106-002-DL & Decant Liquid from SPDL1-C106-002 - Chemical Analysis \\
\hline $96-6103$ & SPD 1-C106-047 & Vendor Product Material - Chemical Analysis \\
\hline $96-6104$ & SPD1-C106-048 & Vendor Product Material - Chemical Analysis \\
\hline $96-6105$ & SPD 1-C106-049 & Vendor Product Material - Chemical Analysis \\
\hline $96-6396$ & SPD1-C106-046 & Vendor Product Material - Physical Testing \\
\hline $96-6653$ & SPD1-C106-046 & Vendor Product Material - Additional Physical Testing \\
\hline $96-6654$ & C106-046-DL & $\begin{array}{l}\text { Decant Liquid from SPDI-C106-046 (96-6653) - Chemical } \\
\text { Analysis }\end{array}$ \\
\hline $97-0108$ & SPD1-C106-046 & $\begin{array}{l}\text { Vendor Product Material - Physical Testing } \\
\text { (Wt\% Solids/Oxide) }\end{array}$ \\
\hline $97-0109$ & SPD1-C106-002 & Original Batch Material - Physical Testing (Wt\% Solids/Oxide) \\
\hline
\end{tabular}




\subsection{SAMPLE PREPARATION}

Four subsamples were collected by the Chemical Separations \& Batch Processing Group in the 324 building for physical and chemical analyses from both the C-106 Original Material and from the bench-scale batch preparation of the C-106 Vendor Product. Three subsamples each from the Original Material and Vendor Product were distributed to the SAL for drying and subsequent analytical sample preparation and/or direct distribution for chemical analysis. The remaining subsamples of the Original Material and Vendor Product were distributed to the HLRF for density, weight loss on drying and firing measurements, and additional physical testing.

\subsection{Preparations for Chemical Analvses}

Vendor Product (See Figure 3.1): The SAL received three containers of Vendor Product for chemical analysis. Each of the three Vendor Product slurries were placed into glass beakers for mixing and drying. The mixed slurries were dried in a oven at $105^{\circ} \mathrm{C}$ until successive weighings yielded less than i percent weight loss between successive dryings. The observed volume reduction of the Vendor Product slurries was approximately 80 percent after drying. In all cases, the remaining dried solids were medium brown in color and the evaporated supernatant left a white coating on the beaker sides and on top of the dried brown solid layer. No indication of spattering, foaming, etc. during drying was noted as evidenced by clean beaker walls above the original fill lines. After drying, each of the three dried slurries were homogenized, and then divided into two halves; forming a sample and a sample duplicate. Homogenization was accomplished by mechanically stirring the beaker contents with a metallic spatula until the contents of each beaker appeared uniform. Analytical sample preparations were conducted on the Vendor Product dried slurries. In addition, analytical sample preparation of a decant supernatant was performed. This decant liquid sample (96-6654) was generated during physical characterization conducted in the HLRF.

Original Material (See Figure 3.2): The SAL received three containers of Original Material for chemical analysis. Each of the three Original Material slurries were placed into glass beakers for mixing and drying. Within minutes after transferring the slurries to beakers, a clear liquid layer was observed forming on top of each slurry material. The layer was suspected to be organic in nature, because the layer separated rapidly, was distinct from the slurry, and coated the beaker walls when swirled. Due to the concern with drying any material having an organic top layer, the suspected layer was removed from the slurries by pipet and placed back into the original containers.

[Note: For each of samples, the clear layer was estimated to be about $10 \%$ of the total volume of the samples (i.e., $10 \mathrm{ml}$ out of $100 \mathrm{ml}$ ), based on the graduates on the beakers.

Approximately 8 to $9 \mathrm{ml}$ of the clear layer was removed; the additional 1 to $2 \mathrm{ml}$ could not be removed from the samples without removing solids. The specific gravity of the clear layer was determined to be $1.06 \mathrm{~g} / \mathrm{mL}$, and investigative organic and radchem screening tests were performed. The major organic components identified were bis(2-ethylhexyl)phosphate and oxalate; organics such as EDTA, HEDTA, TBP and HPH were not observed. This layering was not observed in the preparation of material from the laboratory-scale testing, which may be attributed to the additional water added prior to bench-scale processing. A report detailing the results of the organic and radiochemical screening is provided in a separate letter report (Urie 1996). Based on limited analysis of the remaining supernatant and the volume percent 


\section{HNF-SD-WM-DP-225, REV. 1}

Analytical Summary Report - January 28, 1997

solids, the removal of the 8 to $9 \mathrm{~m}$ l of clear layer is estimated to lower the concentration of the soluble analytes by about $20 \%$, and have no effect on the concentration of the insoluble solids analytes.]

Figure 3.1 Vendor Product Sample Preparation
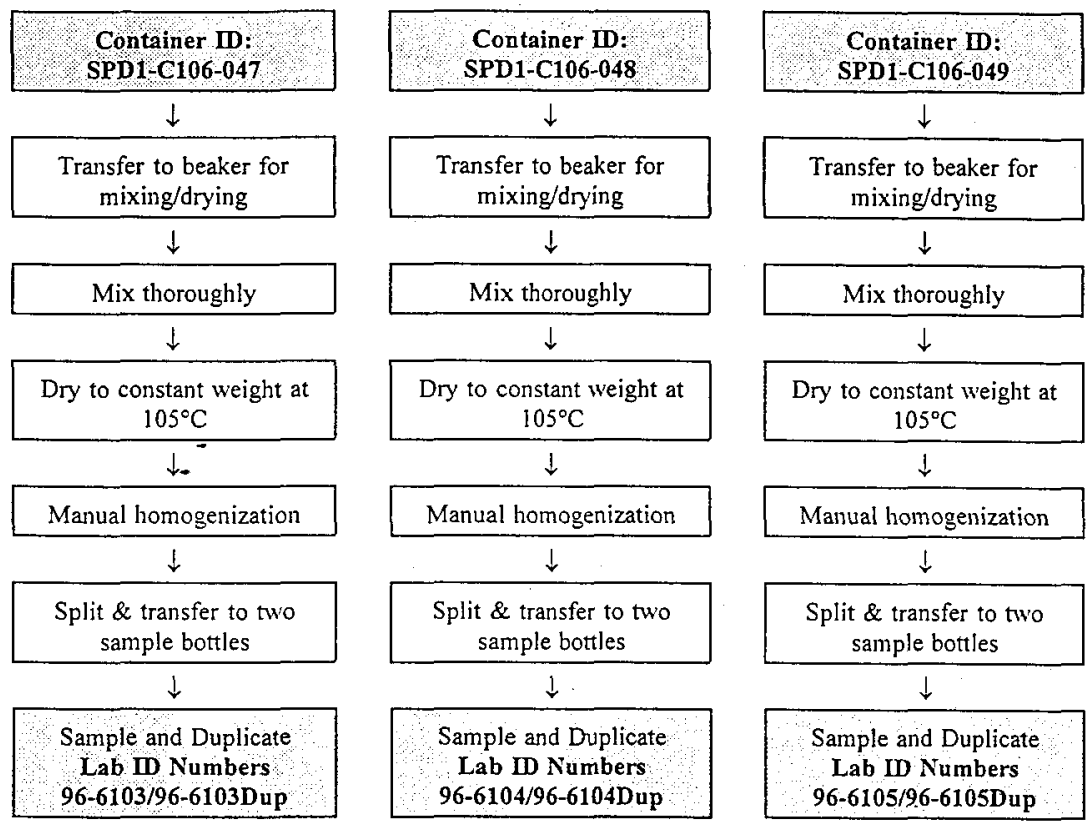
Figure 3.2 Original Material Sample Preparation
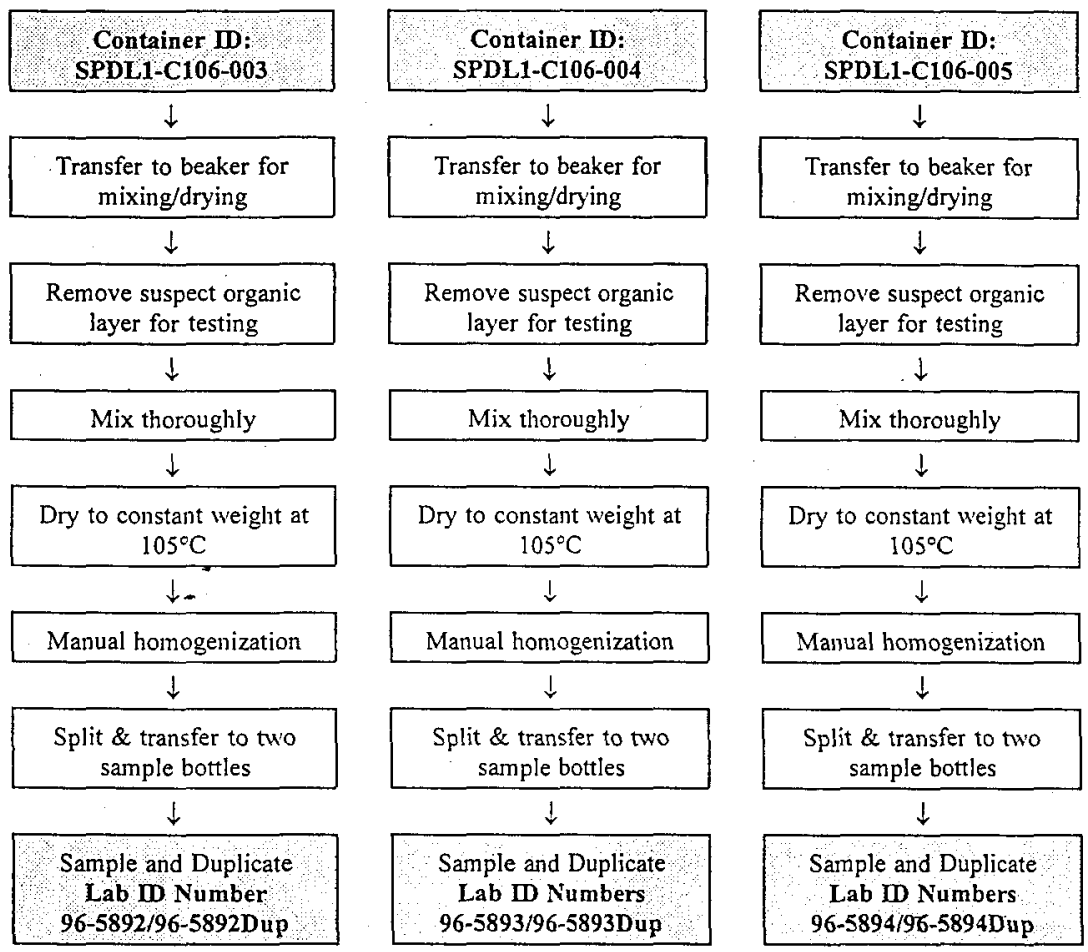

The remainders of the Original Material slurries were dried in a oven at $105^{\circ} \mathrm{C}$ until successive weighings yielded less than 1 percent weight loss between successive dryings. The observed volume reduction of the Original Material slurries was approximately $50 \%$ after drying; however, this result does not include correction for the clear layer removed prior to drying. In all cases, the remaining dried solids were medium brown in color and the evaporated supernatant left a white coating on the beaker sides and on top of the dried brown solid layer. No indication of spattering, foaming, etc. during drying was noted as evidenced by clean beaker walls above the original fill lines. After drying, each of the three dried slurries were homogenized, and then divided into two halves; forming a sample and a sample duplicate. Homogenization was accomplished by mechanically stirring the beaker 
contents with a metallic spatula until the contents of each beaker appeared uniform. Analytical sample preparations were conducted on the Original Material dried slurries, as well as a centrifuged solid and decanted supernatant. The centrifuged solid (96-5946) and decanted liquid (96-5947) samples were generated during physical characterization conducted in the HLRF.

Original Material and Vendor Product: Sample preparations conducted in the SAL included potassium hydroxide fusion utilizing nickel crucibles, sodium peroxide-sodium chloride fusion utilizing zirconium crucibles, nitric acid-hydrochloric acid digestion, and water leaching. All samples were prepared for analysis in duplicate from the dried sluries, based on the subsampling discussed above. Direct analyses were performed in duplicate wherever practical. All results are reported in $\mu \mathrm{g} / \mathrm{g}$ or $\mu \mathrm{Ci} / \mathrm{g}$ of dried solids. Quality control (QC) was applied to analytical batch processing techniques in accordance with the governing quality assurance plan (Kuhl-Klinger 1996). Full QC incorporates the use of preparation blanks, spiked blanks (or laboratory control standards), duplicate samples, matrix spiked samples, and radiochemical tracers, where available and appropriate for use. Figure 3.3 (Vendor Product) and Figure 3.4 (Original Material) identify each of the analytical preparations performed and show the analyses performed on each preparation.

\subsubsection{Acid Digestion Preparation}

Each of the dried samples and sample duplicates were acid digested by method PNL-ALO-129 " $\mathrm{HNO}_{3}-\mathrm{HCl}$ Acid Extraction of Solids Using a Dry-Block Heater," and distributed for ICP-AES, ICP-MS and Radiochemical analyses. Generally, one-half gram samples are digested in about $25 \%$ Nitric/Hydrochlorie acid, and then diluted to a $25 \mathrm{~mL}$ final volume, producing approximately 50 fold analytical processing factors. Full QC, including matrix spikes, was applied with this technique.

\subsubsection{Potassium Hydroxide and Sodium Peroxide Fusion Preparations}

Two fusion techniques, PNL-ALO-115 "Solubilization of Metals from Solids Using $\mathrm{KOH}-\mathrm{KNO}_{3}$ Fusion" and PNL-ALO-116 "Solubilization of Platinum Group Metals using a $\mathrm{Na}_{2} \mathrm{O}_{2}-\mathrm{NaCl}_{\text {Fusion," }}$ were performed and aliquots distributed for ICP-AES, ICP-MS and Radiochemical analyses. The fusion techniques are usually applied where total solubilization of the sample material is required. Typically, one hundred milligram samples are subjected to molten salt and the resulting mixture dissolved with nitric/hydrochloric acid. The final volume for this preparation is $100 \mathrm{~mL}$, thus creating 1000 fold analytical processing factors. The QC is limited to sample duplicates, a preparation blank, and post fusion spiking (i.e., spiking being performed during analysis) for selective analytes.

\subsubsection{Water Leaching Preparation}

Each dried sample and sample duplicate were water leached (PNL-ALO-103 "Water Leach of Sludges, Soils and Other Solid Samples"), filtered, and aliquots distributed for anion analysis by Ion Chromatography (IC) and ammonia determination by Ion Selective Electrode (ISE). The water leaching technique produces a 100 fold analytical processing factor. Full QC was applied for all analytes of interest. Oxalate was noted by IC during analysis of the Vendor Product and Original Material, but is not defined as an analyte of interest in the LOI. Since oxalate was not an analyte of interest, no matrix spiking was performed as per the goveming QA plan requirements. 
HNF-SD-WM-DP-225, REV. 1

Analytical Summary Report - January 28, 1997

Figure 3.3 Vendor Product Preparations and Analyses

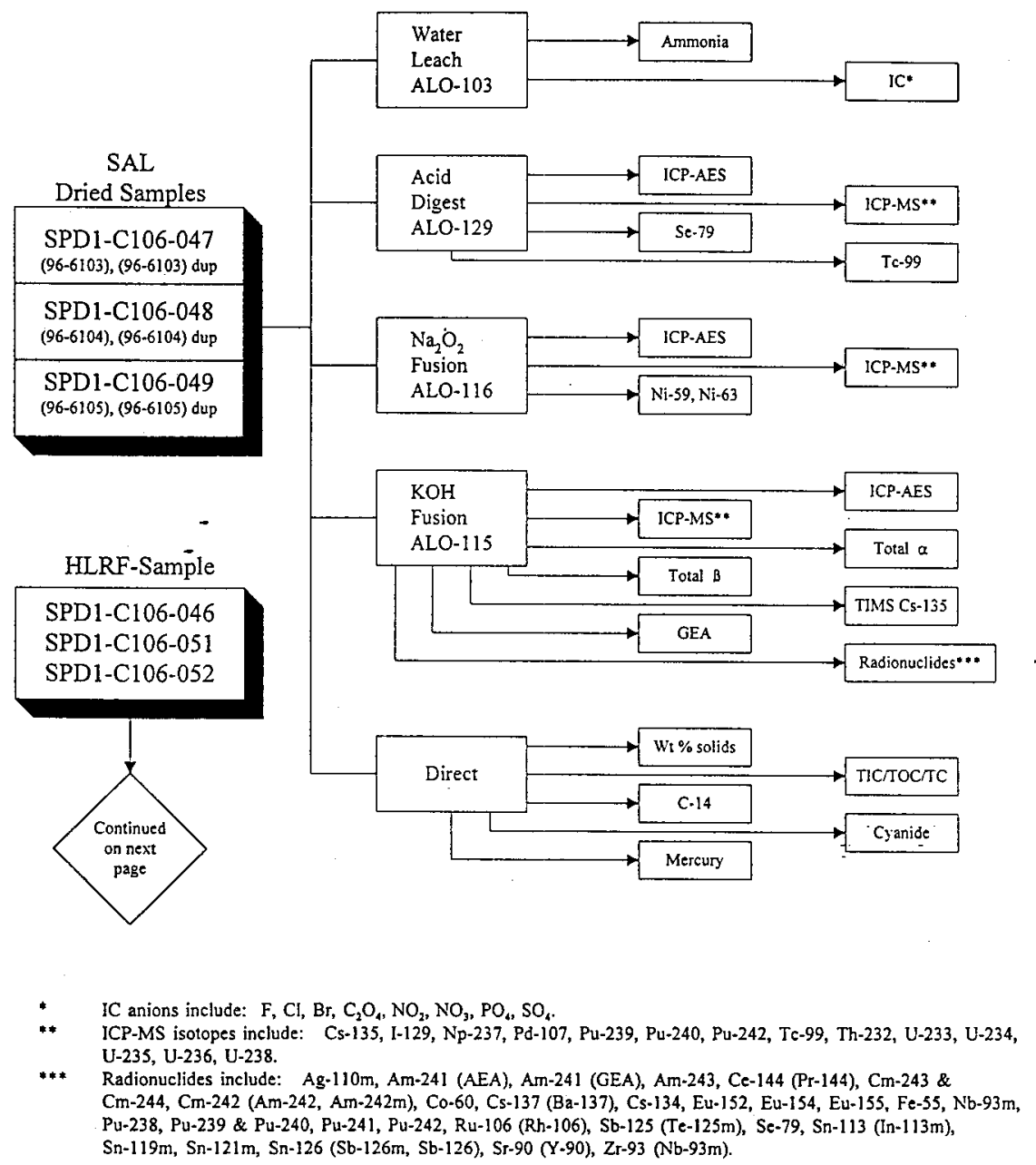


Figure 3.3 Vendor Product Preparations and Analyses (Cont.)

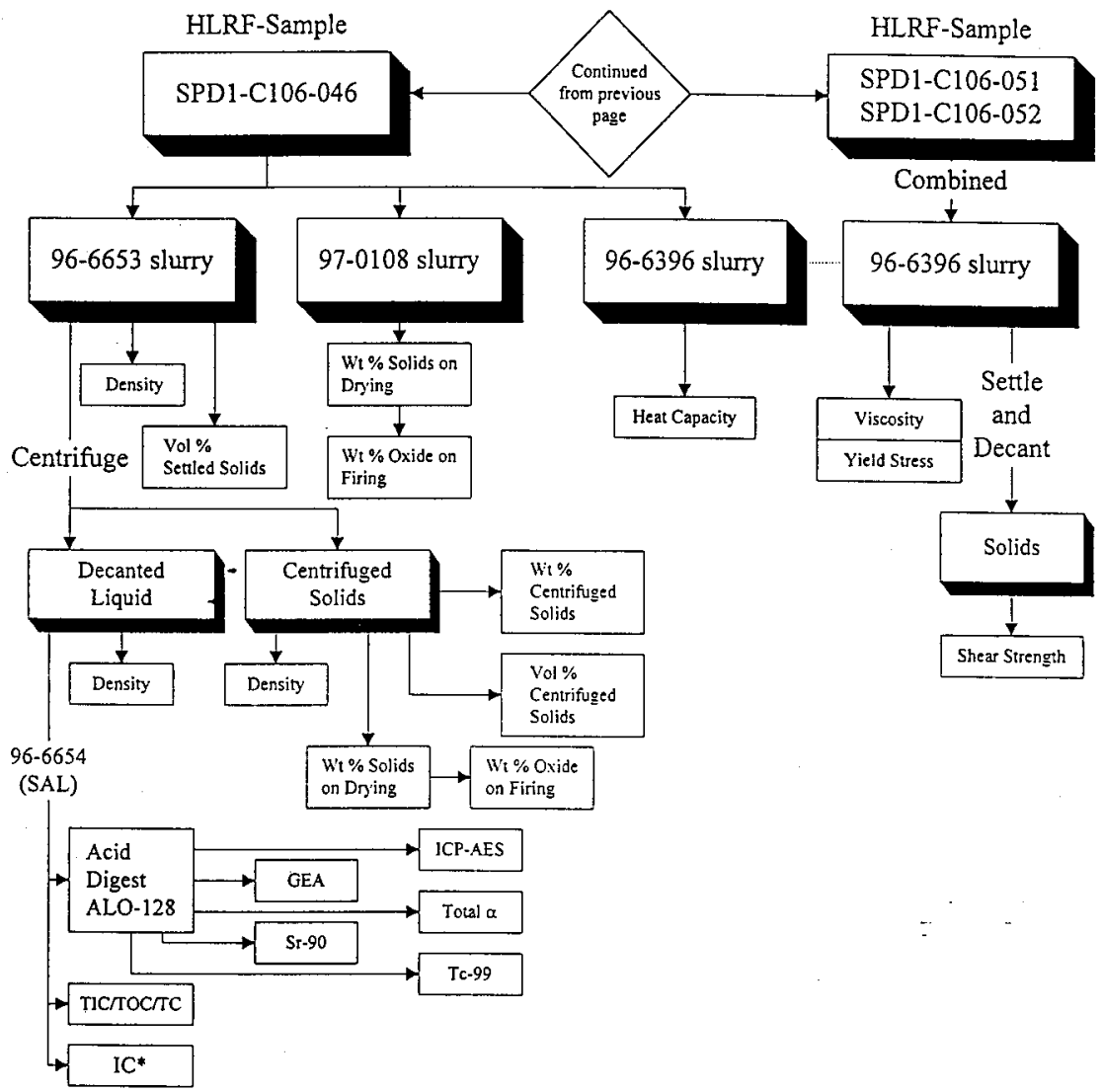

* IC anions include: $\mathrm{F}, \mathrm{Cl}, \mathrm{Br}, \mathrm{C}_{2} \mathrm{O}_{4}, \mathrm{NO}_{2}, \mathrm{NO}_{3}, \mathrm{PO}_{4}, \mathrm{SO}_{4}$. 
HNF-SD-WM-DP-225, REV. 1

Analytical Summary Report - January 28, 1997

\section{Figure 3.4 Original Material Preparations and Analyses}

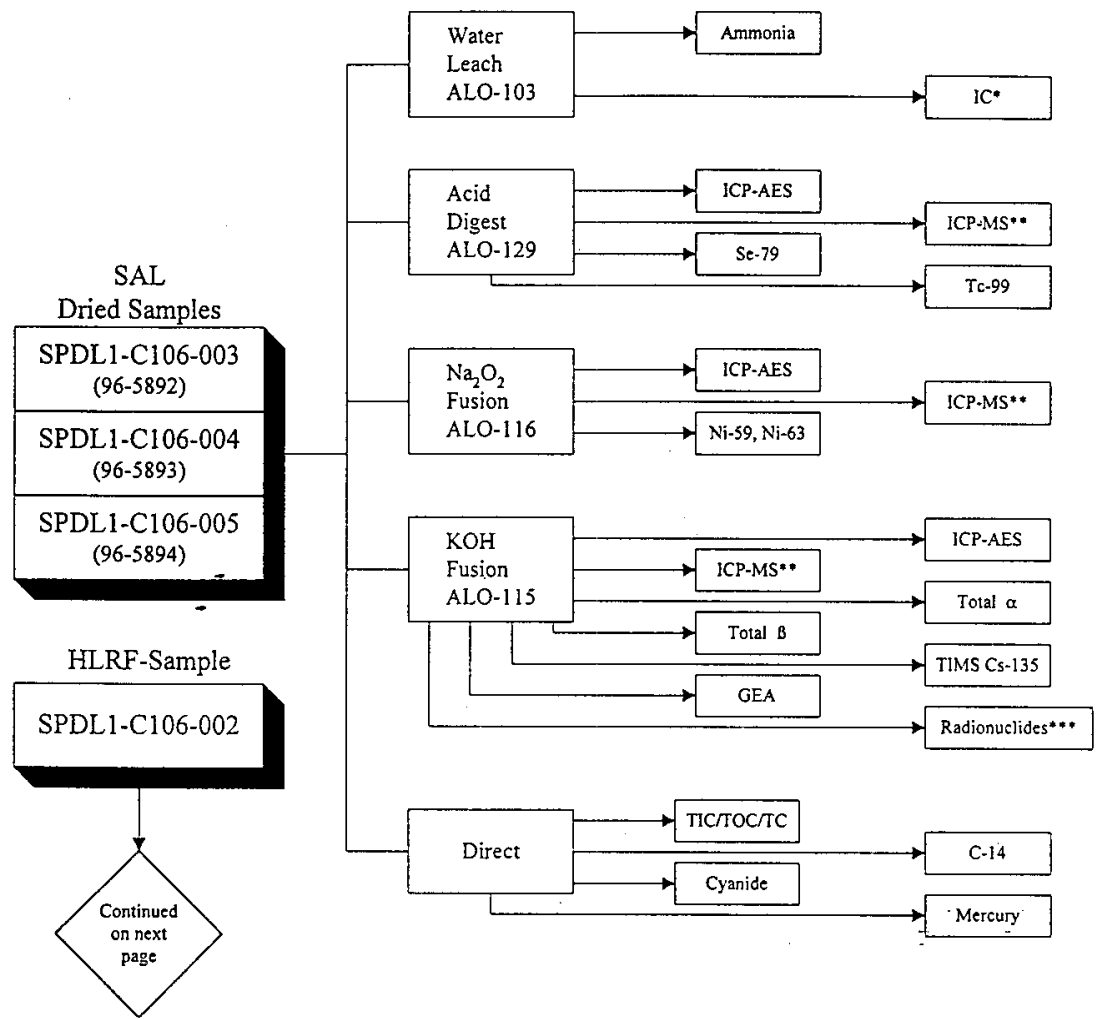

- IC anions include: $\mathrm{F}, \mathrm{Cl}, \mathrm{Br}, \mathrm{C}_{2} \mathrm{O}_{4}, \mathrm{NO}_{2}, \mathrm{NO}_{3}, \mathrm{PO}_{4}, \mathrm{SO}_{4}$.

** ICP-MS isotopes include: Cs-135, I-129, Np-237, Pd-107, Pu-239, Pu-240, Pu-242, Tc-99, Th-232, U-233, U-234, U-235, U-236, U-238.

** Radionuclides include: Ag-110m, Am-241 (AEA), Am-241 (GEA), Am-243, Ce-144 (Pr-144), Cm-243 \& Cm-244, Cm-242 (Am-242, Am-242m), Co-60, Cs-137 (Ba-137), Cs-134, Eu-152, Eu-154, Eu-155, Fe-55, Nb-93m, Pu-238, Pu-239 \& Pu-240, Pu-241, Pu-242, Ru-106 (Rh-106), Sb-125 (Te-125m), Se-79, Sn-113 (In-113m), Sn-119m, Sn-121m, Sn-126 (Sb-126m, Sb-126), Sr-90 (Y-90), Zr-93 (Nb-93m). 
HNF-SD-WM-DP-225, REV. 1

Analytical Summary Report - January 28, 1997

Figure 3.4 Original Material Preparations and Analyses (Cont.)

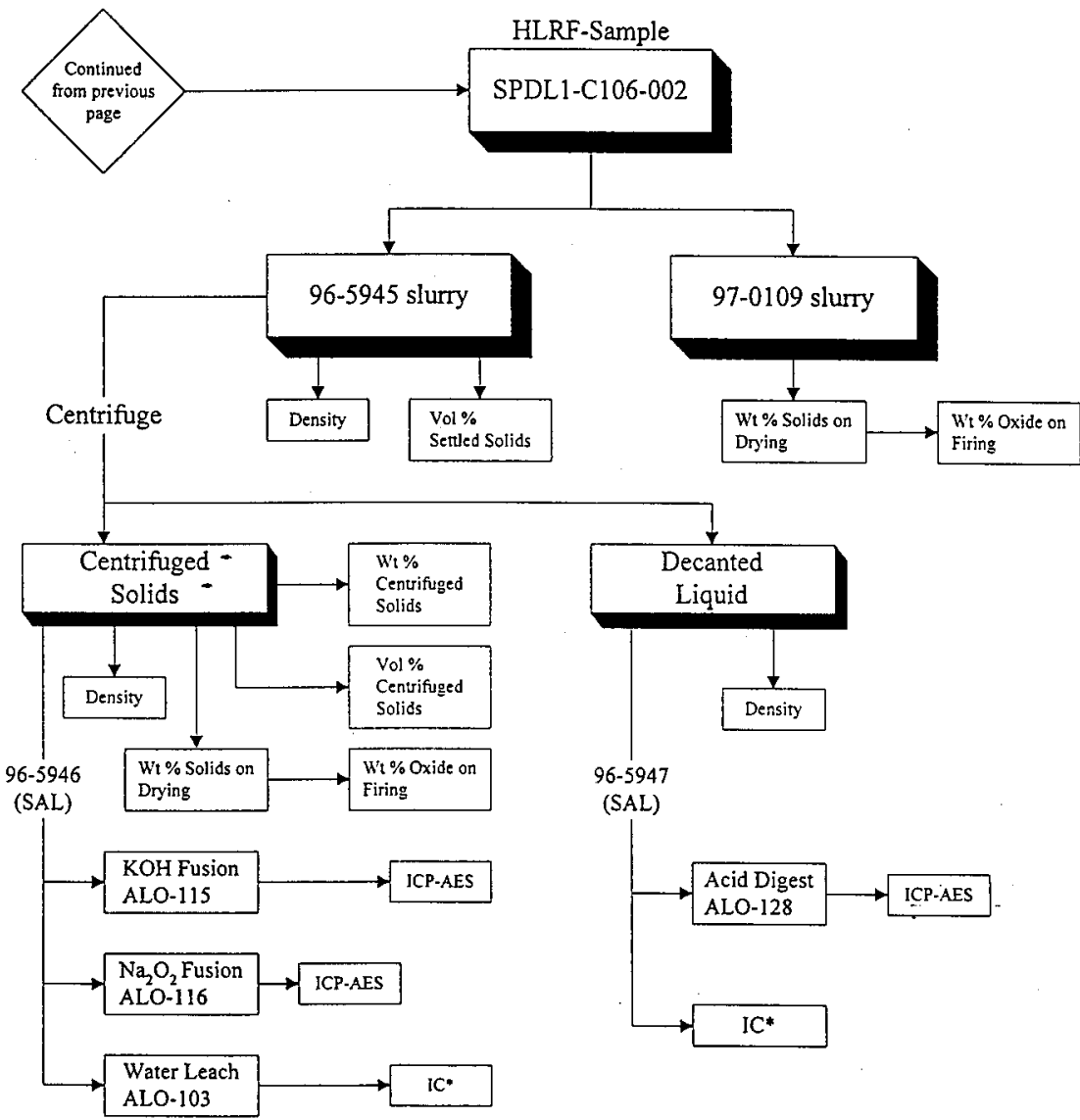

* IC anions include: $\mathrm{F}, \mathrm{Cl}, \mathrm{Br}, \mathrm{C}_{2} \mathrm{O}_{4}, \mathrm{NO}_{2}, \mathrm{NO}_{3}, \mathrm{PO}_{4}, \mathrm{SO}_{4}$ 


\subsection{INORGANIC, RADIOCHEMICAL, AND PHYSICAL ANALYSIS}

The results of the chemical, radionuclide and physical measurement performed on the Vendor Product and Original Material are provided in Tables 4.1 through 4.26. In nearly every case, the analytical results for the sample and duplicate for three samples (either Original Material or Vendor Product), a preparation blank, the average (with standard deviation), and a comparison to the Envelope D specification is reported. The tables are presented in the following order: Physical Measurements, ICP-AES, ICP-MS, Wet Chemistry, Radiochemistry, and Centrifuged Solids/Decant Liquid results. The ICP-AES and ICP-MS results are presented for multiple sample preparations (specifically, potassium bydroxide-potassium nitrate fusion, sodium peroxide-sodium chloride fusion, and nitric/hydrochloric acid digestion). Except where specifically noted, the sample/duplicate results have not been adjusted for the contribution of the preparation blank.

\subsection{Evaluation Vendor Product to Envelope D Specification}

For comparison of the Vendor Product to the Envelope D specification, the result selected is the "most reasonable" averaged result. For many analytes of interest, obtaining this averaged result required selecting a result from either ICP-AES or ICP-MS for one of three different analytical preparations. In selecting the most reasonable result, an effort has been made to evaluate the results based on the limitations of the analytical technique and the preparation method considering quality control performance.

Where the preparation methods are considered equivalent (for this matrix) and the measured value for the analyte is above the EQL, typically the highest reported result is used for comparison to the envelope specification. There are some instances where high preparation blanks may bias the results (e.g., calcium blank in fusion preparations), and in these cases, results from another preparation are selected for comparison. Where the ICP-AES reports results near the EQL, the results from the ICP-MS (which has better sensitivity than ICP-AES) may be selected since the precision is expected to be better. Attempts have been made to select appropriate results for comparison. However, for all analytes the average result from each preparation is compared to the Envelope D specification, and a "greater than specification" flag is provided in the ICP-AES, ICP-MS, Wet Chemistry, and Radiochemistry results tables to indicate when the specification has been exceeded (for the specific method and specific preparation).

\subsubsection{Analytes/Radionuclides/Physical Properties Exceeding Specification}

ICP-AES/ICP-MS (Sodium): As can be seen in the "most reasonable" ICP-AES results from acid digestion, the sodium concentration in the Vendor Product exceeds the Envelope D specification by about five percent. The ICP-MS sodium results from acid digestion is considerably high than the ICP. AES result; exceeding the Envelope D specification by $18 \%$. The ICP-AES sodium results from the $\mathrm{KOH}$ fusion is at approximately $90 \%$ of the specification limit. Based on these analytical results, the Vendor Product can not be verified to be within the specification limit.

ICP-MS (Platinum and ${ }^{233} \mathrm{U}$ ): Based on the ICP-MS results, platinum (KOH fusion only) and ${ }^{233} \mathrm{U}$ (acid digestion only) exceeded the Envelope $\mathrm{D}$ specification by factors of 1.2 and 66, respectively. Platinum is considered a trace component and ${ }^{253} \mathrm{U}$ a trace radionuclide, and the specifications are near 
the EQLMDA. It should be noted that there was a large variation in the platnium results between the two fusion and acid digestion preparations. For platinum, the preparation blank exhibited some contamination.

Radiochemistry $\left({ }^{12 ! m} \mathrm{Sn}\right):$ The measured value of ${ }^{121 \mathrm{~m}} \mathrm{Sn}$ exceeds the specification limit by a factor of 6 .

Physical Measurements: The weight percent solids and total oxide content exceed the LOI specification. The weight percent solids (14.4\%) exceeds the requirement by $10 \%$ and the total oxide $(126.5 \mathrm{~g} / \mathrm{L}$ based on a slurry density of $1.10 \mathrm{~g} / \mathrm{ml})$ exceeds the LOI requirement by $26 \%$. Also, the viscosity is below the lower limit at low shear rates. Compliance with these specifications was not a requirement for shipment of the Vendor Product material to the vendors.

\subsubsection{Radionuclides/Analytes/Properties Unable to be Evaluated Against Specification}

${ }^{110 \mathrm{~m}} \mathrm{Ag},{ }^{242} \mathrm{Pu},{ }^{119 \mathrm{~m}} \mathrm{Sn}:$ There are three cases where no activity was measured; however, the MDA (Minimum Detectable Activity) value exceeded the Envelope D limit; ${ }^{110 m} \mathrm{Ag}$ by a factor of $349,{ }^{242} \mathrm{Pu}$ by a factor of 10.9 , and ${ }^{119 \mathrm{~m}} \mathrm{Sn}$ by a factor of 155 .

${ }^{243} \mathrm{Cm} .{ }^{144 \mathrm{~m}} \mathrm{Pr}$ : Since ${ }^{243} \mathrm{Cm}$ can not be resolved from ${ }^{244} \mathrm{Cm}$ (total activity of $3.13 \mathrm{E}-07 \mathrm{Ci} / \mathrm{L}$ ) and ${ }^{144 \mathrm{~m}} \mathrm{Pr}$ can not be resolved from ${ }^{144} \mathrm{Ce} /{ }^{144} \mathrm{Pr}$ (MDA value of $<7.75 \mathrm{E}-5 \mathrm{Ci} / \mathrm{L}$ ), there exists some possibility that these radionuclides-exceed the Envelope D specification. No estimate of the actual activities (other than that generated from ORIGEN) for these radionuclides can be made at this time.

${ }^{113 \mathrm{~m} C d}$ : Although a method has been proposed, there is currently no approved PNNL method for determining this radionuclide. Therefore, per the Addendum 1 LOI Rev 0 (Jones 1996) no results are reported for this radionuclide. The ${ }^{113 \mathrm{~m}} \mathrm{Cd}$ (half-life of 45 days) is expected to make up less than $0.0002 \%$ of the total activity.

${ }^{3}$ H: Per the Addendum I LOI Rev 0 (Jones 1996) no attempt was made to measure tritium on the dried solids. Tritium (half-life of 12 years) is expected to make up less than $0.0002 \%$ of the total activity.

Sulfur: There is currently no PNNL analytical method for this analyte; therefore, no results are available.

Shear Strength: The shear strength is below detection limit, and the detection limit is above the specification. 


\subsubsection{Radionuclides/Analytes within $50 \%$ of the Specification}

Five analytes have concentrations exceeding $50 \%$ of the Envelope D specification, and three radionuclides have MDA level exceeding $50 \%$ of the specification. These analytes/radionuclides are silver, erbium, iron, thorium, TOC, ${ }^{144} \mathrm{Ce}\left({ }^{144} \mathrm{Pr} /{ }^{144 m} \mathrm{Pr}\right),{ }^{152} \mathrm{Eu}$, and ${ }^{106} \mathrm{Ru}\left({ }^{106} \mathrm{Rh}\right)$. Except for iron and TOC results, the reported concentrations or MDA values may not be sufficiently reliable as to validate that the analyte/radionuclide is not be present above the specification limit. However, the reported concentrations/MDAs are the "most reasonable" values available.

\subsection{Physical Measurements - Vendor Product and Original Material}

With the exception of SAL bulk weight percent solids determination on Vendor Product, all physical testing was performed by the Process Chemistry Group. Physical measurements on both the Original Material and Vendor Product samples included volume percent (Vol\%) settled solids, volume percent centrifuged solids, weight percent (Wt\%) centrifuged solids, slurry density (Original and Vendor), centrifuged solids density, centrifuged supernatant density, weight percent solids on drying and weight percent oxides on firing (Original Material, Vendor Product, and both centrifuged solids), and apparent viscosity as a function of shear rate, yield stress, settled solids shear strength after 2 days, and heat capacity. All analyses were performed in duplicate except for the Original Material and Vendor Product densities which were performed in quadruplicate. The bulk weight percent solids measured in the SAL are presented in Table 4.1 and the physical measurement (percent solids, percent oxides, and density) results from the HLRF are presented in Table 4.2. It is worth noting that there is good agreement between the Vendor Product weight percent solids (on drying) obtained in the HLRF and those obtained in the SAL.

Bulk Weight Percent Solids (Vendor Product Only): Table 4.1 presents the weight percent solids determinations performed on the Vendor Product subsample slurries by SAL at the time of drying the samples for subsequent chemical and radiochemical analysis. Weight percent solids were determined on each of the three Vendor Product subsamples prior to any analytical preparations. Since each sample was taken in its entirety for drying, only one weight percent solids determination could be made for each subsample. Weight percent solids determinations were not performed in the SAL on the Original Material subsample slurries.

Table 4.1 Vendor Product Bulk Weight Percent Solids Measurements

\begin{tabular}{|r|r|c|}
\hline ACL Number & 324 Identifier & Weight Percent Solids \\
\hline $96-06103$ & SPDl-C106-047 & 13.9 \\
\hline $96-06104$ & SPD1-Cl06-048 & 16.9 \\
\hline $96-06105$ & SPD1-Cl06-049 & 13.7 \\
\hline & Average Butk Wt\% Solids & 14.8 \\
\hline & Standard Deviation & 1.8 \\
\hline
\end{tabular}


HNF-SD-WM-DP-225, REV. 1

Analytical Summary Report - January 28, 1997

Table 4.2. Physical Mearusements - Vendor Product and Original Material

\begin{tabular}{|c|c|c|c|c|c|}
\hline Vendor Product Test & $\begin{array}{l}\mathrm{ACL} \\
\mathrm{Lab} \#\end{array}$ & Sample & Duplicate & Average & RPD \\
\hline Vol\% Settled Solids & $96-6653$ & 17.5 & 19.5 & 18.5 & 10.8 \\
\hline Vol\% Centrifuged Solids & $96-6653$ & 12.9 & 11.9 & 12.4 & 8.1 \\
\hline Wt\% Centrifuged Solids & $96-6653$ & 20.3 & 19.5 & 19.9 & 4.0 \\
\hline Density $(g / m)$ Slurry Composite & $96-6653$ & $\begin{array}{l}1.10 \& \\
1.09\end{array}$ & $\begin{array}{c}1.10 \& \\
1.10\end{array}$ & 1.10 & $0.4^{(1)}$ \\
\hline Density ( $g / \mathrm{ml})$ Centrifuged Solids & $96-6653$ & 1.72 & 1.81 & 1.77 & 5.1 \\
\hline Density $(\mathrm{g} / \mathrm{ml})$ Centrifuged Liquid & $96-6653$ & 0.99 & 1.00 & 1.00 & 0.6 \\
\hline Wt\% Solids on Drying Slurry Composite & $97-0108$ & 14.2 & 14.6 & 14.4 & 2.5 \\
\hline Wt\% Oxides on Firing Slurry Composite & $97-0108$ & 11.3 & 11.5 & 11.4 & 1.7 \\
\hline Wt\% Solids on Drying Centrifuged Solids & $96-6653$ & 58.3 & 58.6 & 58.5 & 0.6 \\
\hline Wt\% Oxides on Firing Centifuged Solids & $96-6653$ & 49.5 & 50.0 & 49.8 & 1.0 \\
\hline Original Material Test & $\mathrm{ACL}$ & Sample & Duplicate & Average & RPD \\
\hline Vol\% Settled Solids & $96-5945$ & 40.5 & 43.0 & 41.8 & 6.0 \\
\hline Vol\% Centrifuged Solids & $96-5945$ & 31.6 & 30.9 & 31.3 & 2.2 \\
\hline Wt $\%$ Centrifuged Solids & $96-5945$ & 43.1 & 43.6 & 43.4 & 1.2 \\
\hline Density $(\mathrm{g} / \mathrm{ml})$ Slurry Composite & 95.5945 & $\begin{array}{c}1.25 \& \\
1.26\end{array}$ & $\begin{array}{c}1.24 \& \\
1.25\end{array}$ & 1.25 & $0.3^{(1)}$ \\
\hline Density $(\mathrm{g} / \mathrm{ml})$ Centrifuged Solids & $96-5945$ & 1.71 & 1.76 & 1.74 & 2.9 \\
\hline Density $(\mathrm{g} / \mathrm{ml})$ Centrifuged Liquid & $96 \cdot 5945$ & 1.07 & 1.06 & 1.07 & 0.9 \\
\hline Wt\% Solids on Drying Slurry Composite & $97-0109$ & 28.8 & 29.6 & 29.2 & 2.9 \\
\hline Wt\% Oxides on Firing Slurry Composite & $97-0109$ & 21.6 & 22.1 & 21.9 & 2.1 \\
\hline Wt $\%$ Solids on Drying Centrifuged Soljds & $96-5945$ & 51.7 & 51.7 & 51.7 & 0.1 \\
\hline Wt\% Oxides on Firing Centifuged Solids & $96-5945$ & 33.7 & 34.9 & 34.3 & 3.5 \\
\hline
\end{tabular}

(1)Densities analyzed in quadruplicate; relative standard deviation provided instead of RPD. 
Vol\% Settled Solids. Vol\% Centrifuged Solids. Wt \% Centrifuged Solids: The volume percent (vol\%) settled solids, volume percent (vol\%) centrifuged solids, and the weight percent (wt\%) centrifuged solids of the Vendor Product and Original Material reported in Table 4.2 were measured in duplicate according to technical procedure PNL-ALO-501 (with no deviations). The mass of the samples was measured on a calibrated balance and balance checks were performed during each batch. Volumes were measured using graduated centrifuge cones. These tests were conducted in conjunction with the density measurements for the slurries, centrifuged solids, and centrifuged supernatants.

Subsamples of the Vendor Product and Original Material slurries were centrifuged at high speed in the clinical centrifuge in the HLRF, and the centrifuged supernatant was removed to produce the centrifuged solids samples. At the appropriate times throughout the procedure, the volume percent and weight percent measurements were performed. The data is recorded in Test Instruction C106-2 and the settling of these samples was videotaped.

Densities: The density measurements were performed on the Vendor Product and Original Material slurry samples, as well as all associated centrifuged solids and supernatants, according to teclinical procedure PNL-ALO-501 (with no deviations). Subsamples of the Vendor Product and Original Material slurries were centrifuged at high speed in the clinical centrifuge in the HLRF, and the centrifuged supernatant was removed to produce the centrifuged solids and supernatant samples.

The densities of the Vendor Product and Original Material slurries were measured in quadruplicate, and the densities of the centrifuged solids and supematant were measured in duplicate. The mass of the samples was measured on a calibrated balance and balance checks were performed during each batch. The volume was measured in graduated centrifuge cones. The accuracy of the graduations on these centrifuge cones was measured on a random sampling of this lot of centrifuge cones. The data is recorded in Test Instruction C106-2.

Wt\% Solids \& Wt $\%$ Oxides: Weight loss on drying (to obtain Wt\% Solids) and firing (to obtain Wt\% Oxides) was performed on the Vendor Product and Original Material slurry samples (97-0108 and 97-0109, respectively), as well as the associated centrifuged solids (96-6653 and 96-5945, respectively). Subsamples of the Vendor Product and Original Material slurries were centrifuged at high speed in the clinical centrifuge in the HLRF, and the centrifuged supernatant was removed to produce the centrifuged solid sample.

The weight percent solids and weight percent oxides were measured in duplicate according to technical procedure PNL-ALO-50l (with no deviation). The mass of each sample was measured on a calibrated balance and balance checks were performed during each batch. The data is recorded in Test Instruction C106-2.

The average solids loading for the Vendor Product is 14.4 weight percent, which exceeds the LOI requirement of 2.5 to 13 weight percent. The oxides loading of the Vendor Product based on the measured slurry density of $1.10 \mathrm{~g} / \mathrm{ml}$ is $126.5 \mathrm{~g} / \mathrm{L}$, which is also above the LOI requirement of 25 to $100 \mathrm{~g} / \mathrm{L}$. Compliance with these specifications was not required for shipment of the sample to the vnedor. However, attempts to lower the solids loading during the sodium-reduction treatment of the Vendor Product are detailed in Addendum 1 to this report (Urie 1997). 
Shear Strength: The shear strength was performed on the settled solids resulting from the combination of the two IL Vendor Product containers, SPD1-C106-051 and SPD1-C106-052, because there were insufficient solids in the sample originally assigned for this analysis (SPD1-C106-046). Sufficient quantity of solids from these jars were settled, separated from the overlying liquid, and transferred to a single $125 \mathrm{ml} \mathrm{l}$-Chem jar.

Shear strength, $\tau_{s}$, is a semiquantitative measure of the force required to move the sample. The sample was analyzed for shear strength using a Haake Rotoviscoß viscometer modified for Hot-Cell operation with an MS head and a shear vane manufactured at PNNL. The shear vane had a height of $\mathrm{H}_{\mathrm{v}}=1.588 \mathrm{~cm}$ and a diameter of $\mathrm{D}_{\mathrm{v}}=0.800 \mathrm{~cm}$. The shear strength was then calculated according to Equation 1, where $4.9 \times 10^{5} \mathrm{dyne} c \mathrm{~m}$ is the maximum torque of the M5 head and $\% \tau / 100$ is the ratio of the total torque which is recorded as full scale on the plot of the shear stress. The instrumental detection limit of 1000 dyne/ $\mathrm{cm}^{2}$ exceeds the technical specification of $20-200 \mathrm{dyne} / \mathrm{cm}^{2}$. Due to the relatively high detection limit, it is not possible to determine whether the sample met the technical specification. The analysis was performed in case a measureable shear strength could be detected, thus validating that the specification was exceeded.

$$
\tau_{s}=\frac{\frac{\% \tau}{100} \times S_{\tau} \times 4.90 \times 10^{5}}{\frac{\pi \times H_{v} \times D_{v}{ }^{2}}{2}+\frac{\pi \times D_{v}^{3}}{6}}
$$

The settled solids composite was analyzed in duplicate for shear strength at $29^{\circ} \mathrm{C}$ according to procedure number PNL-ALO-501. These solids were left undisturbed for two days prior to analysis. Following the shear strength analysis the settled solids from the shear strength analysis and remaining settled solids in bottle SPD1-C106-051 were combined, and subsequently subjected to further processing for sodjum reduction (Wiemers 1996, Urie 1997). The decanted supernatant was bottled for archiving. A $51 \mathrm{cP}$ standard was analyzed prior to this sample and met the $\mathrm{QC}$ requirement of $\pm 10 \%$. Both the sample and duplicate results were below the instrumental detection limit of 1000 dyne $/ \mathrm{cm}^{2}$.

Apparent Viscositv and Yield Stress: A $10 \mathrm{~g}$ subsample of the slurry from bottle SPD1-C106-051 was removed for shear stress verses shear rate analysis, as insufficient sample was available from the originally assigned sample, SPD1-C106-046. The subsample was analyzed in replicate (i.e., two individual subsamples were not obtained for analysis) for shear stress as a function of shear rate in at $25^{\circ} \mathrm{C}$. Following the analysis, this material was discarded. At shear rates between 10 and $320 \mathrm{~s}^{\circ}$, the material displayed near Newtonian behavior. The viscosity of the material remained nearly constant at 1 to $3 \mathrm{cP}$ in the range of 10 and $320 \mathrm{~s}^{-1}$.

The samples were stirred with a spatula to combine the separated liquid and solid layers, and analyzed for shear stress as a function of shear rate using a Bohlin CS viscometer modified for glove box operation. Concentric cylinders with a $25 \mathrm{~mm}$ diameter inner cylinder and a $2.5 \mathrm{~mm}$ gap between the cylinders were the measuring geometry used with a $C 25$ measuring sensor. Shear stress as a function of shear rate data was obtained by measuring the shear stress produced at a specific shear rate at $25^{\circ} \mathrm{C}$. 
The shear rate was gradually increased from 0 to $320 \mathrm{~s}^{-1}$ generating the increasing shear rate curve, and then back down to $0 s^{-1}$ generating the decreasing curve. The viscosity was then calculated by dividing the shear stress by the shear rate. Calibration checks were made with a certified $10 \mathrm{cP}$ viscosity standard to ensure that the viscometer was operating properly. Technical Procedure PNL-ALO-501 was used to perform the data acquisition. During data interpretation a deviation was made to Technical Procedure PNL-ALO-501 in determination of the yield stress, the force sufficient to produce fluid behavior in the material. The Bohlin CS viscometer is a controlled stress instrument (versus as controlled rate instrument), therefore the yield stress was determined by plotting the shear stress versus shear rate for the increasing shear rate curve and extrapolating to zero shear rate.

The LOI specification for this material was a viscosity between 6 and $94 \mathrm{cP}, 3$ and $50 \mathrm{cP}$, and 1 and $50 \mathrm{cP}$ at shear rates of 10,25 and $183 \mathrm{~s}^{-1}$, respectively, and a yield stress of between 0.1 and $15 \mathrm{~Pa}$. At $10 \mathrm{~s}^{-1}$, both sample and duplicate had a measured viscosity of $2.1 \mathrm{cP}$, which is below the minimum specification. At $25 \mathrm{~s}^{-1}$, the sample results were 1.3 and $1.6 \mathrm{cP}$, which is also below the minimum specification. At $183 \mathrm{~s}^{-1}$, the sample results were 1.1 and $1.0 \mathrm{cP}$, meeting the low end of the specification. A yield stress was observed, but was well below the quantification limit of $0.1 \mathrm{~Pa}$, which is below the minimum specification. Compliance with these specifications was not required for shipment of the Vendor Product to the vendor. No attempt was made to adjust these properties within the specification.

Heat Capacity: A measure of the heat capacity of the Vendor Product slurry (SPD1-C106-046) was attempted using Differential Scanning Calorimetry (DSC). Heat capacity is a measure of the amount of energy required to produce a given rise in the temperature of the system. Due to several factors (described below), the measurement of the heat capacity of the slurry could not be performed by the DSC method. However, a measurement of the heat capacity of the dried Vendor Product slurry was obtained by this method.

The value for the heat capacity of the dried Vendor Product slurry (heated at $105^{\circ} \mathrm{C}$ until no further mass is lost) was $0.22 \mathrm{cal} / \mathrm{g} \cdot{ }^{\circ} \mathrm{C}$ from 50 to $105^{\circ} \mathrm{C}$. The heat capacity of the dried sample is comparable with the heat capacity of $\mathrm{NaNO}_{3}\left(0.26 \mathrm{cal} / \mathrm{g} \cdot{ }^{\circ} \mathrm{C}\right)$ and $\mathrm{NaNO}_{2}\left(0.24 \mathrm{cal} / \mathrm{g} \cdot{ }^{\circ} \mathrm{C}\right)$. The dried vendor sample may retain water in other than "free" forms. Water will be removed in different amounts from Hanford tank waste samples from ambient temperatures to roughly $325^{\circ} \mathrm{C}$. After drying at $105^{\circ} \mathrm{C}$ no free water will remain, but some adsorbed water and most of the hydrate water is still present. As long as no further loss of water occurs during the DSC run, the heat-capacity $\left(\mathrm{C}_{\mathrm{p}}\right)$ value measured is valid since the heat capacity for many solid hydrates (expressed per gram) is not greatly different (commonly 10 to $20 \%$ ) than for the anhydrous material. The heat capacities of solid hydrates and anhydrous materials are generally much smaller than the heat capacity for water.

A good estimate of the heat capacity of the Vendor Product slurry can be made by averaging the heat capacities per gram of the dried solid and water based on the wt $\%$ of each. This estimate provides a heat capacity of $0.89 \mathrm{cal} / \mathrm{g} \cdot{ }^{\circ} \mathrm{C}$ which is within the RFP specifications listed in Table TS-8.4

$\left(0.79-0.97 \mathrm{cal} / \mathrm{g} \cdot{ }^{\circ} \mathrm{C}\right.$ ). This calculation is based on $14.4 \mathrm{wt} \%$ solids (See Table 4.1 ) and the reported heat capacity for water $1 \mathrm{cal} / \mathrm{g} \cdot{ }^{\circ} \mathrm{C}$. 
The heat capacity was measured on a Seiko Instruments DSC220 with the liquid nitrogen cooling capability. The heat capacity of the sample $\left(C_{p_{s}}\right)$ is calculated according to equation 2 from the difference between the DSC curves of the empty container and sample $\left(Y_{3}\right)$, the difference between the DSC curves of the container and the reference $\left(Y_{r}\right)$, mass of the reference $\left(M_{q}\right)$, mass of the sample $\left(M_{s}\right)$, and heat capacity of the reference $\left(C_{p_{r}}\right)$. The Specific Heat Capacity Calculation Software "DSCCP" supplied by Seiko Instruments Inc. was used to calculate the heat capacity of the sample over the range of the analysis.

$$
C p_{s}=\frac{Y_{s}}{Y_{r}} x \frac{M_{r}}{M_{s}} x C p_{r}
$$

The analysis was performed by performing a DSC analysis on the empty pan (baseline), placing the sapphire standard in that same pan and performing an equivalent analysis (reference), and finally replacing the sapphire with the sample at approximately the same mass of the reference and performing a third equivalent analysis (sample). The baseline is subtracted from both the reference and sample analyses, and the subtracted data is used to calculate the heat capacity.

Several attempts were made to measure the heat capacity of the Vendor Product slurry from $10^{\circ} \mathrm{C}$ above ambient temperature to $105^{\circ} \mathrm{C}$. Because the mass of the sample must be known throughout the analysis and the sample contains water which vaporizes over the temperature range of the analysis, the sample must be placed in a sealed sample pan. A large endotherm from the vaporization of the water in the sample makes it difficult to measure the heat capacity of the sample, because the endothermic transition requires energy to vaporize the water which is not indicative of the heat capacity of the sample. The contribution from the vaporization of water is 540 times the estimated sample heat capacity. When the sample is sealed and water vaporizes, the pressure of the system increases. The heat required to produce a given rise in temperature is dependent upon the conditions under which the system is heated; therefore, heat capacity is generally reported at constant pressure. As the pressure is increasing the measured value of heat capacity is not the heat capacity at constant pressure which is generally reported; therefore, corrections need to be made based on the pressure in the system which is unknown for these samples.

The sample also generates its own heat which could introduce error into the measurement of heat capacity. A calculation of the energy generated (watts) from the sample indicates that this error is minimal for this sample $\left(1 \times 10^{-5} \mathrm{~W} / \mathrm{g}\right.$ of slurry or $2 \times 10^{-7} \mathrm{~W}$ for a $20 \mathrm{mg}$ slurry sample and $1 \times 10^{-5} \mathrm{~W}$ for the $16.5 \mathrm{mg}$ dried sample). The heat capacity was not determined for the Initial Composite Material (i.e., prior to diluting). 


\subsection{Chemical Analysis}

\subsubsection{Inductively Coupled Plasma Atomic Emission Spectrometry (ICP-AES)}

The three Vendor Product and three Original Material samples prepared in duplicate by three different sample preparation procedures (See Section 3) were analyzed by ICP-AES following method PNL-ALO-211 "Determination of Elements by Inductively Coupled Plasma Atomic Emission Spectrometry. Both the Vendor Product and Original Material were analyzed in the same manner. For instance, post digestion spikes were performed on fusion preparations, matrix spikes are included with the acid digestions, and all instrument QC results (e.g., continuing calibration verifications) were within acceptable limits for all reported results. The results from the ICP-AES analysis are reported for any analyte exceeding the instrument detection limit (IDL). Those results above the IDL but below the estimated quantitation limit (EQL) are reported in brackets "[ ]." All reported results have been adjusted for preparation and analytical dilution factors.

In general, the consistency of the results between the various dissolution techniques is very good, except for those analytes which exhibit poor dissolution by specific techniques (e.g., Si/Ti by acid digestion). One notable exception is silver, where the sodium peroxide fusion produced significantly higher results than the potassium hydroxide fusion or acid digestion preparation. However, the precision of the silver analysis was very poor.

\subsubsection{ICP-AES - Vendor Product (Table 4.3 to Table 4.5)}

The analyte concentrations found in the samples above $0.1 \%$ by weight are aluminum, iron, sodium, silicon, silver, calcium, magnesium, manganese, nickel, phosphorus, lead, titanium, and zirconium, and possibly potassium and uranium. For the fusions preparations, the relative percent difference (RPD) between duplicates are less than $20 \%$, except for silver, calcium, lead, phosphorus, and zirconium. Whereas in the acjd digestions, only silver, cadmium, and silicon demonstrate RPD values greater than $20 \%$.

The contribution of the preparation blank to the potassium hydroxide fusion and acid digestion samples is negligible for all analytes, or the blank concentration is insignificant relative to the sample concentration. The exceptions are boron and copper, where the blank accounts for $20-30 \%$ of the sample concentration. However, the preparation blank for the sodium peroxide fusion demonstrated considerable contamination for calcium, copper, potassium, lithium, neodymium, nickel, phosphorus, and lead. This contamination is most likely caused from impurities in the sodium peroxide reagent or cross contamination during sample preparation. The ICP-AES analysis for these analytes from the sodium peroxide fusion should be used with caution. However, since these analytes did not exceed the specification, no additional actions (e.g., reanalysis) were taken.

From the acid digestion preparations, the silver matrix spike and blank spike recoveries are $12 \%$ and $47 \%$, respectively; whereas all other spiked analytes recovered within acceptable limits (75\% to $125 \%$ ). Post spike recoveries for all preparations and all analytes are within acceptable limits (i.e., $75 \%$ to $125 \%$ ). The reported silver value from the acid digestion is considered unreliable, and likely bias low because of precipitation of the silver with chloride present in the sample or from the hydrochloric acid used during preparation. 


\subsubsection{ICP-AES - Original Material (Table 4.6 to Table 4.8)}

The analyte concentrations found in the Original Material above $0.1 \%$ by weight are aluminum, iron, sodium, silicon, silver, calcium, manganese, phosphorus, lead, and zirconium, and possibly tin and uranium. For the fusions preparations, the RPD between duplicates are less than $20 \%$, except for silver. Whereas in the acid digestions, silver, boron, silicon, and zirconium demonstrate very poor precision (i.e., RPD values greater than $20 \%$ ).

The contribution of the preparation blank for the potassium hydroxide fusion and acid digestion samples is negligible for all analytes, or the blank concentration is insignificant relative to the sample concentration. However, the preparation blank for the sodium peroxide fusion demonstrated considerable contamination for bismuth, calcium, cerium, copper, potassium, lithium, magnesium, neodymium, nickel, phosphorus, and lead. The ICP-AES analysis for these analytes from the sodium peroxide fusion are most probably bias high and should be used with caution.

Both the post spike recoveries and matrix spike recoveries for all analytes are within acceptable limits, except for silver. The silver matrix spike recovery is typically about $50 \%$ for the acid digestion process used. The silver matrix spike for the Original Material recovered at only $13 \%$ and, therefore, the silver results from the acid digestion are probably bias very low and are considered unreliable.

\subsubsection{Inductively Coupled Plasma Mass Spectrometry (ICP-MS)}

The three Vendor Product and three Original Material samples prepared in duplicate by three different sample preparation procedures (See Section 3) were analyzed by ICP-MS following methods PNL-ALO-280 "Inductively Coupled Plasma-Mass Spectrometer Analysis," PNL-ALO-281 "ICP-MS Determination of ${ }^{99} \mathrm{Tc}$," and PNL-ALO-282 "Determination of Uranium Concentration/Isotopic Composition Using ICP-MS." Analysis by ICP-MS were performed on one or more of the three preparations. Unlike ICP-AES, the ICP-MS reports only those results which are above the EQL.

\subsubsection{ICP-MS - Vendor Product (Table 4.9 to Table 4.14)}

The Vendor Product samples were analyzed before the Original Material samples. Multiple analyses of the Vendor Product samples were required for some analytes/isotopes of interest in order to obtain acceptable quality control results. Less repeat analyses were required for the Original Material after having increased knowledge based on the Vendor Product results. Analytes that were used for.internal standards (lithium, beryllium, scandium, indium, and thallium) were confirmed not to be present in the samples in measurable concentrations. Isotope values that were calculated from sensitivities of other analytes include ${ }^{240} \mathrm{Pu},{ }^{242} \mathrm{Pu},{ }^{135} \mathrm{Cs},{ }^{107} \mathrm{Pd}$, and ${ }^{232} \mathrm{Th}$. As such, there is no calibration verification for these isotopes.

Quality control data indicate high uncertainties for ICP-MS analysis of calcium, sodium, selenium, zirconium, $\mathrm{m} / \mathrm{z} 93$ (niobium/zirconium), arsenic, rhodium, barium, and antimony. Matrix spike results for sodium, iron, aluminum, and calcium were unusable due to the relatively high sample concentrations (greater than five times that of spike) present for these analytes. 
The $\mathrm{m} / \mathrm{z} 93$ results (the most contribution expected from niobium) may also include a contribution from zirconium. Although ${ }^{93} \mathrm{Zr}$ in not naturally occurring, for these samples there may be an interference from zirconium at $\mathrm{m} / \mathrm{z} 93$. Resolution of the niobium and zirconium is not complete. The same interference problen is experienced also at $\mathrm{m} / \mathrm{z} 241$ with plutonium and americium. Therefore, only one value is reported for $\mathrm{m} / \mathrm{z} 93$ and $\mathrm{m} / \mathrm{z} 241$; possible/probable isotopes contributing to the response observed at these masses are zirconium/niobium and plutonium/americium, respectively.

There does appear to be a small peak at $\mathrm{m} / \mathrm{z} 233$ which indicates the presence of ${ }^{233} \mathrm{U}$. However, this peak may be attributed to a hydride of ${ }^{232} \mathrm{Th}$, which is present in a relatively high concentration.

No modifications were performed to either the fusions or acid digestions to assure the ${ }^{129} \mathrm{I}$ remains in solution and not lost as $I_{2}$. Since iodine was not spiked prior to initiating the preparative process, the reported ICP-MS results for ${ }^{129} \mathrm{I}$ may be biased.

Additional information on general observations made during ICP-MS analyses and analyte/isotope data qualifications is included in Appendix A.

\subsubsection{ICP-MS - Original Material (Table 4.15 to Table 4.20)}

In general, analyses of the Original Material met with fewer problems, required fewer re-runs, and exhibited more consistency in QC performance than that of the Vendor Product. This was primarily due to the experience gained in analyzing the Vendor Product material. Each sample preparation (KOH fusion, acid digestion, and $\mathrm{Na}_{2} \mathrm{O}_{2}$ fusion) was analyzed separately. The samples were analyzed at three different dilutions to obtain valid data for the wide range of analyte concentrations observed. In general, the same data qualifications apply to the Original Material results as to the Vendor Product results (See Section 4.3.2.1 and Appendix A).

\subsubsection{Ion Chromatography (IC) for Anions (Table 4.21 and Table 4.22)}

The IC analyses were performed in duplicate on each of the three Vendor Product and three Original Material samples following leaching with water. The Vendor Product and Original Material were analyzed as a single batch following method PNL-ALO-212 "Determination of Inorganic Anions by Ion Chromatography." Besides the routine suite of anions (i.e., $\mathrm{F}, \mathrm{Cl}, \mathrm{Br}, \mathrm{NO}_{2}, \mathrm{NO}_{3}, \mathrm{SO}_{4}$, and $\mathrm{PO}_{4}$ ), oxalate was also determined on both materials by this method.

In general, the RPD values were within acceptable limits, except for those results at or near the lowest calibration standard concentration. However, the excessively high oxalate is suspected of contributing to the higher than normal RPDs. Most anions were measured near the lowest calibration standard due to the high dilutions required because of the $15 \%$ to $20 \%$ oxalate present. The preparation blank exhibited significant concentrations of chloride, nitrate and sulfate; with the chloride and nitrate being well above $5 \%$ of the sample concentrations. However, since these analytes were below the specification, no further actions were taken.

The blank spikes and matrix spikes associated with the Original Material water leach preparation recovered within the acceptance limits of $\pm 20 \%$. The blank spike associated the Vendor Product water 


\section{HNF-SD-WM-DP-225, REV. 1}

Analytical Summary Report - January 28, 1997

leach preparation was also within acceptance limits; however, fluoride, nitrite, and phosphate spike recoveries failed for the Vendor Product matrix spike. The fluoride recovery (at $144 \%$ ) appears to be bias high due to matrix interferences; these interferences also result in higher reportable detection limits. The nitrite and phosphate spiking levels were less than $25 \%$ of the sample concentration, thus the calculated recoveries are not meaningful. Since these analytes were below the specification, no further actions were taken.

\subsubsection{Ion Specific Electrode for Ammonia (Table 4.21 and Table 4.22)}

Ammonia analyses were performed in duplicate on each of the three Vendor Product and three Original Material samples following leaching with water. The Vendor Product and Original Material were analyzed as separate batches following method PNL-ALO-226 "Ammonia (Nitrogen) in aqueous Samples." Immediately following the water leach preparation, the leached samples were acidified to stabilize the ammonia, then removed from the SAL and analyzed in the laboratory. For all samples analyzed, the ammonia level was below the linear portion of the calibration curve, thus the results are anticipated to have a high uncertainty. The preparation blank concentrations are basically the same as the sample concentration, thus indicating that there is little if any ammonia in the water leached samples.

The RPDs for the sample/duplicate sets were poor; however, this is expected at the low concentrations measured. Also, except for one laboratory double spiking problem, the blank spikes and matrix spikes recovered within acceptable limits. The ammonia method requires post spiking of all samples; the post spikes for the_Vendor Product and Original Material samples ranged from $87 \%$ to $119 \%$.

\subsubsection{Cold Vapor Atomic Absorption for Mercury (Table 4.21 and Table 4.22)}

To obtain mercury results on the dried slurries, small $(0.04 \mathrm{~g})$ subsamples of the dried materials were weighed in the SAL and distributed to the Inorganic Group for cold vapor atomic absorption (CVAA) analysis. Due to analytical instrumentation problems during the analysis of the initial set of samples, an additional set of samples were weighed in the SAL and distributed to the laboratory. Provisions were made for full QC to be applied at the time of analysis (e.g., samples weighed for subsequent laboratory spike additions). The CVAA Mercury analyses were performed in duplicate on each of the three Vendor Product and three Original Material samples. The Vendor Product and Original Material were analyzed as a single batch following Method PNL-ALO-131 for digestion and PNL-ALO-201 for analysis.

Due to the high concentration of mercury in the samples (relative to other Hanford tank materials), the matrix spike could not be recovered (i.e., the matrix spike concentration was less than $20 \%$ of the sample concentration). The RPD values for all the samples were within acceptance limits of $20 \%$, and no mercury was detected in the preparation blank. It should be noted, however, that the continuing calibration verification standards recovered at $82 \%$ to $85 \%$ which is just within the lower acceptance limit of $80 \%$. This suggests that the instrument results may be bias slightly low for all the sample results. However, it the results are adjusted for recovery of both the Vendor Product and the Original Material, the results are well within the Envelope D specification. 


\subsubsection{Cyanide (Table 4.21 and Table 4.22)}

Total cyanide analyses were performed in duplicate on each of the three Vendor Product and three Original Material samples. The Vendor Product and Original Material were analyzed as a single batch. An attempt was made to perform a remote cyanide analysis (PNL-ALO-287 "Midi and Micro Distillation of Cyanide in Liquid and Solid Samples") on the dried slurry materials. The samples became very effervescent with the addition of digesting acid, $2 \mathrm{M} \mathrm{H}_{2} \mathrm{SO}_{4}$. Enough gas was generated to breach the membrane separating the digesting sample from the $\mathrm{NaOH}$ trapping solution ( $\mathrm{HCN}$ distillate). Additionally the samples turned black in color when the acid was applied, and the batch QC failed. Sample sizes were reduced to about 40 milligrams and subsamples distributed to the Inorganic Group for a repeat of the same technique in a fume hood environment; the results were similar. A third set of samples were then weighed and distributed to the Inorganic Group, and an alternate $\mathrm{CN}$ method utilizing a predissolution step and larger distillation tubes (to allow more room for gas expansion during digestion) was performed. This analysis yielded acceptable QC and sample performance. The samples were predigested in an EDTA and ethylenediamine solution prior to distillation, following procedure PNL-ALO-285 "Total Cyanide by Remote Microdistillation and Argentometric Titration." After the predigestion, the distillates were analyzed following method PNL-ALO-289 "Total Cyanide Determination with Spectrophotometry (Manual or Automated)." All batch $\mathrm{QC}$ and instrument $\mathrm{QC}$ are within acceptable limits. The matrix spike ranged in recovery from $98 \%$ to $122 \%$, and the RPD between duplicates ranged from $1 \%$ to $19 \%$.

\subsubsection{Total Carbon - Inorganic and Organic Carbon (Table 4.21 and Table 4.22)}

Total carbon analyses were performed in duplicate in the SAL directly on each of the three Veridor Product and three Original Material samples by the hot persulfate wet oxidation method PNL-ALO-381 "Determination of TC, TOC, and TIC in Radioactive Liquids, Soils, and Sludges." The dried samples and duplicates were acid digested, swept with a pure oxygen stream and the resulting carbon dioxide routed through the coulometry unit of the analyzer. Per the procedure, all sample results have been corrected for the carbon system blank and the control standard recovery. The TIC and TOC are measured directly and the TC is determined by summing the TIC and TOC. Quality Control for the method involves instrumental blanks for IIC and TOC, inorganic and organic standards analysis, and matrix spiked samples.

For the Vendor Product analysis, all batch QC and analytical QC were acceptable, except for one RPD which calculated at $25 \%$; slightly outside the acceptance limit of $20 \%$. For the Original Material, all analytical QC and batch QC were acceptable. However, the range demonstrated by the control standard (i.e., $82 \%$ to $111 \%$ ) is considerably greater than typically experienced. Although the control standards varied more than normal, the sample RPD range $(0.5 \%$ to $25 \%)$ is typical for the matrix type. The oxalate determined by IC analysis accounts for nearly $100 \%$ of the TOC. 


\subsection{Radiochemistry Analysis}

\subsubsection{Radiochemistry - Vendor Product (Table 4.23)}

The Vendor Product radiochemistry analyses were performed on the KOH fusion preparations, unless otherwise noted. Small aliquots of these prepared solutions were then used for the radiochemical analyses. All of the Vendor Product radiochemical results are reported in $\mu \mathrm{Ci} / \mathrm{g}$ dried solids.

Gamma Energy Analyses (GEA): Aliquots of the prepared samples were directly gamma counted following procedure PNL-ALO-450. ${ }^{157} \mathrm{Cs}$ was the dominant gamma emitter. However, ${ }^{60} \mathrm{Co},{ }^{154} \mathrm{Eu}$, ${ }^{155} \mathrm{Eu}$, and ${ }^{241} \mathrm{Am}$ were also detected. None of the other gamma emitters of interest (i.e., ${ }^{106} \mathrm{Ru}\left({ }^{106} \mathrm{Rh}\right)$, ${ }^{110 \mathrm{~m}} \mathrm{Ag},{ }^{125} \mathrm{Sb}\left({ }^{125 \mathrm{~m}} \mathrm{Te}\right),{ }^{154} \mathrm{Cs}$, ${ }^{144} \mathrm{Ce}\left({ }^{144} \mathrm{Pr},{ }^{144 \mathrm{~m}} \mathrm{Pr}\right)$, and $\left.{ }^{152} \mathrm{Eu}\right)$ were detected and MDA values are reported. In some cases, the measured MDA values are orders of magnitude higher than requested. The requested MDA values cannot be obtained due to the very strong interference of Compton background from ${ }^{137} \mathrm{Cs}$. Hence, it would be necessary to chemically separate out the cesium in order to achieve lower detection limits.

The RPD values show good agreement between the samples and duplicates in cases where reasonable counting statistics were achieved. It should also be noted that the three Vendor Product sample splits also show good agreement. There are three cases where the RPD values exceeded the required $20 \%$ limits; ${ }^{241} \mathrm{Am}$ on sample 96-6103 and ${ }^{241} \mathrm{Am}$ and ${ }^{155} \mathrm{Eu}$ on sample 96-6104. In these cases, the counting uncertainties for both the sample and the duplicate are very high; however, the mean difference (MD) values indicate with high confidence that the results are statistically the same. The preparation blank showed only weakk ${ }^{137} \mathrm{Cs}$ activity.

Total Alpha: The total alpha activities were determined following procedures PNL-ALO-420/421. All of the sample analyses give similar results with RPD values below $10 \%$. Weak alpha activity was detected in the preparation blank; however, the value is a factor of about 1,000 less than the samples. No alpha activity was detected in the laboratory blank. Standard and sample spike recoveries were $106 \%$ and $113 \%$, respectively.

Total Beta: The total beta activities were determined following procedures PNL-ALO-430/431. All of the sample analyses give similar results with RPD values below $11 \%$. Weak beta activity was detected in the preparation blank; however, the value is a factor of 10,000 less than the samples. No beta activity was detected in the lab blank. Standard and sample spike recoveries were $97 \%$ and $101 \%$, respectively.

Americium/Curium: The americium and curium isotopes were determined together because they have identical chemical behavior. Analysis proceeded according to procedures PNL-ALO-417/496/422. Sample-specific radiochemical yields/counting efficiencies were determined relative to ${ }^{243} \mathrm{Am}$ tracer.

In order to determine the ${ }^{243} \mathrm{Am}$ activity, a separate aliquot was processed and the indigenous ${ }^{241} \mathrm{Am}$ was used to determine radiochemical yield/counting efficiency, applying the ${ }^{241} \mathrm{Am}$ concentration measured initially. No ${ }^{243} \mathrm{Am}$ was found in the samples so the original premise of using the ${ }^{243} \mathrm{Am}$ to trace yields is valid. The sample-specific ${ }^{243} \mathrm{Am}$ detection limit is driven by the tailing of the ${ }^{241} \mathrm{Am}$ alpha peak into the ${ }^{243} \mathrm{Am}$ alpha energy region. 
The radiochemical yields for the sample preparations were nominally $85 \%$. The RPD values for ${ }^{241} \mathrm{Am}$ activity were below $4 \%$. The ${ }^{243+244} \mathrm{Cm}$ reproducibility was not as tight as that of ${ }^{241} \mathrm{Am}$ with sample 96-6104 at $20.7 \%$ and sample $96-6105$ at $25.6 \%$ RPD. The ${ }^{242} \mathrm{Cm}$ RPD values were also rather high at $31.9 \%(96-6103)$ and $23.7 \%(96-6104)$ however, the errors associated with the results were also high at nominally $20 \%$. In all of these cases, the $\mathrm{MD}$ is less than 1.5 indicating that the results are consistent within the analytical uncertainties. The preparation blank activity for the americium/curium isotopes was well below that of the samples. The highest preparation blank activity is for ${ }^{243+244} \mathrm{Cm}$ which represents $2 \%$ of the sample activity for sample 96-6104. The matrix spike and blank spike yield-corrected recoveries were $93 \%$ and $100 \%$ respectively.

Carbon-14: Radiocarbon determinations were performed in the SAL directly on Vendor Product sample per method PNL-ALO-482 "Determination of Carbon-14 in Radioactive Liquids, Soils, and Sludges." The in-cell UICTM analyzer module was used to digest the samples and sweep the carbon dioxide to a caustic trap located in a fume hood outside the hot cell. The individual sample trap solutions were then combined with scintillation cocktail and counted using a Liquid Scintillation Counter. The QC for this method involves instrumental blanks, analysis of a ${ }^{14} \mathrm{C}$ standard and matrix spiked with the ${ }^{14} \mathrm{C}$ standard material. The reproducibility of the ${ }^{14} \mathrm{C}$ analyses between samples and duplicate was very poor, due to carryover from previously analyzed high standards. Although the matrix spike recoveries are good, the extremely poor sample reproducibility makes the reported results suspect. Even at the highest ${ }^{14} \mathrm{C}$ result, the ${ }^{14} \mathrm{C}$ concentration is only about $25 \%$ of the Envelope D specification; therefore, no additional actions were taken. However, the reason for the carryover from preceding standards is being evaluated.

Cesium Isotopes: The ${ }^{134} \mathrm{Cs}$ and ${ }^{137} \mathrm{Cs}$ activities were measured by GEA. In order to further obtain the activities of ${ }^{135} \mathrm{Cs}$, which has a very long half-life of $2.3 \mathrm{E} 6$ years, the cesium was chemically separated. and then measured by thennal ionization mass spectrometry (TIMS) following procedure PNL-ALO-452. The activities of ${ }^{135} \mathrm{Cs}$ were then calculated from the ${ }^{137} \mathrm{Cs}$ activities, measured by GEA, and the ${ }^{135} \mathrm{Cs} /{ }^{137} \mathrm{Cs}$ mass ratios, measured by TIMS. The results indicate nearly the same activity of ${ }^{135} \mathrm{Cs}$ in all of the samples and RPD values are all less than $5 \%$. The ${ }^{134} \mathrm{Cs}$ could not be detected either by GEA or TIMS measurements and MDA values were reported for the GEA results. The TIMS measurements also detected stable ${ }^{133} \mathrm{Cs}$. Although this isotope was not requested, concentration values are provided for information. Since cesium only has one stable isotope $\left({ }^{133} \mathrm{Cs}\right)$, no appropriate TIMS standards are available. A rubidium standard was thus analyzed instead. The measured ratio of ${ }^{85} \mathrm{Rb} /{ }^{85} \mathrm{Rb}$ was 2.59 , in excellent agreement with the natural isotope ratio of 2.59 .

Fe-55: An iron chemical separation was performed following procedures PNL-ALO-435/450. Stable iron was used to trace the yield. A minor deviation from procedure PNL-ALO-435 was performed to obtain radiochemical yields from stable iron. The iron hydroxide precipitate was dissolved and an aliquot of solution was analyzed by ICP-AES for the iron content. The radiochemical yields averaged $70 \%$.

No ${ }^{5 s} \mathrm{Fe}$ was detected in either the hot cell preparation blank or the lab blank. The blank spike and sample matrix spike yield-corrected recoveries were $115 \%$ and $86 \%$, respectively. The samples demonstrated good agreement with RPD values below $8 \%$. 
Ni-59 and Ni-63: The three Vendor Product samples (including duplicates) from the sodium peroxide fusion process were analyzed for ${ }^{35} \mathrm{Ni}$ and ${ }^{63} \mathrm{Ni}$. Procedure PNL-ALO-495 was used for the nickel purification, PNL-ALO-450 was used for the low-energy photon (LEP) counting, and PNL-ALO-474 was used for beta counting (liquid scintillation). Minor changes were made to the nickel separation procedure. A nickel-specific column from Eichrom Industries was used in place of the house-made columns. The Eichrom column functions with the same chemical principals and would not affect data quality. Another change is the treatment of the nickel-dimethylglyoxime precipitate on the filter. This

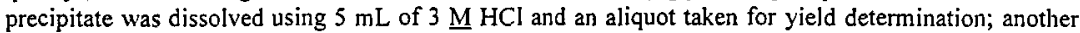
aliquot was taken to dryness, $0.5 \mathrm{~mL}$ water added and $10-\mathrm{mL}$ Ultima Gold added for subsequent liquid scintillation counting. This is a quality improvement in that there is no potential for nickel to be lost due to potential volatilization of the $\left.\mathrm{Ni}_{(} \mathrm{NO}_{3}\right)_{2}-6 \mathrm{H}_{2} \mathrm{O}$.

Radiochemical yields were generally high, nominally $90 \%$. The hot cell preparation blank resulted in no detectable activity for either ${ }^{59} \mathrm{Ni}$ or ${ }^{65} \mathrm{Ni}$. The ${ }^{59} \mathrm{Ni}$ LEP spectra and the ${ }^{63} \mathrm{Ni}$ beta spectra were visually clean from other interferences. The ${ }^{59} \mathrm{Ni}$ activity is barely detectable in some of the samples, at about $2 \mathrm{E}-2 \mu \mathrm{Ci} / \mathrm{g}$. This is virtually at the MDA. The ${ }^{63} \mathrm{Ni}$ concentration is consistent throughout the sample set at nominally $2.4 \mu \mathrm{Ci} / \mathrm{g}$. The matrix spike samples were spiked with ${ }^{63} \mathrm{Ni}$, since ${ }^{59} \mathrm{Ni}$ is not commercially available. The blank spike, blank spike duplicate, and matrix spike recoveries were excellent at $95 \%, 96 \%$ and $101 \%$, respectively.

Nb-93m: The niobium radiochemical separation from other fission products was conducted according to PNL-ALO-4003. (This procedure is currently in the review/approval process.) The final sample preparations were counted by both GEA and LEP according to PNL-ALO-450. Radiochemical yields were traced with ${ }^{95} \mathrm{Nb}$ and averaged about $70 \%$. It appears that ${ }^{125} \mathrm{Sb}$ carried through with the niobium fraction, however this isotope did not adversely interfere with the $16.6 \mathrm{keV}$ photon of ${ }^{93 \mathrm{~m}} \mathrm{Nb}$. The blank spike recovery is low at $71 \%$; the reason for the low recovery is not known. The matrix spike recovery is acceptable at $84 \%$. The RPD values are high at $30 \%$ to $79 \%$; however, the MD shows that within the error of the measurement, the results are consistent.

Plutonium: Plutonium isotopes were separated in sequence after the americium/curium elution according to PNL-ALO-417. One minor modification was made to the procedure to effect this separation/elution. The plutonium was reduced to $\mathrm{a}+3$ oxidation state using $\mathrm{TiCl}_{3}$ solution in $4 \underline{\mathrm{M}}$ $\mathrm{HCl}$. This is a more effective and faster way to elute the plutonium fraction. The Pu fraction then was precipitation-plated directly according to PNL-ALO-496. Alpha spectrometry proceeded according to PNL-ALO-422. The plutonium radiochemical yield/counting efficiency was traced with ${ }^{242} \mathrm{Pu}$.

In order to determine the ${ }^{242} \mathrm{Pu}$ activity, a separate aliquot was processed without added tracer. The indigenous ${ }^{239} \mathrm{Pu}$ in each sample was used to trace the yield. The ${ }^{239} \mathrm{Pu}$ activities were determined using the results of the ${ }^{242} \mathrm{Pu}$-traced aliquot. No ${ }^{242} \mathrm{Pu}$ was found in the samples so the original premise of using the ${ }^{242} \mathrm{Pu}$ to trace yields is valid. The sample-specific ${ }^{242} \mathrm{Pu}$ detection limit is driven by the tailing of the ${ }^{239} \mathrm{Pu}$ alpha peak into the ${ }^{242} \mathrm{Pu}$ alpha energy region.

The radiochemical yields for the plutonium fractions were nominally $85 \%$. The sample precision for ${ }^{239+240} \mathrm{Pu}$ and ${ }^{238} \mathrm{Pu}$, as determined by the RPD, is $9.6 \%$ or better. The preparation blank ${ }^{239+240} \mathrm{Pu}$

\section{0}


activity is less than a factor of 1000 relative to the sample activity. The preparation blank ${ }^{238} \mathrm{Pu}$ activity represents less than $0.5 \%$ of the sample activity.

Overall, the sum of the plutonium and americium/curium data is in excellent agreement with the total alpha results.

Plutonium-241: The sample preparations for plutonium were also used for the subsequent beta analysis of ${ }^{24 \mathrm{I}} \mathrm{Pu}$; a draft procedure was used to perform the ${ }^{241} \mathrm{Pu}$ analysis. The precipitation-plated filter samples were placed in liquid scintillation vials, $10 \mathrm{ml}$ of Ultima Gold were added and the samples were counted by liquid scintillation counting (LSC) following procedure PNL-ALO-474.

The LSC spectra showed both the alpha-peak activity $\left({ }^{258} \mathrm{Pu},{ }^{239+240} \mathrm{Pu}\right.$, and $\left.{ }^{242} \mathrm{Pu}\right)$ and a well-separated beta peak at low energies due to ${ }^{241} \mathrm{Pu}$. The samples and duplicates showed good agreement at a nominal activity level of $7 \mu \mathrm{Ci} / \mathrm{g}$. The preparation blank and laboratory blank activities were well below this level; i.e., at $0.03 \mu \mathrm{Ci} / g$ or less. The blank spike and matrix spike samples were spiked at the time the filter was placed in the LSC vial. The spike recoveries are high at $146 \%$ and $182 \%$, respectively. The blank spike preparation was repeated in duplicate with spike recoveries of 111 and $94 \%$. The reason for the high spike recoveries is not clear; no chemistry was involved in the matrix spike sample preparation.

The ${ }^{24 !} \mathrm{Pu}$ activities were also calculated independently by using the plutonium alpha activities as an internal tracer. The ${ }^{241} \mathrm{Pu}$ activities calculated by this method are in good agreement with the beta calculations and the mean activity values agree within $15 \%$.

Sm-15I and Pm-147: The ${ }^{151} \mathrm{Sm} /{ }^{14} \mathrm{P} m$ screen/determinations were made on the final sample preparations for ${ }^{241} \mathrm{Am}$ analysis. As rare earths, the samarium and promethium radiochemically follow the other rare earths and the $\mathrm{Am}^{-3}$ using the TRU-Spec column chemistry (PNL-ALO-417). The samarium and promethium would also be precipitation-plated (PNL-ALO-496), presumably as efficiently as the americium. Thus the radiochemical yield determined for Am is used to correct for the radiochemical yield of $\mathrm{Sm} / \mathrm{Pm}$. It would have been better to utilize blank and matrix spikes to verify the theory, however the request for ${ }^{151} \mathrm{Sm}$ analysis was made too late (after the Am separation) to utilize the ${ }^{15 t} \mathrm{Sm}$ spike in these analyses.

The precipitation-plated americium preparation was simply placed into LSC cocktail and counted according to PNL-ALO-474 by liquid scintillation. For instrument calibration, ${ }^{63} \mathrm{Ni}$ wäs used because its endpoint energy is similar to that of the ${ }^{151} \mathrm{Sm}$. The ${ }^{147} \mathrm{Pm}$ has a $224 \mathrm{keV} \mathrm{E}$ max which tails under the ${ }^{151} \mathrm{Sm}$ peak at $76 \mathrm{keV} \mathrm{E}$ max ; thus a summation of ${ }^{151} \mathrm{Sm}$ and ${ }^{147} \mathrm{Pm}$ is provided. The resulting beta spectra indicated a large peak due to the ${ }^{151} \mathrm{Sm}$; however, another peak (most probably ${ }^{147} \mathrm{Pm}$ ) tailed under the samarium peak. The alpha peak associated with americium/curium was also evident. The hot cell blank and laboratory blank resulted in a small amount of beta activity relative to the calibration blank; however, their activities are about 3000 times lower than that of the samples. The propagated error associated with these measurements is nominally $4 \%$. A measure of bias cannot be determined without blank spike/matrix spike data.

Along with the rare earths samarium and promethium, europium should also appear in this fraction. The samples were counted by GEA to evaluate the ${ }^{154} \mathrm{Eu}$ and ${ }^{155} \mathrm{Eu}$ activities; however, none was 
detected. The absence of the europium isotopes is not understood at this time. The ${ }^{154} \mathrm{Eu}$ and ${ }^{155} \mathrm{Eu}$ concentrations in the Am-preparation were determined based on the GEA, and the dilution and yield chemistries applied to the samples should have resulted in a detectable quantity of both ${ }^{154} \mathrm{Eu}$ and ${ }^{155} \mathrm{Eu}$. Because they were not detected, no beta interference for ${ }^{154} \mathrm{Eu}$ and ${ }^{155} \mathrm{Eu}$ was present. Had europium been present at the anticipated levels, its contribution would have amounted to nominally $5 \%$ of the beta activity in the $2-90 \mathrm{keV}$ region.

This method must be considered a screening method for ${ }^{15} 1 \mathrm{Sm} /{ }^{147} \mathrm{Pm}$ because the radiochemical separation from europium had not been performed and the radiochemical recovery is based solely on the americium recovery. Europium did not recover as well as the americium, indicating that samarium/promethium may have low recovery also.

Se-79: The ${ }^{79} \mathrm{Se}$ activities were determined following procedures PNL-ALO-440/474. The separation procedure involves an anion/cation exchange to remove most radiochemical interferences followed by a selenium bromide distillation. Since ${ }^{79} \mathrm{Se}$ is not available as a standard, it was not possible to measure standard or sample spike recoveries. Natural selenium metal was added to each sample and the recovered selenium precipitate was used to determine the gravimetric yields.

Acid Digestion: Radiochemical yields were nominally $60 \%$ and all known contaminants were successfully removed, as determined by beta spectral analysis. No ${ }^{79} \mathrm{Se}$ beta activity was seen inceither the samples or the blanks above the calibration blank background counting rate.

Hence, MDA values are reported.

KOH Fustion The radiochemical yields of Se averaged about $50 \%$. No ${ }^{79} \mathrm{Se}$ beta activity was seen in either the samples or the blanks above the calibration blank background counting rate. Hence, MDA values are reported.

Sr-90: The ${ }^{90} \mathrm{Sr}$ activities were determined following procedure PNL-ALO-476/431. Radiochemical yields, traced with ${ }^{85} \mathrm{Sr}$, were excellent averaging $90 \%$. All of the sample analyses gave similar results with RPD values below $19 \%$. Weak ${ }^{90} \mathrm{Sr}$ activity was detected in the preparation blank and the lab blank; however, the values are a factor of about 10,000 less than the activities in the samples. Standard and sample spike recoveries were $93 \%$ and $92 \%$, respectively.

Sn Isotopes: Analyses for ${ }^{113} \mathrm{Sn},{ }^{119 m} \mathrm{Sn},{ }^{121 m} \mathrm{Sn}$, and ${ }^{126} \mathrm{Sn}$ were conducted according to procedure PNL-ALO-453/450. A modification was made to the separation procedure to accommodate ${ }^{119 m} \mathrm{~S} n$ and ${ }^{12} 1 \mathrm{~m} \mathrm{Sn}$ analysis. The final sample mount was a thin $\mathrm{Sn}(\mathrm{OH})_{4}$ precipitate enabling the low-energy photons to be analyzed. The stable tin addition had the additional advantage to be used as a radiochemical yield monitor.

Radiochemical yields were nominally $50 \%$. Purity with respect to other gamma emitters was excellent. The ${ }^{126} \mathrm{Sn}$ is reported in conjunction $w^{1}{ }^{126} \mathrm{Sb}$ and ${ }^{126 m} \mathrm{Sb}$ because these isotopes quickly obtain secular equilibrium. The same reasoning applies to ${ }^{113} \mathrm{Sn}$ and ${ }^{113 \mathrm{~m}} \mathrm{In}$. Only ${ }^{126} \mathrm{Sn}$ (and ${ }^{126 / 126 \mathrm{~m}} \mathrm{Sb}$ ) and ${ }^{121 \mathrm{~m}} \mathrm{Sn}$ were found in the samples. The sample and duplicate values were significantly different in some cases, resulting in high RPD values. However, except for one sample pair, the MD test shows that, within the error of the method, the results are consistent. Sample 96-6105-Dup (KOH fusion) appears to be very different from the other samples in the set. The most likely explanation is that the 
yield determination failed with respect to this sample. A replicate analysis was performed on this sample as part of the analytical batch and its results are consistent with the other samples. The result for the replicate sample $(96-6105 \mathrm{D})$ is reported in Table 4.21 .

At the time of analysis, a tin isotope was not available for spiking a blank spike or matrix spike. A ${ }^{113} \mathrm{Sn}$ isotope was ordered and used for the subsequent analysis of the Original Material. The reagent blank and hot cell preparation blank resulted in no detectable activity for these isotopes.

Zr-93: The ${ }^{95} \mathrm{Zr}$ analyses were performed according to draft procedure PNL-ALO-4007. No ${ }^{93} \mathrm{Zr}$ activity was detected in the Vendor Product samples, and MDA values are reported. The samples were analyzed using a separation procedure modified to improve the zirconium carrier recovery, increase the zironium/niobium separation, and to utilize beta-counting for the final ${ }^{93} \mathrm{Zr}$ determination. In summary, stable zirconium was added as a yield monitor and the samples were passed through two anion exchange columns under varying conditions to separate impurities. The carrier recovery on these samples was reasonably high, but gave only about $80 \%$ decontamination from plutonium, and consequently, the ${ }^{241} \mathrm{Pu}$ beta caused the ${ }^{93} \mathrm{Zr}$ count rate to be biased considerably high. Probably over half the total counts in the ${ }^{93} \mathrm{Zr}$ region are from ${ }^{241} \mathrm{Pu}$. No contaminant other than plutonium is discernable in the ${ }^{93} \mathrm{Zr}$ beta spectrum. The actual ${ }^{93} \mathrm{Zr}$ activity in each of these samples is probably less than half the MDA value reported. The MDA values were conservatively estimated from the total beta count rates in the region of the ${ }^{93} \mathrm{Zr}$ activity.

\subsubsection{Radiochemistry - Original Material (Table 4.24)}

The Original Material radiochemistry analyses were performed on $\mathrm{KOH}$ fusion preparations, unless otherwise noted. Small aliquots of these prepared solutions were then used for the radiochemical analyses. All of the radiochemical results are reported in $\mu \mathrm{Ci} / \mathrm{g}$ dried solids.

Gamma Energy Analyses: Aliquots of the prepared sample were directly gamma counted following procedure PNL-ALO-450. ${ }^{137} \mathrm{Cs}$ was the dominant gamma emitter. However, ${ }^{60} \mathrm{Co},{ }^{154} \mathrm{Eu},{ }^{155} \mathrm{Eu}$, and ${ }^{241} \mathrm{Am}$ were also detected. None of the other gamma emitters of interest (i.e., ${ }^{106} \mathrm{Ru}\left({ }^{106} \mathrm{Rh}\right),{ }^{110 \mathrm{~m}} \mathrm{Ag}$, ${ }^{125} \mathrm{Sb}\left({ }^{125 \mathrm{~m}} \mathrm{Te}\right),{ }^{134} \mathrm{Cs},{ }^{144} \mathrm{Ce}\left({ }^{144} \mathrm{Pr},{ }^{144 \mathrm{mr}}\right)$, and $\left.{ }^{152} \mathrm{Eu}\right)$ were detected and $\mathrm{MDA}$ values are reported. In some cases, the measured MDA values are orders of magnitude higher than requested. The requested MDA values cannot be obtained due to the very strong interference of Compton background from ${ }^{137} \mathrm{Cs}$. Hence, it would be necessary to chemically separate out the cesium in order to achieve lower detection limits.

The RPD values show good agreement between the samples and duplicates in cases where reasonable counting statistics were achieved. It should also be noted that the three Original Material splits also show good agreement. There are three cases where the RPD values exceeded the required $20 \%$ limits; ${ }^{155} \mathrm{Eu}$ on sample $96-5892,{ }^{241} \mathrm{Am}$ on sample 96-5893, and ${ }^{155} \mathrm{Eu}$ on sample 96-5894. In these cases, the counting uncertainties are so high that $M D$ values indicate high confidence that the results are statistically acceptable. The preparation blank showed only weak ${ }^{137} \mathrm{Cs}$ activity. 
Total Alpha: The total alpha activities were determined following procedures PNL-ALO-420/421. All of the sample analyses gave similar results with RPD values below $6 \%$. No alpha activity was detected in either the preparation blank or the laboratory blank. Standard and sample spike recoveries were $110 \%$ and $113 \%$, respectively.

Total Beta: The total beta activities were determined following procedures PNL-ALO-430/431. All of the sample analyses give similar results with RPD values below $10 \%$. Weak beta activity was detected in the preparation blank; however, the value is a factor of 10,000 less than the samples. No beta activity was detected in the laboratory blank. Standard and sample spike recoveries were $96 \%$ and $94 \%$, respectively.

Americium/Curium: The americium and curium isotopes were determined together because they chemically behave identically. Analytical separation proceeded according to PNL-ALO-417/496/422. Sample-specific radiochemical yields/counting efficiency were determined relative to ${ }^{243} \mathrm{Am}$ tracer.

In order to determine the ${ }^{243} \mathrm{Am}$ activity, a separate aliquot was processed and the indigenous ${ }^{241} \mathrm{Am}$ was used to determine radiochemical yield/counting efficiency, applying the ${ }^{241} \mathrm{Am}$ concentration after the initial determination. $\mathrm{No}^{243} \mathrm{Am}$ was found in the samples so the original premise of using the ${ }^{243} \mathrm{Am}$ to trace yields is valid. The sample-specific ${ }^{243} \mathrm{Am}$ detection limit is driven by the tailing of the ${ }^{241} \mathrm{Am}$ alpha peak into the ${ }^{243} \mathrm{Am}$ alpha energy region.

The radiochemical yields for the sample preparations were good at nominally $75 \%$. The RPD values in the calculated ${ }^{241} \mathrm{Am}$ activity were better than $13 \%$. The ${ }^{243+244} \mathrm{Cm}$ and ${ }^{242} \mathrm{Cm}$ reproducibilities were not as tight as that of ${ }^{241} \mathrm{Am}$ with some RPD values at $93 \%$ and $46 \%$. Sample $96-5894$ resulted in 1.6 times the concentration of ${ }^{245 / 244} \mathrm{Cm}$ found in the rest of the sample set. This higher activity was confirmed with the untraced americium sample. The errors associated with the ${ }^{242} \mathrm{Cm}$ results were high, reaching to $41 \%$ due to poor counting statistics. Although the RPD values were not below $20 \%$ on all samples, the MD is below the threshold of 1.96. The preparation blank activity for the Am/Cm isotopes was well below that of the samples. The worst-case effect from the preparation blank ${ }^{243+244} \mathrm{Cm}$ activity is for sample $96-5893$ where it represents $7 \%$ of the sample activity. The matrix spike and blank spike yield-corrected recoveries ranged from $95 \%$ to $106 \%$.

Carbon-14: Radiocarbon determinations were performed in the SAL directly on Original Material sample per method PNL-ALO-482 "Determination of Carbon-14 in Radioactive Liquids, Soils, and Sludges." The in-cell UICTM analyzer module was used to digest the samples and sweep the carbon dioxide to a caustic trap located in a fume hood outside the hot cell. The individual sample trap solutions were then combined with scintillation cocktail and counted using a Liquid Scintillation Counter. The QC for this method involves instrumental blanks, analysis of a ${ }^{14} \mathrm{C}$ standard and matrix spiked with the ${ }^{14} \mathrm{C}$ standard material. The reproducibility of the ${ }^{14} \mathrm{C}$ analyses between samples and duplicate was very poor, due to carryover from previously analyzed high standards. Although the matrix spike recoveries are good, the extremely poor sample reproducibility makes the reported results suspect. The reason for the carryover from high standards is being evaluated.

Cesium Isotopes: The ${ }^{134} \mathrm{Cs}$ and ${ }^{137} \mathrm{Cs}$ activities were measured by GEA. In order to further obtain the activities of ${ }^{135} \mathrm{Cs}$, which has a very long half-life of $2.3 E 6$ years, the cesium was chemically separated and then measured by TIMS, following procedure PNL-ALO-452. The activities of ${ }^{133} \mathrm{Cs}$ were then 
calculated from the ${ }^{137} \mathrm{Cs}$ activities, measured by GEA, and the ${ }^{135} \mathrm{Cs} /{ }^{137} \mathrm{Cs}$ mass ratios, measured by TIMS. The results indicate nearly the same activity of ${ }^{135} \mathrm{Cs}$ in all of the samples and RPD values are all less than $8 \%$. Cesium- 134 could not be detected either by GEA or TIMS, and the GEA MDA values are reported for ${ }^{134} \mathrm{Cs}$. The TIMS measurements also detected stable ${ }^{133} \mathrm{Cs}$. Although this isotope was not requested, concentration values are provided for information only. Since cesium only has one stable isotope, no appropriate mass spectrometry standards are available. A rubidium standard was thus analyzed instead. The measured ratio of ${ }^{85} \mathrm{Rb} /{ }^{37} \mathrm{Rb}$ was 2.61 , in good agreement with the natural isotope ratio of 2.59 .

Fe-55: The iron separations were conducted according to PNL-ALO-435/450. Stable iron was used to trace yield. A minor deviation from procedure PNL-ALO-435 was performed to obtain radiochemical yields from stable iron. The iron hydroxide precipitate was dissolved and an aliquot of solution was analyzed by ICP-AES for the iron content. The radiochemical yields averaged $77 \%$.

No ${ }^{35} \mathrm{Fe}$ was detected in either the hot cell preparation blank or the lab preparation blank. The blank spike and sample matrix spike yield-conected ${ }^{55} \mathrm{Fe}$ recoveries are $92 \%$ and $97 \%$, respectively. The samples demonstrated a fair consistency between the three Original Material samples. One sample, 96-5894/96-5894Dup, resulted in an RPD of 30\%; however, the MD is 0.98 indicating these samples are statistically equal, given the error of the measurements.

Ni-59 and Ni-63: The three Original Material samples and duplicates from the sodium peroxide fusion process were analyzed for ${ }^{59} \mathrm{Ni}$ and ${ }^{63} \mathrm{Ni}$. Procedure PNL-ALO-495 was used for the nickel purification, PNL-ALO-450 was used for the LEP counting, and PNL-ALO-474 was used for beta counting (i.e., liquid scintillation). Minor clanges were made to procedure PNL-ALO-495. A nickel-specific column from Eichrom Industries was used in place of the house-made columns. The Eichrom column functions with the same chemical principals and would not affect data quality. Another change is the treatment of the nickel-dimethylglyoxime precipitate on the filter. This precipitate was dissolved using $5 \mathrm{~mL}$ of $3 \mathrm{M} \mathrm{HCl}$ and an aliquot taken for yield determination; another aliquot was taken to dryness, $0.5 \mathrm{~mL}$ water added and $10 \mathrm{~mL}$ Ultima Gold added for subsequent liquid scintillation counting. This is a quality improvement in that there is no potential for nickel to be lost due to potential volatilization of the $\mathrm{Ni}\left(\mathrm{NO}_{3}\right)_{2} 6 \mathrm{H}_{2} \mathrm{O}$.

Radiochemical yields were generally high, nominally $90 \%$. The hot cell preparation blank resulted in no detectable activity for either ${ }^{39} \mathrm{Ni}$ or ${ }^{63} \mathrm{Ni}$. The ${ }^{59} \mathrm{Ni}$ LEP spectra and the ${ }^{63} \mathrm{Ni}$ beta spectra were visually clean from other interferences. No ${ }^{39} \mathrm{Ni}$ activity is detectable in the samples. - The ${ }^{63} \mathrm{Ni}$ concentration is consistent throughout the sample; nominally $1.2 \mu \mathrm{Ci} / \mathrm{g}$. The matrix spiked samples were spiked only with ${ }^{63} \mathrm{Ni}$, as ${ }^{59} \mathrm{Ni}$ is not commercially available. The blank spike, blank spike duplicate, and matrix spike recoveries were excellent at $101 \%, 98 \%$ and $98 \%$, respectively.

Plutonium: Plutonium isotopes were separated in sequence with americium/curium according to procedure PNL-ALO-417, the plutonium was eluted from the column following elution of americium. One change was made to the procedure to effect this separation/elution. The plutonium was reduced to a +3 oxidation state using $\mathrm{TiCl}_{3}$ solution in $4 \mathrm{M} \mathrm{HCl}$. This is a more effective and faster way to elute the plutonium fraction. The plutonium fraction then was precipitation-plated directly according to PNL-ALO-496, and the plutonium measured by alpha energy analysis according to PNL-ALO-422. The plutonium radiochemical yield/counting efficiency was traced with ${ }^{242} \mathrm{Pu}$. 
In order to determine the ${ }^{24 ?} \mathrm{Pu}$ activity, a separate aliquot was processed without added tracer. The indigenous ${ }^{239} \mathrm{Pu}$ was used to trace the yield/count efficiency. The ${ }^{239} \mathrm{Pu}$ activity was determined based on the results of the ${ }^{242} \mathrm{Pu}$-traced aliquot. $\mathrm{No}^{242} \mathrm{Pu}$ was found in the samples so the original premise of using the ${ }^{242} \mathrm{Pu}$ to trace yields is valid. The sample-specific ${ }^{242} \mathrm{Pu}$ detection limit is driven by the tailing of the ${ }^{239} \mathrm{Pu}$ alpha peak into the ${ }^{242} \mathrm{Pu}$ alpha energy region.

The radiochemical yields for the plutonium fractions were good (nominally $70 \%$ ). The precision for ${ }^{239+240} \mathrm{Pu}$, as determined by the RPD, is $12.2 \%$ or better. The RPD associated with ${ }^{238} \mathrm{Pu}$ is high on one sample pair 96-5894/5894dup. Sample 96-5894 had a higher ${ }^{238} \mathrm{Pu}$ concentration than that of the rest of the set. This higher concentration is confirmed on the untraced sample analysis. It may be worthy to note that this same sample also had a higher ${ }^{243 / 244} \mathrm{Cm}$ activity. However, the MD for sample and duplicate is below 1.96, indicating that the results are statistically equal. For the preparation blank, the ${ }^{239+240} \mathrm{Pu}$ activity represent less than $0.1 \%$ of the sample activity, and the ${ }^{238} \mathrm{Pu}$ activity represents less than $0.4 \%$ of the sample activity. The blank spike recoveries were $96 \%$ or better; the matrix spike recoveries were $95 \%$ or better.

Pu-241: The sample preparations for ${ }^{239+240} \mathrm{Pu}$ and ${ }^{238} \mathrm{Pu}$ were used for the subsequent ${ }^{241} \mathrm{Pu}$ determination. The precipitation-plated samples were placed in LSC vials, $10 \mathrm{~mL}$ Ultima Gold were added and the samples counted by liquid scintillation according to PNL-ALO-474. Yield was determined by counting the ${ }^{242} \mathrm{Pu}$ by alpha spectrometry.

The LSC spectra showed both the alpha-peak activity $\left({ }^{239+240} \mathrm{Pu},{ }^{242} \mathrm{Pu},{ }^{238} \mathrm{Pu}\right)$ and the beta peak from the ${ }^{241} \mathrm{Pu}$. Spectral resolution was excellent. The samples showed good agreement at a nominal $3 \mu \mathrm{Ci} / \mathrm{g}$. Two samples were higher than this nominal activity concentration: $96-5822(5.7 \mu \mathrm{Ci} / \mathrm{g})$ and 96-5894 (4.23 $\mu \mathrm{Ci} / \mathrm{g})$. However, because of the relatively high uncertainties, the MD test indicates the results are not statistically different. The mean ${ }^{24} \mathrm{Pu}$ sample activity is 2.4 times that of the ${ }^{239+240} \mathrm{Pu}$. This is the same trend identified for the Vendor Product, where the ratio is 2.3 .

The preparation blank activity (i.e., $0.07 \mu \mathrm{Ci} / \mathrm{g}$ ) is about 50 times lower than the sample activity. There is not detectable activity in the laboratory blanks. The blank spike and matrix spike recoveries are high at $141 \%( \pm 20 \%)$ and $110 \%( \pm 26 \%)$, respectively. This indicates a possible high bias to the technique, however, the error associated with the spike recoveries are high. These errors may have been lower had the sample been spiked with higher ${ }^{241} \mathrm{Pu}$ activity.

Sm-151 and Pm-147: The ${ }^{151} \mathrm{Sm} /{ }^{147} \mathrm{Pm}$ screen/determinations were made on the final sample preparations for ${ }^{241} \mathrm{Am}$ analysis. The precipitation-plated americium preparation was placed into LSC cocktail and counted according to PNL-ALO-474 by liquid scintillation. For instrument calibration, ${ }^{63} \mathrm{Ni}$ was used because its endpoint energy is similar to that of ${ }^{151} \mathrm{Sm}$. The ${ }^{147} \mathrm{Pm}$ has a $224 \mathrm{keV} \mathrm{E}_{\max }$ which tails under the ${ }^{151} \mathrm{Sm}$ peak at $76 \mathrm{keV} \mathrm{E}_{\text {max }}$; thus a summation of ${ }^{151} \mathrm{Sm}$ and ${ }^{147} \mathrm{Pm}$ is provided. The resulting beta spectra indicated a large peak due to the ${ }^{131} \mathrm{Sm}$; however, another peak (most probably ${ }^{147} \mathrm{Pm}$ ) tailed under the samarium peak. The alpha peak associated with americium/curium was also evident. The preparation blank and laboratory blank resulted in a small amount of beta activity relative to the calibration blank; however, their activities are about 3000 times lower than that of the samples. The propagated error associated with these measurements is nominally $4 \%$. A measure of bias cannot be determined without the blank spike/matrix spike. 


\section{HNF-SD-WM-DP-225, REV. 1}

Analytical Summary Report - January 28, 1997

As rare earths, the samarium and promethium radiochemically follow the other rare earths and the $\mathrm{Am}^{+3}$ using the TRU-Spec column chemistry (PNL-ALO-417). The samarium and promethium would also be precipitation-plated (PNL-ALO-496), presumably as efficiently as the americium. Thus the radiochemical yield determined for americium is used to correct for the radiochemical yield of samarium/promethium. A better approach would be to utilize blank and matrix spikes to verify the yield. [Note: This was not done, since the request for the ${ }^{131} \mathrm{Sm}$ analysis was received after the americium separation processing and the preparation additional samples was not approved.]

Along with the rare earths samarium and promethium, europium should also appear in this fraction. The samples were counted by GEA to evaluate the ${ }^{154} \mathrm{Eu}$ and ${ }^{15 s} \mathrm{Eu}$ activities; however, none was detected. The absence of the europium isotopes is not understood at this time. The ${ }^{154} \mathrm{Eu}$ and ${ }^{155} \mathrm{Eu}$ concentrations in the Am-preparation were determined based on the GEA, and the dilution and yield chemistries applied to the samples should have resulted in a detectable quantities of both ${ }^{154} \mathrm{Eu}$ and ${ }^{155} \mathrm{Eu}$. Because they were not detected, no beta interference for ${ }^{154} \mathrm{Eu}$ and ${ }^{155} \mathrm{Eu}$ was present. Had europium been present at the anticipated levels, its contribution would have amounted to nominally $5 \%$ of the beta activity in the $2-90 \mathrm{keV}$ region.

This method must be considered a screening method for ${ }^{15}{ }^{1} \mathrm{Sm} /{ }^{147} \mathrm{Pm}$ because the radiochemical separation from europium had not been performed and the radiochemical recovery is based solely on the americium recovery. Europium did not recover as well as the americium, indicating that samarium/promethium may have low recovery also.

Se-79: The ${ }^{79}$ Se activities were determined following procedures PNL-ALO-440/474. The separation procedure involves an anion/cation exchange to remove most radiochemical interferences followed by a selenium bromide distillation. Since ${ }^{79} \mathrm{Se}$ is not available as a standard, it was not possible to measure standard or sample spike recoveries. Natural selenium metal was added to each sample and the recovered selenium precipitate was used to determine the gravimetric yields.

Acid Digestion Radiochemical yields were nominally $50 \%$ and all known beta spectral interferences were removed. No ${ }^{79} \mathrm{Se}$ beta activity was seen in either the samples or the blanks above the calibration blank background counting rate. Hence, MDA values are reported.

KOH Fusion Radiochemical yields averaged about $50 \%$. No ${ }^{79} \mathrm{Se}$ beta activity was seen in either the samples or the blanks above the calibration blank background counting rate. Hence, MDA values are reported.

Sr-90: The ${ }^{90} \mathrm{Sr}$ activities were determined following procedures PNL-ALO-476/431. Radiochemical yields, traced with ${ }^{85} \mathrm{Sr}$, were excellent averaging $90 \%$. The sample analyses gave similar results with RPD values below $10 \%$. Weak ${ }^{90} \mathrm{Sr}$ activity was detected in the preparation blank and the lab blank; however, the values are a factor of about 10,000 less than the activities in the samples. Standard and sample spike recoveries were $93 \%$ and $88 \%$, respectively.

Sn Isotopes: Radiochemical separation of tin was conducted according to procedure PNL-ALO-453/450, with modifications to accommodate the low-energy photons of ${ }^{119 m} \mathrm{Sn}$ and ${ }^{121 m} \mathrm{Sn}$. Modifications include use of tin carrier, and final analytical product mounting on a $47 \mathrm{~mm}$ filter, allowing for counting of both low and high energy photons. The carrier recovery was measured by 
dissolving the tin off the filter after the counting was completed, then analyzing the solution for elemental tin by ICP-AES. All the analytical results were below the observed detection limits.

$\mathrm{A}^{113} \mathrm{Sn}$ matrix spike was analyzed with this set. Its yield-corrected recovery was about $50 \%$, which is lower than the known sources of uncertainty can explain. One possible explanation is that the chemical form of the spike was different from that of the carrier (e.g., a fluoride versus a chloride complex) with different distribution coefficients on the anion exchange resin.

$\mathrm{Z}_{\mathrm{r}-93:}$ The ${ }^{93} \mathrm{Zr}$ analyses were performed according to draft procedure PNL-ALO-4007. The ${ }^{93} \mathrm{Zr}$ was analyzed using an analytical procecure modified to improve the cartier recovery and provide complete decontamination from plutonium. In summary, the procedure utilizes zirconium carrier to establish radiochemical yield, the sample is passed through three anion exchange columns under varying conditions to separate the impurities. On these samples, the procedure gave carrier recoveries in the $91-99 \%$ range and beta spectra with very little contamination. The ${ }^{93} \mathrm{Zr}$ results are about 40 times above the MDA.

Because no ${ }^{93} \mathrm{Zr}$ standard is available, no matrix spikes or blank spikes are possible. The ${ }^{93} \mathrm{Zr}$ counting efficiency was estimated from ${ }^{63} \mathrm{Ni}$ counting efficiency under similar counting conditions. ${ }^{93} \mathrm{Zr}$ and ${ }^{63} \mathrm{Ni}$ have very similar beta energies, with no gamma emitters.

The samples gave RPD values of $28 \%, 24 \%$, and $45 \%$. These RPD values are greater than the normal $20 \%$ limit, but in all three cases the MD shows that the duplicates are statistically the same at $95 \%$ confidence. Additionally, two samples were run in replicate; they agree within $2 \%$.

The ${ }^{93} \mathrm{Zr}$ beta spectra obtained from these samples appear to show an alpha emitter - presumably plutonium, the most abundant aipha ernitter in the samples. If plutonium alpha is present (that is, ${ }^{238.239 .240} \mathrm{Pu}$ ), ${ }^{24 t} \mathrm{Pu}$ must also be present, buried in the ${ }^{95} \mathrm{Zr}$ peak. To correct the ${ }^{93} \mathrm{Zr}$ count rate for this bias, the ${ }^{241} \mathrm{Pu}$ count rate in each sample was estimated from the alpha count rate and subtracted from the ${ }^{93} \mathrm{Zr}$ count rate. This correction reduced the ${ }^{93} \mathrm{Zr}$ count rate by roughly $6 \%$ in each sample.

The ${ }^{241} \mathrm{Pu}$ correction has considerable uncertainty. If other alpha emitters besides plutonium are contributing to the alpha activity, then the ${ }^{241} \mathrm{Pu}$ correction is too big. If the true ${ }^{241} \mathrm{Pu}$ counting efficiency is much lower than the estimate used in the calculations, then the ${ }^{241} \mathrm{Pu}$ correction is too small. However, because relatively little ${ }^{241} \mathrm{Pu}$ is present in any case, its influence on the-overall uncertainty is minor.

\subsection{Additional Sample Analyses - Centrifuged Solids and Decanted Liquids}

The results of the various analyses performed on the centrifuged solids and decant liquids are presented in Table 4.24 and Table 4.25 for information only. No attempt has been made to correlate the results with the Original Material and Vendor Product analyses. An evaluation of these results will be presented in the Analytical Final Report (In progress). 


\subsubsection{Preparation of Vendor Product (Decant Liquid)}

The HLRF conducted physical testing on Vendor Product sample SPDL-C106-046. Following the conclusion of physical testing activities, samples from the decanted liquid (obtained following centrifuging) were sent to the SAL for limited chemical characterization. The decanted liquid was subsampled for TOC/TIC/TC and IC, and a portion was acid digested (PNL-ALO-128 "HNO $-\mathrm{HCl}$ Acid Extraction of Liquids for Metals Analysis Using a Dry-Block Heater") and distributed for ICP-AES and limited radiochemical analyses.

\subsubsection{Preparation of Original Material (Centrifuged Solids and Decanted Liquid)}

The HLRF conducted physical testing on Original Material sample SPDL1-C106-002. Following the conclusion of the physical testing activities, samples from the centrifuged solids and decanted liquid were sent to the SAL for limited chemical characterization. Two fusions techniques, PNL-ALO-115 "Solubilization of Metals from Solids Using KOH-KNO ${ }_{3}$ Fusion"; PNL-ALO-116 "Solubilization of Platinum Group Metals using a $\mathrm{Na}_{2} \mathrm{O}_{2}-\mathrm{NaCl}$ Fusion," were performed on the centrifuged solids sample and aliquots distributed for ICP-AES. A portion of the centrifuged solids was water leached per PNL-ALO-103 "Water Leach of Sludges, Soils and Other Solid Samples," filtered, and aliquots distributed for anion analysis by Ion Chromatography. The decanted liquid was subsampled for anion determinations by lon Chromatography, and a portion was acid digested (PNL-ALO-128 " $\mathrm{HNO}_{3}-\mathrm{HCl}$ Acid Extraction of Liquids for Metals Analysis Using a Dry-Block Heater") and distributed for ICP-AES.

\subsubsection{Chemical Änalysis}

The analyses of the Vendor Product decanted liquid (following centrifuging) are presented in Table 4.24. The IC (anions) and TOC analyses were performed in duplicate directly on the decanted liquid. The Vendor Product decanted liquid was subjected to an acid digestion or acid dilution prior to ICP-AES (or radiochemical) analysis.

The analysis of the Original Material centrifuged solids and decanted liquid (following centrifuging) are presented in Table 4.25. The IC analyses were performed in duplicate directly on the decanted liquid, as well as on water leaches from the centrifuged solids. The centrifuged solid sample was prepared in duplicate by potassium hydroxide fusion and sodium peroxide fusion technique prior-to ICP-AES analysis.

\subsubsection{Radiochemical Analysis}

The Vendor Product decant liquid (following centrifuging) was prepared by acid digestion, and analyzed for total alpha, ${ }^{90} \mathrm{Sr}, \mathrm{GEA}$, and ${ }^{99} \mathrm{Tc}$ (by ICP-MS). Weak ${ }^{90} \mathrm{Sr}$ activity $(0.004 \mu \mathrm{Ci} / \mathrm{g}$ of liquid), about 20,000 times less than that measured in the Vendor Product (on a per gram basis), was detected. Cesium-137 was the only gamma emitter detected, and the activity $(2.3 \mu \mathrm{Ci} / \mathrm{g}$ of liquid) is about 200 times less than the activity measured in the Vendor Product. 


\section{HNF-SD-WM-DP-225, REV. 1}

Analytical Summary Report - January 28, 1997

\subsection{REFERENCES}

Brooks, KP, et. al. 1997. Bench-Scale Enhanced Sludge Washing and Gravity Settling of Hanford Tank C-106 Sludge. PNNL-1 1432, Pacific Northwest National Laboratory, Richland, Washington.

Jones, TE. 1996. Addendum 1 of the Letter of Instruction (LOI) for TWRS Privatization Contractor Samples Addressing Waste Envelope D Materials, Revision O. Pacific Northwest National Laboratory, Richland, Washington.

Kuhl-Klinger, KJ. 1996, Quality Assurance Plan for Activities Conducted by the Analytical Chemistry Laboratory (ACL). MCS-033, Pacific Northwest National Laboratory, Richland, Washington.

Kuhl-Klinger KJ and MW Urie. 1997. Tank Waste Remediation System Privatization Contractor Sample Analytical Summary Report, Addendum 1 (January 17). Pacific Northwest National Laboratory, Richland, Washington.

Lumetta, GJ, et. al. 1996. Washing and Caustic Leaching of Hanford Tank C-106 Sludge. PNNL-1 1381, Pacific Northwest National Laboratory, Richland, Washington.

Urie, MW. 1996. Letter Report, MW Urie to CM Seidel, "PNNL ACL Submittal of Organic and Radionuclide Results from Analysis of C-106 Original Material Supernate". October 31, 1996

Wiemers, KD. 1996. Addendum I of the Letter of Instruction (LOI) for TWRS Privatization Contractor Samples Addressing Waste Envelope D Materials, Revision 1. Pacific Northwest National Laboratory, Richland, Washington. 
Table 4.3 ICP-AES KOH Fusion--Vendor Product C106 Privatization Contractor Sample

\begin{tabular}{|c|c|c|c|c|c|c|c|c|c|c|c|c|}
\hline \multirow{2}{*}{ ard } & $\begin{array}{l}\text { Process Blank } \\
\text { 96-6io3.P8. }\end{array}$ & 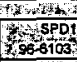 & $\begin{array}{l}304704 \\
0103000\end{array}$ & 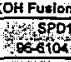 & $\begin{array}{l}\text { bing Ni Cru } \\
6-049 \text { at } \\
6104000\end{array}$ & 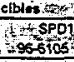 & $\begin{array}{l}6049+409 \\
6105005\end{array}$ & 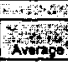 & 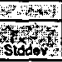 & \multirow{2}{*}{ 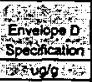 } & \multicolumn{2}{|c|}{ 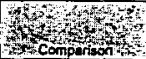 } \\
\hline & 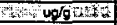 & 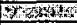 & 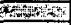 & sints & $x+1+x, 4$ & $6+4 x_{1}^{2}+w_{6}$ & 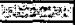 & 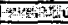 & 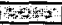 & & Ave/Spec & Rrigot \\
\hline $\begin{array}{l}\text { Ag } \\
\text { A } \\
\text { As } \\
\text { B } \\
\text { Ba }\end{array}$ & $\begin{array}{c}- \\
{[248]} \\
- \\
{[90]} \\
-\end{array}$ & $\begin{array}{c}817 \\
52.600 \\
- \\
{[345]} \\
405\end{array}$ & $\begin{array}{c}1,010 \\
50,200 \\
- \\
1476] \\
395 \\
\end{array}$ & $\begin{array}{c}9,370 \\
34,400 \\
- \\
{[193]} \\
436 \\
\end{array}$ & $\begin{array}{c}920 \\
52,400 \\
- \\
{[259]} \\
422 \\
\end{array}$ & $\begin{array}{c}803 \\
48,500 \\
- \\
2701 \\
384 \\
\end{array}$ & $\begin{array}{c}1260 \\
48,900 \\
- \\
{[267]} \\
397 \\
\end{array}$ & $\begin{array}{l}1.030 \\
51.200 \\
e 479 \\
0.031 \\
407 \\
\end{array}$ & $\begin{array}{c}240 \\
2300 \\
-N / 1- \\
00 \\
19\end{array}$ & $\begin{array}{l}4.38 E+05 \\
1.11 E+05 \\
1.29 E+03 \\
1.03 E+04 \\
3.61 E+04\end{array}$ & $\begin{array}{l}2.35 E-01 \\
4.62 E-01 \\
3.72 E-01 \\
2.24 E-02 \\
1.13 E-02\end{array}$ & \\
\hline $\begin{array}{l}\mathrm{Be} \\
\mathrm{Bi} \\
\mathrm{Ca} \\
\mathrm{Cd} \\
\mathrm{Co}\end{array}$ & $\begin{array}{c}- \\
- \\
{[1,120)} \\
- \\
- \\
\end{array}$ & $\begin{array}{c}- \\
{[552]} \\
7,890 \\
{[83]} \\
- \\
\end{array}$ & $\begin{array}{c}- \\
{[551]} \\
0.250 \\
{[84]} \\
-\end{array}$ & $\begin{array}{l}-\overline{1} \\
{[52 \pi]} \\
6,950 \\
100] \\
-\end{array}$ & $\begin{array}{c}- \\
{[504]} \\
6,600 \\
{[80]} \\
-\end{array}$ & $\begin{array}{c}- \\
{[528)} \\
8,500 \\
{[01]} \\
-\end{array}$ & $\begin{array}{c}- \\
{[324]} \\
5, \pi 70 \\
{[78]} \\
- \\
\end{array}$ & $\begin{array}{r}<0 \\
{[538]} \\
6,690 \\
{[B 1]} \\
<523 \\
\end{array}$ & $\begin{array}{c}-\mathbf{N} / \mathrm{A}- \\
14 \\
720 \\
2 \\
-\mathrm{N} / \mathrm{M} \\
\end{array}$ & $\begin{array}{l}5.15 E+02 \\
2.22 E+04 \\
5.67 E+04 \\
3.61 E+04 \\
5.44 E+03\end{array}$ & $\begin{array}{l}1.69 E-02 \\
2.43 E-02 \\
1.19 E-01 \\
2.25 E-03 \\
8.12 E-02\end{array}$ & \\
\hline $\begin{array}{l}\mathrm{Co} \\
\mathrm{Cr} \\
\mathrm{Cu} \\
\mathrm{Oy} \\
\mathrm{Eu}\end{array}$ & $\begin{array}{l}- \\
{[63]} \\
{[49]} \\
- \\
-\end{array}$ & $\begin{array}{r}- \\
764 \\
- \\
- \\
\end{array}$ & $\begin{array}{c}\overrightarrow{800} \\
{[111]} \\
- \\
- \\
\end{array}$ & $\begin{array}{l}- \\
853 \\
{[113]} \\
= \\
= \\
\end{array}$ & $\begin{array}{c}- \\
025 \\
{[109]} \\
- \\
\end{array}$ & $\begin{array}{c}- \\
700 \\
{[115]} \\
- \\
- \\
\end{array}$ & $\begin{array}{c}- \\
752 \\
{[102]} \\
- \\
=\end{array}$ & $\begin{array}{l}<87 \\
802 \\
{[t 09]} \\
<87 \\
<174 \\
\end{array}$ & $\begin{array}{r}-N / A- \\
30 \\
5 \\
-N / A- \\
-N / A- \\
\end{array}$ & $\begin{array}{l}7.61 E+03 \\
5.41 E+03 \\
3.86 E+03 \\
2.06 E+02 \\
1.29 E+02\end{array}$ & $\begin{array}{l}2.42 \mathrm{E}-02 \\
1.48 \mathrm{E}-01 \\
2.83 \mathrm{E}-02 \\
4.23 \mathrm{E}-01 \\
1.35 \mathrm{E}+\infty 0\end{array}$ & $*$ \\
\hline $\begin{array}{l}\mathrm{F} \\
\mathrm{K} \\
\mathrm{La} \\
\mathrm{Li} \\
\mathrm{Mo}\end{array}$ & $\begin{array}{l}\text { [268] } \\
\mathrm{N} / \mathrm{U} \\
- \\
- \\
- \\
\end{array}$ & $\begin{array}{c}142,000 \\
N N] \\
{[123]} \\
{[86]} \\
{[1,370]} \\
\end{array}$ & $\begin{array}{c}138.000 \\
\mathrm{~N}] \\
{[130]} \\
{[04]} \\
{[1,440]} \\
\end{array}$ & $\begin{array}{c}152,000 \\
N=0 \\
{[118]} \\
-\overline{11,500]} \\
\end{array}$ & $\begin{array}{c}145,000 \\
\text { NU } \\
{[1,4]} \\
- \\
{[1,450]}\end{array}$ & $\begin{array}{c}132.000 \\
N / J \\
{[105]} \\
- \\
{[1,180]}\end{array}$ & $\begin{array}{c}134,000 \\
\mathrm{NU} \\
{[121]} \\
- \\
{[1,210]}\end{array}$ & $\begin{array}{c}141,000 \\
N / 0 \\
{[119]} \\
{[90]} \\
{[1,350]}\end{array}$ & $\begin{array}{c}7600 \\
\text { N/U } \\
0 \\
0 \\
130 \\
\end{array}$ & $\begin{array}{l}2.29 \mathrm{E}+05 \\
1.06 \mathrm{E}+04 \\
2.08 \mathrm{E}+04 \\
1.11 \mathrm{E}+03 \\
1.67 \mathrm{E}+04\end{array}$ & $\begin{array}{c}6.14 E-01 \\
N N \\
5.75 E+03 \\
0.00 E-02 \\
0.11 E-02\end{array}$ & Nu \\
\hline $\begin{array}{l}\text { Mn } \\
\text { Mo } \\
\text { Na } \\
\text { Nd } \\
\text { Ni }\end{array}$ & $\begin{array}{c}181] \\
- \\
2,990 \\
- \\
\text { N/U } \\
\end{array}$ & $\begin{array}{c}3,070 \\
- \\
137,000 \\
102] \\
\mathrm{N} / \mathrm{U} \\
\end{array}$ & $\begin{array}{c}3,260 \\
- \\
145,000 \\
{[427]} \\
\mathrm{N} N] \\
\end{array}$ & $\begin{array}{c}3,330 \\
- \\
131,000 \\
{[373]} \\
\mathrm{NU}\end{array}$ & $\begin{array}{c}3.170 \\
- \\
135.000 \\
{[347} \\
\mathrm{N} \pi\end{array}$ & $\begin{array}{c}3,040 \\
- \\
153,000 \\
{[347} \\
\text { NO }\end{array}$ & $\begin{array}{c}2,960 \\
- \\
151,000 \\
{[376]} \\
\mathrm{NU}\end{array}$ & $\begin{array}{c}3.140 \\
<87 \\
142,000 \\
{[379]} \\
\mathrm{NO} \\
\end{array}$ & $\begin{array}{c}140 \\
-\mathrm{N} / \mathrm{A}- \\
9000 \\
31 \\
\mathrm{NN}\end{array}$ & $\begin{array}{l}5.15 E+04 \\
5.15 E+00 \\
1.55 E+05 \\
1.37 E+04 \\
1.04 E+04\end{array}$ & $\begin{array}{c}0.09 E-02 \\
1.69 E-02 \\
2.19 E-01 \\
2.77 E-02 \\
N W\end{array}$ & NU \\
\hline $\begin{array}{l}P \\
P b \\
P d \\
\text { Rh } \\
\text { Ru }\end{array}$ & $\begin{array}{l}- \\
- \\
- \\
- \\
-\end{array}$ & $\begin{array}{c}3.230 \\
2,040 \\
- \\
- \\
-\end{array}$ & $\begin{array}{c}3,680 \\
2,060 \\
- \\
- \\
-\end{array}$ & $\begin{array}{c}3,620 \\
2,560 \\
- \\
- \\
- \\
\end{array}$ & $\begin{array}{c}2,650 \\
3,510 \\
- \\
- \\
- \\
\end{array}$ & $\begin{array}{c}3,100 \\
3,200 \\
= \\
= \\
=\end{array}$ & $\begin{array}{c}3,530 \\
3,250 \\
- \\
- \\
- \\
\end{array}$ & $\begin{array}{r}3,330 \\
2,950 \\
<1,310 \\
=523 \\
<1,740 \\
\end{array}$ & $\begin{array}{c}370 \\
510 \\
-N / A- \\
-N / A- \\
-N / A-\end{array}$ & $\begin{array}{l}1.39 E+04 \\
5.76 E+03 \\
1.03 E+03 \\
1.03 E+03 \\
2.03 E+03\end{array}$ & $\begin{array}{l}2.39 E-01 \\
3.37 E-01 \\
1.27 E+\infty 0 \\
5.07 E-01 \\
5.15 E-01\end{array}$ & - \\
\hline $\begin{array}{l}\mathrm{Sb} \\
\text { Se } \\
\text { Si } \\
\text { Sn }\end{array}$ & $\begin{array}{l}- \\
- \\
-\end{array}$ & $\begin{array}{c}- \\
\overline{6} \\
\overline{-} \\
-\end{array}$ & $\begin{array}{c}- \\
- \\
57.300 \\
=\end{array}$ & $\begin{array}{c}- \\
- \\
75,100 \\
- \\
\end{array}$ & $\begin{array}{c}- \\
72.500 \\
- \\
\end{array}$ & $\begin{array}{c}- \\
- \\
0+200 \\
=\end{array}$ & $\begin{array}{c}\overline{-} \\
\overline{5} .900 \\
-\end{array}$ & $\begin{array}{r}<871 \\
\times 810 \\
68.900 \\
<3.920 \\
\end{array}$ & $\begin{array}{l}\text {-NNA- } \\
\text {-NAA } \\
4500 \\
\text {-NNA- }\end{array}$ & $\begin{array}{l}6.70 E+03 \\
4.12 E+03 \\
1.49 E+05 \\
2.83 E+02\end{array}$ & $\begin{array}{l}1.30 E-01 \\
\text { S.48E-01 } \\
4.61 E .01 \\
\text { t.20E+01 }\end{array}$ & \\
\hline $\begin{array}{l}\text { St } \\
\text { Te } \\
\text { Th } \\
\text { Ti } \\
\pi\end{array}$ & $\begin{array}{l}- \\
- \\
- \\
-\end{array}$ & $\begin{array}{c}79] \\
- \\
- \\
1,010 \\
- \\
\end{array}$ & $\begin{array}{c}{[78]} \\
- \\
- \\
065 \\
- \\
\end{array}$ & $\begin{array}{c}{[37]} \\
- \\
- \\
1,100 \\
- \\
\end{array}$ & $\begin{array}{c}{[B]} \\
- \\
- \\
1,150 \\
- \\
\end{array}$ & $\begin{array}{l}{[73]} \\
- \\
- \\
959 \\
-\end{array}$ & $\begin{array}{c}74 \\
- \\
- \\
954 \\
- \\
\end{array}$ & $\begin{array}{r}79 ! \\
<2.810 \\
=1,740 \\
1,030 \\
<071 \\
\end{array}$ & $\begin{array}{c}5 \\
-N A- \\
-N / A- \\
79 \\
-N / A- \\
\end{array}$ & $\begin{array}{l}4.12 E+03 \\
1.03 E+03 \\
4.12 E+03 \\
1.03 E+04 \\
3.61 E+03\end{array}$ & $\begin{array}{l}1,02 \mathrm{E}-02 \\
2.54 \mathrm{E}+00 \\
4.23 \mathrm{E}-01 \\
0.98 \mathrm{E}-02 \\
2,42 \mathrm{E}-01\end{array}$ & • \\
\hline $\begin{array}{l} \\
V \\
W \\
Y \\
Z n \\
Z r\end{array}$ & $\begin{array}{l}- \\
- \\
- \\
- \\
- \\
-\end{array}$ & $\begin{array}{l}- \\
- \\
- \\
- \\
{[203]} \\
1,090\end{array}$ & $\begin{array}{l}- \\
- \\
= \\
- \\
{[188]} \\
1.500\end{array}$ & $\begin{array}{c}- \\
- \\
- \\
- \\
{[174]} \\
1.260\end{array}$ & $\begin{array}{c}- \\
- \\
- \\
- \\
{[181]} \\
{[515]}\end{array}$ & $\begin{array}{c}- \\
- \\
- \\
- \\
{[210]} \\
{[470]}\end{array}$ & $\begin{array}{l}- \\
- \\
= \\
- \\
1141] \\
1.790\end{array}$ & $\begin{array}{l}<4,360 \\
<87 \\
<3,480 \\
<131 \\
{[183]} \\
1,110 \\
\end{array}$ & $\begin{array}{l}-N / A- \\
-N / A- \\
-N / A- \\
-N / A- \\
25 \\
530 \\
\end{array}$ & $\begin{array}{l}1.05 E+05 \\
2.58 E+02 \\
1.01 E+03 \\
1.29 E+03 \\
3.35 E+03 \\
1.19 E+05\end{array}$ & $\begin{array}{l}4,03 E-02 \\
3,30 E-01 \\
1.03 E+00 \\
1,01 E-01 \\
5,46 E-02 \\
0,35 E-03\end{array}$ & " \\
\hline
\end{tabular}

- Analyte not dotected above the IDL: only averago lDL reported as less than (4) value. NA Not Applicabla

NUU Mot Usable

Results in brackets are qualified but they ara included in the cakulation of the average. 
Table 4.4 ICP-AES Na2O2 Fusion-Vendor Product C106 Privatization Contractor Sample

\begin{tabular}{|c|c|c|c|c|c|c|c|c|c|c|c|c|}
\hline \multirow{2}{*}{ 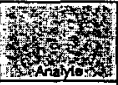 } & \multirow{2}{*}{ 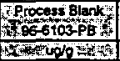 } & \multirow{2}{*}{\multicolumn{2}{|c|}{ 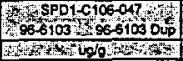 }} & \multirow{2}{*}{\multicolumn{2}{|c|}{ 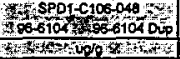 }} & \multirow{2}{*}{\multicolumn{2}{|c|}{ 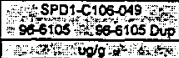 }} & \multirow{2}{*}{\multicolumn{2}{|c|}{ 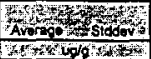 }} & 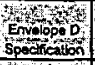 & \multicolumn{2}{|c|}{ 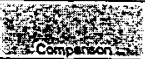 } \\
\hline & & & & & & & & & & $34 \log / 9 \mathrm{x}$ & Aver'spect & 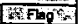 \\
\hline $\mathrm{Ag}$ & [50] & 1,890 & 3,580 & 3,450 & 3,080 & 3,350 & 3,420 & 3,400 & 880 & $4.398+03$ & $7.28 E-01$ & \\
\hline A) & [21日] & 48,000 & 47,300 & 51,400 & $\$ 3,200$ & 49,800 & 47,500 & 49.500 & 2400 & $1.11 E+05$ & 4.47E-01 & \\
\hline As & - & - & - & - & - & - & - & $<510$ & $-N A-$ & $1,29 E+03$ & $3.98 \mathrm{E}-01$ & \\
\hline$B$ & - & [186] & [159] & - & $=$ & - & [116] & {$[154]$} & 35 & $1.03 E+04$ & $1,49 E-22$ & . \\
\hline $\mathrm{Ba}$ & [25] & 414 & 490 & 457 & 470 & 431 & 410 & 437 & 22 & $3.61 E+04$ & $1.21 E-02$ & \\
\hline Be & - & - & - & - & - & - & - & $<9$ & $-\mathrm{N} / \mathrm{A}$ & $5.15 E+02$ & $1.80 E-02$ & \\
\hline $\mathrm{Bi}$ & - & {$[506]$} & [B39] & {$[681]$} & [569] & {$[580]$} & [554] & {$[s: 2]$} & so & $2.22 E+04$ & $2.52 E-02$ & \\
\hline $\mathrm{Ca}$ & 14.18 아 & 0,350 & 11,000 & 10.300 & 9.000 & 0.970 & 10,700 & 9,050 & 1000 & $\$ .67 E+04$ & 1.74E-01 & \\
\hline $\mathrm{Cd}$ & - & กบ & \{Bit & leg & $(8 \pi)$ & [B1 & $|\mathrm{a} 1|$ & [1] & 5 & $3.81 \mathrm{E}+04$ & $2.24 E-03$ & \\
\hline C. & - & - & - & $=$ & $=$ & - & - & $<1,500$ & $-\mathrm{N} / \mathrm{A}$ & 6.44E 403 & 2-ASE-01 & \\
\hline Co & - & - & - & - & - & - & - & $<93$ & $-N / A \sim$ & $3.61 E+03$ & $2.57 E-02$ & \\
\hline $\mathrm{Cr}$ & [45] & 47 & 573 & 737 & $n 1$ & 695 & 675 & 700 & 48 & $5.4 \pm E+03$ & 129E-01 & \\
\hline $\mathrm{Cu}$ & [49] & [84] & [110] & [126] & [119] & {$[101]$} & {$[111]$} & [109] & 15 & $3.86 E+03$ & $2.81 E-02$ & \\
\hline Dy & - & - & - & - & - & - & - & $<3$ & $-\mathrm{N} / \mathrm{A}$ & $2.06 E+02$ & 4.50E-D1 & \\
\hline Eu & - & $=$ & - & - & - & - & $=$ & $<185$ & $-\mathrm{N} / \mathrm{A}=$ & $1.29 E+02$ & $1.40 \varepsilon+\infty)$ & $\div$ \\
\hline $\mathrm{Fa}$ & [270] & 124.000 & 126,000 & 138,000 & 142,000 & 130,000 & 123,000 & $1 \longdiv { 3 1 . 0 0 0 }$ & 7800 & $2.29 E+05$ & 5.59E-D1 & \\
\hline$K$. & {$[8,930]$} & {$[4,540]$} & {$[B, 7 \% 0]$} & {$[9,250]$} & {$[8,830]$} & {$[7,580]$} & {$[9,420]$} & {$[7,590]$} & 1700 & $1.08 E+06$ & 7.18E-01 & \\
\hline La & - & [118] & {$[176]$} & [?56] & [171] & {$[166]$} & {$[157]$} & {$[184]$} & 26 & $206 E+04$ & $7.00=03$ & \\
\hline Lì & [59] & [105] & [139] & [54] & [71] & $\Gamma \theta$ & |99| & [94! & 25 & $1.11 E+03$ & $8.47 E-02$ & \\
\hline $\mathrm{Mg}$ & - & {$[1,210]$} & {$[1,3990]$} & {$[1,380]$} & {$[1,410]$} & {$[1,250]$} & {$[1,150]$} & {$[1,310]$} & ย9 & $1.67 E+04$ & 7.79E- 02 & \\
\hline $\mathrm{Mn}$ & - & 2,470 & 2,500 & 2,720 & 2,520 & 2.590 & 2.470 & 2.590 & 180 & $5.15 E+04$ & 5.02E-02 & \\
\hline Mo & - & - & - & - & - & - & - & $<93$ & $-N / A--$ & $5.15 E+03$ & 1. $.00 E-02$ & \\
\hline $\mathrm{Na}$ & NN & NU & No & NN & No & No & Nus & MN & NOS & $1.55 E+05$ & NN & NAS \\
\hline Nd & [254] & {$[400]$} & [5.6] & {$[562]$} & [525] & [518] & [550] & {$[517]$} & 59 & $4.37 E+C A$ & $3.78 E-02$ & \\
\hline $\mathrm{Ni}$ & 597 & 1,470 & 1.580 & 1,780 & 1,790 & 1.530 & 1,700 & 1,660 & 120 & $1.8 \mathrm{~g} E+04$ & 8. $02 E-02$ & \\
\hline$P$ & [239] & 3,650 & 3.920 & 4,570 & 4,560 & 3,520 & 2.000 & 3,40 & 670 & $1.39 E+04$ & $2.78 E-21$ & \\
\hline $\mathrm{Pb}$ & [203] & 3,230 & 3,450 & 3,780 & 3,760 & 3,580 & 3.390 & 3.530 & 210 & $6.76 \mathrm{E}+03$ & $4.03 E-01$ & \\
\hline $\mathrm{Pd}$ & - & - & - & - & - & - & - & $<1,390$ & -N/A- & $1.03 E+03$ & $1.35 E+00$ & - \\
\hline$R h$ & - & - & - & - & - & - & - & $<5 s$ & $-\mathrm{N} / \mathrm{A}$ & $1.03 E+03$ & $5.40 E-01$ & \\
\hline Ru & $=$ & $=$ & $=$ & - & - & $=$ & - & $\propto 1,850$ & $-\mathrm{N} / \mathrm{A}-$ & $2.43 E+03$ & 5.54E-01 & \\
\hline Sb & $=$ & - & - & - & - & - & - & $<927$ & $-\mathrm{N} / \mathrm{A}-$ & $6.705+0.3$ & $1,38 \mathrm{E}-01$ & \\
\hline Se & - & - & - & - & - & - & - & $<0$ & $-N / A$ & $4,12 E+03$ & $1.57 E-01$ & \\
\hline $\mathrm{Si}$ & - & 82.000 & 82.200 & 67,600 & 71.200 & 63.500 & 61,300 & 04,600 & 3900 & $1.49 \varepsilon+05$ & 4. $33 E-01$ & \\
\hline Sn & $=$ & - & - & $=$ & $=$ & $=$ & - & $\times 4,170$ & -NAA- & $2.63 E+02$ & 1.47E & - \\
\hline Sr & RII & {$[127]$} & [456] & {$[146]$} & {$[147]$} & {$[134]$} & [159] & {$[145]$} & 12 & $4.12 E+03$ & $3.51 E-02$ & \\
\hline Te & - & - & - & - & - & - & - & $<2,780$ & $-N / A$ & $1.03 E+03^{\circ}$ & $2.70 E+00$ & - \\
\hline$T h$ & - & - & - & - & - & - & - & $<1,050$ & $-N / A$ & $4.12 \bar{E}+00$ & $4.50 \vec{E}-0 \mathrm{~T}$ & \\
\hline$\pi$ & - & Eso & 979 & 1,130 & 1,170 & 987 & 922 & 1.020 & 100 & $1.03 E+04$ & S.9AE-02 & \\
\hline$\pi$ & - & - & - & - & - & - & - & $<927$ & $-\mathrm{N} / \mathrm{A}-$ & $3.81 E+03$ & 2.57E-01 & \\
\hline $\mathbf{U}$ & - & - & - & - & - & - & - & $<6,950$ & $-N / A-$ & $1.00 E+05$ & $8.12 E-02$ & \\
\hline v & - & - & - & - & - & - & - & $<93$ & $-\mathrm{NA}$ & $2.58 E+02$ & $3.50 E-01$ & \\
\hline$w$ & - & - & - & - & - & - & - & 23.710 & - Wu & $1.8 t E+03$ & $1.24 E+\infty$ & - \\
\hline$y$ & - & - & - & - & - & - & - & $<130$ & -NA & $1.29 E+03$ & $1.08 \varepsilon-01$ & \\
\hline Zn & - & [150] & [159) & [151] & {$[107$} & [182] & (15s) & [181] & 14 & $3.35 E+03$ & $4.80 E-02$ & \\
\hline $2 r$ & Nos & NUS & No & $\mathrm{N} / \mathrm{N}$ & NO & NN & $\mathrm{NNO}$ & Nu & No & $1,10 \varepsilon+05$ & NU & NU \\
\hline
\end{tabular}

- Analyte not detected above the IDL; only average IDL reported as less than (s) value. NA Not Applicable

MU Not Usable

Results in brackets are qualfied but they are included in the calaulation of the average. 
Table 4.5 ICP-AES Acid Digestion-Vendor Product C106 Privatization Contractor Samplo

\begin{tabular}{|c|c|c|c|c|c|c|c|c|c|c|c|c|}
\hline \multirow{2}{*}{ 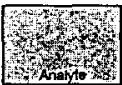 } & \multirow{2}{*}{ 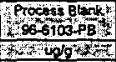 } & \multirow{2}{*}{\multicolumn{2}{|c|}{ 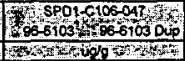 }} & \multirow{2}{*}{\multicolumn{2}{|c|}{ 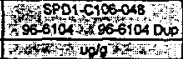 }} & \multirow{2}{*}{\multicolumn{2}{|c|}{ 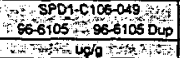 }} & \multirow{2}{*}{\multicolumn{2}{|c|}{ 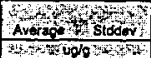 }} & \multirow{2}{*}{ 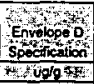 } & \multicolumn{2}{|c|}{ 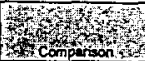 } \\
\hline & & & & & & & & & & & Ave/Spict & Fing \\
\hline$A g$ & - & 75.8 & 75.8 & 78.1 & 78.1 & 68.4 & 81.3 & 78 & 3 & $4.38 E+03$ & $1.73 E-02$ & \\
\hline$A$ & [15] & 48,900 & 46,500 & 48.100 & 48.100 & 47.500 & 50,100 & 47.900 & 1300 & $1.11 E+05$ & $4.33 E-01$ & \\
\hline As & - & - & - & - & - & - & - & $<123$ & $-N / 2$ & $1.29 \mathrm{E}+03$ & O.SSE-02 & \\
\hline$B$ & [581] & 310 & 292 & 153 & 103 & 201 & 211 & 225 & 62 & $1.03 E+04$ & $2.9 B E-02$ & \\
\hline $\mathrm{Be}$ & $=$ & 408 & 403 & 417 & 416 & 411 & 427 & 415 & 10 & $3.61 E+04$ & $1.15 E-02$ & \\
\hline Be & - & - & - & - & - & - & - & $<1$ & $-N A$ & $5.15 E+02$ & $2.3 \mathrm{~d} E-03$ & \\
\hline$B i$ & - & 426 & 414 & 430 & 429 & 426 & $4 T$ & 430 & 11 & $2.22 E+04$ & $1.94 E-02$ & \\
\hline $\mathrm{Ca}$ & $\nabla \eta$ & 3,760 & 3,740 & 3,900 & 3,910 & 3,780 & 3,000 & 3,040 & 93 & $5.57 \varepsilon+04$ & $6.785-02$ & \\
\hline $\mathrm{Cd}$ & - & 882 & 06.3 & 64.7 & $8 d 4$ & 66.5 & 99.7 & 73 & 13 & $3.81 E+0$. & $2.025-03$ & \\
\hline Ce & - & [307] & 297 & 2901 & [28a; & 295 & {$[\mathrm{~B}: \mathrm{B}]$} & (299) & 11 & 6.4AE E.03 & $4.65 E-02$ & \\
\hline Co & - & {$[281$} & [27] & [23] & (2) & [28) & [29] & [28] & 1 & $3.51 E+03$ & $7.80 E-03$ & \\
\hline $\mathrm{Cr}$ & - & 327 & 520 & 535 & 533 & 527 & $\$ 58$ & 533 & 12 & $5.41 E+03$ & $9.85 E-02$ & \\
\hline $\mathrm{Cu}$ & - & 77.1 & 76.2 & 78.6 & $\pi .7$ & 74.9 & $\infty 0.3$ & 78 & 2 & $3.46 \mathrm{E}+03$ & $2.00 \mathrm{E}-02$ & \\
\hline Dy & - & - & - & - & - & - & - & $<12$ & -NIA- & $2.06 E+02$ & \$.9TE-02 & \\
\hline Eu & - & - & - & $=$ & - & $=$ & - & $\times 25$ & $-\mathrm{N} / \mathrm{A}-$ & $1.25 \mathrm{E}+02$ & $1.915-01$ & \\
\hline$F 0$ & 间 & 135,000 & 134,000 & 140,000 & 139.000 & 133,000 & 140,000 & 137,000 & 3200 & $2.29 \mathrm{E}=0.05$ & S.97E-01 & \\
\hline$k$ & - & - & - & - & - & - & - & $<492$ & -Mia- & $1.06 E \$ 4$ & $4.65 E-02$ & \\
\hline La & - & [F8] & [94] & [95; & [54] & [\&4] & [102] & [98] & 3 & $2.0 E E+04$ & $4.68 E-03$ & \\
\hline $\mathrm{Li}$ & - & 85.5 & 842 & [26] & [26] & [25] & {$[27]$} & 48 & 30 & $1.9 t E+03$ & 4.14E-C2 & \\
\hline $\mathrm{Mg}$ & - & $\begin{array}{r}752 \\
\end{array}$ & 741 & 733 & 771 & 718 & 780 & 754 & 23 & $1.67 E+04$ & 4.5QE- 02 & \\
\hline $\mathrm{Mr}$ & - & 2.580 & 2.530 & 2,620 & 2,800 & 2.550 & 2,700 & 2.600 & 60 & $5.15 E+04$ & S.04E-02 & \\
\hline Mo & - & [27] & [25] & $2 T$ & [06] & {$[2 \pi]$} & [20] & [2]] & 1 & $5.15 E+03$ & $5.19 E-03$ & \\
\hline Na & [181] & 168,000 & 165,000 & 149.000 & 158,000 & 164,000 & 170,000 & 162,000 & 8000 & $1.55 E+05$ & $1.0 s \varepsilon+00$ & - \\
\hline Nd & - & 235 & 278 & 279 & 276 & 277 & 297 & 282 & . & $1.37 E+04$ & $2.08 E-02$ & \\
\hline $\mathrm{Ni}$ & - & 1,130 & 1,100 & 1,130 & 1,120 & 1,120 & 1,190 & 1,130 & 31 & $1.20 E+04$ & $6.025-02$ & \\
\hline $\mathbf{P}$ & - & 1,450 & 1,380 & 1,290 & 1,270 & 1,310 & 1,350 & 1,340 & 86 & $1.39 E+04$ & $9.64 E-02$ & \\
\hline $\mathrm{Pb}$ & - & 3,370 & 1,340 & 3,460 & 3,450 & 3,400 & 3.560 & 3,430 & 70 & $8.76 E+03$ & $3.92 \mathrm{E}-01$ & \\
\hline $\mathrm{Pd}$ & - & - & - & - & - & - & - & $<369$ & -NAA- & $1.03 E * 03$ & J.SaE-01 & \\
\hline Rh & - & . & - & - & - & - & - & $\times 74$ & -NAS- & $1.02 \varepsilon+03$ & $7.16 E-02$ & \\
\hline$R u$ & - & $=$ & $=$ & $=$ & - & $=$ & - & c 738 & -NiA- & $2.85 \mathrm{E}+03$ & 2.81E-01 & \\
\hline So & - & - & - & $=$ & - & - & - & $<74$ & -N/A- & $5.70 E+03$ & $1.10 E-02$ & \\
\hline Se & - & - & - & - & - & - & - & $<369$ & -Nin- & $4.12 E+03$ & 1.96E-02 & \\
\hline $\mathrm{Si}$ & [165] & 3,150 & 2,360 & 2,290 & 2,930 & 2.750 & 3,310 & 2,800 & 430 & $1,45 E+05$ & $1.04 E-02$ & \\
\hline Sn & - & - & - & $=$ & - & - & - & $<4,060$ & $-N / A-$ & $2.93 \mathrm{E}+02$ & $1.43 E+01$ & $\cdot$ \\
\hline Sr & - & 53.2 & 52.5 & 54.1 & 540 & 930 & 562 & 54 & 1 & $4,12 E+03$. & $1.31 E-02$ & \\
\hline Te & - & 317 & 509 & 526 & 322 & 510 & S45 & 523 & 12 & $1.03 E=03$ & S.0re-01 & \\
\hline in & - & [356] & [340] & [349] & [350] & [362] & [350] & [349] & 5 & $4.12 E \mp 03$ & 1. $45 E-02$ & \\
\hline $\mathrm{Ti}$ & - & 340 & 337 & 357 & $3 s$ & 334 & 365 & 350 & 11 & $1.03 E+04$ & $3.40 E-02$ & \\
\hline$\pi$ & - & - & - & - & - & - & - & $<123$ & -N/A- & $3.81 E+03$ & $3.41 E-02$ & \\
\hline U & - & {$[1,940]$} & $\{1,220\}$ & {$[1,250]$} & {$[1,940]$} & {$[1,329$} & 2,020 & {$[1.950]$} & 37 & $1.08 E+05$ & $1.005-02$ & \\
\hline IV & - & - & - & - & - & - & - & $<37$ & $-\mathrm{NAS}$ & $2.58 E+02$ & $1.43 E-01$ & \\
\hline$w$ & - & - & - & - & - & - & - & $<492$ & $-\mathrm{N} / \mathrm{A}-$ & $1.21 \varepsilon+03$ & 2.68E-01 & \\
\hline$y$ & - & - & - & - & - & - & - & $<4$ & $-\mathrm{N} / \mathrm{A}-$ & $1.28 \varepsilon+03$ & 5.60E-02 & \\
\hline Zn & [18] & [116] & [1:14] & [119] & [10] & [11:] & [110] & [116] & 3 & $3.35 E \div 03$ & 3.45E-02 & \\
\hline $\mathrm{Zr}$ & - & 037 & 674 & 400 & 455 & 724 & $6 \% 1$ & so1 1 & 200 & $1.10 E+05$ & $5.5 \Delta E-03$ & \\
\hline
\end{tabular}

- Arabte not delected above the IDL; only average LLL reported as less than (s) vatue. N/A Not Appicable

Rosults in brackets are quatified but they are included in the calculation of the average. 
Table 4.6 ICP-AES KOH Fusion-Original Material C106 Privatization Contractor Sample

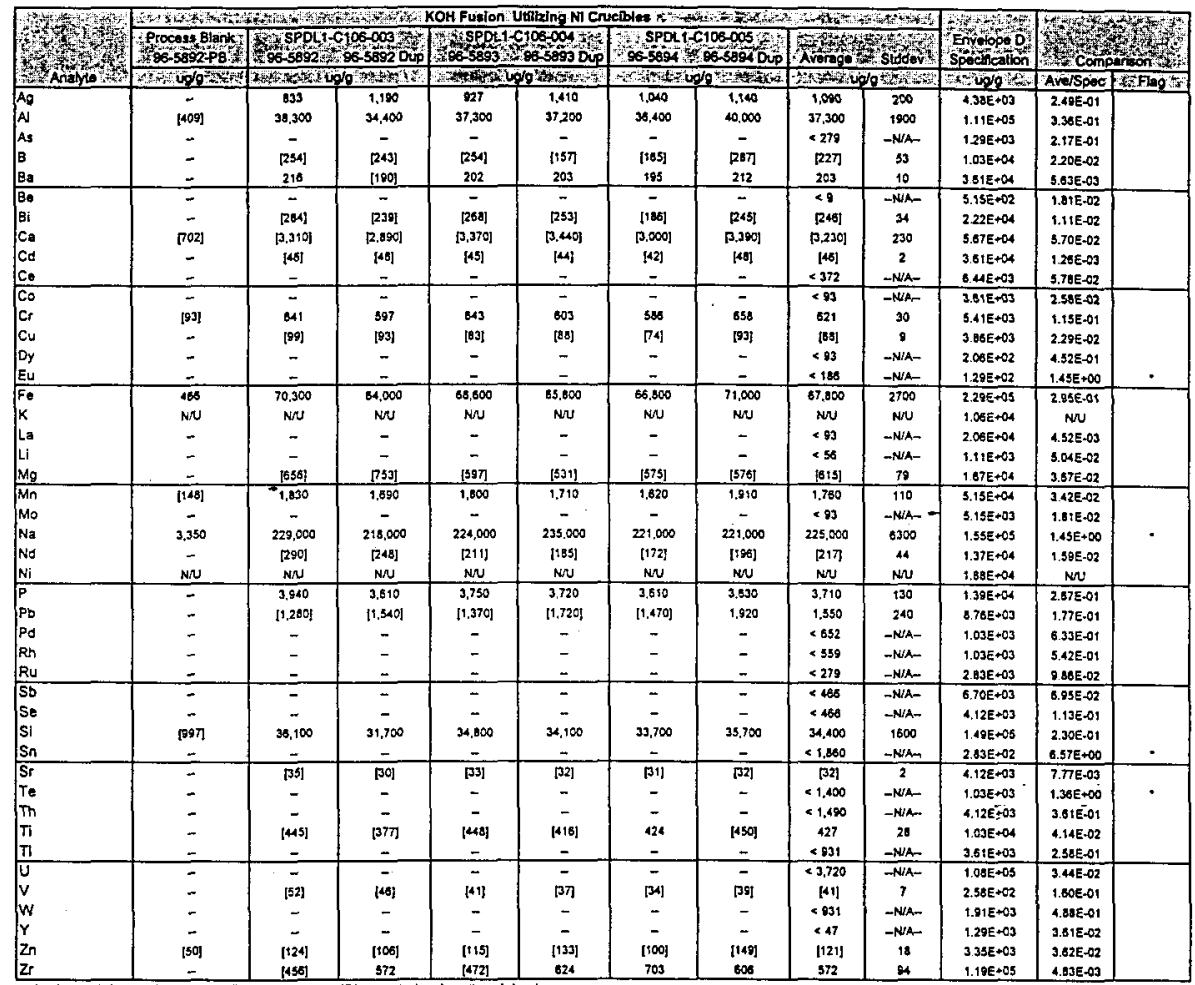

- Analyte not detected above the IDL; only average IDL reported as less than (\&) value N/A Not Applicabla NU Not Usmble

Results in brackets are çualified but they are included in the calculation of the average. 
Table 4.7 ICP-AES Na2O2 Fusion-Original Material C106 Privatization Contractor Sample

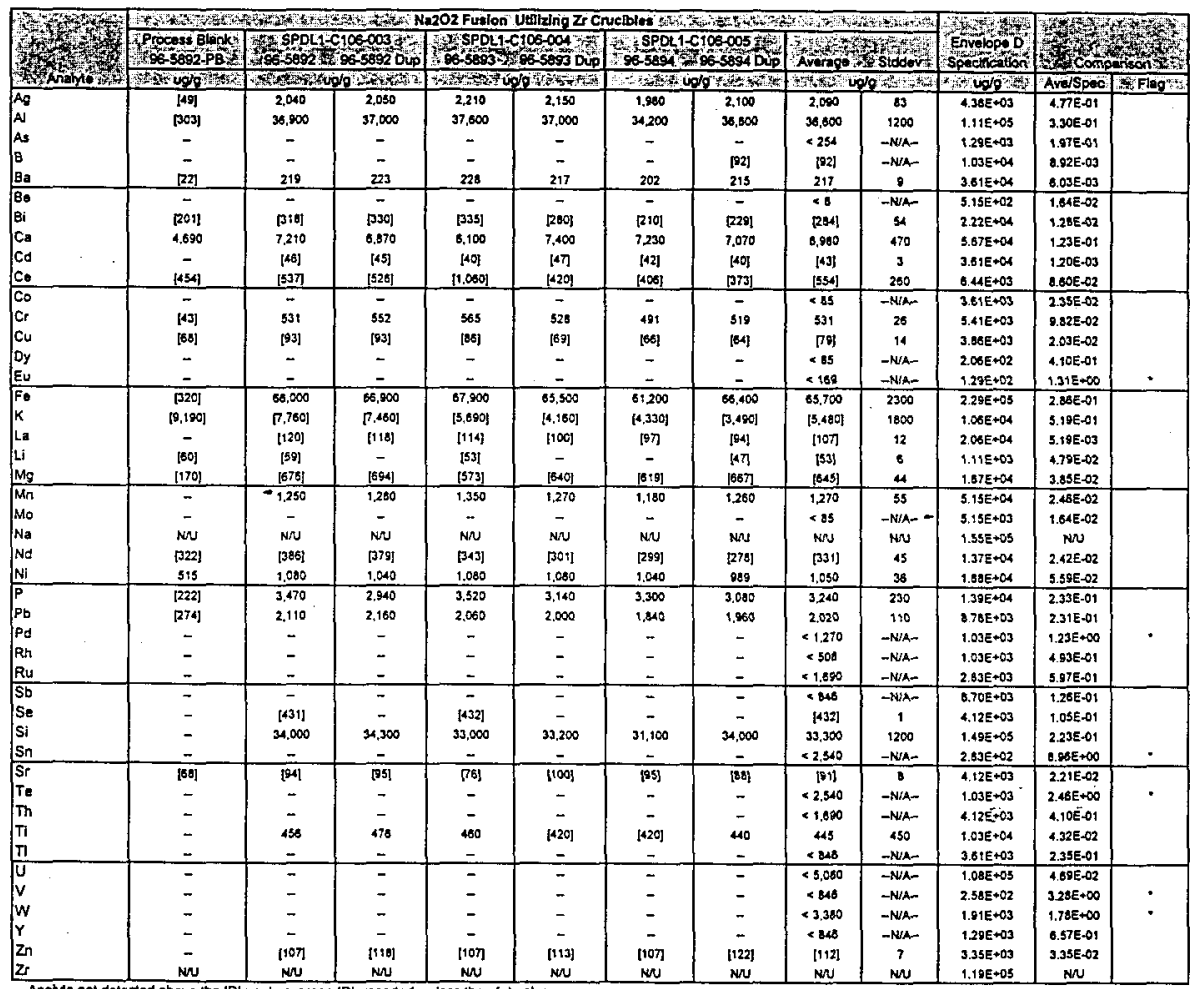

- Analye not detected abovo the IDL; anty average IDL reported as less than ( $c$ ) valub. N/A Not Applicable

NN Not Usable

Results in brackets are qua lified but they are inchuded in the caleulation of the averogo. 
Analytical Summary Report - January 28, 1997

Table 4.8 ICP-AES Acid Digestion--Original Material C106 Privatization Contractor Sample

\begin{tabular}{|c|c|c|c|c|c|c|c|c|c|c|c|c|}
\hline \multirow{2}{*}{ 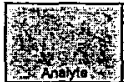 } & \multirow{2}{*}{\multicolumn{3}{|c|}{ 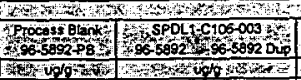 }} & \multirow{2}{*}{\multicolumn{2}{|c|}{ 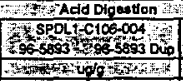 }} & \multicolumn{2}{|c|}{$\begin{array}{l}\text { SPDL }-C 108-005 \\
\text { ss-5884 }\end{array}$} & \multicolumn{2}{|c|}{ Why } & \multirow{2}{*}{ 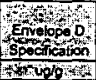 } & \multicolumn{2}{|c|}{ 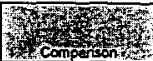 } \\
\hline & & & & & & 549 & 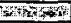 & 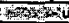 & 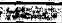 & & Avalspot & Aflog \\
\hline $\mathrm{AO}_{0}$ & - & 175 & 141 & 144 & 140 & 155 & 150 & 152 & 12 & $4.30 E+03$ & $3.48 E-02$ & \\
\hline A & Eᄇ & 37,200 & 33.500 & 35,400 & 34,600 & 33.200 & 32,500 & 34,400 & 1700 & $1.11 E+0,5$ & $3.11 \varepsilon-01$ & \\
\hline As & - & [74] & [D] & [7s] & [79] & [6] & {$[73]$} & [P4] & 4 & $1.29 E+0.3$ & $5.72 \mathrm{E}-02$ & \\
\hline B & $\$ 5.8$ & 150 & 120 & 129 & 139 & 149 & 135 & 138 & 13 & $1.03 E+06$ & $1.34 \mathrm{E}-02$ & \\
\hline Ba & $=$ & 208 & 188 & 195 & iss & ses & 180 & 192 & 10 & $3.6 \mathrm{tE}+0 \mathrm{~d}$ & $5.32 E-00$ & \\
\hline Be & - & $=$ & - & - & - & - & - & $<0.2$ & $-N N_{-}$ & $3.15 E+02$ & $4.55 E-04$ & \\
\hline Bi & - & {$[180]$} & [:68] & [175] & [179] & [13:1 & 1137 & [162] & 20 & $2225+04$ & $7.33 E-03$ & \\
\hline $\mathrm{Ca}$ & (39) & 1,700 & 1,550 & 1,840 & 1,840 & 1,500 & 1.850 & 1,700 & 120 & $5.87 E+0 d$ & $3.01 E-02$ & \\
\hline Cod & - & 39.0 & 35.7 & 37.4 & 36.8 & [36] & [35] & 37 & 2 & $3.61 E+04$ & $1.01 E-003$ & \\
\hline Co & - & [193] & [192] & [190] & 1900 & [179] & [18:1] & [185] & B & $0.44 E+03$ & $2.89 E-02$ & \\
\hline $\mathrm{Co}$ & - & [15] & [14] & [15] & [14] & [14] & (14) & [14] & 0 & $3.61 E+0 \mathrm{~J}$ & $3.94 E-03$ & \\
\hline $\mathrm{Cr}$ & - & 451 & 408 & 431 & 422 & 402 & 395 & $4 \%$ & 21 & $5.41 E+0 S$ & $7.73 E-02$ & \\
\hline $\mathrm{Cu}$ & - & [39] & [38] & [39] & [38] & {$[37]$} & [30] & [30] & 1 & $3.86 E+03$ & $0.06 E-03$ & \\
\hline Dy & - & - & - & - & - & - & - & $<2.4$ & $-\mathrm{N} / \mathrm{A}$ & $2.06 E+02$ & $1.14 E-02$ & \\
\hline Eu & - & - & - & $=$ & $=$ & $=$ & - & $<4.7$ & $-\mathrm{NA}-$ & $1.29 E+02$ & $3.04 E-02$ & \\
\hline $\mathrm{F} \cdot$ & - & 71,100 & 63,400 & 67,600 & 65,800 & 84,100 & 63,400 & 65,900 & 3000 & $2.29 E+05$ & $2.87 E-01$ & \\
\hline$k$ & - & [513]. & [1523\} & [773] & $\{85\}$ & [382\} & {$[09]$} & [B1] & 110 & $1.06 \mathrm{E}+04$ & $7.69 \mathrm{E}-02$ & \\
\hline La & - & $15 \pi$ & [55] & {$[5 \pi]$} & [5] & [54] & [54] & [56] & 2 & $2.06 \mathrm{E}+0.4$ & $2.695-03$ & \\
\hline Li & - & - & $\pi$ & [a! & [B] & [3] & [9] & (d) & 1 & $1.11 E+05$ & $7,405-03$ & \\
\hline$M_{3}$ & - & 345 & 319 & 34 & 331 & 321 & 328 & 331 & 11 & $1.67 \mathrm{E}+04$ & $1.8 a=-02$ & \\
\hline$M n$ & - & 1,370 & 1,240 & 0.320 & 1.250 & 9.230 & 1.220 & 1,280 & 59 & $5.15 E+04$ & $2.44 E-02$ & \\
\hline Mo & - & [30] & [30! & [29] & [9:1 & [32] & Bo] & [30] & 1 & S.15E+CB & $5.90 \mathrm{E}-03$ & \\
\hline $\mathrm{Na}$ & 100 & 228,000 & 223,000 & 215.000 & 228.000 & 230,000 & 217.000 & 225,000 & 8300 & $1.55 E+05$ & $1.45 E \div 00$ & • \\
\hline Nd & - & [18d] & {$[157]$} & {$[162]$} & {$[161]$} & [153] & [155; & [159] & 4 & $1.37 \mathrm{E}=0 \mathrm{~d}$ & $1.16 E-02$ & \\
\hline $\mathrm{Ni}$ & [2] & 549 & 509 & 531 & 524 & 491 & 407 & 515 & 24 & $1.88 E+04$ & $2.74 E-02$ & \\
\hline$P$ & - & 2,080 & 1.910 & 2.020 & 2,000 & 1.930 & 1,880 & 1,970 & 76 & $1.39 \mathrm{E}+04$ & $1.42 E-01$ & \\
\hline $\mathrm{Pb}$ & - & 1,940 & 1.780 & 1.870 & 1,830 & 1,760 & 1,720 & 1,010 & e3 & b.73E.01 & $2.07 E-01$ & \\
\hline Pd & - & - & - & - & - & - & - & $<35.2$ & $-N / 2$ & $1.03 E+03$ & $3.41 E-02$ & \\
\hline R & - & - & - & - & - & - & - & $<14.1$ & $-N / A-$ & $1.03 E+03$ & 1.JTE-OZ & \\
\hline Ru & - & - & - & - & - & $=$ & $=$ & $<48.9$ & $-\mathrm{N} / \mathrm{A}-$ & 2. $23 E+03$ & $1.66 \mathrm{E}-02$ & \\
\hline $5 b$ & - & - & - & - & - & - & - & $<23.5$ & $-N / A-$ & $6.70 E+03$ & $3.50 E-03$ & \\
\hline $5 e$ & - & $\{221\}$ & [202] & {$[297$} & [210] & [205] & [205] & {$[210]$} & a & $4.12 E+03$ & $5.09 E-02$ & \\
\hline $\mathbf{S i}$ & [163] & 25,200 & 10,200 & 29,000 & 22.900 & 22,500 & 20,700 & 20,800 & 5400 & $1,49 E+05$ & $1.395-01$ & \\
\hline Sn & - & {$[1,40]$} & {$[1,3 \in 0]$} & {$[1,470]$} & {$[1,40]$} & $1: 450\}$ & {$[1,460]$} & {$[t, 4 \infty 0]$} & 32 & $2.03 E+02$ & $5.08 E+00$ & * \\
\hline Sr & - & {$[i n]$} & $\{1 / 4\}$ & ini & in & {$[16]$} & 113] & [16] & 1 & $4.12 E+03$ & $394 E-03$ & \\
\hline To & - & - & - & - & - & - & - & $<70.4$ & $-\mathrm{N} / \mathrm{Am}$ & $1.03 E \times 03$ & C.83E-02 & \\
\hline Th & - & - & - & - & - & - & - & $<46.0$ & N/A & $4.12 E+03$ & $1.14 E-02$ & \\
\hline$T$ & - & 15 & 134 & 147 & 144 & 100 & 14 & 144 & 7 & $1.03 E+04$ & $1.40 E-02$ & \\
\hline $\mathrm{TI}$ & $=$ & - & - & - & - & - & - & $<23.5$ & $-N / A_{-}$ & $3.61 E 403$ & $6.50 \mathrm{E}-03$ & \\
\hline $\bar{U}$ & - & {$[1,090]$} & {$[1,370]$} & $\lceil 1,270\rceil$ & {$[1.500]$} & {$[1,150]$} & {$[1,460]$} & $\{1,380\}$ & 150 & $1.04 E+05$ & $1.25 E-02$ & \\
\hline$v$ & - & [25] & [24] & 25) & [2S] & [24] & [R4] & [25] & 1 & $2.50 E+02$ & $9.50 \$-02$ & \\
\hline$w$ & - & . - & - & - & - & - & - & $<93.0$ & -N/A- & $1.01 \mathrm{E}-03$ & $4.92 \mathrm{E}-02$ & \\
\hline$Y$ & - & [41] & [39] & B年 & 阝 & [D8] & [BT] & $p$ p & 2 & $1.29 E+03$ & $3.02 E-02$ & \\
\hline $2 n$ & $=$ & $15 \pi$ & [53] & {$[50]$} & is & (3)] & [5T & [5E] & 2 & $3.35 E+03$ & $1,67 \mathrm{E}-02$ & \\
\hline$z$ & - & 469 & 237 & 353 & 423 & $3 x$ & 357 & 356 & 79 & & $305 E-03$ & \\
\hline
\end{tabular}

- Anelyte not detected abova the IDL; only averags IDL reported as less than (<) valus. NIA Not Applicable

Results in brackets are quelified but they are included in the calcilation ef the average. 
HNF-SD-WM-DP-225, REV. 1

Analytical Summary Report - January 28, 1997

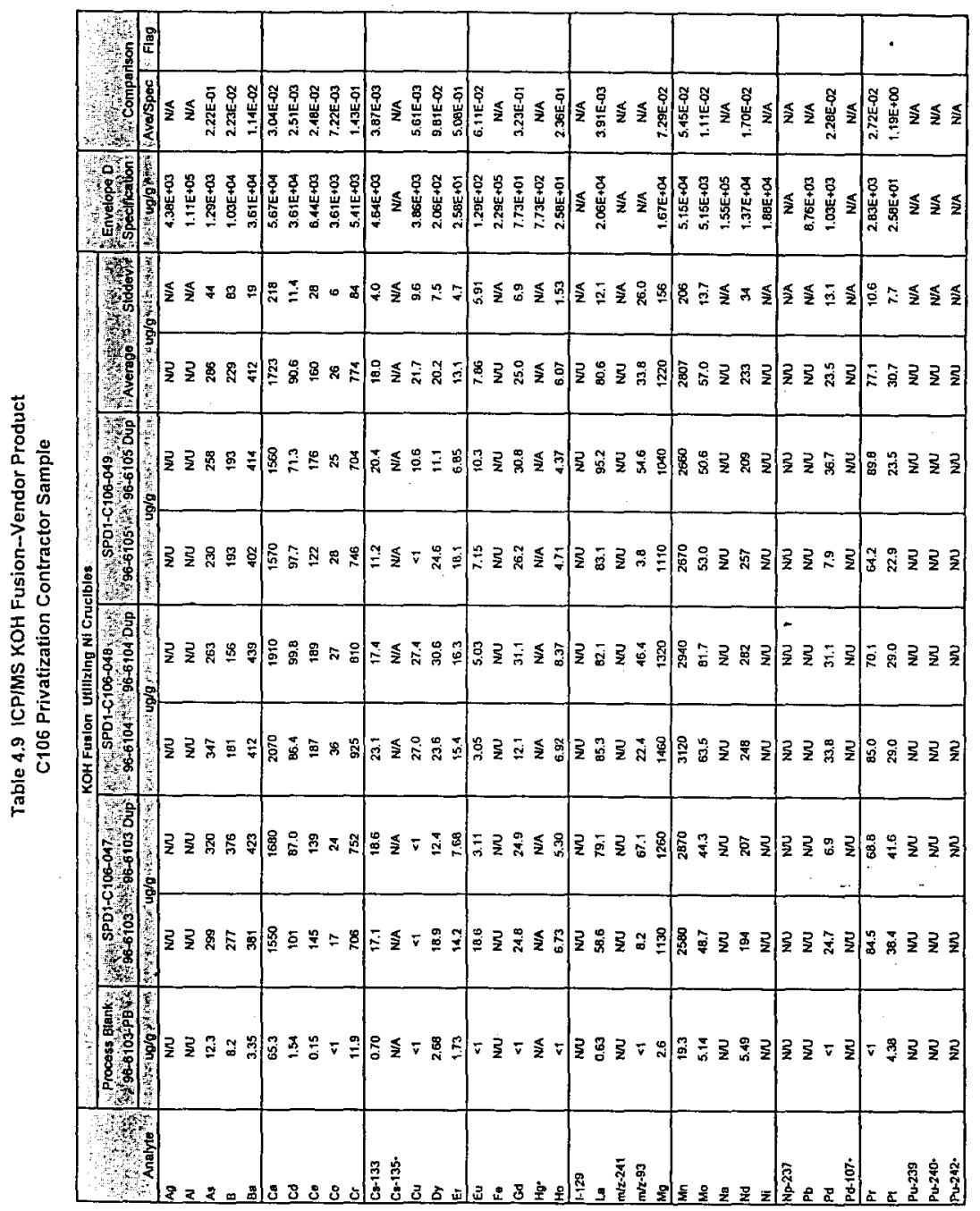


Table 4.9 ICPIMS KOH Fusion--Vendor Product C106 Privatization Contractor Sample

\begin{tabular}{|c|c|c|c|c|c|c|c|c|c|c|c|c|}
\hline \multirow{3}{*}{ 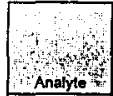 } & \multicolumn{9}{|c|}{\begin{tabular}{|c} 
\\
KOH Fuslon UtIlling NI Gruclbles
\end{tabular}} & \multirow{3}{*}{$\begin{array}{l}\text { Envelope o } \\
\text { specifeation } \\
\text { stoug/g }\end{array}$} & \multirow{2}{*}{\multicolumn{2}{|c|}{ Comparison }} \\
\hline & \multirow{2}{*}{ 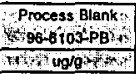 } & \multicolumn{2}{|c|}{ 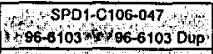 } & \multicolumn{2}{|c|}{$\begin{array}{l}\text { SPD1-C106-048 } \\
\text { 7o6-6104 }\end{array}$} & \multicolumn{2}{|c|}{ 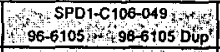 } & \multicolumn{2}{|c|}{ Avorago 3 siddev } & & & \\
\hline & & ingaty & renting & 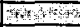 & $4 x^{2}$ ahy & istaming & 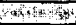 & $2 x+2$ & has? & & Ave/Spec & Fíflag \\
\hline$R \mathbf{b}$ & NN & N/ & NU & NN & NNU & NN & NUS & Nus & N/A & $1.55 E+03$ & N/A & \\
\hline Re & 1.16 & 5.00 & 8.26 & 8.03 & 8.79 & 5.92 & 3.68 & 6.61 & 2.06 & NA & N/A & \\
\hline Rh & $<1$ & $<1$ & $<1$ & 15.4 & 84.3 & $<1$ & 11.2 & 36.9 & 11.0 & $1.03 E+03$ & 3.59E-02 & \\
\hline Ru & $<1$ & 91 & & 103 & & 93 & & 95.7 & 6.5 & $2.83 E+03$ & $3.38 \varepsilon-02$ & \\
\hline $5 b$ & $<1$ & $<1$ & $<1$ & $<1$ & $<1$ & $\leq 1$ & $<1$ & $<1$ & N/A & B. $70 E+03$ & 1.49E-04 & \\
\hline Se & $\mathrm{N} N \mathrm{~J}$ & N/U & $\mathrm{N} / \mathrm{U}$ & NNS & $\mathrm{N} / \mathrm{U}$ & $\mathrm{N} / \mathrm{U}$ & NeN & N/N & N/A & $4.12 E+03$ & $\mathbf{N A}$ & \\
\hline Sn & $<1$ & 5,44 & 31.6 & 83.2 & 24.7 & 24.6 & 6.1 & 29.3 & 28.5 & $2.63 E+02$ & $1.03 E-01$ & \\
\hline Sr & $<1$ & 45 & 54 & 77 & $7 a$ & 49 & 50 & 59 & 15 & 4. $12 E+03$ & $1.42 E-02$ & \\
\hline Ta & 1.84 & 15.3 & 26.8 & 32.7 & 29.6 & 6.91 & 16.6 & 21.3 & 9.9 & $2.06 \mathrm{E}+02$ & t.03E-01 & \\
\hline $\mathbf{T b}$ & $<1$ & 7.41 & 8.46 & 9.75 & 9.86 & 5.71 & 7.99 & 8.20 & 1.55 & 2.58E+01 & 3.1BE-01 & \\
\hline Te-99 & $<1$ & $<1$ & & $<1$ & & $<1$ & & $<1$ & N/A & $\mathrm{N} / \mathrm{A}$ & N/A & \\
\hline Te & 220 & 322 & 511 & 472 & 515 & 353 & 372 & 424 & 85 & $1.03 E+03$ & 4.11E-01 & \\
\hline The 232 & NN & NתI & N/S & NNS & No & $N / U$ & NU & Nu & NA & NA & NUA & \\
\hline$n$ & 4.4 & 1003 & 1040 & 1260 & 1290 & 1050 & 1100 & 1124 & 121 & $1.03 \varepsilon+04$ & $1.09 \mathrm{E}-01$ & \\
\hline $\mathrm{Im}$ & $<1$ & 2.41 & 4.10 & $<1$ & 1.40 & $<1$ & $<1$ & 2.64 & 1.36 & $2.58 \mathrm{E}+01$ & t.02E-0t & \\
\hline Tolal U & $<1$ & 1760 & 2010 & 2060 & 1810 & 1770 & 1970 & 1897 & 132 & $1.00 E+05$ & $1,75 \mathrm{E}-02$ & \\
\hline U-233. & Nu & Nת & NN & NU & NoU & NN & NN & NM & N/A & N/A & N/A & \\
\hline$U-234$. & N/N & Mus & Nu & $\mathrm{N} N$ & NN & NNS & NN & N/U & N/A & NA & NA & \\
\hline U-235. & $\mathbf{N U}$ & $\mathrm{N} / \mathrm{U}$ & NU & NN & No & NU & $\mathrm{N} N$ & $N N$ & N/A & N/A & N/A & \\
\hline$u-236$. & NNS & Nus & NAS & $\mathrm{NM}$ & NYU & Nos & $\mathrm{N} / \mathrm{U}$ & NN & N/A & N/A & NIA & \\
\hline $0-238$ & $<1$ & 1750 & 2000 & 2050 & 1800 & 1760 & 1960 & 1897 & 132 & N/A & N/A & \\
\hline $\mathbf{v}$ & NN & NUS & wn & NN & NN & NN & NN & NN & N/A & $2.58 \mathrm{E}+02$ & N/A & \\
\hline$w$ & 11.6 & 474 & 541 & 618 & 451 & 407 & 500 & 496 & 74 & $1.91 E+03$ & $2.61 \varepsilon-01$ & \\
\hline Yo & 1.39 & 14.9 & 20.4 & 15.8 & 13.5 & 2.69 & 8.26 & 12.6 & 6.2 & $2.58 E+01$ & 4. $.99 E-01$ & \\
\hline zn & 5.24 & 258 & 444 & 281 & 320 & 268 & 268 & 306 & 71 & $3.35 E+03$ & $9.15 \varepsilon-02$ & \\
\hline$z r$ & 1.99 & 260 & 762 & 343 & 621 & 147 & 165 & 133 & 230 & 1.19E +05 & $3.66 E-03$ & \\
\hline
\end{tabular}

NIU Not Usable

- Wo standerds used; responso taken from different Isolope. 
HNF-SD-WM-DP-225, REV. 1

Analytical Summary Report - January 28, 1997

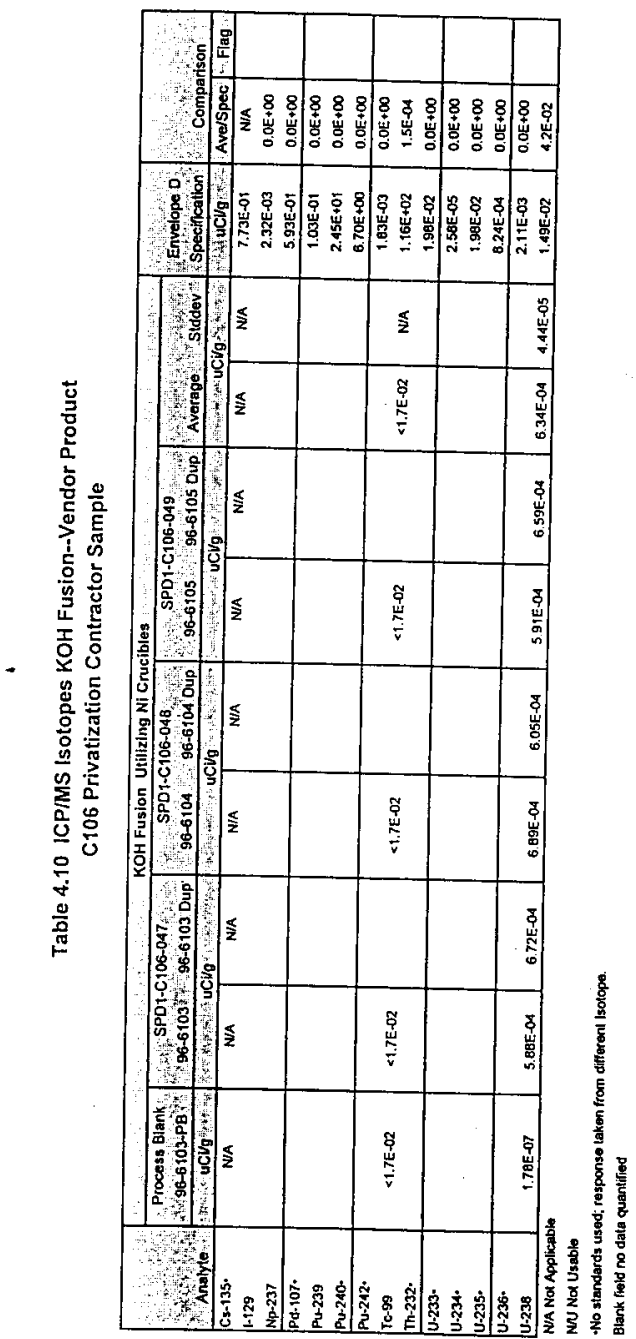


Table 4.11 ICP/MS Na2O2 Fusion-Vendor Product

C106 Privatization Contractor Sample

\begin{tabular}{|c|c|c|c|c|c|c|c|c|c|c|c|c|}
\hline \multirow{3}{*}{$\begin{array}{c}{ }^{n} \\
\text { Analyte }\end{array}$} & \multicolumn{9}{|c|}{ NazO2 Fusion Uilling Zr Criclbles } & \multirow{3}{*}{ 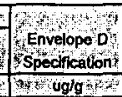 } & \multirow{2}{*}{\multicolumn{2}{|c|}{ Comparison }} \\
\hline & \multirow{2}{*}{ 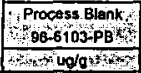 } & \multirow{2}{*}{\multicolumn{2}{|c|}{ 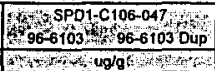 }} & \multirow{2}{*}{\multicolumn{2}{|c|}{ 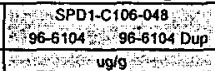 }} & \multicolumn{2}{|c|}{$\begin{array}{l}\text { SPO -C106-049) } \\
\text { 96-6105\% }\end{array}$} & \multicolumn{2}{|c|}{ Averagon of stodov } & & & \\
\hline & & & & & & mistom & 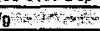 & की & 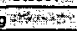 & & Ave/Spec & $\times$ Flag: \\
\hline$\overline{A_{B}}$ & NN & NN & NU & NN & Nou & NN & NIU & NU & N/A & $4.38 \mathrm{E}+03$ & $\mathbf{N} / \mathbf{A}$ & \\
\hline A & NNS & Mu & NN & NN & No & No & wu & Mu & NAA & $1.11 E+05$ & N/A & \\
\hline As & 6.6 & 219 & 192 & 310 & 334 & 228 & 250 & 256 & 55 & $1.29 E+03$ & $1.98 E-01$ & \\
\hline B & 2.2 & 196 & 160 & 67 & 48 & 51 & 63 & 101 & 63 & $1.03 E+04$ & $9.82 \mathrm{E}-03$ & \\
\hline Ba & $<1$ & 417 & 446 & 410 & 436 & 420 & 432 & 427 & 14 & $3.61 E+04$ & 1.18E-02 & \\
\hline $\overrightarrow{\mathrm{Ca}}$ & 133 & $\mathbf{3 3 4 0}$ & 3920 & 4210 & 4080 & 3500 & 3650 & 3767 & 336 & $5.67 E+04$ & $6.65 E-02$ & \\
\hline cod & 4 & 04.8 & 78.0 & 81.0 & 71.0 & 67.0 & 62.6 & 74.1 & 8.6 & $3,61 E+04$ & 2.05E-03 & \\
\hline ce & $\& 1$ & 198 & 216 & 210 & 235 & 181 & 174 & 202 & 23 & $6.44 E+03$ & $3.14 E-02$ & \\
\hline co & $<1$ & 18.6 & 20.0 & 23.7 & 23.6 & 23.9 & 21.0 & 21.8 & 2.3 & $3.61 \mathrm{E}+03$ & 6.0-1E-03 & \\
\hline cr & 5.99 & 696 & 705 & 783 & 809 & 735 & 706 & 739 & 47 & $5.41 E+03$ & 1.37E-01 & \\
\hline Cs-133 & $<1$ & 9.30 & 6.77 & 6.50 & 9.70 & 7.10 & 3.70 & 7.18 & 2.17 & $4.64 E+03$ & 1.55E-03 & \\
\hline Cs.135. & N/A & NA & N/A & $\mathrm{N} / \mathrm{A}$ & N/A & $\mathrm{N} / \mathrm{A}$ & N/A & $\mathrm{NAA}$ & N/A & NA & NA & \\
\hline Cu & 1.2 & 154.0 & 136.1 & 160.0 & 175.8 & 156.4 & 155.3 & 156.3 & 12.7 & $3.86 E+03$ & 4.04E-02 & \\
\hline Dy & $<1$ & 1.70 & 1.00 & 4.00 & 4.30 & 5.50 & 5.12 & 3,60 & 1.84 & $2.06 E+02$ & $1.75 E-02$ & \\
\hline Er & $\leqslant 1$ & $\leq 1$ & $\leq 1$ & $<1$ & 0.3 & $<1$ & $\leqslant 1$ & 0.3 & N/A & $2.58 \mathrm{E}+01$ & $1.16 E-02$ & \\
\hline Eu & $<1$ & 7.30 & 8.00 & 7.20 & 9.60 & 6.10 & a.86 & 7.84 & 1.26 & $1.29 E+02$ & 6.09E.02 & \\
\hline $\mathrm{Fe}$ & NU & NN & No & Nת & Nu & Nos & $N N$ & MN & $N / A$ & $2.20 E+05$ & N/A & \\
\hline Gd & $<1$ & 140 & 140 & 14.0 & 15,0 & 8.50 & 6.50 & 12.1 & 3,4 & $7.73 E+01$ & $1.57 E-01$ & \\
\hline $\mathrm{Hg} \cdot$ & NVA & N/A & NA & $N / A$ & N/A & $N / A$ & N/A & N/A & $N / A$ & $7,73 E+02$ & N/A & \\
\hline Ho & $<1$ & $<1$ & 1.11 & $\leq 1$ & $<1$ & $<1$ & $<1$ & 1.11 & $\mathrm{~N} / \mathrm{A}$ & $2.58 E+01$ & 4.31E-02 & \\
\hline 1.129 & $<20$ & $<20$ & $<20$ & $<20$ & $<20$ & $<20$ & $<20$ & $<20$ & N/A & N/A & N/A & \\
\hline La & $<1$ & 79.9 & 88.9 & 110 & 109 & 90.4 & 74.9 & 92.1 & 14.5 & $2.06 E+04$ & 4.47E-03 & \\
\hline$m / 2-241$ & $<1$ & 1.10 & 1.64 & 2.14 & $t .60$ & 1.53 & 1.70 & 1.65 & 0.34 & N/A & NA & \\
\hline$m / 2-93$ & 41 & 257 & 256 & 297 & 323 & 157 & 117 & 234 & 81 & N/A & NA & \\
\hline $\mathrm{Mg}$ & 7.05 & 1230 & 1330 & 1260 & 1370 & 1190 & 1160 & 1257 & 81 & 1.67E+04 & 7.50E- 02 & \\
\hline Mn & 3.63 & 2500 & 2460 & 2640 & 2760 & 2570 & 2420 & 2558 & 126 & $5.15 E+04$ & $4.97 E-02$ & \\
\hline Mo & 1.30 & 21.0 & 24.2 & 32.0 & 32.0 & 28.0 & 30.0 & 27.9 & 4.5 & 5.15E+03 & $5.41 \varepsilon-03$ & \\
\hline $\mathrm{Na}$ & NN & NN & NU & Nons & $\mathrm{NN}$ & NN & Non. & Nos & NA & 1.55E +05 & NIA & \\
\hline Nd & 49 & 202 & 241 & 220 & 260 & 210 & 213 & 224 & 22 & $1.37 E+04$ & $1,64 \varepsilon-02$ & \\
\hline $\mathrm{Ni}$ & 30.5 & 1500 & 1560 & 1650 & 1650 & 1620 & 1650 & 1605 & 62 & $1.88 E+04$ & 8.53E-02 & \\
\hline$N p-237$ & $<1$ & 6.70 & 4.70 & 6.92 & 5.89, & 5.00 & 4.18 & 5.57 & 0.12 & N/A & $\mathrm{N} / \mathrm{A}$ & \\
\hline $\mathrm{Pb}$ & 2.76 & 2470 & 2540 & 2660 & $2 G 40$ & 2590 & 2420 & 2553 & 95 & B.76E+03 & $2.92 E-01$ & \\
\hline Pd & $<1$ & 22,0 & 29.0 & 36.2 & 23.0 & 26.0 & 28.2 & 27.4 & 5.1 & $1.03 E+03$ & 2.66E.02 & \\
\hline Pd-107. & NU & $\mathrm{NU}$ & 1 NU & NU & $\mathrm{Nu}$ & NU & NU & NU & N/A & $\mathrm{N} / \mathrm{A}$ & $\mathrm{N} / \mathrm{A}$ & \\
\hline Pr & $<1$ & 83.6 & 84,4 & 87.2 & 91.3 & 76.4 & 81.0 & 84.1 & 5.3 & $2.83 E+03$ & $2.97 E-02$ & \\
\hline Pt & $<1$ & 5.21 & 4.00 & 3.50 & 5.27 & 4,90 & 8.26 & 5. 19 & 1.66 & $2.58 E+01$ & 2.01E-01 & \\
\hline Pu-239 & $<1$ & 22.6 & 25.0 & 26.0 & 26.5 & 22.4 & 24,9 & 24.6 & 1.7 & N/A & $N / A$ & \\
\hline$P u-240$ & $<1$ & 41 & $1 \quad 4$ & $\ll 1$ & $<1$ & $<1$ & 21 & $<1$ & N/A & N/A & $N / A$ & \\
\hline Pu-242. & $<1$ & 0.01 & $<1$ & 0.03 & $<1$ & 0.03 & 0.02 & 0.02 & 0.01 & N/A & N/A & \\
\hline
\end{tabular}


Table 4.11 ICPIMS Na2O2 Fusion--Vendor Product C106 Privatization Contractor Sample

\begin{tabular}{|c|c|c|c|c|c|c|c|c|c|c|c|c|}
\hline \multirow{3}{*}{ 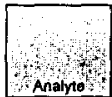 } & \multicolumn{9}{|c|}{$\mathrm{Na} 2 \mathrm{O} 2$ Fusion Utilizing Zr Crucibles } & \multirow{3}{*}{$\begin{array}{l}\text { Envelope } 0 \\
\text { specification } \\
\text { Spe } \\
\text { rog/g } 1\end{array}$} & & \\
\hline & \multirow{2}{*}{ 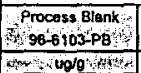 } & \multirow{2}{*}{\multicolumn{2}{|c|}{ 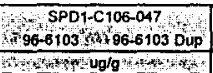 }} & \multirow{2}{*}{\multicolumn{2}{|c|}{ 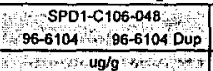 }} & \multicolumn{2}{|c|}{\begin{tabular}{|} 
SPD1-C106-049 \\
96-6105: $96-6105$ Dup
\end{tabular}} & \multicolumn{2}{|c|}{ 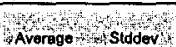 } & & \multicolumn{2}{|c|}{ Comparison } \\
\hline & & & & & & wats & $5+4 \sin$ & Hats & What & & Ave/Spec & Flag \\
\hline $\mathbf{R b}$ & $<1$ & 4.90 & 7.70 & 13.00 & 6.00 & 5.17 & 7.30 & 7.68 & 2.92 & $1.55 E+03$ & $4.97 \mathrm{E}-03$ & \\
\hline Ra & $<1$ & $<1$ & 1.00 & $<1$ & $\Leftrightarrow$ & $<1$ & $<1$ & 1.00 & N/A & N/A & N/A & \\
\hline Rh & 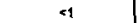 & 143 & 120 & 147 & 170 & 144 & 141 & 144 & 16 & $1.03 E+03$ & $1.40 E-01$ & \\
\hline Ru & N/A & NAA & N/A & NAA & N/A & NA & N/A & N/A & N/A & $2.83 E+03$ & NA & \\
\hline $\mathbf{S b}$ & $\leqslant 1$ & $<1$ & $\Leftrightarrow 1$ & $<1$ & $<1$ & 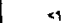 & 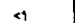 & $\leftrightarrow$ & $N / A$ & $6.70 E+03$ & 1.49E-04 & \\
\hline Se & NU & NU & Nars & NN & NN & NUS & $\mathrm{NM}$ & $\mathbf{N u}$ & N/A & $4.12 E+03$ & N/A & \\
\hline Sn & 1.73 & 75.0 & 100 & 130 & 138 & 105 & 64.6 & 102 & 29 & $2.03 E+02$ & $3.60 \mathrm{E}-01$ & \\
\hline Sr & 6.87 & 137 & 160 & 152 & 151 & 134 & 168 & 150 & 13 & $4.12 E+03$ & $3.65 E-02$ & \\
\hline Ta & $<1$ & 28.8 & 33.0 & 39.0 & 38.0 & 17,70 & 15.1 & 28.6 & 10.2 & $2.05 E+02$ & 1.39E- 04 & \\
\hline To & $<1$ & 1.00 & 2.00 & 2.85 & 2.80 & 2.23 & 3.15 & 2.34 & 0.78 & $2.58 \mathrm{E}+01$ & $9.08 E-02$ & \\
\hline Te-99 & Nu & Nu & WN & NN & NN & NN & $\mathrm{N} / \mathrm{N}$ & NN & N/A & N/A & N/A & \\
\hline T. & 4.4 & 340 & 160 & 430 & 340 & 218 & 240 & 288 & 99 & $1.03 E+03$ & 2.80E-01 & \\
\hline Th-232. & $<1$ & 461 & 575 & 644 & 685 & 290 & 336 & 499 & 163 & N/A & N/A & \\
\hline Iit & 3.20 & 1270 & 1240 & 1500 & 1490 & 1240 & $\$ 140$ & 1313 & 147 & $1.03 E+04$ & 1.27E-01 & \\
\hline$T m$ & $<1$ & $<1$ & $<1$ & 1.24 & $<1$ & $<1$ & 1,10 & 1.17 & 0.10 & $2.58 E+01$ & $\triangle 54 E-02$ & \\
\hline Total U & $<1$ & 1940 & 2030 & 1990 & 2120 & 2030 & 1950 & 2010 & 66 & $1.08 E+05$ & $1,86 \mathrm{E}-02$ & \\
\hline U-233. & NN & NM & NNS & NN & NN & NN & No & NN & Nos & N/A & N/A & \\
\hline U.234. & NN & NN & NOS & NN & NN & NU & NN & WN & No & NA & N/A & \\
\hline U.235. & NN & NO & NN & Nת & NU & Nu & NN & Nos & NN & N/A & NA & \\
\hline U.236. & $\mathrm{NN}$ & NUS & NN & NN & $\mathrm{N} N$ & NII & Nus & Nas & wn & NA & NIA & \\
\hline $\mathrm{U}-238$ & $<1$ & 1930 & 2020 & $1980^{-}$ & 2100 & 2020 & 1940 & 1906 & 63 & NA & N/A & \\
\hline$v$ & No & NOS & N/N & NN & $N n$ & NN & Nov & wo & NM & $2.50 E+02$ & N/A & \\
\hline$w$ & 6.6 & 388 & 371 & 455 & 474 & 268 & 183 & 357 & 112 & $1.99 E+03$ & $1.87 \mathrm{E}-01$ & \\
\hline $\mathrm{Yb}$ & $<1$ & 200 & 3.50 & 1.80 & 5.30 & 4.61 & 2.20 & 3.24 & 1.48 & $2.58 E+01$ & 1.26E-01 & \\
\hline $2 n$ & 1.50 & 228 & 251 & 263 & 288 & 164 & 200 & 234 & 44 & 3. $35 E+03$ & $6.97 E-02$ & \\
\hline $\mathrm{z}$ & $\mathrm{N} N$ & NN & NN & NNS & N/U & $\mathrm{N} / \mathrm{N}$ & $\mathrm{NUS}$ & NN & N/A & $1.19 E+05$ & N/A & \\
\hline
\end{tabular}

N/A Not Applicable

NNU Nol Usable

-No standards used; response taken from different Isotope. 
HNF-SD-WM-DP-225, REV, 1

Analytical Summary Report - January 28, 1997

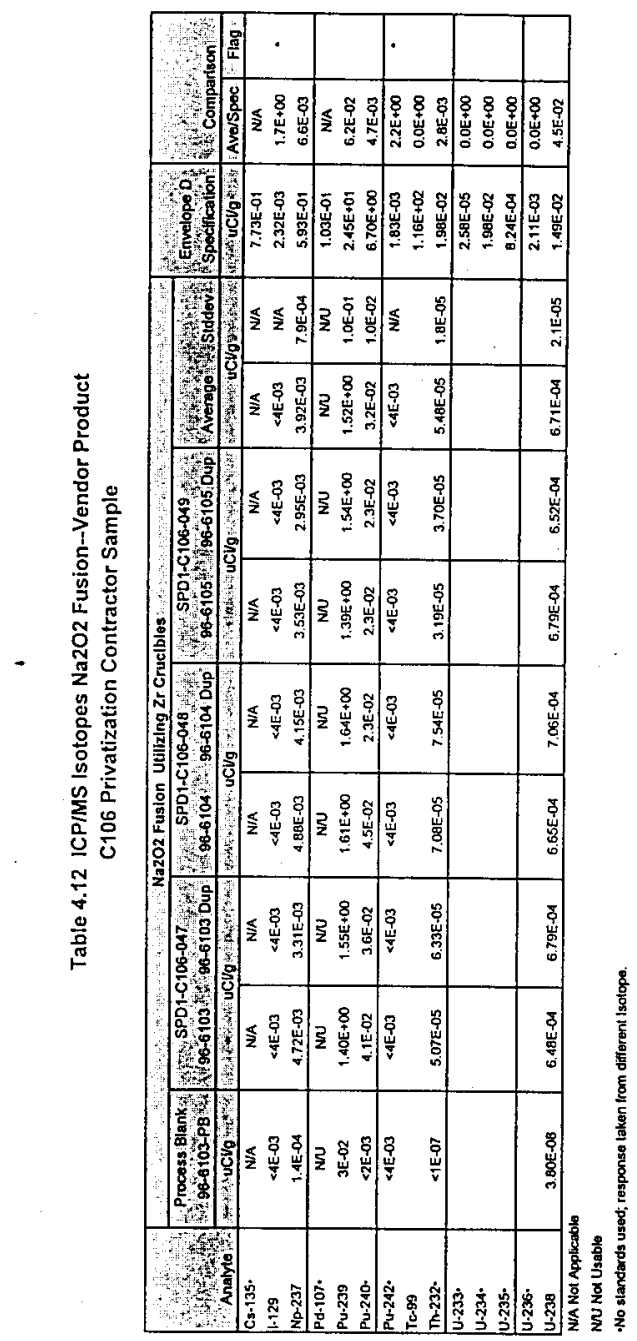


Table 4.13 ICP/MS Acid Digestion--Vendor Product C106 Privatization Contractor Sample

\begin{tabular}{|c|c|c|c|c|c|c|c|c|c|c|c|c|}
\hline \multirow{3}{*}{ Analyie } & \multicolumn{3}{|c|}{$\therefore \ldots \cdots: \because \cdots, \cdots$} & \multicolumn{6}{|c|}{ Acid Digestion } & \multirow{3}{*}{$\begin{array}{l}\text { Envelope D } \\
\text { \% Specifications } \\
\text { Stintug/g } \\
\end{array}$} & \multirow{2}{*}{\multicolumn{2}{|c|}{ Comparison }} \\
\hline & \multirow{2}{*}{\begin{tabular}{|} 
Process Blank \\
$96-6103-\mathrm{PB}$ \\
ug/gw
\end{tabular}} & \multicolumn{2}{|c|}{ 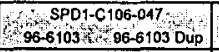 } & \multicolumn{2}{|c|}{$\begin{array}{l}\text { SPD1-C106-048 } \\
\text { 96-6104 96-6104 Dup }\end{array}$} & \multicolumn{2}{|c|}{$\begin{array}{c}\text { SPO1-C106-049 } \\
\text { 96-6105 }\end{array}$} & \multicolumn{2}{|c|}{ Averagak 6 Siddov } & & & \\
\hline & & \multicolumn{2}{|c|}{ 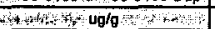 } & \multicolumn{2}{|c|}{ 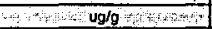 } & \multicolumn{2}{|c|}{ 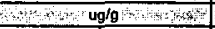 } & \multicolumn{2}{|c|}{ 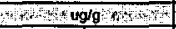 } & & Ave/Spec & Ftag \\
\hline$A_{g}$ & $\mathrm{~N} N \mathrm{~N}$ & NN & $\mathrm{Nu}$ & NN & NIU & N/U & NNS & No & N/A & $4.38 E+03$ & N/A & \\
\hline Al & 8.22 & 32000 & 32000 & 32300 & 34100 & 32100 & 38100 & 33433 & 2425 & $1.11 E+05$ & $3.02 E-01$ & \\
\hline As & 0.97 & 172 & 163 & 158 & 138 & 145 & 154 & 155 & 12 & $1.29 \mathrm{E}+03$ & $1.20 \mathrm{E}-01$ & \\
\hline$B$ & 50.3 & 267 & 259 & 121 & 142 & 158 & 169 & 186 & 62 & $1.03 E+04$ & $1.00 E-02$ & \\
\hline Ba & 0.10 & 348 & 362 & 365 & 358 & 359 & 378 & 362 & 10 & $3.61 E+04$ & $1.00 E-02$ & \\
\hline $\mathrm{ca}$ & 84,0 & 11300 & 8950 & 8360 & 9140 & 9090 & 10600 & 9573 & 1124 & $5.67 E+04$ & $1,69 \mathrm{E}-01$ & \\
\hline Cd & 0.05 & 58.4 & 60.0 & 58.5 & 55.9 & 56.0 & 59.5 & 50.1 & 1.7 & $3.61 E+04$ & $1.61 E-03$ & \\
\hline co & 0.03 & 161 & 164 & 157 & 151 & 156 & 167 & 160 & 6 & $6.44 E+C 3$ & $2,48 E-02$ & \\
\hline co & 0.03 & 15.6 & 16.0 & 16.1 & 15.6 & $14 . a$ & 15.9 & 15.7 & 0.5 & $3.61 E+03$ & 4.35E-03 & \\
\hline $\mathrm{Cr}$ & 0.67 & 429 & 433 & 429 & 412 & 414 & 440 & 426 & 11 & $5.41 E+03$ & 7.8BE-02 & \\
\hline Cs-133 & $<0.02$ & 10.9 & 11.6 & 10.1 & 10.0 & 10.2 & 9.88 & 10.4 & 0.7 & $4.64 E+03$ & $2.25 \mathrm{E}-03$ & \\
\hline Cs-135. & N/A & N/A & N/A & N/A & N/A & N/A & N/A & N/A & N/A & N/A & N/A & \\
\hline $\mathrm{Cu}$ & 0.4 & 105.7 & 109.0 & 112.3 & 109.5 & 107.1 & 112.3 & 110.0 & 20 & $3.86 E+03$ & $2.85 E-02$ & \\
\hline Dy & 0.04 & 1.52 & 1.40 & 1.45 & 1.60 & 1.34 & 1.50 & 1.47 & 0.09 & $2.06 E+02$ & $7.13 \mathrm{E}-03$ & \\
\hline Er & $<0.02$ & 0.7 & 0.80 & 0.8 & 0.7 & 0.8 & 0.80 & 0.8 & 0.0 & $2,58 E+01$ & $2.99 E-02$ & \\
\hline Eu & 0.02 & 6.20 & 6.40 & 6.45 & 6.70 & 6.23 & 6.77 & 6.46 & 0.24 & 1.29E+02 & 5.01E-02 & \\
\hline$F_{a}$ & 20.2 & 96200 & 110000 & 115000 & $\$ 14000$ & 103000 & 120000 & 109700 & 8717 & $2.29 E+05$ & $478 E-01$ & \\
\hline$G d$ & $<0.02$ & 14.9 & 13.3 & 12.2 & 13.1 & 13.3 & 14.8 & 13.6 & 1.0 & 7.73E+01 & $1.76 E-01$ & \\
\hline $\mathrm{Hg} \cdot$ & N/A & N/A & N/A & N/A & N/A & N/A & N/A & N/A & $\mathrm{N} / \mathrm{A}$ & $7.73 E+02$ & NA & \\
\hline $\mathrm{Ho}$ & 80.02 & 0.15 & 0.14 & 0.16 & 0.10 & 0.20 & 0.10 & 0.14 & 0.04 & $2.58 E+01$ & $5,50 E-03$ & \\
\hline $\mid-129$ & $\ll 1$ & $1 . \infty$ & $<1$ & $<1$ & 1.20 & 1.27 & 1.60 & 1.27 & 0.25 & NAA & NA & \\
\hline Le & $<0.02$ & 64.7 & 96.3 & 84.2 & 80.3 & 83.7 & 90.5 & 84.9 & 3.4 & $2.06 E+04$ & 4.12E-03 & \\
\hline$m / z-241$ & $<0.02$ & 0.90 & 0.77 & 0.70 & 0.77 & 0.74 & 0.76 & 0.77 & 0.07 & N/A & NAA & \\
\hline$m / z-93$ & $<0.02$ & 60.5 & 65.2 & 34.3 & 36.7 & 52.6 & 50.4 & 49.9 & 12.4 & N/A & N/A & \\
\hline$M_{g}$ & 2.82 & 472 & 452 & 436 & 423 & 428 & 455 & 446 & 17 & $1.87 \mathrm{E}+04$ & 2.66E- 02 & \\
\hline$M n$ & 4.00 & 2060 & 1930 & 2130 & 2040 & 1900 & 2200 & 2043 & 115 & 5. $15 E+04$ & $3.97 E-02$ & \\
\hline Mo & 0.07 & 10.7 & 9.23 & 9.29 & 9.60 & 8.99 & 9.78 & 9.60 & 0.60 & $5.15 E+03$ & $1.86 E-03$ & \\
\hline $\mathrm{Na}$ & 27.9 & 150000 & 208000 & 186000 & 148000 & 188000 & 210000 & 181667 & 27171 & 1.55E+05 & $1.18 E+\infty$ & - \\
\hline Nd & 0.07 & 194 & 199 & 192 & 187 & 191 & 197 & 193 & 4 & $1.37 E+04$ & $1.42 \mathrm{E}-02$ & \\
\hline $\mathrm{Ni}$ & 7.40 & 963 & 912 & 993 & 939 & 864 & 1020 & 948 & 56 & 1. $188 E+04$ & $5.04 E-02$ & \\
\hline Np-237 & $<0.02$ & 4.22 & 4.52 & 4.39 & 4.78 & 4.43 & 4.55 & 4.48 & 0.19 & NA & NUA & \\
\hline $\mathrm{Pb}$ & 8.31 & 2700 & 2740 & 2960 & 2620 & 3100 & 3000 & 2853 & 192 & $8.76 \mathrm{E}+03$ & $3.26 E-01$ & \\
\hline Pd & 0.1 & 9.12 & 10.4 & 10.8 & 10.0 & 9.29 & 10.3 & 10.0 & 0.7 & $1,03 E+03$ & $9.71 E-03$ & \\
\hline Pd-107. & $<0.5$ & 5.00 & $! \quad 1.00$ & $<0.5$ & $<0.5$ & 0.60 & $<0.5$ & 2.20 & 2.43 & N/A & N/A & \\
\hline$\overline{\mathbf{P r}}$ & 0.02 & 70.8 & .72 .3 & 69.4 & 67.9 & 69.5 & 73.2 & 70.5 & 20 & $2.83 E+03$ & $2.49 E-02$ & \\
\hline $\mathbf{P I}$ & 0.10 & 0.2 & 0.3 & 0.2 & 0.2 & 0.1 & 0.4 & 0.2 & 0.1 & $2.50 E+01$ & 8.86E-03 & \\
\hline Pu-239 & $<0.02$ & 7.52 & 7.65 & 6.66 & 6.87 & 7.52 & 7.59 & 7.34 & 0.37 & N/A & N/A & \\
\hline Pu-240. & $<0.02$ & 0.60 & 0.59 & 0.48 & 0.55 & 0.58 & 0.55 & 0.56 & 0.04 & NA & N/A & \\
\hline$P u-242$ & $<0.02$ & 0.02 & 0.02 & $<0.02$ & $<0.02$ & $<002$ & $<0.02$ & 0.02 & 0.00 & $N / A$ & N/A & \\
\hline
\end{tabular}


Table 4.13 ICP/MS Acid Digestion--Vendor Product C106 Privatization Contractor Sample

\begin{tabular}{|c|c|c|c|c|c|c|c|c|c|c|c|c|}
\hline \multirow{3}{*}{ 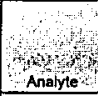 } & \multicolumn{3}{|c|}{ 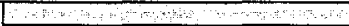 } & \multicolumn{2}{|c|}{ Aclid Digestion } & \multicolumn{4}{|c|}{ 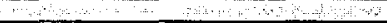 } & \multirow{3}{*}{ 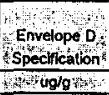 } & \multirow{2}{*}{\multicolumn{2}{|c|}{ Pots }} \\
\hline & \multirow{2}{*}{$\begin{array}{l}\text { Process Blank } \\
\text { p6-6to3-PB } \\
\end{array}$} & \multirow{2}{*}{\multicolumn{2}{|c|}{ 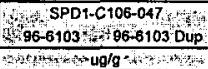 }} & \multirow{2}{*}{\multicolumn{2}{|c|}{ 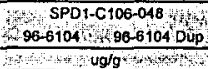 }} & \multirow{2}{*}{\multicolumn{2}{|c|}{ 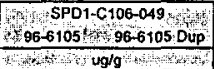 }} & \multicolumn{2}{|c|}{ Averago W X Stdderg } & & & \\
\hline & & & & & & & & menta & Whath & & Ave/Spec & Flag \\
\hline $\mathrm{Rb}$ & 0.2 & 1.21 & 1.07 & 1.25 & 1.10 & 1.00 & 1.00 & 1.10 & 0.11 & $1.55 E+03$ & $7.15 E-04$ & \\
\hline $\mathbf{R e}$ & 0.02 & 0.03 & 0.12 & 0.08 & 0.10 & 0.05 & 0.09 & 0.08 & 0.03 & N/A & NA & \\
\hline $\mathbf{R h}$ & $<0,02$ & 108.6 & 107.7 & 100.4 & 101.9 & 101.6 & 107.6 & 104.7 & 3.7 & $1.03 E+03$ & $1.02 E-01$ & \\
\hline Ru & 0.1 & 55.7 & 56.3 & 53.3 & 52.6 & 53.3 & 53.8 & 54.2 & 1.5 & $2.83 E+03$ & $1.91 E-02$ & \\
\hline sb & $<0.02$ & 1,00 & $<0.02$ & $<0.02$ & $<0.02$ & 0.70 & 0.50 & 0.73 & 0.25 & $6.70 E+03$ & $1.09 E-04$ & \\
\hline So & NN & NNS & Non & NN & NUU & Non & NN & Nu & N/A & 4.12E+03 & N/A & \\
\hline sn & $<0.02$ & 36.8 & 35.5 & 35,4 & 33.5 & 32.8 & 34.6 & 34.8 & 1.5 & $2,83 E+02$ & 1.23E-01 & \\
\hline sr & 0.07 & 44.9 & 45.6 & 45.3 & 44.6 & 43.7 & 46.3 & 45.1 & 0.9 & $4.12 E+03$ & $1.09 E-02$ & \\
\hline Ta & 0.09 & 15.3 & 9.7 & 8.2 & 8.4 & 8.50 & 12.4 & 10.4 & 2.9 & $2.06 E+02$ & 5.06E-02 & \\
\hline Db & 0.02 & 1,52 & 1.46 & 1.42 & 1.55 & 1.50 & 1.30 & 1.46 & 0.09 & $2.58 \mathrm{E}+01$ & $5.66 \mathrm{E}-02$ & \\
\hline TC-99 & 0.048 & 0.396 & 0.399 & 0.260 & 0.293 & 0.333 & 0.297 & 0.330 & 0.058 & N/A & $N / A$ & \\
\hline To & 9.74 & 206 & 202 & 213 & 193 & 196 & 210 & 203 & 8 & $1.03 E+03$ & 1.97E-01 & \\
\hline Th-232. & $<0.2$ & 194 & 178 & 185 & 186 & 180 & 185 & 185 & 6 & NA & N/A & \\
\hline $\mathrm{Ti}$ & 0.4 & 366 & 362 & 365 & 351 & 338 & 368 & 358 & 12 & $1.03 E+04$ & $3.48 E-02$ & \\
\hline $\mathrm{Im}$ & $<0.02$ & 0.10 & 0.05 & 0.06 & 0.06 & 0.05 & 0.05 & 0.06 & 0.02 & $2.58 \mathrm{E}+01$ & 2.39E- 03 & \\
\hline Total U & 2.12 & 1910 & 1910 & 1910 & 1770 & 1950 & 1980 & 1905 & 72 & $1.08 E+05$ & $1.76 \mathrm{E}-02$ & \\
\hline U-233. & $<0.02$ & 0.19 & 0.18 & 0.13 & 0.14 & 0.18 & 0.22 & 0.17 & 0.03 & N/A & NA & \\
\hline U.234. & $<0.02$ & 0.13 & 0.11 & 0.11 & 0.11 & 0.11 & 0.17 & 0.12 & 0.02 & N/A & $N / A$ & \\
\hline U-235. & $<0.02$ & 13.1 & 13.8 & 12.8 & 11.7 & 13.? & 12.8 & 13.0 & 0.8 & NAA & N/A & \\
\hline U-236. & $<0.02$ & 0.34 & 0.28 & 0.25 & 0.24 & 0.33 & 0.28 & 0.29 & 0.04 & N/A & N/A & \\
\hline U-238 & 2,11 & 1900 & 1900 & 1900 & 1760 & 1940 & 1970 & 1895 & 72 & N/A & N/A & \\
\hline $\mathrm{v}$ & 4 & 44.4 & 42.6 & 38.4 & 32.4 & 26.3 & 39.5 & 37.2 & 6.8 & $2.58 \mathrm{E}+02$ & $1.45 E-0 t$ & \\
\hline w & 0.9 & 237 & 240 & 210 & 205 & 214 & 224 & 222 & 15 & $1.91 E+03$ & $1.16 \mathrm{E}-01$ & \\
\hline$r$ & 0.02 & 0.40 & 0.30 & 0.20 & 0.29 & 0.20 & 0.10 & 0.2 & 0.1 & $2.50 E+01$ & 9.63E-03 & \\
\hline $2 n$ & 5.95 & 110 & 100 & 103 & 93.4 & 91.7 & 97.1 & 99.3 & 6.7 & $3.35 E+03$ & $2.96 \mathrm{E}-02$ & \\
\hline$z$ & 0.25 & 307 & 340 & $17 f$ & 183 & 276 & 250 & 256 & 67 & 1.19E+05 & $2.16 \mathrm{E}-03$ & \\
\hline
\end{tabular}

NAA Nol Applicablo

NU Not Usabto

-No standards used, response laken from different Isolope. 
HNF-SD-WM-DP-225, REV ?

Analytical Summary Report - January 28, 1997

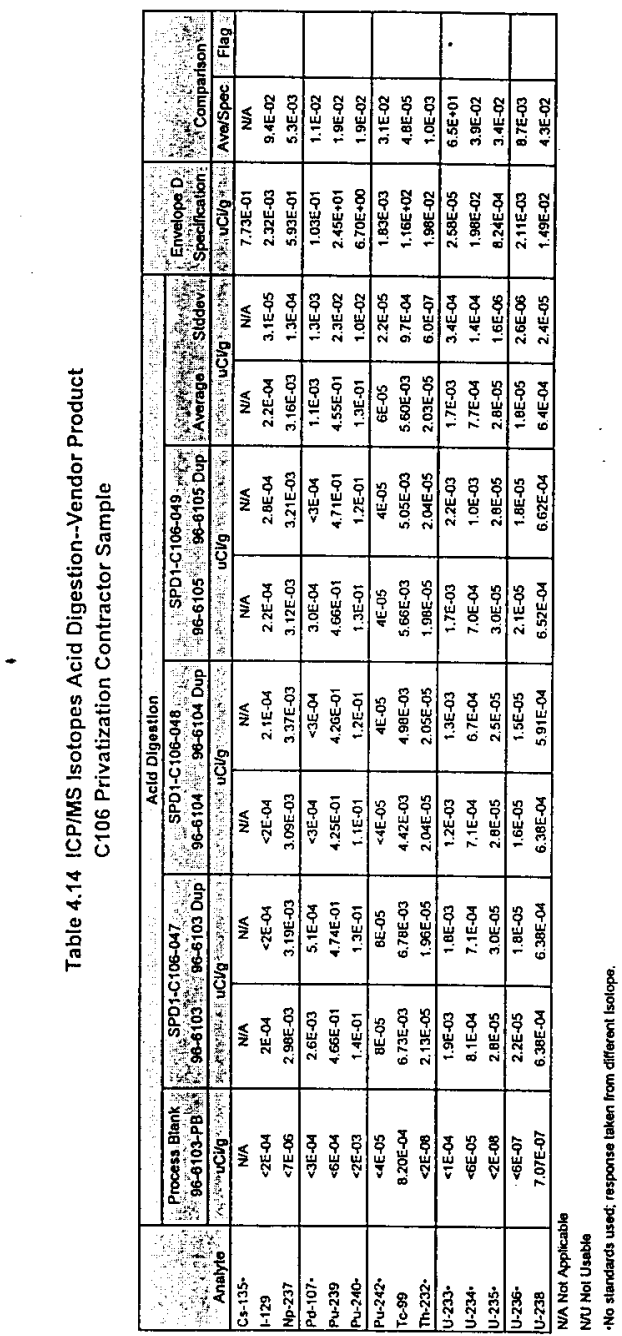


HNF-SD-WM-DP-225, REV ?

Analytical Summary Report - January 28, 1997

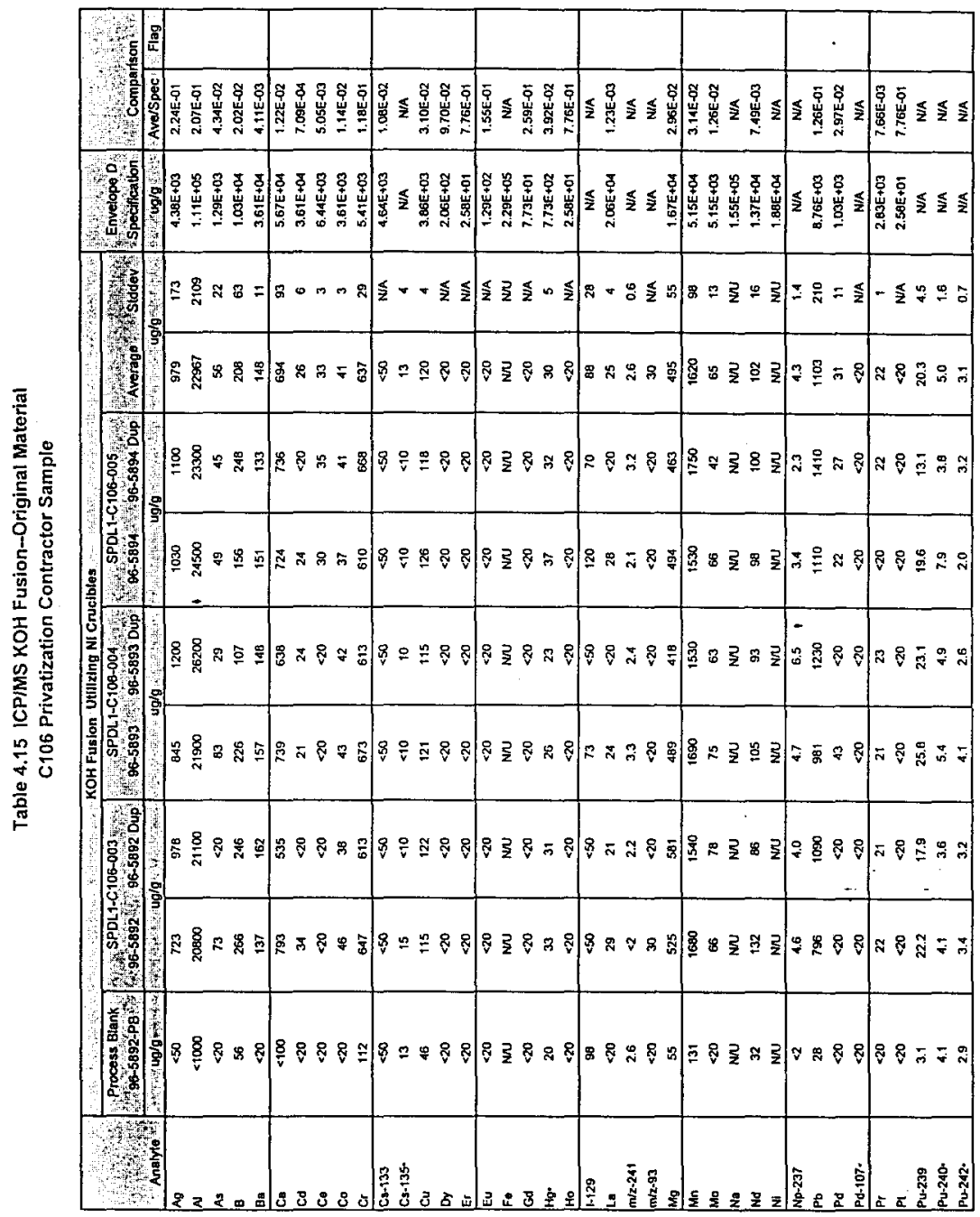


Table 4.15 ICPIMS KOH Fusion--Original Material C106 Privatization Contractor Sample

\begin{tabular}{|c|c|c|c|c|c|c|c|c|c|c|c|c|}
\hline \multirow{3}{*}{\begin{tabular}{|c|} 
\\
$y$ \\
Analyte \\
And
\end{tabular}} & \multicolumn{3}{|c|}{$(\ldots,+\cdots$} & \multicolumn{6}{|c|}{ KOH Fuslon Utillzing NI Cruclbles } & \multirow{3}{*}{ 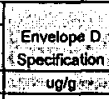 } & \multirow{2}{*}{\multicolumn{2}{|c|}{ Wr, comparison }} \\
\hline & \multirow{2}{*}{ 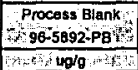 } & \multicolumn{2}{|c|}{$\begin{array}{l}\text { SPDL 1-C106-003 } \\
\text { 96-5892 }\end{array}$} & \multicolumn{2}{|c|}{$\begin{array}{l}\text { SPOL1-C106-004 } \\
96-5893 \quad 96-5893 \text { Dup }\end{array}$} & \multicolumn{2}{|c|}{$\begin{array}{l}\text { SPDL1-C100-005 } \\
\text { 96-5894 } \\
\text { 96-5894 Dup }\end{array}$} & \multicolumn{2}{|c|}{ 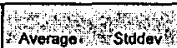 } & & & \\
\hline & & 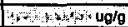 & weptats & ton $r$ an & oglg $n$ n $\psi$ & Wry +4 & 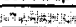 & mithotits & 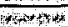 & & Ave/Spec & Flag \\
\hline Rb & 330 & 445 & 405 & 429 & 365 & 369 & 420 & 412 & 24 & $1.55 E+03$ & $2.67 \mathrm{E}-01$ & \\
\hline Re & N/A & NA & N/A & $N / A$ & N/A & N/A & N/A & NA & N/A & Na & N/A & \\
\hline Rh & $<20$ & $<20$ & $<20$ & $<20$ & $<20$ & $<20$ & $<20$ & $<20$ & NAA & $1.03 E+03$ & $1.94 E-02$ & \\
\hline Ru & $<20$ & 107 & 101 & 113 & $\$ 10$ & 99 & 100 & 105 & 6 & $2.83 E+03$ & $3.70 E-02$ & \\
\hline sb & $<20$ & 30 & 29 & $<20$ & 27 & $<20$ & 35 & 30 & 3 & $6.70 E+03$ & 4.53E-03 & \\
\hline Se & $<100$ & 400 & $<100$ & $<100$ & $<100$ & $<100$ & $<100$ & $<100$ & N/A & $4.12 E+03$ & $2.43 E-02$ & \\
\hline $\mathrm{sn}$ & $<20$ & $<20$ & $<20$ & $<20$ & $<20$ & $<20$ & $<20$ & $<20$ & N/A & $2,83 E+02$ & $7,06 \mathrm{E}-02$ & \\
\hline sr & $<20$ & 26 & $\leqslant 20$ & 30 & 22 & 24 & 23 & 25 & 3 & 4.12E +03 & $6.07 E-03$ & \\
\hline$T_{B}$ & $<20$ & $<20$ & 20 & $<20$ & $<20$ & $<20$ & $<20$ & $<20$ & N/A & $2.06 E+02$ & $9.70 E-02$ & \\
\hline $\mathrm{Tb}$ & $<20$ & $<20$ & $<20$ & $<20$ & $<20$ & $<20$ & $<20$ & $<20$ & N/A & $2.56 E+01$ & $7.76 \mathrm{E}-01$ & \\
\hline Te-99 & 4 & $<5$ & 6.0 & $<5$ & 5.2 & 45 & $<5$ & 5.6 & 0.6 & NA & N/A & \\
\hline To & $<50$ & $<50$ & $<50$ & $<50$ & $<50$ & 66 & $<50$ & 66 & N/A & $1.03 E+03$ & $6.39 \varepsilon-02$ & \\
\hline Th-232- & $<20$ & 44 & 36 & 30 & 46 & 23 & 33 & 35 & 9 & $N / A$ & N/A & \\
\hline$\pi$ & 43 & 664 & 528 & 532 & 541 & 583 & 539 & 564 & 53 & $1.03 E+04$ & $5.46 E-02$ & \\
\hline Im & $<20$ & $<20$ & $<20$ & $\times 20$ & $<20$ & $<20$ & $<20$ & $<20$ & N/A & $2,58 \mathrm{E}+01$ & $7.76 E-01$ & \\
\hline Total U & $<2$ & 1250 & 1170 & 1220 & 1270 & 1210 & 856 & 1163 & 154 & $1.06 E+05$ & $1.07 \mathrm{E}-02$ & \\
\hline U-233. & $<2$ & $<2$ & $<2$ & $<2$ & $<2$ & $<2$ & 3 & 3 & N/A & NA & N/A & \\
\hline$u-234$. & 2.9 & 2.3 & $<2$ & 3.8 & $<2$ & 2.0 & $<2$ & 2.7 & 1.0 & NA & N/A & \\
\hline U-235. & $<2$ & 10.8 & 7.5 & 10.5 & 9,0 & 9.3 & 6.2 & B. 9 & 1.8 & N/A & N/A & \\
\hline U-236* & 2.4 & 22 & $<2$ & 4.2 & 2.6 & 2.0 & 2.2 & 2.8 & 1.0 & N/A & N/A & \\
\hline$u-238$ & $<2$ & 1240 & 1160 & 1210 & 1260 & 1200 & 850 & 1153 & 152 & NA & N'A & \\
\hline v & 125 & 315 & 415 & 346 & 428 & 397 & 391 & 382 & 43 & $2.50 E+02$ & $1.48 E+\infty$ & • \\
\hline$w$ & 22 & 432 & 368 & 436 & 420 & 387 & 453 & 416 & 32 & $1.91 \mathrm{E}+03$ & $2,18 E-01$ & \\
\hline ro & $<0$ & $<20$ & $<20$ & $<20$ & $<20$ & $<20$ & $<20$ & $<20$ & N/A & $2.58 E+01$ & 7.7GE-01 & \\
\hline $2 n$ & 98 & 195 & 157 & 183 & 202 & 174 & 209 & 187 & 19 & $3.35 E+03$ & $5.58 E-02$ & \\
\hline$z r$ & $<20$ & 319 & 256 & 230 & 140 & 193 & 130 & 211 & 72 & $1.19 E+05$ & $1.7 \mathrm{BE}-03$ & \\
\hline
\end{tabular}

\section{N/A Nor Applicablo}

NU Nor Usabio

-No standards used, response taken fion different Isotope. 
HNF-SD-WM-DP-225, REV. 1

Analytical Summary Report - January 28, 1997

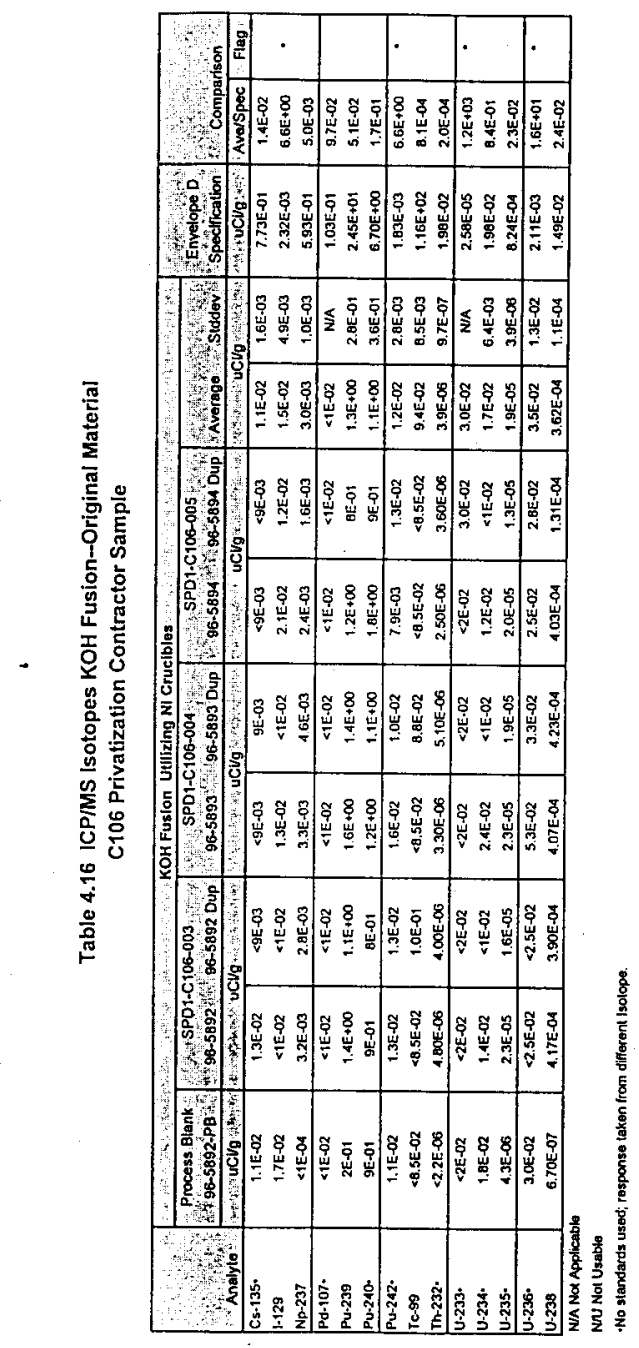

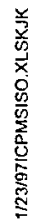


HNF-SD-WM-DP-225, REV. 1

Analytical Summary Report - January 28, 1997

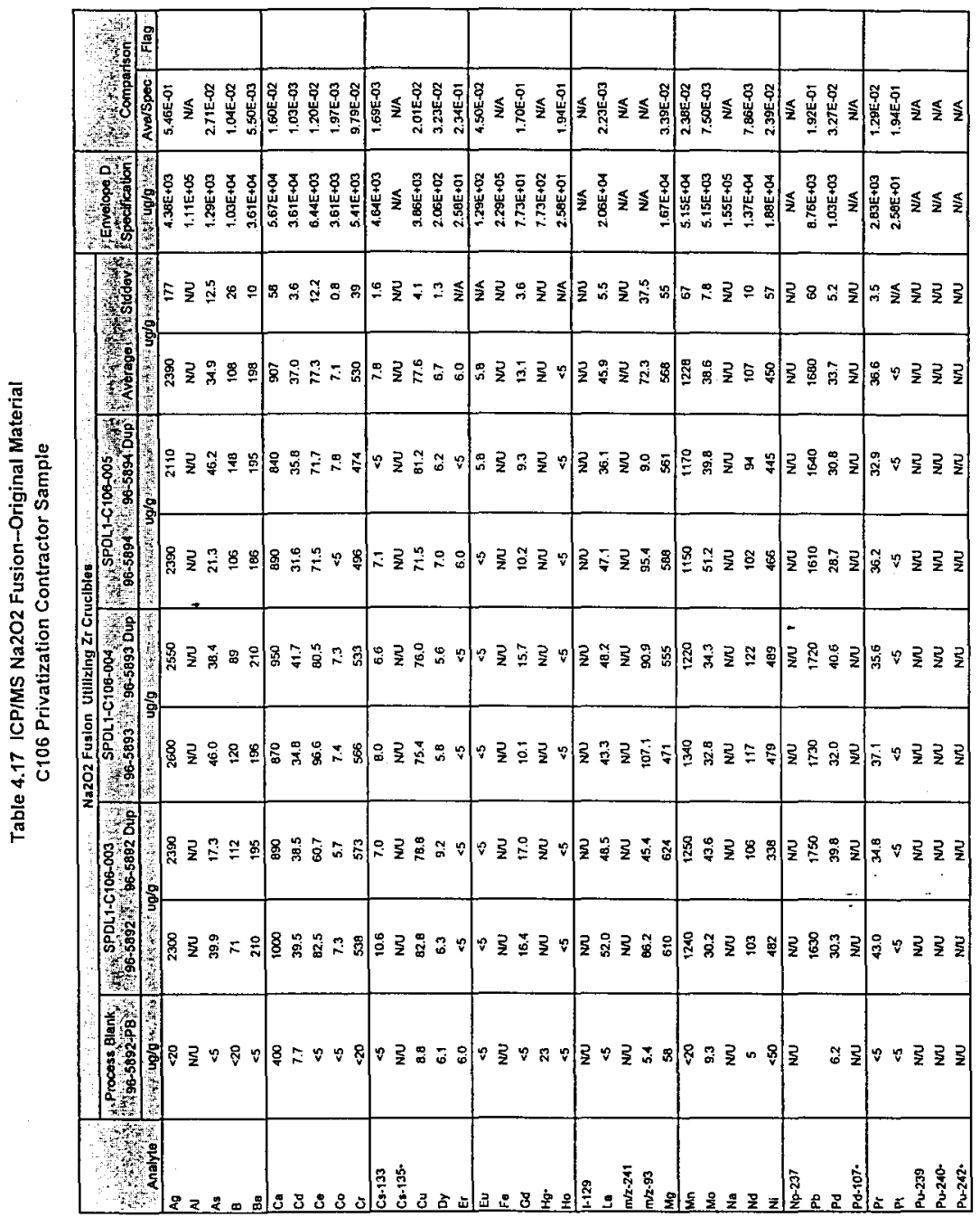


Table 4.17 ICP/MS Na2O2 Fusion-Original Material

C106 Privatization Contractor Sample

\begin{tabular}{|c|c|c|c|c|c|c|c|c|c|c|c|c|}
\hline \multirow{3}{*}{$\begin{array}{l}\text { ons } \\
\text { Analye }\end{array}$} & \multicolumn{9}{|c|}{ W... } & \multirow{3}{*}{$\begin{array}{l}\text { Envolope o } \\
\text { Specification } \\
\text { ug/g }\end{array}$} & \multirow{2}{*}{\multicolumn{2}{|c|}{ Comparison }} \\
\hline & \multirow{2}{*}{ 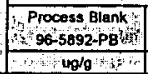 } & \multirow{2}{*}{\multicolumn{2}{|c|}{ 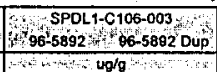 }} & \multirow{2}{*}{\multicolumn{2}{|c|}{$\begin{array}{r}\text { SPDL1-C106-004 } \\
96-5893 \text { 96-5893 Dup } \\
\text { ug/g }\end{array}$}} & \multicolumn{2}{|c|}{ 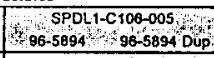 } & \multicolumn{2}{|c|}{ 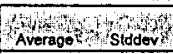 } & & & \\
\hline & & & & & & $-\ldots+4$ & 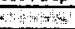 & 90 & the & & Ave/Spec & Flag \\
\hline $\mathrm{Rb}$ & 3.3 & 6.2 & 5.7 & 3.6 & 5.4 & 2.9 & 3.6 & 4.6 & 1.4 & $1.55 E+03$ & $2.95 \mathrm{E}-03$ & \\
\hline Re & N/A & NA & N/A & N/A & $N / A$ & N/A & N/A & N/A & N/A & N/A & N/A & \\
\hline Rh & $<20$ & 20 & $<20$ & $<20$ & $<20$ & $<20$ & $<20$ & $<20$ & $N / A$ & $1.03 E+03$ & $1.94 \mathrm{E}-02$ & \\
\hline Ru & No & $\mathrm{N}$ & NNS & NN & $\mathrm{N} / \mathrm{U}$ & NNS & NN & Nu & $\mathrm{MNS}$ & $2.83 E+03$ & N/A & \\
\hline sb & $<5$ & 6.1 & 7.0 & 7.1 & 6.4 & 7.1 & 6.6 & 6.7 & 0.4 & $6.70 E+03$ & $1.00 \mathrm{E}-03$ & \\
\hline Sa & $<50$ & $<50$ & $<50$ & $<50$ & $<50$ & 56 & $<50$ & 56 & N/A & $4.12 E+03$ & 1.35E- 02 & \\
\hline Sn & 144.6 & 97.2 & 48.6 & 99.1 & 86.9 & 70.0 & 71.1 & 78.8 & 19.3 & $2.83 E+02$ & $2.78 E-01$ & \\
\hline Sr & 73.9 & 76.5 & 88.2 & 65.7 & 82.7 & e3.9 & 75.0 & 78.7 & 8.0 & 4.12E+03 & $1.91 \mathrm{E}-02$ & \\
\hline Ta & $<5$ & 13.8 & 5.1 & 17.6 & $\$ 4.9$ & 13.7 & $<5$ & 13.0 & 4.7 & $2.06 E+02$ & G.32E-02 & \\
\hline $\mathbf{B b}$ & $<5$ & $<5$ & 45 & $<5$ & $<5$ & $<5$ & $<5$ & $<5$ & N/A & $2.58 \mathrm{BE}+01$ & 1,94ع-01 & \\
\hline Te-99 & $\mathrm{NU}$ & NNU & NN & NUU & $\mathrm{NOU}$ & NIU & $\mathrm{NM}$ & NN & NNS & $\mathrm{N} / \mathrm{A}$ & N/A & \\
\hline Te. & 137.5 & 102.7 & 63.6 & 134.0 & 77.5 & 100.6 & 55.7 & 89.0 & 29.1 & $1.03 E+03$ & 8.64E.02 & \\
\hline Th-232. & Nu & Nu & $\mathrm{N} N$ & NN & $\mathrm{N} N$ & NN & NN & No & $\mathbf{N O}$ & $N / A$ & NA & \\
\hline$\pi$ & 416 & 780 & 800 & 671 & 736 & 734 & 689 & 735 & 50 & $1.03 E+04$ & 7.13E-02 & \\
\hline Im & 45 & $<5$ & $<5$ & $<5$ & $<5$ & 5 & $<5$ & $<5$ & NA & $2.58 E+01$ & 1.94E-01 & \\
\hline Tolat U & $<5$ & 962 & 1180 & 1070 & 1090 & 1380 & 1110 & $\$ 132$ & 141 & $1.08 E+05$ & $1.05 E-02$ & \\
\hline U.233. & NO & NUI & NY & $N n$ & $\mathrm{~N} N$ & NN & $\mathrm{N} N \mathrm{U}$ & NN & NN & $N / A$ & N/A & \\
\hline U-234. & NU & NN & NN & NN & NN & NN & NU & NN & NN & WA & N/A & \\
\hline U.235. & NN & N/U & $\mathrm{N} N$ & $\mathrm{~N} N$ & NU & N/U & NU & $\mathrm{NNS}$ & NNS & NA & $\mathrm{N} / \mathrm{A}$ & \\
\hline U-236- & Nu & $\mathrm{NNU}$ & $\mathrm{NN}$ & Nos & NnS & N/U & No & NU & Nu & $N / A$ & N/A & \\
\hline U-238 & $\mathrm{NN}$ & N/U & NU & NNS & $\mathrm{N} \Omega \mathrm{U}$ & N/U & $\mathrm{NN}$ & Mn & NUS & $\mathrm{N} / \mathrm{A}$ & N/A & \\
\hline$v$ & 507 & 646 & 895 & 942 & 941 & 1030 & 843 & 883 & 131 & $2,58 E+02$ & $3.43 E+\infty 0$ & - \\
\hline$w$ & $<5$ & 259 & 260 & 339 & 368 & 403 & 255 & 314 & 65 & $1.91 E+03$ & $1.65 \mathrm{E}-01$ & \\
\hline ro & $<5$ & $<5$ & $<5$ & $<5$ & $<5$ & $<5$ & $\times 5$ & $<5$ & N/A & $2.58 E+01$ & $1.94 E-01$ & \\
\hline$z n$ & 114 & 136 & 105 & 123 & 120 & 163 & 118 & 127 & 20 & $3.35 E+03$ & $3.60 E-02$ & \\
\hline$z$ & NO & NN & $\mathrm{N} N$ & NNU & NN & NOU & NN & NU & NMU & $1.19 E+05$ & N/A & \\
\hline
\end{tabular}

Nu Nol Usabte

-No standards used; responsa laken from diflerent Isolope. 
HNF-SD-WM-DP-225, REV. 1

Analytical Summary Report - January 28, 1997

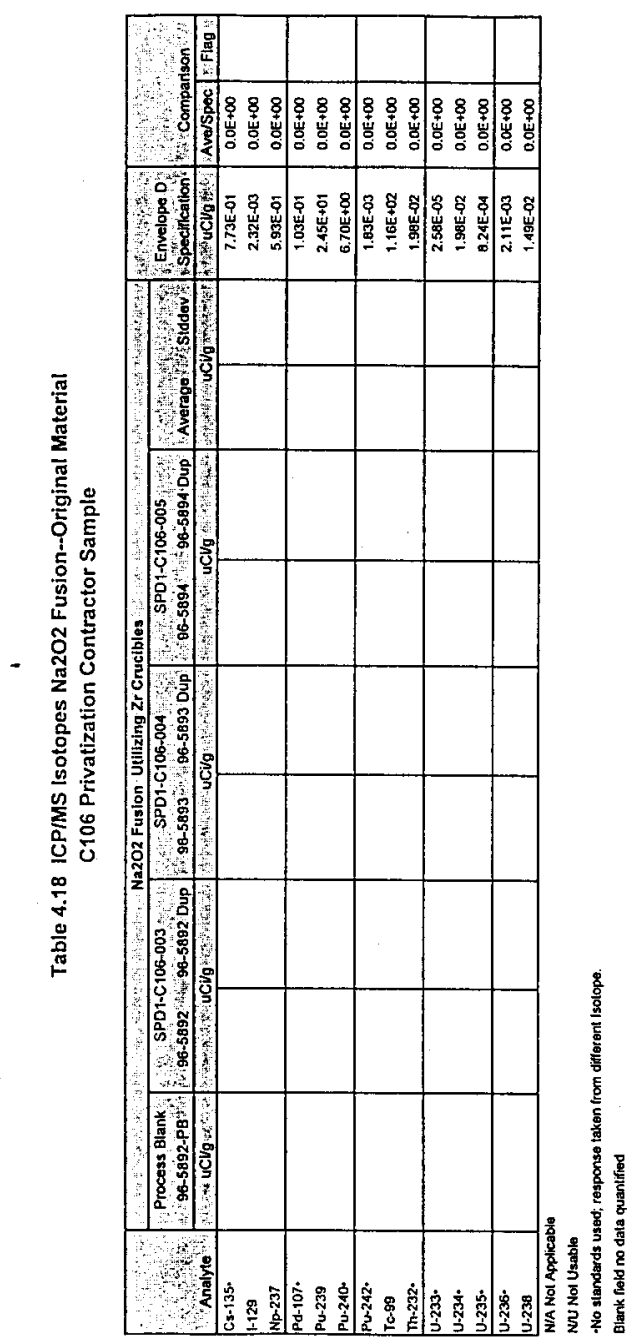


Table 4.19 ICPIMS Acid Digestion-Original Material C106 Privatization Contractor Sample

\begin{tabular}{|c|c|c|c|c|c|c|c|c|c|c|c|c|}
\hline \multirow{2}{*}{ 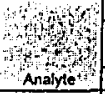 } & \multicolumn{3}{|c|}{ 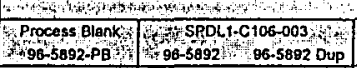 } & \multicolumn{2}{|c|}{$\begin{array}{l}\text { acld digestion } \\
\text { 96-5893 SPDL-C106-004 } \\
\text { 96-5893 Dup }\end{array}$} & \multicolumn{2}{|c|}{$\begin{array}{l}\text { SPOL1-C106-005 } \\
\text { 96-5894 } \quad \text { 96-5894 Dup }\end{array}$} & \multicolumn{2}{|c|}{$\begin{array}{l}\text { ath } \\
\text { Average }\end{array}$} & \multirow{2}{*}{$\begin{array}{l}\text { Envelope D } \\
\text { Speciricalion } \\
\text { Yetug/g }\end{array}$} & \multicolumn{2}{|c|}{ 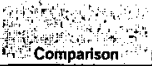 } \\
\hline & 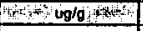 & 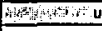 & $9 \operatorname{lit}^{2}$ & की & जिएक & afofent & $1+40+1$ & and & 9.tong & & Ave/Spec & Flag. \\
\hline$A_{0}$ & $<5$ & 94.8 & 71.4 & 79.6 & 79.6 & 70.0 & 80.4 & 79.3 & 8.8 & $4.38 E+03$ & 1.81E-02 & \\
\hline A & $<50$ & 30100 & 28400 & 28500 & $3+000$ & 28900 . & 26900 & 28967 & 1431 & $1.11 E+05$ & $2.61 E-01$ & \\
\hline As & $\$ 5.2$ & 29.6 & 35.4 & 32.2 & 39.0 & 18.0 & 22.1 & 29.4 & 8.0 . & $1.29 E+03$ & $2.20 \mathrm{E}-02$ & \\
\hline B & 82 & 123 & 106 & 97 & 107 & 126 & 103 & 111 & 11 & $1.03 E+04$ & t. $08 E-02$ & \\
\hline $\mathrm{Ba}$ & $<6$ & 199 & $19 a$ & 199 & 185 & 179 & 201 & 194 & 9 & $3.61 E+04$ & 5. $37 E-03$ & \\
\hline $\mathrm{Ca}$ & 28 & 110 & 310 & 388 & 302 & 403 & 400 & 383 & 38 & $5.67 E+04$ & $6.76 \mathrm{E}-03$ & \\
\hline Cd & $<5$ & 31,6 & 32.0 & 27.3 & 26.2 & 25.8 & 31.8 & 29.1 & 3.0 & $3.61 E+04$ & Q.07E-04 & \\
\hline C* & $<5$ & 903,1 & 96.8 & 97.9 & 85.7 & 90.7 & 93.4 & 94.6 & 6.1 & $6.44 E+03$ & $1.47 E-02$ & \\
\hline Co & $<5$ & 8.2 & 7.4 & 7.7 & 7.2 & 8.4 & 7.3 & 7.7 & 0.5 & $3.61 E+03$ & $2.13 E-03$ & \\
\hline $\mathrm{Cr}$ & 10 & 402 & 393 & 379 & 365 & 369 & 382 & 382 & 14 & $5.41 E+03$ & $7.05 E-02$ & \\
\hline Cs-133 & 45 & 8.6 & 9.4 & 7.5 & 7.7 & 7.9 & 7.5 & 8.1 & 0.8 & $4.64 E+03$ & $1.75 E-03$ & \\
\hline Cs-135. & $<0.5$ & $<0.5$ & $<0.5$ & $<0,5$ & $<0.5$ & $<0.5$ & $<0.5$ & $<0.5$ & N/A & N/A & N/A & \\
\hline cu & $<5$ & 71.2 & 70.6 & 60.9 & 66.4 & 67.8 & 67.5 & 69.4 & 2.0 & $3,86 \mathrm{E}+03$ & $1,77 \mathrm{E}-02$ & \\
\hline Dy & 45 & $<5$ & $<5$ & $<5$ & 25 & 45 & .5 & 45 & $N \wedge$ & $2.0 G E+02$ & 2. 13E-02 & \\
\hline Fr & $<5$ & $<5$ & 45 & $\leq 5$ & 55 & 5.5 & 4 & $<5$ & N/A & 2.505:-01 & 1.94E-01 & \\
\hline Eu & 45 & 25 & $<5$ & $<5$ & 45 & $<5$ & 45 & 45 & NUN & $1.29 E+02$ & J.00E-02 & \\
\hline Fe & $<\infty 000$ & 97000 & 95700 & 97000 & $B G B 00$ & 95600 & 96500 & 95033 & 4148 & $2.20 E+05$ & $4.14 E-01$ & \\
\hline Gd & $<5$ & 11.0 & 9.1 & 15.2 & 8.9 & 9.5 & 9.4 & 10.5 & 2.4 & $7.73 E+01$ & $1,36 E \cdot 01$ & \\
\hline $\mathrm{Hg} \cdot$ & $<5$ & 50 & 59 & 60 & 57 & 49 & 54 & 56 & 1 & $7.73 E+02$ & $7.22 E-02$ & \\
\hline Ho & 45 & -5 & $\times 5$ & 45 & 45 & $<5$ & 45 & $<5$ & N/A & $2.58 \mathrm{BE}+01$ & $1.94 \mathrm{E}-01$ & \\
\hline $1-129$ & $<5$ & 7.8 & 9.2 & 11.8 & 9.8 & 10.4 & 14.8 & 10.6 & 2.4 & $\mathrm{~N} / \mathrm{A}$ & N/A & \\
\hline La & $<5$ & 52.6 & 51.8 & 52.2 & 51.7 & 47.5 & 31.5 & 51.2 & 1.9 & $2.06 E+04$ & $2.48 E-03$ & \\
\hline$m / z \cdot 241$ & $<0.2$ & 0.6 & 0.5 & 0.6 & $<0.5$ & $<0.5$ & $<0.5$ & 0.6 & 0.1 & N/A & $\mathrm{N} / \mathrm{A}$ & \\
\hline$m / 2-93$ & $<5$ & 31.8 & 15,4 & 36.0 & 47.6 & 42.4 & 19.8 & 32.2 & 12.6 & NA & NA & \\
\hline$M g$ & $<5$ & 278 & 262 & 277 & 249 & 261 & 273 & 267 & 11 & $1.67 E+04$ & $1.59 E .02$ & \\
\hline $\mathrm{Mn}$ & 45 & 1150 & 1120 & 1140 & 1020 & 1090 & 1110 & 1105 & 47 & $5.15 E+04$ & $2.14 E-02$ & \\
\hline Mo & $<5$ & 18.8 & 23.2 & 99.7 & 20.6 & 21.1 & 21.8 & 20.9 & 1.6 & $5.15 E+03$ & $4.05 E-03$ & \\
\hline $\mathrm{Na}$ & $<200$ & 178000 & 185000 & 169000 & 199000 & 181000 & 167000 & 179833 & 11669 & $1.55 E+05$ & $1.16 E+00$ & • \\
\hline Nd & 4 & 148 & 108 & 116 & 106 & 128 & 136 & 124 & 17 & $1.37 E+04$ & $9.06 E-03$ & \\
\hline $\mathrm{Ni}$ & 31 & 241 & 259 & 222 & 191 & 181 & 199 & 216 & 30 & $1.8 B E+04$ & $1,15 E-02$ & \\
\hline Np.237 & 0.2 & 1.6 & 2.2 & 2,1 & $2.4 ?$ & 2.0 & 2.1 & 2.1 & 0.3 & N/A & N/A & \\
\hline $\mathrm{Pb}$ & 6 & 1217 & 1191 & 1176 & $t 100$ & 1105 & 1137 & 1154 & 48 & $8.76 \mathrm{E} \cdot 03$ & $1.32 E-01$ & \\
\hline$P d$ & $<5$ & 0.0 & 8.0 & 6.6 & 8.5 & 8.0 & 8.7 & 8.3 & 0.9 & $1.03 E+03$ & B. $03 E-03$ & \\
\hline Pd-107. & $<5$ & 16.5 & 6.1 & $<5$ & $\times 5$ & $<5$ & .5 & 11.3 & 7.4 & N/A & N/A & \\
\hline Pr & 45 & 43.2 & 43.6 & 43.6 & 35.2 & 37.1 & 37.8 & 40.1 & 3.8 & $2.83 E+03$ & $1.41 \mathrm{E}-02$ & \\
\hline $\mathrm{PI}$ & $<5$ & $<5$ & $<5$ & $<5$ & $<5$ & $<5$ & 45 & $<5$ & N/A & $2.58 E+01$ & $1.94 E-01$ & \\
\hline Pu-239 & $<0.2$ & 4.9 & 6.5 & 3.7 & 4.6 & 5.3 & 6.9 & 5.3 & 1.2 & NA & N/A & \\
\hline Pu-240. & $<0.4$ & 0.9 & 0.9 & $<0,4$ & 0.7 & 0.7 & 0.5 & 0.7 & 0.2 & NAA & N/A & \\
\hline$P_{11-242 .}$ & $<0.6$ & $\leqslant 0.6$ & $\times 0.6$ & $<0.6$ & $\leq 0.6$ & $<0.0$ & $=0.6$ & $<0.6$ & N/A & N/A & N/A & \\
\hline
\end{tabular}


HNF-SD-WM-DP-225, REV. 1

Analytical Summary Report - January 28, 1997

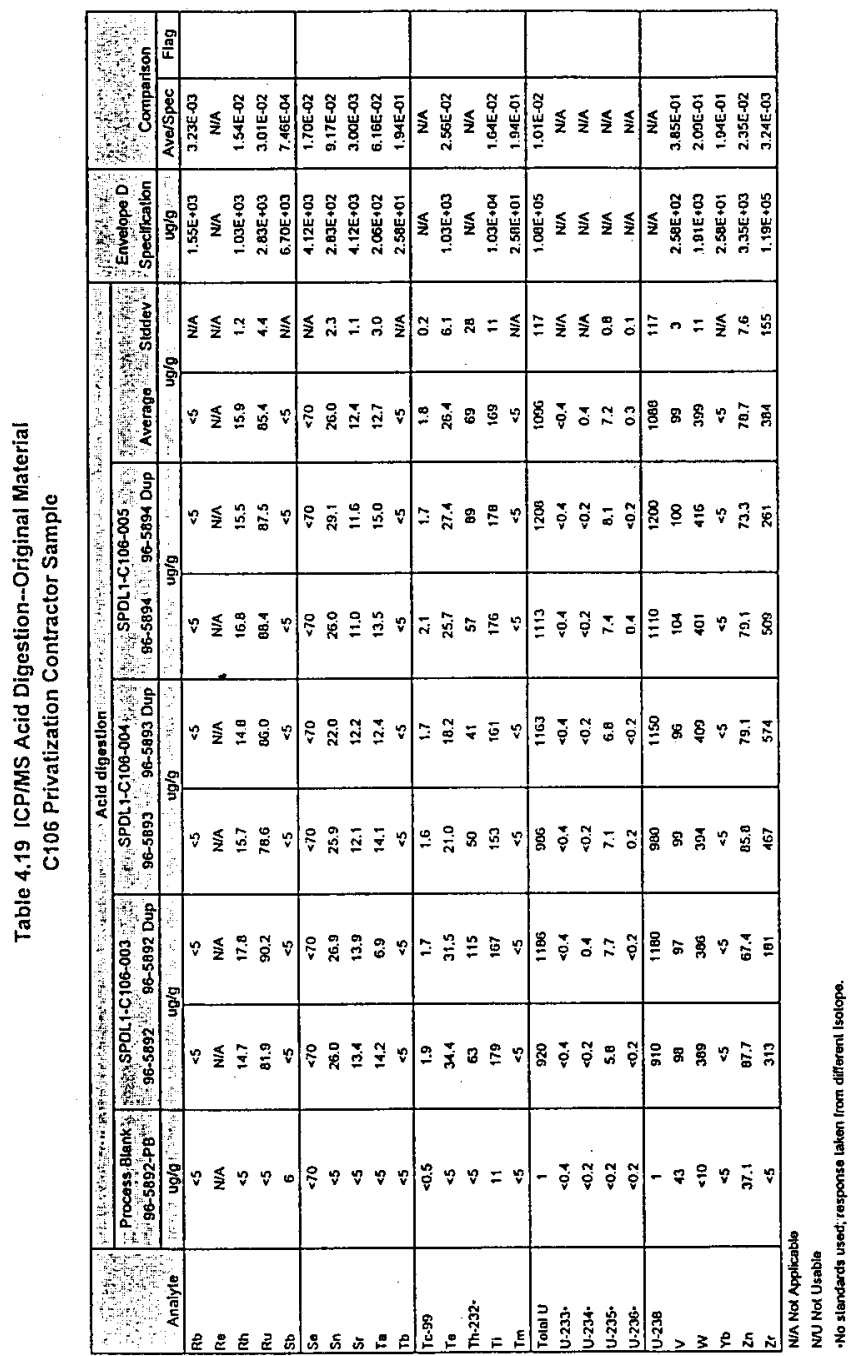


Table 4.20 ICP/MS Isotopes Acid Digestion-Original Material C106 Privatization Contractor Sample

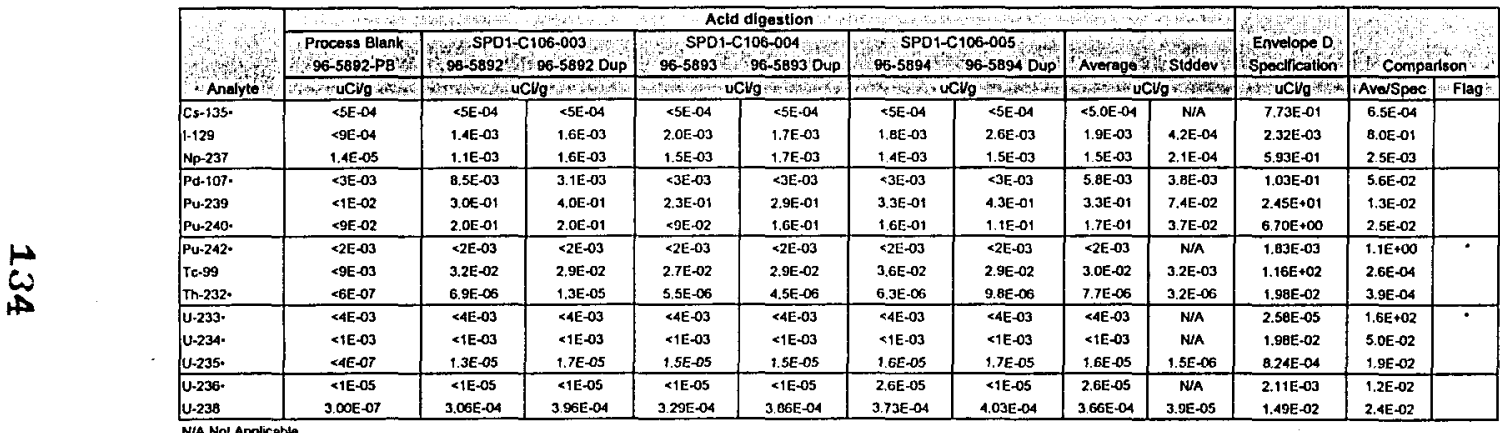

NN Nol Usable

-No standards usod, responso taken from differenl lsolope. 
HNF-SD-WMI-DP-225, REV. I

Analytical Summary Report - January 28, 1997
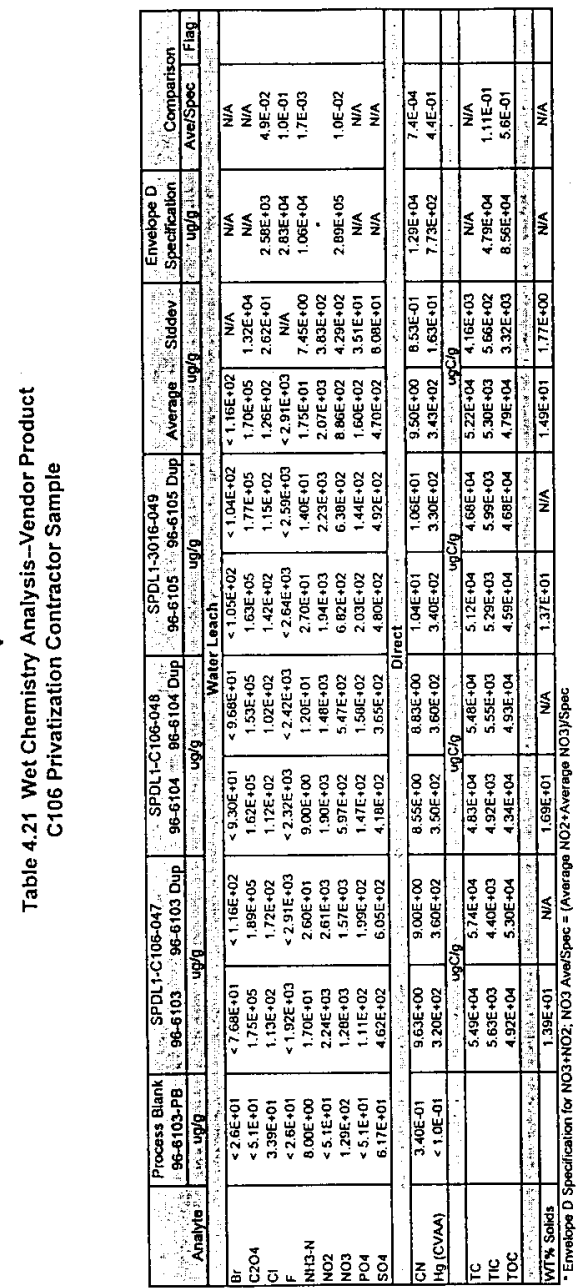
Table 4.22 Wet Chemistry Analysis-Original Material C106 Privatization Contractor Sample

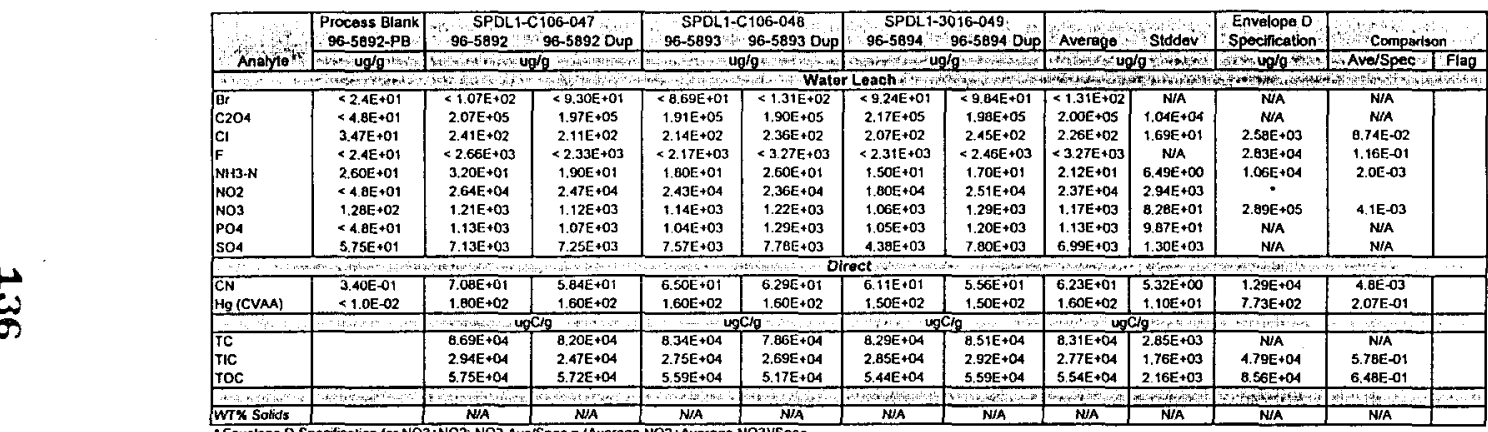

Envelope o Specificalion for $\mathrm{NO}^{3+\mathrm{NO} 2}$ NO3 Ave/Spec = (Average NO2+Average NO3)/Spec 
HNF-SD-WM-DP-225, REV. 1

Analytical Summary Report - January 28, 1997

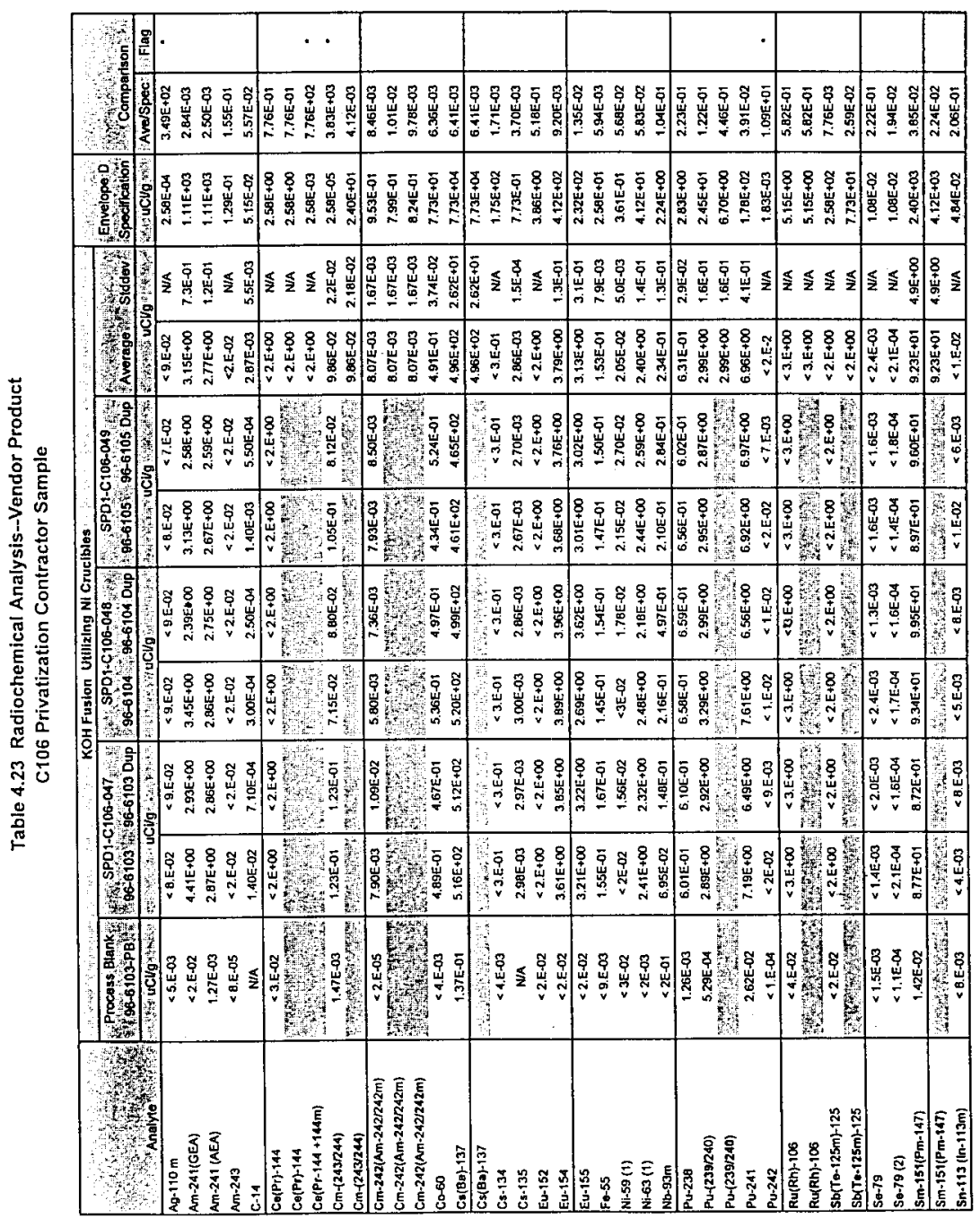

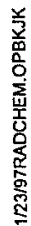


HNF-SD-WM-DP-225, REV. i

Analytical Summary Report - January 28, 1997

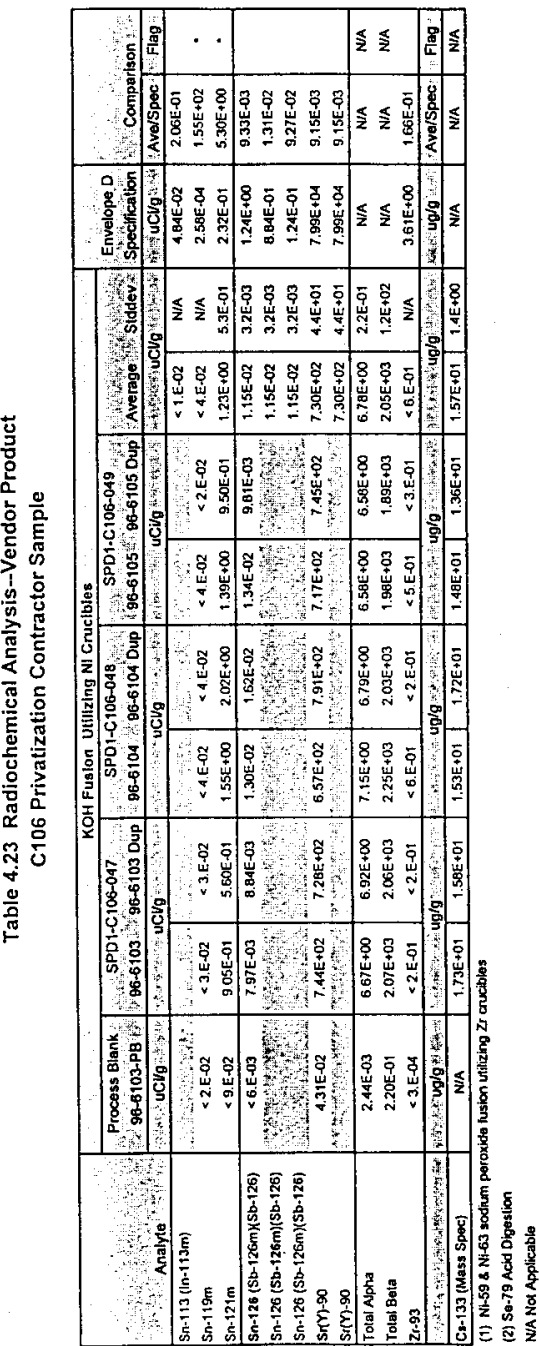


Table 4.24 Radiochemical Analysis--Original Material

C106 Privatization Contractor Sample

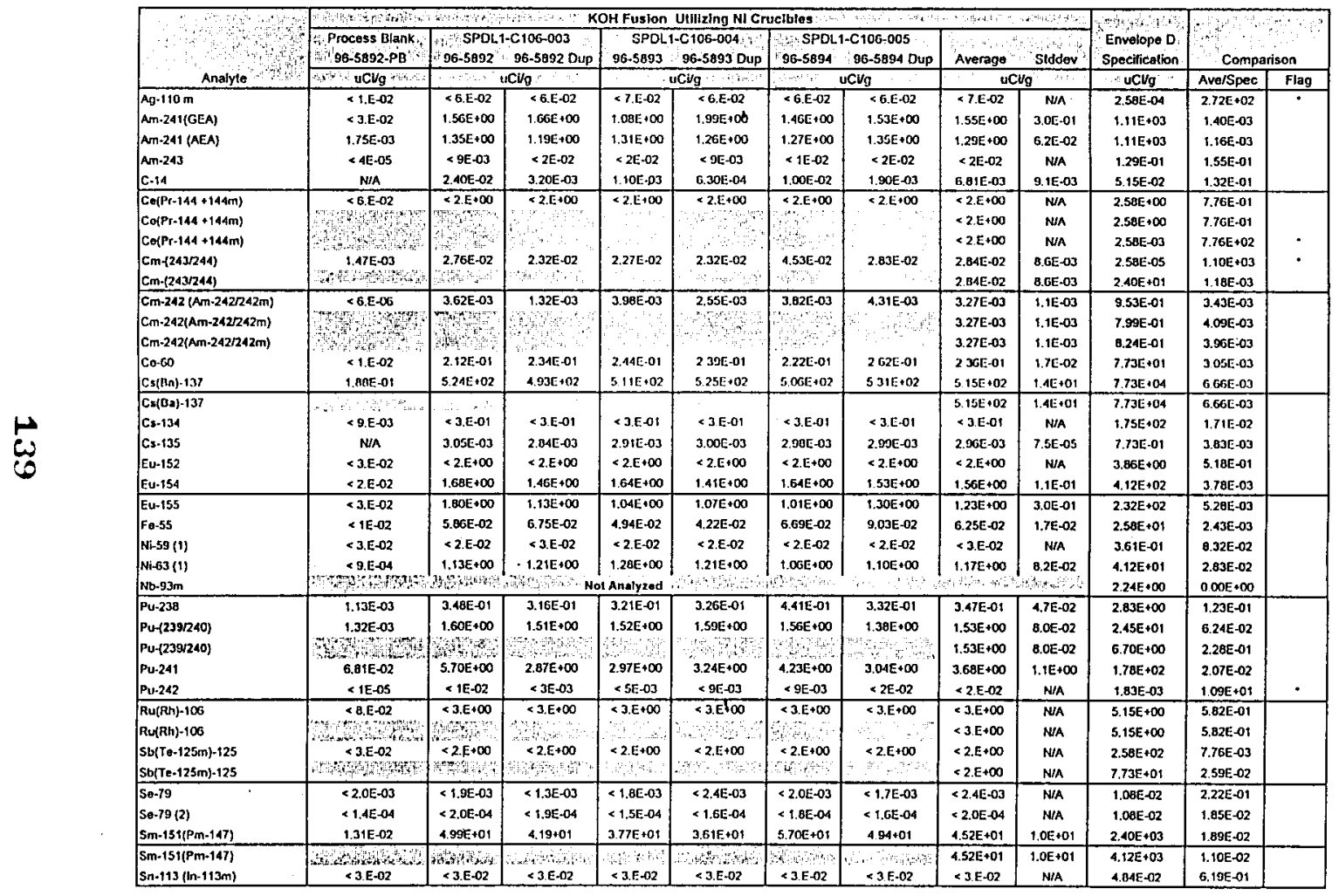


$+$

-Table 4.24 Radiochemical Analysis--Original Material C106 Privatization Contractor Sample

\begin{tabular}{|c|c|c|c|c|c|c|c|c|c|c|c|c|}
\hline \multirow{3}{*}{$\begin{array}{r} \\
\text { Analyie }\end{array}$} & \multicolumn{9}{|c|}{ KOH Fusion Uillizing NI Griclbles } & \multirow{3}{*}{$\begin{array}{l}\text { Envelope } D \\
\text { Specitication } \\
\text { uCvg }\end{array}$} & \multirow{2}{*}{\multicolumn{2}{|c|}{ Comparison }} \\
\hline & $\begin{array}{c}\text { Procoss Blank } \\
\text { 9G-5892-PB }\end{array}$ & \multicolumn{2}{|c|}{$\begin{array}{l}\text { SPOL1-C106-003 } \\
96-5892 \text { 96-5892 Dup }\end{array}$} & \multicolumn{2}{|c|}{ SPOL1-C106-004 } & \multicolumn{2}{|c|}{ SPDL1-C106-005 } & \multicolumn{2}{|c|}{ Averago SIddov } & & & \\
\hline & uCvg & 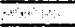 & $\mathrm{Cl}_{\mathrm{g}} \mathrm{a}$ & $\therefore$ & $\sqrt[n+\infty]{n+m}$ & 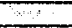 & $\overline{v g}$ & \multicolumn{2}{|c|}{ uCvg } & & Ave/Spec & Flag \\
\hline $\begin{array}{l}S n-113(\ln -113 m) \\
S n-119 m\end{array}$ & $\begin{array}{l}14 \mathrm{Ch} \\
\times 2 \mathrm{E}-01\end{array}$ & $\begin{array}{l}<1, E-01 \\
<4\end{array}$ & $\begin{array}{r}\times 1 \\
\times 3 E-01\end{array}$ & $<4,5-01$ & $\begin{array}{l}0.0 .01 \\
C 3.01\end{array}$ & $<1, E \cdot 01$ & < A.E.01 & $\begin{array}{l}<3 . E-02 \\
<4.5-01\end{array}$ & N/A & $\begin{array}{l}4.84 E-02 \\
2.50 E-01\end{array}$ & $\begin{array}{l}6.19 E-01 \\
1.55 E \cdot 03\end{array}$ & $\cdot$ \\
\hline $5 n+121 m$ & $=2.1 .00$ & $<2, F, 00$ & $=3 F+\infty$ & $<3 E+00$ & $-2 E+00$ & $<3 E+\infty 0$ & $<3[.000$ & $<x \in+0$ & N/A & 2.37E-01 & 1295.01 & - \\
\hline $\begin{array}{l}\mathrm{Sn}-126(\mathrm{Sb}-126 \mathrm{~m})(\mathrm{Su}-126) \\
\mathrm{Sn}-126(\mathrm{Sb}-126 \mathrm{~m})(\mathrm{Sb}-126) \\
\operatorname{Sn}-126(\mathrm{Sb}-126 \mathrm{~m})(\mathrm{Sb}-126) \\
\operatorname{Sr}(Y)-90 \\
\mathrm{Sr}(Y) \cdot 90\end{array}$ & 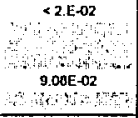 & $\begin{array}{r}<2 . E-02 \\
1 \\
3,60 E \cdot 02 \\
\quad \\
\end{array}$ & $\begin{array}{c}<2 . E-02 \\
\cdots \\
3.35 E+02 \\
\end{array}$ & $<3 . E-02$ & $\begin{array}{r}<2, \mathrm{E}-02 \\
3.50 E+02 \\
\therefore \quad+\end{array}$ & $\begin{array}{l}<2 . E-02 \\
\cdots \\
3.65 E+02\end{array}$ & $3.57 E+02$ & $\begin{array}{l}<3 . E-02 \\
<3 . E-02 \\
<3 . E-02 \\
3.54 E+02 \\
3.54 E+02 \\
\end{array}$ & $\begin{array}{c}N / A \\
N / A \\
N / A \\
1.1 E+01 \\
1,1 E+01 \\
\end{array}$ & $\begin{array}{l}1.24 E-01 \\
0.84 E-01 \\
1.24 E-0 t \\
7.99 E+04 \\
7.99 E+04\end{array}$ & $\begin{array}{l}2.4 J E-01 \\
3.40 E-02 \\
2.4 t E-01 \\
4.43 E-03 \\
4.43 E-03\end{array}$ & \\
\hline Tolal Apha & $<5 . E-03$ & $2.83 E+00$ & $2.80 E+00$ & $3.05 E+00$ & 3.01E +00 & $3.87 E+00$ & $3.67 E+00$ & $3.21 E+00$ & $4,5 E-01$ & N/A & NAA & N/A \\
\hline Total Beta & 4,34E-01 & $1.29 E \cdot 03$ & $1.17 E+03$ & $1.19 E+03$ & $1.22 E+03$ & $1.196+03$ & $1.24 E+03$ & $1.22 E+03$ & $4.4 E+01$ & N/A & $\mathrm{N} / \mathrm{A}$ & N/A \\
\hline $27 \cdot 93$ & $<2 . E-03$ & $5.78 E-02$ & 7.63E-02 & 4.15E- -02 & $3.25 E-02$ & 4.59E. .02 & 2.91E.02 & $4.72 \mathrm{E}-02$ & $10 \mathrm{E}-02$ & $3.61 E+00$ & $1.31 E .02$ & \\
\hline 3 & $\sim \mu g / g$ & 100 & p/g & & log & $\therefore$ & 19 & & & ug/g & Ave/Spec & Flag \\
\hline Cs-133 (Mass Spec) & N/A & $1.53 E \cdot 01$ & $1.39 \mathrm{E}+01$ & $1,54 \mathrm{5}+01$ & $1.64 E+01$ & $1.53 E+01$ & $2.80 E+01$ & $1.75[+01$ & $56 t+00$ & N/A & N/A & N/A \\
\hline
\end{tabular}

\section{(1) Ni-59 \& Ni-63 sodwun peroxido lusion ulliking Zr crucibies}

(2) So-79 Acid Digostion

NIA Not Appikable 
HNF-SD-WM-DP-225, REV. 1

Analytical Summary Report - January 28, 1997

Table 4.25 Decant Liquid--Vendor Product C106 Privatization Contractor Sample

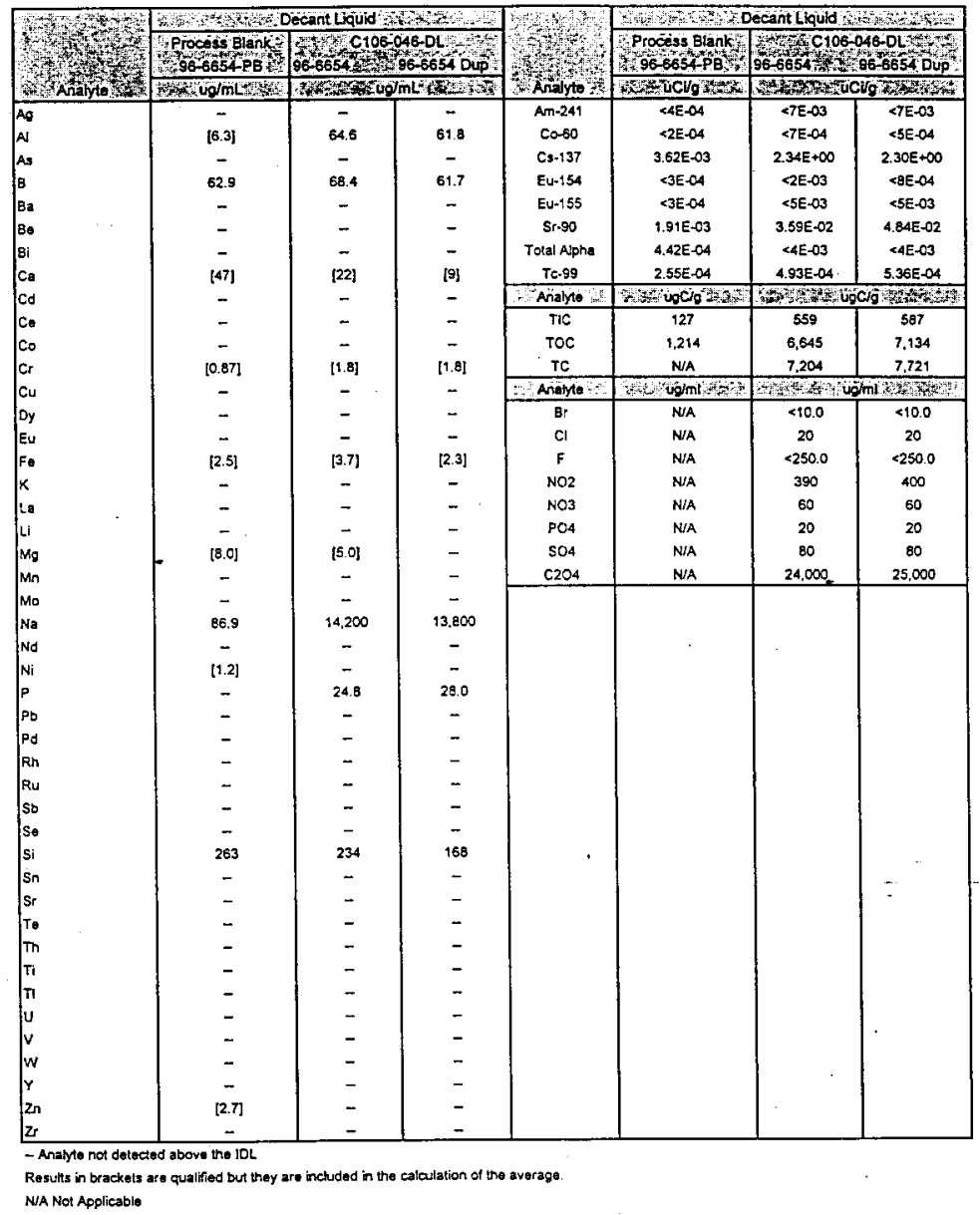


HNF-SD-WM-DP-225, REV. 1

Analytical Summary Report - January 28, 1997

Table 4.26. Centrifuged Solids/Decant Liquid-Original Material C106 Privatization Contractor Sample

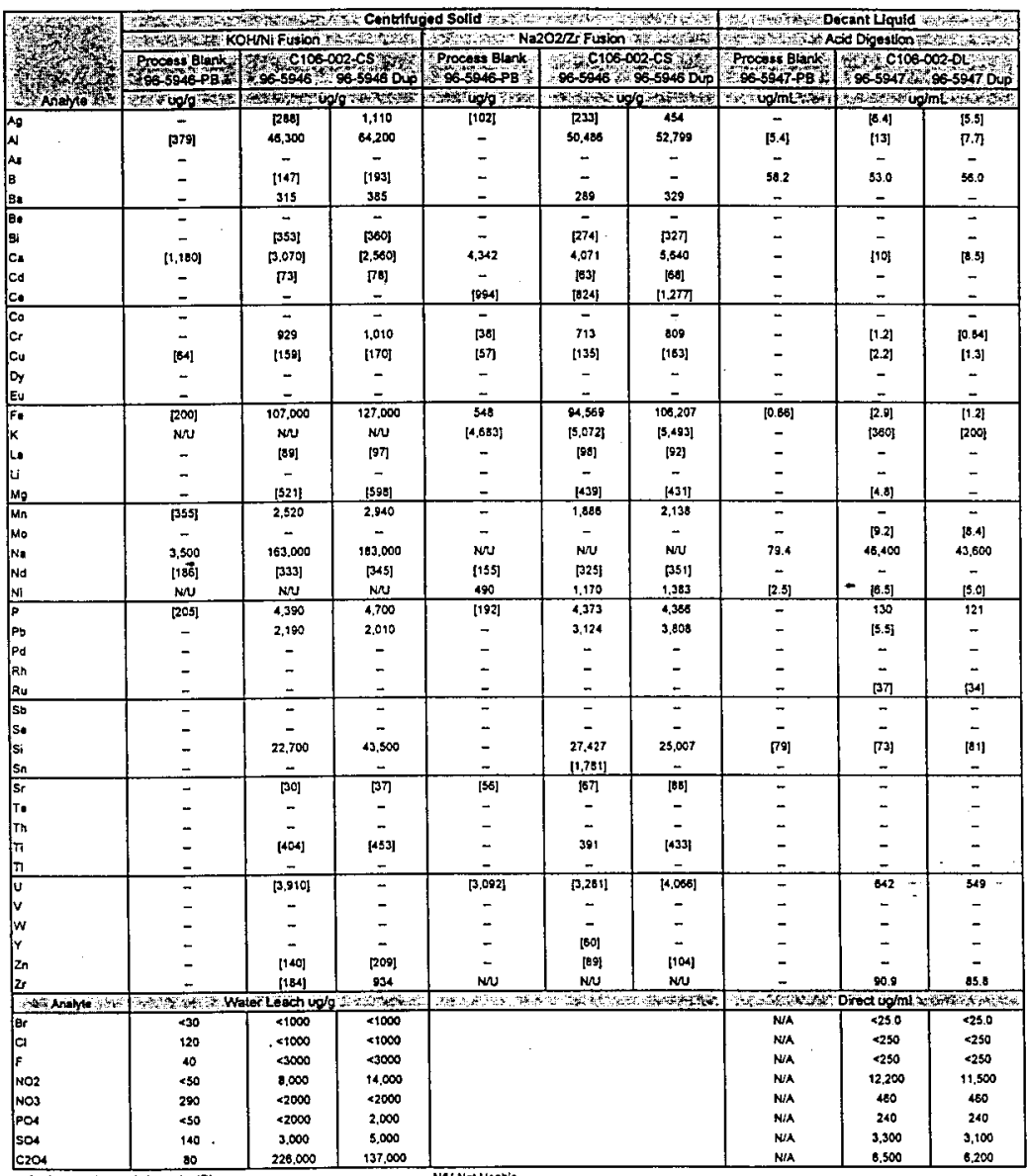

- Anatre nor cletected soove the $10 \mathrm{~L}$

N/A Not Appliende

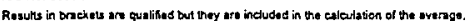




\section{APPENDIX A}

\section{ICP-MS DATA QUALIFICATIONS AND OBSERVATIONS - VENDOR PRODUCT}

\section{$\underline{\text { Selenium }}$}

There were significant disparities observed between the different preparations for nearly every element. One element for which this was especially apparent was selenium. It was not immediately clear if the software misinterpreted the mass spectra when integrating and calculating, but none of the spectra for any of the preparations indicate a measurable quantity of selenium. The isotope used for these calculations ( $\mathrm{m} / \mathrm{z} 78$ ) yielded the best calibration curve; however, it is typically interfered with by the ${ }^{38} \mathrm{Ar}{ }^{40} \mathrm{Ar}$ dimer.

\section{Vanadium}

There were also disparities observed between vanadium results. Visual inspection of the data indicated $\mathrm{ClO}^{+}$interference peaks for both fusion preparations; the presence of this interference in the acid digestion preparation could not be confirmed.

\section{Mass $93(\mathrm{Zr} / \mathrm{Nb})$ and Mass $241(\mathrm{Pu} / \mathrm{Am})$}

The ICP-MS $\mathrm{m} / \mathrm{z} 93$ (probably $\mathrm{Nb}$ ) results also include a contribution from zirconium, since there is an interference from zirconium at $\mathrm{m} / \mathrm{z} 93$ by ICP-MS. Resolution of the niobium and zirconium is not complete. The same interference problem is experienced at $\mathrm{m} / \mathrm{z} 241$ with plutonium and americium. Therefore, only one value is reported for $\mathrm{m} / \mathrm{z} 93$ and $\mathrm{m} / \mathrm{z} 241$; possible/probable isotopes contributing to the response observed at these masses are zirconium/niobium and plutonium/americium, respectively. However, it should be noted that ${ }^{93 \mathrm{~m}} \mathrm{Nb},{ }^{93} \mathrm{Zr},{ }^{24 t} \mathrm{Pu}$, and ${ }^{241} \mathrm{Am}$ were measured separately by conventional radiochemistry techniques.

\section{Mass $242(\mathrm{Pu})$}

While there appear to be measurable peaks at $\mathrm{m} / \mathrm{z} 240$ and $\mathrm{m} / \mathrm{z} 241$, the values reported for $\mathrm{m} / \mathrm{z} 242$ are estimated quantitation levels. The lowest ${ }^{24} \mathrm{Pu}$ standard analyzed was $0.1 \mathrm{ppb}$, which would amount to approximately $0.125 \mathrm{ppm}$ in the sample. Because a response was obtained for this standard, $0.1 \mathrm{ppm}$ is a reasonable estimate for a lower limit of detection'quantitation for ${ }^{242} \mathrm{Pu}$.

\section{Rubidium}

The rubidium results on the $\mathrm{KOH}$ fusions are questionable. Based on the preparation blank result, it is possible that the $\mathrm{KOH}$ was contaminated with rubidium; multiplying by a total dilution factor of 25,000 would amplify this affect. The other two sample preparation results agreed reasonably well, and did not have as high a dilution factor, making those values more reliable. 


\section{Palladium-107}

The ${ }^{107} \mathrm{Pd}$ results show a questionably high concentration in the $\mathrm{Na}_{2} \mathrm{O}_{2}$ fusions. These values (in the mid-200 ppm range) may be due to a $\mathrm{ZrO}^{+}$interference. Also, the relatively high concentration of $\mathrm{Ag}$ in these samples makes for a very large correction factor, necessarily resulting in a large uncertainty. Between $1-5 \mathrm{ppm}{ }^{107} \mathrm{Pd}$ was observed in the acid digestion samples. Because the silver and zirconium concentrations are significantly lower in the acid digested samples, the results are considered more reliable.

\section{Zirconium}

Because the reported value for zirconium was calculated from the response at $m / z 90$, it includes a contribution from ${ }^{90} \mathrm{Sr}$, likely making the ICP-MS zirconium value bias high.

\section{Isotopic and Fission-product Considerations}

Ba: The barium isotopic abundance appears to be significantly altered. This is most notable for 138, whereas all isotopes below 137 agree with each other. The $\mathrm{m} / \mathrm{z} 138$ was used to measure the Ba concentration, which means that the reported values may be bias high. It is unclear whether the 138 peak is barium or perhaps fission-produced cerium.

$\mathrm{Cu}$ : Copper appears to be completely natural, with no apparent contribution from ${ }^{63} \mathrm{Ni}$. The reported result was obtained from $\mathrm{m} / \mathrm{z} 65$.

Cs: The cesium values reported are for the concentration of ${ }^{133} \mathrm{Cs}$ ONLY, and does not include ${ }^{135} \mathrm{Cs}$ or ${ }^{137} \mathrm{Cs}$.

Pd: There seems to be slight enrichment at 106 and 107, which may be fission-product palladium, or may be $\mathrm{ZrO}^{+}$. Confirmation is not possible without a separation; however, little change in the result would be anticipated. The $\mathrm{m} / \mathrm{z} 105$ peak used agrees well with $\mathrm{m} / \mathrm{z} 108$.

Ru: The ruthenium present appears to be exclusively fission-produced. The concentration was calculated using the response at $\mathrm{m} / \mathrm{z} 102$, with an assumed isotopic abundance of $-47 \%$ based on the relative abundance observed of $\mathrm{m} / \mathrm{z} 101$ and 102 .

Te: The tellurium present in the samples resembles that of fission-product tellurium." The most apparent indication that at most (if not all) of the tellurium present is fission produced is the presence of a prominent $\mathrm{m} / \mathrm{z} 130$ peak, which appears to be almost 5 times that of the $\mathrm{m} / \mathrm{z} 128$ peak; for natural abundance the responses should be nearly equal. In addition, the responses observed where the natural lower-mass tellurium isotopes should be found (i.e., $\mathrm{m} / \mathrm{z} 122$, $\mathrm{m} / \mathrm{z} 123, \mathrm{~m} / \mathrm{z} 124$ ) appear to be due to natural tin and antimony.

$\mathrm{Zr}$ : The $\mathrm{m} / \mathrm{z} 90$ was used in calculating the reported zirconium result, which in retrospect was probably not the best choice due to ${ }^{90} \mathrm{Sr}$. However when the spectra were reviewed, it was observed that the zirconium abundance appeared to be completely natural, with $\mathrm{m} / \mathrm{z} 90$ being just slightly low rather than high. It appears that the results reported are representative of the total zirconium concentration, but with a possible low bias. 


\section{ICP-MS DATA QUALIFICATIONS AND OBSERVATIONS - ORIGINAL MATERIAL}

\section{Mass $93(\mathrm{Zr} / \mathrm{Nb})$ and Mass $241(\mathrm{Pu} / \mathrm{Am})$}

As in the Vendor Product, only one value is reported for $\mathrm{m} / \mathrm{z} 93$ and 241. The possible/probable isotopes contributing to the response observed at these masses are zirconium/niobium, and plutonium/americium, respectively. The $\mathrm{m} / \mathrm{z}-93$ results from the $\mathrm{Na}_{2} \mathrm{O}_{2}$ fusion do not appear to be usable due to high levels of zirconium and possible niobium contamination (from the zirconium). There are large disparities observed between the samples and their duplicates.

\section{Mass 240/242}

There appears to be a blank problem at $\mathrm{m} / \mathrm{z} 240$ and $\mathrm{m} / \mathrm{z} 242$ for the KOH fusion; the concentrations found in the samples are virtually identical to those found in the preparation blank. The acid digestion was analyzed at a lower dilution and has a lower detection limit. Based on this, the acid digestion results are considered the most reliable.

\section{Vanadium}

As in the Vendor Product analysis, both fusion preparations appear to have a significant $\mathrm{ClO}^{+}$response at $\mathrm{m} / \mathrm{z} 51$, which interferes with vanadium. This could not be confirmed with the acid digestion preparation.

\section{Rubidium}

The rubidium results on the $\mathrm{KOH}$ fusions are questionable, due to an inexplicably high blank result. The suspected cause of the high rubidium concentration in this preparation is contamination.

\section{Palladium-107}

The ${ }^{107} \mathrm{Pd}$ was not reported for the $\mathrm{Na}_{2} \mathrm{O}_{2}$ fusions due to problems observed in obtaining reliable results from this preparation. The ${ }^{107} \mathrm{Pd}$ measured relatively high due to a $\mathrm{ZrO}+$ interference. Also, the relatively high concentration of silver in these samples makes for a very large correction-factor, necessarily resulting in a large uncertainty. Because of lower silver and zirconium concentrations, the acid digestion preparation results are considered more reliable.

\section{$\underline{\text { Zirconium }}$}

The reported value for zirconium was calculated from the response at $\mathrm{m} / \mathrm{z} 90$ and included a contribution from ${ }^{90} \mathrm{Sr}$ that made the zirconium value bias high. The zirconium results reported were corrected for this ${ }^{90} \mathrm{Sr}$ contribution, which was found to be approximately $10 \mathrm{ppm}$. 


\section{Isotopic and fission-product considerations}

Ba: The isotopic abundance is significantly altered, most notably for 138 ; however, all isotopes below 137 agree with each other. For calculations, $\mathrm{m} / \mathrm{z} 138$ was used, which means that the reported results may be biased high. It is unclear whether the 138 peak is barium or perhaps fission-produced cerium.

$\mathrm{Cu}$ : Copper appears to be completely natural, with no apparent contribution from ${ }^{63} \mathrm{Ni}$. Result was obtained from $\mathrm{m} / \mathrm{z} 65$.

Pd: There seems to be slight enrichment at 106 and 107, which may be fission-product palladium, or may be $\mathrm{ZrO}+$. This cannot be confirmed without a separation, but it should not make a significant difference even if the additional response were included. The $\mathrm{m} / \mathrm{z} 105$ peak used agrees well with $\mathrm{m} / \mathrm{z} 108$.

Ru: The ruthenium present appears to be exclusively fission-produced. The concentration was calculated using the response at $\mathrm{m} / \mathrm{z} 102$, with an assumed isotopic abundance of $\sim 47 \%$ based on the relative abundance observed of $\mathrm{m} / \mathrm{z} 101$ and 102 .

Te: The tellurium present in the samples resembles that of fission-product tellurium. The most apparent indication that most (if not all) of the tellurium present is fission produced is the presence of a prominent $\mathrm{m} / \mathrm{z} 130$ peak, which appears to be almost 5 times that of the $\mathrm{m} / \mathrm{z} 128$ peak; for natural abundance the responses should be nearly equal. In addition, the responses observed where the natural lower-mass tellurium isotopes should be found (i.e., $\mathrm{m} / \mathrm{z} 122$, $\mathrm{m} / \mathrm{z} 123, \mathrm{~m} / \mathrm{z} 124$ ) appear to be due to natural tin and antimony. 


\title{
TANK WASTE REMEDIATION SYSTEM (TWRS) PRIVATIZATION CONTRACTOR
}

\author{
ENVELOPE D SAMPLES \\ (C-106)
}

ATTACHMENT 2

ANALYTICAL SUMMARY REPORT
ADDENDUM 1, REVISION 2

March 1997

Pacific Northwest National Laboratory 
HNF-SD-WM-DP-225, REV. 1

TANK WASTE REMEDIATION SYSTEM (TWRS) PRIVATIZATION CONTRACTOR SAMPLES

WASTE ENVELOPE D MATERIAL 241-C-106

ANALYTICAL SUMMARY REPORT

ADDENDUM 1 - Revision 2

Prepared by: Kris Kubl-Klinger and Michael W. Urie

Pacific Northwest National Laboratory (PNNL)

Analytical Chemistry Laboratory (ACL)

C-106 Project Manager

March 7, 1997 


\subsection{INTRODUCTION}

This report represents Addendum 1 to the Analytical Summary Report on TWRS Privatization Contractor Samples conducted in support of the Tank Waste Remediation System (TWRS) Privatization Contractor Samples, Waste Envelope D, C-106 characterization testing. All work performed was conducted in accordance with "Addendum 1 of the Letter of Instruction (LOI) for TWRS Privatization Contractor Samples Addressing Waste Envelope D Materials - Revision 0, Revision 1, and Revision 2." [Jones 1996, Wiemers 1996 (a), Wiemers 1996 (b)]

The Initial Vendor Product' resulting from the treatment defined in the LOI Revision 0 exceeded the Envelope D specifications for $\mathrm{Na}$ and ${ }^{121 \mathrm{~m}} \mathrm{~S}$. Additionally, a trace component $(\mathrm{Pt})$ and a trace radionuclide $\left({ }^{233} \mathrm{U}\right)$ were found to exceed the envelope specification, and some radionuclides ( ${ }^{110 \mathrm{~m}} \mathrm{Ag}$, ${ }^{242} \mathrm{Pu}$, and ${ }^{119 \mathrm{~m}} \mathrm{Sn}$ ) could not be verified due to analytical detection limitations. Also, the result for ${ }^{243} \mathrm{Cm} /{ }^{244} \mathrm{Cm}$ and ${ }^{144} \mathrm{Ce} /{ }^{144} \mathrm{Pr} /{ }^{144} \mathrm{Pr}$ were not within the specification; but only if the results are compared to the Envelope D specifications for ${ }^{243} \mathrm{Cm}$ (as a trace radionuclide) and for ${ }^{144 \mathrm{~m}} \mathrm{Pr}$, respectively. Primarily due to failure of $\mathrm{Na}$ to meet the specification, further treatment of the Initial Vendor Product was requested, as defined by the LOI Revision 1. A decant/dilute treatment was performed to bring the sodium concentration within specification limits; the details of this treatment. are described in Section 2.

Following the sodium adjustment treatment, the Final Vendor Product was transferred to twenty individual containers for shipping to the privatization vendors. However, the percent solids and percent oxide measurements performed on this material were inconsistent with expected results. Visual observation of the individual containers noted inconsistent levels of settled solids in each container. Based on these measurements and observations, additional blending, sampling/analyses, and packaging were requested, as defined by the LOI Revision 2. The reblending and repackaging are described in Section 2 and the sampling/analysis results are discussed in Section 4. Table A1-1

\footnotetext{
'Within this report "Initial Vendor Product" is used to jdentify product material resulting from the bench-scale processing (i.e., LOI Rev. 0) and the "Final Vendor Product" is used to identify material resulting from the decant/dilute treatment to reduce the Na content (i.e., LOI Rev. 1 and LOI Rev. 2).
} 


\section{HNF-SD-WM-DP-225, REV. 1}

Analytical Summary Report Addendum 1, Revision 2 - March 7, 1997

presents the "best" value for analytes measured in the Initial Vendor Product materials and the measured or "adjusted" (i.e., calculated estimates) values for the Final Vendor Product. The analysis and calculations are further described in Section 3 and Section 4.

Following the decant/dilute treatment the C106 Final Vendor Product is within specification (including sodium), except one inorganic analyte of interest $(\mathrm{Pt})$ and two radionuclides $\left({ }^{121 \mathrm{~m}} \mathrm{Sn}\right.$ and $\left.{ }^{233} \mathrm{U}\right)$ have measured concentrations above the Envelope D specification, and three radionuclides $\left({ }^{110 \mathrm{~m}} \mathrm{Ag},{ }^{199 \mathrm{~m}} \mathrm{Sn}\right.$, ${ }^{242} \mathrm{Pu}$ ) have analytical detection limits above the specification. And, as with the Initial Vendor Product, ${ }^{243} \mathrm{Cm} /{ }^{44} \mathrm{Cm}$ and ${ }^{144} \mathrm{Ce} /{ }^{144} \mathrm{Pr} /{ }^{144 m} \mathrm{Pr}$ exceed the specification if the results are compared to the Envelope D specifications for ${ }^{243} \mathrm{Cm}$ (as a trace radionuclide) and ${ }^{144 \mathrm{~m}} \mathrm{Pr}$, respectively. Statistical analysis of the product analytical results is reported in the "Preliminary Statistical Report" and "Final Statistical Report" (in progress).

Prior to completing all treatment physical measurements (e.g., weight percent oxide on firing), a preliminary estimate of the Final Vendor Product composition was provided to DOE-RL by Wiemers. ${ }^{2}$ The composition reported by Wiemers was estimated based on a calculated adjusted oxide content (i.e., oxide adjusted for loss of sodium by decanting) and preliminary data for the Initial Vendor Product and Initial Vendor Product supernatant composition. The results provided in this report supersede the composition estimates reported by Wiemers.

${ }^{2}$ Internal memo, $\mathrm{K}$ Wiemers to R Gilbert and N Brown, "Composition of Envelope D Private Contractor Sample," dated 8 November 96. This memo was provided to J Dickey, LMAES by R Gilbert, "Transfer of Envelope D Samples - Contract Number DE-RP06-96RL13309", DOE-RL letter \#96-WDD-190, dated 8 Nov 96. 
Table A1-1. Comparision of Adjusted and Measured Final Vendor Product Results with Envelope D Specificatlon

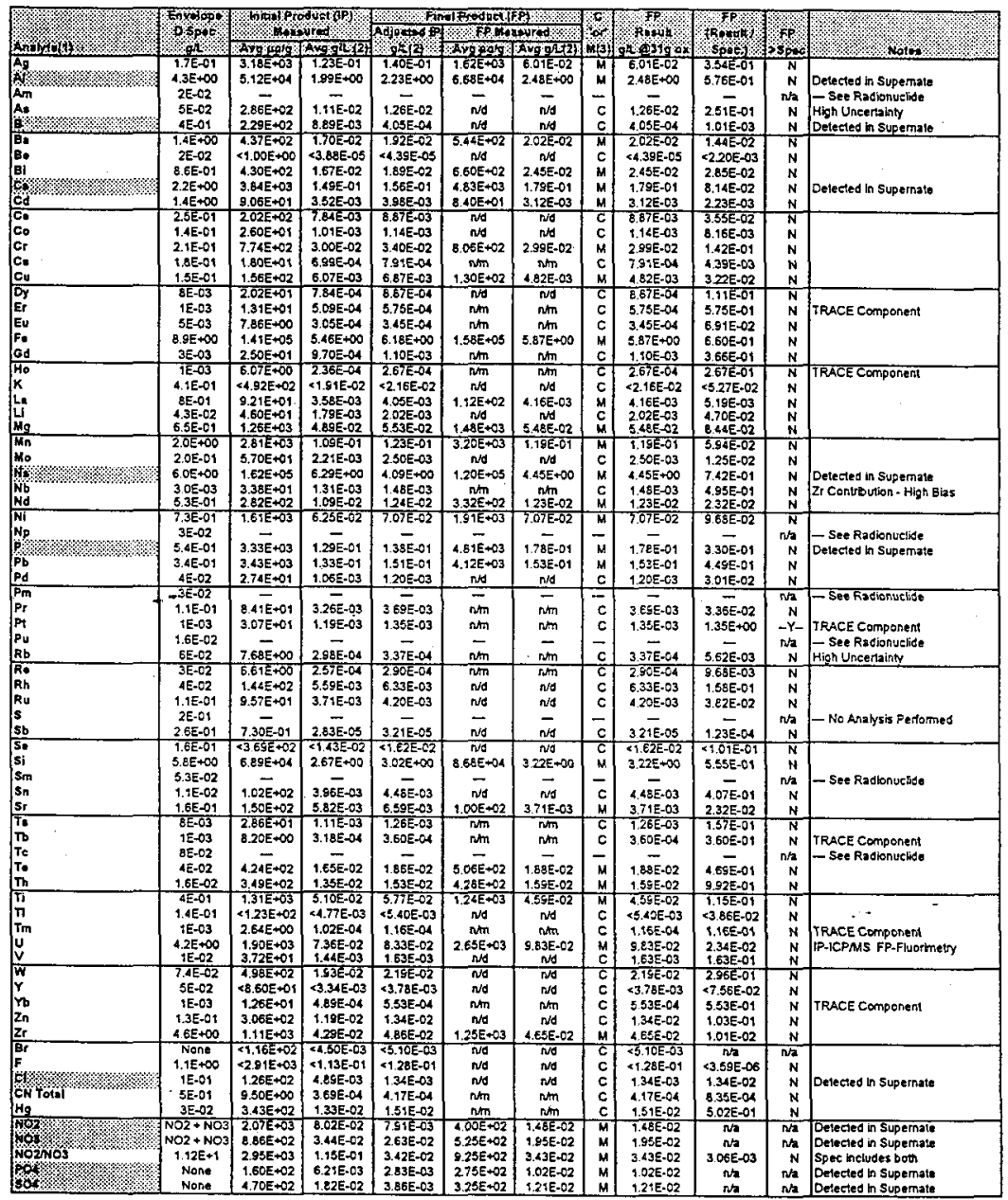


Table A1-9. Comparision of Adjusted and Measured Final Vendor Product Results with Envelope D Specification

\begin{tabular}{|c|c|c|c|c|c|c|c|c|c|c|c|}
\hline & Ensop: & bispt & $80 \mathrm{~s}$ & Adjortar & alpodtald & F,PXF & \%8 & Pro & \% & in & \\
\hline Ans & 8 & Sorgigit: & Ays: ort 23 & 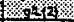 & 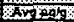 & Aw & (15) & Ho13 $0 x$ & Wsper & $\mathrm{s}_{2} \mathrm{~s}$ & Noto \\
\hline 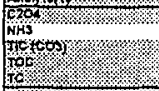 & $\begin{array}{l}\text { Non } \\
5 E-01 \\
1.86 E+0 \\
3.4 E 400 \\
\text { None }\end{array}$ & $\begin{array}{l}1.70 E+05 \\
2.13 E+01 \\
5.30 E+03 \\
4.79 E+04 \\
5.22 E+04\end{array}$ & $\begin{array}{l}5.5 \mathrm{EE}+00 \\
8.25 \mathrm{E}-04 \\
2.06 \mathrm{E}-01 \\
1.86 \mathrm{E}+\infty \\
2.03 \mathrm{E}+00 \\
\end{array}$ & $\begin{array}{l}2.37 E+\infty \\
0.33 E-04 \\
1.13 E-01 \\
6.58 E-01 \\
7.27 E-01\end{array}$ & $\begin{array}{l}8.33 E+0 / 4 \\
\mathrm{nhm} \\
\mathrm{mm} \\
\mathrm{nm}\end{array}$ & $\begin{array}{c}3.09 \mathrm{E}+00 \\
\mathrm{~nm} \\
\mathrm{~mm} \\
\mathrm{~nm} \\
\mathrm{~nm}\end{array}$ & $\begin{array}{l}M \\
c \\
c \\
c \\
c\end{array}$ & $\begin{array}{l}3.09 E+00 \\
9.33 E-04 \\
1.13 E-01 \\
5.58 E-01 \\
7.27 E-01\end{array}$ & $\begin{array}{c}n= \\
1.07 E-03 \\
6.05 E-02 \\
1.94 E-01 \\
n \pi\end{array}$ & $\begin{array}{c}n \\
N \\
N \\
N \\
N\end{array}$ & $\begin{array}{l}\text { Defocted in Supomate } \\
\text { N Resuk * (17MA) - High Blank } \\
\text { Delected in Supemato } \\
\text { Detoeted in Supernate } \\
\text { Delected in Supernate (TIC-TOC) }\end{array}$ \\
\hline 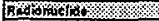 & EWt & 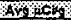 & Argotsto & anis & A\& Hois & Ancted & & & & & \\
\hline $\begin{array}{l}A g-110 m \\
A m-241 \\
A m-243\end{array}$ & $\begin{array}{l}1.00 E-08 \\
4.30 E-02 \\
5.00 E-06\end{array}$ & $\begin{array}{l}<9 E-02 \\
3.15 E+\infty \\
<2 E-02\end{array}$ & $\mid \begin{array}{c}<.49 \mathrm{E}-06 \\
1.22 \mathrm{E}-04 \\
\times 7.76 \mathrm{E}-07\end{array}$ & $\begin{array}{r}\times .95 E-06 \\
1.38 E-04 \\
\times 8.79 E-07\end{array}$ & $\sum_{n \rightarrow n}$ & $n=m$ & \begin{tabular}{l|l}
$c$ \\
$c$ \\
$c$
\end{tabular} & $\begin{array}{l}\times 3.95 E-06 \\
1.38 E-04 \\
<8.79 E-07\end{array}$ & $\begin{array}{l}3.95 E+02 \\
3.20 E-03 \\
5176 E-01\end{array}$ & $\begin{array}{c}-1-1 \\
N \\
N\end{array}$ & \\
\hline En & $2.00 E-06$ & $2.87 E-03$ & $1.11 E-07$ & $126 \mathrm{E}-07$ & $M n$ & $\frac{1 \mathrm{man}}{\mathrm{Mm}}$ & c & $\frac{0.18 E-07}{1.26 E-07}$ & $\frac{1.76 E-01}{6.30 E-02}$ & $\frac{\mathbf{N}}{\mathbf{N}}$ & Poor Analysis Ptecision \\
\hline $\begin{array}{l}C *-144(\operatorname{Pr}-144+144 m) \\
c o-144(P r-144+144 m)\end{array}$ & $1.00 E-04$ & $<2 E+00$ & $\begin{array}{l}<7.76 E-05 \\
\times 776 E-05\end{array}$ & $<8.79 E-05$ & m & $n / m$ & c & 8.75E-0S & <.79E-C1 & $\mathbf{N}$ & Ca- 144 Specification \\
\hline $\begin{array}{l}\text { Co-144(Pr-144+144m) } \\
\text { Co-144(Pr-144+14Am) }\end{array}$ & $\begin{array}{l}1.00 E-04 \\
1.00 E-07\end{array}$ & $<2 E+\infty 0$ & $\mid \begin{array}{r}\times 7.76 \mathrm{E}-05 \\
\times 7.76 \mathrm{E}-05\end{array}$ & $\begin{array}{l}<.79 E-05 \\
<8.79 E-05\end{array}$ & inth & $n / m$ & $\begin{array}{l}c \\
c\end{array}$ & $\begin{array}{l}<8.79 E-05 \\
<8.79 E-05\end{array}$ & $\begin{array}{l}<8.79 E-01 \\
<8.79 E+02\end{array}$ & ${ }_{-}^{N}$ & PF- $\{44$ Specrication \\
\hline$c_{m-2} 2(A m-242+242 m)$ & $3.70 E-05$ & $8.07 E-03$ & $3.135-07$ & 3.55E-07 & $\min$ & nm & $\mathrm{c}$ & $3.55 \mathrm{E}-07$ & 9.58E-03 & -4 & \\
\hline$\overline{C m-242(A m-242+242 m)}$ & $3.10 E-05$ & 8.07E-03 & $3.13 E-07$ & 3.55E-07 & hath & $n m$ & $\mathrm{C}$ & 3.55E-07 & $1.14 E=02$ & N & Ant-242 specication \\
\hline$(C m+242(A m-242+242 m)$ & $3.20 E-05$ & 8.07E-03 & 3.13E-07 & 3.55E-07 & $\sin$ & $n, n$ & c & $3.55 E-07$ & $1.11 E-02$ & $N$ & Am-242m Specification \\
\hline $\begin{array}{l}C m-243+244 \\
C_{m-243+244} \\
C_{0-10}\end{array}$ & $\begin{array}{l}1.005-09 \\
9.30 E-04 \\
3.00 E-03\end{array}$ & $\begin{array}{l}9.85 E-02 \\
9.86 E-02 \\
4.91 E-01\end{array}$ & $\begin{array}{l}3.83 E-06 \\
3.83 E-06 \\
1.01 E-05\end{array}$ & $\begin{array}{l}4.33 E-06 \\
4.33 E-06 \\
2.16 E-05\end{array}$ & $\begin{array}{l}\text { nhth } \\
\text { nin } \\
\text { nim }\end{array}$ & $\min _{n \rightarrow n}$ & $\begin{array}{l}c \\
c \\
c\end{array}$ & $\begin{array}{r}4.33 E-06 \\
-433 E-06 \\
2.16 E-05\end{array}$ & $\begin{array}{r}4.33 E+03 \\
4.66 E-03 \\
7.19 E-03\end{array}$ & $\underset{\mathbf{N}}{-\mathbf{Y}-}$ & $\begin{array}{l}\text { Con-243 spec:Trace Rastion. } \\
\text { Cmi-244 Speclification }\end{array}$ \\
\hline Co-134 & E. $80 E-03$ & $\angle 3 E-01$ & ब1.16E-05 & $\triangle 1.32 E-05$ & $\overrightarrow{n t n}$ & $n$ & c & $41,32 \mathrm{E}-05$ & C.9TE-03 & $\frac{1}{N}$ & \\
\hline Cn-135 & $3.00 \mathrm{E}-\mathrm{OS}$ & $2.86 \pm-03$ & 1.11E-07 & $1.265-07$ & $\mathrm{~nm}$ & $\mathrm{~mm}$ & C & $1.26 E-07$ & 4.19E-03 & $N$ & \\
\hline 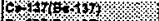 & $3.00 E+\infty 0$ & $1,96 E-02$ & $1.93 \mathrm{E}-02$ & 2.93E-02 & $n+m$ & nutin & c & 2.13E-C2 & $7,10 \mathrm{E}-03$ & N & Cs-137 specodot \\
\hline $\mathrm{E}_{\mathrm{U}-152} \mathrm{O}_{2}$ & $\begin{array}{l}3.00 \mathrm{E}+00 \\
1.50 \mathrm{E}+04\end{array}$ & $\begin{array}{c}4.96 E+02 \\
<2 E+\infty 0 \\
\end{array}$ & $\begin{array}{r}1.93 E-02 \\
47.76 E-03\end{array}$ & $\begin{array}{r}2.13 E-02 \\
<6.79 E+05 \\
\end{array}$ & $\begin{array}{l}n / m \\
n / m\end{array}$ & $\begin{array}{l}\text { nitn } \\
\text { nimen }\end{array}$ & c & $\begin{array}{r}2.13 E-02 \\
<4,79 E-05\end{array}$ & $\begin{array}{l}7.10 E-03 \\
4.8 E E-01\end{array}$ & $\begin{array}{l}N \\
N\end{array}$ & Bo- 137 Spectoe \\
\hline EN.134 & $1,60 \mathrm{E}-02$ & $3.795+\infty 0$ & $1.47 \mathrm{E}-04$ & 1.66E-04 & $\sin$ & $a m$ & C & $1.66 \mathrm{E}-04$ & 1.04E-02 & $\mathrm{N}$ & \\
\hline $\mid \begin{array}{l}E v-155 \\
F *-55\end{array}$ & $\begin{array}{l}9.00 E-03 \\
1.00 E-03\end{array}$ & $\begin{array}{l}3.13 E+00 \\
1.53 E-01\end{array}$ & $\begin{array}{l}1.21 E-04 \\
5.54 E-06\end{array}$ & $\begin{array}{l}1.37 E-04 \\
6.72 E-06\end{array}$ & $n$ & $n_{n \rightarrow m}$ & $\begin{array}{l}c \\
c\end{array}$ & $\begin{array}{l}1.37 E-04 \\
6.72 E-06\end{array}$ & $\begin{array}{l}1.53 \mathrm{E}-02 \\
6.72 \mathrm{E}-0.3\end{array}$ & $\begin{array}{l}\mathrm{N} \\
\mathrm{N}\end{array}$ & \\
\hline $1-120$ & $9.00 \mathrm{E}-08$ & 2.20E-04 & 8.54E-OS & $9.66 E-09$ & $\mathrm{n} / \mathrm{m}$ & $n \pi n$ & c & $9.66 E-09$ & $1.07 E-01$ & N & Polental Prep Losses - Low Blas \\
\hline$N b-93 m$ & $8.70 \mathrm{E} .05$ & 2.34 E-01 & 9.08E-05 & $1.03 \mathrm{E}-05$ & $\sin$ & $n \pi n$ & $\mathrm{c}$ & $1.03 \mathrm{E}-05$ & 1.1BE-01 & $\mathrm{N}$ & \\
\hline $\mathrm{Ni}_{\mathrm{i}-35}$ & $1.405=05$ & $2.05 E-02$ & $7.965-07$ & 9.01E-07 & $n$ & $\mathrm{nth}$. & C & S.01E-07 & $6,43 E-02$ & $\mathrm{~N}$ & \\
\hline $\mathrm{Ni}_{\mathrm{i}-53}$ & $1.60 E-03$ & $2.40 E+\infty 0$ & $9.32 E-05$ & $1.05 E-04$ & 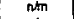 & IAn & c & $1.05 E-04$ & $6.59 \mathrm{E}-02$ & $\mathbf{N}$ & \\
\hline $\mathrm{Np}+237$ & $2.30 \mathrm{E}-05$ & -03 & $1.53 E-07$ & $1.73 E-07$ & $n / n$ & notn & $c$ & $1.73 E-07$ & 7.51E-03 & $\mathbf{N}$ & \\
\hline $\begin{array}{l}P d-107 \\
P u=238\end{array}$ & $400 \mathrm{E}-06$ & $1,10 E-03$ & 4.27E-08 & 4 A3E-08 & ntin & Wh & c & 4.83E-08 & $1.21 E-02$ & $\mathbf{N}$ & . \\
\hline$\frac{P u=238}{P U-239}$ & $\begin{array}{l}1.10 \mathrm{E}-04 \\
050 \mathrm{0}-04\end{array}$ & $\frac{6.31 E-01}{1.53 E+00}$ & $\frac{2.45 E-05}{5.94 E-0.9}$ & $\frac{2.77 \mathrm{E}-05}{6.72 \mathrm{E}-0.5}$ & $\frac{M m}{n m}$ & ntm & $c$ & 2.77E-05 & 2.52E-01 & $N$ & \\
\hline Pu-240 & $2.50 \mathrm{E}-04$ & $1.275-01$ & $4.93 E-05$ & $6.68 E-05$ & $n+m$ & $\begin{array}{l}n / \pi n \\
n / n\end{array}$ & C & $\begin{array}{l}6.72 E=03 \\
5.58 E-06\end{array}$ & $\begin{array}{l}7,07 E-02 \\
2,15 E-02\end{array}$ & $\begin{array}{l}\mathrm{N} \\
\mathrm{N}\end{array}$ & \\
\hline Pu-241 & $6.905-03$ & $6.96 E+\infty 0$ & $2.70 \mathrm{E}-0 \mathrm{~A}$ & $3.00 E-D A$ & $n / m$ & $n$ inn & c & $3.05 E-04$ & $4.43 E-02$ & $\mathrm{~N}$ & \\
\hline Pu.242. & $3.10 \mathrm{E}-08$ & $<2 E-02$ & <.76E-07 & $<8.79 E-07$ & $\mathrm{~nm}$ & nh & c & 48.79E-07 & $<1.24 E+01$ & $-Y=$ & \\
\hline Ru-105(Rh-10s\} & $2.005-04$ & $<3 E+\infty$ & $\leq 1,16 \mathrm{E}-0,4$ & $=9.32 E-04$ & $\sin$ & $\mathrm{nm}$ & C & $\$ 1,32 E-04$ & $<6.59 E-01$ & $\mathrm{~N}$ & Ru-106 Spectirsation \\
\hline$R N-106(R h-106)$ & $2.00 E-04$ & $<3 E+\infty 0$ & 21.16E-0A & 7.32E-04 & $n / m$ & $n / T$ & c & $<1, \overline{32 E-04}$ & $<6.59 \mathrm{E}-01$ & N & Rn-105 Specifreation \\
\hline $5 b-125(T *-125 m)$ & $1.00 E-02$ & $<2 E+\infty$ & $47,76 E-05$ & $<8.79 E-03$ & atn & $n / n$ & c & $<8,79 E-05$ & $<8.79 \mathrm{E}-03$ & N & Sb-125 Specification \\
\hline $\int_{50-79}^{S b-125(T a-123 m)}$ & $3.00 E-03$ & $<2 E+\infty$ & $6 E-05$ & $\angle 6.79 E-05$ & nth & $\mathrm{nm}$ & c & $\angle 8.79 E-05$ & $<2.93 E-02$ & N & Te-125m Spechicatien \\
\hline $\begin{array}{l}5 m-79 \\
\$ m-151(P m-147\}\end{array}$ & $\begin{array}{l}4.20 \mathrm{E}-07 \\
9.30 \mathrm{E}+02\end{array}$ & $\begin{array}{c}<2 \mathrm{E}-04 \\
9.23 \mathrm{E}+0.1\end{array}$ & $\mid \begin{array}{c}<.15 \mathrm{E}-09 \\
3.58 \mathrm{E}-03\end{array}$ & $\begin{array}{c}29.22 E-09 \\
4.05 E-03\end{array}$ & $\lim _{n \rightarrow m}$ & חדוד & $\begin{array}{l}c \\
c\end{array}$ & $\begin{array}{l}<9.22 \mathrm{E}-09 \\
4.05 \mathrm{E}-03\end{array}$ & $\begin{array}{l}<20 E-02 \\
4.36 E-02\end{array}$ & $\begin{array}{l}\mathbf{N} \\
\mathbf{N}\end{array}$ & Sm-151 Specifieation \\
\hline $5 m-151(P m-147)$ & $1.60 E-01$ & $9.23 E+01$ & $3.58 E-03$ & $4.05 E-03$ & $\mathrm{~mm}$ & $\sin$ & c & $4.05 E-03$ & $2.53 E-02$ & $\frac{N}{N}$ & Pm-147 Specirication \\
\hline $5 n-113(\ln -113)$ & 1.68E-05 & $<1 E-02$ & $43.88 E-07$ & <439E-07 & $a n$ & nth & c & $<4.39 E-0\rangle$ & $<2.34 \mathrm{E}-01$ & N & Sn-113 Speclication \\
\hline $5 n+113(\ln -113)$ & 1. $88 E-06$ & $\quad<1 E-02$ & $<3.8 B E-07$ & $<4.39 E-07$ & ntn & nm & c & C4.39E-07 & $<2.34 \mathrm{E}-01$ & $N$ & In-113 Specification \\
\hline $\begin{array}{l}s n-118 m \\
\$ n=121 m\end{array}$ & $\begin{array}{l}1.00 \mathrm{E}-08 \\
9.00 \mathrm{E}-06\end{array}$ & $\begin{array}{c}<4 E-02 \\
1,23 E+00\end{array}$ & $\begin{array}{l}6.55 \mathrm{E}-06 \\
4,77 \mathrm{E}-05\end{array}$ & $\begin{array}{c}<1.76 E-06 \\
\$ .40 E-05\end{array}$ & $\operatorname{mim}$ & $\mathrm{nm}$ & $\begin{array}{l}\mathrm{C} \\
\mathrm{C}\end{array}$ & $\begin{array}{l}\text { 1.76E-08 } \\
5.40 E-05\end{array}$ & $\begin{array}{c}1.76 E+02 \\
6.00 E+00\end{array}$ & $\begin{array}{l}-Y- \\
-Y-\end{array}$ & \\
\hline $5 n-126(5 b-126 m, 5 b-126)$ & $4.80 E-05$ & $1.95 E-02$ & $4.46 E-07$ & 5.05E-07 & $\sin$ & $n$ & $\mathrm{C}$ & $5.05 E-07$ & $1.03 E+02$ & $N$ & Sn-125 Spectication \\
\hline $5 n-126(5 b-126 m, 5 b-126)$ & $3.43 E-05$ & 1.15E-02 & 4.46E- 07 & $5.05 E-07$ & ntm & ntin & c & 5.05E-07 & $1.47 E-02$ & N & Sb-126m Spectication \\
\hline$(5 n-126(5 b-125 m, 5 b-126)$ & 4.83E-06 & $1.15 E-02$ & 4.46E 07 & $5.05 E-07$ & $n+m$ & $\min$ & c & \$.DSE-07 & $1.05 E-01$ & $\mathrm{~N}$ & St-126 Speefincation \\
\hline 510901 & $3.10 \mathrm{E}+00$ & $7,30 \mathrm{E}+02$ & $2.83 E-02$ & $3.21 E-02$ & 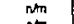 & $\min$ & c & $321 E-02$ & $1.03 E-02$ & $N$ & \$1-90 Spiedel in Sup. \\
\hline 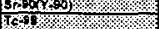 & $\frac{3.105+\infty}{4.50 E-03}$ & $\frac{7,30 E+02}{1,32 E-02}$ & $\frac{2.83 E-02}{5.12 E-07}$ & $\frac{3,21 E-02}{4.70 E-07}$ & $\mathrm{Nm}$ & $\sin$ & $\mathrm{C}$ & $3.21 E-02$ & $1.03 E-02$ & $\mathbf{N}$ & t in Sup. \\
\hline$T h-232$ & See Th" & 5. $48 E-05$ & $2.13 E-09$ & $2.41 E-09$ & 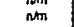 & $n$ & C & $\begin{array}{l}\text { 4.70E-07 } \\
2.4 t E-09\end{array}$ & $\begin{array}{c}1.05 E-04 \\
\mathrm{na}\end{array}$ & ${ }_{n h}^{N}$ & Sperfficatio \\
\hline $\mathbf{u}$ & $1.00 E-09$ & $1.70 E-03$ & $6.6 E-08$ & $7.47 E-08$ & nth & in & c & $7,47 E-08$ & $7,47 E+01$ & $-Y-$ & TRACE Comp - Th Interference \\
\hline U.23 & $7.70 E-07$ & $7.70 \mathrm{E}-04$ & $2.99 E-08$ & $3.39 E+0 \mathrm{~s}$ & nh & nhn & c & $3.38 E-08$ & 4.39E-02 & $\mathbf{N}$ & \\
\hline$\frac{0.235}{0.236}$ & $3.20 E-08$ & 2.80E-05 & 1,08E-09 & $1,23 E-09$ & nm & $\mathrm{ntm}$ & C & $123 E-09$ & 3.84E-02 & $N$ & \\
\hline $\begin{array}{l}0.236 \\
U-239\end{array}$ & $\begin{array}{l}8.20 E-08 \\
5.80 E-07\end{array}$ & $\begin{array}{l}1.80 E-05 \\
6.37 E-04\end{array}$ & $\begin{array}{l}6.99 E-10 \\
2+7 E+00\end{array}$ & $\begin{array}{l}7.99 E-10 \\
2.80 E-50\end{array}$ & 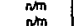 & inth & c & $791 \mathrm{E}-10$ & Q.64E-03 & $N$ & 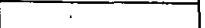 \\
\hline Zinas & $1.40 \mathrm{E}-04$ & $<6 \varepsilon-01$ & $<2.33 E-05$ & $<2.64 \mathrm{E}-05$ & $n h m$ & nm & c & $\begin{array}{l}2.80 E-08 \\
<2.64 E-05\end{array}$ & $\begin{array}{r}4.82 E-02 \\
<1.88 E-01\end{array}$ & $\begin{array}{l}\mathbf{N} \\
\mathbf{N}\end{array}$ & \\
\hline $\begin{array}{l}\text { Totnl Alphe } \\
\text { Total Bote }\end{array}$ & $\begin{array}{l}\text { none } \\
\text { none }\end{array}$ & $\begin{array}{l}6.78 E+00 \\
2.05 E+03\end{array}$ & $\begin{array}{l}2.63 \mathrm{E}-04 \\
7.95 \mathrm{E}-02 \\
\end{array}$ & $\begin{array}{l}2.995-04 \\
9.01 E-02\end{array}$ & nem & nhm & C & $\begin{array}{l}2.98 E-04 \\
9.01 E-02\end{array}$ & nh & na & \\
\hline 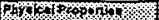 & & Antista & sodut & & 12400 & & & & & & \\
\hline & & & ALO & (1) & & 4 & & & & & \\
\hline 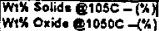 & $2.5 \cdot 13$ & $\begin{array}{l}14.4 \\
11.5\end{array}$ & 14.8 & $\begin{array}{l}152 \\
127\end{array}$ & 11 & & & & & & Sten note 4 \\
\hline Totnl Oxide-(gI) & 25.100 & 126.5 & $\mathbf{m} \mathbf{m}$ & 139.4 & $\mathrm{~m}$ & tho & & & & & \\
\hline slurry Donsiby - (g/m) & $1.02-1.10$ & 1.10 & Mm & 1.05 & $M$ & & & & & & \\
\hline $\begin{array}{l}\text { Sotlied solide - Norh) } \\
\text { oH }\end{array}$ & $\begin{array}{l}7-95 \\
>10\end{array}$ & $\begin{array}{l}18.90 \\
\mathrm{~nm}\end{array}$ & $\begin{array}{l}\text { nth } \\
\text { ntm }\end{array}$ & nin & 11 & & & & & & \\
\hline
\end{tabular}

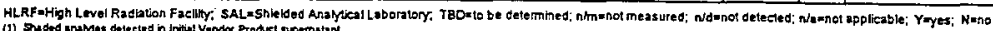

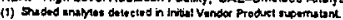

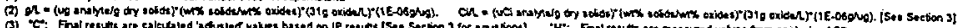

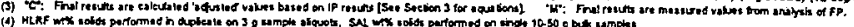




\section{HNF-SD-WM-DP-225, REV. 1}

Analytical Summary Report Addendum 1, Revision 2 - March 7, 1997

\subsection{FINAL VENDOR PRODUCT SODIUM-REDUCTION TREATMENT}

A flowchart of the treatment and analysis scheme is presented in Figure Al-1. The Initial Vendor Product was received from the 324 building in two containers, labeled SPDI-C106-051 (1624 g) and SPD1-C106-052 (1523 g) [Brooks 1997]. Since the sodium concentration of the Initial Vendor Product exceeded the Envelope D specification, a sodium-reduction treatment was performed on the material. The treatment as described in LOI Revision 1 consisted of decanting a known quantity of supernatant containing sodium and replacing it with deionized water in excess of the decant supernatant volume. Water was added in excess of the volume of supernatant decanted with the intent to bring the product into specification with respect to weight percent solids and weight percent oxide. However, meeting the weight percent solids/oxides specification is not a requirement for shipment of the product.

\subsection{DECANT/DILUTE OPERATION - LOI Revision 1}

The supernatant from the containers SPD1-C106-051 and SPD1-C106-052 was decanted after gravity settling, combined, weighed, and poured into two $1 \mathrm{~L}$ polyethylene bottles (C106-Supernatant-1, C106-Supematant-2) for archiving. The total mass of supernatant decanted was 2002.32 grams. A subsample of the combined, decanted supematant was collected for supematant density measurement and ICP-AES analysis. The residual solids from each of these two containers of Initial Vendor Product were combined together into a 4 liter plastic beaker. The total mass of the blended solids was 908.60 grams. A known mass of distilled water was used to rinse the Initial Vendor Product from the containers as well as for final dilution of the solids. The total mass of distilled water added to the solids was 2602.85 grams. Table A1-2.1 presents the weights obtained for the decanted supernatant, distilled water and resulting Final Vendor Product. The weight of the Initial Vendor Product is calculated from the weight of the Final Vendor Product adjusted for the weight differences between the decant liquid and added water. This weight is approximately $200 \mathrm{~g}$ less than the quantity of material received from the 324 building (i.e., $3147 \mathrm{~g}$ ). This weight discrepancy is not anticipated to have any significant impact on the final product results; however, this is to be further evaluated and conclusion reported in the "Analytical Final Report" which is to be issued in March 1997. 
Figure A1-1. Initial Product Analysis and Sodium Adjustment Treatment

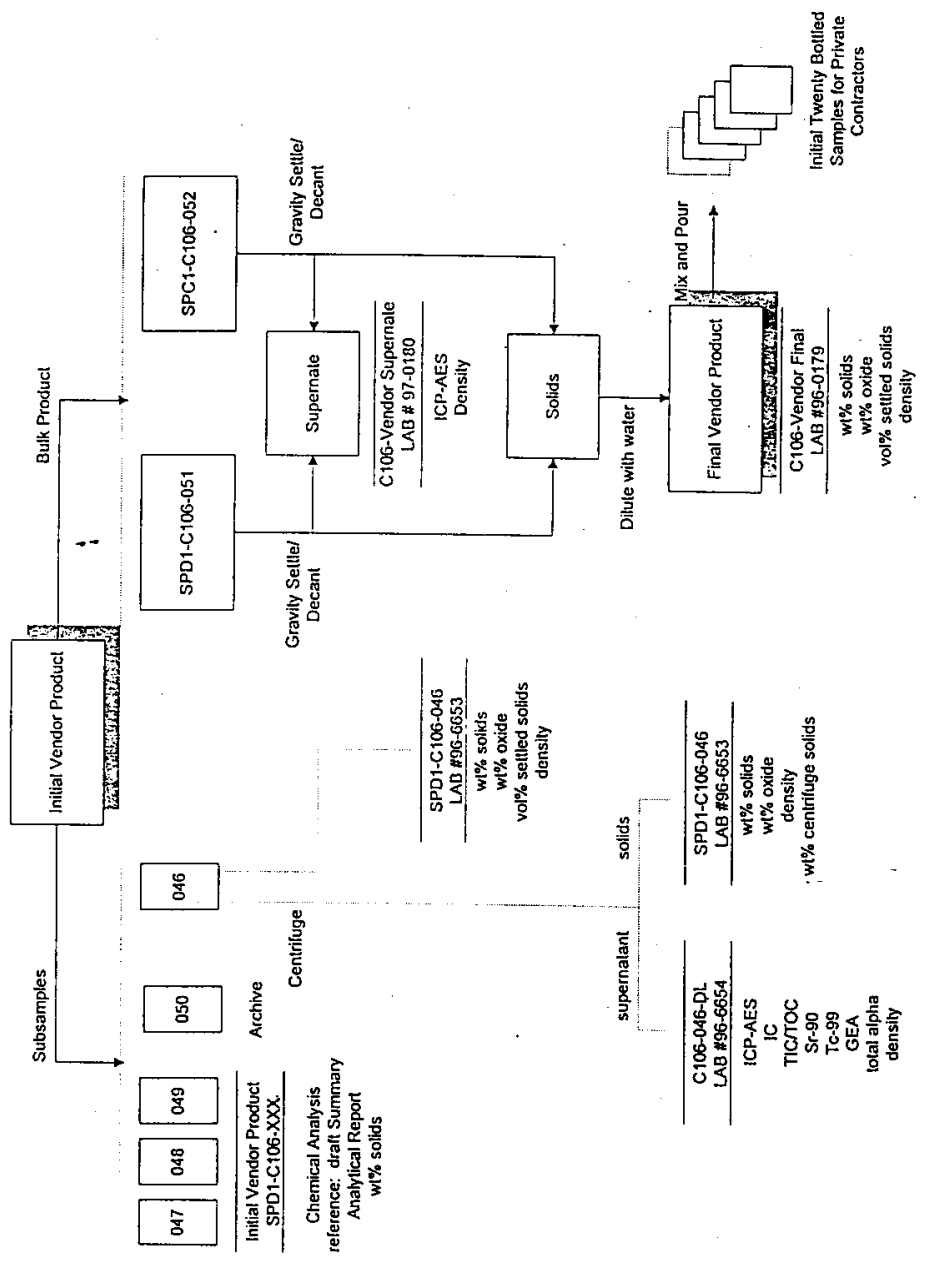


HNF-SD-WM-DP-225, REV. 1

Analytical Summary Report Addendum 1, Revision 2 - March 7, 1997

Table A1-2.1. DecantDilution Weights

\begin{tabular}{||l|c|c|c||}
\hline Description & Sample ID & Lab Number & Weight (g) \\
\hline \hline Initial Vendor Product & $\begin{array}{c}\text { SPDI-C106-051 and } \\
\text { SPD1-C106-052 }\end{array}$ & N/A & $\begin{array}{c}2910.92 \\
\text { (Calculated) }\end{array}$ \\
\hline Decanted Liquid & C106 Vendor Supernate & $97-0180$ & 2002.32 \\
\hline Distilled Water Added & N/A & N/A & 2602.85 \\
\hline Final Vendor Product & C106 Vendor Final & $97-0179$ & 3511.45 \\
\hline
\end{tabular}

\subsubsection{Physical Measurements}

The Final Vendor Product was sampled for physical testing measurements by pouring while stiring mechanically just prior to filling a sample bottle (Container ID 97-0179). These measurements consisted of weight percent solids, weight percent oxide, slurry density, and volume percent settled solid, and the results are presented in Table A1-2.2. The subsamples for each of the physical measurements were extracted from the bottle while stirring; however, both the sample and duplicate were extracted from the same bottle. The resulting weight percent solids $(21.5 \%)$ and weight percent oxide (18.6\%) were inconsistent with expectations. Since water in excess of the decanted supernatant volume was added during the decant/dilute treatment, the weight percent solids should have been lower than the Initial Vendor Product result (i.e., <14.4\%). Resampling of the material with Lab Number 97-0179 and measurement of the weight percent solids confirmed the high result. The higher than expected percent solids has been attributed to the difficulty in stirring/pouring the-slurries:

Although there was no immediate visual clue that the subsampling was not representative, after settling it was observed that the settled solids heights were significantly different among the twenty bottles prepared for shipping to the vendors. The physical measurement results from this subsample are included for information only, since these results are superseded by the measurements conducted following reblending, resampling, and rebottling operations [See Section 2.2; Note - The weight percent solids and weight percent oxides presented in Table Al-2.2 are not used to calculate any "adjusted" concentrations.] 
HNF-SD-WM-DP-225, REV. $?$

Analytical Summary Report Addendum 1, Revision 2 - March 7, 1997

Table A1-2.2. Settled Solids and Density Measurements per LOI Revision 1 (for Information Only)

\begin{tabular}{|c|c|c|c|c|c|}
\hline Sample ID & $\begin{array}{l}\text { Settled } \\
\text { Solids } \\
\text { (Vol\%) }\end{array}$ & Solids & Oxides & $\begin{array}{l}\text { oxides } \\
(\mathrm{g} / \mathrm{L})\end{array}$ & $\begin{array}{l}\text { Density } \\
(\mathrm{g} / \mathrm{ml})\end{array}$ \\
\hline 97-0179 (Final Vendor Product) & $39.1^{(0)}$ & 21.2 & 18.5 & 206 & 1.11 \\
\hline 97-0179. Dup (Final Vendor Product) & $39.4^{(\sqrt{)})}$ & 21.7 & 18.6 & 214 & 1.15 \\
\hline Final Vendor Product - Average & 39.2 & 21.5 & 18.6 & 210 & 1.13 \\
\hline Final Vendor Product - RPD (\%) & 0.8 & 2.3 & 0.3 & 3.8 & 3.6 \\
\hline
\end{tabular}

(a) Greater than $95 \%$ of the solids have settled in less than eight hours.

\subsubsection{Initial Bottling of Final Vendor Product}

Following the decant/dilute treatment, twenty vendor subsamples (ten for each vendor) were aliquotted into glass bottles; this operation was performed by pouring while stirring mechanically prior to filling each bottle. This initial bottling of the Final Vendor Product resulted in bottles containing variable quantities of solids, as observed from the settled solids height in each bottle. Based on the inconsistency in the settled solids level, as well as the unexpectedly high weight percent solids, additional processing of the Final Vendor Product was necessary in order to establish credible solids and oxides concentrations [See Section 2.2]. 


\subsection{REBLENDING, RESAMPLING, REBOTTLING OPERATION - LOI Revision 2}

\subsection{1 $\quad$ Reblending}

All Final Vendor Product material including the material initially bottled for each vendor and the remaining archive material was composited into a $4 \mathrm{~L}$ plastic beaker and blended by mechanical stirring. A flowchart detailing the reblending, rebottling, and reanalysis is presented in Figure A1-2. This operation was performed per the instructions provided in the LOI Revision 2.

\subsubsection{Bottling of Final Vendor Product}

Following compositing and mechanical stirring to thoroughly blend the Final Vendor Product slurry, twenty vendor bottles were prepared. While mechanically stirring the slurry contents of the $4 \mathrm{~L}$ beaker, the Final Vendor Product was transferred to the vendor bottles by pipetting. Each vendor bottle was filled with two $50 \mathrm{ml}$ pipet aliquots. Two additional bottles (97-0179-1 and 97-0179-2) were filled to provide material for any future physical testing, if required. Table A1-2.3 provides the vendor container identification numbers, the transferring sequence numbers (i.e., I through 20), and the quantities transferred to each container. Observation of the solids level following settling indicate no visual difference in the quantities of solids transferred to each vendor bottle. Four of these bottles, i.e., C106-FP-V1-1, C106-FP-V1-6, C106-FP-V2-3, and C106-FP-V2-8, were selected for further physical and chemical analyses (See Section 2.2 .3 and Section 4). 


\section{HNF-SD-WM-DP-225, REV. 1}

Analytical Summary Report Addendum 1, Revision 2 - March 7, 1997

Figure A1-2. Reblending, Rebottling, and Reanalysis of Final Vendor Product

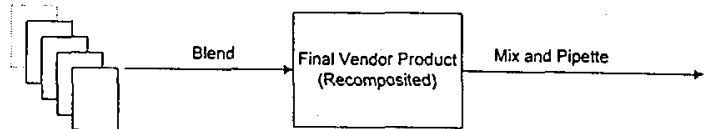

Initial Twenty Bottled
Samples for Private Samples for Private

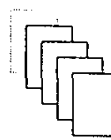

Twenty Bottled Samples for Privale Contractors. C106-FP-V1-1 to -10 and $-v_{2}-1$ to -10

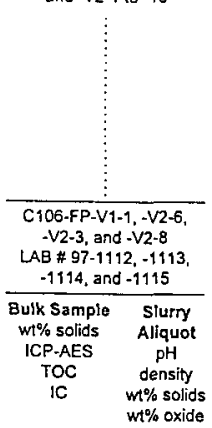

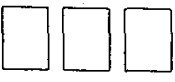

Remaining Final Vendor Product 
HNF-SD-WM-DP-225, REV. 1

Analytical Summary Report Addendum 1, Revision 2 - March 7, 1997

Table A1-2.3 Vendor Subsampling Container $\mathrm{ID}$ and Quantity

\begin{tabular}{|c|c|c|c|c|c|}
\hline Sampling & Container ID & $\begin{array}{l}\text { Subsample } \\
\text { Weight (g) }\end{array}$ & Sampling & Container ID & $\begin{array}{l}\text { Subsample } \\
\text { Weight (g) }\end{array}$ \\
\hline 1 & C106-FP-V1-1* & 99.62 & 2 & C106-FP-V2-1 & 97.64 \\
\hline 3 & C106-FP-V1-2 & 99.02 & 4 & C106-FP-V2-2 & 102.13 \\
\hline 5 & C106-FP-VL-3 & 98.51 & 6 & C106-FP-V2-3* & 98.74 \\
\hline 7 & C106-FP-VI-4 & 100.31 & 8 & C106-FP-V2-4 & 102.49 \\
\hline 9 & C106-FP-V1-5 & 99.06 & 10 & C106-FP-V2-5 & 89.93 \\
\hline 11 & C106-FP-V1-6* & 100.02 & 12 & C106-FP-V2-6 & 96.21 \\
\hline 13 & C106-FP-V1-7 & 95.74 & 14 & C106-FP-V2-7 & 88.13 \\
\hline 15 & C106-FP-V1-8 & 99.25 & 16 & C106-FP-V2-8* & 97.00 \\
\hline 17 & C106-FP-V1-9 & 89.14 & 18 & C106-FP-V2-9 & 106.12 \\
\hline 19 & C106-FP-V1-10 & 103.61 & 20 & C106-FP-V2-10 & 99.10 \\
\hline
\end{tabular}

* Samples selected for additional physical and chemical analyses

\subsubsection{Physical Measurement}

The physical measurements conducted on the reblended Final Vendor Product composite are presented in Table A1-2.4, and consist of weight percent solids, weight percent oxide, and slurry density. The weight percent solids on drying and the oxide concentration on firing of the Final Vendor Product remain slightly higher than the values obtained on the Initial Vendor Product (see Table A1-1). The use of small sample aliquots (i.e., $3 \mathrm{~g}$ ) collected by pipetting for measuring the percent solids in this material appears to bias the results high relative to the percent solids determined from large quantity, 
bulk samples (See Section 4). The percent solids and percent oxides results from Table A1-2.4 (i.e., $15.2 \%$ and $12.7 \%$ ) are used to convert the Final Vendor Product "measured" results from $\mu \mathrm{g} / \mathrm{g}$ dried solids to $\mathrm{g} / \mathrm{L}$ at $31 \mathrm{~g}$ oxide.

Table A1-2.4. Wt\% Solids and Wt\% Oxide Measurements per LOI Revision 2

\begin{tabular}{|c|c|c|c|c|c|}
\hline $\mathrm{Lab} \mathrm{Number}$ & $\begin{array}{l}\text { Sample/Container } \\
\text { ID }\end{array}$ & $\begin{array}{l}\text { Density } \\
(\mathrm{g} / \mathrm{ml})\end{array}$ & $\begin{array}{l}\text { Solids } \\
(W t \%)\end{array}$ & $\begin{array}{l}\text { Oxides } \\
(w t \%)\end{array}$ & $\begin{array}{l}\text { Oxides } \\
(\mathrm{g} / \mathrm{L})\end{array}$ \\
\hline $97-1112$ & C106-FP-V1-I & 1.06 & 14.4 & 12.0 & 126 \\
\hline $97-1113$ & C106-FP-V1-6 & 1.04 & 13.9 & 11.6 & 122 \\
\hline $97-1114$ & C106-FP-V2-3 & 1.06 & 16.3 & 13.9 & 146 \\
\hline $97-1115$ & C106-FP-V2-8 & 1.06 & 15.9 & 13.3 & 140 \\
\hline \multicolumn{2}{|c|}{ Final Vendor Product - Average } & 1.05 & 15.2 & 12.7 & 133.5 \\
\hline \multicolumn{2}{|c|}{ Final Vendor Product - Std Dev } & \pm 0.01 & \pm 1.2 & \pm 1.1 & \pm 12 \\
\hline
\end{tabular}

\subsection{ADJUSTED ANALYTE CONCENTRATIONS FOLLOWING SODIUM REDUCTION}

The source and type of analysis used to estimate the composition of the Final Vendor Product is shown in Figure A1-1. The Final Vendor Product concentration is calculated from the Initial Vendor Product concentration, the oxide content of the Initial Vendor Product and Final Vendor Product, and the analyte/radionuclide concentrations of the decanted supematant (where measured and detected). 


\subsection{INITIAL VENDOR PRODUCT ANALYSIS}

The Initial Vendor Product was analyzed per the requirements of LOI Revision 0 and all chemical, radionuclide, and physical measurement results have been presented in the Analytical Summary Report [Urie 1997]. The analytical results are summarized in Table A1-1 (Initial Product Measured).

\subsection{INITIAL VENDOR PRODUCT SUPERNATANT ANALXSIS}

The Initial Vendor Product supernatant was analyzed twice. The first set of analyses (sample C106-046-DL, 96-6654) were performed on centrifuged decant liquid of the Initial Vendor Product. Limited chemical and radionuclide analyses were performed on this supernatant. Since this supernatant was decanted nearly a month prior to performing the sodium adjustment treatment process, there was concern that the analyses might not accurately reflect the composition of the Initial Vendor Product just prior to the decant/dilute treatment for Final Vendor Product generation. Therefore, following gravity settling of Initial Vendor Product, another sample of supernatant (sample C-106-Vendor Supernate, 97-0180) was taken just prior to decanting. This second sample was analyzed by ICP-AES (only) and compared to the original ICP-AES results.

A summary of the chemical and radionuclide analyses performed on the two Initial Vendor Product supernatant samples is provided in Table A1-3.1. Besides density shown in Table A1-3.2, ICP-AES was the only analysis performed on both supernatant samples. As can be seen from the ICP-AES results, there appears to be little change in the chemical composition of the supernatant with time, which is expected from a saturated supernatant. Sodium is clearly the primary ICP-AES component in both supernatants. Aluminum was present in quantitative levels; whereas, Boron, Calcium, and Phosphorus were present in low, semi-quantitative levels and are subject to large errors. For these analytes (i.e., sodium, aluminum, boron, calcium, and phosphorus), only the ICP-AES results from the second analysis (sample C-106-Vendor Supernate, 97-0180) are used to calculate the quantity of analyte decanted. 
Analytical Summary Report Addendum 1, Revision 2 - March 7, 1997

Table A1-3.1. Initial Vendor Product Supernatant Analytical Results

\begin{tabular}{|c|c|c|c|c|c|c|}
\hline \multirow[t]{2}{*}{ 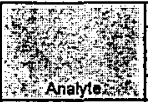 } & \multirow[t]{2}{*}{ 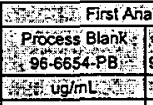 } & \multirow{2}{*}{\multicolumn{2}{|c|}{ 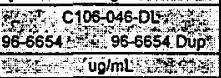 }} & \multirow[t]{2}{*}{ 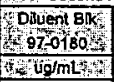 } & \multirow{2}{*}{\multicolumn{2}{|c|}{ 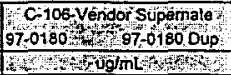 }} \\
\hline & & & & & & \\
\hline $\overrightarrow{A g}$ & $\overline{-}$ & - & - & - & - & - \\
\hline Al & [5.3] & 64.6 & 61.8 & {$[0.1]$} & 91.8 & 94.6 \\
\hline As & - & - & - & - & - & - \\
\hline$B$ & 62.9 & 68.4 & 61.7 & 2.84 & [44] & 51.3 \\
\hline Ba & - & - & - & - & - & - \\
\hline Be & - & - & - & - & - & - \\
\hline$B i$ & - & - & - & - & - & - \\
\hline $\mathrm{Ca}$ & {$[47]$} & [22] & [9] & - & {$[78]$} & [49] \\
\hline $\mathrm{cd}$ & - & - & - & - & - & - \\
\hline $\mathrm{Ce}$ & - & - & - & - & - & - \\
\hline co & - & - & - & - & - & - \\
\hline $\mathrm{Cr}$ & {$[0.87]$} & [1.8] & {$[1.8]$} & - & [2.4] & - \\
\hline Cu & - & - & - & - & - & - \\
\hline Dy & - & - & - & - & - & - \\
\hline Ev & - & - & - & - & & - \\
\hline $\mathrm{Fe}$ & [2.5] & [3.7] & {$[2,3]$} & - & - & - \\
\hline$k$ & - & - & - & - & - & - \\
\hline La & - & - & - & - & - & - \\
\hline Li & - & - & - & - & - & - \\
\hline $\mathrm{M}_{1}$. & [8.0] & [5.0] & - & - & - & - \\
\hline$M n$ & - & - & - & - & - & - \\
\hline Mo & - & - & -- & - & - & - \\
\hline $\mathrm{Na}$ & 86.9 & 14,200 & 13,800 & 3.29 & 14,700 & 15,200 \\
\hline Nd & - & - & - & - & - & - \\
\hline $\mathrm{Ni}$ & [1.2] & - & - & - & - & - \\
\hline$P$ & - & 24.8 & 28.0 & - & [39] & [41] \\
\hline $\mathrm{Pb}$ & - & - & - & - & - & - \\
\hline Pd & - & - & - & - & - & - \\
\hline Rh & - & - & - & - & - & - \\
\hline Ru & - & - & - & - & - & - \\
\hline Sb & - & - & - & - & - & - \\
\hline Se & - & - & - & - & - & - \\
\hline $\mathbf{S i}$ & 263 & $234^{\circ}$ & 168 & {$[0.60]$} & - & - \\
\hline & - & - & - & - & - & $-\quad-$ \\
\hline Sr & - & - & - & - & - & - \\
\hline Te & - & - & - & - & - & - \\
\hline Th & - & - & - & - & - & - \\
\hline$\pi$ & - & - & - & - & - & - \\
\hline$|T|$ & - & - & - & - & - & - \\
\hline $\mathrm{u}$ & - & - & - & - & - & - \\
\hline V & - & - & - & - & - & - \\
\hline$w$ & - & - & - & - & - & - \\
\hline Y & - & - & - & - & - & - \\
\hline $\mathrm{Zn}$ & {$[2.7]$} & - & - & - & - & - \\
\hline $\mathrm{Zr}$ & - & - & - & - & - & - \\
\hline
\end{tabular}

"- - Analyle not delected above the instrument Defection Limit

Results in brackets are below the Estimated Quantitation Limit but above the Instument Detection Limit. 
Table A1-3.1 (Cont). Initial Vendor Product Supernatant Analytical Results

\begin{tabular}{|c|c|c|c|c|c|c|}
\hline \multirow{3}{*}{ 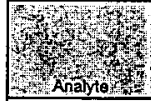 } & \multicolumn{3}{|c|}{ 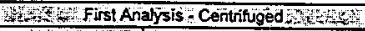 } & \multicolumn{3}{|c|}{ in Second Anaysis Gravity Settled } \\
\hline & $\begin{array}{l}\text { Process Blank } \\
y^{2}\end{array}$ & \multicolumn{2}{|c|}{ 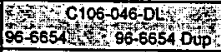 } & 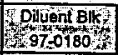 & \multicolumn{2}{|c|}{ 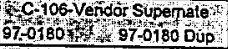 } \\
\hline & 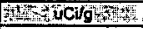 & \multicolumn{2}{|c|}{ Fi:s } & \multirow{2}{*}{\multicolumn{3}{|c|}{ 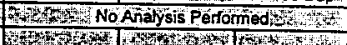 }} \\
\hline Am-241 & CAE-04 & $<7 E-03$ & $<7 E-03$ & & & \\
\hline Co-60 & $<2 \mathrm{E}-04$ & $<7 E-04$ & $<5 E-04$ & & & \\
\hline Cs-137 & $3.62 \mathrm{E}-03$ & $2.34 E+00$ & $2.30 E+00$ & & & \\
\hline$E u-154$ & $<3 E-04$ & $<2 E-03$ & $\angle 8 E-04$ & & & \\
\hline Eu-155 & *3E-04 & $<5 E-03$ & $<5 E-03$ & 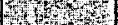 & 19 & \\
\hline Sr-90 & $1.91 \mathrm{E}-03$ & $3.59 E-02$ & $4.84 E-02$ & $3+x$ & 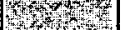 & \\
\hline Total Alpha & 4.42E-O4 & $<4 E-03$ & C4E-03 & tor. & & \\
\hline Te-99 & 2.55E-04 & $4.93 E-04$ & $5.36 E-04$ & & & \\
\hline L Y Aralyle I & $+\mathrm{ugClo}$ & 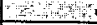 & $1+x+4$ & & Hon & \\
\hline TIC & 127 & 559 & 587 & 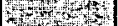 & 13 & \\
\hline TOC & 1214 & 6645 & 7134 & & Wart & \\
\hline TC & N/A & 7204 & 7721 & & -6 & \\
\hline Analyte 1 . & के ught & $2^{2}-9$ & (1) & 87 & 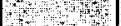 & \\
\hline $\mathrm{Br}$ & $N / A$ & $<10.0$ & $<10.0$ & & $\frac{4}{3}$ & \\
\hline $\mathrm{Cl}$ & N/A & 20 & 20 & & & \\
\hline$F \cdot$ & N/A & $<250.0$ & $<250.0$ & & & \\
\hline $\mathrm{NO2}$ & N/A & 390 & 400 & & of & \\
\hline NO3 & N/A & 60 & 60 & & n+m, & \\
\hline PO4 & N/A & 20 & 20 & $y^{2}+4$ & 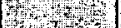 & \\
\hline $\mathrm{SO4}$ & N/A & Bo & 80 & thintor & 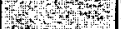 & $4=3$ \\
\hline $\mathrm{C} 2 \mathrm{O} 4$ & N/A & 24000 & 25000 & $1+1 \%, y+$ & mos & \\
\hline
\end{tabular}

No radiochemistry, TOC, or anion analyses perfomed on 97-0180.

" $<$ " - Analyte not detected above the Instrument Detection Limit N/A - Not Applicable 
HNF-SD-WM-DP-225, REV :

Analytical Summary Report Addendum 1, Revision 2 - March 7, 1997

Table A1-3.2. Initial Vendor Product Supernatant Density Measurements per LOI Revision 1

\begin{tabular}{|l|c|}
\hline $\begin{array}{l}\text { Laboratory Number (Sample ID) } \\
\text { Lensity }\end{array}$ & $\begin{array}{c}\text { Densit } \\
(\mathrm{g} / \mathrm{ml})\end{array}$ \\
\hline $\begin{array}{l}\text { 96-6653 (C106-046-DL, First Analysis Initial Vendor } \\
\text { Product Supernatant) -- Centrifuged }\end{array}$ & 1.000 \\
\hline $\begin{array}{l}\text { 97-0180 (C-106-Vendor Product, Second Analysis } \\
\text { Initial Vendor Product Supematant) -- Gravity Settled }\end{array}$ & 1.036 \\
\hline
\end{tabular}

\subsection{CALCULATED "ADJUSTED" FINAL VENDOR PRODUCT CONCENTRATION}

Based on the consisistency demonstrated by the sodium, and assuming other analytes behave similarly, the Final Vendor Product results have been adjusted for the reduced sodium concentration and loss of analytes/radionuclides measured in the decanted supernatants, where applicable. The "adjusted" concentration is presented in Table A1-1 as the Final Product results, whenever Final Vendor Product measured results (See Section 4) are not available (i.e., either not measured or not detected). 


\subsubsection{Adjusted Concentrations for Analytes/Radionuclides NOT Detected or Measured in the Initial} Vendor Product Supematant

For analytes/radionuclides not measured or detected in the Initial Vendor Product supernatant, the reported Final Vendor Product concentration is the Initial Vendor Product concentration adjusted for the reduction in the oxide loading following the sodium reduction treatment (Equation 1). For explanation of calculation of Final Vendor Product "total oxide" (FO) contents, see Appendix A.

$$
\begin{aligned}
& \mathrm{FVP}=(\mathrm{IVP}) *(\mathrm{PS} / \mathrm{PO}) *(\mathrm{IO} / \mathrm{FO}) *(31) *(1 \mathrm{e}-6) \quad \text { (Equation } 1) \\
& \text { Where, } \mathrm{FVP}=\text { Final Vendor Product concentration in } \mathrm{g} / \mathrm{L}(\text { or } \mathrm{Ci} / \mathrm{L}) \\
& \mathrm{IVP}=\text { Initial Vendor Product concentration in } \mu \mathrm{g} / \mathrm{g}(\text { or } \mu \mathrm{Ci} / \mathrm{g})-\text { See Table } \mathrm{Al}-1 \\
& \mathrm{PS}=\text { Weight percent solids for Initial Vendor Product }(14.4 \%) \\
& \mathrm{PO}=\text { Weight percent oxide for Initial Vendor Product }(11.5 \%) \\
& \mathrm{IO}=\text { Initial Vendor Product total oxide content }(334.76 \mathrm{~g}) \\
& \mathrm{FO}=\text { Final Vendor Product estimated total oxide content }(295.81 \mathrm{~g}) \\
& 31=\text { Gram oxide per } 31 \mathrm{~g} \text { oxide/L } \\
& 1 \mathrm{e}-6=\text { Gram per microgram }
\end{aligned}
$$

\subsubsection{Adjusted Concentrations for Analytes/Radionuclides. Detected or Measured in the Initial Vendor Product Supernatant}

For analytes/radionuclides measured in the Initial Vendor Product supernatant, the reported Final Vendor Product concentration is the Initial Vendor Product concentration adjusted for the reduction in the oxide loading following the sodium reduction treatment and the quantity of analyte/radionuclide removed during the decanting process (Equation 2). For explanation of the calculation of total masses (i.e., dried solids and decant liquid) and final total oxide, see Appendix A. 
HNF-SD-WM-DP-225, REV. 1

Analytical Summary Report Addendum 1, Revision 2 - March 7, 1997

\author{
FVP $=[($ IVP * MD) $-($ IVS * MS) $] *(1 / \mathrm{FO}) *(31) *(1 \mathrm{e}-6) \quad$ (Equation 2) \\ Where, FVP = Final Vendor Product concentration in $\mathrm{g} / \mathrm{L}$ (or $\mathrm{Ci} / \mathrm{L}$ ) \\ IVP $=$ Initial Vendor Product concentration in $\mu \mathrm{g} / \mathrm{g}$ (or $\mu \mathrm{Ci} / \mathrm{g}$ )--See Table A1-1) \\ $\mathrm{MD}=$ Total mass of dried Initial Vendor Product (419.17 g) \\ IVS $=$ Initial Vendor Supernatant concentration in $\mu \mathrm{g} / \mathrm{g}$ (or $\mu \mathrm{Ci} / \mathrm{g}$ ) (See Table A1-3.1, \\ adjusted for supernatant density of $1.036 \mathrm{~g} / \mathrm{ml}$ or $1.000 \mathrm{~g} / \mathrm{ml}$, where \\ appropriate) \\ MS = Total Mass of decanted Supernatant $(2002.3 \mathrm{~g})$ \\ FO $=$ Final Vendor Product estimated total oxide content $(295.81 \mathrm{~g})$ \\ $31=$ Gram oxide per $31 \mathrm{~g}$ oxide $L$ \\ le-6 $=$ Gram per microgram
}

\title{
4.0 FINAL VENDOR PRODUCT ANALYSES - LOI Revision 2
}

Following the compositing and rebottling of the Final Vendor Product, four bottles were selected for additional analyses. The analyses performed were slurry density (See Table A1-2.5), bulk weight percent solids, total organic carbon, $\mathrm{pH} / \mathrm{OH}$, anions by IC, uranium by laser fluorimetry, and metals by ICP-AES. The results for the chemical analyses are presented in Tables A1-4.1 through A1-4.5. Due to balance capacity limitations (i.e., weighing container and sample was limited to $200 \mathrm{~g}$ ) in the Shielded Analytical Laboratory (SAL) and the requirement to perform weight percent solids on the entire contents of the containers, the contents of the four containers were split in High Level Radiation Facility, resulting in eight containers being submitted to the SAL for additional analyses. One split (i.e., C106-FP-V1-6b) was lost during handling. The C106 Final Vendor Product samples received from the HLRF for preparation and analysis consisted of seven, 30-50 g Final Vendor Product slurries and four, $10 \mathrm{ml}$ aliquots for $\mathrm{pH}$ or $\mathrm{OH}$. The $\mathrm{pH} / \mathrm{OH}$ aliquots were allowed to settle and $5 \mathrm{mls}$ of the supernatant from each was taken out of the hot cell and delivered to the ACL Sample Receiving and Prep Laboratory for analysis. 
HNF-SD-WM-DP-225, REV. 1

Analytical Summary Report Addendum 1, Revision 2 - March 7, 1997

\subsection{SAMPLE DRYING AND BLENDING}

Each of the seven Final Product slurries was weighed upon receipt and dried in a oven at $105^{\circ} \mathrm{C}$ until successive weighings yielded less than 1 percent weight loss between successive dryings. The bulk sample weight percent solids on drying are presented in Table A1-4.6. The observed volume reduction ranged from $55 \%$ to $70 \%$ after drying. The average reduction was $60 \%$ with a relative standard deviation of about $10 \%$. In all cases, the remaining dried solids were medium brown in color and the evaporated supernatant left a white coating on the container sides and on top of the dried brown solid layer. No indication of spattering, foaming, etc. during drying was noted as evidenced by clean container walls above the original fill lines. After drying, all seven dried slurries were individually homogenized by manual stirring, and then sample splits were recombined and blended together to produce a total of four samples for subsequent chemical analysis.

Table A1-4.6 Bulk Weight Percent Solids

\begin{tabular}{|c|c|}
\hline \begin{tabular}{|c|c|}
\hline Lab Number/Sample Identification \\
\hline $97-01112 /$ C106-FP-V1-1
\end{tabular} & 12.35 \\
\hline C106-FP-V1-1-B & 11.31 \\
\hline $97-01113 /$ Weight Percent Solids \\
\hline C106-FP-V1-1-B (lost) & 11.61 \\
\hline $97-01114 /$ C106-FP-V2-3 & $n / a$ \\
\hline C106-FP-V2-3-B & 12.58 \\
\hline C106-FP-V2-8-B & 11.24 \\
\hline Final Vendor Product - Average & 12.45 \\
\hline Final Vendor Product - Std Dev & 11.47 \\
\hline
\end{tabular}




\subsection{CHEMICAL PREPARATION FOR ANALYSIS}

Full Quality Control (QC) was applied to all batch processing techniques in accordance with the Quality Assurance Plan MCS-033. Full QC incorporates the use of methods blanks, spiked blanks, duplicate samples, matrix spiked samples and radiochemical tracers where available.

\subsubsection{Acid Digestion}

Each dried slurry sample was acid digested (PNL-ALO-129 "HNO $-\mathrm{HCl}$ Acid Extraction of Solids Using a Dry-Block Heater") and distributed for ICP/AES. It was noted during sample blending that the stir bars introduced into the containers by the HLRF were covered with magnetic solids. The stir bars were subjected to the same acid digestion treatment as the samples and the resulting solutions provided for ICP/AES.

\subsubsection{Fusion}

Caustic fusions (PNL-ALO-114 "Solubilization of Metals from Solids Using a $\mathrm{Na}_{2} \mathrm{O}_{2}-\mathrm{NaOH}$ Fusion", PNL-ALO-1 15 "Solubilization of Metals from Solids Using $\mathrm{KOH}-\mathrm{KNO}_{3}$ Fusion") were performed and aliquots distributed for ICP/AES and uranium analyses. The fusion technique is usually applied where total solubilization of the sample material is required, $Q C$ is limited to sample duplicates, a methods blank and post fusion spiking for selective analytes applied at the bench.

\subsubsection{Water Leach}

Each dried slurry sample was water leached (PNL-ALO-103 "Water Leach of Sludges, Soils and Other Solid Samples") filtered and aliquots distributed for anion analysis by Ion Chromatography. 


\subsubsection{Carbon Analysis}

Total Carbon, Total Inorganic Carbon and Total Organic Carbon analyses (PNL-ALO-381 "Direct Detemination of TC,TOC, and TIC in Radioactive Sludges and Liquids by Hot Persulfate Method") is usually performed remotely using a UIC Coulemetrics Carbon Analyzer. However, due to hardware failures, the TOC analysis can not be reported at this time.

\subsection{MEASURED ANALYTICAL RESULTS}

The results of the analyses conducted on the Final Vendor Product are summarized in Table Al-1 (FP Measured). Table A1-4.1 shows the average ICP-AES results from each sample preparation and the "most reasonable" result selected for inclusion in Table A1-1. Tables A1-4.2 through A1-4.5 contain the chemical results obtained on the four selected Final Vendor Product samples.

-

\subsubsection{ICP-AES Analysis}

ICP-MS and ICP-AES were both used to analyze the Initial Vendor Product and the majority of the results summarized in Table Al-1 are from the ICP-MS analysis; primarily since the ICP-MS provides significant higher sensitivity. For the Final Vendor Product only ICP-AES analyses were performed since the ICP/MS was unavailable. Due to the limited sensitivity of the ICP-AES, only a subset of the analytes of interest are reported for the Final Vendor Product. However, the agreement between the calculated "adjusted" value and the ICP-AES measured value are very good. Except for silver, phosphorus and Strontium, the measured results is with $\pm 30 \%$ (and most analytes are within $\pm 10 \%$ ) of the calculated result. The Final Vendor Product silver result is only half of that calculated, which is attributed to difficulties of the specific dissolution procedure to maintain silver in solution. Strontium in the Initial Vendor Product was report by ICP-MS. Although ${ }^{90} \mathrm{Sr}$ was detected in the Initial Vendor Product decanted supernatant, the ICP-AES analysis of the supernatant detected no strontium and no correction for strontium in the decant supernatant could be made resulting in a high estimate for the 
"adjusted" value. The phosphorus result is higher for the Final Vendor Product than the Initial Vendor Product; the reason for this is unclear but appears to be related to difference in fusion dissolution (See Table A1-4.1 for phosphorus values obtained from each preparatory technique).

In most cases, results from.ICP-AES (Initial and Final Vendor Product) and ICP-MS (Initial Product Only) were obtained from three different preparation techniques; specifically $\mathrm{KOH}$ fusion, $\mathrm{Na}_{2} \mathrm{O}_{2}$ fusion and $\mathrm{HNO}_{3}-\mathrm{HCl}$ acid digestion. In selecting the most reasonable result (Table A1-4.1), an effort has been made to evaluate the results based on the characteristics of the analytical technique and the limitation of the preparation method. A description of the selection basis for the Initial Vendor Product characterization is provided in the Analytical Summary Report'. For the analysis of the Final Vendor Product, only the limitation of the preparation method needs to be considered, since ICP-MS was not available. Where the preparation methods are considered equivalent and the measured value for analyte is above the EQL, the highest reported result is used for comparison to the envelope specification. This applies to 16 of the 23 analytes detected by ICP-AES in the Final Vendor Product. Results from the acid digest preparations were selected for the other seven analytes, primarily because of better sample sensitivity (i.e., the samples prepared by fusion methods require 10 to 40 times higher dilutions than samples prepared by acid digestion, and most of the results from the fusion methods were below the EQL). Due to excessively high and variable calcium results for the fusion processing blanks (evident in both the Initial Vendor Product and Final Vendor Product analyses), the calcium results from the acid digestion are considered more reliable, and therefore selected for reporting.

\subsubsection{IC Analysis}

Following the water leaching of the Final Vendor Product, IC analyses were performed to determine the anion concentrations. Except for nitrate+nitrite, the calculated "adjusted" anion concentrations do not agree with the measure Final Vendor Product results. This is to be expected, since the water leaching does not provide $100 \%$ dissolutions, some anion such as oxalate are at solubility limits, and other anions are at the measurement quantitation limit. The Final Vendor Product results represent the anion solution concentrations for product material leached at a 1:100 ratio. 
Table A1-4.1 ICP-AES Summary of Results for Final Vendor Product

**All Results in ug/g - dry weight"**

\begin{tabular}{|c|c|c|c|c|c|c|c|c|}
\hline $\begin{array}{l}\text { Anghing } \\
\text { Ang } \\
\text { Al } \\
\text { As } \\
\text { B } \\
\text { Ba }\end{array}$ & 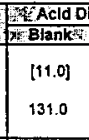 & \begin{tabular}{|c|} 
gestlon \\
Average: \\
126 \\
59,050 \\
\\
145 \\
487 \\
\end{tabular} & 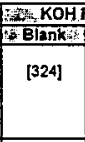 & $\begin{array}{c}\text { uslonys } \\
\text { Average } \\
1,620 \\
66,800 \\
\begin{array}{c}159] \\
527\end{array} \\
\end{array}$ & $\begin{array}{c}\text { Elank } \mathrm{Na}_{2} \mathrm{O}_{2} \\
\text { [65] }\end{array}$ & $\begin{array}{c}\text { Auslonisy } \\
\text { Average: } \\
749 \\
64,100 \\
544\end{array}$ & \begin{tabular}{|c|} 
Result \\
1,6 Selected \\
1,620 \\
66.800 \\
\\
\\
544 \\
\end{tabular} & $\begin{array}{l}\text { Pow } \\
\text { Highest selected } \\
\text { Highest selected } \\
\text { not detected } \\
\text { not detected - high blank'uncertainty } \\
\text { Highest selected }\end{array}$ \\
\hline $\begin{array}{l}\mathrm{Be} \\
\mathrm{Bl} \\
\mathrm{Ca} \\
\mathrm{Cd} \\
\mathrm{Ce}\end{array}$ & [54.0] & $\begin{array}{c}530 \\
4,825 \\
84 \\
{[328]}\end{array}$ & {$[1,570]$} & $\begin{array}{l}{[633]} \\
8,963 \\
{[110]} \\
{[577]}\end{array}$ & $\begin{array}{l}{[2,180]} \\
{[428]}\end{array}$ & $\begin{array}{c}{[660]} \\
12,075 \\
{[99]} \\
{[1,820]}\end{array}$ & $\begin{array}{c}{[660]} \\
4,825 \\
84\end{array}$ & $\begin{array}{l}\text { not detected } \\
\text { High uncertainty - below EQL } \\
\text { Fusions have high blanks } \\
\text { Fusions are below EQL } \\
\text { not detected - too close to IDL to use }\end{array}$ \\
\hline $\begin{array}{l}\text { Co } \\
C_{r} \\
C_{u} \\
\text { Dy } \\
\text { Fe }\end{array}$ & $\begin{array}{l}{[2.0]} \\
{[2.0]}\end{array}$ & $\begin{array}{r}{[31]} \\
570 \\
130 \\
145,000 \\
\end{array}$ & [91] & $\begin{array}{c}806 \\
{[205]} \\
158,250 \\
\end{array}$ & [84] & $\begin{array}{c}765 \\
{[201]} \\
+53,000 \\
\end{array}$ & $\begin{array}{r}806 \\
130 \\
158.250 \\
\end{array}$ & $\begin{array}{l}\text { not detected } \\
\text { Highest selected } \\
\text { Fusions are below EQL } \\
\text { not delected } \\
\text { Highest selected }\end{array}$ \\
\hline $\begin{array}{l}\mathrm{K} \\
\mathrm{La} \\
\mathrm{LI} \\
\mathrm{Mg} \\
\mathrm{Mn}\end{array}$ & & $\begin{array}{c}{[112]} \\
{[23]} \\
803 \\
3,010 \\
\end{array}$ & $(189)$ & $\begin{array}{l}{[162]} \\
{[1.478]} \\
3.820 \\
\end{array}$ & & $\begin{array}{c}{[5.103]} \\
{[175]} \\
{[1,375]} \\
3,200 \\
\end{array}$ & $\begin{array}{l}{[112]} \\
{[1,478]} \\
3,200\end{array}$ & $\begin{array}{l}\text { not detected - fusion high uncertainty } \\
\text { High uncertainty - below EQL } \\
\text { not delected - to close to IDL to use } \\
\text { High uncertainty - below EQL } \\
\text { Highest selected }\end{array}$ \\
\hline $\begin{array}{l}\mathrm{Mo} \\
\mathrm{Na} \\
\mathrm{No} \\
\mathrm{Ni} \\
\mathrm{P}\end{array}$ & $\begin{array}{l}1520= \\
{[10.0]}\end{array}$ & $\begin{array}{c}{[22]} \\
120,000 \\
332 \\
1.300 \\
1,653\end{array}$ & $\begin{array}{l}2,710 \\
{[172]}\end{array}$ & $\begin{array}{c}120,250 \\
{[539]} \\
3.070\end{array}$ & [98] & $\begin{array}{l}{[550]} \\
1,905 \\
4,805\end{array}$ & $\begin{array}{c}120,000 \\
332 \\
1,905 \\
4,805\end{array}$ & $\begin{array}{l}\text { not detected - to close to ID to use } \\
\text { Fusion has high blank } \\
\text { Fusion has high uncertainty } \\
\text { Highest selected } \\
\text { Highest selected }\end{array}$ \\
\hline $\begin{array}{l}\mathrm{Pb} \\
\mathrm{Pd} \\
\mathrm{Rh} \\
\mathrm{Ru} \\
\mathrm{Sb}\end{array}$ & & 3,893 & & 3,473 & & 4,118 & 4,118 & $\begin{array}{l}\text { Highest selected } \\
\text { not detected } \\
\text { not detected } \\
\text { not detected } \\
\text { not detected }\end{array}$ \\
\hline $\begin{array}{l}\text { Se } \\
\text { Si } \\
\text { Sn } \\
\text { Sr } \\
\text { Te } \\
\end{array}$ & {$[197.0]$} & $\begin{array}{c}{[159]} \\
2,678 \\
62 \\
{[506]} \\
\end{array}$ & & $\begin{array}{c}86,800 \\
{[100]}\end{array}$ & [41] & $\begin{array}{r}78,850 \\
{[163]}\end{array}$ & $\begin{array}{r}86,800 \\
\\
{[100]} \\
{[506]} \\
\end{array}$ & $\begin{array}{l}\text { not detected } \\
\text { Highest selected } \\
\text { not detected } \\
\text { Fusion have higher uncertainty } \\
\text { High uncertainty }\end{array}$ \\
\hline $\begin{array}{l}\text { Th } \\
\mathrm{TI} \\
\mathrm{TI} \\
\mathrm{U} \\
\mathrm{V}\end{array}$ & & $\begin{array}{c}{[428]} \\
364 \\
{[2,530]}\end{array}$ & & {$[5,602\}$} & & $\begin{array}{l}1,238 \\
{[4,802]}\end{array}$ & $\begin{array}{l}{[428]} \\
1,238\end{array}$ & $\begin{array}{l}\text { High uncertainty } \\
\text { Highest selected } \\
\text { not detected } \\
\text { Use Laser Fluorimetry Results } \\
\text { not delected }\end{array}$ \\
\hline $\begin{array}{l}\mathrm{W} \\
\mathbf{Y} \\
\mathrm{Zn} \\
\mathbf{Z r}\end{array}$ & [5.0] & $\begin{array}{l}{[86] .} \\
{[111]} \\
{[85]}\end{array}$ & & $\begin{array}{l}{[105]} \\
{[195]} \\
1.252\end{array}$ & & $\begin{array}{l}{[100]} \\
{[209]}\end{array}$ & 1,252 & $\begin{array}{l}\text { not delected } \\
\text { not detected - to near IDL to use } \\
\text { not detected - to near IDL to use } \\
\text { Highest selected }\end{array}$ \\
\hline
\end{tabular}

Results for each preparation (KOH/Na2O2/Acid) are average of 4 samples 97-1112 through 97-1115

Results in brackets are below Estimated Quantitation Limit but above instrument Detection Limit

Acid digestion results selected for $\mathrm{Ca}$ due to excessively high and variable processing blank from Shielded Analytical Laboratory 


\section{HNF-SD-WM-DP-225, REV.1}

Analytical Summary Report Addendum 1, Revision 2 - March 7, 1997

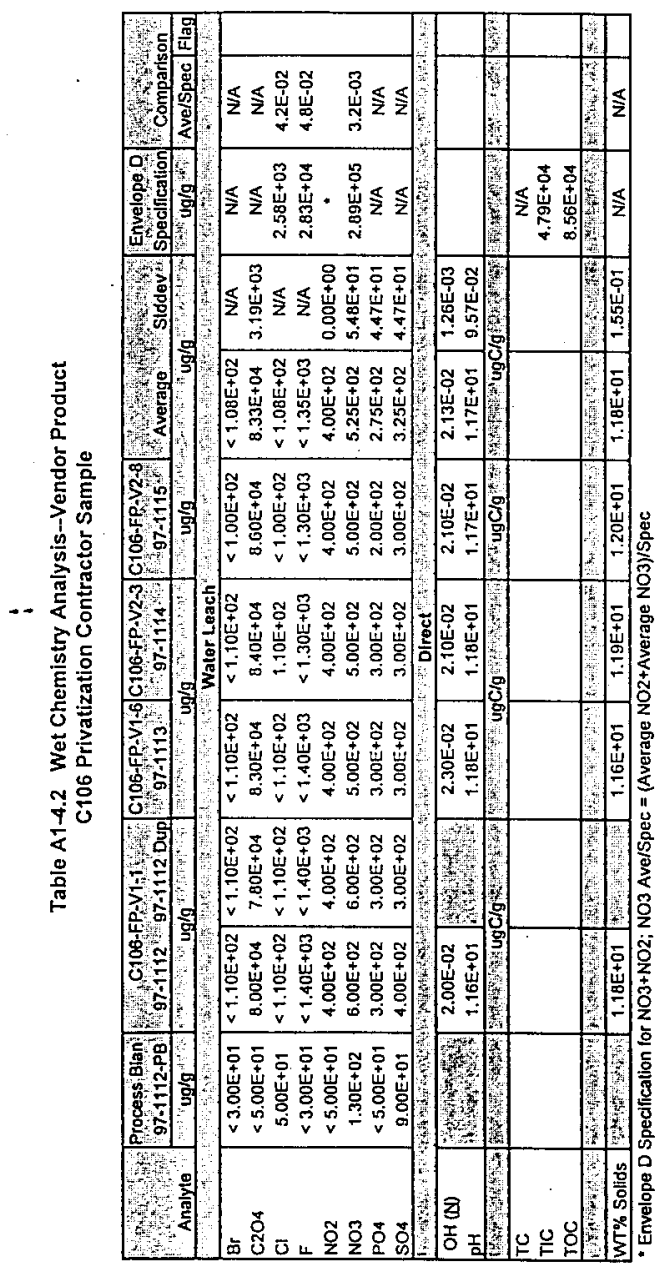


Table A1-4.3. ICP-AES KOH Fusion-Vendor Product C106 Privatization Contractor Sample

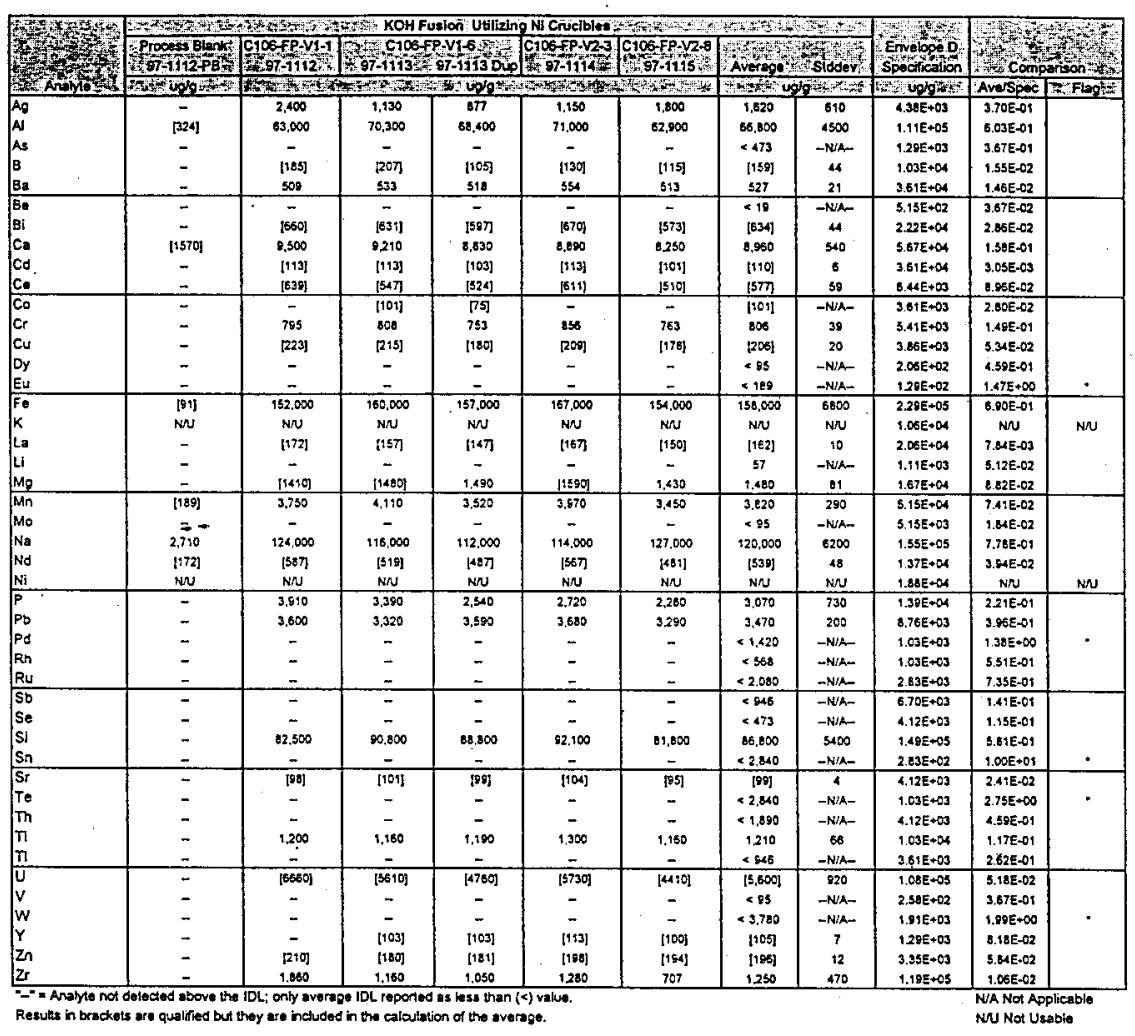


Table A1-4.4, ICP-AES Na2O2 Fusion-Vendor Product C106 Privatization Contractor Sample

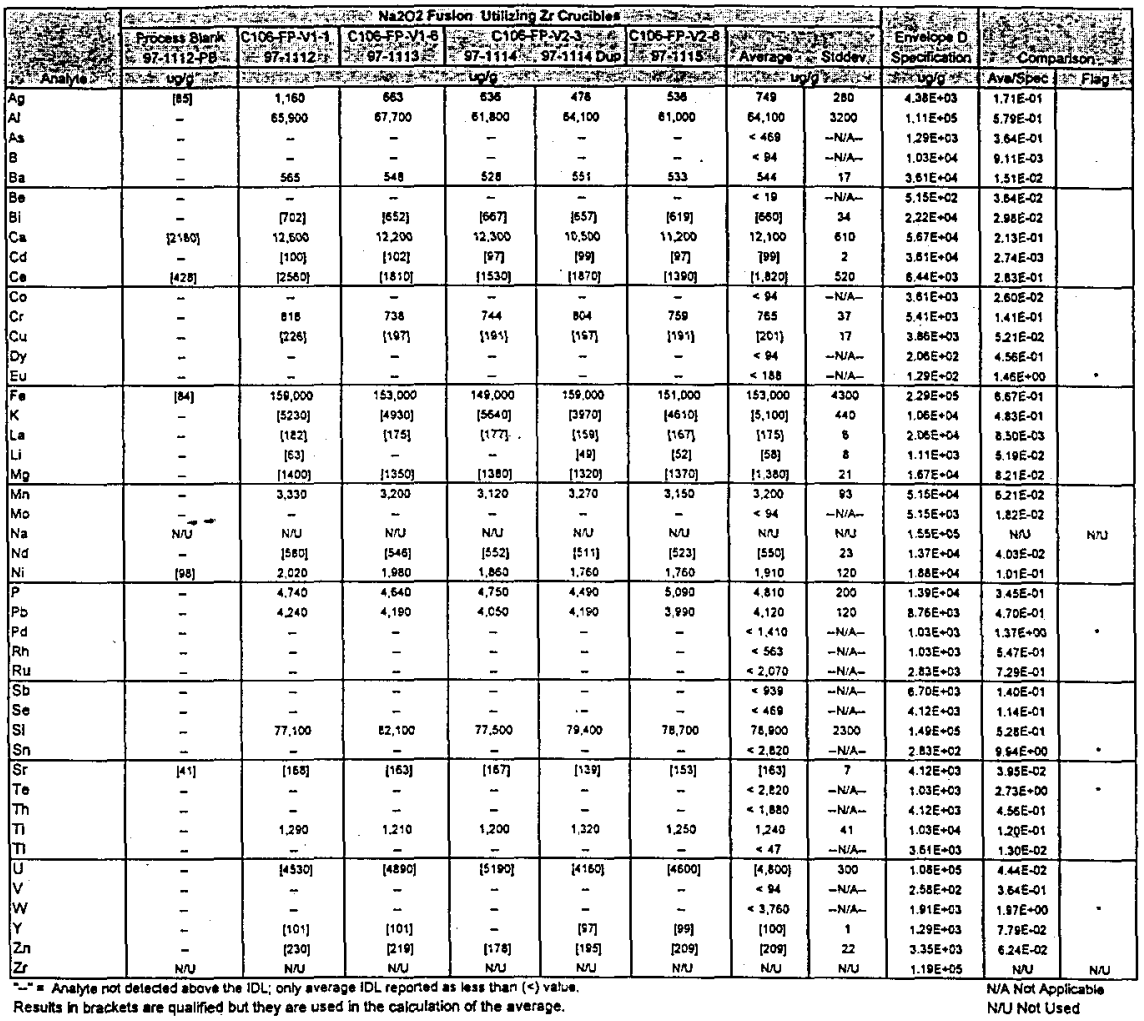


Table A14.5. ICP.AES Acid Digestion-Vendor Produet C106 Privatization Contractor Sample

\begin{tabular}{|c|c|c|c|c|c|c|c|c|c|c|c|}
\hline \multirow{2}{*}{ 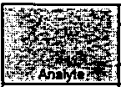 } & \multirow{2}{*}{ 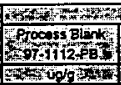 } & 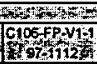 & 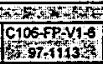 & 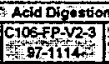 & 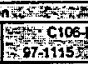 & 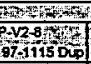 & 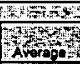 & stody & \multirow{2}{*}{ 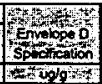 } & \multicolumn{2}{|c|}{ 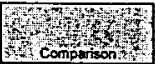 } \\
\hline & & $7 x+4+4$ & 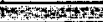 & ADAt uog & 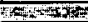 & 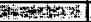 & Hats & ondyint & & Averspoct & If Flogtat \\
\hline$A D$ & - & 110 & 110 & 134 & 112 & 107 & 128 & 13 & $4.38 \mathrm{E}+03$ & $2.00 E-02$ & \\
\hline Al & [11] & 60,400 & 60,300 & 62,200 & 53,300 & $\$ 1,400$ & 59,100 & 3900 & $1.11 E+05$ & $5.33 E=01$ & \\
\hline As & - & - & - & - & - & - & $<62$ & $-\mathrm{N} / \mathrm{A}-$ & $1.29 E \div 03$ & 4.84E-02 & \\
\hline$B$ & $\$ 31$ & 138 & 165 & [120] & 156 & 140 & 145 & 20 & $1.03 E=04$ & $1,40 E-02$ & \\
\hline Ba & - & 500 & 495 & 505 & 49 & 432 & 497 & 26 & $3.61 E+04$ & $1.35 \mathrm{E}-02$ & \\
\hline B. & $i$ & - & - & - & - & - & $<2$ & $-N / A-$ & $3.15 E+C 2$ & 4.4AE-03 & \\
\hline $\mathrm{Bi}$ & - & 340 & 538 & 348 & 453 & AEI & 530 & 25 & $222 E+04$ & $2.39 \mathrm{E}-02$ & \\
\hline $\mathrm{Ca}$ & [54] & 4,780 & 8,060 & 5,130 & 4,330 & 4.170 & 4,830 & 360 & $3.67 E+0 \times$ & B.51E-C2 & \\
\hline Cd & - & 86.3 & Q4.8 & es.s & 79.4 & 70 & 84 & 3 & $3.61 E+04$ & $2.32 \mathrm{E}-\mathrm{CS}$ & \\
\hline C* & - & [332] & [344] & [321] & 1516] & {$[308]$} & [328] & 12 & B. $44 \mathrm{E}+03$ & $5.10 \mathrm{E} \sim \mathrm{C2}$ & \\
\hline Co & - & [31] & 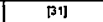 & [31] & [29] & [2ㅁ] & [31] & 1 & $3.61 E+03$ & B.46E-OS & \\
\hline cr & - & Sto & 569 & 594 & 520 & 500 & 570 & 29 & $5.41 \mathrm{E}+03$ & 1. OSED 1 & \\
\hline $\mathrm{Cu}$ & [2] & 140 & 120 & 130 & 121 & 117 & 130 & a & $3.66 \mathrm{E}+03$ & 3.38E-02 & \\
\hline Dy & - & - & - & - & - & - & $<13$ & $-N / A$ & $2.05 E+02$ & 6.0SE-D2 & \\
\hline Ev & - & - & - & $=$ & - & - & $<25$ & $-N / A=$ & $129 E+02$ & $1.20 E-01$ & \\
\hline$F \bullet$ & [2] & 949.000 & $\$ 45,000$ & 150.000 & 135,000 & 131,000 & $\$ 45,000$ & 6900 & $2295+05$ & B.32E-01 & \\
\hline$k$ & - & - & - & - & - & - & $<489$ & $-N_{A} /-$ & $1.06 E+04$ & $4,72 E-02$ & \\
\hline Le & - & 114 & [116] & [109] & [100] & [105] & 112 & 4 & $2.06 E+04$ & $5.42 E-03$ & \\
\hline $\mathrm{Li}$ & - & [23] & $\{23 \mid$ & {$[25]$} & [21] & [21] & [23] & 2 & $1.11 E+03$ & $2.08 \mathrm{E}-02$ & \\
\hline Mo & - & 827 & 795 & ass & 730 & 720 & 003 & 10 & $1.67 E+04$ & $4.79 \mathrm{E}-02$ & \\
\hline$M n$ & -7 & 3,110 & 3,040 & 3,090 & 2.810 & 2,700 & 3,010 & 140 & $3.15 E+04$ & 5.045 .02 & \\
\hline Mo & - & [23] & [22] & [22] & [21] & [21] & [22] & 1 & $5.15 E+03$ & $4.27 E-03$ & \\
\hline $\mathrm{Na}$ & 152 & 128,000 & 111,000 & 123,000 & 190,000 & 108,000 & 120,000 & 7600 & $1.55 E+05$ & $7,75 \mathrm{E}=01$ & \\
\hline Nd & - & 340 & 342 & 333 & 314 & 305 & 392 & 13 & $1.37 E+O A$ & $2.49 E-02$ & \\
\hline $\mathrm{Ni}$ & [10] & 1,550 & 1.320 & 1.300 & 1.220 & 1,180 & $1,300^{\circ}$ & 59 & $1.88 E+04$ & E.51E-02 & \\
\hline $\bar{P}$ & - & 1,670 & 1,670 & 1.760 & 1.510 & 1.490 & 1,650 & 100 & $1.39 E+04$ & $1.19 E-01$ & \\
\hline PD & - & 3,980 & 3,970 & 3.990 & 5.630 & 3.320 & 3,000 & 180 & $8.75 E+03$ & $4.44 E-01$ & \\
\hline Po & - & {$[201]$} & - & - & - & - & \{20:1\} & $-N / A-$ & $1.03 E+03$ & 1.85E-01 & \\
\hline Rh & - & - & - & - & - & - & $<75$ & $-N / A-$ & $1.03 E+03$ & $7.265-02$ & \\
\hline$R_{u}$ & - & $1557]$ & - & - & - & - & $1557]$ & -N/A- & $2.83 \mathrm{E}+\mathrm{CH}$ & 1.57E-01 & \\
\hline Sb & - & - & - & - & - & - & $<125$ & $-\mathrm{N} / \mathrm{s}-$ & 6.705403 & $1.865-02$ & \\
\hline se & - & [161] & {$[15 \pi]$} & [165] & {$[150]$} & [147] & [159] & . & $4.12 E+03$ & $3.25 E-02$ & \\
\hline Si & {$[187$} & 1,580 & 2,200 & 5,440 & $1,030^{\circ}$ & [1170] & 2,680 & 1800 & $1.49 E * 05$ & $1.79 E-02$ & \\
\hline Sn & - & [1590] & - & [685] & $=$ & - & $\{1,140\}$ & 640 & 2. $83 \mathrm{E}+02$ & $4.02 E+00$ & $=$ \\
\hline $\mathrm{Sr}$ & - & 63.7 & 63.4 & 63.2 & 57.7 & 85.6 & 52 & 3 & $4,12 E+03$ & $1.50 E-02$ & \\
\hline To & - & [\$14] & 1517 & [522] & [472] & [45.] & [508] & 23 & $1.03 \mathrm{E}+03$ & $4,91 E-01$ & \\
\hline Th & - & {$[457]$} & |《s & {$[410]$} & [401] & (375) & [428] & 27 & $4.12 E+03$ & $1.04 E-01$ & \\
\hline$T$ & - & $360^{\circ}$ & $350^{\circ}$ & 34 & 334 & 314 & 364 & 25 & $1.03 \varepsilon+04$ & $3.53 E-02$ & \\
\hline TI & - & - & - & - & $=$ & - & $<125$ & $-N / A-$ & $3.61 E+03$ & 3.4.8E-02 & \\
\hline $\mathrm{U}$ & - & [2539] & [2620] & [2540] & [2530] & [2270] & {$[2,530]$} & 140 & $1.08 E+05$ & 2.34E-02 & \\
\hline$v$ & - & [15] & - & - & - & - & {$[15]$} & $-N / A-$ & $2.58 E+02$ & $5.71 E-62$ & \\
\hline$W$ & - & - & - & - & - & - & $<498$ & $-N / A_{-}$ & $1.81 E+03$ & $2.62 E-0 t$ & \\
\hline$Y$ & - & [Bs] & [88] & [Es] & $\nabla 9$ & [75] & {$[106]$} & 4 & $1.29 \mathrm{E}+03$ & $6.66 \mathrm{E} \cdot 02$ & \\
\hline Zn & [5] & 114 & [111] & [114] & {$[10]$} & {$[109]$} & [191] & 5 & $3.35 E+03$ & 3.31E-O2 & \\
\hline$z$ & - & [82] & [105] & {$[104]$} & (65) & BSE & tes & 23 & $1.19 \Xi+05$ & $7.15 E-04$ & \\
\hline
\end{tabular}
Resulas in brackets are qualified but they are included in the caleuletion of the sverage. 


\subsection{REFERENCES}

Brooks, KP, et al. 1997. Bench-Scale Enhancement Sludge Washing and Gravity Settling of Hanford Tank C-106 Sludge. PNNL-1 1432. Pacific Northwest National Laboratory, Richland, Washington.

Jones, TE. 1996. Addendum 1 of the Letter of Instruction (LOI) for TWRS Privatization Contractor Samples Addressing Waste Envelope D Materials, Revision 0. Pacific Northwest National Laboratory, Richland, Washington.

Urie, MW. 1997. Tank Waste Remediation System (TWRS) Privatization Contractor Samples, Waste Envelope D Material, 241-C-106, Analytical Summary Report, Revision 0. Pacific Northwest National Laboratory, Richland, Washington.

Wiemers, KD. 1996a. Addendum 1 of the Letter of Instruction (LOI) for TWRS Privatization Contractor Sampiés Addressing Waste Envelope D Materials, Revision I. Pacific Northwest National Laboratory, Richland, Washington.

Wiemers, KD. 1996b. Addendun 1 of the Letter of Instruction (LOI) for TWRS Privatization Contractor Samples Addressing Waste Envelope D Materials, Revision 2. Pacific Northwest National Laboratory, Richland, Washington. 


\section{HNF-SD-WM-DP-225, REV. 1}

Analytical Summary Report Addendum 1, Revision 2 - March 7, 1997

Appendix A:

C106 Calculatlons for the Final Vendor Product Adjustment - After Decant/Dilute Treatment

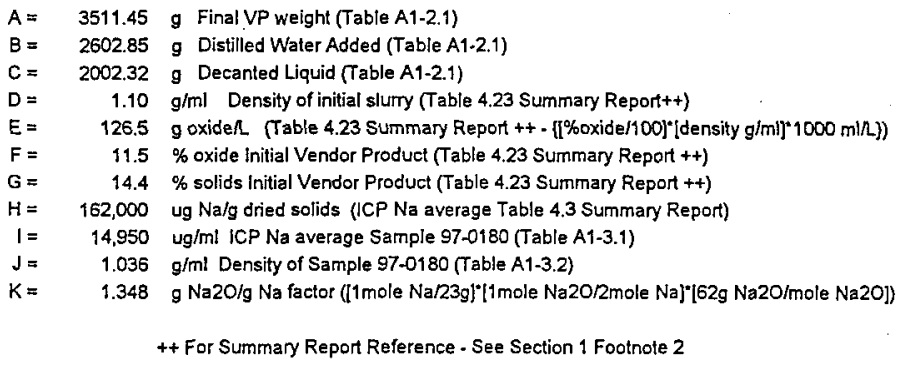

Weight of inital slurry $(A-B+C)$

Volume of initlal sluriy ([R/D]/1e3)

Total Oxide Initial Vendor Product (E* $S$ )

Total Mass of Dried Solids Inltial Vendor Product (T * [G/F])

Total Sodium $[[\mathrm{H} * \mathrm{U}] / \mathrm{1e6})$

\section{$\rightarrow-$}

Sodium in Supernate $\left[[\mathrm{JJ}]{ }^{*}[\mathrm{C} / \mathrm{Te}]\right\}$

Sodium in Final Vendor Product $(\mathrm{V}-\mathrm{W})$

Final Oxide - Adjusted only for Sodium Removal $\left(T-\left[W^{*} K\right]\right)$

$\begin{array}{rrl}R= & 2910.92 & \mathrm{~g} \\ \mathrm{~S}= & 2.646 & \mathrm{~L} \\ \mathrm{~T}= & 334.76 & \text { g oxide } \\ \mathrm{U}= & 419.17 & \mathrm{~g} \text { dried solids } \\ \mathrm{V}= & 67.91 & \mathrm{~g} \mathrm{Na} \\ \mathrm{W}= & 28.89 \mathrm{~g} \mathrm{Na} \\ \mathrm{X}= & 39.01 \mathrm{~g} \mathrm{Na} \\ \mathrm{Y}= & 295.81 & \text { goxide }\end{array}$

Adfustment calculation for each analyte measured in supernate $\gg \quad$ g Analyte/31 g oxide:

g Analyte $/ 31 \mathrm{~g}$ oxide $=\quad\left(M 1 * \mathrm{U}-M 2^{*} \mathrm{C}\right) \cdot(M 3 \mathrm{M}) * 1 \mathrm{e}-6$

M1 Value of analyte concentration in dried slurry materia! ( $\mathrm{wg} / \mathrm{g}$ )

U "Total Mass of Dried Solids Initial Vendor Product" (g)

M2 Value of analyte concentration in supernate (ug/g)

C "Decanted Liquid Mass" (g)

M3 31 (number of $g$ oxide $/ 31 \mathrm{~g}$ oxide)

Y "Final Oxide Adjusted for Na Removal" (g oxide)

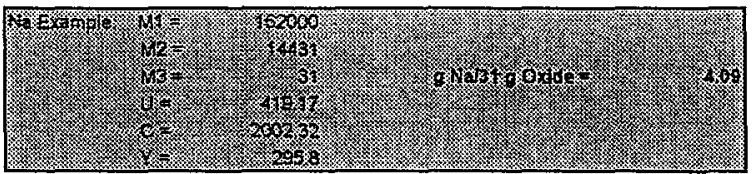




\title{
TANK WASTE REMEDIATION SYSTEM (TWRS) PRIVATIZATION CONTRACTOR
}

\author{
ENVELOPE D SAMPLES \\ (C-106)
}

ATTACHMENT 3

PNNL ACL SUBMITTAL OF ORGANIC AND RADIONUCLIDE RESULTS FROM ANALYSIS OF

C-106 ORIGINAL MATERIAL SUPERNATE

\section{March 1997}

Pacific Northwest National Laboratory 
HNF-SD-WM-DP-225, REV. 1

October 31, 1996

Mr. Cary M. Seidel

Rust Federal Services, Hanford

Mail Stop T6-04

Richland, Washington 99352

Dear Cary:

\section{PACIFIC NORTHWEST NATIONAL LABORATORY ANALYTICAL CHEMISTRY LABORATORY SUBMITTAL OF ORGANIC AND RADIONUCLIDE RESULTS FROM ANALYSIS OF C-106 ORIGINAL MATERIAL SUPERNATE}

Attached is a copy of the ACL results from the organic and radiochemical analysis of the supernate from the C106 Original Material. Please recall that this additional scope was added in early September.

In late August the $\mathrm{ACL}$ received the $\mathrm{C}-106$ Original Material for testing. The three subsamples received were mixed and transferred to three beakers in preparation for drying. It was observed that a clear layer developed rapidly. This layer appeared to be organic in nature and thus raised concern for the drying process. The decision was therefore made to remove the clear layer of supernate and save it for potential analysis at a later date. In early September the decision was made to go forward with limited analysis on the clear layer. A portion of the supernate was aliquoted and transferred for total alpha and beta analysis and organic characterization. These results are included herein. You will find a table (Table X) containing the alpha and beta results, performed in duplicate, and an organic report prepared by Gary Mong and Jim Campbell.

If you have any questions or concerns please contact me on 376-9454.

Very truly yours,

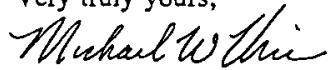

Michael W. Urie, Envelope D, C106 Project Manager

Analytical Chemistry Laboratory

cc: Karyn D. Wiemers, PNNL

MWU/ean

Neil R. Brown, DOE

Attachments (2)

Battelle Boulevard = P.0. Box 999 a Richland, WA 99352

Telephone (509) 376-9454 E Email mw_urie@pnl.gov * Fax (509) 372-2624 
Cary Seidel

October 31, 1996

Page 2

bcc: KJ Kuhl-Klinger

File/LB 


\author{
Analysis of C-106 Sample \\ G.M. Mong and J.A. Campbell
}

\title{
Introduction
}

The TWRS Privatization Envelope D starting composite material (96-05892, SPDL1-C106003), was transfered from $150 \mathrm{ml}$ bottles to $150 \mathrm{ml}$ beakers for drying. The bottles were shaken vigorously before the materials were transferred to the beakers in an effort for a quantitative transfer with no additional liquid added. Ten to fifteen minutes after transferring to the beaker; the slurry solids settled. This resulted in an approximately $10 \mathrm{ml}$ transparent liquid layer on top of the slurry. A concern that this layer might contain concentrated insoluble organics lead to a decision to pipette the clear supernatant before drying the solids. A portion of this transparent layer was submitted for organic analyses.

This report describes the organic analyses regime which was conducted on the separated supernate.

The analysis of potential major organic components related to tank wastes cannot be accomplished via a single analytical scheme. The carboxylates and chelators may be screened using gas chromatography/mass spectrometry (GC/MS) after methylation with $\mathrm{BF}_{3}$-methanol; acidic phosphate esters (such as bis(2-ethylhexyl)phosplate) require diazomethane derivatization for analysis. Nonpolar organics are commonly extracted with methylene chloride and identified directly using GC/MS analysis. The supernatant sample was analyzed in this fashion for chelators and chelator fragments, phosphate-containing components, and organic-soluble carbon using GCAMS.

Methylene chloride extracts of the sludge oil resulting from C-106 waste treatments have been previously analyzed ${ }^{1}$, and the major component identified using derivatization gas chromatography/mass spectrometry(GC/MS) was bis(2-ethylhexyl)phosphate.

\section{Experimental Methods}

A portion of the sample was stirred with water to dissolve the salts and then extracted with measured volumes of methylene chloride. The methylene chloride extracts were separated, dried with a bed of sodium sulfate to remove residual aqueous radioactive components, taken to a minimum volume $(300 \mu \mathrm{L})$ using a gentle stream of dry nitrogen and reconstituted to a known volume with methylene chloride. Solvent extracted samples were acidified and treated with an unquantified excess of ethereal diazomethane (formed from base treatment of $\mathrm{N}$ methyl- $\mathrm{N}$-nitrosourea) to simultaneously determine phosphate-containing organic components and organically soluble materials (e.g. TBP) by GCMS analysis

Aliquots of the aqueous portion were taken to dryness for analysis of chelators and chelator fragments. These samples were treated with $\mathrm{BF}_{3} /$ methanol complex $(2 \mathrm{~mL})$ for $1 \mathrm{~h}$ at $100^{\circ} \mathrm{C}$ to derivatize potential chelator components such as EDTA, NTA, and HEDTA for subsequent analysis by GCMS. 
Mass spectrometric data were obtained on low-resolution GC/MS systems (HP 5988A and 5987) that were tuned with perfluorotributylamine (PFTBA) before use. Additional GC/MS data were obtained using a HP 5970 mass selective detector system, tuned with PFTBA before use.

\section{Results and Discussion}

Methylene chloride extraction of the C-106 sample did not afford appreciable residual material upon evaporation of the solvent This minor residual was treated with ethereal $\mathrm{HCl}$, evaporated a second time, and quenched with diazomethane. Very little of the diazomethane was consumed, indicating acidic phosphates at minimal concentration. Gas chromatography/mass spectrometry analysis of this extract indicates a value of about 60 $\mu \mathrm{g} / \mathrm{gram}$ bis(2-ethylhexyl)phosphate (DE2HP) to be present in the liquid supernatant as delivered from the bot cell. This value is based on a single point calibration and imperfect measurement of the amount of solvents used in the work-up of the small sample. More sample is required to refine this number. The mass spectrum of the analyte (methyl-DE2HP) appears to contain one major ion which may be assigned as $\mathrm{m} / \mathrm{z} 125$ or $\mathrm{m} / \mathrm{z} 126$ depending upon instrumental parameters. We will be pursuing a high-resolution mass spectrometric investigation of this material to further understand the mass spectral properties of DE2HP.

The remainder of the sample (after a $0.1 \mathrm{~mL}$ aliquot for potential TOC analysis and an additional subsample were placed in reserve for HPLC analysis), was dried, weakly acidified, and dried again. Boron trifluoride/methanol was added, the derivatized-material separated, and subsequently analyzed using GCMS. The only organic of any consequence was oxalic acid, to the extent of about $6 \mathrm{mg} / \mathrm{g}$ of sample supernatant.

Typical organic components such as tributyl phosphate or hormal paraffin hydrocarbons identified in other tanks were not present in any of the samples examined.

In summary, the major organic components identified and quantitated in the supernatant sample were bis(2-ethylhexyl)phosphate and oxalic acid. The concentrations were $60 \mu \mathrm{g} / \mathrm{g}$ and $6 \mathrm{mg} / \mathrm{g}$, respectively. Other organics such as EDTA, NTA, IDA, ED3A, and HEDTA were not observed. In addition, organically soluble components such as IBP and NPH were not observed.

\section{Reference}

Campbell, JA et al. 1996. Organic Tanks Safety Program: Advanced Organic Analysis FY 1996 Progress Report. PNNL-11309, Pacific Northwest National Laboratory, Richland, Washington. 
Table X: Tank C106 Total Alpha and Beta Results (Original Material, Liquid Layer)

\begin{tabular}{|c|c|c|c|c|c|}
\hline ALO \# & CORE ID & $\begin{array}{l}\text { Total Alpha } \\
\text { UCi } / \mathrm{ml}\end{array}$ & $\begin{array}{l}+/-\% \\
\text { Error }\end{array}$ & $\begin{array}{c}\text { Total Beta } \\
\mathrm{uCi} / \mathrm{ml}\end{array}$ & $\begin{array}{l}+1-\% \\
\text { Error }\end{array}$ \\
\hline $96-5893-\mathrm{Ni}$ & $\begin{array}{l}\text { SPOL1-C106-004 } \\
\text { Liquid Layer }\end{array}$ & $2.21 E-01$ & $.4 \%$ & $3.34^{\prime} E+01$ & $4 \%$ \\
\hline 96-5893-Ni-Rcp & $\begin{array}{l}\text { SPDL1-C106-004 } \\
\text { Liquid Layer } \\
\text { RPD }\end{array}$ & $\begin{array}{c}2.35 E-01 \\
6.1 \%\end{array}$ & $4 \%$ & $\begin{array}{c}3.37 E+01 \\
0.9 \%\end{array}$ & $4 \%$ \\
\hline Sample Spikc & & & & $\mathbf{9 8 \%}$ & \\
\hline Slandard & & $110 \%$ & & $102 \%$ & \\
\hline Blank & & $<2 . E-4$ & & $<2$.E-2 & \\
\hline
\end{tabular}

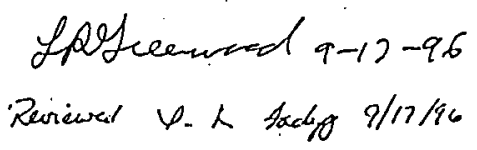




\title{
TANK WASTE REMEDIATION SYSTEM (TWRS) PRIVATIZATION CONTRACTOR
}

\author{
ENVELOPE D SAMPLES \\ (C-106)
}

\section{ATTACHMENT 4}

\section{FINAL STATISTICAL REPORT}

March 1997

Pacific Northwest National Laboratory 
HNF-SD-WM-DP-225, REV. 1

ATTACHMENT TO RFSH-9752732

TANK WASTE REMEDIATION SYSTEM PRIVATIZATION

PRIVATE CONTRACTOR SAMPLES

FOR HIGH-LEVEL WASTE FEED ENYELOPE D, TANK 241-C-106

FINAL STATISTICAL REPORT

Consisting of 45 pages

(including cover page) 
HNF-SD-WM-DP-225, REV. 1

97012-RFSH

ATTACHMENT

TANK WASTE REMEDIATION SYSTEM PRIVATIZATION

CONTRACTOR SAMPLES

WASTE ENVELOPE D MATERIAL

Tank 241-C-106

Final Statistical Analysis Report

Prepared by: Terri L WeTsh Babcock \& Wilcox Protec, Inc.

Safeguards Material Control

March 27, 1997

Consisting of 44 pages

including the cover page 


\section{LIST OF TERMS}

Acid

ANOVA

CI

Ci/L

CVAA

$d f$

DQO

EL

EQL

FS.Ni

FS.Zr

GEA

$\mathrm{g} / \mathrm{L}$

HLW

IC

ICP/AES

ICP/MS

IDL

IPT

$\mathrm{LL}$

LOI

$\mu \mathrm{Ci} / \mathrm{g} \mathrm{d}$

$\mu \mathrm{g} / \mathrm{g} \mathrm{d}$

$\mathrm{m} / \mathrm{z}$

NA

$\mathrm{NH}_{3}-\mathrm{N}$

PNNL

PHMC

Rad

REML.

RSD

SAL

SYS

TIC

TOC

TWRS

UL

Water

WtChem

. W

.wo

$\hat{\mu}$
$\hat{\sigma}_{\hat{\hat{\sigma}}}$
$\hat{\sigma}_{\text {total }}$

nitric acid-hydrochloric acid digestion

analys is of variance

confidence interval

curies per liter (at 31 gram waste oxide)

cold vapor atomic absorption

degrees of freedom

data quality objective

"D" Envelope Limit

estimated quantitation limit

potassium hydroxide fusion utilizing $\mathrm{Ni}$ crucibles

sodium peroxide-sodium chloride fusion utilizing zirconium crucibles

gamma energy analys is

grams per liter (at 31 gram waste oxide)

high level waste

ion chromatography

inductively coupled plasma/atomic emission spectrometry

inductively coupled plasma/mass spectrometry

instrument detection limit

integrated process product team

lower limit

letter of instruction

microcuries per gram dried slurry

miçrogram per gram dried slurry

mass-to-charge ratio (ICP/MS)

not available or not applicable

the ammonia procedure reports the results as nitrogen

Pacific Northwest National Laboratory

Project Manford Management Contract

Radiochemical analysis

restricted maximum likelihood estimation

relative standard deviation

Shielded Analytical Laboratory

systematic variability

total inorganic carbon

total organic carbon

tank waste remediation system

upper 1 imit

water leaching

wet chemistry

indicates that the "less than" values were used in the

statistical analysis at the upper value (e.g. 3 for $<3$ ).

indicates that the "less than" values were deleted from the statistical analysis

estimate of the mean

standard deviation associated with the mean, random variability

standard deviation associated with the mean, combined random and systematic variability 
HNF-SD-WM-DP-225, REV. 1

\title{
TANK WASTE REMEDIATION SYSTEM PRIVATIZATION CONTRACTOR SAMPLES
}

\author{
WASTE ENVELOPE D MATERIAL \\ Tank 241-C-106
}

Final Statistical Analysis Report

\subsection{INTRODUCTION}

This statistical analysis report presents the evaluation of a set of analytical data generated for the Tank Waste Remediation System (TWRS) Privatization Contractor Samples, High-Activity Waste Feed Envelope D, Tank 241-C-106. Samples were received at the 325 building High Level Radiochemistry Facility and composited. The composited material was then transferred to the 324 bujlding for Envelope D batch processing (Enhanced sludge Washing). Subsamples of the original composited materials were collected from the batch processing system for subsequent chemical, radiological, and physical property measurements. Following batch processing, the resulting material (Vendor Product) was subsampled. The subsamples were sent to the 325 building for analysis in accordance with Addendum 1 of the Letter of Instruction (LOI) (Jones, 1996). Further treatment was required to meet the envelope D sodium specification. A limited set of analyses were completed for the resultant "treated" materials (Final Vendor Product). The Final Vendor Product was then bottled for shipment to the private contractors. This report uses data collected for the Vendor Product materials.

Twenty grab samples were taken from tank 241-C-106 between June 21, 1996, and June 24, 1996. All of the samples were taken from Riser 7 at sample depths between 406 and 414 inches. Sample depth is measured from the top of the riser to the mouth of the sample bottle. Each grab sample was individually homogenized. The homogenized samples were then transferred to a 2 liter carboy. The details regarding the compositing of the grab samples are provided by.Urie (1997).

Following the batch processing as described by Brooks (1997), five subsamples of the Vendor Product were obtained for subsequent physical and chemical characterization and archive. Three of the five samples were submitted to the Shielded Analytical Laboratory (SAL) for analysis of chemical and radionuclide constituents. Each of the three subsamples $(-125 \mathrm{~mL})$ were placed into glass beakers for mixing and drying. After drying, each of the three dried slurries were homogenized, and then divided into two halves; forming a sample and a sample duplicate. Analytical sample preparations were conducted on these Vendor Product dried slurries. Additional details regarding the subsampling and sample preparations are provided by Urie (1997).

\subsection{OBJECTIVES}

The objectives of the statistical analysis were

- to provide estimates of the subsample variability for the analytes, 
- to provide estimates of the analytical variability for the analytes,

- to compare the upper limit of a $95 \%$ confidence interval (CI) for the mean analyte concentration with the respective envelope limit.

Both the subsample variability and the analytical variability, as determined from the analytical measurements, are used to estimate the compositional ranges of the sample concentrations. The magnitude of the subsample variability also identifies possible sample inhomogeneity where the inhomogeneity could have been caused by entrained solids. The magnitude of the analytical variability identifies, for this sample matrix, those analytes with large analytical uncertainties. The large analytical uncertainty could be due to sample inhomogeneity, matrix interferences, or analyte concentrations close to the detection limit of the analytical procedure. The magnitude of the analytical uncertainties will be used to develop the integrated process product team. (IPT) feed validation procedures and the data quality objectives (DQO) for high level waste (HLW) feed staging.

The upper limit of a one-sided $95 \%$ CI on the mean concentration takes into account the sampling and analytical uncertainties. Comparing the upper limit of this $95 \%$ CI for the mean concentration with the respective envelope limit is one indicator of the Project Hanford Management Contract's (PHMC) risk of delivering feed within the envelope specification.

\subsection{STATISTICAE METHODOLOGY}

The data used in the statistical analysis were obtained from Urie (1997). The differences between the three subsample results are used in computing the random "sampling" variability. The differences between the two measurements per subsample are used in computing the random "analytical" variability.

The data were statistically evaluated using one-way analysis of variance (ANOVA): the data are identified by one variable (the subsample). The one-way analysis of variance statistical model used to describe the structure of the data is

where

$$
\begin{gathered}
Y_{i j}=\mu+S_{i}+A_{i j}, \\
i=1,2, \ldots, a, j=1,2, \ldots, n_{i},
\end{gathered}
$$

$\begin{array}{ll}Y_{i j} & =\quad \begin{array}{l}\text { concentration from the } j^{\text {th }} \text { analytical result from the } \\ i^{\text {th }} \text { subsample }\end{array} \\ S_{i} & =\text { the grand mean of the population } \\ A_{i j} & =\quad \text { the effect of the } i^{\text {th }} \text { subsample } \\ & \quad \text { the effect of the } j^{\text {th }} \text { analytical result from the } j^{\text {th }}\end{array}$




$\begin{array}{ll}\text { a } & =\text { the number of subsamples } \\ n_{i} & =\begin{array}{l}\text { the number of analytical results from the } i^{\text {th }} \\ \text { subsample. }\end{array}\end{array}$

The variable $S_{i}$, which represents the difference between the concentration of the $i^{\text {th }}$ sample and the average content over the population, is assumed to be a random effect. This variable, as well as $A_{i j}$ (which represents the analytical variability), are assumed to be uncorrelated and normally distributed with means zero and variances $\sigma^{2}(S)$ and $\sigma^{2}(A)$, respectively. Since every sample has its value of $S_{i}, S_{i}$ is considered to be a random variable with a distribution over the population. This distribution has mean 0 , since the $S_{i}$ are defined as deviations from the population mean.

Estimates of $\sigma^{2}(S)$ and $\sigma^{2}(A)$ were obtained using Restricted Maximum Likelihood Estimation (REML) techniques. This method, applied to variance component estimation, is described in Harville, 1977. The results using the REML techniques were obtained using the statistical analysis package S-PLUS (Statistical Sciences, 1993).

The following paragraphs describe how the mean analyte concentration $(\hat{\mu})$, the standard deviation of the mean $\left(\hat{\sigma}_{\dot{\mu}}\right)$, and the degrees of freedom $(d f)$ were determined using the results from the statistical model fit to the data.

\section{Mean Analyte Concentration}

The estimator, $\hat{\mu}$, is the maximum likelihood estimate of the mean. This estimator was determined by the structure of the data reflected by the statistical model. The estimate of $\hat{\mu}$ was obtained using REML techniques in S-PLUS.

Standard Deviation of the Mean

The estimated standard deviation of the mean (where the mean is the maximum likelihood estimate), $\hat{\sigma}_{\hat{\mu}}$, is the square root of a function of the variance estimates:

$$
\hat{\sigma}^{2}(S) \text { and } \hat{\sigma}^{2}(A) \text {. }
$$

These estimates were obtained using REML techniques. For unbalanced data, $\hat{\sigma}_{\vec{\mu}}$ is a complicated function of these variances.

\section{Degrees of Freedom}

The degrees of freedom ( $d f$ ) are dependent on the data structure or the statistical model used. The $d f$ associated with $\hat{\mu}$, the mean analyte concentration, for balanced data (every subsample has the same number of analytical measurements) is the number of subsamples minus one. For unbalanced data the $d f$ associated with $\hat{\mu}$ is approximated by the number of subsamples minus one.

Reference standards were analyzed as specified in the analytical procedures. The majority of the reference standards were within quality control limits. The reference standards and spike recoveries provide assurances that the analytical procedures are producing valid results. The majority of the spike 
recoveries were within the customer specified limits. The analytes which had reference standard recoveries or spike recoveries outside the customer specified limits will be identified in the Final Analytical Report.

\subsection{STATISTICAL RESULTS - Random Variability}

The results of the statistical analyses for the Vendor Product data provided in Urie (1997) are lissted in Table 4.l. The mean analyte concentration ( $\hat{\mu})$, the relative standard deviation of the mean (RSD), the degrees of freedom $(d f)$, and the upper limit to the one-sided $95 \%$ confidence interval on the mean are included in Table 4.l. The RSD of the mean $\left(\hat{\sigma}_{\hat{\mu}} / \hat{\mu}\right)$ is defined as the standard deviation associated with the mean $\left(\hat{\sigma}_{\hat{i}}\right)$ divided by the mean.

The standard deviation for each analyte listed in Urie (1997) was computed by pooling all the analytical results (up to 6 measurements, 3 samples in duplicate). The standard deviation of the mean is then computed by dividing the standard deviation by the square root of $n$, where $n$ is the number of measurements. The standard deviation of the mean in Table 4.1 was calculated using the sampling variability and the analytical variability (square root of $\left[\hat{\sigma}^{2}(S) / 3+\hat{\sigma}^{2}(A) / 6\right]$ in the balanced case). The two values for the standard deviation of the mean will be different due to the assumptions used for the calculations.

The mean results provided in Urie (1997) may a] so be different from those 7isted in Table 4.1 due to the treatment of the data (especially the "less than" values)..

The upper limit (UL) to a one-sided $95 \%$ confidence interval for the mean is

$$
U L=\hat{\beta}+t_{(d t, 0.05)} \times \hat{\sigma}_{\beta} .
$$

In this equation, $t_{(d f, 0,05)}$ is the quantile from Student's $t$ distribution with $d f$ degrees of freedom'for a one-sided $95 \%$ confidence interval. The upper limit of the one-sided confidence interval on the mean in Table 4.1 takes into account both the random sampling variability and the random analytical variability.

The analytical data for several anaiytes consisted of both quantitative values and "less than" values. A statistical analysis of the data was performed if at least $50 \%$ of the data were quantitative values. The ANOVA was computed using two different methodologies for these data.

The upper value of the "less than" (e.g., 3.5 for $<3.5$ ) was used to represent all "less than" analytical values in the first computation. This produces a bias of unknown magnitude in both the mean analyte concentration and the variance associated with the mean; the mean analyte concentration is biased high. The extension ". w" was added to the analyte name in the tables to distinguish which analyte was statistically analyzed using "less than" values. 
The "less than" values were deleted in the second computation. Deleting data produces unbalanced data sets which complicates the statistical analysis. Deleting data decreases the number of degrees of freedom. Deleting data also produces a bias of unknown magnitude in both the mean analyte concentration and the variance associated with the mean. The .extension ". wo" was added to the analyte name in the tables to distinguish which analyte was statistically analyzed with the "less than" values deleted.

The statistical analysis was not performed for those analytes which had more than $50 \%$ of the analytical results reported as "less than" values. These analytes are listed in Table 4.2. Table 4.2 cites the largest value listed from the six analytical results.

The random variance component estimates were determined from ANOVA using REML techniques. These variance component estimates (from which the standard deviation associated with the mean concentration were calculated) are provided in Table 4.3. The RSD for each variance component has also been provided in Table 4.3. The RSDs give an indication of the magnitude of the random sampling variability and the magnitude of the random analytical variability.

The anaiytes which have a large (>20\%) sampling variability and a large $(>20 \%)$ analytical variability are listed in Table 4.4 . The concentration of many of these analytes are near the measurement detection limits causing a large variability in their results. These analytes are marked with a $n * 11$ in the table. The analytical measurements are discussed in Urie (1997). Since the anatyses were performed on "slurry" samples, the large sampling variability may be attributed to inhomogeneity in the suspended solids composition and reproducibility of the solids fraction sampled.

\subsection{STATISTICAL RESULTS - Random and Systematic Variabijity}

There are two sources of variability; random variability and systematic variability: Random variability (the chance variability encountered in all measurement work) is the variability that affects each member of a set of data (measurements) in a different manner. For this data set, the random analytical variability is estimated from the duplicate results obtained from each subsample (within-subsample differences) and the random sampling variability is estimated from the between-subsample differences.

Systematic variability (sometimes referred to as accuracy) is a variability that affects all members of a data set (the variability remains constant over a series of replicated measurements). Spike recoveries or reference standard recoveries are used to estimate the systematic analytical variability. associated with analytical procedures for a particular sample matrix. Assigned values of $10 \%, 25 \%, 50 \%, 75 \%$, and $100 \%$ were used as estimates of the systematic analytical variability. It is not possible to estimate the systematic sampling variability.

The total measurement uncertainty (random and systematic variability combined) was estimated using the following equation 


$$
\hat{\sigma}_{\text {total }}=\hat{\sigma}_{\text {rasdom }}^{2}+\hat{\sigma}^{2}{ }_{\text {systematic }}
$$

where $\hat{\sigma}_{\text {random }}$ is the relative standard deviation of the mean provided in Table 4.1 and $\hat{\sigma}_{\text {systematic }}$ is the systematic variability (in terms of RSD).

For the analytes listed in Table 4.1 , the RSD of the mean, $\hat{\sigma}_{\text {total }} / \hat{\mu}$, was determined using each assigned systematic variability estimate (in terms of RSD, $\hat{\sigma}_{\text {systematic }}=10 \%, 25 \%, 50 \%, 75 \%$, and $100 \%$ ). The upper 1 imit of the one-sided $95 \%$ confidence interval on the mean was then calculated for each analyte using the $d f$ associated with the random variability. The upper 1 imit (UL) to a one-sided $95 \%$ confidence interval for the mean is

$$
U L=\hat{\beta}+t_{(d f, 0.05)} \times \hat{\sigma}_{\text {total }} .
$$

In this equation, $t_{(d f, 0,05)}$ is the quantile from Student's $t$ distribution with df degrees of freedom for a one-sided $95 \%$ confidence interval. The upper limit to a one-sided confidence interval on the mean using $\hat{\sigma}_{\text {total }}$ takes into account the random sampling variability, the random analytical variability, and the systematic analytical variability.

The use of "assigned" systematic variability estimates was to determine the effect of the total measurement uncertainty on the ratio UL/EL (UL is the upper limit of the one-sided $95 \% \mathrm{CI}$ on the mean concentration and $\mathrm{EL}$ is the envelope $D$ specification limit). A ratio of less than one indicates that the upper limit to the one-sided $95 \%$ CI on the mean analyte concentration is with in the envelope $D$ specification.

The UL/EL ratios, determined from varying the systematic variability, indicate when the upper limit to the one-sided $95 \%$ CI on the mean analyte concentration might exceed the envelope criterion. For those analyte concentrations which exceed the envelope $D$ specifications (UL/EL $>1$ ), the observed systematic variability is compared to the "assigned" systematic variability. If the observed systematic variability is less than this "assigned" systematic variability, the upper limit to the one-sided $95 \%$ CI on the mean concentration is below the envelope $D$ specifications. The analytes flagged in this assessment have a higher risk with respect to exceeding the envelope criterion. Further discussion of analytes "at risk" is provided in Section 6.0 .

\subsection{COMPARISON OF ANALYTICAL RESULTS TO THE ENVELOPE D SPECIFICATION}

The envelope " $D$ " specification limits are given in terms of $\mathrm{g} / \mathrm{L}$ (at 31 gram waste oxide). The analytes reported with units of $\mu \mathrm{g} / \mathrm{g}$ dried slurry were converted to $\mathrm{g} / \mathrm{L}$ (at $31 \mathrm{gram}$ waste oxide) using equation 4 . The analytes reported with units of $\mu \mathrm{C} i / g$ dried siurry were converted to $\mathrm{Ci} / \mathrm{L}$ (at $3 \mathrm{l} \mathrm{gram}$ waste oxide) using equation 5 . The weight percent dried slurry and the weight percent oxide were obtained from laboratory measurements and are listed in Kuhl-Klinger (1997). 


$$
\begin{aligned}
& \frac{g}{L}=\frac{\mu g_{\text {analyze }}}{g_{\text {dried slurry }}} \times \frac{g}{1000000 \mu g} \times \frac{\frac{14.4 g_{\text {dried slurxy }}}{\text { total } g}}{\frac{11.5 g_{\text {oxide }}}{\text { total } g}} \times \frac{31 g_{\text {oxide }}}{L} \\
& \frac{C i}{L}=\frac{\mu C i_{\text {andiyte }}}{g_{\text {dried slurry }}} \times \frac{C i}{1000000 \mu i} \times \frac{\frac{14.4 g_{\text {dried sluryy }}}{\text { total } g}}{\frac{11.5 g_{\text {oxide }}}{\text { totalg }}} \times \frac{31 g_{\text {oxide }}}{L}
\end{aligned}
$$

Many of the analyte concentrations were determined using two analytical procedures (ICP/AES and ICP/MS) with multiple sample preparations (Acid, FS.Ni, and FS.Zr). The summary statistics were reported for each measurement method and sample preparation in Table 4.1.

The Analytical Summary Report (Urie, 1997) lists the "most reasonable" average per analyte for comparison to the Envelope D specifications. In most cases, the "most reasonable" estimate reflects a specific sample preparation and analysis method. The results are presented in units of $g / L$ and $\mathrm{Ci} / \mathrm{L}$. (at 31 gram waste oxide) for comparison with the envelope specifications. The statistical evaluation of the analytical data selected as "most reasonable" is presented in Tables 6.1 and 6.2. These tables contain (if applicable) the mean analyte concentration $(\hat{\mu})$, the RSD of the mean $\left(\hat{\sigma}_{\bar{\mu}} / \hat{\mu}\right)$, the upper limit to the one-sided $95 \%$ confidence interval on the mean (random variability only), and the ratio of the upper limit to the one-sided $95 \%$ CI on the mean to the envelope "D" specification limit.

The ratio of the upper limit to the one-sided $95 \%$ CI on the mean concentration to the envelope $D$ specification limits provides a method of comparing the analyte concentration to the envelope criterion. A ratio less than 1.0 indicates that the mean analyte concentration is below the envelope criterion. For the analytes which had concentrations reported as "less than" values, the ratio of the analyte to the envelope specification limit was calculated using the upper limit of the less than value listed; a conservative approach.

The upper limits of the $95 \%$ CI for the mean concentration were below the respective envelope "D" maximum specification limits for the majority of the analytes. The Vendor Product analytes for which the UL of the $95 \%$ CI for the mean concentration exceeded the Envelope $D$ specifications are $\mathrm{Na}$, Pt (a trace element) ${ }^{12 \mathrm{~m}_{\mathrm{S}} \mathrm{n} \text {, and }} 233_{\mathrm{U}}$ (a trace radionuclide). Three radionuclides $\left({ }^{110 \mathrm{~m}} \mathrm{Ag}\right.$, ${ }^{242} \mathrm{Pu}$, and ${ }^{119 m} \mathrm{Sn}$ ) exceeded the Envelope $\mathrm{D}$ specifications if the maximum detection limits are used as the results.

The result for ${ }^{243 / 244} \mathrm{Cm}$ exceeds the Envelope $\mathrm{D}$ specification if compared to the ${ }^{243} \mathrm{Cm}$ specification. The result for ${ }^{144} \mathrm{Ce} /{ }^{144} \mathrm{Pr} /{ }^{144 \mathrm{~m}} \mathrm{Pr}$ exceeds the Envelope D specification if compared to the ${ }^{44 m} \mathrm{Pr}$ specification.

These results do not differ from those presented in Urie (1997). 
Five analytes ( $\mathrm{Al}, \mathrm{Fe}, \mathrm{Na}, \mathrm{Ni}$, and $\mathrm{CO}_{3}$ ) have Envelope $\mathrm{D}$ minimum specification limits. Compliance with the minimum specification limits is not addressed in this report.

The comparison of the $95 \%$ UL with the envelope 1 imit provides an indicator of the risk to be assumed by the PHMC in delivering feed that meets the envelope specification. It is not intended by this comparison to imply that the $95 \%$ UL will be the basis for feed composition verification. Current U.S. Department of Energy, Richland Operations Office strategy is to use the mean concentration rather than the $95 \%$ UL for the feed composition verification.

Table 6.3 contains the ratio of the upper Timit to the one-sided $95 \%$ CI on the mean to the envelope specification limit where the total measurement uncertainty $\left(\hat{\sigma}_{\text {total }}\right)$ was used in calculating UL (the upper Timit to the one-sided $95 \%$ CI on the mean). Five ratios (as explained in Section 5.0 ) are presented in Table 6.3 since $\dot{\sigma}_{\text {total }}$ was calculated using the five assigned values for the systematic variability. The five ratios indicate the effect of the total measurement uncertainty on the UL.

Using a total measurement uncertainty of $-100 \%$ (calculated from equation 2 using the random variability listed in Table 4.1 and a systematic variability of $100 \%$ ), the UL is below the envelope D specification for 52 of the 71 comparisons in Table 6.3. The systematic variability at which the remaining 19 comparisons have a ratio greater than one is presented in Table 6.4. This table also includes the observed systematic variability.

The UL based on $\hat{\sigma}_{0}$ (the random sampling and analytical variability) identified $\mathrm{Na}, \mathrm{Pt}$ (a trace etement), ${ }^{221 m} \mathrm{Sn}$, and ${ }^{233} \mathrm{U}$ (a trace radioniclide) as anaiytes exceeding the envelope D specifications. The UL based on $\hat{\sigma}_{\text {total }}$ (the total measurement uncertainty) adds $\mathrm{Ag}$ and $\mathrm{Yb}$ (a trace element) to the list of analytes whose UL exceeds the envelope D specifications. 


\subsection{REFERENCES}

Brooks, K. P., et. al., 1997, Bench-Scale Enhanced S7udge Washing and Gravity Settling of Hanford Tank C-106 Sludge, PNNL-11432, Pacific Northwest National Laboratory, Richland, WA 99352.

Kuhl-Klinger, K.J. and M. K. Urie, 1997, Tank Waste Remediation System Privatization Contractor Sample Analytical Summary Report, Addendum 1 (January 17), Pacific Northwest National Laboratory, Richiand, WA 99352.

Urie, M. W., 1997, Tank Waste Remediation System (TWRS) Privatization Contractor Samples Waste Envelope D Material Tank 241-C-106, HNF-SD-WM-DP-225, Rev. 0, Rust Federal Services of Hanford, Inc., Richland, WA 99352.

Jones, T.E., 1996, "Addendum 1 of the Letter of Instruction (LOI) for TWRS Privatization Contractor Samples Addressing Waste Envelope D Materials, Revision 0.", Pacific Northwest National Laboratory, Richland, WA 99352.

Book, Statistical Sciences, Inc. S-PLUS Reference Manual, Version 3.2, Seattle: StatSci, a division of MathSoft, Inc., 1993.

Book, Snedecor, G. W., and W. G. Cochran, 1980, Statistical Methods, 7th Eơition, Iowa State University Press, Ames, Iowa.

Harville, D. A., 1977, "Maximum Likel ihood Approaches to Variance Component Estimation and to Related Problems," Journal of the American Statistical Association, Vol. 72, pp. 320-340. 


\section{HNF-SD-WM-DP-225, REV. 1}

Tabie 4.1. Tank 241-C-106 Vendor Product Summary Statistics - Random Variability. (page 1 of 9)

\begin{tabular}{|c|c|c|c|c|c|c|c|c|}
\hline Analyte a & Method & Digest & Units $s$ & $\vec{A}$ & & $\begin{array}{c}\operatorname{RSD}(\bar{\mu}) \\
(X)\end{array}$ & $d f$ & $95 \%$ UL \\
\hline Ag & ICP/AES & Acid & $\mu g / 9 d$ & $7.56 \varepsilon+01$ & & 2.69 & 2 & $8.15 E+01$ \\
\hline Ag & ICP/AES & FS.Ni & $\mu g / g d$ & $1.03 E+03$ & & 9.34 & 2 & $1.31 E+03$ \\
\hline Ag & ICP/AES & Fs.Zr & $\mu g / 9 d$ & $3.18 E+03$ & & 8.76 & 2 & $3.99 E+03$ \\
\hline Al & ICP/AES & Acid & $\mu g / g d$ & $4.79 E+04$ & & 1.40 & 2 & $4.99 E+04$ \\
\hline Al & ICP/AES & FS.Ni & $\mu g / g d$ & $5.12 E+04$ & & 2.63 & 2 & $5.51 E+04$ \\
\hline Al & ICP/AES & FS.Zr & $\mu g / g d$ & $4.95 E+04$ & & 2.88 & 2 & $5.37 E+04$ \\
\hline Al & ICP/MS & Acid & $\mu g / g d$ & $3.34 E+04$ & & 2.96 & 2 & $3.63 E+04$ \\
\hline${ }^{249_{\text {Am. aea }}}$ & Rad & FS.Ni & $\mu \mathrm{Ci} / \mathrm{g} d$ & $2.77 E+00$ & & 2.55 & 2 & $2.97 E+00$ \\
\hline $249_{\text {Am.gea }}$ & . Rad & FS.Ni & $\mu C i / g d$ & $3.15 E+00$ & & 9.40 & 2 & $4.01 E+00$ \\
\hline As & ICP/MS & Acid & $\mu g / g d$ & $1.55 E+02$ & & 4.04 & 2 & $1.73 E+02$ \\
\hline As & ICP/MS & FS.Ni & $\mu g / g d$ & $2.86 E+02$ & & 7.38 & 2 & $3.48 \mathrm{E}+02$ \\
\hline As & ICP/MS & $\mathrm{FS} . \mathrm{Zr}$ & $\mu g / 9 d$ & $2.56 E+02$ & & 13.55 & 2 & $3.57 E+02$ \\
\hline B & ICP/AES & Acid & $\mu g / g d$ & $2.25 \varepsilon+02$ & & 17.58 & 2 & . $\quad 3.40 E+02$ \\
\hline B & ICP/AES & FS.Ni & $\mu g / 9 d$ & $3.03 E+02$ & * & 18.37 & 2 & $4.66 E+02$ \\
\hline B & $1 \mathrm{CP} / \mathrm{NS}$ & Acid & $\mu g / g d$ & $1.86 E+02$ & & 21.29 & 2 & $3.02 E+02$ \\
\hline B & $\mathrm{ICP} / \mathrm{MS}$ & FS.Ni & $\mu g / g d$ & $2.29 E+02$ & & 21.41 & 2 & $3.75 \mathrm{E}+02$ \\
\hline $\mathrm{B}$ & ICP/MS & FS.Zr & $\mu g / g d$ & $1.01 E+02$ & & 38.58 & 2 & $2.15 E+02$ \\
\hline $\mathrm{Ba}$ & ICP/AES & Acid & $\mu g / g d$ & $4.15 E+02$ & & 1.17 & 2 & $4.29 E+02$ \\
\hline $\mathrm{Sa}$ & ICP/AES & FS.Ni & $\mu g / g d$ & $4.07 E+02$ & & 2.85 & 2 & $4.40 E+02$ \\
\hline $\mathrm{Ba}$ & ICP/AES & Fs.2r & $\mu g / g d$ & $4.37 E+02$ & & 3.08 & 2 & $4.76 E+02$ \\
\hline $\mathrm{Ba}$ & $1 \mathrm{CP} / \mathrm{MS}$ & Acid & $\mu g / 9 d$ & $3.62 E+02$ & & 1.11 & 2 & $3.73 E+02$ \\
\hline $\mathrm{Ba}$ & $I \mathrm{CP} / \mathrm{MS}$ & $\mathrm{FS.Ni}$ & $\mu g / 9 d$ & $4.12 \mathrm{E}+02$ & & 1.94 & 2 & $4.35 E+02$ \\
\hline $\mathrm{Ba}$ & $I C P / M S$ & FS.Zr & $\mu g / g d$ & $4.27 E+02$ & & 1.29 & 2 & $4.43 \mathrm{E}+02$ \\
\hline $\mathrm{Bi}$ & ICP/AES & Acid & $\mu \mathrm{g} / \mathrm{g} d$ & $4.30 \mathrm{E}+02$ & & 1.18 & 2 & $4.45 E+02$ \\
\hline $\mathrm{Bi}$ & ICP/AES & FS.Ni & $\mu g / g d$ & $5.38 E+02$ & $\star$ & 1.42 & -2 & $5.60 \mathrm{E}+02$ \\
\hline$B i$ & ICP/AES & $\mathrm{FS.2t}$ & $\mu \mathrm{g} / \mathrm{g} \mathrm{d}$ & $5.82 E+02$ & 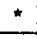 & 4.06 & 2 & $6.50 E+02$ \\
\hline $\mathrm{C}_{2} \mathrm{O}_{4}$ & IC & Water & $\mu g / g d$ & $1.70 \mathrm{E}+05$ & . & 4.24 & 2 & $1.91 E+05$ \\
\hline${ }^{14} \mathrm{C}$ & Rad & FS.Ni & $\mu \mathrm{Ci} / \mathrm{g} d$ & $2.87 E-03$ & & 78.53 & 2 & $9.45 E-03$ \\
\hline $\mathrm{CN}^{-}$ & wtChen & Direet & $\mu g / g d$ & $9.50 \mathrm{E}+00$ & & 5.59 & 2 & $1.11 E+01$ \\
\hline $\mathrm{Ca}$ & ICP/AES & Acid & $\mu g / g d$ & $3.84 E+03$ & & 9.22 & 2 & $3.98 E+03$ \\
\hline $\mathrm{Ca}$ & ICP/AES & FS.Ni & $\mu g / 9 \mathrm{~d}$ & $6.69 E+03$ & & 4.37 & 2 & $7.54 E+03$ \\
\hline $\mathrm{Ca}$ & ICP/AES & FS.Zr & $\mu g / 9$ o & $9.85 E+03$ & & 4.26 & 2 & $1.11 E+04$ \\
\hline
\end{tabular}

a = Table does not include analytes where more than $50 \%$ of the data are "less than" values.

$\$=\mu g / g d$ is $\mu \mathrm{g} / \mathrm{g}$ dried slurry: $\mu \mathrm{Ci} / \mathrm{g} d$ is $\mu \mathrm{Ci} / \mathrm{g}$ dried slurry.

* At least one analytical result was above the id but below the EQL. 
HNF-SD-WM-DP-225, REV. 1

Table 4.1. Tank 241-C-106 Vendor Product

Sumary Statistics - Random Variability. (page 2 of 9)

\begin{tabular}{|c|c|c|c|c|c|c|c|c|}
\hline Analyte a & Method & Digest & Units $s$ & $\dot{A}$ & & $\begin{array}{c}\operatorname{RSD}(\beta) \\
(\%)\end{array}$ & $d f$ & $95 \%$ UL \\
\hline $\mathrm{Ca}$ & ICP/MS & Acid & $\mu g / g d$ & $9.57 E+03$ & & 4.79 & 2 & $1.09 E+04$ \\
\hline $\mathrm{Ca}$ & ICP/MS & FS.Ni & $\mu g / g d$ & $1.72 E+03$ & & 7.78 & 2 & $2.11 E+03$ \\
\hline $\mathrm{Ca}$ & ICP /MS & FS.Zr & $\mu g / g d$ & $3.77 E+03$ & & 5.02 & 2 & $4.32 E+03$ \\
\hline cd & ICP/AES & Acid & $\mu g / g d$ & $7.30 E+01$ & & 7.35 & 2 & $8.86 E+01$ \\
\hline $\mathrm{Cd}$ & ICP/AES & ES.Ni & $\mu g / g d$ & $8.10 E+01$ & $\star$ & 1.71 & 2 & $8.51 E+01$ \\
\hline $\mathrm{Cd}$ & ICP/AES & fS.Zr & $49 / g d$ & $8.08 E+01$ & $\star$ & 2.32 & 2 & $8.62 E+01$ \\
\hline cd & ICP/MS & Acid & $\mu g / g d$ & $5.81 E+01$ & & 1.22 & 2 & $6.01 E+01$ \\
\hline $\mathrm{cd}$ & ICP /MS & ES.Ni & $\mu \mathrm{g} / \mathrm{g} d$ & $9.05 E+01$ & & 5.13 & 2 & $1.04 E+02$ \\
\hline cd & ICP/MS & FS.Zr & $\mu g / g d$ & $7.41 E+01$ & & 6.60 & 2 & $8.83 E+01$ \\
\hline $\mathrm{Ce}$ & ICP/AES & Acid & $\mu \mathrm{g} / \mathrm{g} d$ & $2.99 \xi+02$ & $\star$ & 1.75 & 2 & $3.14 E+02$ \\
\hline $\mathrm{Ce}$ & ICP/MS & Acid & $\mu g / g d$ & $1.59 E+02$ & & 1.68 & 2 & $1.67 E+02$ \\
\hline $\mathrm{Ce}$ & ICP/MS & FS.Ni & $\mu g / g d$ & $1.60 E+02$ & & 8.96 & 2 & $2.01 E+02$ \\
\hline $\mathrm{Ce}$ & ICP/NS & FS.2r & $\mu g / g d$ & $2.02 E+02$ & & 6.52 & 2 & $2.41 \varepsilon+02$ \\
\hline $\mathrm{Cl}^{-}$ & IC & Water & $\mu g / g d$ & $1.26 E+02$ & & 8.49 & 2 & $1.57 E+02$ \\
\hline${ }^{242} \mathrm{Cm} / 242 \mathrm{Am}^{242 \mathrm{~m}_{\mathrm{Am}}}$ & Rad & FS.Ni & $\mu \mathrm{Ci} / \mathrm{s} d$ & $8.07 \mathrm{E}-03$ & & 10.16 & 2 & $1.05 E-02$ \\
\hline $243 / 244 \mathrm{~cm}$ & Rad & FS.Ni & $\mu \mathrm{Ci} / \mathrm{g} \mathrm{d}$ & $9.86 \mathrm{E}-02$ & & 12.97 & 2 & $1.36 \mathrm{E}-01$ \\
\hline $\mathrm{Co}$ & ICPIAES & Acid & $\mu g / 9 d$ & $2.81 E+01$ & \pm & 1.06 & 2 & $2.90 \mathrm{E}+01$ \\
\hline $\mathrm{Co}$ & ICP/MS & Acid & $\mu g / g d$ & $1.57 E+01$ & & 1.23 & 2 & $1.62 \mathrm{E}+01$ \\
\hline $\mathrm{Co}$ & ICP/MS & FS.Ni & $\mu g / g d$ & $2.62 E+01$ & & 12.15 & 2 & $3.55 \varepsilon+01$ \\
\hline Co & ICP/MS & FS.Zr & $\mu g / g d$ & $2.18 E+01$ & & 5.95 & 2 & $2.56 E+01$ \\
\hline${ }^{60} \mathrm{CO}$ & Rad & FS.Ni & $\mu \mathrm{Ci} / \mathrm{g} \mathrm{d}$ & 4.91E-01 & & 3.11 & 2 & $5.36 E-01$ \\
\hline & ICP/AES & Acid & $\mu g / g \mathrm{~d}$ & $5.33 E+02$ & & 0.98 & 2 & $5.48 E+02$ \\
\hline $\mathrm{Cr}$ & ICP/AES & FS.Ni & $\mu g / g d$ & $8.02 E+02$ & & 2.70 & 2 & $8.66 \Xi+02$ \\
\hline $\mathrm{Cr}$ & ICP/AES & FS.Zr & $\mu g / g d$ & $7.00 \mathrm{E}+02$ & & 4.02 & 2 & $7.82 E+02$ \\
\hline $\mathrm{Cr}$ & $1 \mathrm{CP} / \mathrm{MS}$ & Acid & $\mu g / g d$ & $4.26 \mathrm{E}+02$ & & 1.05 & 2 & $4: 39 E+02$ \\
\hline $\mathrm{Cr}$ & ICP/MS & FS.Ni & $\mu g / g d$ & $7.74 \varepsilon+02$ & & 6.05 & 2 & $9.11 \mathrm{E}+02$ \\
\hline $\mathrm{Cr}$ & $1 \mathrm{CP} / \mathrm{MS}$ & $\mathrm{Fs.2r}$ & $\mu g / g d$ & $7.39 \mathrm{E}+02$ & & 3.93 & 2 & $8.24 E+02$ \\
\hline${ }^{133} \mathrm{cs}$ & ICP/MS & Acid & $\mu \mathrm{g} / \mathrm{g} d$ & $1.04 E+01$ & & 3.85 & 2 & $1.96 E+09$ \\
\hline${ }^{133} \mathrm{cs}$ & ICP/MS & FS.Ni & $\mu g / g d$ & $1.80 E+01$ & & 9.06 & 2 & $2.27 E+01$ \\
\hline${ }^{133} \mathrm{cs}$ & I CP/MS & FS.Zr & $\mu g / g d$ & $7.18 \mathrm{E}+00$ & & 12.39 & 2 & $9.78 E+00$ \\
\hline${ }^{133} \mathrm{cs}$ & $\operatorname{Rad}(M S)$ & FS.Ni & $\mu g / g d$ & $1.57 \mathrm{E}+01$ & & 4.71 & 2 & $1.78 \mathrm{E}+01$ \\
\hline${ }^{135} \mathrm{cs}$ & Rad & ES.Ni & $\mu \mathrm{Ci} / \mathrm{g} d$ & $2.86 \mathrm{E}-03$ & & 3.15 & 2 & $3.13 E-03$ \\
\hline${ }^{137} \mathrm{Cs} / \mathrm{Ba}$ & Rad & IS.Ni & $\mu C \mathrm{i} / \mathrm{g} \mathrm{d}$ & $4.96 E+02$ & & 3.29 & 2 & $5.43 E+02$ \\
\hline
\end{tabular}

$a=$ Table does not include analytes where more than $50 \%$ of the data are "less than" values.

$\$=\mu \mathrm{g} / \mathrm{g} \mathrm{d}$ is $\mu \mathrm{g} / \mathrm{g}$ dried sturry; $\mu \mathrm{Ci} / \mathrm{g}$ d is $\mu \mathrm{Ci} / 9 \mathrm{dried}$ slurry.

* At least one analytical result was above the IOL but below the EOL. 
HNF-SD-WM-DP-225, REV. 1

Table 4.1. Tank 241-C-106 Vendor Product

Summary Statistics - Random Variability. (page 3 of 9)

\begin{tabular}{|c|c|c|c|c|c|c|c|c|}
\hline Analyte a & Method & Digest & Units s & $\ddot{\mu}$ & & $\begin{array}{c}\operatorname{RSD}(\hat{\mu}) \\
(\%)\end{array}$ & $d f$ & $95 \%$ UL \\
\hline Cu & ICP/AES & Acid & $\mu g / g d$ & $7.75 E+01$ & & 0.99 & 2 & $7.97 \mathrm{E}+01$ \\
\hline Cu & ICP/AES & FS.Ni & $\mu g / g d$ & $1.09 E+02$ & $*$ & 1.78 & 2 & $1.95 E+02$ \\
\hline $\mathrm{Cu}$ & ICP/AES & $\mathrm{FS} . \mathrm{Zr}$ & $\mu g / g d$ & $1.09 \mathrm{E}+02$ & $\star$ & 6.83 & 2 & $1.30 E+02$ \\
\hline $\mathrm{Cu}$ & ICP/MS & Acid & $\mu g / g d$ & $1.10 E+02$ & & 0.75 & 2 & $1.12 E+02$ \\
\hline $\mathrm{Cu}$ & ICP/MS & $\mathrm{Fs} .2 \mathrm{r}$ & $\mu g / g d$ & $1.56 E+02$ & & 4.22 & 2 & $1.76 \mathrm{E}+02$ \\
\hline CU.H & ICP/MS & FS.Ni & $\mu g / g d$ & $1.13 E+01$ & & 71.06 & 2 & $3.48 \mathrm{E}+01$ \\
\hline CU.HO & ICP/MS & FS.Ni & $\mu g / g \quad d$ & $1.89 E+01$ & & 43.91 & 1 & $7.13 E+01$ \\
\hline Dy & ICP/MS & Acid & $\mu g / g d$ & $1.47 E+00$ & & 2.56 & 2 & $1.58 \mathrm{E}+00$ \\
\hline Dy & ICP/MS & F5.Ni & $\mu g / s d$ & $2.02 E+01$ & & 17.37 & 2 & $3.04 E+01$ \\
\hline Dy & ICP/MS & FS.Zr & $\mu g / g d$ & $3.60 \mathrm{E}+00$ & & 32.62 & 2 & $7.04 E+00$ \\
\hline Er & ICP/MS & Acid & $\mu g / g d$ & $7.67 \mathrm{E}-01$ & & 2.75 & 2 & $8.28 \mathrm{E}-01$ \\
\hline$E_{r}$ & $I C P / M S$ & FS.Ni & $\mu g / g d$ & $1.31 E+01$ & & 14.64 & 2 & $1.87 \mathrm{E}+01$ \\
\hline Eu & ICP/MS & Rcid & $\mu g / 9 d$ & $6.46 E+00$ & & 1.49 & 2 & $6.74 E+00$ \\
\hline Eu & ICP/MS & FS.Ni & $\mu g / g d$ & $7.87 E+00$ & & 30.72 . & 2 & $1.49 E+01$ \\
\hline Eu & ICP/MS & fs.2r & $\mu g / g d$ & $7.84 E+00$ & & 6.54 & 2 & $9.34 E+00$ \\
\hline $154 \mathrm{EU}$ & Rad & FS.Ni & $\mu \mathrm{Ci} / \mathrm{g} d$ & $3.79 \mathrm{E}+00$ & & 1.76 & 2 & $3.99 \bar{E}+00$ \\
\hline${ }^{155} \mathrm{Eu}$ & Rad & $\mathrm{FS.Mi}$ & $\mu \mathrm{Ci} / \mathrm{g} d$ & $3.13 E+00$ & & 4.02 & 2 & $3.50 \varepsilon+00$ \\
\hline $\mathrm{Fe}$ & ICP/AES & Acid & $\mu g / g d$ & $1.37 E+05$ & & 1.06 & 2 & $1.41 E+05$ \\
\hline $\mathrm{Fe}$ & ICP/AES & ES.Ni & $\mu g / g d$ & $1.41 E+05$ & & 3.29 & 2 & $1.54 E+05$ \\
\hline $\mathrm{Fe}$ & ICP/AES & Fs.zr & $\mu g / g d$ & $1.31 E+05$ & & 3.65 & 2 & $1,44 E+05$ \\
\hline $\mathrm{Fe}$ & [CP/MS & Acid & $\mu g / g d$ & $1.10 E+05$ & & 3.24 & 2 & $1.20 E+05$ \\
\hline${ }^{55} \mathrm{Fe}$ & Rad & FS.Ni & $\mu \mathrm{Ci} / \mathrm{gd}$ & $1.53 E-01$ & & 2.62 & 2 & $1.65 E-01$ \\
\hline Gd & ICP/MS & Acid & $\mu g / g d$ & $1.36 E+01$ & & 3.49 & 2 & $1.50 E+01$ \\
\hline Gd & ICP/MS & FS.Ni & $\mu g / g$ of & $2.50 E+01$ & & 11.29 & 2 & $3.32 E+01$ \\
\hline Gd & ICP/MS & FS.2r & $\mu \mathrm{g} / \mathrm{g} \mathrm{d}$ & $1.21 \varepsilon+01$ & & 17.49 & .2 & 1. $83 E+01$ \\
\hline $\mathrm{Hg}$ & CVAA & Direct & $\mu g / g d$ & $3.43 E+02$ & & 1.94 & 2 & $3.63 E+02$ \\
\hline Ho & ICP/MS & Acid & $\mu \mathrm{g} / \mathrm{g} \mathrm{d}$ & $1.42 \varepsilon-01$ & & 11.00 & 2 & $1.87 \varepsilon-01$ \\
\hline Ho & ICP/NS & FS.Ni & $\mu \mathrm{g} / \mathrm{g} \mathrm{d}$ & $6.07 E+00$ & & 14.78 & 2 & $8.69 E+00$ \\
\hline $129_{1 . W}$ & ICP/MS & Acid & $\mu g / g d$ & $1.98 E+00$ & & 11.16 & 2 & $1.56 E+00$ \\
\hline $129_{1 . W}$ & ICP/MS & Acid & $\mu \mathrm{ci} / \mathrm{g} d$ & $2.18 E-04$ & & 7.28 & 2 & $2.65 E-04$ \\
\hline 129 1. Wo & ICP/MS & Acid & $\mu g / g d$ & $1.25 E+00$ & & 10.80 & 2 & $1.64 E+00$ \\
\hline${ }^{129}$ I. Wo & ICP/MS & Acid & $\mu C i / g d$ & $2.37 E-04$ & & 9.24 & 2 & $3.00 E \cdot 04$ \\
\hline$k$ & ICP/AES & Fs.zr & $\mu g / g d$ & $7.59 \mathrm{E}+03$ & * & 8.91 & 2 & $9.56 E+03$ \\
\hline
\end{tabular}

$a=$ Table does not include analytes where more than $50 \%$ of the data are "less than" values.

$\$=\mu \mathrm{g} / \mathrm{g} d$ is $\mu \mathrm{g} / \mathrm{g}$ dried $\mathrm{slurry} / \mu \mathrm{Ci} / \mathrm{g} d$ is $\mu \mathrm{Ci} / \mathrm{g}$ dried slurry.

* = Ar least one arialytical result was above the $10 \mathrm{~L}$ but below the EOL. 
Table 4.1. Tank 241-C-106 Vendor Product Summary Statistics - Random Variability. (page 4 of 9)

\begin{tabular}{|c|c|c|c|c|c|c|c|c|}
\hline Analyte a & Method & Digest & Units $\mathbf{s}$ & $\dot{\mu}$ & & $\begin{array}{c}\operatorname{RSD}(\dot{\mu}) \\
(\%)\end{array}$ & $d f$ & $95 \%$ UL \\
\hline La & ICP/AES & Acid & $\mu 9 / 9$ d & $9.61 E+01$ & 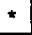 & 1.37 & 2 & $8.91 E+01$ \\
\hline La & ICP/AES & FS.Ni & $\mu g / g d$ & $1.19 \mathrm{E}+02$ & 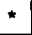 & 3.45 & 2 & $1.30 E+02$ \\
\hline La & ICP/AES & Fs.zr & $\mu g / g d$ & $1.64 E+02$ & 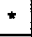 & 6.48 & 2 & $1.95 E+02$ \\
\hline La & ICP/NS & Acid & $\mu g / g d$ & $8.50 E+01$ & & 1.68 & 2 & $8.91 E+01$ \\
\hline La & ICP/NS & FS.Ni & $\mu g / g d$ & $8.06 E+01$ & & 7.53 & 2 & $9.83 \mathrm{E}+01$ \\
\hline $\mathrm{La}$ & ICP/MS & FS.Zr & $\mu g / g d$ & $9.22 E+09$ & & 9.41 & 2 & $1.18 E+02$ \\
\hline Li & ICP/AES & Acid & $\mu g / g d$ & $4.59 E+01$ & & 42.50 & 2 & $1.03 E+02$ \\
\hline Li & ICP/AES & $\mathrm{FS} .2 \mathrm{r}$ & $\mu g / g d$ & $9.39 E+01$ & * & 14.81 & 2 & $1.34 E+02$ \\
\hline $\mathrm{Mg}$ & ICP/AES & Acid & $\mu g / g d$ & $7.54 E+02$ & & 1.53 & 2 & $7.88 E+02$ \\
\hline $\mathrm{Mg}$ & ICP/AES & FS.Ni & $\mu g / 9 d$ & $1.36 E+03$ & $\star$ & 6.19 & 2 & $1.60 E+03$ \\
\hline $\mathrm{Mg}$ & ICP/AES & FS.Zr & $\mu g / g d$ & $1.31 E+03$ & $\star$ & 3.88 & 2 & $1.45 E+03$ \\
\hline $\mathrm{Mg}$ & $I C P / H S$ & Acid & $\mu g / g d$ & $4.45 E+02$ & & 1.96 & 2 & $4.71 E+02$ \\
\hline $\mathrm{Mg}$ & ICP/MS & FS.Ni & $\mathrm{Hg} / \mathrm{g} \mathrm{d}$ & $1.22 E+03$ & & 7.52 & 2 & $1.49 E+03$ \\
\hline $\mathrm{Mg}$ & ICP/MS & FS.Zr & $\mu g / g d$ & $1.26 E+03$ & & 3.35 & 2 & $1.52 E+03$ \\
\hline$M n$ & ICP/AES & Acid & $\mu 9 / 9 d$ & $2.60 \mathrm{E}+03$ & & 0.95 & 2 & $2.67 E+03$ \\
\hline Mn & ICP/AES & FS.Ni & $\mu g / g d$ & $3.14 E+03$ & & 2.34 & 2 & $3.35 E+03$ \\
\hline Mn & ICP/AES & FS.2r & $\mu g / g d$ & $2.59 E+03$ & & 3.58 & 2 & $2.86 E+03$ \\
\hline$M n$ & I CP/HS & Acid & $\mu g / g d$ & $2.04 E+03$ & & 2.29 & 2 & $2.18 E+03$ \\
\hline$M_{n}$ & $I \mathrm{CP} / \mathrm{MS}$ & FS.Ni & $\mu g / g d$ & $2.81 E+03$ & & 4.03 & 2 & $3.14 E+03$ \\
\hline $\mathrm{Mn}$ & $1 \mathrm{CP} / \mathrm{MS}$ & FS.2r & $\mu g / g d$ & $2.56 \mathrm{E}+03$ & & 2.77 & 2 & $2.77 E+03$ \\
\hline Ho & ICP/AES & Acid & $\mu g / g d$ & $2.67 E+01$ & $\star$ & 0.90 & 2 & $2.74 E+01$ \\
\hline Mo & $1 \mathrm{CP} / \mathrm{MS}$ & Acid & $\mu g / g d$ & $9.60 E+00$ & & 2.59 & 2 & $1.03 E+01$ \\
\hline Ho & $1 \mathrm{CP} / \mathrm{MS}$ & FS.Ni & $\mu g / g d$ & $5.70 E+01$ & & 13.98 & 2 & $8.02 E+01$ \\
\hline Mo & ICP/NS & FS.Zr & $\mu g / g d$ & $2.79 E+01$ & & 9.95 & 2 & $3.60 E+01$ \\
\hline $\mathrm{NH}_{3}-\mathrm{N}$ & wtchem & Water & $\mu g / g d$ & $1.75 E+01$ & & 20.07 & 2 & $2.78 E+01$ \\
\hline $\mathrm{NO}_{2}^{-}$ & IC & Water & $\mu g / g d$ & $2.07 E+03$ & & 10.20 & 2 & $2.68 E+03$ \\
\hline $\mathrm{NO}_{3}^{-}$ & IC & Water & $\mu g / g d$ & $8.86 E+02$ & & 30.56 & 2 & $1.68 \mathrm{E}+03$ \\
\hline $\mathrm{Na}$ & ICP/AES & Acid & $\mu g / g d$ & $1.62 E+05$ & & 2.93 & 2 & $1.76 E+05$ \\
\hline $\mathrm{Na}$ & ICP/AES & FS.Ni & $\mu g / g d$ & $1.42 E+05$ & & 3.88 & 2 & $1.58 \mathrm{E}+05$ \\
\hline $\mathrm{Na}$ & IEP/MS & Acid & $\mu g / 9 d$ & $1.82 E+05$ & & 6.11 & 2 & $2.14 E+05$ \\
\hline $93 \mathrm{~m}_{\mathrm{Nb}}$ & Rad & FS.Ni & $\mu C i / g d$ & $2.37 \mathrm{E}-01$ & & 30.19 & 2 & $4.47 E-01$ \\
\hline Nd & ICP/AES & Acid & $\mu \mathrm{g} / \mathrm{g} d$ & $2.82 E+02$ & & 1.19 & 2 & $2.91 \mathrm{E}+02$ \\
\hline $\mathrm{Nd}$ & ICP/AES & $\mathrm{FS.Ni}$ & $\mathrm{Hg} / \mathrm{g} \mathrm{d}$ & $3.79 E+02$ & 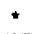 & 4.73 & 2 & $4.31 E+02$ \\
\hline
\end{tabular}

$a=$ Table does not include analytes where more than $50 \%$ of the data are "less than" values.

$\$=\mu g / g d$ is $\mu g / g$ dried slurry; $\mu \mathrm{Ci} / g$ o is $\mu \mathrm{Ci} / \mathrm{g}$ dried slurry.

* = At least one analytical result was above the IDL but below the EOL. 
Table 4.1. Tank 241-C-106 Vendor Product

Summary Statistics - Random Variability. (page 5 of 9)

\begin{tabular}{|c|c|c|c|c|c|c|c|c|}
\hline Analyte a & Method & Digest & Units & $\dot{\mu}$ & & $\begin{array}{c}R S O(\not i) \\
(\%)\end{array}$ & $d f$ & $95 \%$ UL \\
\hline $\mathrm{Nd}$ & ICP/AES & $\mathrm{FS} . \mathrm{Zr}$ & $\mu g / g d$ & $5.17 E+02$ & $\star$ & 4.69 & 2 & $5.87 E+02$ \\
\hline Nd & ICP/MS & Acid & $\mu g / g d$ & $1.93 E+02$ & & 1.06 & 2 & $1.99 E+02$ \\
\hline Nd & ICP/MS & FS.Ni & $\mu g / g d$ & $2.33 E+02$ & & 8.00 & 2 & $2.87 E+02$ \\
\hline Nd & ICP/MS & $\mathrm{FS} .2 \mathrm{r}$ & $\mu g / g d$ & $2.24 E+02$ & & 3.99 & 2 & $2.50 E+02$ \\
\hline $\mathrm{Ni}$ & ICP/AES & Acid & $\mu g / g d$ & $1.13 E+03$ & & 1.10 & 2 & 1. $17 E+03$ \\
\hline $\mathrm{Ni}$ & ICP/AES & Fs.2r & $\mu g / 9 \mathrm{~d}$ & $1.66 E+03$ & & 4.53 & 2 & $1.88 \mathrm{E}+03$ \\
\hline $\mathrm{Ni}$ & ICP/MS & Acid & $\mu g / g d$ & $9.49 E+02$ & & 2.42 & 2 & $1.02 E+03$ \\
\hline $\mathrm{Ki}$ & ICP/MS & FS.2r & $\mu g / g d$ & $1.61 E+03$ & & 2.35 & 2 & $1.72 \mathrm{E}+03$ \\
\hline $59 \mathrm{Ni.H}$ & Rad & FS.Zr & $\mu C i / g d$ & $2.20 E-02$ & & 10.23 & 2 & $2.86 \mathrm{E}-02$ \\
\hline $59 \mathrm{Mi.HO}$ & Rad & FS.Zr & $\mu \mathrm{Ci} / \mathrm{gd}$ & $1.97 \mathrm{E}-02$ & & 14.16 & 2 & $2.79 \mathrm{E}-02$ \\
\hline${ }^{63} \mathrm{Ni}$ & Rad & FS.Zr & $\mu C i / g d$ & $2.40 E+00$ & & 2.39 & 2 & $2.57 E+00$ \\
\hline $237_{\mathrm{Kp}}$ & ICP/MS & Acid & $\mathrm{Hg} / \mathrm{gd}$ & $4.48 E+00$ & & 1.70 & 2 & $4.70 E+00$ \\
\hline $237_{\mathrm{NP}}$ & $I C P / M S$ & Acid & $\mu \mathrm{Ci} / 9 \mathrm{~d}$ & $3.16 E-03$ & & 1.70 & 2 & $3.32 \varepsilon-03$ \\
\hline $237_{\mathrm{NP}}$ & ICP/MS & $\mathrm{FS.Zr}$ & $\mu g / g d$ & $5.57 E+00$ & & 9.49 & 2 & $7.11 E+00$ \\
\hline $237_{\mathrm{NP}}$ & $\mathrm{ICP} / \mathrm{MS}$ & FS.2r & $\mu \mathrm{Ci} / \mathrm{g} d$ & $3.92 E-03$ & & 9.49 & 2 & $5.01 \mathrm{E}-03$ \\
\hline$P$ & ICP/AES & Acid & $\mu g / g d$ & $1.34 E+03$ & & 2.94 & 2 & $1.46 \mathrm{E}+03$ \\
\hline$p$ & ICP/AES & ES.Ni & $\mu g / g d$ & $3.33 E+03$ & & 4.58 & 2 & $3.77 E+03$ \\
\hline$P$ & ICP/AES & $\mathrm{FS.2r}$ & $\mu g / g d$ & $3.84 E+03$ & & 10.59 & 2 & $5.02 E+03$ \\
\hline $\mathrm{PO}_{4}^{3-}$ & IC & Hater & $\mu g / g d$ & $1.60 E+02$ & & 8.94 & 2 & $2.02 E+02$ \\
\hline $\mathrm{Pb}$ & ICP/AES & Acid & $\mu g / g d$ & $3.43 \varepsilon+03$ & & 1.11 & 2 & $3.54 E+03$ \\
\hline $\mathrm{Pb}$ & ICP/AES & FS.Ni & $\mu g / g d$ & $2.95 E+03$ & & 8.53 & 2 & $3.69 \mathrm{E}+03$ \\
\hline $\mathrm{Pb}$ & ICP/AES & F5.2r & $\mu g / g d$ & $3.53 E+03$ & & 3.49 & 2 & $3.89 E+03$ \\
\hline $\mathrm{Pb}$ & ICP/MS & Acid & $\mu g / g d$ & $2.85 E+03$ & & 3.52 & 2 & $3.15 E+03$ \\
\hline $\mathrm{Pb}$ & ICP/MS & FS.2r & $\mu g / g d$ & $2.55 E+03$ & & 1.89 & 2 & $2.69 E+03$ \\
\hline $\mathrm{Pd}$ & ICP/MS & Acid & $\mu g / g d$ & $9.99 E+00$ & & 2.69 & 2 & $1: 08 E+01$ \\
\hline $\mathrm{Pd}$ & ICP/MS & FS.Ni & $\mu g / g d$ & $2.35 E+01$ & & 22.75 & 2 & $3.91 E+01$ \\
\hline Pd & ICP/MS & $\mathrm{fS.Zr}$ & $\mu g / g d$ & $2.74 E+01$ & & 7.63 & 2 & $3.35 E+01$ \\
\hline $107_{\text {Pd.w }}$ & ICP/MS & Acid & $\mu g / g d$ & $1.35 E+00$ & & 61.12 & 2 & $3.76 E+00$ \\
\hline${ }^{107}$ Pd.W & $I C P / M S$ & Acid & $\mu c i / g d$ & $7.18 \mathrm{E}-04$ & & 58.24 & 2 & $1.94 E-03$ \\
\hline${ }^{107}$ Pd.wo & ICP/MS & Acid & $\mu g / 9$ o & $2.20 E+00$ & & 63.85 & 1 & $1.11 E+01$ \\
\hline 107 Pd.wo & ICP/MS & Acid & $H C i / g d$ & $1.14 E-03$ & & 64.59 & 1 & 5.77E-03 \\
\hline
\end{tabular}

$\mathrm{a}=$ Table does not include analytes where more than $50 \%$ of the data are "less than" values.

$\$=\mu \mathrm{g} / \mathrm{g} d$ is $\mu \mathrm{g} / \mathrm{g}$ dried slurry; $\mu \mathrm{Ci} / \mathrm{g}$ o is $\mu \mathrm{Ci} / \mathrm{g}$ dried slurry.

- At least one analytical result was above the IDL but below the EOL. 
Table 4.1. Tank 241-C-106 Vendor Product

Summary Statistics - Random Variability. (page 6 of 9 )

\begin{tabular}{|c|c|c|c|c|c|c|c|}
\hline Analyte a & Method & Digest & Units 5 & $\ddot{j}$ & $\begin{array}{c}\operatorname{RSD}(\bar{\mu}) \\
(\%)\end{array}$ & $d f$ & $95 \%$ UL \\
\hline $\mathrm{Pr}$ & ICP/MS & Acid & $\mu g / g d$ & $7.05 E+01$ & 1.33 & 2 & $7.32 E+01$ \\
\hline $\mathrm{Pr}$ & $1 \mathrm{CP} / \mathrm{MS}$ & FS.Ni & $\mu g / 9 d$ & $7.71 \mathrm{E}+01$ & 5.62 & 2 & $8.97 E+01$ \\
\hline $\mathrm{Pr}$ & $1 \mathrm{CP} / \mathrm{MS}$ & FS.Zr & $\mu g / g d$ & $8.41 E+01$ & 3.73 & 2 & $9.32 E+01$ \\
\hline$P t$ & $1 \mathrm{CP} / \mathrm{MS}$ & Acid & $\mu g / g d$ & $2.33 \mathrm{E}-01$ & 18.07 & 2 & $3.56 \mathrm{E}-01$ \\
\hline$P t$ & ICP/MS & FS.Ni & $\mathrm{\mu g} / \mathrm{g} d$ & $3.07 E+01$ & 16.03 & 2 & $4.51 E+01$ \\
\hline $\mathrm{Pt}$ & ICP/MS & FS.2r & $\mu g / g d$ & $5.19 \mathrm{E}+00$ & 13.45 & 2 & $7.23 E+00$ \\
\hline${ }^{238} \mathrm{Pu}$ & Rad & FS.Ni & $\mu \mathrm{Ci} / \mathrm{g} \mathrm{d}$ & $6.31 E-01$ & 2.43 & 2 & $6.76 E-01$ \\
\hline${ }^{239} \mathrm{Pu}$ & $I C P / H S$ & Acid & $\mu g / g d$ & $7.34 E+00$ & 3.21 & 2 & $8.02 E+00$ \\
\hline $239 \mathrm{Pu}$ & ICP/MS & Acid & $\mu \mathrm{Ci} / \mathrm{g} \mathrm{O}^{\circ}$ & 4.55E-01 & 3.21 & 2 & $4.97 E-01$ \\
\hline${ }^{239} \mathrm{Pu}$ & ICP/MS & FS.2r & $\mu g / g d$ & $2.46 E+01$ & 3.43 & 2 & $2.70 E+01$ \\
\hline${ }^{239} \mathrm{Pu}$ & ICP/MS & FS.Zr & $\mu \mathrm{Ci} / \mathrm{g} d$ & $1.52 E+00$ & 3.38 & 2 & $1.67 \mathrm{E}+00$ \\
\hline $239 / 240_{\mathrm{Pu}}$ & Rad & FS.Ni & $\mu \mathrm{Ci} / \mathrm{g} d$ & $2.99 E+00$ & 2.60 & 2 & $3.21 E+00$ \\
\hline${ }^{260} \mathrm{Pu}$ & ICP/MS & Acid & $\mu g / s d$ & $5.58 \mathrm{E}-01$ & 4.18 & 2 & $6.26 E-01$ \\
\hline $240_{\mathrm{Pu}}$ & $1 \mathrm{CP} / \mathrm{MS}^{\circ}$ & Acid & $\mu C i / g d$ & $1.25 \mathrm{E}-01$ & .4 .62 & 2 & $1.42 E-01$ \\
\hline${ }^{241} \mathrm{Pu}$ & Rad & $\mathrm{FS.Ni}$ & $\mu C i / g d$ & $6.96 \mathrm{E}+00$ & 2.43 & 2 & $7.45 E+00$ \\
\hline${ }^{242}$ PU.W & ICP/MS & Acid & $\mu \mathrm{Ci} / \mathrm{g} d$ & $5.33 E-05$ & 25.00 & 2 & $9.23 E-05$ \\
\hline $\mathrm{Rb}$ & ICP/MS & Acid & $\mu g / g d$ & $1.11 E+00$ & 4.84 & 2 & $1.26 E+00$ \\
\hline $\mathrm{Rb}$ & ICP/MS & fs.zr & $\mu g / 9 d$ & $7.68 E+00$ & 18.38 & 2 & $1.18 E+01$ \\
\hline $\mathrm{Re}$ & ICP /MS & Acid & $\mu g / g d$ & $7.83 \mathrm{E} \cdot 02$ & 17.26 & 2 & $1.18 E-01$ \\
\hline Re & ICP/MS & FS.Ni & $\mu g / g d$ & $6.61 E+00$ & 15.76 & 2 & $9.66 \mathrm{E}+00$ \\
\hline $\mathrm{Rh}$ & ICP/MS & Acid & $\mu g / g d$ & $1.05 E+02$ & 1.93 & 2 & $1.11 E+02$ \\
\hline Rh & ICP/MS & FS.Zr & $\mu g / g d$ & $1.44 E+02$ & 5.44 & 2 & $1.67 E+02$ \\
\hline Rh.w & ICP/MS & FS.Ni & $\mu g / g d$ & $1.90 \mathrm{E}+01$ & 81.67 & 2 & $6.43 \mathrm{E}+01$ \\
\hline Rh.wo & ICP/MS & FS.Ni & $\mu g / g d$ & $3.70 E+01$ & 64.11 & 1. & $1.87 E+02$ \\
\hline Ru & ICP/MS & FS.Ni & $\mu g / g d$ & $9.57 \mathrm{E}+02$ & 3.88 & 2 & $1.07 E+02$ \\
\hline $\mathrm{SO}_{4}{ }^{2-}$ & IC & Hater & $\mu g / g d$ & $4.70 E+02$ & 8.85 & 2 & $5.92 E+02$ \\
\hline Sb.H & ICP/NS & Acid & $\mu g / g d$ & $3.77 \mathrm{E}-01$ & 47.84 & 2 & $9.03 E-01$ \\
\hline Sb.wo & $1 \mathrm{CP} / \mathrm{MS}$ & Acid & $\mu g / g d$ & $7.88 E-01$ & 25.35 & 1 & $2.05 E+00$ \\
\hline si & ICP/AES & Acid. & $\mu g / g d$ & $2.80 E+03$ & 6.19 & $\dot{2}$ & $3.31 E+03$ \\
\hline si & ICP/AES & FS.Ni & $\mu g / 9 d$ & $6.89 E+04$ & 3.91 & 2 & $7.67 \mathrm{E}+04$ \\
\hline si & ICP/AES & FS.Zr & $\mu g / g d$ & $6.46 E+04$ & 3.69 & 2 & $7.16 E+04$ \\
\hline${ }^{151_{\mathrm{Sm}} /{ }^{147_{\mathrm{Pm}}}}$ & Rad & FS.NI & $\mu \mathrm{Ci} / \mathrm{g} d$ & $9.23 E+01$ & 2.84 & 2 & $9.99 E+01$ \\
\hline
\end{tabular}

$a=$ Table does not include analytes where more than $50 \%$ of the data are "less than" values.

$s=\mu g / g$ d is $\mu \mathrm{g} / \mathrm{g}$ dried slurry; $\mu \mathrm{Ci} / \mathrm{g}$ o is $\mu \mathrm{Ci} / \mathrm{g}$ dried slurry.

* = At least one analytical result was above the $10 \mathrm{~L}$ but below the EOL. 


\section{HNF-SD-WM-DP-225, REV 1}

Table 4.1. Tank 241-C-106 Vendor Product

Summary Statistics - Random Variability. (page 7 of 9)

\begin{tabular}{|c|c|c|c|c|c|c|c|c|}
\hline Analyte a & Method & Digest & Units S & $\dot{\mu}$ & & $\begin{array}{c}\operatorname{RSD}(\bar{\mu}) \\
(\%)\end{array}$ & $d f$ & $95 \%$ UL \\
\hline Sn & ICP/MS & Acid & $\mu g / g d$ & $3.48 E+01$ & & 2.08 & 2 & $3.69 \mathrm{E}+01$ \\
\hline sn & $1 \mathrm{CP} / \mathrm{MS}$ & FS.Ni & $\mu g / g d$ & $2.93 E+01$ & & 42.26 & 2 & $6.54 E+01$ \\
\hline $5 \pi$ & ICP/MS & FS.Zr & $\mu g / g d$ & $1.02 E+02$ & & 15.64 & 2 & $1.49 E+02$ \\
\hline $121 \pi_{\mathrm{Sn}}$ & Rad & FS.Ni & $\mu \mathrm{Ci} / \mathrm{s} d$ & $1.23 \mathrm{E}+00$ & & 24.84 & 2 & $2.12 E+00$ \\
\hline${ }^{126} \mathrm{sn} / /^{126 n} \mathrm{sb} /{ }^{126} \mathrm{sb}$ & Rad & FS.Ni & $\mu \mathrm{Ci} / \mathrm{g} d$ & $1.15 E-02$ & & 15.50 & 2 & $1.68 \mathrm{E}-02$ \\
\hline $\mathrm{sr}$ & ICP/AES & Acid & $\mu g / 9 d$ & $5.38 E+01$ & & 0.99 & 2 & $5.54 E+01$ \\
\hline $\mathrm{Sr}$ & ICP/AES & FS.Ni & $\mu g / g d$ & $7.91 E+01$ & $\star$ & 4.23 & 2 & $8.89 E+01$ \\
\hline Sr & JCP/AES & FS.Zr & $\mu g / g d$ & $1.45 E+02$ & $\star$ & 3.49 & 2 & $1.60 E+02$ \\
\hline$S r$ & ICP/MS & Acid & $\mu 9 / 9 d$ & $4.51 E+01$ & & 0.81 & 2 & $6.61 E+01$ \\
\hline Sr & $1 \mathrm{CP} / \mathrm{MS}$ & FS.Ni & $\mu g / 9 d$ & $5.88 E+01$ & & 15.86 & 2 & $8.61 E+01$ \\
\hline $\mathrm{Sr}$ & ICP/MS & $\mathrm{FS.Zr}$ & $\mu g / g d$ & $1.50 E+02$ & & 3.55 & 2 & $1.66 \varepsilon+02$ \\
\hline $90_{S r / Y}$ & Rad & FS.Ni & $\mu \subset \mathbf{i} / g d$ & $7.30 \mathrm{E}+02$ & & 2.45 & 2 & $7.83 E+02$ \\
\hline TIC & Wtchem & Direct & $\mathrm{ugC} / \mathrm{g} d$ & $5.30 E+03$ & & 4.36 & 2 & $5.97 E+03$ \\
\hline TOC & wichem & Direct & ugc/gd & $4.79 \mathrm{E}+04$ & & 3.30 & 2 & $5.26 E+04$ \\
\hline $\mathrm{Ta}$ & $I C P / M S$ & Acid & $\mu g / 9 d$ & $1.04 E+01$ & & 11.64 & 2 & $1.40 E+01$ \\
\hline $\mathrm{Ta}$ & ICP/MS & FS.Ni & $\mu g / g d$ & $2.13 E+01$ & & 26.27 & 2 & $3.77 E+01$ \\
\hline $\mathrm{Ta}$ & ICP/MS & FS.Zr & $\mu g / 9 d$ & $2.86 \mathrm{E}+01$ & & 22.67 & 2 & $4.75 E+01$ \\
\hline To & $I C P / M S$ & Acid & $\mu g / g d$ & $1.46 E+00$ & & .2 .52 & 2 & $1.57 E+00$ \\
\hline Ib & ICP/WS & FS.Ni & $\mu g / g d$ & $8.20 E+00$ & & 10.53 & 2 & $1.07 E+01$ \\
\hline$T b$ & I CP/NS & FS.2r & $\mu g / g d$ & $2.34 E+00$ & & 18.00 & 2 & $3.57 E+00$ \\
\hline${ }^{99}$ TC & $I C P / M S$ & Acid & $\mu g / g d$ & $3.30 E-01$ & & 10.83 & 2 & $4.34 E-01$ \\
\hline $99 \mathrm{TC}$ & ICP/MS & Acid & $\mu \mathrm{Ci} / \mathrm{g} d$ & $5.60 \mathrm{E}-03$ & & 10.83 & 2 & $7.37 \mathrm{E}-03$ \\
\hline Te & ICP/AES & Acid & $\mu g / g d$ & $5.23 E+02$ & & 1.03 & 2 & $5.39 E+02$ \\
\hline Te & ICP/NS & Acid & $\mu g / g d$ & $2.03 E+02$ & & 1.57 & 2 & $2.13 E+02$ \\
\hline Te & ICP/MS & FS.Ni & $\mu g / g d$ & $4.24 E+02$ & & 8.96 & 2 & $5: 35 E+02$ \\
\hline Te & ICP/MS & FS.Zr & $\mu g / g d$ & $2.88 E+02$ & & 16.97 & 2 & $4.31 \mathrm{E}+02$ \\
\hline Th & ICP/AES & Acid & $\mu g / g d$ & $3.49 E+02$ & 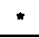 & 0.55 & 2 & $3.54 E+02$ \\
\hline${ }^{232}$ Th & $I C P / M S$ & scid & $\mu g / g d$ & $1.85 E+02$ & & 1.23 & 2 & $1.91 E+02$ \\
\hline $232_{\text {th }}$ & ICP/WS & Acid & $\mu \mathrm{Ci} / \mathrm{gd}$ & $2.03 E-05$ & & 1.20 & 2 & $2.10 E-05$ \\
\hline $232 \mathrm{Th}$ & ICP/MS & Fs.2r & $\mu g / g d$ & $4.995+02$ & & 20.45 & 2 & $7.96 E+02$ \\
\hline $232_{\text {Th }}$ & ICP/MS & Es.zr & $\mu \mathrm{Ci} / \mathrm{g} d$ & $5.48 E-05$ & & 20.45 & 2 & $8.76 \mathrm{E}-05$ \\
\hline
\end{tabular}

$a=$ Table does not include analytes where more than $50 \%$ of the data are "less than" values.

$s=\mu g / g$ d is $\mu g / g$ dried slurry; $\mu C i / g$ d is $\mu C i / g$ dried slurry.

* = At least one analytical result was above the $10 \mathrm{~L}$ but below the EQL. 
HNF-SD-WM-DP-225, REV. 1

Table 4.1. Tank 241-C-106 Vendor Product

Summary Statistics - Random Variability. (page 8 of 9)

\begin{tabular}{|c|c|c|c|c|c|c|c|c|}
\hline Analyte a & Method & Digest & Units s & . it & & $\begin{array}{c}\operatorname{RSD}(\bar{\mu}) \\
(\%)\end{array}$ & $d t$ & $95 \%$ UL \\
\hline$T \mathbf{i}$ & ICP/AES & Acid & $\mu g / g d$ & $3.50 E+02$ & & 1.28 & 2 & $3.63 E+02$ \\
\hline$T i$ & ICP/AES & FS.Ni & $\mu g / g d$ & $1.03 E+03$ & & 4.74 & 2 & $1.17 \mathrm{E}+03$ \\
\hline$T i$ & ICP/AES & FS.Zr & $\mathrm{Hg} / \mathrm{g} d$ & $1.02 E+03$ & & 6.13 & 2 & $1.21 E+03$ \\
\hline $\mathrm{Ti}$ & ICP/MS & Acid & $\mu g / g d$ & $3.58 E+02$ & & 1.33 & 2 & $3.72 E+02$ \\
\hline $\mathrm{Ti}$ & ICP/MS & FS.Ni & $\mu g / g d$ & $1.12 E+03$ & & 6.86 & 2 & $1.35 E+03$ \\
\hline$T \mathbf{j}$ & ICP/MS & FS.Zr & $\mu g / g d$ & $1.31 E+03$ & & 7.06 & 2 & $1.58 E+03$ \\
\hline $\mathrm{Tm}$ & $1 \mathrm{CP} / \mathrm{MS}$ & Acid & $\mu g / g d$ & $6.17 \mathrm{E}-02$ & & 12.85 & 2 & $8.48 E-02$ \\
\hline Tm. & $I C P / N S$ & FS.Hi & $\mu g / g d$ & $1.82 E+00$ & & 39.63 & 2 & $3.92 E+00$ \\
\hline Tm.wo & ICP/MS & FS.Ni & $\mu \mathrm{g} / \mathrm{g} d$ & $2.52 E+00$ & & 36.01 & 2 & $5.17 E+00$ \\
\hline Total Alpha & Rad & FS.Ni & $\mu \mathrm{Ci} / \mathrm{g} \mathrm{d}$ & $6.78 E+00$ & & 1.66 & 2 & $7.11 \mathrm{E}+00$ \\
\hline Total Beta & Rad & FS.Ni & $\mu \mathrm{Ci} / \mathrm{g} d$ & $2.05 \mathrm{E}+03$ & & 2.93 & 2 & $2.22 \mathrm{E}+03$ \\
\hline Total Carbon & wtchen & Water & ugc/g d & $5.22 E+04$ & & 4.01 & 2 & $5.83 E+04$ \\
\hline Total U & ICP/MS & Acid & $\mu g / g d$ & $1.91 E+03$ & & 1.90 & 2 & $2.01 E+03$ \\
\hline Total U & ICP/MS & FS.Ni & $\mu g / g d$ & $1.90 E+03$ & & 2.84 & 2 & $2.05 E+03$ \\
\hline Total U & ICP/MS & FS.Zr & $\mu g / 9 d$ & $2.01 \mathrm{E}+03$ & & 1.36 & 2 & $2.095+03$ \\
\hline$U$ & ICP/AES & Aeid & $\mu g / g d$ & $1.95 E+03$ & $\star$ & 0.78 & 2 & $1.99 E+03$ \\
\hline $233 \mathrm{u}$ & ICP/MS & Acid & $\mu g / g d$ & $1.73 E-01$ & & 11.34 & 2 & $2.31 E-01$ \\
\hline $233 \mathrm{U}$ & ICP/MS & Acid & $\mu \mathrm{Ci} / \mathrm{g} \sigma$ & $1.68 \mathrm{E}-03$ & & 91.56 & 2 & $2.25 E-03$ \\
\hline $234 \mathrm{U}$ & ICP/MS & Acid & $\mu g / g d$ & $1.23 \mathrm{E}-01$ & & 8.02 & 2 & $1.52 \mathrm{E}-01$ \\
\hline $234 \mathrm{U}$ & ICP/MS & Acid & $\mu C i / g d$ & $7.73 E-04$ & & 7.41 & 2 & $9.40 E-04$ \\
\hline $235 \mathrm{u}$ & ICP/MS & Acid & $\mu g / g d$ & $1.30 E+01$ & & 2.86 & 2 & $1.41 E+01$ \\
\hline $235 \mathrm{U}$ & ICP/MS & Acid & $\mu C i / 9 d$ & $2.80 \mathrm{E}-05$ & & 2.86 & 2 & $3.04 E-05$ \\
\hline $236_{U}$ & $1 \mathrm{CP} / \mathrm{MS}$ & Acid & $\mu g / g d$ & $2.87 \mathrm{E}-01$ & & 7.28 & 2 & $3.48 \varepsilon-01$ \\
\hline $236 \mathrm{v}$ & $1 \mathrm{CP} / \mathrm{MS}$ & Acid & $\mu[\mathbf{i} / g d$ & $1.85 \mathrm{E}-05$ & . & 7.11 & 2 & $2.23 \mathrm{E}-05$ \\
\hline $238 \mathrm{U}$ & ICP/MS & Acid & $\mu g / g d$ & $1.90 E+03$ & & 1.91 & 2 & $2.00 E+03$ \\
\hline $23 B_{U}$ & $1 \mathrm{CP} / \mathrm{HS}$ & Acid & $\mu[\mathbf{i} / g d$ & $6.37 \mathrm{E}-04$ & & 1.91 & 2 & $6.72 E-04$ \\
\hline $238 \mathrm{U}$ & $1 \mathrm{CP} / \mathrm{MS}$ & FS.Ni & $\mu g / g d$ & $1.89 E+03$ & & 2.86 & 2 & $2.04 E+03$ \\
\hline $238_{i}$ & ICP/MS & FS.Mi & $\mu \mathrm{Ci} / \mathrm{g} d$ & $6.34 E-04$ & & 2.86 & 2 & $6.87 E-04$ \\
\hline $238_{\mathrm{u}}$ & 1CP/MS & FS.Zr & $\mu g / g d$ & $2.00 E+03$ & & 1.28 & 2 & $2.07 E+03$ \\
\hline $238_{\mathrm{U}}$ & ICP/MS & FS.zr & $\mu \mathrm{Ci} / \mathrm{gd}$ & $6.71 E-04$ & & 1.28 & 2 & $6.97 \mathrm{E} \cdot 04$ \\
\hline$v$ & ICP/MS & Acid & $\mu g / g d$ & $3.73 E+01$ & & 8.58 & 2 & $4.66 E+01$ \\
\hline
\end{tabular}

a = rable does not include analytes where more than $50 \%$ of the data are "less than" values.

$\$=\mu g / g$ d is $\mu g / g$ dried slurry; $\mu \mathrm{Ci} / \mathrm{g}$ d is $\mu \mathrm{Ci} / \mathrm{g}$ dried slurry.

* = At least one analytical result wes above the IDL but below the EOL. 
Table 4.1. Tank 241-C-106 Vendor Product Summary Statistics - Random Variability. (page 9 of 9)

\begin{tabular}{|c|c|c|c|c|c|c|c|c|}
\hline Anatyte a & $\begin{array}{c}\text { Method } \\
\end{array}$ & Digest & Units $s$ & $\bar{\mu}$ & & $\begin{array}{r}\operatorname{RSD}(\bar{\mu}) \\
(\%) \\
\end{array}$ & $d f$ & $95 \%$ UL \\
\hline$H$ & ICP/MS & Acid & $\mu g / g d$ & $2.22 \mathrm{E}+02$ & & 4.08 & 2 & $2.48 \mathrm{E}+02$ \\
\hline H & ICP/MS & FS.Mi & $\mu g / \rho d$ & $4.99 E+02$ & & 6.05 & 2 & $5.87 \mathrm{E}+02$ \\
\hline$H$ & ICP/HS & FS.Zr & $\mu g / g d$ & $3.57 E+02$ & & 19.62 & 2 & $5.61 E+02$ \\
\hline Yo & ICP/MS & Acid. & $\mu g / g d$ & $2.50 \mathrm{E}-01$ & & 23.09 & 2 & $4.19 E-01$ \\
\hline $\mathrm{Yb}$ & ICP/MS & FS.Ni & $\mu g / g d$ & $1.26 E+01$ & & 29.08 & 2 & $2.33 \mathrm{E}+01$ \\
\hline $\mathrm{rb}$ & ICP/MS & $\mathrm{FS.Zr}$ & $\mu g / g d$ & $3.24 E+00$ & & 18.62 & 2 & $4.99 E+00$ \\
\hline $\mathrm{Zn}$ & ICP/AES & Acid & $\mu g / g d$ & $1.16 \mathrm{E}+02$ & $\star$ & 1.28 & 2 & $1.20 \mathrm{E}+02$ \\
\hline $\mathbf{Z n}$ & ICP/AES & FS.Mi & $\mu g / g d$ & $1.83 \mathrm{E}+02$ & $\star$ & 5.47 & 2 & $2.12 E+02$ \\
\hline $\mathrm{Zn}$ & ICP/AES & FS.Zr & $\mu g / g d$ & $1.61 E+02$ & $\star$ & 3.48 & 2 & $1.77 \mathrm{E}+02$ \\
\hline $\mathrm{Zn}$ & ICP/MS & Acid & $\mu g / g d$ & $9.92 \varepsilon+01$ & & 3.13 & 2 & $1.08 \mathrm{E}+02$ \\
\hline $\mathrm{Zn}$ & ICP/MS & FS.Ni & $\mu \mathrm{g} / \mathrm{g} d$ & $3.07 E+02$ & & 9.43 & 2 & $3.81 E+02$ \\
\hline $\mathrm{Zn}$ & $1 \mathrm{CP} / \mathrm{MS}$ & FS.Zr & $\mu g / g d$ & $2.34 E+02$ & & 11.13 & 2 & $3.10 E+02$ \\
\hline $2 r$ & ICP/AES & Acid & $\mu g / g d$ & $6.61 E+02$ & & 18.92 & 2 & $1.03 E+03$ \\
\hline $2 r$ & ICP/AES & FS.Ni & $\mu g / g d$ & $1.11 \varepsilon+03$ & & 19.48 & 2 & $1.73 E+03$ \\
\hline $2 r$ & ICP/MS & Acid & $\mu g / g d$ & $2.56 E+02$ & & 16.67 & 2 & $3.80 E+02$ \\
\hline $2 r$ & ICP/NS & FS.Ni & $\mu g / g d$ & $4.33 E+02$ & & 21.68 & 2 & $7.07 \mathrm{E}+02$ \\
\hline$m / 2241$ & ICP/MS & Acid & $\mu g / g d$ & $7.73 E-01$ & & 4.03 & 2 & 8.64E-01 \\
\hline$m / 2241$ & ICP/MS & $\mathrm{FS.2 \textrm {r }}$ & $\mu g / 9 \mathrm{~d}$ & $1.65 E+00$ & & 8.55 & 2 & $2.06 E+00$ \\
\hline $\mathrm{m} / 293$ & $I C P / M S$ & Acid & $\mu g / g d$ & $5.00 E+01$ & & 15.88 & 2 & $7.31 E+01$ \\
\hline $\mathrm{m} / 293$ & $I C P / M S$ & FS.Ni & $\mu g / g d$ & $3.38 \mathrm{E}+01$ & & 31.48 & 2 & $6.48 E+01$ \\
\hline$\pi / 293$ & $1 \mathrm{CP} / \mathrm{MS}$ & Fs.Zr & $\mu g / g d$ & $2.35 E+02$ & & 21.81 & 2 & $3.84 E+02$ \\
\hline
\end{tabular}

$\mathrm{a}=$ Table does not include analytes where more than $50 \%$ of the data are "less than" values.

$\$=\mu g / g d$ is $\mu g / g$ dried slurry; $\mu \mathrm{Ci} / \mathrm{g} d$ is $\mu \mathrm{Ci} / \mathrm{g} d r i e d$ slurry.

* At least one analytical result was above the $10 \mathrm{~L}$ but below the EOL. 
HNF-SD-WM-DP-225, REV. 1

Table 4.2. Tank 241-C-106 Vendor Product

Analytes with More Than 50\% "Less Than" Resuits. (page 1 of 3 )

\begin{tabular}{|c|c|c|c|c|c|}
\hline Analyte & Method & Digest & Units $\$$ & \multicolumn{2}{|c|}{ Largest Result } \\
\hline $110 \mathrm{~m}_{\mathrm{Ag}}$ & Rad & FS.Ni & $\mu \mathrm{Ci} / \mathrm{g} d$ & & 0.09 \\
\hline $243_{A M}$ & Rad & FS.Ni & $\mathrm{HCi} / \mathrm{g} d$ & $<$ & 0.02 \\
\hline As & ICP/AES & Acid & $\mu g / g d$ & $<$ & 123 \\
\hline As & ICP/AES & ES.Ni & $\mu g / g d$ & $<$ & 479 \\
\hline As & ICP/AES & $\mathrm{FS} .2 \mathrm{r}$ & $\mu g / g d$ & $<$ & 510 \\
\hline B & ICP/RES & FS. Zr & $\mu g / g d$ & & 186 \\
\hline Be & ICP/AES & Acid & $\mu g / 9 d$ & $<$ & 1 \\
\hline $\mathrm{Be}$ & ICP/AES & FS.Mi & $\mu g / g d$ & $<$ & 9 \\
\hline $\mathrm{Be}$ & ICP/AES & $\mathrm{FS} . \mathrm{Zr}$ & $\mu g / g d$ & $<$ & 9 \\
\hline $\mathrm{Br}$ & IC & Water & $\mu g / g d$ & $<$ & 116 \\
\hline Ce & ICP/AES & FS.Ni & $\mu g / g d$ & $<$ & 523 \\
\hline $\mathrm{Ce}$ & ICP/AES & FS.Zr & $\mu g / g d$ & $<$ & 1580 \\
\hline${ }^{144} \mathrm{Ce} /{ }^{144} \mathrm{Pr} /{ }^{144 \mathrm{~m}} \mathrm{Pr}$ & Rad & FS.Ni & $\mu \mathrm{Ci} / \mathrm{g} \mathrm{d}$ & $<$ & 2 \\
\hline Co & ICP/RES & $\mathrm{FS} . \mathrm{Ni}$ & $\mu g / g d$ & $<$ & 87 \\
\hline Co & ICP/AES & $\mathrm{FS} .2 \mathrm{r}$ & $\mu g / g d$ & $<$ & 93 \\
\hline${ }^{134} \mathrm{cs}$ & Rad & FS.Ni & $\mu \mathrm{Ci} / \mathrm{g} d$ & $<$ & 0.3 \\
\hline Dy & ICP/AES & Acid & $\mu s / g d$ & $<$ & 12 \\
\hline Dy & I CP/AES & FS.Ni & $\mu g / g d$ & $<$ & 87 \\
\hline Dy & ICP/AES & FS.2r & $\mu g / g d$ & $<$ & 93 \\
\hline Er & $I C P / M S$ & $\mathrm{FS.25}$ & $\mu g / g d$ & $<$ & 1 \\
\hline Eu & ICP/AES & Acid & $\mu g / g d$ & $<$ & 25 \\
\hline Eu & ICP/AES & FS.Ni & $\mu g / 9 d$ & $<$ & 174 \\
\hline Eu & ICP/AES & FS.Zr & $\mu g / g d$ & $<$ & 185 \\
\hline${ }^{152} \mathrm{Eu}$ & Rad & FS.Ni & $\mu C i / g d$ & $<$ & 2 \\
\hline $\mathrm{F}^{-}$ & IC & Water & $\mu g / g d$ & $<$ & 2910 \\
\hline Ko & $I C P / M S$ & $\mathrm{F5.2r}$ & $\mu g / g d$ & & 1.11 \\
\hline 129 & ICP/MS & FS.Zr & $\mu g / g d$ & $<$ & 20 \\
\hline $129_{1}$ & ICP/HS & F S.Zr & $\mu C i / g d$ & $<$ & 0.004 \\
\hline$x$ & ICP/AES & Acid & $\mathrm{Hg} / \mathrm{g} d$ & $<$ & 492 \\
\hline $\mathrm{Li}$ & ICP/AES & FS.Mi & $\mu g / g d$ & & 96 \\
\hline Mo & ICP/AES & FS.Ni & $\mu g / g d$ & $<$ & 87 \\
\hline Mo & ICP/AES & $\mathrm{FS} . \mathrm{Zr}$ & $\mu g / 9 d$ & $<$ & 93 \\
\hline $\mathrm{Pd}$ & ICP/AES & Acid & $\mathrm{Hg} / \mathrm{g} d$ & $<$ & 359 \\
\hline $\mathrm{Pd}$ & ICP/AES & FS.Ni & $\mu g / g d$ & $<$ & 1310 \\
\hline $\mathrm{Pd}$ & ICP/AES & FS.Zr & $\mathrm{kg} / \mathrm{g} \mathrm{d}$ & $<$ & 1390 \\
\hline
\end{tabular}

$\Phi=\mu g / g$ d is $\mu g / g$ dried slurry; $\mu C i / g d$ is $\mu C i / g$ dried slurry. 
Table 4.2. Tank 241-C-I06 Vendor Product

Analytes with More Than 50\% "Less Than" Results. (page 2 of 3 )

\begin{tabular}{|c|c|c|c|c|c|c|}
\hline Analyte & Method & Digest & Units s & \multicolumn{3}{|c|}{ Largest Result } \\
\hline $240_{P U}$ & $I C P / M S$ & $\mathrm{FS} .2 \mathrm{r}$ & $\mu g / 9 d$ & $<$ & 1 & \\
\hline $240 \mathrm{Pu}$ & ICP/MS & FS.Zr & $\mu C i / g d$ & $<$ & 0.2 & \\
\hline $242 \mathrm{Pu}$ & ICP/MS & Acid & $\mu g / g d$ & $<<$ & 0.02 & \\
\hline $242 \mathrm{pu}$ & ICP/MS & Acis & $\mu \mathrm{Ci} / \mathrm{g} d$ & $<$ & 0.00008 & \\
\hline${ }^{242} \mathrm{Pu}$ & IEP/HS & FS.2r & $\mu g / g d$ & $<$ & 1 & \\
\hline $242 \mathrm{pu}$ & $\mathrm{ICP} / \mathrm{HS}$ & FS.Zr & $\mu \mathrm{Ci} / \mathrm{g} d$ & $<$ & 0.004 & \\
\hline $242 \mathrm{Pu}$ & Rad & FS.Ni & $\mu \mathrm{Ci} / \mathrm{gd}$ & $<$ & 0.02 & \\
\hline $\mathrm{Re}$ & ICP/MS & $\mathrm{FS} .2 \mathrm{r}$ & $\mu g / g d$ & $<$ & 9 & \\
\hline $\mathrm{Rh}$ & ICP/AES & Acid & $\mu g / g d$ & $<$ & 74 & \\
\hline Rh & ICP/AES & FS.Ni & $\mu g / g d$ & $<$ & 523 & \\
\hline$R h$ & ICP/AES & FS.Zr & $\mu g / g d$ & $<$ & 556 & \\
\hline Ru & ICP/AES & Acid & $\mu g / g d$ & $<$ & 738 & \\
\hline Ru & ICP/AES & FS.Ni & $\mu g / g d$ & $<$ & 1740 & \\
\hline$R u$ & ICP/AES & F5.2r & $\mu g / g d$ & $<$ & 1850 & \\
\hline${ }^{106_{R U / R h}}$ & Rad & $\mathrm{FS} . \mathrm{Ni}$ & $\mu \mathrm{Ci} / \mathrm{g} d$ & $<$ & 3 & \\
\hline sb & ICP/AES & Acid & $\mu g / g d$ & $<$ & 74 & \\
\hline sb & ICP/AES & FS.Ni & $\mu g / g d$ & $<$ & 871 & \\
\hline $5 b$ & ICP/AES & FS.Zr & $\mu g / g d$ & $<$ & 927 & \\
\hline sb & ICP/KS & FS.Ni & $\mu g / g d$ & $<$ & 1 & \\
\hline sb & $1 \mathrm{CP} / \mathrm{KS}$ & f5.2r & $\mu g / g d$ & $<$ & 1 & \\
\hline${ }^{125} \mathrm{Sb} /{ }^{125 \mathrm{~m}_{\mathrm{Te}}}$ & Red & FS, Ni & $\mu \mathrm{Ci} / \mathrm{g} \mathrm{d}$ & $<$ & 2 & \\
\hline $\mathrm{Se}$ & ICP/AES & Acid & $\mu g / g d$ & $<$ & 369 & \\
\hline Se & JCP/AES & FS.Ni & $\mu g / g d$ & $<$ & 610 & · \\
\hline Se & ICP/AES & FS.Zr & $\mu g / g d$ & $<$ & 649 & \\
\hline${ }^{79} \mathrm{se}$ & Rad & FS.Ni & $\mu \mathrm{Ci} / \mathrm{gd}$ & $<$ & 0.0024 & \\
\hline${ }^{79} \mathrm{se}$ & Rad & Acid & $\mu C i / g d$ & $\therefore$ & 0.00021 & \\
\hline Sn & ICP/AES & Acid & $\mu g / g$ d & $<$ & 4060. & - \\
\hline Sn & ICP/AES & FS.Ni & $\mu g / g d$ & $\leq$ & 3920 & \\
\hline sn & ICP/AES & FS.Zr & $\mu g / g d$ & $<$ & 4170 & \\
\hline${ }^{113_{\mathrm{sn}}}{ }^{113 \mathrm{~m}_{\mathrm{in}}}$ & Rad & FS.Ni & $\mu \mathrm{Ci} / \mathrm{g} d$ & $\cdot$ & 0.01 & \\
\hline $119 \mathrm{~m} \mathrm{sn}$ & Rad & FS.Mi & $\mu \mathrm{Ci} / \mathrm{gd}$ & $<$ & 0.04 & \\
\hline${ }^{99} \mathrm{Tc}$ & $I C P / M S$ & FS.NI & $\mu \mathrm{g} / \mathrm{g} \mathrm{d}$ & $<$ & 1 & \\
\hline${ }^{99} \mathrm{Tc}$ & ICP/MS & FS.Ni & $\mu \mathrm{Ci} / \mathrm{g} d$ & $<$ & 0.017 & \\
\hline Te & ICP/AES & FS.Ni & $\mu g / 9 d$ & 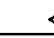 & 2610 & \\
\hline Te & ICP/AES & $\mathrm{FS.2r}$ & $\mu g / g d$ & $<$ & 2780 & \\
\hline
\end{tabular}

$\$=\mu \mathrm{g} / \mathrm{g} d$ is $\mu \mathrm{g} / \mathrm{g}$ dried sturry; $\mu \mathrm{Ci} / \mathrm{g} d$ is $\mu \mathrm{Ci} / \mathrm{g}$ dried slurry. 


\section{HNF-SD-WM-DP-225, REV. 1}

Table 4.2. Tank 241-C-106 Vendor Product

Analytes with More Than 50\% "Less Than" Results. (page 3 of 3 )

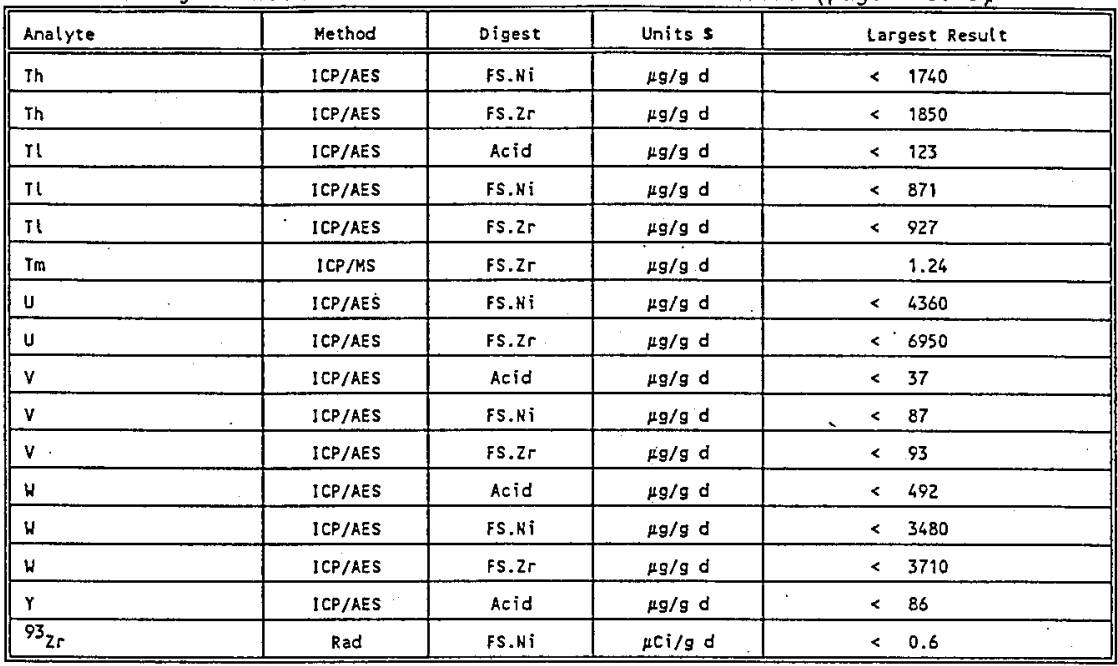

$s=\mu g / g$ of is $\mu g / g$ dried slurry; $\mu \mathrm{Ci} / \mathrm{g}$ d is $\mu \mathrm{Ci} / \mathrm{g}$ dried slurry. 
HNF-SD-WM-DP-225, REV 1

Table 4.3. Tank 241-C-106 Vendor Product - Variance Components. (page 1 of 9)

\begin{tabular}{|c|c|c|c|c|c|c|}
\hline Analyte a & Method & Digest & Mean & Units s & $\begin{array}{l}\text { Randon } \\
\text { Sampl ing } \\
\text { RSD } \% \\
\end{array}$ & $\begin{array}{c}\text { Random } \\
\text { Analytical } \\
\text { RSD } \mathbf{z} \\
\end{array}$ \\
\hline $\mathrm{Ag}$ & ICP/AES & Acid & $7.56 E+01$ & $\mu \mathrm{g} / \mathrm{g} d$ & $0.00 *$ & 6.58 \\
\hline Ag & ICP/AES & FS.Ni & $1.03 E+03$ & $\mu g / g d$ & $0.00 *$ & 22.89 \\
\hline Ag & ICP/AES & Fs.zr & $3.18 E+03$ & $\mu \mathrm{g} / \mathrm{g} d$ & $0.00 *$ & 21.45 \\
\hline Al & ICP/AES & Acid & $4.79 E+04$ & $\mu g / g$ d & 2.01 & 1.90 \\
\hline Al & ICP/AES & FS.Ni & $5.12 \mathrm{E}+04$ & $\mu g / g d$ & 4.20 & 2.50 \\
\hline Al & ICP/AES & Fs.2r & $4.95 E+04$ & $\mu \mathrm{g} / \mathrm{g} \mathrm{d}$ & 4.70 & 2.35 \\
\hline Al & ICP/MS & Acid & $3.34 \mathrm{E}+04$ & $\mu g / g d$ & $0.00 * 1$ & 7.25 \\
\hline $241_{\text {Arr. aea }}$ & Rad & FS.Ni & $2.77 E+00$ & $\mu[\mathrm{C} / \mathrm{g} d$ & 4.18 & 2.01 \\
\hline $241_{\text {Arn.gea }}$ & Rad & FS.Ni & $3.15 E+00$ & $\mu \mathrm{Ci} / \mathrm{gd}$ & $0.00 *$ & 23.03 \\
\hline As & ICP/MS & Acid & $1.55 E+02$ & $\mu g / g d$ & 5.43 & 6.24 \\
\hline As & ICP/MS & FS.Ni & $2.86 E+02$ & $\mu \mathrm{g} / \mathrm{g} \mathrm{d}$ & 8.90 & 12.98 \\
\hline As & ICP/MS & FS.Zr & $2.56 \Xi+02$ & $\mu \mathrm{g} / \mathrm{g} \mathrm{d}$ & 22.98 & 6.76 \\
\hline B & ICP/AES & Acid & $2.25 E+02$ & $\mu \mathrm{g} / \mathrm{s} \mathrm{d}$ & 30.09 & 6.60 \\
\hline$B$ & ICP/AES & FS.Ni & $3.03 E+02$ & $\mu g / g d$ & 28.49 & 20.04 \\
\hline$B$ & ICP/MS & Acid & $1.86 E+02$ & $\mu g / g d$ & 36.66 & 5.49 \\
\hline B & ICP/NS & FS.Ni & $2.29 E+02$ & $\mu g / g d$ & 34.78 & 18.18 \\
\hline- & ICP/MS & $\mathrm{Fs} .2 \mathrm{r}$ & $1.01 E+02$ & $\mu g / g d$ & 64.91 & 22.50 \\
\hline $\mathrm{Ba}$ & ICP/AES & Acid & $4.15 E+02$ & $\mu g / 9 d$ & 1.28 & 2.22 \\
\hline $\mathrm{Ba}$ & ICP/AES & FS.Ni & $4.07 E+02$ & $\mathrm{Hg} / \mathrm{g} d$ & 4.69 & 2.17 \\
\hline $\mathrm{Ba}$ & ICP/AES & $\mathrm{FS} . \mathrm{Zr}$ & $4.37 E+02$ & $\mu \mathrm{g} / \mathrm{g} d$ & 5.08 & 2.28 \\
\hline $\mathrm{Ba}$ & ICP/MS & Acid & $3.62 E+02$ & $\mu \mathrm{g} / \mathrm{g} d$ & $0.00 *$ & 2.72 \\
\hline $\mathrm{Ba}$ & $I C P / M S$ & FS.Ni & $4.12 E+02$ & $\mathrm{Fg} / \mathrm{g} \mathrm{d}$ & $0.00 *$ & 4.75 \\
\hline $\mathrm{Ba}$ & ICP/MS & Fs.zr & $4.27 E+02$ & $\mu g / g d$ & $0.00 *$ & 3.15 \\
\hline $\mathbf{B i}$ & ICP/AES & Acid & $4.30 E+02$ & $\mu g / g d$ & 1.08 & 2.45 \\
\hline $\mathrm{Bi}$ & ICP/AES & $\mathrm{FS.Hi}$ & $5.38 E+02$ & $\mu \mathrm{g} / \mathrm{g} \mathrm{d}$ & 2.19 & 1.60 \\
\hline$B \mathbf{i}$ & ICP/AES & FS.Zr & $5.82 E+02$ & $\mu \mathrm{g} / \mathrm{g} \mathrm{d}$ & $0.00 *$ & 9.94 \\
\hline $\mathrm{C}_{2} \mathrm{O}_{4}$ & IC & Water & $1.70 E+05$ & $\mathrm{~kg} / \mathrm{g} \mathrm{d}$ & 6.30 & 5.33 \\
\hline${ }^{14} c$ & Rad & FS.Ni & $2.87 \mathrm{E}-03$ & $\mu C i / g d$ & 23.15 & 189.54 \\
\hline $\mathrm{CN}^{-}$ & wechem & Direct & $9.50 E+00$ & $\mu g / \mathrm{g} \mathrm{d}$ & 9.43 & 3.08 \\
\hline $\mathrm{Ca}$ & ICP/AES & Acid & $3.84 E+03$ & $\mu g / g$ d & 1.62 & 1.93 \\
\hline $\mathrm{Ca}$ & ICP/AES & FS.Ni & $6.69 \mathrm{E}+03$ & $\mu g / g d$ & $0.00 *$ & 10.70 \\
\hline $\mathbf{C a}$ & ICP/AES & FS.zr & $9.85 E+03$ & $\mu g / g d$ & $0.00 *$ & 10.42 \\
\hline
\end{tabular}

$a=$ Table does not incluzie analytes where more than $50 \%$ of the data are "less than" values.

$\$=\mu g / g d$ is $\mu \mathrm{g} / \mathrm{g}$ dried slurry; $\mu \mathrm{Ci} / \mathrm{g} d$ is $\mu \mathrm{Ci} / \mathrm{g}$ dried slurry.

* = A RSD(S) of $0.00 \%$ indicates that the variability between duplicate analytical measurements was greater than the variability between subsamples. 
Table 4.3. Tank 241-C-105 Vendor Product - Variance Components. (page 2 of 9)

\begin{tabular}{|c|c|c|c|c|c|c|}
\hline Analyte a & Method & Digest & Hean & Units $\$$ & $\begin{array}{l}\text { Random } \\
\text { Sampling } \\
\text { RSD \% }\end{array}$ & $\begin{array}{c}\text { Random } \\
\text { Analyt ical } \\
\text { RSO \% }\end{array}$ \\
\hline $\mathrm{Ca}$ & ICP/HS & Acid & $9.57 E+03$ & $\mu \mathrm{g} / \mathrm{g} \mathrm{d}$ & $0.00 *$ & 11.74 \\
\hline $\mathrm{Ca}$ & ICP/MS & FS.Ni & $1.72 E+03$ & $\mu \mathrm{g} / \mathrm{g} \mathrm{d}$ & 13.03 & 4.89 \\
\hline $\mathrm{Ca}$ & ICP/HS & FS.Zr & $3.77 E+03$ & $\mu g / g d$ & 7.73 & 5.63 \\
\hline cd & ICP/AES & Acid & $7.30 \mathrm{E}+01$ & $\mu g / g \quad 0$ & $0.00 *$ & 18.00 \\
\hline cd & ICP/AES & FS.Ni & 8. $10 E+01$ & $\mu g / s d$ & 2.79 & 1.36 \\
\hline $\mathrm{Cd}$ & ICP/AES & fs.zr & $8.08 E+01$ & $\mu g / 9 d$ & 1.23 & 5.40 \\
\hline cd & ICP JMS & Acid & $5.81 E+01$ & $\mu g / g d$ & $0.00 *$ & 2.99 \\
\hline cd & $1 \mathrm{CP} / \mathrm{MS}$ & FS.Ni & $9.05 E+01$ & $\mu g / g$ d & $0.00 *$ & 12.56 \\
\hline cd & ICP/MS & $\mathrm{Fs} . \mathrm{Zr}$ & $7.41 \mathrm{E}+01$ & $\mu g / g d$ & 10.27 & 7.09 \\
\hline $\mathrm{Ce}$ & ICP/AES & Acid & $2.99 E+02$ & $\mu g / g d$ & 1.83 & 3.43 \\
\hline $\mathrm{Ce}$ & $I C P / M S$ & Acid & $1.59 E+02$ & $\mu g / g d$ & 1.75 & 3.30 \\
\hline $\mathrm{Ce}$ & ICP/MS & FS.Ni & $1.60 E+02$ & $\mu g / g d$ & 12.01 & 13.90 \\
\hline $\mathrm{Ce}$ & ICP/MS & $\mathrm{Fs} .2 \mathrm{r}$ & $2.02 E+02$ & $\mu g / g d$ & 10.36 & 6.37 \\
\hline $\mathrm{Cl}^{-}$ & IC & Water & $1.26 \varepsilon+02$ & $\mu \mathrm{g} / \mathrm{g} \mathrm{d}$ & $0.00 *$ & 20.80 \\
\hline${ }^{242} \mathrm{Cm}{ }^{242} \mathrm{Am} /{ }^{242 \mathrm{~m}_{\mathrm{Am}}}$ & Rad & FS.Ni & $8.07 E-03$ & $\mu \mathrm{Ci} / \mathrm{g} \mathrm{d}$ & 12.55 & 17.36 \\
\hline $243 / 244 \mathrm{~cm}$ & Rad & FS.Ni & $9.86 E-02$ & $\mu \mathrm{Ci} / \mathrm{gd}$ & 20.80 & 11.89 \\
\hline Co & ICP/AES & Acid & $2.81 E+01$ & $\mu g / 9 \mathrm{~d}$ & $0.00 *$ & 2.60 \\
\hline Co & ICP/MS & Acid & $1.57 E+01$ & $\mu g / g d$ & $0.00 *$ & 3.01 \\
\hline Co & ICP/MS & FS.Ni & $2.62 E+01$ & $\mu \mathrm{g} / \mathrm{g} \mathrm{d}$ & 16.55 & 18.39 \\
\hline Co & ICP/MS & FS.Zr & $2.18 E+01$ & $\mu g / g d$ & 9.38 & 6.03 \\
\hline${ }^{60} \mathrm{co}$ & Rad & FS.Ni & $4.91 \mathrm{E}-01$ & $\mu[i / g d$ & $0.00 *$ & 7.61 \\
\hline $\mathrm{Cr}$ & ICP/AES & Acid & $5.33 E+02$ & $\mu g / g$ o & 0.51 & 2.29 \\
\hline $\mathrm{Cr}$ & ICP/AES & FS.Ni & $8.02 E+02$ & $\mu g / g$ o & 4.23 & 2.85 \\
\hline $\mathrm{Cr}$ & ICP/AES & FS.Zr & $7.00 E+02$ & $\mu g / g d$ & 6.68 & 2.76 \\
\hline $\mathrm{Cr}$ & ICP/HS & Acid & $4.26 E+02$ & $\mu g / g d$ & $0.00 *$ & 2.58 \\
\hline $\mathrm{Cr}$ & $1 \mathrm{CP} / \mathrm{MS}$ & FS.Ni & $7.74 \varepsilon+02$ & $\mu g / g d$ & 9.28 & 6.90 \\
\hline $\mathrm{cr}$ & ICP/MS & Fs.25 & $7.39 E+02$ & $\mu g / g d$ & 6.63 & 2.21 \\
\hline${ }^{133} \mathrm{cs}$ & ICP/MS & Acid & $1.04 E+01$ & $\mu g / 9 d$ & 6.30 & 3.03 \\
\hline $133 \mathrm{cs}$ & ICP/NS & FS.Ni & $1.80 E+01$ & $\mu \mathrm{g} / \mathrm{g} d$ & $0.00 *$ & 22.20 \\
\hline${ }^{133} \mathrm{cs}$ & $1 \mathrm{CP} / \mathrm{MS}$ & $\mathrm{FS.2r}$ & $7.18 E+00$ & $\mu 9 / 9$ d. & 2.11 & 30.20 \\
\hline${ }^{133} \mathrm{cs}$ & Rad & FS.Ni & $1.57 E+01$ & $\mu \mathrm{Ci} / \mathrm{gd}$ & 6.47 & 7.04 \\
\hline${ }^{135} \mathrm{cs}$ & Rad & FS.Ni & $2.86 \mathrm{E}-03$ & $\mu \mathrm{ci} / \mathrm{g} d$ & 5.26 & 2.05 \\
\hline${ }^{137} \mathrm{Cs} / \mathrm{Bz}$ & Rad & FS.Ni & $4.96 E+02$ & $\mu \mathrm{Ci} / \mathrm{gd}$ & 5.56 & 1.79 \\
\hline
\end{tabular}

$a=$ Table does not include analytes where more than $50 \%$ of the data are "less than" values.

$\$=\mu g / g$ of is $\mu g / g$ dried slurry; $\mu \mathrm{Ci} / \mathrm{g}$ d is $\mu \mathrm{Ci} / \mathrm{g}$ dried slurry.

* = ARSD(S) of $0.00 \%$ indicates that the variability between duplicate analytical measurements was greater than the variability between subsamples. 
HNF-SD-WM-DP-225, REV. 1

Table 4.3. Tank 241-C-106 Vendor Product - Variance Components. (page 3 of 9)

\begin{tabular}{|c|c|c|c|c|c|c|}
\hline Analyte a & Method & Digest & Mean & Units $s$ & $\begin{array}{l}\text { Randon } \\
\text { Sampl ing } \\
\text { RSD \% }\end{array}$ & $\begin{array}{l}\text { Random } \\
\text { Analytical } \\
\text { RSD } \%\end{array}$ \\
\hline $\mathrm{Cu}$ & ICP/AES & Acid & $7.75 E+01$ & $\mu g / g d$ & $0.00 \star$ & 2.43 \\
\hline $\mathrm{Cu}$ & ICP/AES & FS.Ni & $1.09 E+02$ & $\mu 9 / 9 d$ & $0.00 *$ & 4.35 \\
\hline $\mathrm{Cu}$ & ICP/AES & $\mathrm{FS.ZR}$ & $1.09 E+02$ & $\mu g / 9 \mathrm{~d}$ & 9.12 & 10.66 \\
\hline $\mathrm{Cu}$ & ICP/MS & Acid & $1.10 E+02$ & $\mu g / g d$ & $0.00 *$ & 1.83 \\
\hline $\mathrm{Cu}$ & ICP/MS & FS.2r & $1.56 E+02$ & $\mu g / g d$ & 5.83 & 6.24 \\
\hline $\mathrm{Cu} . \mathrm{H}$ & ICP/MS & $\mathrm{FS}, \mathrm{Ni}$ & $1.13 E+01$ & $\mu g / g d$ & 120.62 & 34.61 \\
\hline Cu.wo & ICP/MS & FS.Ni & $1.89 E+01$ & $\mu g / g$ d & 62.09 & 1.50 \\
\hline Dy & $1 C P / N S$ & Acid & $1.47 E+00$ & $\mu g / g d$ & $0.00 *$ & 6.28 \\
\hline Dy & ICP/MS & FS.Ni & $2.02 E+01$ & $\mu g / g d$ & 18.61 & 33.42 \\
\hline Dy & ICP/MS & FS.Zr & $3.60 E+00$ & $\mu g / g d$ & 56.09 & 9.64 \\
\hline $\mathrm{Er}$ & ICP/NS & Acid & $7.67 E-01$ & $\mu g / g d$ & $0.00 \star$ & 6.74 \\
\hline $\mathrm{Er}$ & ICP/MS & FS.Ni & $1.31 E+01$ & $\mu g / g$ d & $0.00 *$ & 35.87 \\
\hline Eu & $1 \mathrm{CP} / \mathrm{MS}$ & Acid & $6.46 E+00$ & $\mu g / g d$ & $0.00 *$ & 3.65 \\
\hline Eu & $1 \mathrm{CP} / \mathrm{MS}$ & FS.Ni & $7.87 \mathrm{E}+00$ & $\mu g / g d$ & $0.00 \star$ & 75.25 \\
\hline Eu & $I C P / M S$ & FS.Zr & $7.84 E+00$ & $\mu g / g \triangle$ & $0.00 *$ & 16.02 \\
\hline${ }^{154} \mathrm{EU}$ & Rad & FS.Ni & $3.79 E+00$ & $\mu \mathrm{Ci} / \mathrm{gd}$ & 2.30 & 2.83 \\
\hline${ }^{155_{\mathrm{EU}}}$ & Rad & FS.Ni & $3.13 \bar{c}+00$ & $\mathrm{HCi} / \mathrm{g} d$ & $0.00 *$ & 9.85 \\
\hline $\mathrm{Fe}$ & ICP/AES & Acid & $1.37 E+05$ & $\mu g / g d$ & 1.05 & 2.13 \\
\hline $\mathrm{Fe}$ & ICP/AES & FS.Ni & $1.41 E+05$ & $\mu g / g d$ & 5.49 & 2.17 \\
\hline $\mathrm{Fe}$ & ICP/AES & FS.Zr & $1.31 E+05$ & $\mu g / 9 d$ & 6.06 & 2.60 \\
\hline $\mathrm{Fe}$ & $I C P / N S$ & Acid & $1.10 E+05$ & $\mathrm{Hg} / \mathrm{g} d$ & $0.00 *$ & 7.95 \\
\hline${ }^{55} \mathrm{Fe}$ & Rad & FS.Ki & $1.53 E-01$ & $\mu \mathrm{Ci} / \mathrm{gd}$ & 3.50 & 4.08 \\
\hline Gd & $I C P / M S$ & Acid & $1.36 E+01$ & $\mu g / g d$ & 3.36 & 7.12 \\
\hline Gd & ICP/MS & FS.Ni & $2.50 E+01$ & $\mu \mathrm{g} / \mathrm{s} d$ & $0.00 \star$ & 27.66 \\
\hline Gd & $1 \mathrm{CP} / \mathrm{MS}$ & FS.Zr & $1.21 E+01$ & $\mu g / g d$ & 29.81 & 7.52 \\
\hline $\mathrm{Hg}$ & CVAA & Direct & $3.43 E+02$ & $\mu g / 9 d$ & $0.00 *$ & 4.76 \\
\hline ho & ICP/MS & Acid & $1.42 E-01$ & $\mu g / g d$ & $0.00 *$ & 26.94 \\
\hline Ho & $I C P / M S$ & FS.Ni & $6.07 \mathrm{E}+00$ & $\mu g / g d$ & 23.64 & 13.89 \\
\hline${ }^{129}{ }_{1 . \mathrm{H}}$ & ICP/MS & Acid & $1.18 E+00$ & $\mu g / g d$ & 16.87 & 13.37 \\
\hline 129 I.W & ICP/MS & Acid & $2.18 E-04$ & $\mu \mathrm{Ci} / \mathrm{g} d$ & 9.72 & 11.37 \\
\hline 129 , wo & $I C P / M S$ & Acid & $1.25 E+00$ & $\mu g / g d$ & 10.75 & 17.33 \\
\hline 129 I. WO & ICP/NS & Acid & $2.37 \mathrm{E} \cdot 04$ & $\mu C i / g d$ & $0.00 *$ & 16.00 \\
\hline$x$ & ICP/AES & FS.2r & $7.59 E+03$ & $\mu g / g d$ & $0.00 *$ & 21.84 \\
\hline
\end{tabular}

a = Table does not include analytes where nore than $50 \%$ of the data are "less than" values.

$s=\mu g / g$ d is $\mu \mathrm{g} / \mathrm{g}$ dried slurry; $\mu \mathrm{Ci} / \mathrm{g} d$ is $\mu \mathrm{Ci} / \mathrm{g}$ dried slurry.

* = A RSO(S) of $0.00 \%$ indicates that the variability between duplicate analytical measurements was greater than the variability between subsamples. 


\section{HNF-SD-WM-DP-225, REV 1}

Table 4.3. Tank 241-C-106 Vendor Product - Variance Components. (page 4 of 9)

\begin{tabular}{|c|c|c|c|c|c|c|}
\hline Analyte a & Method & Digest & Mean & Units $s$. & $\begin{array}{l}\text { Random } \\
\text { Sampl ing } \\
\text { RSD } \%\end{array}$ & $\begin{array}{c}\text { Random } \\
\text { Analytical } \\
\text { RSD } \%\end{array}$ \\
\hline La & ICP/AES & Acid & $9.61 E+01$ & $\mu g / g d$ & $0.00 *$ & 3.36 \\
\hline La & ICP/AES & FS.Ni & $1.19 E+02$ & $\mu g / 9 \mathrm{~d}$ & 4.09 & 6.17 \\
\hline $\mathrm{La}$ & ICP/AES & FS.2r & $1.64 E+02$ & $\mu g / g d$ & $0.00 *$ & 15.87 \\
\hline La & ICP/MS & Acid & $8.50 \mathrm{E}+01$ & $H g / g d$ & 1.04 & 3.84 \\
\hline $\mathrm{La}$ & ICP/MS & FS.Ni & $8.06 E+01$ & $\mu g / g d$ & 9.80 & 12.17 \\
\hline La & ICP/NS & fs. $2 r$ & $9.22 E+01$ & $\mu g / g d$ & 15.30 & 7.95 \\
\hline Li & ICP/AES & Acid & $4.59 \mathrm{E}+01$ & $\mu g / g d$ & 73.60 & 1.67 \\
\hline Li & ICP/AES & FS.Zr & $9.39 E+01$ & $\mu g / g d$ & 22.88 & 16.42 \\
\hline $\mathrm{Mg}$ & ICP/AES & Acid & $7.54 E+02$ & $\mu g / g d$ & 2.03 & 2.39 \\
\hline $\mathrm{Mg}$ & ICP/AES & FS.Ni & $1.36 E+03$ & $\mu g / g d$ & 10.55 & 2.74 \\
\hline $\mathrm{Mg}$ & ICP/AES & FS.Zr & $1.31 E+03$ & $\mu g / 9 d$ & 5.20 & 6.01 \\
\hline $\mathrm{Mg}$ & ICP/MS & Acid & $4.45 \mathrm{E}+02$ & $\mu g / 9 d$ & 2.56 & 3.15 \\
\hline $\mathrm{Mg}$ & ICP/MS & FS.Ni & $1.22 \varepsilon+03$ & $\mu \mathrm{g} / \mathrm{g} d$ & 12.11 & 6.81 \\
\hline $\mathrm{Mg}$ & $1 \mathrm{CP} / \mathrm{MS}$ & FS.Zr & $1.26 E+03$ & $\mu g / 9 d$ & 4.63 & 4.93 \\
\hline $\mathrm{Nn}$ & ICP/AES & Acid & $2.60 E+03$ & $\mu g / 9 d$ & $0.00 *$ & 2.32 \\
\hline Mn & ICP/AES & FS.Ni & $3.14 E+03$ & $\mu g / g d$ & 3.26 & 3.39 \\
\hline $\mathrm{Mn}$ & ICP/AES & $\mathrm{FS} . \mathrm{Zr}$ & $2.59 E+03$ & $\mu g / g d$ & 5.75 & 3.29 \\
\hline Mn & ICP/MS & Acid & $2.04 E+03$ & $\mu g / g d$ & $0.00 *$ & 5.61 \\
\hline$M n$ & ICP/MS & FS.Ni & $2.81 E+03$ & $\mu \mathrm{g} / \mathrm{g} \mathrm{d}$ & 6.02 & 4.97 \\
\hline Mn & ICP/MS & FS.Zr & $2.56 E+03$ & $\mu g / g d$ & 4.26 & 3.13 \\
\hline Ho & ICP/AES & Acid & $2.67 E+01$ & $\mu g / g d$ & 0.31 & 2.16 \\
\hline Mo & ICP/MS & Acid & $9.60 E+00$ & $\mu g / 9 d$ & $0.00 *$ & 6.33 \\
\hline Mo & ICP/MS & FS.Ni & $5.70 \varepsilon+01$ & $\mu g / g d$ & 22.25 & 13.53 \\
\hline Mo & ICP/MS & FS. $2 \mathrm{r}$ & $2.79 \mathrm{E}+01$ & $\mu g / g d$ & 16.78 & 5.53 \\
\hline $\mathrm{NH}_{3}-\mathrm{N}$ & WrChem & Water & $1.75 E+01$ & $\mu g / g d$ & 22.44 & 37.54 \\
\hline $\mathrm{NO}_{2}^{-}$ & 10 & Water & $2.07 E+03$ & $\mu g / 9 d$ & 15.28 & 12.55 \\
\hline $\mathrm{NO}_{3}$ & IC & Water & $8.86 E+02$ & $\mu g / g d$ & 52.06 & 13.50 \\
\hline $\mathrm{Na}$ & ICP/AES & Acid & $1.62 E+05$ & $\mu g / g d$ & 4.78 & 2.44 \\
\hline $\mathrm{Na}$ & ICP/AES & FS.Ni & $1.42 E+05$ & $\mu g / g d$ & 6.45 & 2.63 \\
\hline $\mathrm{Na}$ & $1 \mathrm{CP} / \mathrm{KS}$ & Acid & $1.82 E+05$ & $\mu g / g$ d & $0.00 *$ & 14.96 \\
\hline $93 \mathrm{~m}_{\mathrm{Nb}}$ & Rad & FS.Ni & $2.37 E-01$ & $\mu[\mathrm{i} / \mathrm{g} d$ & 37.35 & 51.76 \\
\hline
\end{tabular}

a = Table does not include analytes where more than $50 \%$ of the data are "less than" values.

$\$=\mu g / g$ d is $\mu g / g$ dried slurry; $\mu \mathrm{Ci} / \mathrm{g} d \mathrm{is} \mu \mathrm{Ci} / \mathrm{g}$ dried slurry.

* A RSD(S) of $0.00 \%$ indicates that the variability between duplicate analytical measurements was greater than the variability between subsamples. 
Table 4.3. Tank 24l-C-105 Vendor Product - Variance Components. (page 5 of 9)

\begin{tabular}{|c|c|c|c|c|c|c|}
\hline Analyte a & Method & Digest & Mean & Units $s$ & $\begin{array}{l}\text { Random } \\
\text { Sampling } \\
\text { RSD \% }\end{array}$ & $\begin{array}{l}\text { Random } \\
\text { Analytical } \\
\text { RSD \% }\end{array}$ \\
\hline NO & ICP/AES & Acid & $2.82 E+02$ & $\mu g / g d$ & $0.00 *$ & 2.92 \\
\hline Nd & ICP/AES & FS.Hi & $3.79 E+02$ & $\mu g / g d$ & 7.40 & 4.99 \\
\hline Nd & ICP/AES & FS.Zr & $5.17 E+02$ & $\mu g / g d$ & $0.00 *$ & 11.49 \\
\hline Nd & ICP/MS & Acid & $1.93 E+02$ & $\mu \mathrm{g} / \mathrm{g} d$ & 1.20 & 1.96 \\
\hline Nd & ICP/MS & FS.Ni & $2.33 E+02$ & $\mu g / g d$ & 11.67 & 10.56 \\
\hline Nd & ICP/MS & FS.zr & $2.24 E+02$ & $\mu g / g d$ & $0.00 *$ & 9.77 \\
\hline $\mathrm{Ni}$ & ICP/AES & Acid & $1.13 E+03$ & $\mu g / g d$ & $0.00 *$ & 2.70 \\
\hline $\mathrm{Ni}$ & ICP/AES & $\mathrm{Fs.2r}$ & $1.65 \mathrm{E}+03$ & $\mu g / 9 d$ & 7.51 & 3.22 \\
\hline $\mathrm{Ni}$ & ICP/MS & Acid & $9.49 E+02$ & $\mu \mathrm{g} / \mathrm{g} \mathrm{d}$ & $0.00 *$ & 5.94 \\
\hline $\mathrm{Ni}$ & ICP/MS & FS.zr & $1.61 E+03$ & $\mu \mathrm{g} / \mathrm{g} d$ & 3.89 & 1.71 \\
\hline $59_{\mathrm{Ni} . \mathrm{K}}$ & Rad & FS.zr & $2.20 E-02$ & $\mu \mathrm{Ci} / \mathrm{gd}$ & $0.00 *$ & 25.07 \\
\hline${ }^{59} \mathrm{Ni}$.wo & Rad & Es.2r & $1.97 \mathrm{E}-02$ & $\mu \mathrm{Ci} / \mathrm{g} d$ & 18.21 & 18.37 \\
\hline${ }^{63} \mathrm{Ni}$ & Rad & Fs.2r & $2.40 E+00$ & $\mu C i / g d$ & $0.00 *$ & 5.85 \\
\hline $237_{\mathrm{Np}}$ & ICP/MS & Acid & $4.48 E+00$ & $\mu \mathrm{g} / \mathrm{g} \mathrm{d}$ & $0.00 *$ & 4.17 \\
\hline $237_{\mathrm{Np}}$ & $1 \mathrm{CP} / \mathrm{MS}$ & Acid & $3.16 E-03$ & $\mu \mathrm{Ci} / \mathrm{gd}$ & $0.00 *$ & 4.16 \\
\hline $237_{\mathrm{Np}}$ & ICP/KS & FS.Zr & $5.57 E+00$ & $\mu g / g d$ & 10.77 & 17.57 \\
\hline $237_{\mathrm{NP}}$ & ICP/MS & fS.Zr & $3.92 \mathrm{E}-03$ & $\mathrm{HCi} / \mathrm{go}$ & 10.75 & 17.58 \\
\hline $\mathbf{P}$ & ICP/AES & Acid & $1.34 E+03$ & $\mu g / g d$ & 4.76 & 2.53 \\
\hline $\mathbf{P}$ & ICP/AES & FS.Ni & $3.33 E+03$ & $\mu g / s d$ & $0.00 *$ & 11.23 \\
\hline $\mathbf{P}$ & ICP/AES & Es. $2 r$ & $3.84 E+03$ & $\mu g / g d$ & $17: 41$ & 8.18 \\
\hline $\mathrm{PO}_{4}^{3-}$ & IC & Water. & $1.60 \mathrm{E}+02$ & $\mu g / g d$ & $0.00 *$ & 29.89 \\
\hline $\mathrm{Pb}$ & ICP/AES & Acid & $3.43 E+03$ & $\mu g / g d$ & 1.35 & 1.94 \\
\hline $\mathrm{Pb}$ & ICP/AES & FS.Ni & $2.95 E+03$ & $\mu g / 90$ & 10.93 & 14.05 \\
\hline $\mathrm{Pb}$ & ICP/AES & FS.2r & $3.53 \mathrm{E}+03$ & $\mu g / g d$ & 5.56 & 3.36 \\
\hline $\mathrm{Pb}$ & ICP/MS & Acid & $2.85 \mathrm{E}+03$ & $\mu g / g d$ & 4.91 & 5.70 \\
\hline $\mathrm{Pb}$ & ICP/MS & FS.Zr & $2.55 \varepsilon+03$ & $\mathrm{Hg} / \mathrm{g} \mathrm{d}$ & 2.53 & 2.96 \\
\hline Pd & ICP/MS & Acid & $9.99 \mathrm{E}+00$ & $\mathrm{Hg} / \mathrm{g} \mathrm{d}$ & $0.00 *$ & 6.59 \\
\hline Pd & ICP/MS & FS.Ni & $2.35 \mathrm{E}+01$ & $\mu g / g$ d & $0.00 *$ & 55.72 \\
\hline Pd & ICP/MS & Fs.zr & $2.74 E+01$ & $\mu \mathrm{g} / \mathrm{g} d$ & $0.00 *$ & 18.69 \\
\hline${ }^{107_{\text {Pd.W }}}$ & ICP/NS & Acid & $1.35 E+00$ & $\mu 9 / 9 d$ & 62.34 & 121.00 \\
\hline $107_{\text {Pd.w }}$ & ICP/MS & Acid & $7.18 E-04$ & $\mu \mathrm{Ci} / \mathrm{s} \mathrm{d}$ & 55.86 & 118.78 \\
\hline${ }^{107}$ Pd. wo & ICP/KS & Acid & $2.20 E+00$ & $\mu \mathrm{g} / \mathrm{gd}$ & $0.00 \div$ & 110.60 \\
\hline $107_{\text {Pd.WO }}$ & ICP/KS & Acid & $1.14 E-03$ & $\mu \mathrm{Ci} / \mathrm{g} \mathrm{d}$ & $0.00 *$ & 111.87 \\
\hline
\end{tabular}

a = Table does not inclute analytes where more than $50 \%$ of the cata are "less than" values.

$S=\mu \mathrm{g} / \mathrm{g} d$ is $\mu \mathrm{g} / \mathrm{g}$ dried slurry; $\mu \mathrm{Ci} / \mathrm{g} \mathrm{d}$ is $\mu \mathrm{Ci} / \mathrm{g} \mathrm{dried}$ slurry.

* = A RSD(S) of $0.00 \%$ indicates that the variability between duplicate analyticat measurements was greater than the variability between subsamples. 
Table 4.3. Tank 241-C-106 Vendor Product - Variance Components. (page 6 of 9)

\begin{tabular}{|c|c|c|c|c|c|c|}
\hline Analyte a & Method & Digest & $\begin{array}{c}\text { Hean } \\
. \\
\end{array}$ & Units $\$$ & $\begin{array}{c}\text { Randon } \\
\text { Sampl ing } \\
\text { RSD \% }\end{array}$ & $\begin{array}{c}\text { Random } \\
\text { Analytical } \\
\text { RSO \% }\end{array}$ \\
\hline $\mathrm{Pr}$ & ICP/MS & Acid & $7.05 E+09$ & $\mu \mathrm{g} / \mathrm{g} d$ & 1.49 & 2.47 \\
\hline $\mathrm{Pr}$ & ICP/MS & FS.Ni & $7.71 \mathrm{E}+01$ & $\mu g / g d$ & 0.00 & 13.77 \\
\hline $\mathrm{Pr}$ & $I C P / M S$ & FS.ZF & $8.41 \mathrm{E}+01$ & $\mu g / g d$ & 6.04 & 3.22 \\
\hline $\mathrm{Pt}$ & ICP/MS & Acid & $2.33 E-01$ & $\mu g / g d$ & $0.00 *$ & 44.26 \\
\hline $\mathrm{Pt}$ & IEP/NS & FS.Ni & $3.07 E+01$ & $\mu g / g d$ & 27.60 & 4.32 \\
\hline $\mathrm{Pt}$ & ICP/MS & $\mathrm{FS.2 \textrm {r }}$ & $5.19 E+00$ & $\mu g / g d$ & 7.14 & 31.35 \\
\hline $238_{\mathrm{Pu}}$ & $\operatorname{Rad}$ & FS.Ni & $6.31 E-01$ & $\mu \mathrm{Ci} / 9 d$ & 3.38 & 3.54 \\
\hline${ }^{239} \mathrm{Pu}$ & ICP/MS & Acid & $7.34 E+00$ & $\mu g / g$ d & 5.52 & 0.82 \\
\hline${ }^{239} \mathrm{Pu}$ & ICP/MS & Acid & $4.55 \mathrm{E}-01$ & $\mu \mathrm{Ci} / \mathrm{g} d$ & 5.53 & 0.85 \\
\hline $239 \mathrm{Pu}$ & ICP/MS & FS.2r & $2.46 \mathrm{E}+01$ & Fg/g d & 4.29 & 5.82 \\
\hline${ }^{239} \mathrm{Pu}$ & $1 \mathrm{CP} / \mathrm{MS}$ & FS.Zr & $1.52 E+00$ & $\mu \mathrm{Ci} / \mathrm{g} d$ & 4.24 & 5.71 \\
\hline $239 / 240_{\mathrm{Pu}}$ & Rad & FS.Ni & $2.99 E+00$ & $\mu[i / g d$ & 3.34 & 4.27 \\
\hline $240 \mathrm{Pu}$ & $1 \mathrm{CP} / \mathrm{MS}$ & Acid & $5.58=-01$ & $\mu g / g d$ & 6.05 & 5.62 \\
\hline${ }^{240} \mathrm{Pu}$ & ICP/MS & Acid & $1.25 \mathrm{E} \cdot 01$ & $\mu \mathrm{Ci} / \mathrm{g} \mathrm{d}$ & 6.93 & 5.66 \\
\hline $241_{\mathrm{Pu}}$ & Rad & FS.Ni & $6.96 E+00$ & $\mu \mathrm{Ci} / \mathrm{g} d$ & $0.00 *$ & 5.95 \\
\hline $\mathrm{Rb}$ & ICP/MS & Acid & $1.11 E+00$ & $\mu g / g d$ & 6.44 & 7.58 \\
\hline $\mathrm{Rb}$ & ICP/MS & FS.Zr & $7.68 E+00$ & $k g / 9 \mathrm{~d}$ & 22.02 & 32.51 \\
\hline $\mathrm{Re}$ & $1 \mathrm{CP} / \mathrm{MS}$ & Acid & $7.83 E-02$ & $\mu g / g d$ & $0.00 *$ & 42.28 \\
\hline $\mathrm{Re}$ & ICP/MS & FS.Ni & $6.61 E+00$ & $\mu g / g d$ & 20.88 & 24.86 \\
\hline $\mathbf{R h}$ & ICP/MS & Acid & $1.05 E+02$ & $\mu g / 9 \mathrm{~d}$ & 2.87 & 2.44 \\
\hline Rh & ICP/MS & FS.Zr & $1.44 E+02$ & $\mu g / 9 d$ & 6.78 & 9.25 \\
\hline Rh.w & ICP/MS & FS.Ni & $1.90 E+01$ & $\mu g / g d$ & 93.76 & 149.79 \\
\hline Rh.wo & ICP/MS & FS.Ni & $3.70 E+01$ & $H 9 / 9 d$ & $0.00 *$ & 111.03 \\
\hline $\mathrm{Ru}$ & ICP/MS & FS.Ni & $9.57 E+02$ & $\mu g / g d$ & $6.72 \#$ & NA \\
\hline $\mathrm{SO}_{4}^{2-}$ & JC & Hater & $4.70 E+02$ & $\mu 8 / 9 d$ & 12.09 & 13.34 \\
\hline Sb.w & ICP/MS & Acid & $3.77 \mathrm{E}-01$ & $\mu g / g d$ & 31.49 & 108.41 \\
\hline Sb.Ho & ICP/MS & Acid & $7.88 \varepsilon-01$ & $\quad k g / 9 d$ & 32.37 & 17.96 \\
\hline si & ICP/AES & Acid & $2.80 E+03$ & $\mu g / g d$ & $0.00 *$ & 15.17 \\
\hline si & ICP/AES & FS.Ni & $6.89 E+04$ & $\mu g / g d$ & 6.63 & 1.92 \\
\hline $5 i$ & ICP/AES & FS.2r & $6.46 E+04$ & $\mu g / g d$ & 6.11 & 2.67 \\
\hline${ }^{151} \mathrm{Sm} /{ }^{147} \mathrm{Pm}$ & $\operatorname{Rad}$ & FS.Ni & $9.23 E+01$ & $\mu \mathrm{Ci} / \mathrm{gd}$ & 4.07 & 3.89 \\
\hline
\end{tabular}

$a=$ Table does not include analytes where more than $50 \%$ of the data are "less than" values.

$\$=\mu g / g$ d is $\mu \mathrm{g} / \mathrm{g}$ dried slurry; $\mu \mathrm{Ci} / \mathrm{g}$ d is $\mu \mathrm{Ci} / \mathrm{g}$ dried slurry.

* = ARSO(S) of $0.00 \%$ indicates that the variability between duplicate analytical measurements was greater than the variability between subsimples.

$\omega$ = Duplicate measurements were not available, the sampling and analytical variability cannot be separated from each other. 
Table 4.3. Tank 241-C-106 Vendor Product - Variance Components. (page 7 of 9 )

\begin{tabular}{|c|c|c|c|c|c|c|}
\hline Analyte a & Method & Digest & Mean & Units $\mathbf{s}$ & $\begin{array}{l}\text { Random } \\
\text { Sampl ing } \\
\text { RSD \% }\end{array}$ & $\begin{array}{c}\text { Random } \\
\text { Analytical } \\
\text { RSO } x\end{array}$ \\
\hline $\mathrm{Sn}$ & ICP/KS & Acid & $3.48 E+01$ & $\mu g / g d$ & 2.67 & 3.43 \\
\hline Sn & ICP/MS & FS.Ni & $2.93 E+01$ & $\mu g / g d$ & 32.13 & 93.02 \\
\hline Sn & $1 \mathrm{CP} / \mathrm{MS}$ & FS.Zr & $1.02 E+02$ & $\mu g / g d$ & 23.42 & 19.26 \\
\hline $129 \mathrm{~m}_{\mathrm{sn}}$ & Rad & FS.Ni & $1.23 \bar{E}+00$ & $\mu \mathrm{Ci} / \mathrm{g} d$ & 39.45 & 24.26 \\
\hline${ }^{126} \mathrm{sn} /{ }^{126 m_{s b}}{ }^{126} \mathrm{sb}$ & Rad & FS.Ni & $1.15 \mathrm{E}-02$ & $\mu[i / g d$ & 23.91 & 17.29 \\
\hline $\mathrm{Sr}$ & ICP/AES & Acid & $5.38 E+01$ & $\mu g / g d$ & $0.00 \pm$ & 2.43 \\
\hline $\mathrm{Sr}$ & ICP/AES & FS.Ni & $7.91 E+01$ & $\mu g / 9 d$ & 7.25 & 1.51 \\
\hline $\mathrm{Sr}$ & ICP/AES & FS.Zr & $1.45 E+02$ & $\mu g / g d$ & $0.00 *$ & 8.55 \\
\hline $\mathrm{Sr}$ & ICP/NS & Acid & $4.51 E+01$ & $\mu g / g d$ & $0.00 *$ & 1.98 \\
\hline $\mathrm{Sr}$ & ICP/MS & FS.Ni & $5.88 \varepsilon+01$ & $\mu g / g \&$ & 27.11 & 6.32 \\
\hline $\mathrm{Sr}$ & ICP/MS & $\mathrm{FS} .2 \mathrm{r}$ & $1.50 \mp+02$ & $\mu g / g d$ & $0.00 *$ & 8.69 \\
\hline${ }^{90} \operatorname{sir} / Y$ & Rad & FS.Ni & $7.30 E+02$ & $\mu \mathrm{Ci} / \mathrm{g} d$ & $0.00 *$ & 6.01 \\
\hline TIC & Wtchen & Direct & $5.30 E+03$ & $\mathrm{ugC} / 9 \mathrm{~g}$ & $0.00 *$ & 10.69 \\
\hline TOC & Wtchen & Direct & $4.795+04$ & $u g c / g d$ & 3.82 & 6.03 \\
\hline $\mathrm{Ta}$ & ICP/MS & Acid & $1.04 E+01$ & $\mu g / g \circ$ & 6.97 & 26.76 \\
\hline $\mathrm{Ta}$ & ICP/MS & FS.Ni & $2.13 E+01$ & $p g / 9$ d & 40.47 & 29.40 \\
\hline Ta & ICP/MS & FS.2r & $2.86 E+01$ & $\mu g / g d$ & 38.93 & 7.19 \\
\hline Ib & ICP/MS & Acid & $1.46 E+00$ & $\mu g / g d$ & $0.00 *$ & 6.17 \\
\hline $\mathrm{Tb}$ & ICP/MS & FS.Ni & $8.205+00$ & $\mu \mathrm{g} / \mathrm{g} d$ & 15.95 & 12.51 \\
\hline Tb & ICP/MS & $\mathrm{FS.2r}$ & $2.34 E+00$ & $\mu g / g d$ & 26.28 & 23.74 \\
\hline${ }^{99} \mathrm{TC}$ & ICP/MS & Acid & $3.30 E-01$ & $\mu g / g d$ & 18.26 & 6.06 \\
\hline${ }^{99} \mathrm{TC}$ & ICP/MS & Acid & $5.60 E-03$ & $\mu c i / g d$ & 18.24 & 6.04 \\
\hline Te & ICP/AES & Acid & $5.23 E+02$ & $\mu g / g d$ & 0.84 & 2.22 \\
\hline Te & ICP/MS & Acid & $2.03 E+02$ & $\mu g / g d$ & 0.00 & 3.86 \\
\hline Te & ICP/NS & FS.Ni & $4.24 E+02$ & $\mu g / g d$ & 8.08 & 18.74 \\
\hline te & ICP/MS & FS.Zr & $2.88 E+02$ & $\mu s / g d$ & 21.27 & 28.70 \\
\hline Th & ICP/AES & Acid & $3.49 E+02$ & $\mu g / g d$ & 0.00 & 1.34 \\
\hline $232^{7 h}$ & ICP/MS & Acid & $1.85 \mathrm{E}+02$ & $\mu g / g d$ & $0.00 \star$ & 3.02 \\
\hline $232 \mathrm{Th}$ & ICP/MS & Acid & $2.03 E-05$ & $\mu \mathrm{Ci} / \mathrm{g} d$ & $0.00 *$ & 2.95 \\
\hline 232 Th & ICP/NS & FS.Zr & $4.99 E+02$ & $\mu g / g d$ & 34.61 & 10.61 \\
\hline${ }^{232} \mathrm{Th}$ & ICP/NS & $\mathrm{FS} . \mathrm{Zr}$ & $5.48 E-05$ & $\mu \mathrm{Ci} / \mathrm{g} d$ & 34.61 & 10.61 \\
\hline
\end{tabular}

$a=$ Table does not include analytes where more than $50 \%$ of the data are "less than" vatues.

$\$=\mu g / g d$ is $\mu \mathrm{g} / \mathrm{g}$ dried slurry; $\mu \mathrm{Ci} / \mathrm{g} d$ is $\mu \mathrm{Ci} / \mathrm{g}$ dried slurry.

* A RSD (S) of $0.00 \%$ indicates that the variability between duplicate analytical measurements was greater than the variability between subsemples. 
HNF-SD-WM-DP-225, REV. 1

Table 4.3. Tank 241-C-106 Vendor Product - Variance Components. (page 8 of 9)

\begin{tabular}{|c|c|c|c|c|c|c|}
\hline Analyte a & Method & oigest & Mean & Units $\$$ & $\begin{array}{l}\text { Random } \\
\text { Sampl ing } \\
\text { RSD } \mathbf{x}\end{array}$ & $\begin{array}{c}\text { Random } \\
\text { Analytical } \\
\text { RSD } \%\end{array}$ \\
\hline$T i$ & ICP/AES & Acid & $3.50 E+02$ & $\mu g / g d$ & $0.00 *$ & 3.14 \\
\hline $\mathbf{T i}$ & ICP/AES & $\mathrm{FS.Ni}$ & $1.03 E+03$ & $\mu g / g d$ & 7.96 & 2.85 \\
\hline $\mathbf{T i}$ & ICP/AES & $\mathrm{FS} .2 \mathrm{r}$ & $1.02 E+03$ & $\mu g / g d$ & 10.38 & 3.13 \\
\hline $\mathbf{I} \mathbf{i}$ & ICP/MS & Acid & $3.58 E+02$ & $\mu g / g d$ & $0.00 *$ & 3.25 \\
\hline $\mathbf{T i}$ & I CP/MS & FS.Ni & $1.12 E+03$ & $\mu g / g d$ & 11.76 & 2.51 \\
\hline $\mathbf{T i}$ & ICP/MS & FS.2r & $1.31 E+03$ & $\mu \mathrm{g} / \mathrm{g} \mathrm{d}$ & 12.01 & 3.26 \\
\hline $\mathrm{Im}$ & ICP/MS & Acid & $6.17 E-02$ & $\mu g / g d$ & $0.00 *$ & 31.47 \\
\hline Im.w & ICP/MS & FS.Ni & $1.82 E+00$ & $\mu g / g \mathrm{~d}$ & 62.86 & 38.99 \\
\hline Tm.wo & ICP/MS & $\mathrm{FS.Ni}$ & $2.52 E+00$ & $\mu g / g d$ & 31.98 & 47.42 \\
\hline Total Alpha & $\mathrm{Rad}$ & FS.Ni & $6.78 E+00$ & $\mu \mathrm{Ci} / \mathrm{gd}$ & 2.19 & 2.64 \\
\hline Total Beta & $\operatorname{Rad}$ & FS.Ni & $2.05 E+03$ & $\mu \mathrm{Ci} / \mathrm{g} d$ & 3.80 & 4.75 \\
\hline Total Carbon & Wtchem & Direct & $5.22 E+04$ & $\mathrm{ugC} / \mathrm{g} \mathrm{d}$ & 5.23 & 6.44 \\
\hline Total U & $I \mathrm{CP} / \mathrm{MS}$ & Acid & $1.91 E+03$ & $\mu g / g d$ & 2.47 & 3.07 \\
\hline Total U & ICP/MS & FS.Ni & $1.905+03$ & $\mu g / g d$ & $0.00 \star$ & 6.96 \\
\hline Total U & $1 C P / H S$ & $\mathrm{FS.2r}$ & $2.01 E+03$ & $\mu g / g d$ & $0.00 *$ & 3.29 \\
\hline $\mathrm{u}$ & ICP/AES & Acid & $1.95 E+03$ & $\mu g / g d$ & $0.00 *$ & 1.90 \\
\hline $233_{U}$ & ICP/MS & Acid & $1.73 E-01$ & $\mu g / g d$ & 18.32 & 9.99 \\
\hline $233 \mathrm{u}$ & ICP/MS & Acid & $1.68 \mathrm{E}-03$ & $\mu C i / g d$ & 18.24 & 11.69 \\
\hline $234 \mathrm{v}$ & $I C P / M S$ & Acid & $1.23 E \cdot 01$ & $\mu g / g d$ & $0.00 \star$ & 19.64 \\
\hline $234 \mathrm{U}$ & ICP/MS & Acid & 7.73E-04 & $\mu C i / s d$ & $0.00 *$ & 18.15 \\
\hline $235 u$ & ICP/MS & Acid & $1.30 E+01$ & $\mu g / g d$ & 3.48 & 4.98 \\
\hline $235_{v}$ & ICP/MS & Acid & $2.80 E+05$ & $\mathrm{HCi} / \mathrm{gd}$ & 3.48 & 4.98 \\
\hline $236 \mathrm{u}$ & ICP/MS & Acid & $2.87 E-01$ & $\mu g / g d$ & 9.82 & 11.21 \\
\hline $236_{\mathrm{U}}$ & ICP /MS & Acid & $1.85 E-05$ & $\mu \mathrm{Ci} / \mathrm{g} d$ & 9.18 & 11.61 \\
\hline $238_{u}$ & ICP/MS & Acid & $1.90 E+03$ & $\mu g / g d$ & 2.48 & 3.08 \\
\hline $238 \mathrm{u}$ & ICP/MS & Acid & $6.37 E-04$ & $\mu \mathrm{Ci} / \mathrm{g} d$ & 2.48 & 3.08 \\
\hline $238 \mathrm{v}$ & ICP/MS & $\mathrm{FS.Ni}$ & $1.89 E+03^{\circ}$ & $H g / g d$ & $0.00 *$ & 7.00 \\
\hline $238_{u}$ & $1 \mathrm{CP} / \mathrm{MS}$ & FS.Ni & $6.34 \mathrm{E}-04$ & $\mu \mathrm{Ci} / \mathrm{g} d$ & $0.00 *$ & 7.00 \\
\hline $238_{U}$ & ICP/MS & FS.2r & $2.00 E+03$ & $\mu g / 9 \mathrm{~d}$ & $0.00 *$ & 3.14 \\
\hline${ }^{238} \mathrm{U}$ & ICP/MS & FS.Zr & $6.71 E-04$ & $\mu \mathrm{Ci} / \mathrm{g} d$ & $0.00 \star$ & 3.14 \\
\hline$v$ & $I C P / N S$ & Acid & $3.73 E+01$ & $\mu g / g d$ & 9.64 & 16.01 \\
\hline
\end{tabular}

$\mathrm{a}=$ Table does not include analytes where more than $50 \%$ of the data are "less than" values.

$\$=\mu g / g$ d is $\mu g / g$ dried slurry; $\mu \mathrm{Ci} / \mathrm{g} d$ is $\mu \mathrm{Ci} / \mathrm{g}$ dried slurry.

* = A RSO(S) of $0.00 \%$ indicates that the veriability between duplicate analytical measurements was greater then the variability between subsamples. 


\section{HNF-SD-WM-DP-225, REV. 1}

Table 4.3. Tank 241-C-106 Vendor Product - Variance Components. (page 9 of 9)

\begin{tabular}{|c|c|c|c|c|c|c|}
\hline Analyte a & Method & Digest & Mean & Units $\$$ & $\begin{array}{c}\text { Random } \\
\text { Sampling } \\
\text { RSD \% }\end{array}$ & $\begin{array}{c}\text { Random } \\
\text { Analytical } \\
\text { RSo \% }\end{array}$ \\
\hline$W$ & ICP/MS & Acid & $2.22 E+02$ & $\mu g / g d$ & 6.91 & 2.13 \\
\hline$H$ & . $1 \mathrm{CP} / \mathrm{MS}$ & FS. $\mathrm{Ni}$ & $4.99 \mathrm{E}+02$ & $\mu g / g d$ & $0.00-$ & 14.83 \\
\hline $\mathrm{H} \cdot$ & ICP/MS & FS.Zr & $3.57 E+02$ & $\mu g / g d$ & 33.21 & 10.16 \\
\hline Yb & ICP/MS & Acid & $2.50 \mathrm{E}-01$ & $\mu g / g d$ & 34.64 & 28.28 \\
\hline Yb & ICP/MS & FS.Ni & $1.26 E+01$ & $\mu g / 9 d$ & 46.77 & 26.45 \\
\hline Yb & ICP/MS & FS.ZF & $3.24 E+00$ & $\mu g / 9 d$ & $0.00 *$ & 45.60 \\
\hline $\mathrm{Zn}$ & ICP/AES & Acid & $1.16 \mathrm{E}+02$ & $\mu g / g d$ & 1.75 & 1.93 \\
\hline $2 n$ & ICP/AĖS & FS.Ni & $1.83 E+02$ & $\mu g / g d$ & 0.00 & 13.40 \\
\hline $2 n$ & ICP/AES & Fs.2r & $1.61 E+02$ & $\mu g / g d$ & $0.00 \star$ & 8.52 \\
\hline $\mathrm{Zn}$ & $1 \mathrm{CP} / \mathrm{MS}$ & Acid & $9.92 E+01$ & $\mu g / g d$ & 3.25 & $\quad 6.12$ \\
\hline $2 n$ & ICP/MS & FS.Ni & $3.07 E+02$ & $\mu g / 9 d$ & $0.00 \star$ & 23.10 \\
\hline $\mathrm{Zn}$ & ICP/MS & FS.Zr & $2.34 E+02$ & $\mu g / g d$ & $18.0 \%$ & 9.71 \\
\hline $2 r$ & ICP/AES & Acid & $6.61 E+02$ & $\mu g / g d$ & 32.55 & 5.44 \\
\hline $2 r$ & ICP/AES & $\mathrm{FS}, \mathrm{Ni}$ & $1.11 E+03$ & $\mu g / g d$ & $0.00 *$ & 47.71 \\
\hline $2 r$ & ICP/MS & Acid & $2.56 E+02$ & $\mu g / 9 d$ & 28.54 & 6.30 \\
\hline $2 r$ & ICP/MS & FS.Ni & $4.33 E+02$ & $\mu g / g d$ & $0.00 \star$ & 53.11 \\
\hline$\pi / 2241$ & ICP/MS & Acid & 7.73E-01 & $\mu g / g d$ & 4.21 & 7.87 \\
\hline $\mathrm{m} / 2249$ & ICP/MS & FS.2r & $1.65 E+00^{\circ}$ & $\mu g / g d$ & $0.00=$ & 20.94 \\
\hline $\mathrm{m} / 293$ & $1 \mathrm{CP} / \mathrm{MS}$ & Acid & $5.00 E+01$ & $\mu g / g d$ & 27.31 & 4.67 \\
\hline $\mathrm{m} / \mathrm{z} 93$ & ICP/MS & FS.Ni & $3.38 E+01$ & $\mu g / 9 d$ & $0.00 *$ & 77.10 \\
\hline$\pi / 293$ & $1 \mathrm{CP} / \mathrm{MS}$ & FS.Zr & $2.35 E+02$ & $\mu g / g d$ & 37.31 & 8.31 \\
\hline
\end{tabular}

a = Table does not include analytes where more than $50 \%$ of the data are "less than" yalues.

$s=\mu g / g$ d is $\mu g / g$ dried slurry; $\mu \mathrm{Ci} / \mathrm{g}$ d is $\mu \mathrm{Ci} / \mathrm{g}$ dried slurry.

* = A RSD(S) of $0.00 \%$ indicates that the variability between duplicate analytical measurements was greater than the variability between subsamples. 
Table 4.4. Tank 241-C-106 Vendor Product Analytes with Sampling Variability $>20 \%$ and Analytical Variability $>20 \%$. (page 1 of 2)

\begin{tabular}{|c|c|}
\hline Sample Variability $>20 \%$ & Analytical Variability $>20 \%$ \\
\hline As (ICP/MS.FS.Zr) & $\mathrm{Ag}$ (ICP/AES.FS.Ni) \\
\hline B (ICP/AES.Acid) & $\mathrm{Ag}$ (ICP/AES.FS.Zr) \\
\hline$B(I C P / A E S . F S . N i)$ & ${ }^{241}$ Am.gea (Rad.FS.Ni) * \\
\hline B (ICP/MS.Acid) & $B($ ICP/AES.FS.Ni) * \\
\hline B (ICP/MS.FS.Ni) & $B(I C P / M S . F S . Z r) *$ \\
\hline 8 (ICP/MS.FS.Zr) & $\mathrm{Cl}^{-}$(IC.Water)* \\
\hline${ }^{243 / 244} \mathrm{Cm}$ (Rad.FS.Ni) & ${ }^{133} \mathrm{CS}$ (ICP/MS.FS.Ni) * \\
\hline${ }^{14} \mathrm{C}$ (Rad.FS.Ni) & ${ }^{133}$ CS (ICP/MS.FS.Zr) * \\
\hline CU.W (ICP/MS.FS.Ni) & CU.W (ICP/MS.FS.Ni)* \\
\hline CU.Wo (ICP/MS.FS.Ni) & ${ }^{14} \mathrm{C}$ (Rad.FS.Ni) * \\
\hline Dy (ICP/MS.FS.Zr) & Dy (ICP/MS.FS.Ni) * \\
\hline Gd (ICP/MS.FS.Zr) & $\operatorname{Er}($ ICP/MS.FS.Ni) * \\
\hline Ho (ICP/MS.FS.Ni) & Eu (ICP/MS.FS.Ni) * \\
\hline Li (ICP/AES:Acid) & Gd (ICP/MS.FS.Ni) \\
\hline $\mathrm{Li}(\mathrm{ICP} / \mathrm{AES} . \mathrm{FS} . \mathrm{Zr}$ ) & Ho (ICP/MS.Acid) * \\
\hline Mo (ICP/MS.FS.Ni) & $K($ ICP/AES.FS.Zr $) *$ \\
\hline $\mathrm{NH}_{3}-\mathrm{N}$ (WtChem. Water) & $\mathrm{NH}_{3}-\mathrm{N}$ (WtChem. Water) * \\
\hline $\mathrm{NO}_{3}^{-}$(IC.Water) & ${ }^{93 \mathrm{~m}} \mathrm{Nb}$ (Rad.FS.Ni) * \\
\hline 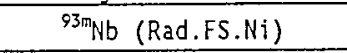 & ${ }^{59} \mathrm{Ni} . \mathrm{W}$ (Rad.FS.Zr) * \\
\hline${ }^{107} \mathrm{Pd} . \mathrm{W}$ (ICP/MS.ACid) & $\mathrm{PO}_{4}^{3-}$ (IC.Water) * \\
\hline Pt (ICP/MS.FS.Ni) & $\mathrm{Pd}$ (ICP/MS.FS.Ni) * \\
\hline $\mathrm{Rb}$ (ICP/MS.FS.Zr) & ${ }^{107} \mathrm{Pd} . \mathrm{w}$ (ICP/MS.Acid) * \\
\hline $\operatorname{Re}(I C P / M S . F S . N i)$ & ${ }^{107} \mathrm{Pd}$.wo (ICP/MS.Acid) * \\
\hline Rh.w (ICP/MS.FS.Ni) & Pt (ICP/MS.Acid) * \\
\hline Sb.W (ICP/MS.Acid) & Pt (ICP/MS.FS.Zr) * \\
\hline \multirow[t]{3}{*}{ Sb.wo (ICP/MS.Acid) } & $\mathrm{Rb}$ (ICP/MS.FS.Zr) * \\
\hline & $\operatorname{Re}($ ICP/MS.Acid) * \\
\hline & $\cdot \operatorname{Re}($ ICP/MS.FS.Ni) * \\
\hline
\end{tabular}

* = Large anaiytical variability is due to the results being close to the detection limits. 
HNF-SD-WM-DP-225, REV: 1

Table 4.4. Tank 241-C-106 Vendor Product Analytes with Sampling Variability $>20 \%$ and Analytical Variability $>20 \%$. (page 2 of 2 )

\begin{tabular}{|c|c|}
\hline Sample Variability > $20 \%$. & Analytical Variability $>20 \%$ \\
\hline Sn (ICP/MS.FS.Ni) & Rh.w (ICP/MS.FS.Ni) \\
\hline Sn (ICP/MS.FS.Zr) & Rh.wo (ICP/MS.FS.Ni) \\
\hline${ }^{121 m} \mathrm{Sn}$ (Rad.FS.Ni) & Sb.w (ICP/MS.ACid) * \\
\hline${ }^{126} \mathrm{Sn} /{ }^{126 \mathrm{~m}} \mathrm{Sb} /{ }^{126} \mathrm{Sb}$ (Rad.FS.Ni) & Sn (ICP/MS.FS.Ni) \\
\hline Sr (ICP/MS.FS.Ni) & ${ }^{12 \mathrm{~m}} \mathrm{Sn}$ (Rad.FS.Ni) * \\
\hline $\mathrm{Ta}$ (ICP/MS.FS.Ni) & Ta (ICP/MS.Acid) \\
\hline Ta (ICP/MS.FS.Zr) & Ta (ICP/MS.FS.Ni) \\
\hline Tb (ICP/MS.FS.Zr) & Tb (ICP/MS.FS.Zr) * \\
\hline Te (ICP/MS.FS.Zr) & Te (ICP/MS/FS.Zr) \\
\hline${ }^{232}$ Th (ICP/MS.FS.Zr) & $\operatorname{Tm}($ ICP/MS.ACid) * \\
\hline TM.W (ICP/MS.FS.Ni) & Tm.w (ICP/MS.FS.Ni) * \\
\hline Tm.wo (ICP/MS.FS.Ni) & Tm.wo (ICP/MS.FS.Ni) * \\
\hline$W(I C P / M S . F S . Z r)$ & Yb (ICP/MS.Acid) * \\
\hline $\mathrm{Yb}$ (ICP/MS.Acid) & $\mathrm{Yb}$ (ICP/MS.FS.Ni) * \\
\hline $\mathrm{Yb}$ (ICP/MS.FS.Ni) & Yb (ICP/MS.FS.Zr) * \\
\hline $\operatorname{Zr}($ ICP/AES.ACid $)$ & Zn (ICP/MS.FS.Ni) \\
\hline $\mathrm{Zr}$ (ICP/MS.Acid) & $\operatorname{Zr}(\mathrm{ICP} / \mathrm{AES} . \mathrm{FS} . \mathrm{Ni}) *$ \\
\hline $\mathrm{m} / 293$ (ICP/MS.Acid) & $\operatorname{Zr}$ (ICP/MS.FS.Ni) * \\
\hline \multirow[t]{2}{*}{$\mathrm{m} / 293$ (ICP/MS.FS.Zr) } & $\mathrm{m} / \mathrm{z241}$ (ICP/MS.FS.Zr) * \\
\hline & $\mathrm{m} / \mathrm{Z} 93$ (ICP/MS.FS.Ni) * \\
\hline
\end{tabular}

* = Large analytical variability is due to the results being close to the detection limits. 
Table 6.1. Tank 241-C-106 Vendor Product

Envelope "D" Specification Analytes - Random Variability. (page 1 of 4 )

\begin{tabular}{|c|c|c|c|c|c|c|c|c|}
\hline Analyte a & Method & Digest & Units \& & $\ddot{A}$ & $\begin{array}{c}\operatorname{RSD}(\mu) \\
(\%)\end{array}$ & $95 \%$ UL & $\begin{array}{l}\text { "D" Envelope } \\
\text { Limit (EL) }\end{array}$ & UL/EL $\mathbf{S}$ \\
\hline Ag & ICP/AES & FS.2r & $g / L$ & $1.23 E-01$ & 8.76 & $1.55 \mathrm{E}-01$ & 0.17 & 0.911 \\
\hline At & ICP/AES & FS.Ni & $g / L$ & $1.99 E+00$ & 2.63 & $2.14 E+00$ & 4.3 & 0.498 \\
\hline $241_{\text {An.gea }}$ & Rad & FS.Ni & $\mathrm{Ci} / \mathrm{b}$ & $1.22 E-04$ & 9.40 & $1.56 \mathrm{E}-04$ & 0.043 & 0.004 \\
\hline As & ICP/MS & FS.Ni & $g / L$ & $1.11 E \cdot 02$ & 7.38 & $1.35 \mathrm{E}-02$ & 0.05 & 0.270 \\
\hline B & ICP/MS & FS.Ni & $g / L$ & $8.90 E-03$ & 21.41 & $1.45 E-02$ & 0.4 & 0.036 \\
\hline $\mathrm{Ba}$ & ICP/AES & $\mathrm{FS} .2 \mathrm{r}$ & $g / L$ & $1.70 E-02$ & 3.08 & $1.85 \mathrm{E}-02$ & 1.4 & 0.013 \\
\hline $8 i$ & ICP/AES & Acid & $g / L$ & $1.67 \mathrm{E}-02$ & 1.18 & $1.73 \mathrm{E}-02$ & 0.86 & 0.020 \\
\hline${ }^{14} \mathrm{c}$ & Rad & FS.Ni & $\mathrm{Ci} / \mathrm{L}$ & $1.11 E-07$ & 78.53 & $9.45 \mathrm{E}-03$ & $2 E-06$ & 0.183 \\
\hline $\mathrm{CN}^{-}$ & Wtchem & Direst & $g / L$ & $3.69 \mathrm{E} \cdot 04$ & 5.59 & $4.29 E-04$ & 0.5 & 0.001 \\
\hline $\mathrm{Ca}$ & ICP/AES & Acid & $g / L$ & $1.49 E-09$ & 1.22 & $1.54 E-01$ & 2.2 & 0.070 \\
\hline $\mathrm{Cd}$ & $1 \mathrm{CP} / \mathrm{MS}$ & ES.Ni & $g / L$ & $3.51 \mathrm{E}-03$ & 5.13 & 4.04E-03 & 1.4 & 0.003 \\
\hline $\mathrm{Ce}$ & ICP/NS & $\mathrm{F} 5.2 \mathrm{r}$ & $g / L$ & $7.85 E-03$ & 6.52 & $9.35 E-03$ & 0.25 & 0.037 \\
\hline $\mathrm{Cl}^{\circ}$ & $1 \mathrm{c}$ & Water & $9 / 1$ & $4.89 E-03$ & 8.49 & $6.10 E-03$ & 0.1 & 0.061 \\
\hline $24 \mathrm{~cm}^{242} \mathrm{Am}_{\mathrm{Am}} 242 \mathrm{~m}_{\mathrm{Am}}$ & Rad & FS.Ni & $\mathrm{Ci} / \mathrm{L}$ & $3.13 E-07$ & 10.14 & 4.06E-07 & 0.000037 & 0.011 \\
\hline${ }^{242} \mathrm{~cm} / 242{ }_{\mathrm{Am} /} 242 \pi_{\mathrm{Am}}$ & Rad & FS.Ni & $\mathrm{Ci} / \mathrm{L}$ & $3.13 \mathrm{E}-07$ & 10.14 & $4.06 E-07$ & 3.1E-05 & 0.013 \\
\hline${ }^{242} \mathrm{~cm} / 242 \mathrm{Am}^{242 \mathrm{~m}_{\mathrm{Am}}}$ & Rad & FS.Ni & $\mathrm{Ci} / \mathrm{L}$ & $3.13 E-07$ & 10.14 & $4.06 E-07$ & 0.000032 & 0.013 \\
\hline${ }^{243} \mathrm{~cm} /{ }^{244} \mathrm{~cm}$ & Rad & Fs. Ni & $\mathrm{Ci} / \mathrm{L}$ & $3.83 \mathrm{E}-06$ & 12.97 & $5.28 \varepsilon-06$ & $1.00 E-09$ & 5277 \\
\hline${ }^{243} \mathrm{cmv}{ }^{244} \mathrm{~cm}$ & Rad & FS.Ni & $\mathrm{Ci} / \mathrm{L}$ & $3.83 E-06$ & 12.97 & $5.28 \mathrm{E}-06$ & $9.3 E-04$ & 0.006 \\
\hline Co & ICP/MS & FS.Ni & $g / L$ & $1.02 \mathrm{E}-03$ & 12.15 & $1.38 \mathrm{E}-03$ & 0.14 & 0.010 \\
\hline${ }^{60} \mathrm{Co}$ & Rad & FS.Ni & $\mathrm{Ci} / \mathrm{L}$ & $1.91 E-05$ & 3.11 & $2.08 \bar{E}-05$ & 0.003 & 0.007 \\
\hline $\mathrm{Cr}$ & ICP/MS & FS.Ni & $g / L$ & $3.00 E-02$ & 6.05 & $3.53 E-02$ & 0.21 & 0.168 \\
\hline${ }^{135} \mathrm{Cs}$ & Rad & FS.Ni & $\mathrm{Ci} / \mathrm{L}$ & $1.11 \mathrm{E}-07$ & 3.15 & $1.21 E-07$ & 0.00003 & 0.004 \\
\hline${ }^{137} \mathrm{Cs} /{ }^{137} \mathrm{Ba}$ & $\mathrm{Rad}$ & FS.Ni & $\mathrm{ci} / \mathrm{L}$ & $1.92 E-02$ & 3.29 & $2.11 E \cdot 02$ & 3 & 0.007 \\
\hline${ }^{137} \mathrm{Cs} /{ }^{137} \mathrm{Ba}$ & Rad & FS.Ni & $\mathrm{Ci} / \mathrm{L}$ & $1.92 E-02$ & 3.29 & $2.11 E-02$ & 3 & 0.007 \\
\hline $\mathrm{Cu}$ & $1 \mathrm{CP} / \mathrm{MS}$ & $\mathrm{FS} .2 \mathrm{r}$ & $\mathrm{g} / \mathrm{L}$ & $6.07 E-03$ & 74.22 & $6.81 E \cdot 03$ & .0 .15 & 0.045 \\
\hline Dy & ICP/MS & FS.Ni & $g / L$ & $7.84 E-04$ & 17.37 & $1.18 E-03$ & 0.008 & 0.148 \\
\hline Er & ICP/MS & FS.Ni & $g / L$ & $5.085-04$ & 14.64 & $7.25 E-04$ & 0.001 & 0.725 \\
\hline Eu & ICP/NS & FS.Hi & $g / L$ & $3.06 E-04$ & 30.72 & $5.80 E-04$ & 0.005 & 0.116 \\
\hline${ }^{154} \mathrm{EU}$ & Rad & FS.Ni & $\mathrm{Ci} / \mathrm{L}$ & $1.47 \mathrm{E}-04$ & 1.76 & $1.55 E-04$ & 0.016 & 0.010 \\
\hline${ }^{155_{E U}}$ & Rad & FS.Ni & $\mathrm{Ci} / \mathrm{L}$ & $1.21 E-04$ & 4.02 & $1.36 \mathrm{E}-04$ & 0.009 & 0.015 \\
\hline $\mathrm{Fe}$ & ICP/AES & FS.Ni & $9 / L$ & $5.46 E+00$ & 3.29 & $5.99 E+00$ & 8.9 & 0.672 \\
\hline $55_{\mathrm{Fe}}$ & Rad & FS.Ni & $\mathrm{Ci} / \mathrm{L}$ & $5.94 \mathrm{E} \cdot 06$ & 2.62 & $6.39 \mathrm{E}-06$ & 0.001 & 0.006 \\
\hline
\end{tabular}

a = "most reasonable" analyte ss described in urie (1997).

$\&=g / L$ or $\mathrm{Ci} / \mathrm{L}$ is $\mathrm{g} / \mathrm{L}$ (at 31 gram waste oxide) $\mathrm{Cr} \mathrm{Ci} / \mathrm{L}$ (at $31 \mathrm{gram}$ weste exide).

* = At least one of the six aralytical results is above the IDL but below the EOL.

$s=$ If more than one radionuclide is $l$ isted, then the UL is compared to the specification for each radionuclide.

$\#=\mathrm{NH}_{3}+\mathrm{N}$ converted to $\mathrm{NH}_{3}$ by multiplying by $(17 / 14)$. 
Table 6.1. Tank 241-C-106 Vendor Product

Envelope "D" Specification Analytes - Random Variability. (page 2 of 4)

\begin{tabular}{|c|c|c|c|c|c|c|c|c|}
\hline Analyte a & Method & Digest & Units \& & ii & $\begin{array}{c}\operatorname{RSO}(\bar{\mu}) \\
(\%)\end{array}$ & $95 \%$ U & $\begin{array}{l}\text { "D" Envelope } \\
\text { Limit (EL) }\end{array}$ & $U L / E L \$$ \\
\hline Gd & $1 \mathrm{CP} / \mathrm{MS}$ & $\mathrm{FS} . \mathrm{Ni}$ & $9 / L$ & $9.70 E-04$ & 11.29 & $1.29 E-03$ & 0.003 & 0.430 \\
\hline $\mathrm{Hg}$ & CVAA & Direct & $g / L$ & $1.33 E-02$ & 1.94 & $1.41 E-02$ & 0.03 & 0.669 \\
\hline Ho & ICP/MS & FS.Ni & $g / L$ & $2.35 E-04$ & 14.78 & $3.37 E-04$ & 0.001 & 0.337 \\
\hline${ }^{129} 1.4$ & ICP/MS & Acid & $\mathrm{Ci} / \mathrm{L}$ & $8.48 E-09$ & 7.28 & $1.03 E-08$ & $9.00 E \cdot 08$ & 0.114 \\
\hline 1291.40 & ICP/MS & Acid & $\mathrm{Ci} / \mathrm{L}$ & $9.19 E-09$ & 9.24 & $1.17 \mathrm{E}-\mathrm{CB}$ & $9.00 E-08$ & 0.130 \\
\hline La & ICP/MS & FS.2r & $g / L$ & $3.58 \mathrm{E}-03$ & 9.41 & $4.56 \mathrm{E}-03$ & 0.8 & 0.006 \\
\hline Li & ICP/AES & Acid & $\mathrm{g} / \mathrm{L}$ & $1.78 E-03$ & 42.50 & $3.99 \mathrm{E}-03$ & 0.043 & 0.093 \\
\hline $\mathrm{Mg}$ & ICP/MS & FS.Zr & $g / L$ & 4.88E-02 & 3.35 & $5.91 \mathrm{E}-02$ & 0.65 & 0.091 \\
\hline Mn & ICP/MS & FS.Ni & $g / L$ & $1.09 E-01$ & 4.03 & $1.22 E-01$ & 2 & 0.061 \\
\hline Mo & ICP/MS & FS.Ni & $\mathrm{g} / \mathrm{L}$ & $2.21 E-03$ & 13.98 & $3.11 E-03$ & 0.2 & 0.016 \\
\hline $\mathrm{NH}_{3} \#$ & WrChen & Water & $g / 4$ & $8.25 E-04$ & 20.07 & $1.31 E-03$ & 0.5 & 0.003 \\
\hline $\mathrm{NO}_{2}^{-}+\mathrm{NO}_{3}^{-}$ & 10 & Water & $\mathrm{g} / \mathrm{L}$ & $1.42 E-01$ & 10.69 & $1.87 \mathrm{E}-01$ & 11.2 & 0.017 \\
\hline $\mathrm{Na}$ & ICP/AES & Acid & $\mathrm{g} / \mathrm{L}$ & $6.29 E+00$ & 2.93 & $6.83 E+00$ & 6 & 1.138 \\
\hline $93 \mathrm{mb}$ & Rad & FS.Ni & $\mathrm{cis}$ & $9.22 E-06$ & 30.19 & $1.73 E-05$ & $8.7 E-05$ & 0.199 \\
\hline Nd & ICP/AES & Acid & $\mathrm{g} / \mathrm{L}$ & $1.095-02$ & 1.19 & $1.13 E-02$ & 0.53 & 0.021 \\
\hline $\mathrm{Ni}$ & $I C P / M S$ & FS.Zr & $g / L$ & $6.23 E-02$ & 2.35 & $6.66 \mathrm{E}-02$ & 0.73 & 0.091 \\
\hline${ }^{59} \mathrm{Ki.W}$ & $\mathrm{Rad}$ & FS.Zr & $\mathrm{Ci} / \mathrm{L}$ & $8.53 E-07$ & 10.23 & $1.11 E-06$ & 0.000014 & 0.079 \\
\hline${ }^{59} \mathrm{Ni.HO}$ & Rad & FS.Zr & $\mathrm{Ci} / \mathrm{L}$ & $7.68 \mathrm{E}-07$ & 14.16 & $1.085-06$ & 0.000014 & 0.077 \\
\hline${ }^{63} \mathrm{Ni}$ & Rad & FS.Zr & $\mathrm{Ci} / \mathrm{L}$ & $3.33 E-05$ & 2.39 & $9.98 E-05$ & 0.0016 & 0.062 \\
\hline $237_{\mathrm{NP}}$ & ICP/MS & $\mathrm{FS} . \mathrm{Zr}$ & $\mathrm{Ci} / \mathrm{L}$ & $1.52 E-07$ & 9.49 & $1.94 \mathrm{E}-07$ & 0.000023 & 0.008 \\
\hline$P$ & ICP/AES & FS.Ni & $g / L$ & $1.29 E-01$ & 4.58 & $1.46 E-01$ & 0.54 & 0.271 \\
\hline $\mathrm{Pb}$ & ICP/AES & Acid & $\mathrm{g} / \mathrm{L}$ & $1.33 E \cdot 01$ & 1.11 & $1.37 E-01$ & 0.34 & 0.404 \\
\hline Pd & ICP/MS & $5 S .2 \mathrm{r}$ & $s / 6$ & $1.06 E-03$ & 7.63 & $1.30 E-03$ & 0.04 & 0.033 \\
\hline 107 Pd.h & $I C P / M S$ & Acid & $\mathrm{Ci} / \mathrm{L}$ & $2.79 \mathrm{E}-08$ & 58.24 & $7.53 \mathrm{E}-08$ & 0.000004 & 0.019 \\
\hline 107 Pd.wo & $1 \mathrm{CP} / \mathrm{MS}$ & Acid & $\mathrm{Ci} / \mathrm{L}$ & 4.41E-08 & 64.59 & $2.24 \mathrm{E}-07$ & 0.000004 & 0.056 \\
\hline $\mathrm{Pr}$ & ICP/MS & FS.ir & $\mathrm{g} / \mathrm{L}$ & $3.26 E \cdot 03$ & 3.73 & $3.62 E-03$ & 0.11 & 0.033 \\
\hline $\mathrm{Pt}$ & ICP/MS & FS.Ni & $g / L$ & 1.19E-03 & 16.03 & $1.75 \mathrm{E}-03$ & 0.001 & 1.751 \\
\hline $238_{\mathrm{PU}}$ & Rad & FS.Ni & $\mathrm{Cill}$ & $2.45 \mathrm{E}-05$ & 2.43 & $2.62 E-05$ & 0.00011 & 0.238 \\
\hline $239 \mathrm{Pu}$ & ICP/MS & FS.Zr & $\mathrm{Ci} / \mathrm{L}$ & $5.91 \mathrm{E}-05$ & 3.38 & $6.49 \mathrm{E} \cdot 05$ & 0.00095 & 0.068 \\
\hline $240 \mathrm{Pu}$ & ICP/MS & Acid & $\mathrm{Ci} / \mathrm{L}$ & $4.85 \mathrm{E}-06$ & 4.62 & $5.51 E-06$ & 0.00026 & 0.021 \\
\hline $241_{\mathrm{PU}}$ & Rad & FS.Ni & $\mathrm{Ci} / \mathrm{L}$ & $2.70 E-04$ & 2.43 & $2.89 E-04$ & 0.0069 & 0.042 \\
\hline
\end{tabular}

$\mathrm{a}=$ "most reasonable" analyte es described in Urie (1997).

$\&=g / L$ or $\mathrm{Ci} / \mathrm{L}$ is $\mathrm{g} / \mathrm{L}$ (at 31 gran kaste oxide) or $\mathrm{Ci} / \mathrm{L}$ (at 31 gram waste oxide).

* = At least one of the six analytical results is above the $10 \mathrm{~L}$ but below the EOL:

$s=$ if more than one radionuclide is listed, then the UL is compared to the specification for each radionuelide.

$\#=\mathrm{KH}_{3}-\mathrm{H}$ converted to $\mathrm{NH}_{3}$ by multiplying by $(17 / 14)$. 
HNF-SD-WM-DP-225, REV. i

Table 6.1. Tank 241-C-106 Vendor Product

Envelope "D" Specification Analytes - Random Variability. (page 3 of 4)

\begin{tabular}{|c|c|c|c|c|c|c|c|c|}
\hline Analyte a & Method & Digest & Units : & $\dot{H}$ & $\begin{array}{c}\mathrm{RSD}(i) \\
(\%)\end{array}$ & $95 \%$ UL & $\begin{array}{l}\text { "D" Envelope } \\
\text { Limit (EL) }\end{array}$ & UL/EL \$ \\
\hline $\mathrm{Rb}$ & $I C P / M S$ & FS.Zr & $\mathrm{g} / \mathrm{L}$ & $2.985-04$ & 18.38 & $4.58 \mathrm{E}-04$ & 0.06 & 0.008 \\
\hline $\mathrm{Re}$ & ICP/MS & FS.Ni & $\mathrm{g} / \mathrm{L}$ & $2.57 E \cdot 04$ & 15.76 & $3.75 E-04$ & 0.03 & 0.012 \\
\hline $\mathrm{Rh}$ & $1 \mathrm{CP} / \mathrm{MS}$ & Fs.zr & $\mathrm{g} / \mathrm{L}$ & $5.60 E-03$ & 5.44 & $6.48 E-03$ & 0.04 & 0.162 \\
\hline Ru & ICP/MS & FS.Ni & $g / L$ & $3.71 E-03$ & 3.88 & $4.13 E-03$ & 0.11 & 0.038 \\
\hline Sb.H & $1 \mathrm{CP} / \mathrm{MS}$ & Acid & $g / L$ & $1.46 \mathrm{E}-05$ & 47.84 & $3.50 E-05$ & 0.26 & 0.000 \\
\hline Sb.wo & $I C P / M S$ & Acid & $g / L$ & $3.06 E+05$ & 25.35 & $7.95 E-05$ & 0.26 & 0.000 \\
\hline si & ICP/AES & FS.Ni & $\mathrm{g} / \mathrm{L}$ & $2.67 E+00$ & 3.91 & $2.98 E+00$ & 5.8 & 0.513 \\
\hline${ }^{159} \mathrm{Sm} /{ }^{147} \mathrm{Pm}$ & Rad & FS.Ni & $\mathrm{Ci} / \mathrm{L}$ & $3.58 E-03$ & 2.84 & $3.88 E-03$ & 0.093 & 0.042 \\
\hline${ }^{15} 1_{\mathrm{Sm} /} /{ }^{147_{\mathrm{Pm}}}$ & Rad & FS.Ni & $\mathrm{Ci} / \mathrm{L}$ & $3.58 \mathrm{E}-03$ & 2.84 & $3.88 E \cdot 03$ & 0.16 & 0.024 \\
\hline \$n & ICP/MS & FS.2r & $g / L$ & $3.96 E-03$ & 15.64 & $5.77 E \cdot 03$ & 0.011 & 0.525 \\
\hline $21 \ln _{5 n}$ & Rad & FS.Ni & $\mathrm{Ci} / \mathrm{L}$ & $4.77 \mathrm{E}-05$ & 24.84 & $8.23 E-05$ & 0.000009 & 9.146 \\
\hline${ }^{126} \mathrm{sn} /{ }^{126 \pi} \mathrm{sb} j^{126} \mathrm{sb}$ & Rad & FS.Ni & $\mathrm{Ci} / \mathrm{L}$ & $4.48 E-07$ & 15.50 & $6.51 E-07$ & 0.000048 & 0.014 \\
\hline${ }^{126} \mathrm{sn} /^{126 m} \mathrm{sb} /{ }^{126} \mathrm{sb}$ & Rad & ES.Ni & $\mathrm{Ci} / \mathrm{L}$ & $4.48 E \cdot 07$ & 15.50 & $6.51 E-07$ & 0.000034 & 0.019 \\
\hline$\left.{ }^{126} \mathrm{sn}\right)^{126 m} \mathrm{sb} /^{126} \mathrm{sb}$ & Rad & ES. Hi & $\mathrm{Ci} / \mathrm{L}$ & $4.48 E-07$ & 15.50 & $6.51 E-07$ & 0.000005 & 0.135 \\
\hline $5 r$ & ICP/MS & $\mathrm{FS} . \mathrm{Zr}$ & $g / L$ & $5.84 \mathrm{E} \cdot 03$ & 3.55 & $6.44 E-03$ & 0.16 & 0.040 \\
\hline $90_{\mathrm{Sr} /} 90_{\mathrm{Y}}$ & Rad. & FS.Ni & $\mathrm{Ci} / \mathrm{L}$ & $2.83 E-02$ & 2.45 & $3.04 E-02$ & 3.1 & 0.010 \\
\hline${ }^{90} \mathrm{Sr}^{90} \mathrm{Y}$ & $\vec{R}$ ad & FS.Ni & $\mathrm{Ci} / \mathrm{L}$ & $2.83 E-02$ & 2.45 & $3.04 E-02$ & 3.1 & 0.010 \\
\hline TIC & Wrchern & Direct & $g / L$ & $2.06 E-01$ & 4.36 & $2.32 \mathrm{E} \cdot 01$ & 1.86 & 0.125 \\
\hline TOC & withem & Direct & $\mathrm{g} / \mathrm{L}$ & $1.86 \mathrm{E}+00$ & 3.30 & $2.04 E+00$ & 3.4 & 0.600 \\
\hline $\mathrm{Ta}$ & ICP/MS & $\mathrm{FS} .2 \mathrm{r}$ & $g / L$ & $1.11 E-03$ & 22.67 & $1.84 E-03$ & 0.008 & 0.231 \\
\hline Tb & ICP/MS & FS.Ni & $g / L$ & $3.18 E-04$ & 10.53 & $4.16 \mathrm{E} \cdot 04$ & 0.001 & 0.416 \\
\hline${ }^{99} \mathrm{Te}$ & ICP/NS & Acid & $\mathrm{ci} / \mathrm{L}$ & $2.18 E-07$ & 10.82 & $2.86 E \cdot 07$ & 0.0045 & 0.000 \\
\hline Te & ICP/MS & FS.Ni & $g / L$ & $1.65 E-02$ & 8.96 & $2.08 \mathrm{E}-02$ & 0.04 & 0.519 \\
\hline Th * & ICP/AES & Acid & $g / L$ & $1.35 \mathrm{E}-02$ & 0.55 & $1.38 E-02$ & 0.16 & 0.086 \\
\hline $\mathrm{Ti}$ & ICP/MS & FS.Zr & $\mathrm{s} / \mathrm{L}$ & $5.10 \mathrm{E}-02$ & 7.06 & $6.15 E \cdot 02$ & 0.4 & 0.154 \\
\hline Tm. H & ICP/MS & FS.Ni & $g / L$ & $7.06 E-05$ & 39.63 & $1.52 E-04$ & 0.001 & 0.152 \\
\hline Tm. Wo & ICP/NS & FS.Ni & $g / L$ & $9.78 E-05$ & 36.01 & $2.01 E-04$ & 0.001 & 0.201 \\
\hline Total U & $1 C P / M S$ & FS.Ni & $g / L$ & $7.36 \mathrm{E}-02$ & 2.84 & $7.97 \mathrm{E}-02$ & 4.2 & 0.019 \\
\hline $233_{u}$ & ICP/MS & Acid & $\mathrm{Ci} / \mathrm{L}$ & $6.52 E-08$ & 11.56 & $8.72 \mathrm{E}-08$ & $1.00 E-09$ & 87.2 \\
\hline $234 \mathrm{U}$ & ICP/MS & Acid & $\mathrm{Ci} / \mathrm{L}$ & $3.00 E-08$ & 7.41 & $3.65 E-08$ & $7.70 E-07$ & 0.047 \\
\hline $235 \mathrm{u}$ & ICP/MS & Reid & $\mathrm{Ci} / \mathrm{L}$ & $1.09 E-09$ & 2.86 & $1.18 \mathrm{E}-09$ & $3.20 E-08$ & 0.037 \\
\hline
\end{tabular}

$a=$ "most reasonable analyte as described in Urie (1997).

$\&=g / L$ or $C i / L$ is $g / L$ (at 31 gran waste oxide) or $C i / L$ (at 31 gram waste oxide).

* = At least one of the six arialytical results is above the IDL but below the ECL.

$s=$ If more than one radionuclide is listed, then the UL is compared to the specification for each radionuclide.

$\#=\mathrm{NH}_{3}-\mathrm{N}$ converted to $\mathrm{NH}_{3}$ by multiplying by $(17 / 14)$. 
HNF-SD-WM-DP-225, REV. 1

Table 6.1. Tank 241-C-106 Vendor Product

Envelope "D" Specification Analytes - Random Variability. (page 4 of 4)

\begin{tabular}{|c|c|c|c|c|c|c|c|c|}
\hline Analyte a & Hethod & Digest & Units \& & $\ddot{\mu}$ & $\begin{array}{c}\operatorname{RSD}(\bar{\mu}) \\
(\%)\end{array}$ & $95 \%$ UL & $\begin{array}{l}\text { "D" Envelope } \\
\text { Limit (EL) }\end{array}$ & UL/EL \$ \\
\hline${ }^{236} \mathrm{U}$ & $I C P / M S$ & Acid & $\mathrm{Ci} / \mathrm{L}$ & $7.17 \varepsilon-10$ & 7.11 & $8.66 E-10$ & $8.20 E-08$ & 0.011 \\
\hline $238 \mathrm{u}$ & ICP/MS & FS.2r & $\mathrm{Ci} / \mathrm{L}$ & $2.61 \varepsilon-08$ & 1.28 & $2.70 E-08$ & $5.80 \mathrm{E} \cdot 07$ & 0.047 \\
\hline$v$ & ICP/MS & Aeid & $\mathrm{g} / \mathrm{L}$ & $1.45 \varepsilon-03$ & 8.58 & $1.81 E-03$ & 0.01 & 0.181 \\
\hline$w$ & ICP/MS & ES.Ni & $g / L$ & $1.94 E-02$ & 6.05 & $2.28 \mathrm{E}-02$ & 0.074 & 0.308 \\
\hline Yb & ICP/MS & ES.Ni & $g / L$ & $4.89 E=04$ & 29.08 & $9.04 E-04$ & 0.001 & 0.904 \\
\hline $\mathrm{Zn}$ & ICP/MS & FS.Ni & g/L & $1.19 \mathrm{E}-02$ & 9.43 & $1.52 E-02$ & 0.13 & 0.117 \\
\hline $\mathrm{Zr}$ & ICP/AES & FS.Ni & $g / L$ & $4.29 \mathrm{E}-02$ & 19.48 & $6.73 E-02$ & 4.6 & 0.015 \\
\hline
\end{tabular}

$a=$ "most reasonable" analyte as described in Urie (1997).

$z=g / L$ or $\mathrm{Ci} / \mathrm{L}$ is $\mathrm{g} / \mathrm{L}$ (at $31 \mathrm{gran}$ waste oxide) or $\mathrm{Ci} / \mathrm{L}$ (at 31 gram waste oxide).

* = At least one of the six analytical results is above the IDL but below the EOL.

$s=$ If more than one radionuclide is $l i s t e d$, then the $U L$ is compared to the specification for each radionuclide.

$\#=\mathrm{NH}_{3}-\mathrm{N}$ converted to $\mathrm{NH}_{3}$ by multiplying by $(17 / 14)$. 
Table 6.2. Tank 241-C-106 Vendor Product

Envelope "D" Specification Analytes - Less Than Values.

\begin{tabular}{|c|c|c|c|c|c|c|c|}
\hline Analyte a & Method & Digest & Units \& & \multicolumn{2}{|c|}{ Largest Result } & $\begin{array}{l}\text { "D" Envelope } \\
\text { Limit (EL) }\end{array}$ & Largest/EL 5 \\
\hline $110 \mathrm{~m}_{\mathrm{Ag}}$ & Rad & FS.Ni & $\mathrm{EI} / \mathrm{L}$ & $<$ & $3.49 E-06$ & $1.00 \mathrm{E}-08$ & 349 \\
\hline $243_{\mathrm{Am}}$ & Rad & FS.Ni & $\mathrm{Ci} / \mathrm{L}$ & & $7.76 E \cdot 07$ & 0.000005 & 0.155 \\
\hline Be & ICP/AES & Acid & $g / h$ & $<$ & $3.88 E-05$ & 0.02 & 0.002 \\
\hline${ }^{144} \mathrm{Ce} /{ }^{144} \mathrm{Pr} /{ }^{144 \mathrm{~m}} \mathrm{Pr}$ & Rad & FS.Ni & $\mathrm{Ci} / \mathrm{L}$ & $<$ & $7.76 E=05$ & 0.0001 & 0.776 \\
\hline${ }^{144} \mathrm{Ce} / /^{144} \mathrm{Pr} /{ }^{144 \pi \mathrm{mr}}$ & Rad & FS.Ni & $\mathrm{Ci} / \mathrm{L}$ & $<$ & $7.76 \mathrm{E}-05$ & 0.0001 & 0.776 \\
\hline${ }^{144} \mathrm{Ce} /{ }^{144} \mathrm{Pr} /{ }^{144 \mathrm{~m}} \mathrm{Pr}$ & Rad & FS.Ni & $\mathrm{Ci} / \mathrm{L}$ & $<$ & $7.76 E-05$ & $1.0 \mathrm{E}-07$ & 776 \\
\hline${ }^{134} \mathrm{cs}$ & Rad & FS.Ni & $\mathrm{Ci} / \mathrm{L}$ & $<$ & $1.16 E-05$ & 0.0068 & 0.002 \\
\hline $1522_{\mathrm{EU}}$ & Rad & FS.Ni & $\mathrm{Ci} / \mathrm{L}$ & $<$ & $7.76 E-05$ & 0.00014 & 0.555 \\
\hline $\mathrm{F}^{-}$ & Wetchem & Water & $g / L$ & $<$ & $1.13 E-01$ & 1.1 & 0.103 \\
\hline$k$ & ICP/AES & Acid & $g / L$ & $<$ & $1.91 E-02$ & 0.41 & 0.047 \\
\hline $242_{P U}$ & Rad & FS.Ni & $\mathrm{Ci} / \mathrm{L}$ & $<$ & $7.76 E \cdot 07$ & $7.10 \mathrm{E}-08$ & 10.936 \\
\hline${ }^{106} \mathrm{RU}_{\mathrm{R}}{ }^{106} \mathrm{Rh}$ & Rad & ES.Ni & $\mathrm{Ci} / \mathrm{L}$ & $<$ & $1.16 \mathrm{E}-04$ & 0.0002 & 0.582 \\
\hline${ }^{106} \mathrm{Ru}_{1}{ }^{106_{\mathrm{Rh}}}$ & Rad & FS.Ni & $\mathrm{Ci} / \mathrm{L}$ & $<$ & $1.16 E-04$ & 0.0002 & 0.582 \\
\hline${ }^{125} \mathrm{sb}{ }^{125 m_{\mathrm{Te}}}$ & Rad & FS.Ni & {$[i / L$} & $<$ & $7.76 E-05$ & 0.01 & 0.008 \\
\hline $125_{\mathrm{sb} /}{ }^{125 \mathrm{~m}_{\mathrm{Te}}}$ & Rad & FS.Ni & $\mathrm{Ci} / \mathrm{L}$ & $<$ & $7.76 E \cdot 05$ & 0.003 & 0.026 \\
\hline se & ICP/AES & Acid & $g / L$ & $<$ & $1.43 E-02$ & 0.16 & 0.090 \\
\hline $79 \mathrm{se}$ & Rad & Acid & $\mathrm{Ci} / \mathrm{L}$ & $<$ & $8.15 E-09$ & 4.20E-07 & 0.019 \\
\hline${ }^{113} \mathrm{sn} /^{113 m_{1 n}}$ & Rad & FS.Ni & $\mathrm{Ci} / \mathrm{h}$ & $<$ & $3.88 E-07$ & $1.18 \mathrm{E} \cdot 06$ & 0.206 \\
\hline${ }^{113} \mathrm{sn} /^{113 \mathrm{~min}} \mathrm{In}$ & Rad & FS.Ni & $\mathrm{Ci} / \mathrm{L}$ & $<$ & $3.88 E-07$ & $1.18 \mathrm{E}-06$ & 0.206 \\
\hline $119 \mathrm{~m}_{\mathrm{sn}}$ & Rad & FS.Ni & $\mathrm{Ci} / \mathrm{L}$ & $<$ & $1.55 \mathrm{E}-06$ & $1.00 E \cdot 08$ & 155.270 \\
\hline $\mathrm{Tt}$ & ICP/AES & Acid & $g / L$ & $<$ & $4.77 \mathrm{E} \cdot 03$ & 0.14 & 0.034 \\
\hline$Y$ & ICP/AES & Acid & $g / L$ & $<$ & $3.30 E-03$ & 0.05 & 0.066 \\
\hline${ }^{93} \mathrm{Zr}$ & Rad & FS.Ni & $\mathrm{Ei} / \mathrm{L}$ & $\therefore$ & $2.33 \mathrm{E}-05$ & $1.4 \mathrm{E}-04$ & 0.166 \\
\hline
\end{tabular}

$a$ = "most reasonable" anatyte as described in Urie (1997).

$\$$ = If more than one radionuclide is listed, then the UL is compared to the specification for each radionuclide.

$\&=g / L$ or $\mathrm{Ci} / \mathrm{L}$ is $\mathrm{g} / \mathrm{L}$ (at $31 \mathrm{gram}$ waste oxide) or $\mathrm{Ci} / \mathrm{L}$ (at $31 \mathrm{gram}$ waste oxide). 
HNF-SD-WM-DP-225, REV.

Table 6.3. Tank 241-C-106 Vendor Product

Envelope "D" Specification Analytes - Total Variability. (page 1 of 4)

\begin{tabular}{|c|c|c|c|c|c|c|c|c|c|}
\hline Analyte 2 & Method & Digest & Units \& & $\ddot{\mu}$ & $\begin{array}{l}\text { UL/EL } \$ \\
S Y S=10 \% \\
\end{array}$ & $\begin{array}{l}U L / E L \$ \\
S Y S=25 \%\end{array}$ & $\begin{array}{l}\text { UL/EL } 5 \\
\text { SYS }=50 \%\end{array}$ & $\begin{array}{l}\text { UL/EL } \$ \\
\text { SYS }=75 \%\end{array}$ & $\begin{array}{l}\text { UL/EL } \$ \\
S Y S=100 \%\end{array}$ \\
\hline $\mathrm{Ag}$ & ICP/AES & FS.2r & $g / L$ & $1.23 \mathrm{E}-01$ & 1.007 & 1.287 & 1.801 & 2.326 & 2.853 \\
\hline$A L$ & ICP/AES & FS.Ni & $g / L$ & $1 . \varsigma \subseteq E+00$ & 0.602 & 0.801 & 1.138 & 1.475 & 1.812 \\
\hline $241_{\text {Am.gea }}$ & Rad & $\mathrm{FS.Ni}$ & $\mathrm{Ci} / \mathrm{L}$ & $1.22 E-04$ & 0.004 & 0.005 & 0.007 & 0.009 & 0.011 \\
\hline As & ICP/MS & FS.Ni & $g / L$ & $1.11 \mathrm{E}-02$ & 0.303 & 0.391 & 0.550 & 0.711 & 0.873 \\
\hline B & ICP/MS & FS.Ni & $g / L$ & $8.90 \mathrm{E}-03$ & 0.038 & 0.044 & 0.058 & 0.073 & 0.089 \\
\hline $\mathrm{Ba}$ & ICP/AES & Es.zr & $g / L$ & $1.70 E-02$ & 0.016 & 0.021 & 0.030 & 0.039 & 0.047 \\
\hline $\mathrm{Bi}$ & ICP/AES & Acid & $g / L$ & $1.67 \mathrm{E}-02$ & 0.025 & 0.034 & 0.048 & 0.062 & 0.076 \\
\hline${ }^{14} \mathrm{C}$ & Rad & FS.Ni & $\mathrm{Ci} / \mathrm{L}$ & $1.11 E-07$ & 0.184 & 0.190 & 0.207 & 0.232 & 0.262 \\
\hline $\mathrm{CN}^{*}$ & Wtchem & Direct & $g / L$ & $3.69 \mathrm{E}-04$ & 0.001 & 0.001 & 0.002 & 0.002 & 0.003 \\
\hline $\mathrm{Ca}$ & ICP/AES & Acid & $g / L$ & $1.45 E-01$ & 0.088 & 0.117 & 0.167 & 0.216 & 0.286 \\
\hline cd & ICP/MS & FS.Ni & $g / L$ & $3.51 E-03$ & 0.003 & 0.004 & 0.006 & 0.008 & 0.010 \\
\hline $\mathrm{Ce}$ & ICP/MS & FS.Zr & $g / L$ & $7.85 E \cdot 03$ & 0.042 & 0.055 & 0.078 & 0.100 & 0.123 \\
\hline $\mathrm{Cl}^{-}$ & IC & Water & $g / L$ & $4.895-03$ & 0.068 & 0.087 & 0.121 & 0.157 & 0.192 \\
\hline${ }^{242} \mathrm{~cm} / 242 \mathrm{Am} /{ }^{242 m_{\mathrm{An}}}$ & Rad & FS.Ni & $\mathrm{Ci} / \mathrm{L}$ & $3.13 E-07$ & 0.012 & 0.015 & 0.021 & 0.027 & 0.033 \\
\hline${ }^{242}\left[m / 242{ }_{A m /}{ }^{242 m_{A m}}\right.$ & Rad & FS.Ni & $\mathrm{Ci} / \mathrm{L}$ & $3.13 E-07$ & 0.014 & 0.018 & 0.025 & 0.032 & 0.040 \\
\hline${ }^{242} \mathrm{~cm} /{ }^{242} \mathrm{Am} /{ }^{242 m_{\mathrm{Am}}}$ & Rad & FS.Ni & $\mathrm{Ci} / \mathrm{L}$ & $3.13 \mathrm{E} \cdot 07$ & 0.014 & 0.017 & 0.024 & 0.031 & 0.038 \\
\hline${ }^{243} \mathrm{~cm} /{ }^{244} \mathrm{~cm}$ & $\operatorname{Rad}$ & $\mathrm{FS} . \mathrm{Ni}$ & $\mathrm{ci} / \mathrm{L}$ & $3.83 E-06$ & 5658 & 6975 & 9601 & 12335 & 15099 \\
\hline${ }^{243} \mathrm{Cm} /{ }^{244} \mathrm{Cm}$ & Rad & FS.HI & $\mathrm{Ci} / \mathrm{L}$ & $3.83 E-06$ & 0.006 & 0.008 & 0.010 & 0.013 & 0.016 \\
\hline Co & $I \mathrm{CP} / \mathrm{MS}$ & FS.Ni & $g / 1$ & $1.02 E-03$ & 0.011 & 0.013 & 0.018 & 0.023 & 0.029 \\
\hline${ }^{60} \mathrm{CO}$ & Rad & FS.Ni & $\mathrm{Ci} / \mathrm{L}$ & 1.91E-05 & 0.008 & 0.011 & 0.016 & 0.020 & 0.025 \\
\hline $\mathrm{Cr}$ & ICP/MS & FS. $\mathrm{Hi}$ & $g / L$ & $3.00 E \cdot 02$ & 0.192 & 0.250 & 0.353 & 0.457 & 0.561 \\
\hline${ }^{135} \mathrm{Cs}$ & Rad & FS.Ni & $\mathrm{Ci/L}$ & $1.11 \mathrm{E}-07$ & 0.005 & 0.006 & 0.009 & 0.012 & 0.015 \\
\hline${ }^{137} 7_{\mathrm{Cs} /}{ }^{137_{\mathrm{Ba}}}$ & Red & FS.Ni & {$[i / L$} & $1.92 E-02$ & 0.008 & 0.011 & 0.016 & 0.020 & 0.025 \\
\hline${ }^{137} \mathrm{Cs} /{ }^{137} \mathrm{Ba}$ & Rad & FS.Mi & $C i / L$ & $1.92 E-02$ & 0.008 & 0.011 & 0.016 & 0.020 & 0.025 \\
\hline $\mathrm{Cu}$ & ICP/MS & FS.Zr & $g / L$ & $6.07 E-03$ & 0.053 & 0.070 & 0.100 & 0.929 & 0.159 \\
\hline Dy & ICP/MS & FS.Ni & $g / \mathrm{t}$ & 7.84 E-04 & 0.155 & 0.185 & 0.249 & 0.318 & 0.388 \\
\hline $\mathrm{Er}$ & ICP/MS & FS.Ni & $g / L$ & $5.08 E .04$ & 0.771 & 0.938 & 1.281 & 1.642 & 2.007 \\
\hline Eu & ICP/NS & FS.Ni & $g / L$ & $3.06 E-04$ & 0.119 & 0.932 & 0.166 & 0.206 & 0.248 \\
\hline${ }^{154} \mathrm{Eu}$ & Rad & FS.Ni & $\mathrm{Ci} / \mathrm{L}$ & $1.47 \mathrm{E}-04$ & 0.012 & 0.016 & 0.023 & 0.029 & 0.036 \\
\hline${ }^{155} \mathrm{Eu}$ & Rad & FS.Ni & $\mathrm{Ci} / \mathrm{L}$ & $1.21 E \cdot 04$ & 0.018 & 0.023 & 0.033 & 0.043 & 0.053 \\
\hline Fe & ICP/AES & FS.Ni & $g / L$ & $5.46 E+00$ & 0.802 & 1.065 & 1.511 & 1.958 & 2.406 \\
\hline$\overline{55 \mathrm{Fe}}$ & Rad & FS.Ni & $\mathrm{Ci} / \mathrm{L}$ & 5.545 .06 & 0.008 & 0.010 & 0.015 & 0.019 & 0.023 \\
\hline
\end{tabular}

$a=$ "most rezscriable" arialyte as deseribed in Urie (1997).

$\&=g / L$ or $C i / L$ is $g / L$ (at 31 gren waste oxide) or Ci/L (at 31 gram waste oxide).

$S=$ If more than one radionuclide is listed, then the UL is compared to the specification for each radionuclide. 
Tabie 6.3. Tank 241-C-105 Vendor Product

Envelope "D" Specification Analytes - Total Variability. (page 2 of 4)

\begin{tabular}{|c|c|c|c|c|c|c|c|c|c|}
\hline Analyte a & Method & Digest & Units \& & $\dot{\mu}$ & $\begin{array}{l}U L / E L \$ \\
S Y S=10 \%\end{array}$ & $\begin{array}{l}\text { UL/EL } \$ \\
\text { SYS }=25 \%\end{array}$ & $\begin{array}{l}U L / E L \$ \\
S Y S=50 \%\end{array}$ & $\begin{array}{l}\text { UL/EL \$ } \\
\text { SYS }=75 \%\end{array}$ & $\begin{array}{c}U L / E L \text { S } \\
\text { SYS }=100 \%\end{array}$ \\
\hline Gd & ICP/NS & ES.Ni & $g / 6$ & $9.70 E-04$ & 0.466 & 0.582 & 0.807 & 1.039 & 1.273 \\
\hline $\mathrm{Hg}$ & CVAA & Direct & $g / L$ & $1.33 E-02$ & 0.576 & 0.770 & 1.093 & 1.417 & 1.742 \\
\hline Ho & ICP/MS & FS.Ni & $\mathrm{g} / \mathrm{L}$ & $2.35 E-04$ & 0.358 & 0.435 & 0.594 & 0.769 & 0.931 \\
\hline $129 \mathrm{~L} . \mathrm{H}$ & ICP/MS & Acid & $\mathrm{Ci} / \mathrm{L}$ & $8.48 E-09$ & 0.128 & 0.166 & 0.233 & 0.301 & 0.370 \\
\hline 129 I.Ko & ICP/MS & Acid & $\mathrm{Ci} / \mathrm{L}$ & $9.19 E-09$ & 0.143 & 0.182 & 0.254 & 0.327 & 0.401 \\
\hline La & $1 \mathrm{CP} / \mathrm{MS}$ & FS.Zr & $9 / 1$ & $3.58 E \cdot 03$ & 0.006 & 0.008 & 0.011 & 0.014 & 0.018 \\
\hline$L i$ & ICP/AES & Acid & $g / L$ & $1.78 E-03$ & 0.094 & 0.101 & 0.121 & 0.146 & 0.173 \\
\hline $\mathrm{Hg}$ & 1CP/MS & $\mathrm{FS.2r}$ & $\mathrm{g} / \mathrm{L}$ & $4.88 E-02$ & 0.125 & 0.195 & 0.312 & 0.431 & 0.549 \\
\hline$M n$ & ICP/MS & FS.Ni & $g / L$ & $1.09 E-01$ & 0.072 & 0.095 & 0.134 & 0.174 & 0.214 \\
\hline Mo & ICP/MS & FS.Ni & $g / L$ & $2.21 E-03$ & 0.017 & 0.020 & 0.028 & 0.036 & 0.044 \\
\hline $\mathrm{NH}_{3}=$ & WtChem & Water & $g / L$ & $8.25 \mathrm{E}-04$ & 0.003 & 0.003 & 0.004 & 0.005 & 0.007 \\
\hline $\mathrm{NO}_{2}^{-}+\mathrm{NO}_{3}^{-}$ & IC & Water & $g / L$ & $1.42 E-01$ & 0.018 & 0.021 & 0.028 & 0.035 & 0.043 \\
\hline $\mathrm{Na}$ & ICP/AES & Acid & $\S / L$ & $6.29 E+00$ & 1.367 & 1.818 & 2.581 & 3.345 & 4.110 \\
\hline $93 \mathrm{mb}_{\mathrm{Nb}}$ & $\operatorname{Rad}$ & FS.Ni & $\mathrm{Ci} / \mathrm{L}$ & $9.22 E-06$ & 0.204 & 0.227 & 0.287 & 0.232 & 0.429 \\
\hline Nd & ICP/AES & Acid & $g / L$ & $1.09 E-02$ & 0.027 & 0.036 & 0.051 & 0.066 & 0.081 \\
\hline $\mathrm{Ni}$ & JCP/MS & Fs.2r & $g / L$ & $6.23 E-02$ & 0.111 & 0.148 & 0.210 & 0.272 & 0.335 \\
\hline $59 \mathrm{Ni.H}$ & $\operatorname{Rad}$ & FS.Zr & $\mathrm{Ci} / \mathrm{L}$ & $8.53 E-07$ & 0.086 & 0.109 & 0.152 & 0.196 & 0.240 \\
\hline $59 \mathrm{Ni}$.HO & Rad & FS.Zr & $\mathrm{Cj} / \mathrm{L}$ & $7.66 E-07$ & 0.082 & 0.101 & 0.138 & 0.177 & 0.216 \\
\hline${ }^{63} \mathrm{Ni}$ & Rad & FS.Zr & $\mathrm{CI} / \mathrm{L}$ & $9.33 E-05$ & 0.076 & 0.101 & 0.144 & 0.186 & 0.229 \\
\hline $237_{\mathrm{Np}}$ & $1 \mathrm{CP} / \mathrm{MS}$ & FS. Zr & $\mathrm{ci/h}$ & $1.52 \mathrm{E}-07$ & 0.009 & 0.012 & 0.016 & 0.021 & 0.026 \\
\hline$P$ & ICP/AES & FS.Ni & $g / L$ & $1.25 \mathrm{E}-01$ & 0.316 & 0.416 & 0.589 & 0.763 & 0.938 \\
\hline $\mathrm{Pb}$ & ICP/AES & Acid & $g / L$ & $1.33 E-01$ & 0.507 & 0.678 & 0.953 & 1.249 & 1.535 \\
\hline Pd & ICP/MS & FS.ZF & $\mathrm{g} / \mathrm{L}$ & $1.06 \mathrm{E} \cdot 03$ & 0.036 & 0.047 & 0.066 & 0.085 & 0.104 \\
\hline $107_{\text {Pd.H }}$ & $I C P / M S$ & Acid & $\mathrm{ci} / \mathrm{L}$ & $2.79 E-08$ & 0.019 & 0.020 & 0.023 & 0.026 & 0.031 \\
\hline 107 Pd.wo & $I C P / M S$ & Acid & $\mathrm{Ci} / \mathrm{L}$ & $4.41 E-08$ & 0.057 & 0.059 & 0.068 & 0.080 & 0.094 \\
\hline $\mathrm{Pr}$ & ICP/NS & $\mathrm{FS} . \mathrm{Zr}$ & $g / L$ & $3.26 \mathrm{E}-03$ & 0.039 & 0.052 & 0.073 & 0.095 & 0.116 \\
\hline $\mathrm{Pt}$ & ICP/MS & FS.Ni & $g / L$ & $1.19 \mathrm{E}-03$ & 1.851 & 2.228 & 3.022 & 3.865 & 4.721 \\
\hline $238_{\mathrm{Pu}}$ & Rad & FS.Ni & $\mathrm{Ci} / \mathrm{L}$ & $2.45 E-05$ & 0.290 & 0.386 & 0.548 & 0.711 & 0.873 \\
\hline $239 \mathrm{Pu}$ & ICP/MS & FS.2r & $\mathrm{Ci} / \mathrm{L}$ & $5.91 E-05$ & 0.081 & 0.108 & 0.153 & 0.199 & 0.244 \\
\hline${ }^{240} \mathrm{Pu}$ & ICP/MS & Acid. & $\mathrm{Ci} / \mathrm{L}$ & $4.85 E-06$ & 0.025 & 0.033 & 0.046 & 0.060 & 0.073 \\
\hline $241 \mathrm{Pu}$ & Rad & FS.Ni & $\mathrm{ci} / \mathrm{L}$ & $2.70 \mathrm{E}-04$ & 0.051 & 0.058 & 0.096 & 0.125 & 0.153 \\
\hline $\mathrm{Rb}$ & ICP/MS & $\mathrm{F} 5.2 \mathrm{r}$ & $g / L$ & $2.98 E-04$ & 0.008 & 0.009 & 0.013 & 0.016 & 0.020 \\
\hline
\end{tabular}

a = "most reasonable" analyte as described in Urie (1997).

$\&=g / L$ or $C i / L$ is $g / L$ (at 31 gram haste oxide) or $[i / L$ (at 31 gram waste cxide).

$S$ = If more than one radionuclide is listed, then the LL is compared to the specification for each radionuclide. 
Table 6.3. Tank 241-C-106 Vendor Product

Envelope "D" Specification Analytes - Total Variability. (page 3 of 4)

\begin{tabular}{|c|c|c|c|c|c|c|c|c|c|}
\hline Analyte a & Method & Digest & Units \& & $\hat{\mu}$ & $\begin{array}{l}\text { UL /EL \$ } \\
\text { SYS }=10 \%\end{array}$ & $\begin{array}{l}U L / E L S \\
S Y S=25 \% \\
\end{array}$ & $\begin{array}{l}\text { UL/EL S } \\
\text { SYS }=50 \%\end{array}$ & $\begin{array}{l}\text { UL/EL } 5 \\
\text { SYS }=75 \%\end{array}$ & $\begin{array}{l}\text { UL/EL } \$ \\
\text { SYS }=900 \%\end{array}$ \\
\hline $\mathrm{Re}$ & $I C P / M S$ & FS.Ni & $g / h$ & $2.57 E-04$ & 0.013 & 0.016 & 0.022 & 0.028 & 0.034 \\
\hline$R h$ & ICP/MS & FS.Zr & $g / 6$ & $5.60 E-03$ & 0.186 & 0.244 & 0.345 & 0.447 & 0.549 \\
\hline Ru & ICP/MS & FS.Ni & $g / L$ & $3.71 \mathrm{E}-03$ & 0.044 & 0.059 & 0.083 & 0.108 & 0.132 \\
\hline Sb.W & ICP/MS & Acid & $9 / \mathrm{L}$ & $1.46 E-05$ & 0.000 & 0.000 & 0.000 & 0.000 & 0.000 \\
\hline Sb.wo & ICP/MS & Acid & $\mathrm{g} / \mathrm{L}$ & $3.06 E-05$ & 0.000 & 0.000 & 0.001 & 0.001 & 0.001 \\
\hline $\mathrm{si}$ & ICP/AES & FS.Ni & $\mathrm{g} / \mathrm{L}$ & $2.67 \mathrm{E}+00$ & 0.605 & 0.801 & 1.136 & 1.471 & 1.807 \\
\hline${ }^{151} \mathrm{sm} /{ }^{147} \mathrm{Pm}$ & $\mathrm{Rad}$ & $\mathrm{FS.Ni}$ & $\mathrm{Ci} / \mathrm{L}$ & $3.58 E-03$ & 0.050 & 0.067 & 0.095 & 0.123 & 0.151 \\
\hline${ }^{151_{5 \mathrm{~m} /}}{ }^{147_{\mathrm{Pm}}}$ & Rad & FS.Ni & $\mathrm{Ci} / \mathrm{L}$ & $3.58 \mathrm{E}-03$ & 0.029 & 0.039 & 0.055 & 0.071 & 0.088 \\
\hline $\mathrm{s} \pi$ & ICP/MS & f5.2r & $g / L$ & $3.96 E \cdot 03$ & 0.556 & 0.671 & 0.911 & 1.166 & 1.425 \\
\hline $121 \mathrm{~m}_{\mathrm{Sn}}$ & Rad & FS.Ni & $\mathrm{Ci} / \mathrm{L}$ & $4.77 \mathrm{E}-05$ & 9.45 & 10.76 & 13.94 & 17.53 & 21.25 \\
\hline 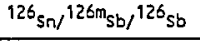 & Rad & FS.Ni & $\mathrm{Ci} / \mathrm{L}$ & $4.48 E-07$ & 0.014 & 0.017 & 0.024 & 0.030 & 0.037 \\
\hline${ }^{126} \mathrm{sn} /{ }^{126 m_{\mathrm{sb}} /^{126} \mathrm{sb}}$ & $\operatorname{Rad}$ & FS.Ni & $\mathrm{Ci} / \mathrm{L}$ & $4.48 \varepsilon-07$ & 0.020 & 0.024 & 0.033 & 0.042 & 0.052 \\
\hline 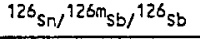 & Rad & FS.Ni & $\mathrm{Ci} / \mathrm{L}$ & $4.48 E \cdot 07$ & 0.143 & 0.172 & 0.234 & 0.300 & 0.367 \\
\hline Sr & $I C P / M S$ & FS.Zr & $g / L$ & $5.8 \div E-03$ & 0.048 & 0.063 & 0.090 & 0.116 & 0.143 \\
\hline${ }^{80^{2 r} / 1} 0^{\lambda}$ & Rad & FS.Ni & $\mathrm{Ci} / \mathrm{L}$ & $2.83 \equiv-02$ & 0.012 & 0.016 & 0.023 & 0.029 & 0.036 \\
\hline${ }_{20}{ }^{2 t}, 10^{4}$ & Rad & FS.Ni & $\mathrm{Ci} / \mathrm{L}$ & $2.83 \equiv-02$ & 0.012 & 0.016 & 0.023 & 0.029 & 0.036 \\
\hline 110 & Wichen & Direct & $g / L$ & $2.05 E-01$ & 0.146 & 0.192 & 0.273 & 0.353 & 0.434 \\
\hline 100 & Wtchen & Direct & $g / L$ & $1.86 E+00$ & 0.716 & 0.950 & 1.348 & 1.747 & 2.146 \\
\hline $\mathrm{Ta}$ & ICP/MS & $\mathrm{Fs} .2 \mathrm{r}$ & $g / L$ & $1.11 E-03$ & 0.239 & 0.276 & 0.361 & 0.456 & 0.554 \\
\hline Ib & ICP/MS & FS.Ni & $g / 6$ & $3.18 E-04$ & 0.453 & 0.570 & 0.793 & 1.022 & 1.252 \\
\hline${ }^{99}$ IC & ICP/MS & Acid & $\mathrm{CI} / \mathrm{L}$ & $2.18 E-07$ & 0.000 & 0.000 & 0.000 & 0.000 & 0.000 \\
\hline Te & ICP/MS & FS.Ni & $9 / L$ & $1.65 \mathrm{E}-02$ & 0.573 & 0.731 & 1.022 & 1.319 & 1.618 \\
\hline Th & ICP/AES & Acid & $5 / 6$ & $1.35 \mathrm{E}-02$ & 0.109 & 0.146 & 0.208 & 0.270 & 0.332 \\
\hline$I i$ & $I C P / M S$ & $\mathrm{Fs.2r}$ & $\mathrm{g} / \mathrm{L}$ & $5.10 \mathrm{E} \cdot 02$ & 0.173 & 0.224 & 0.315 & 0.408 & 0.501 \\
\hline Tก.H & ICP/MS & FS.Ni & $g / L$ & $7.06 \mathrm{E}-05$ & 0.155 & 0.167 & 0.202 & 0.245 & 0.292 \\
\hline Tm.wo & $I C P / M S$ & FS.Ni & $g / L$ & $9.78 E-05$ & 0.205 & 0.223 & 0.274 & 0.335 & 0.401 \\
\hline Total U & ICP/MS & FS.Ni & $9 / L$ & $7.36 E-02$ & 0.023 & 0.030 & 0.043 & 0.056 & 0.069 \\
\hline${ }^{233} \mathrm{u}$ & ICP/MS & Acid & $\mathrm{Ci} / \mathrm{L}$ & $6.52 E-08$ & 94.26 & 117.59 & 162.84 & 209.59 & 256.75 \\
\hline $234 \mathrm{u}$ & $I C P / M S$ & Acid & $\mathrm{Ci} / \mathrm{L}$ & $3.00 \mathrm{E}-08$ & 0.053 & 0.069 & 0.096 & 0.125 & 0.153 \\
\hline $235 \mathrm{u}$ & ICP/MS & Acid & $\mathrm{Ci} / \mathrm{L}$ & $1.09 \mathrm{E}-09$ & 0.044 & 0.059 & 0.084 & 0.109 & 0.133 \\
\hline${ }^{236} \mathrm{u}$ & ICP/MS & Acid & $\mathrm{Ci} / \mathrm{L}$ & $7.17 \mathrm{E}-10$ & 0.012 & 0.015 & 0.022 & 0.028 & 0.036 \\
\hline $23 \varepsilon_{\mathrm{U}}$ & ICP/MS & $\mathrm{Fs} .2 \mathrm{r}$ & $\mathrm{Ci} / \mathrm{L}$ & $2.61 \mathrm{E}-08$ & 0.058 & 0.078 & 0.111 & 0.143 & 0.176 \\
\hline
\end{tabular}

a = "most reasenable" analyte as deseribed in Urie (1997).

$\&=g / L$ or $C i / L$ is $g / L$ (at 31 gran heste oxide) or Ci/L (at 31 gram waste oxide).

$\$=$ If more then one redionuclide is listed, then the Ut is compared to the specification for each radionuclide. 
HNF-SD-WM-DP-225, REV. 1

Table 6.3. Tank 241-C-105 Vendor Product

Envelope "D" Specification Analytes - Total Variability. (page 4 of 4)

\begin{tabular}{|c|c|c|c|c|c|c|c|c|c|}
\hline Analyte a & Me thod & Digest & Units \& & $\dot{\mu}$ & $\begin{array}{l}\text { UL/EL } \$ \\
\text { SYS=10\% }\end{array}$ & $\begin{array}{l}\text { UL/EL } \$ \\
\text { SYS }=25 \%\end{array}$ & $\begin{array}{l}\text { UL/EL } \$ \\
\text { SYS=50\% }\end{array}$ & $\begin{array}{l}U L / E L \$ \\
S Y S=75 \%\end{array}$ & $\begin{array}{l}\text { UL/EL } \$ \\
S Y S=100 \%\end{array}$ \\
\hline$v$ & ICP/MS & Acid & $g / L$ & $1.45 \mathrm{E}-03$ & 0.200 & 0.256 & 0.359 & 0.464 & 0.569 \\
\hline$H$ & ICP/MS & FS.Ni & $g / L$ & $1.94 E-02$ & 0.351 & 0.458 & 0.646 & 0.836 & 1.026 \\
\hline$Y b$ & ICP/MS & FS.Ki & $g / L$ & $4.89 E-04$ & 0.928 & 1.036 & 1.314 & 1.637 & 1.975 \\
\hline $2 n$ & ICP/MS & FS.Hi & $g / L$ & $1.19 E-02$ & 0.128 & 0.163 & 0.227 & 0.294 & 0.360 \\
\hline $2 r$ & ICP/AES & FS.Ni & $g / L$ & $4.29 E-02$ & 0.015 & 0.018 & 0.024 & 0.030 & 0.037 \\
\hline
\end{tabular}

$a=$ "most reasonable" analyte as described in Urie (1997).

$\&=\mathrm{g} / \mathrm{L}$ or $\mathrm{Ci} / \mathrm{L}$ is $\mathrm{g} / \mathrm{L}$ (at $31 \mathrm{gram}$ waste oxide) or $\mathrm{Ci} / \mathrm{L}$ (at 31 gram waste oxide).

$S=$ If more than one radionuclide is listed, then the UL is compared to the specification for each radionuclide. 
HNF-SD-WM-DP-225, REV. 1

Table 6.4. Tank 241-C-106 Vendor Product - Ratio $(U L / E L)>1$.

\begin{tabular}{|c|c|c|}
\hline Analyte & $\begin{array}{l}\text { Systematic Variability } \\
\text { Estimatee at which the } \\
\text { Ratio (UL/EL) }>1\end{array}$ & $\begin{array}{c}\text { Observed } \\
\text { Systematic Variability }\end{array}$ \\
\hline $\mathrm{Na}$ & $0 \%$ & $6 \%-s d$ \\
\hline $\mathrm{Pt}$ & $0 \%$ & $42 \%-r s$ \\
\hline${ }^{121 m} 5 n$ & $0 \%$ & $30 \%-c t$ \\
\hline${ }^{233} \mathrm{U}$ & $0 \%$ & NA \\
\hline $243 / 244 \mathrm{Cm}$ & $0 \%\left({ }^{243} \mathrm{~cm} \mathrm{EL}\right)$ & $7 \%-\left({ }^{241} \mathrm{Am}\right.$ sp $)$ \\
\hline $\mathrm{Ag}$ & between $0 \% \& \quad 10 \%$ & $11.5 \%-s p$ \\
\hline Al & between $25 \% \& \quad 50 \%$ & $<10 \%-5 d$ \\
\hline $\mathrm{Er}$ & between $25 \%$ \& $50 \%$ & $1 \%-r s$ \\
\hline $\mathrm{Fe}$ & between $10 \%$ \& $25 \%$ & $0.6 \%-s p$ \\
\hline Gd & between $50 \% \& \quad 75 \%$ & $8 \%-r s$ \\
\hline $\mathrm{Hg}$ & between $25 \%$ \& $50 \%$ & $<25 \% .-s p$ \\
\hline $\mathrm{Pb}$ & between $50 \% \& \quad 75 \%$ & $<25 \%-s p$ \\
\hline Si & between $25 \% \& \quad 50 \%$ & $<25 \%-s p$ \\
\hline Sn & between $50 \% \& \quad 75 \%$ & $<25 \%-s p$ \\
\hline TOC & between $25 \%$ \& $75 \%$ & $<25 \%-s p$ \\
\hline Tb & between $50 \%$ \& $75 \%$ & $<25 \%-s p$ \\
\hline $\mathrm{Te}$ & between $25 \%$ \& $50 \%$ & $<25 \%-s p$ \\
\hline$W$ & between $75 \% \& 100 \%$ & $<25 \%-$ sp $\$$ \\
\hline$\overline{Y b}$ & between $10 \% \& 25 \%$ & $<25 \%-\operatorname{sp~} \$$ \\
\hline
\end{tabular}

$s p=$ systematic variapijity estimated from spike analyses

sd = systematic varjapijty estimated from serial dilutions.

$r s=$ systematic variapijity estimated from reference standards.

$c t=$ systematic variability estimated from counting statistics:

= spike analysis is within the specified limits, but the control standard was outside the control 1 imits. 


\section{DISTRIBUTION SHEET}

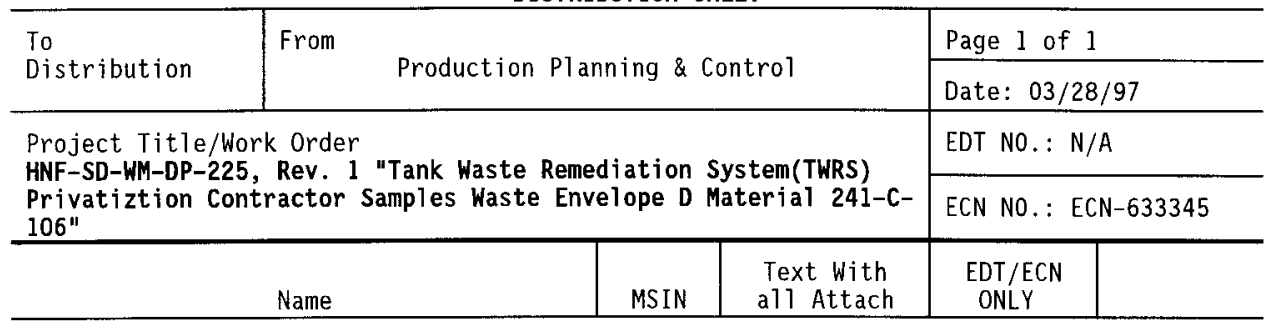

B\&W Protec. Inc.

T. L. Welsh

T4-40 X

U.S. Department of Energy, RL

N. R. Brown

K6-51 X

Lockheed Martin Hanford Corp.

J. N. Appel

G3-21

R2-12

G3-2]

J. G. Berry

G3-21

K. A. Gasper

R2-12

K. M. Hall

A. E. Young

R1-08

$X$

A3-88 $X$

Lockheed Martin Services, Inc.

Central Files

Los Alamos Technical Associates

M. T. Ellsworth

T6-06

$X^{*}$

Pacific Northwest National Laboratory

K. D. Wiemers

$K 6-51 \quad 2$

Rust Federal Services of Hanford. Inc.

R. A. Esch

C. M. Seide1

$\begin{array}{ll}\text { T6-06 } & X \\ \text { T6-04 } & X\end{array}$

Numatec Hanford Corp.

J. Y. Bourges

B. A. Crawford

T6-07

T6-50

T6-50

$x$

$x$

$x$

$x$

W. I. Winters

$x$

$x$

* Needs only releasing paperwork, not a copy of the released document. 